\title{
ANÁLISIS DE SOPORTES MIXTOS SOMETIDOS A FLEXOCOMPRESIÓN ESVIADA
}

Ernesto Jesús Fenollosa Forner 


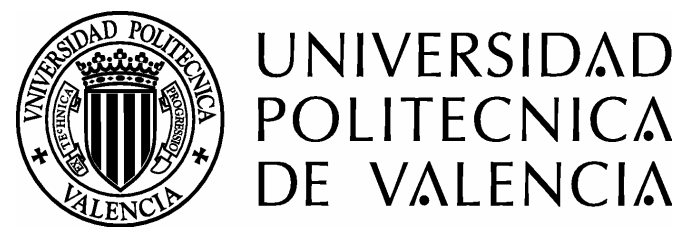
DEPARTAMENTO DE MECÁNICA DE LOS MEDIOS CONTINUOS
Y TEORÍA DE ESTRUCTURAS

\title{
ANALISIS DE SOPORTES MIXTOS SOMETIDOS A FLEXOCOMPRESION ESVIADA
}

\section{TESIS DOCTORAL}

\author{
Presentada por: Ernesto Fenollosa Forner \\ Dirigida por: José Monfort Lleonart \\ Enrique Gil Benso \\ Adolfo Alonso Durá
}




\section{Esta editorial es miembro de la UNE, lo que garantiza la difusión y comercialización de sus publicaciones a nivel

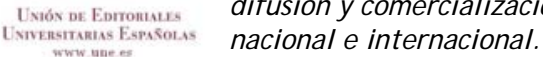

\section{(c) Ernesto J esús Fenollosa Forner, 2011}

Primera edición, 2011

(c) de la presente edición:

Editorial Universitat Politècnica de València

www.editorial.upv.es

ISBN: $-978-84-694-9393-9$

Ref. editorial: 5508

Queda prohibida la reproducción, distribución, comercialización, transformación, y en general, cualquier otra forma de explotación, por cualquier procedimiento, de todo o parte de los contenidos de esta obra sin autorización expresa y por escrito de sus autores. 
Quiero dedicar esta Tesis Doctoral:

A Liliana, mi esposa, mi Luz y mi Camino. La persona que más me ayuda, más apoyo me dá. Quien alegra la vida y me la hace feliz. A quien difícilmente podré devolverle el tiempo que le he robado para dedicarme a este trabajo.

A Saul y Aida, mis hijos, que en incontables ocasiones han mostrado su sincero interés por el trabajo de su padre y su preocupación por saber cuándo lo terminará. Ahora ya conocemos la respuesta.

Agradecimientos:

Quisiera dar las gracias a mis directores de tesis por todo lo que me han enseñado y animado. A Pepe Monfort, Enrique Gil y Adolfo Durá, por sus sabios consejos, su permanente optimismo y su cooperación desinteresada.

A mis estimados compañeros de docencia: Arturo Martínez, Iván Cabrera, Begoña Serrano, Ana Almerich y Verónica Llopis, por la ayuda prestada en todo momento, especialmente en los difíciles. $Y$ al resto de compañeros por su interés y apoyo.

$\mathrm{Y}$, como no, mi más sincero agradecimiento a toda mi familia por la dedicación y paciencia que me han mostrado. 
L'essentiel est invisible pour les yeux

Saint-Exupéry "El principito" 


\section{RESUMEN}

\section{SOPORTES MIXTOS SOMETIDOS A FLEXOCOMPRESIÓN ESVIADA}

Esta investigación aborda el estudio de los soportes mixtos formados por un perfil metálico recubierto de hormigón con armaduras, sometidos a flexocompresión esviada, aplicando un procedimiento de cálculo incluido en el denominado "Método General" del Eurocódigo 4

El interés del tema de la presente tesis consiste en el acercamiento a un elemento estructural con un comportamiento de complejo análisis y a la vez de creciente aplicación en las estructuras de edificación, especialmente en los edificios en altura.

La identificación de la multitud de variables que les afecta se ha realizado a partir de una exhaustiva revisión bibliográfica y el planteamiento de las hipótesis y métodos de cálculo se plantea y desarrolla a la vista de la normativa específica.

En cuanto al procedimiento, el trabajo sobre soportes mixtos se inicia con el estudio del comportamiento de su sección, continúa con la barra como elemento aislado, y finaliza con el análisis del conjunto de la estructura.

En la evaluación de la capacidad resistente de la sección se han tenido en cuenta las características de los materiales que los componen mediante la consideración de las relaciones constitutivas del hormigón y del acero. A efectos de barra se ha analizado la influencia de su longitud, de las imperfecciones iniciales o de las excentricidades en la aplicación de la carga. En cuanto a la estructura, se ha estudiado, fundamentalmente, la influencia de la ausencia de arriostramientos. En el cálculo de la barra y de la 
estructura se han tenido en cuenta los efectos de segundo orden tanto geométricos como mecánicos.

Tras el estudio teórico y dada la elevada complejidad de cálculo derivada de la existencia de materiales con distintos comportamientos y de las numerosas variables contempladas, se ha optado por el desarrollo de un programa informático que permite el estudio de la sección mixta sometida a flexocompresión esviada. Este módulo se ha integrado en un programa de análisis no lineal de estructuras.

Se consideran como principales aportaciones del presente trabajo:

- La obtención de los diagramas de interacción de distintas secciones mixtas usuales, sometidas a flexocompresión plana y esviada, que facilitan su dimensionamiento.

- La cuantificación de la influencia de los efectos reológicos del hormigón sobre la capacidad resistente de los soportes mixtos.

- La elaboración de un conjunto de gráficas de soportes mixtos que consideran distintas longitudes de la barra y distintas excentricidades en la aplicación de la carga. A partir de ellas se han obtenidos las correspondientes superficies mecánicas.

- La evaluación del efecto, sobre las deformaciones y solicitaciones, de la traslacionalidad de distintas estructuras con soportes mixtos

Así pues, se ha conseguido el desarrollo de un programa informático de cálculo de soportes mixtos en régimen no lineal que supone una potente herramienta para posteriores investigaciones, a la vez que facilita el diseño y cálculo de este tipo de piezas en estructuras tridimensionales. 
RESUM

\section{SUPORTS MIXTES SOTMESOS A FLEXOCOMPRESSIÓ ESBIAIXADA}

Aquesta investigació aborda l'estudi dels suports mixtes formats per un perfil metàl-lic recobert de formigó amb armadures, sotmesos a flexocompressió esbiaixada, aplicant un procediment de càlcul inclòs a l'anomenat "Mètode General" de l'Eurocodi 4.

L'interès del tema de la present tesi consisteix a l'apropament a un element estructural amb un comportament d'anàlisi complex i, paral-lelament, d'aplicació creixent a les estructures d'edificació, especialment als edificis en alçada.

La identificació de la multitud de variables que els afecta s'ha realitzat a partir d'una exhaustiva revisió bibliogràfica i el plantejament de les hipòtesis i mètodes de càlcul es planteja i desenvolupa a la vita de la normativa específica.

Pel que fa al procediment, el treball sobre suports mixtes s'inicia amb l'estudi del comportament de la seua secció, continua amb la barra com a element aïllat i finalitza amb l'anàlisi del conjunt de l'estructura.

A l'avaluació de la capacitat resistent de la secció s'han tingut en compte les característiques dels materials que els composen mitjançant la consideració de les relacions constitutives del formigó i de l'acer. Pel que fa a la barra s'ha analitzat la influència de la llargària, de les imperfeccions inicials $o$ de les excentricitats en l'aplicació de la càrrega. Pel que fa a l'estructura, s'ha estudiat, fonamentalment, la influència de l'absència d'arrostraments. Al càlcul de la barra i de l'estructura s'han tingut en compte els efectes de segon ordre tant geomètrics com mecànics. 
Després de l'estudi teòric i arran de l'elevada complexitat del càlcul derivada de l'existència de materials amb distints comportaments i de les nombroses variables contemplades, s'ha optat pel desenvolupament d'un programa informàtic que permet l'estudi de la secció mixta sotmesa a flexocompressió esbiaixada. Aquest mòdul s'ha integrat en un programa d'anàlisi no lineal d'estructures.

Es consideren com a principals aportacions del present treball:

- L'obtenció dels diagrames d'interacció de diferents seccions mixtes usuals, sotmeses a flexocompressió plana i esbiaixada, que faciliten el seu dimensionat.

- La quantificació de la influència dels efectes reològics del formigó sobre la capacitat resistent dels suports mixtos.

- L'elaboració d'un conjunt de gràfiques de suports mixtos que consideren diferents llargàries de la barra i distintes excentricitats en l'aplicació de la càrrega. A partir d'elles s'han obtingut les corresponents superfícies mecàniques.

- L'avaluació de l'efecte, sobre les deformacions i sol-licitacions, de la traslacionalitat de diferents estructures amb suports mixtos.

Així doncs, s'ha aconseguit el desenvolupament d'un programa informàtic de càlcul de suports mixtes en règim no lineal que suposa una potent ferramenta per posteriors investigacions, a l'hora que facilita el disseny i càlcul d'aquest tipus de peces en estructures tridimensionals. 
COMPOSITE COLUMNS UNDER AXIAL COMPRESSION AND BIAXIAL BENDING MOMENTS

This research approaches the study of composite columns consisting of metal profiles coated with reinforced concrete, under the effects of axial compression and bi-axial bending moments applying a design procedure included in the "General Method" in Eurocode 4.

This PhD Thesis' main interest is based on the approach to a structural element with both a rather complex analysis of its behavior and an increasing application in building structures, especially in tall buildings.

Identification of numerous variables affecting them has been made from a thorough review in bibliography. Approach of departing assumptions and design methods has been raised and developed following the specific legislation.

In terms of procedure, composite supports analysis began studying its section behavior, continued studying the bar as an isolated element and ended with an analysis of the entire structure.

When evaluating the section's bearing capacity, characteristics of the materials which constitute it have been taking into account by considering the constitutive relations for concrete and steel. Regarding the bar behavior, its length influence has been analyzed in addition to its initial imperfection or eccentricities when applying the loads. As for the whole structure, lack of bracing has been the main topic studied. When designing isolated bars or the whole structure, second order effects, both geometric and mechanical, have been considered. 
After this theoretical study and due to the high computational complexity arising from not only the coexistence of materials with different behaviors but also the numerous variables taken into account, it was decided to develop a software which permits the user studying composite sections under the effects of axial compression and bi-axial bending moments. This module has been integrated into a more general software where nonlinear analysis of structures can be studied.

This work's main contributions are:

- Obtaining the interaction diagrams of usual composite sections under axial compression and bi-axial bending, facilitating their sizing.

- Quantification of the influence of the rheological effects of concrete on the bearing capacity of composite columns.

- Development of a set of graphs for composite columns considering different bar lengths and different eccentricities when applying the load. As a result corresponding mechanical areas have been obtained.

- Evaluation of the effect which translationality (considered on different types of structures designed with composite columns) has on deformations and efforts.

Thus, a new software for designing composite columns under a nonlinear behavior has been developed, turning into a powerful tool for further research and making easier the design of this kind of threedimensional structures. 
ÍNDICE

1. EL ESTADO DEL ARTE ................................................ 19

1.1. LOS ORÍGENES DE LAS ESTRUCTURAS MIXTAS .....21

1.2. EL PROBLEMA DE LA "VIGA-COLUMNA" .................... 34

1.2.1. INTRODUCCION ..............................................................

1.2.2. PARAMETROS QUE INTERVIENEN EN LOS EFECTOS DE SEGUNDO ORDEN ……………………………………....

1.2.3. METODOS DE ANALISIS .....................................................44

1.3. EVOLUCIÓN DE LOS METOdOS DE CÁLCULO DE SOPORTES MIXTOS …........................................ 46

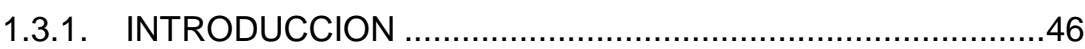

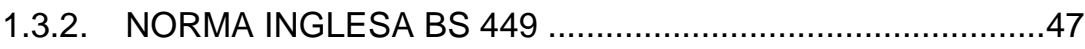

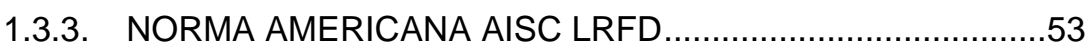

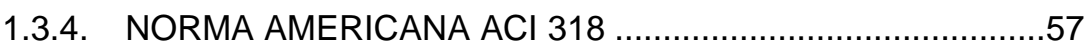

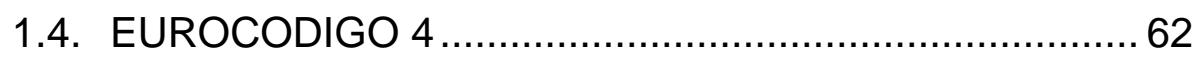

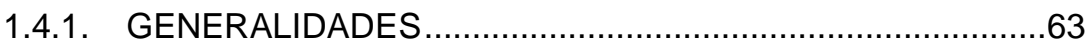

1.4.1.1. CAMPO DE APLICACIÓN ...................................63

1.4.1.2. CLASIFICACIÓN DE SECCIONES MIXTAS...........64

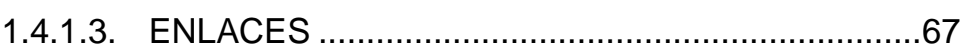

1.4.2. MÉTODO GENERAL .......................................................

1.4.2.1. GENERALIDADES ...........................................72

1.4.2.2. PROCESO DE CÁLCULO …………………….....72

1.4.2.3. IMPERFECCIONES.............................................74

1.4.2.4. PANDEO LOCAL DE ELEMENTOS DE ACERO...74

1.4.2.5. RECUBRIMIENTO Y ARMADURA. .75 
1.4.2.6. RASANTE ENTRE LOS COMPONENTES DE ACERO Y HORMIGON ....................................... 79

1.4.2.7. RESISTENCIA AL ESFUERZO RASANTE ........... 80

1.4.2.8. PERNOS DE CONEXION UNIDOS AL ALMA DE UN PILAR MIXTO ................................................. 81

1.4.3. MÉTODO SIMPLIFICADO DE CÁLCULO ........................... 82

1.4.3.1. CAMPO DE APLICACIÓN ................................... 82

1.4.3.2. COEFICIENTES DE SEGURIDAD PARCIALES ... 85

1.4.3.3. RESISTENCIA PLÁSTICA A COMPRESIÓN DE LA

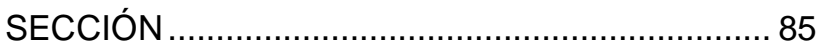

1.4.3.4. FACTOR DE CONTRIBUCIÓN DEL ACERO........ 87

1.4.3.5. RIGIDEZ A FLEXIÓN ELÁSTICA EFICAZ (A CORTO PLAZO) ................................................... 88

1.4.3.6. LONGITUD DE PANDEO DEL PILAR ................... 90

1.4.3.7. ESBELTEZ RELATIVA Y ADIMENSIONAL........... 90

1.4.3.8. COMPRESIÓN SIMPLE ........................................ 91

1.4.3.9. FLEXOCOMPRESIÓN .......................................... 91

1.4.3.10. RESISTENCIA DE SECCIONES SOMETIDAS A FLEXO-COMPRESIÓN MONOAXIAL ................... 93

1.4.3.11. INFLUENCIA DEL ESFUERZO CORTANTE ........99

1.4.3.12. RESISTENCIA DE ELEMENTOS SOMETIDOS A COMPRESIÓN Y FLEXIÓN MONOAXIAL ........... 100

1.4.3.13. COMPRESIÓN Y FLEXIÓN BIAXIAL ................... 103

2. OBJETIVOS DEL TRABAJO …..................................107

2.1. ESTUDIO DE LA SECCION MIXTA ...........................109

2.2. EFECTOS REOLÓGICOS ....................................111

2.3. ANÁLISIS DE LA BARRA .....................................112

2.4. EL CONJUNTO ESTRUCTURAL ..............................115 
3. PLANTEAMIENTO TEÓRICO.................................. 117

3.1. HIPÓTESIS DE CÁLCULO ..................................... 119

3.1.1. SOBRE EL ELEMENTO...............................................119

3.1.2. COMBINACION DE ACCIONES .......................................120

3.1.3. VALORES DE CÁlCULO DE LAS ACCIONES PERMANENTES .............................................................122

3.1.4. COEFICIENTES DE SEGURIDAD ..................................123

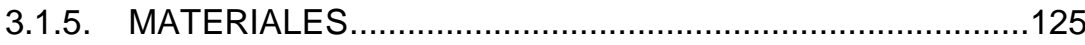

3.1.6. RESISTENCIA AL PANDEO LATERAL............................132

3.1.7. INTRODUCCIÓN DE CARGAS .........................................132

3.1.8. RESISTENCIA A CORTANTE .......................................133

4. ANÁLISIS DE LA SECCIÓN MIXTA ............................ 135

4.1. INTRODUCCIÓN. ................................................... 137

4.2. HIPÓTESIS COMPRENDIDAS EN EL PROGRAMA DE CÁLCULO..................................................... 138

4.3. PLANTEAMIENTO CONCEPTUAL DEL PROGRAMA. 139

4.4. ENTRADA DE DATOS. ..................................... 143

4.4.1. DATOS DE LA SECCIÓN ................................................143

4.4.2. CARACTERÍSTICAS DE LOS MATERIALES .....................146

4.4.3. OPCIONES DE CÁLCULO ..............................................148

4.5. ANÁLISIS DE LA SECCIÓN .................................. 149

4.5.1. COMPROBACIÓN DE LA SECCIÓN ................................153 
4.6. RESULTADOS: TABLAS DE DIMENSIONAMIENTO DE SECCIONES MIXTAS.

4.6.1. ANÁLISIS COMPARATIVO DIAGRAMAS INTERACCIÓN 155

4.6.2. TABLAS DE DIMENSIONAMIENTO EN FLEXOCOMPRESIÓN RECTA ........................................... 161

4.6.3. FLEXOCOMPRESIÓN ESVIADA....................................... 165

4.6.4. TABLAS DE DIMENSIONAMIENTO EN FLEXOCOMPRESIÓN ESVIADA...................................... 169

5. EFECTOS REOLÓGICOS .........................................177

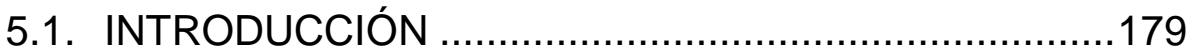

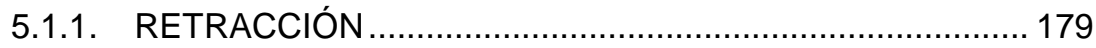

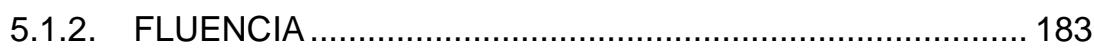

5.2. CRITERIO DE LA EHE-08 ...................................... 190

5.3. CRITERIO DE LOS EUROCÓDIGOS ........................192

5.4. EVALUACIÓN DE LOS EFECTOS REOLÓGICOS ......195

5.5. VALORES ADOPTADOS ........................................199

5.6. RESULTADOS: ESTUDIO COMPARATIVO DEL EFECTO REOLÓGICO ............................................203

6. ANÁLISIS ESTRUCTURAL........................................207

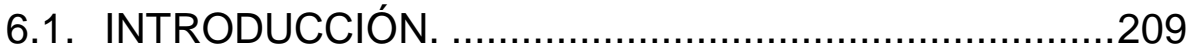

6.2. EL PROGRAMA AV_CID....................................210

6.3. INTERVENCIONES REALIZADAS. .........................212 
7. ANÁLISIS DE LA BARRA ....................................... 221

7.1. INTRODUCCIÓN. RESEÑA HISTORICA ..................... 223

7.2. INESTABILIDAD Y AGOTAMIENTO ......................... 235

7.3. HIPÓTESIS SOBRE LA DEFORMADA DE LA DIRECTRIZ ....................................................... 240

7.4. DISTINTOS MÉTODOS DE ANÁLISIS .......................247

7.4.1. MÉTODOS DE INTEGRACIÓN NUMÉRICA......................248

7.4.2. MÉTODOS BASADOS EN LAS CURVAS DE

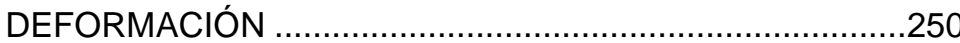

7.4.3. MÉTODOS BASADOS EN LAS FUNCIONES DE

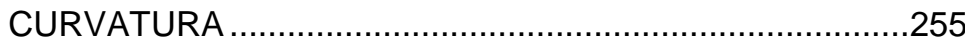

7.4.4. MÉTODOS BASADOS EN TÉCNICAS ITERATIVAS .........259

7.5. MÉTODO DE ANÁLISIS ADOPTADO ..........................260

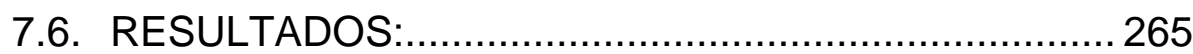

7.6.1. CURVAS DE INTERACCION DE LA BARRA.....................265

7.6.2. EFECTO DE LA NO LINEALIDAD DEL MATERIAL............276

7.6.3. INFLUENCIA DE LOS EFECTOS REOLOGICOS ..............280

8. EL CONJUNTO ESTRUCTURAL ................................... 283

8.1. INTRODUCCIÓN ................................................... 285

8.2. CRITERIO DE LOS EUROCÓDIGOS .......................... 286

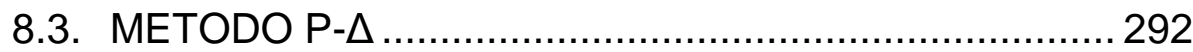


8.4. MÉTODOS DE ANÁLISIS MATRICIAL DE LOS EFECTOS DE SEGUNDO ORDEN. 298

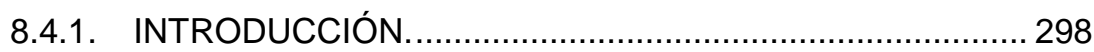

8.4.2. MÉTODOS INCREMENTALES DE PASO ÚNICO .............. 301

8.4.3. MÉTODOS INCREMENTALES ITERATIVOS .................... 304

8.5. MÉTODO ADOPTADO EN ESTE TRABAJO .................308

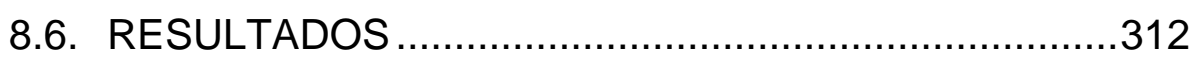

8.6.1. ANÁLISIS COMPARATIVO DE LOS EFECTOS DE SEGUNDO ORDEN …………………………………........ 312

8.6.2. DIMENSIONAMIENTO DE SOPORTES MIXTOS ............... 329

9. CONCLUSIONES …....................................................33

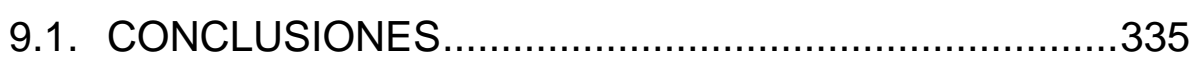

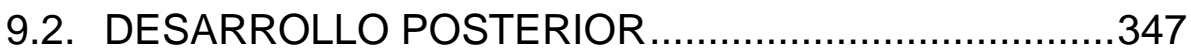

10. ANEXO 1 CARACTERISTICAS AV_CID .....................349

11. ANEXO 2 LISTADO PROGRAMA SOPORTES MIXTOS377

12. REFERENCIAS BIBLIOGRÁFICAS ............................413 
1. EL ESTADO DEL ARTE 


\subsection{LOS ORÍGENES DE LAS ESTRUCTURAS MIXTAS}

\section{$\underline{\text { La estructura metálica }}$}

Aunque el origen de los primitivos altos hornos $\mathrm{y}$, por tanto, la fabricación de los primeros elementos estructurales de hierro, fundición o forja, datan de mediados del siglo XVII, el material fue inicialmente demasiado valioso para su uso en la industria de la construcción. Entre las primeras construcciones dignas de mención ${ }^{1}$, realizadas con elementos de fundición, cabe citar la cúpula sobre el patio circular de alrededor de 36,50 metros de diámetro, del Hall aux Blés en Paris, fechada en 1809, del arquitecto François-Joseph Bélanger. En la década de 1830 se desarrollan las vigas con sección en doble $\mathrm{T}$ y en la de los 40 se realizan las primeras barras laminadas, todavía de hierro.

Aunque el uso soportes y vigas de fundición o forja comenzaba a extenderse, los edificios seguían conservando sus pesadas fachadas de piedra, para, entre otros motivos, proporcionar estabilidad y rigidez estructural. Se atribuye al Hungerford Fish Market de Londres $^{2}$ (1831-1833) proyectado por Charles Fowler, ser el primer edificio realizado con pórticos de nudos rígidos entre soportes y vigas.

Ya de la segunda mitad del siglo, y también situada en Londres, es el Palacio de Cristal de Joseph Paxton, realizado para la primera exposición universal de 1851. Su estructura está formada por vigas en dos direcciones unidas rígidamente a los soportes. La estabilidad lateral se conseguía mediante la propia rigidez de los pórticos en dos

\footnotetext{
1 "Technics and architecture" Cecil D. Elliot. (1992)

2 "Building: 3000 years of design engineering and construction". Bill Addis (2007)
} 
direcciones y con la disposición de arriostramientos mediante cables en diagonal.

La invención del convertidor Bessemer en 1855 y el desarrollo y perfeccionamiento, en los años siguientes, del proceso que lleva el mismo nombre, permitió el rápido crecimiento del suministro de acero. Su uso en la construcción tuvo su primera aplicación importante en la realización de puentes, como por ejemplo el Glasgow Bridge sobre el rio Missuri, iniciado en 1878, con cinco vanos de 95,70 m. entre apoyos, realizado con celosías Whipple. En edificación ${ }^{3}$ cabe citar el Home Insurance Building en Chicago, proyectado por William LeBaron Jenny. Su construcción se inició en 1884 con vigas de hierro forjado en las primeras seis plantas y finalizado con vigas de acero Bessemer. Por último citar la Galeria de las Máquinas, proyectada por el ingeniero Victor Contamin y el arquitecto Ferdinand Dutert. Construida para la exposición universal de Paris de 1889, sus arcos triarticulados de acero cubrían una luz de $114 \mathrm{~m}$.

Así, a finales del siglo XIX, las herramientas y materiales para la construcción de estructuras de acero se habían generalizado.

\section{Las estructuras de hormigón armado}

Respecto de las estructuras de hormigón, un hecho significativo es la invención en Inglaterra del cemento Portland en 1824 por el constructor Joseph Aspdin. En 1871 David Saylor obtiene la patente para la fabricación del cemento Portland en Estados Unidos ${ }^{4}$. La construcción de hornos propicia, a finales de la década de 1880, la

3 "Building: 3000 years of design engineering and construction". Bill Addis (2007)

4 "Composite construction design for buildings". Ivan M. Viest, Joseph P. Colaco, Richard W. Furlong, Lawrence G. Griffis, Roberto T. Leon, Loring A. Wyllie, Jr. (1997) 
producción en masa del cemento en EEUU, con unos 20 años de retraso respecto de Europa.

Hacia finales de los años 30, el material obtenido a partir de cemento, arena, grava y agua comenzó a denominarse "hormigón". Al principio fue utilizado como hormigón en masa, fundamentalmente en cimentaciones combinado con la piedra hasta que terminó por sustituirla. El primero en comprender la naturaleza del hormigón fue W. Wilkinson, quien, en 1854, patentó su sistema de forjados resistentes al fuego, que se muestra en la Figura 1.1-1, consistentes en vigas de hormigón reforzadas con barras de hierro, situadas en la parte inferior en la zona central del vano y que se elevan en los extremos ${ }^{5}$.

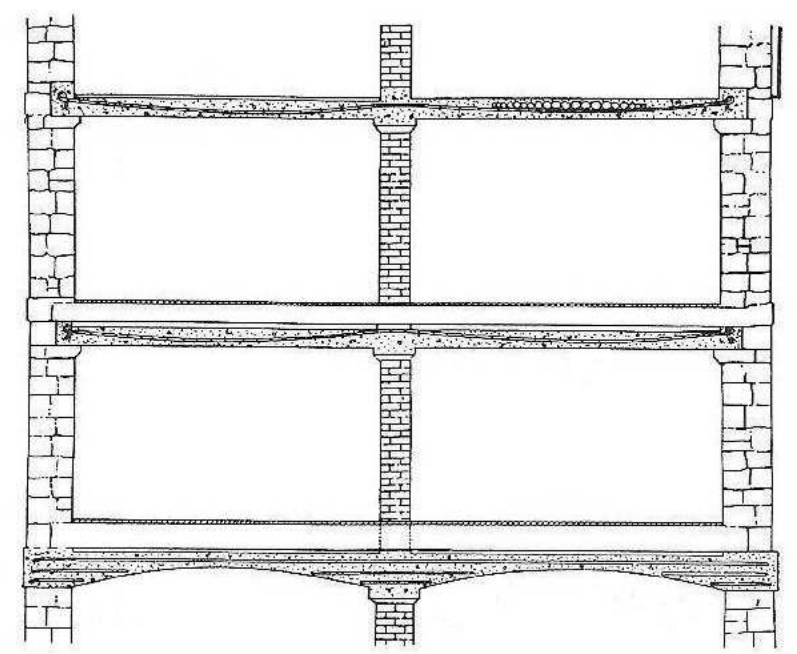

Figura 1.1-1 Sistema de viga y forjado patentado por Wilkinson

En 1867, el francés Joseph Monier patenta su sistema de hormigón reforzado $^{6}$, que se utilizará, años después, en la construcción de depósitos de agua. En 1878 obtiene una patente sobre vigas de

5 "Building: 3000 years of design engineering and construction". Bill Addis (2007)

6 "Technics and architecture". Elliot, C.D. (1992) 
hormigón reforzadas con barras de hierro que absorben las fuerzas de tracción producidas tanto por la flexión como por el cortante.

En 1886, Matthias Koenen publica en una revista de construcción alemana el primer análisis fundamental sobre el comportamiento estructural del hormigón armado.

A finales de la década de 1880, tanto el alemán Gustav Wayss como el francés François Hennebique, conciben la idea de un sistema estructural completo realizado en hormigón armado: cimentaciones, soportes, vigas y forjados. Este hecho, junto con el desarrollo científico, propicia que la construcción en hormigón armado vaya progresivamente desplazando a la madera y la mampostería en la construcción de edificios.

Como primeras construcciones de interés ${ }^{7}$ cabe citar el edificio Ingalls, en Cincinnati, Ohio, proyectado por los arquitectos Elzner y Anderson. Se trata de un edificio de 16 plantas, construido entre 1902-03 con una estructura consistente en pórticos y losas de hormigón armado. Tambien en Europa, en 1903, August Perret levantaba un edificio de apartamentos en la calle Franklin de Paris con un sistema estructural de similares características.

\section{Las estructuras mixtas}

Casi tan pronto como los dos materiales, acero y hormigón, estuvieron disponibles de forma generalizada para su uso por los ingenieros como sistemas estructurales, surgieron las primeras estructuras mixtas combinando ambos materiales, como a continuación se expondrá.

Si bien desde primeros del siglo $\mathrm{XX}$, las estructuras de hormigón armado predominan como sistemas sometidos a flexión (vigas y

7 “Technics and architecture”. Elliot, C.D. (1992) 
losas) en edificios de luces moderadas debido a que el hormigón combina una alta capacidad resistente, reducido coste y aceptable resistencia a la corrosión, a la abrasión y al fuego, cuando las luces superan los $10 \mathrm{~m}$, las vigas métalicas comienzan a ser competitivas. Cuando la estructura metálica requirió cumplir exigencias de protección frente al fuego, los perfiles comenzaron a ser recubiertos de hormigón. Probablemente sea este el motivo que propició la aparición de las primeras estructuras mixtas ${ }^{8}$.

Es, de hecho, un incendio quien publicita una de las bondades de este sistema. En 1897, el Methodist Building de Pittsburgh padeció un incendio que consumió todo su contenido. Su estructura, realizada en 1894 mediante perfiles metálicos recubiertos de hormigón, no sufrió demasiados desperfectos ${ }^{9}$. En los siguientes años, varias empresas presentaron sus patentes de estructuras horizontales resistentes al fuego.

En la construcción de puentes es significativa la propuesta realizada en 1894 por el ingeniero austriaco Josef Melan, consistente en una serie de perfiles de acero tipo $\mathrm{H}$, curvados en forma de arco embebidos en hormigón. La serie de cálculos presentada por Melan para mostrar que hormigón y acero actuaban conjuntamente, pueden considerarse de los primeros cálculos de estructuras mixtas.

Entre los primeros edificios con soportes mixtos (columnas de acero embebidas en hormigón) se pueden citar los almacenes Druecker, construidos en Chicago en 1898.

\footnotetext{
8 "Composite structures of steel and concrete. Volume 1: Beams, columns, frames and applications in building". Johnson, R.P. 3르. ed. (2004)

9 "Composite construction design for buildings". Ivan M. Viest, Joseph P. Colaco, Richard W. Furlong, Lawrence G. Griffis, Roberto T. Leon, Loring A. Wyllie, Jr. (1997)
} 
Pioneros de la tecnología del hormigón armado como Emperger, Burr, Withey, Talbot y Lord o Mench utilizaron hormigón reforzado con tubos y perfiles, tanto de acero como de hierro fundido.

Inicialmente, el hormigón utilizado como recubrimiento era de baja resistencia y era habitual dimensionar las vigas y los soportes para que toda la carga fuera soportada por el elemento metálico, sin contar con el incremento de capacidad resistente aportado por el hormigón. Si bien en las vigas este incremento era pequeño, el tiempo demostró que en el caso de los soportes el incremento en resistencia y rigidez aportado por el hormigón sería importante.

Los primeros ensayos sobre soportes mixtos fueron realizados por Burr en $1912^{10}$. Se elaboran las primeras reglas de cálculo de soportes mixtos que se incluyen en las normativas de hormigón armado. Estas normas vienen caracterizadas por permitir tensiones muy bajas en el hormigón, ser muy conservadoras respecto a la estabilidad del soporte y no contemplar el caso de la flexión.

Los resultados de los ensayos realizados por Faber ${ }^{11}$ (1956), Stevens (1959 y 1965) y Jones y Rizk ${ }^{12}$ (1963) demostraron la importancia del incremento de la capacidad resistente aportada por el hormigón. Si en lugar de utilizar como recubrimiento de los perfiles un hormigón de mala calidad, se utilizaba un hormigón de mayor resistencia y su contribución se tenía en cuenta en el cálculo del soporte, se podía conseguir un ahorro en el dimensionamiento.

De estos primeros estudios se observó que la capacidad resistente de un soporte mixto corto se podía obtener mediante la suma de la

\footnotetext{
10 "Composite columns of concrete and steel" Burr, WH. (1912)

11 "Savings to be effected by the more rational design of cased stanchions as a result of recent full size tests" Faber, O.; Mech, M.I. (1956)

12 "An investigation on the behaviour of encased steel columns under load" Royston Jones y A.A. Rizk. (1963)
} 
resistencia de cada uno de sus materiales. Asimismo, se comprobó que la resistencia del soporte se reducía conforme aumentaba la esbeltez.

Se establece la clasificación de soportes mixtos que se mantiene en la actualidad: perfiles metálicos, generalmente en forma de $\mathrm{H}$, parcial o totalmente embebidos en la sección de hormigón y perfiles huecos, de sección rectangular o circular, rellenos de hormigón ${ }^{13}$.

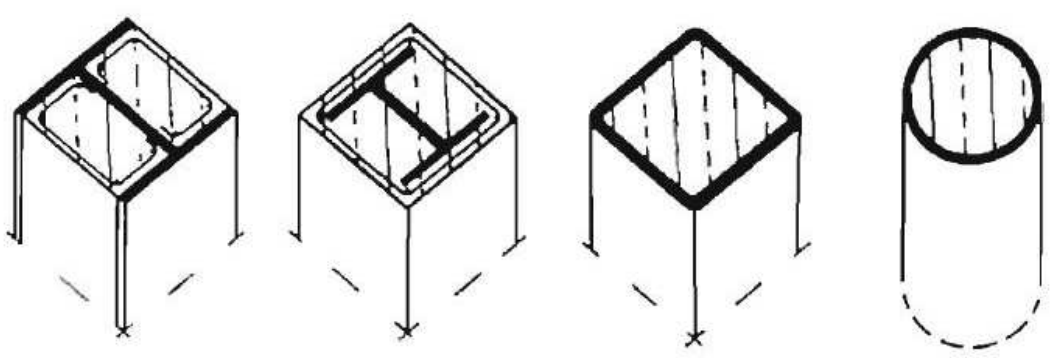

Figura 1.1-2 Tipología de soportes mixtos

Las características, ventajas e inconvenientes de los distintos tipos de soportes mixtos, así como el diseño de distintos nudos rígidos y articulados, según el tipo del soporte y de viga a enlazar se describen en los apartados 1.4.1.2 y 1.4.1.3, respectivamente.

Desde la década de 1970 se suceden numerosos ensayos de los distintos tipos de soportes mixtos en varios países ${ }^{14}$ : Estados Unidos, Canada, Inglaterra, Alemania, Japón,... de los que se obtienen experiencias para la elaboración de las normativas estatales o bien para calibrarlas.

13 "Composite beams and columns to Eurocode 4". European Convention for Constructional Steelwork. Technical committee 11. Composite structures. (1993)

14 "Tesis doctoral: A database for composite columns". Kim, D.K. (2005) 
Entre ellos cabe citar los ensayos de soportes mixtos formados por perfiles embebidos realizados en Canadá por Mirza y Skrabek ${ }^{15,16}$ en 1991 y 1992 para investigar el efecto sobre la resistencia del soporte de distintos parámetros: la resistencia del hormigón y del acero, las dimensiones de la sección tranversal de hormigón y el tamaño del perfil metálico, el refuerzo de la sección con barras de acero, la esbeltez del soporte y la excentricidad de la carga.

Tambien los ensayos de soportes mixtos formados por perfiles rectangulares rellenos de hormigón realizados en Inglaterra por Shakir Khalii ${ }^{17,18}$ en 1989 y 1990 sometidos a tres estados de carga distintos: axil centrado, flexocompresión uniaxial respecto tanto del eje fuerte como del eje débil y flexocompresión esviada.

Las investigaciones mas recientes en soportes mixtos se orientan en las siguientes direcciones ${ }^{19}$ :

- Estudio del efecto del uso de hormigones de alta resistencia.

- El efecto del empleo de conectores mecánicos entre el perfil de acero y el hormigón, en el caso de la presencia de importantes esfuerzos cortantes o la aplicación de cargas dinámicas.

- Comportamiento de los soportes mixtos sometidos a cargas cíclicas y dinámicas.

\footnotetext{
15 "Reliability of short composite beam-column strength interaction" Mirza, SA.; Skrabek BW. (1991)

16 "Statistical analysis of slender composite beam-column strength" Mirza, SA.; Skrabek BW. (1992)

17 "Experimental behaviour of concrete-filled rolled rectangular hollowsection columns" Shakir-Khalil, H.; Zeghiche, J. (1989)

18 "Further tests on concrete-filled rectangular hollow section columns" Shakir-Khalil, H.; Mouli, M. (1990)

19 "State of the art report on steel-concrete composite columns". Shanmugam, N.E., Lakshmi, B. (2001)
} 
- Determinación de la ductilidad de un soporte mixto.

- Determinación del efecto de confinamiento en el hormigón, especialmente presente en el caso de secciones rellenas pero existente también en distintos grados en el hormigón de las secciones embebidas.

Paralelamente los conceptos de viga y forjado mixtos sufren también una transformación. Ya desde los primeros ensayos de vigas mixtas, perfiles metálicos totalmente embebidos en la losa de hormigón, realizados en las primeras décadas del siglo $X X$ se observó una buena interacción entre los dos materiales y que realmente podían actuar de forma conjunta. Como variante de la sección tradicional, se ensayan también varias vigas metálicas únicamente con su ala superior embebida en la losa de hormigón.

Las primeras tablas y métodos de cálculo de vigas mixtas se publican en Inglaterra entre 1910 y 1920. El American Institute of Steel Construction (AISC) incluye en 1936 una nueva sección dedicada a "vigas mixtas" dentro de sus "Especificaciones para el cálculo, fabricación y montaje de acero estructural en edificios"20.

Durante estos primeros años del siglo $X X$ se publican numerosos estudios sobre vigas mixtas todas ellas formadas por perfiles metálicos totalmente embebidos en hormigón sin conectores entre ambos. Hay que esperar hasta 1939 para que se publique un completo estudio que incluye perfiles metálicos total y parcialmente embebidas en el hormigón con algunas de ellas incluyendo conectores de cortante formados por angulares.

20 "Composite construction design for buildings". Ivan M. Viest, Joseph P. Colaco, Richard W. Furlong, Lawrence G. Griffis, Roberto T. Leon, Loring A. Wyllie, Jr. (1997) 
Desde este momento se estudian y patentan diversos tipos de conectores: conectores de corte en espiral, conectores con barras en forma de gancho o lazo, conectores rígidos realizados con barras o perfiles de acero, pernos. El empleo de conectores entre el perfil metálico y la cabeza de hormigón se generaliza, garantizando un trabajo conjunto de los dos materiales. Se establece así el término de "viga mixta" tal como habitualmente se utiliza en la actualidad: sección en forma de $\mathrm{T}$ formada por un perfil inferior, generalmente en forma de $\mathrm{H} \circ \mathrm{I}$, conectado con un bloque superior rectangular de hormigón armado $^{21}$.

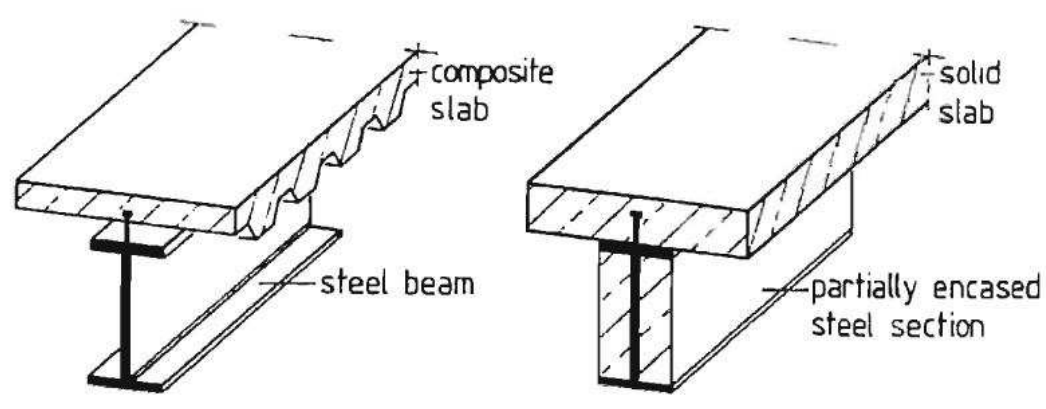

Figura 1.1-3 Ejemplos de vigas mixtas

El forjado mixto, constituido por una chapa de acero conformada en frío y una losa superior de hormigón armado, tampoco contaba inicialmente con la contribución resistente de la chapa, que intervenía únicamente como encofrado perdido. Al igual que en la viga, los conectores evitan el deslizamiento entre los dos materiales y permiten el trabajo conjunto de ambos. Este sistema, utilizado en Estados Unidos desde los primeros años 50, especialmente en el caso de los edificios en altura, comienza a generalizarse en Europa

21 "Composite beams and columns to Eurocode 4". European Convention for Constructional Steelwork. Technical committee 11. Composite structures. (1993) 
en los años 70. En España es frecuente su denominación como "forjado de chapa colaborante".

La Figura 1.1-4 ${ }^{22}$ muestra la mencionada tipología, junto con otras variantes de forjados compuestos, formados por losas de hormigón prefabricado y una capa superior de hormigón in situ.
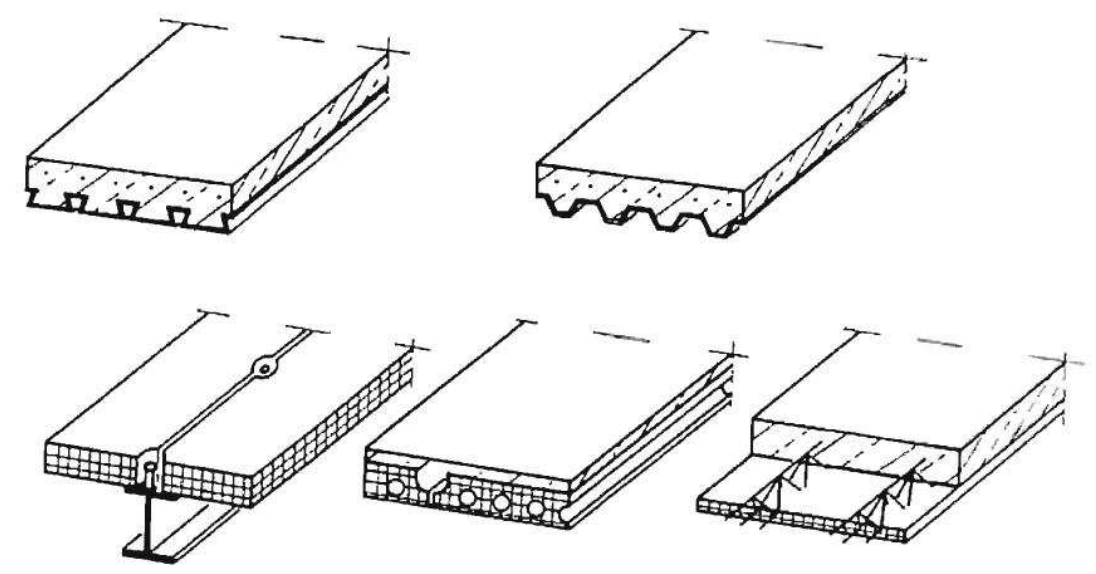

Figura 1.1-4 Distintos tipos de forjados mixtos

Cuando el tiempo de ejecución juega un papel importante en el coste de la edificación, es habitual recurrir a estructuras porticadas metálicas que permiten un rápido ensamblaje de sus elementos, junto a los forjados mixtos cuya chapa colaborante actúa como encofrado perdido ${ }^{23}$.

En el caso de los soportes, aunque la relación entre la capacidad resistente a compresión y el coste es favorable a los soportes hormigón armado en edificios de escasa altura, los soportes mixtos, tanto de perfiles embebidos como de secciones rellenas, empiezan a

22 "Composite beams and columns to Eurocode 4". European Convention for Constructional Steelwork. Technical committee 11. Composite structures. (1993)

${ }^{23}$ "The civil engineering handbook". Chen, W.F. y Richard Liew, J.Y. 
ser competitivos en edificios a partir de 30 plantas y la relación se vuelve favorable a partir de las 50 plantas $^{24}$.

Si además las exigencias de protección contra el fuego requieren un eficaz recubrimiento de los perfiles métálicos, la solución de soportes mixtos puede ser más eficaz y económica que la de hormigón armado, sobre todo si se tiene en cuenta su menor ocupación en planta.

La estructura mixta en su máxima expresión (vigas y soportes mixtos acompañados de forjados con chapa colaborante) tiene su especial campo de aplicación en los edificios en altura, donde se combinan todos los parámetros que les son favorables: elevadas cargas de compresión (especialmente sobre los soportes de las plantas bajas), el empleo de luces medias-grandes, la necesidad de utilizar sistemas estructurales a flexión ligeros con el objeto de minimizar la carga gravitatoria global, requerimiento de protección contra el fuego y la conveniencia del diseño de sistemas constructivos que permitan una rápida ejecución.

Dos ejemplos de esta tipología de edificación ilustran la evolución de las estructuras mixtas:

Por un lado el Empire State Building construido en Nueva York entre 1929 y 1931 con sus 102 plantas y $380 \mathrm{~m}$ de altura fue, durante 40 años, el edificio más alto de Estados Unidos. En el cálculo de su estructura formada por pórticos metálicos embebidos en hormigón no se tuvo en cuenta la capacidad resistente del hormigón, aunque si el incremento de rigidez que le aportaba ${ }^{25}$.

24 "Composite concrete filled steel tube columns". Webb, J. y Peyton, J.J. (1990)

25 "Composite construction design for buildings". Ivan M. Viest, Joseph P. Colaco, Richard W. Furlong, Lawrence G. Griffis, Roberto T. Leon, Loring A. Wyllie, Jr. (1997) 
Por otro lado el Banco de China, proyectado por el arquitecto Ming Pei y construido en Hong Kong entre 1982 y 1990 con 70 plantas y $315 \mathrm{~m}$ de altura fue, en su momento, el edificio más alto construido fuera de Estados Unidos. Su mega-estructura exterior formada por perfiles rellenos y recubiertos de hormigón demuestra el progreso tecnológico conseguido a lo largo del siglo XX.

También la filosofía de cálculo ha ido evolucionando a lo largo del tiempo ${ }^{26}$. Del tradicional "método elástico", donde la "tensión admisible" se obtiene dividiendo la resistencia del material por un coeficiente de seguridad que tiene en cuenta todos los tipos de incertidumbres, se ha pasado a un método que contempla las deformaciones plásticas en el análisis de la sección, sobre todo en el caso del hormigón. Finalmente el método de cálculo en "estados límite" utiliza los denominados valores característicos, a partir de los cuales se obtienen los valores de cálculo mediante la aplicación de los oportunos coeficientes parciales de seguridad. Éstos se aplican donde realmente corresponden, utilizando distintos valores para los distintos materiales y para las cargas, distinguiendo éstas según su naturaleza.

Se distinguen dos tipos de estados límites: Ios denominados Estados Límites Últimos, asociados al colapso total o parcial de la estructura (incapacidad resistente, inestabilidad, etc) y los Estados Límites de Servicio, relacionados con el deterioro del edificio (excesivas deformaciones, fisuración, corrosión, etc.) aunque no implican la imposibilidad de su uso. Los valores de los coeficientes de seguridad utilizados son distintos en cada "estado límite" e identifican los

26 "Composite structures of steel and concrete.Volume 1: Beams, columns, frames and applications in building". Johnson, R.P.,. $3^{a}$ ed. (2004) 
grados de riesgo, incertidumbres, tecnología de los materiales, calidad de ejecución, etc.

La primera normativa que recoge en su articulado este nuevo método de cálculo es el Code of Practice, publicado en Gran Bretaña en 1972.

\subsection{EL PROBLEMA DE LA "VIGA-COLUMNA"}

Es frecuente denominar como "problema de la viga-columna" al análisis de una pieza real sometida a flexocompresión recta 0 esviada. Su estudio exige la consideración conjunta de todos los aspectos que intervienen tanto en la evaluación de la capacidad resistente de la sección como los que afectan a la estabilidad de la barra, tales como los casos de flexión esviada, las condiciones de enlace entre las barras de los entramados, imperfecciones geométricas de barras y pórticos, efectos reológicos en el hormigón y tensiones residuales en el acero,...

Dada la importancia de este tema se ha considerado oportuno dedicar de forma completa el capítulo 7 del presente trabajo, (donde se remite para una mayor profundización en el tema) para realizar una revisión histórica del estudio del problema con el planteamiento de distintas aproximaciones a su resolución, el análisis de los factores mas importantes que intervienen en los efectos de segundo orden, el establecimiento de hipótesis sobre la deformada de la barra y la exposición de varios métodos de resolución del problema no lineal.

No obstante, no se ha querido dejar pasar la ocasión, en esta introducción al análisis de soportes mixtos, de exponer, cuanto menos, el planteamiento general del problema. 


\subsubsection{INTRODUCCION}

Aunque Musschenbroek ${ }^{27}$ ya había realizado años antes los primeros ensayos de compresión sobre columnas esbeltas de madera, fue Euler quien en 1744 plantea de forma teórica el análisis del efecto del pandeo sobre una "barra ideal" bajo las siguientes hipótesis:

- El material del que esta formado la barra se comporta de forma perfectamente elástica y lineal, es decir, se cumple la ley de Hooke entre tensiones y deformaciones

- La directriz de la barra es idealmente recta, sin imperfecciones.

- El esfuerzo axil está centrado, exactamente aplicado sobre el centro de gravedad de la sección.

- Los vínculos exteriores son teóricos, constituidos por un apoyo fijo y otro deslizante paralelo a la directriz de la barra.

Matemáticamente se puede deducir que el menor valor de la carga que produce una bifurcación en el estado de equilibrio vale ${ }^{28}$.

$$
N_{c r i t}=\frac{\pi^{2} \cdot E \cdot I}{L^{2}}
$$

Esta expresión, obtenida para el caso de la barra biarticulada sometida a esfuerzo axil centrado, se puede generalizar para otros casos de sustentación de la barra a partir del concepto de la longitud de pandeo $\left(L_{p}\right)$ :

$$
N_{c r i t}=\frac{\pi^{2} \cdot E \cdot I}{L_{p}^{2}}
$$

27 "Coulomb's memoir on statics. An essay in the history of civil engineering". Jacques Heyman (1972)

28 "Resistencia de materiales". Luis Ortiz Berrocal. (1991)

"Resistencia de materiales". Manuel Vazquez (1994) 
Se entiende la longitud de pandeo $\left(L_{p}\right)$ de una barra con cualquier tipo de sustentación, como la longitud de una barra ficticia con la misma sección transversal, articulada en sus extremos y que tenga igual carga crítica en compresión simple ${ }^{29}$.

Si dividimos la carga crítica por el área de la sección podremos expresar el problema en términos de tensión:

$$
\sigma_{\text {crit }}=\frac{N_{\text {crit }}}{A}=\frac{\pi^{2} \cdot E \cdot I / A}{L^{2}}=\frac{\pi^{2} \cdot E}{(L / i)^{2}}=\frac{\pi^{2} \cdot E}{\lambda^{2}}
$$

Siendo $\lambda$ la esbeltez mecánica de la barra, definida como la relación entre la longitud de la barra y el radio de giro de la sección, en el plano de pandeo considerado.

Puesto que la "tensión crítica de Euler" se ha obtenido bajo la hipótesis de la validez de la ley de Hooke, ésta sólo será válida si es menor que el límite elástico del material $\left(\sigma_{c r i t} \leq \sigma_{p}\right)$, de donde se deduce que existen dos límites que no se pueden superar: la capacidad resistente de la sección (su límite elástico si nos encontramos en dimensionamiento en régimen elástico) y la carga que produce la inestabilidad de la barra (carga o tensión crítica).

Es decir, que el colapso de una barra sometida a un esfuerzo de compresión excéntrica se puede producir de dos formas: por agotamiento de su sección más solicitada o por inestabilidad ${ }^{30}$ de la barra.

Si se observan las posibles trayectorias hasta el agotamiento, producidas por un incremento progresivo de las solicitaciones $(N-M)$,

\footnotetext{
29 "Estructuras metálicas para edificación: adaptado al CTE." Monfort Lleonart, J. (2006)

30 "Design of slender concrete columns" Mac Gregor, J.G; Breen, J.E. y Pfrang, E.O. (1970)
} 
respecto de la curva de interacción de la sección, Figura 1.2-1, los caminos Ol y Oll, corresponden al agotamiento de la sección, el primero en el caso de una barra infinitamente rígida y en el segundo para una barra flexible, mientras que el camino Oll corresponde al agotamiento de la barra por inestabilidad (el colapso se produce sin que la curva alcance al diagrama de interacción) ${ }^{31}$.
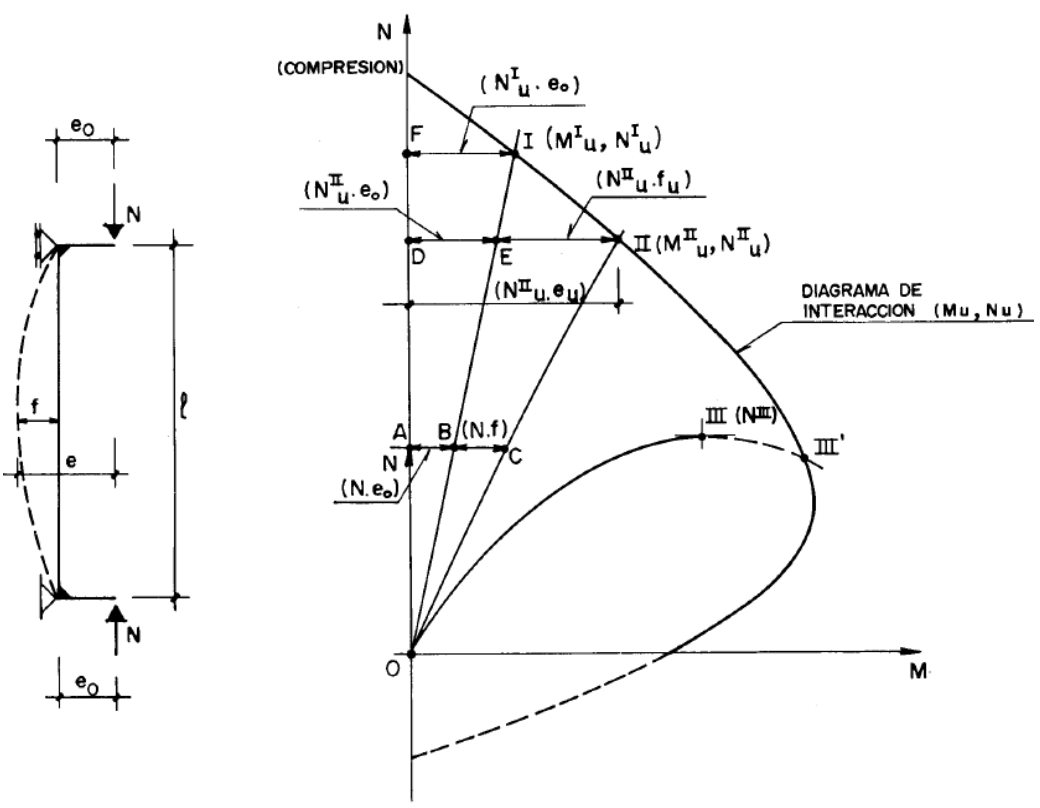

Figura 1.2-1 Agotamiento de una barra sometida a flexocompresión

Puesto que el valor de la tensión crítica es inversamente proporcional al cuadrado de la esbeltez de la barra, en el caso de "soportes esbeltos" la condición de inestabilidad será más restrictiva, mientras que los "soportes cortos" colapsarán por agotamiento de la sección.

Una exposición más detallada de este tema se puede consultar en el apartado 7.2 del presente trabajo.

31 "Cálculo de soportes rectangulares de hormigón armado en teoría de segundo orden". Juan J. Arenas de Pablo (1980) 


\subsubsection{PARAMETROS QUE INTERVIENEN EN LOS EFECTOS DE SEGUNDO ORDEN}

Los trabajos de Pfrang y Siess ${ }^{32}$ sobre los factores que intervienen en la resistencia de soportes biarticulados, muestran que entre los más importantes se encuentran: la esbeltez $(L / h)$, la excentricidad de la carga en el extremo $(e / h)$ y la relación de excentricidades en ambos extremos $\left(e_{1} / e_{2}\right)^{33}$, siendo $L$ la altura del soporte, $h$ el canto de la sección y e la excentricidad del axil respecto de la directriz de la barra.

A continuación se relacionarán éstos y otros de menor importancia que, directa o indirectamente, también afectan a la magnitud de los efectos de segundo orden, no sólo en el caso de soportes aislados de extremos articulados e indesplazables sino para soportes que forman parte de entramados de nudos rígidos.

\section{Longitud de Pandeo}

La longitud de pandeo, definida en el apartado anterior, depende fundamentalmente de tres factores:

- Longitud real de la barra

- Condiciones de contorno ${ }^{34}:$ La coacción del giro de los extremos de la barra condiciona la forma de la deformada del soporte y por lo tanto su longitud de pandeo.

32 "Behavior of restrained reinforced concrete columns" Pfrang, E.O. y Siess, C.P. (1965)

33 "Design of slender concrete columns" Mac Gregor, J.G; Breen, J.E. y Pfrang, E.O. (1970)

34 "Pandeo de estructuras de hormigón armado". Jordi Maristany. (1996) 
Cuando el soporte se encuentra unido al exterior mediante vínculos teóricos, el valor de la longitud de pandeo es sencilla de determinar (Figura 1.2-2 a y b), pero cuando se encuentra unido a un entramado mediante nudos rígidos, en su determinación también interviene la rigidez de las vigas respecto de la de los soportes que concurren en el nudo (Figura 1.2-2 c).

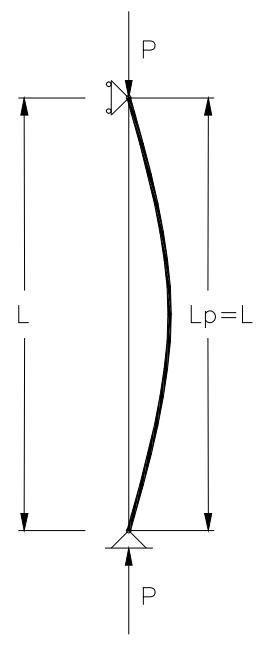

a

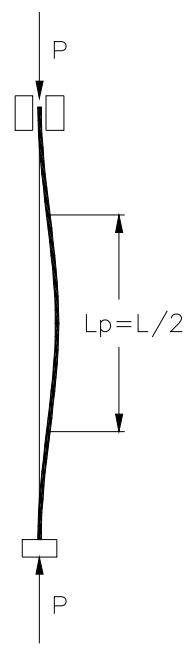

b

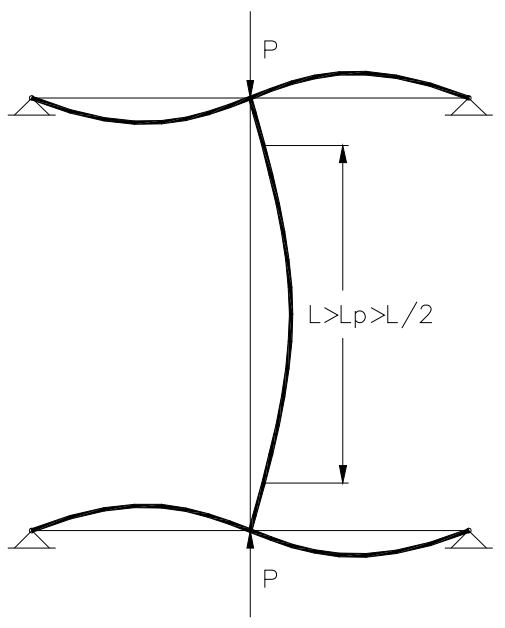

C

Figura 1.2-2 Longitud de pandeo en barras intraslacionales

- Traslacionalidad: También la libertad de desplazamiento de los extremos de la barra condiciona a la deformada del soporte, tanto cuando se trata de vínculos exteriores teóricos como cuando se trata de soportes de entramados de nudos rígidos (Figura 1.2-3). 

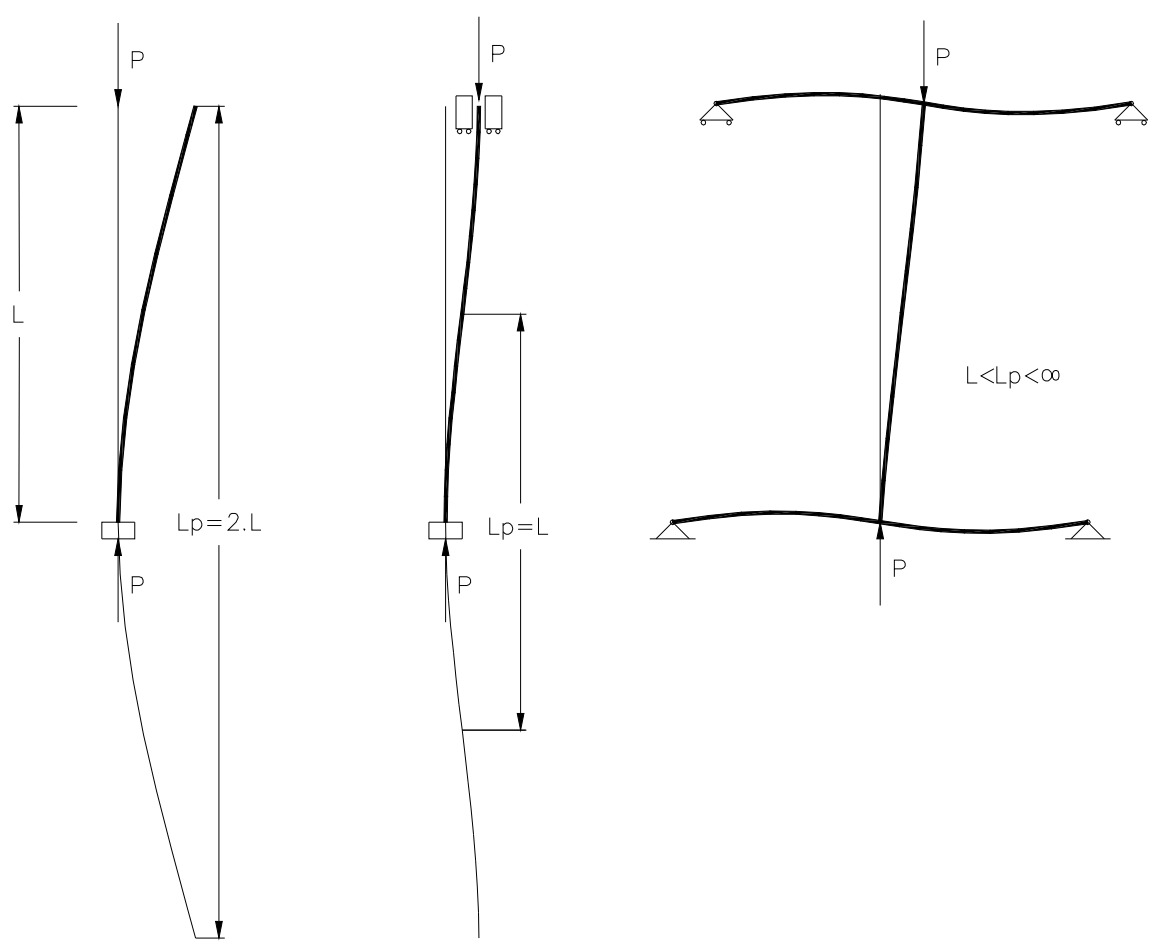

Figura 1.2-3 Longitud de pandeo en barras traslacionales

\section{Esbeltez}

Se denomina "esbeltez" a la relación entre la altura del soporte y las dimensiones de su sección transversal. En función del parámetro de la sección utilizado se puede definir como:

- Esbeltez geométrica: Relación entre la longitud de la barra y la dimensión de la sección en el eje considerado:

$$
\lambda_{g}=L_{p} / h
$$

- Esbeltez mecánica: Relación entre la longitud de la barra y el radio de giro de la sección en el eje considerado: 


$$
\lambda_{m}=L_{p} / i
$$

En función de la esbeltez los soportes se clasifican en "cortos" y "esbeltos", lo cual interviene en gran medida en la forma de agotamiento de la barra (véase el Apartado 7.2 del presente trabajo).

\section{Momentos en los extremos}

Puesto que la relación, tanto de valor como de signo, de los momentos en los extremos de la barra condiciona su deformada, serán un parámetro que tendrá gran influencia en el pandeo del soporte.

La situación más desfavorable se producirá cuando en un mismo punto coincida el momento máximo de primer orden con el desplazamiento máximo (que a su vez producirá el mayor momento de segundo orden). A continuación se describen varias situaciones, suponiendo siempre que no existen cargas transversales a la barra.

Por ello, la peor de las combinaciones se produce para dos momentos iguales y de signo contrario (misma excentricidad) en los dos extremos tal como se grafía en el esquema a de la Figura 1.2-4. En este caso, al momento de primer orden, esquema c, constante a lo largo del soporte, hay que añadir el momento de segundo orden cuyo máximo se produce en el punto medio de la barra donde se produce el máximo desplazamiento, (esquema b). La consideración de los efectos de segundo orden siempre incrementa el valor del momento máximo del soporte, (esquema d), reduciéndose su capacidad resistente. 


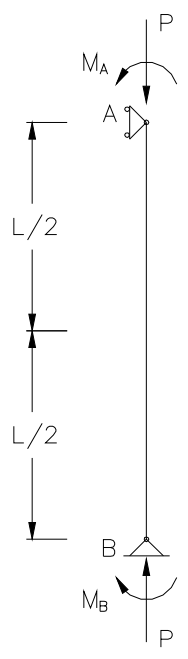

a

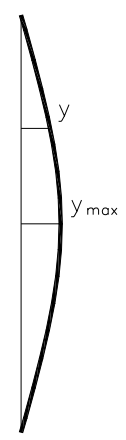

b

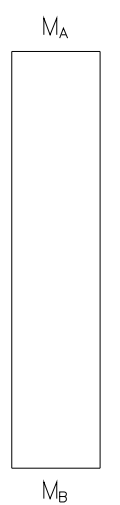

C

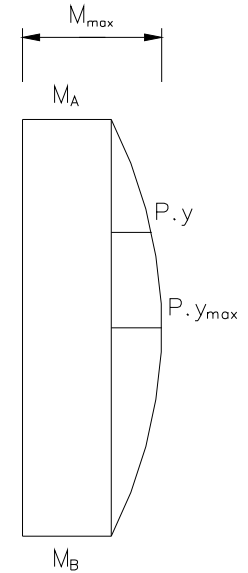

d

Figura 1.2-4 Soporte sometido a dos momentos contrarios en los extremos

La mejor de las situaciones consiste en una barra intraslacional sometida a flexocompresión con momentos del mismo signo (excentricidades contrarias) en ambos extremos, tal como se grafía en la Figura 1.2-5 (esquema a). Así, el momento máximo derivado del análisis de primer orden se produce en un extremo de la barra (esquema c), mientras que el desplazamiento máximo se produce en un punto intermedio de la barra (esquema b). Dependiendo de la esbeltez y de la rigidez de la barra, el momento máximo considerando los efectos de segundo orden se producirá en el punto de momento máximo de primer orden (esquema d) o en el punto de desplazamiento máximo (esquema e). La consideración de los efectos de segundo orden únicamente reducirá la capacidad resistente del soporte a partir de cierta esbeltez. 


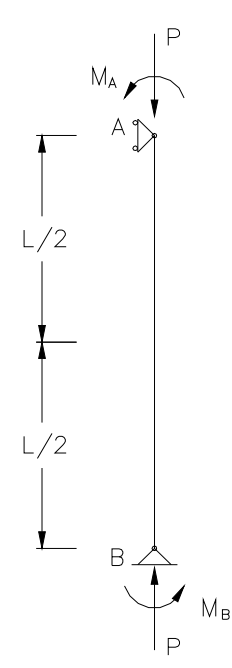

a

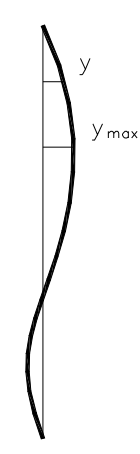

b

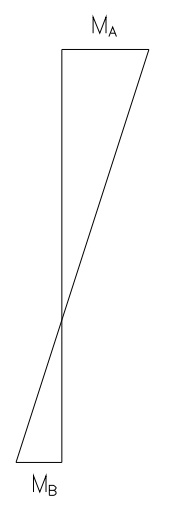

C

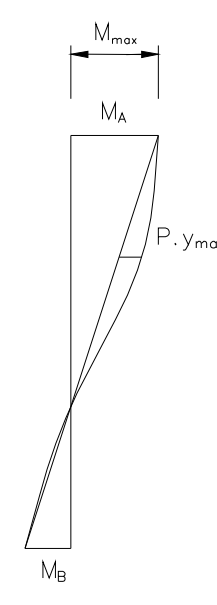

d

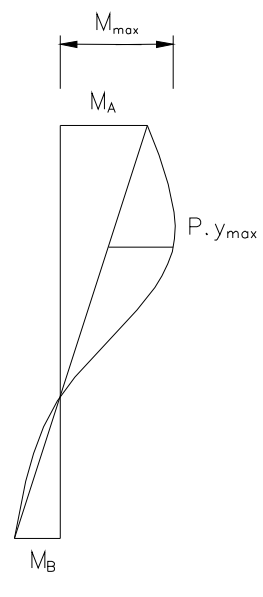

e

Figura 1.2-5 Soporte sometido a dos momentos del mismo sentido de giro en los extremos

La situación en la que el momento en uno de los extremos es nulo representa un estado intermedio entre los dos descritos.

Se puede encontrar mayor documentación sobre este tema en los Apartados 7.2 y 7.3 del presente trabajo.

\section{Duración de la carga.}

Algunos materiales, como el hormigón, incrementan su deformación a lo largo del tiempo por el hecho de estar sometidos a una carga permanente. A este fenómeno se le denomina fluencia reológica del hormigón.

Un soporte de hormigón armado o mixto sometido a un esfuerzo de flexocompresión mantenido en el tiempo, como es el caso habital en las estructuras de edificación, incrementará progresivamente su deformación, por lo que aumentará la excentricidad de la carga axil, reduciéndose la capacidad resistente del soporte. 
Se ofrece una mayor información sobre este tema en el Apartado 7.2 y, especialmente, en el Capítulo 5 del presente trabajo.

\subsubsection{METODOS DE ANALISIS}

El estudio de barras sometidas a flexocompresión recta o esviada exige, cuanto menos un análisis no lineal geométrico. Algunas de las hipótesis simplificadoras utilizadas por la resistencia de materiales como la de la pequeñez de las deformaciones o la de proporcionalidad entre acciones y deformaciones, ya no son aceptables.

El equilibrio de la estructura ya no puede plantearse con la geometría inicial sino que se deben tener en cuenta las deformaciones obtenidas del análisis en primer orden. Según el método de cálculo utilizado, las solicitaciones y deformaciones finales tendrán que obtenerse mediante un proceso iterativo.

Se puede conseguir una mayor aproximación a la realidad si se tiene en cuenta la verdadera relación entre tensiones y deformaciones de los materiales empleados, es decir, si además de la no linealidad geométrica también se considera una no linealidad en el comportamiento del material $^{35}$.

La no proporcionalidad entre tensiones y deformaciones es especialmente significativa en materiales como el hormigón que presentan un comportamiento anisótropo y no lineal desde el inicio de su puesta en carga. Además, la reducida capacidad resistente a tracción de este material produce la aparición de fisuras en las zonas traccionadas, que reducen su inercia original. Es por ello que ni el

35 "Estudio no lineal del comportamiento resistente de soportes de hormigón armado solicitados a flexo-compresión esviada" J.R. Atienza, R. Irles. (1985) 
módulo de elasticidad ni la inercia pueden considerarse constantes a lo largo del proceso.

En el caso del acero, Engesser estudio el comportamiento teórico de soportes comprimidos sometidos a tensiones superiores al límite de proporcionalidad, bajo las mismas premisas que fueron adoptadas por Euler, salvo el mantener constante el valor del módulo de elasticidad $(E)$. Propuso dos teorías ${ }^{36}$ para la obtención de la carga crítica, una basada en el "módulo tangente" y otra en el "doble módulo".

La discusión entre Engesser, Considère y von Kármán sobre el valor del módulo a emplear se expone en el apartado 7.1, mientras que distintos métodos de resolución del problema no lineal se describen en el apartado 7.4 .

El método de cálculo propuesto y desarrollado en el presente trabajo, (ver apartado 7.5) pretende tener en cuenta no sólo las no linealidades geométricas y del material, sino también otros aspectos que influyen en la capacidad resistente de los soportes mixtos sometidos a flexocompresión, como son las imperfecciones iniciales de la barra o los efectos reológicos del hormigón.

36 "Contribution a l'etude du flambement dans le domaine plastique" Virlogeux. Annales de l'Institute Technique du Batiment et des Travaux Publics. № 312 


\subsection{EVOLUCIÓN DE LOS METODOS DE CÁLCULO DE SOPORTES MIXTOS}

\subsubsection{INTRODUCCION}

Tanto las investigaciones como los ensayos llevados a cabo durante las primeras décadas del siglo permitieron entender el comportamiento de las estructuras mixtas y condujeron a la publicación de numerosos artículos y libros sobre el tema. Muchos de ellos contenían sus propias teorías y métodos de cálculo de los aspectos particulares que trataban: soportes, vigas, conectores, etc. Algunos de estos métodos de cálculo son posteriormente incluidos en las normativas nacionales como es el caso del método de Basu y Sommerville recogido por la norma inglesa "The use of structural steel in buildings" BS 449 (British Standards Institution) ${ }^{37}$.

En los primeros años de la década de 1960 los países tecnológicamente mas avanzados disponen ya de sus propias normativas y códigos de cálculo de estructuras mixtas que se irán progresivamente actualizando a lo largo del siglo XX, incorporando las técnicas mas avanzadas.

Por ejemplo, el método de cálculo de estructuras mixtas contenido en la edición de 1948 de la mencionada norma inglesa BS 449, basado en la teoría de las "tensiones admisibles" fue sustituido por el código BS 5950 basado en la teoría de los "estados límites".

En Japón, donde ya se conocen edificaciones realizadas con estructuras mixtas desde la década de 1910, el Architectural Institute of Japan (AIJ) publica su primer código denominado "Steel Reinforced Concrete" (SRC) en $1951^{38}$. La experiencia del terremoto

37 "Composite structures of steel and concrete. Volume 1: Beams, columns, frames and applications in building". Johnson, R.P.,. $3^{\underline{a}}$ ed. (2004)

38 "Composite construction". David A. Nethercot.(2003) 
de Tokachi-oki de 1968, que provocó fallos por esfuerzo cortante en numerosos soportes mixtos, condujo a la revisión de este código en 1975.

En Alemania, las primeras reglas para el cálculo de vigas mixtas adoptaron el carácter de norma en 1954 publicada como DIN 1078. Ésta ha sido actualizada por la norma DIN 18806.

En Estados Unidos, dos institutos disponen desde 1961 de métodos de cálculo de estructuras mixtas para la edificación. Se trata del American Institute of Steel (AISC) que realiza una aproximación a la estructura mixta desde el punto de vista de las estructuras metálicas, y el American Concrete Institute (ACl) que la interpreta como una estructura de hormigón con refuerzos a base de perfiles metálicos.

En los siguientes apartados se exponen los métodos de cálculo de soportes mixtos de algunas de estas normativas ${ }^{39}$

\subsubsection{NORMA INGLESA BS 449}

Basada en el método de Basu y Sommerville ${ }^{40}$, es aplicable soportes de sección mixta simétrica respecto de dos ejes ortogonales, sometidos a flexocompresión, en cualquiera de los dos planos de simetría.

La hipótesis fundamental en que se basa, consiste en suponer que las secciones inicialmente planas permacen planas tras la aplicación de las cargas.

\footnotetext{
39 "Colonnes mixtes acier-béton". Glück, R., Clenin, D. (1987)

40 "Composite structures of steel and concrete. Volume 1: Beams, columns, frames and applications in building". Johnson, R.P.,. $3^{\underline{a}}$ ed. (2004)
} 
Como valores característicos de las resistencias de los materiales se adoptan el límite elástico del acero $\left(f_{s d}\right)$, de las armaduras $\left(f_{y d}\right)$ y del hormigón $\left(f_{c d}\right)$

El proceso de aplicación de este método comienza con la representación del diagrama de interacción de la barra (curva de resistencia última de una barra sometida a esfuerzo axil excéntrico), a partir de la deteminación de tres puntos A, B y C (ver Figura 1.3-1 $)^{41}$. El punto $A$ representa la capacidad resistente última a esfuerzo axil centrado (momento flector nulo) y el punto $C$ la capacidad resistente última a flexión simple (axil nulo).

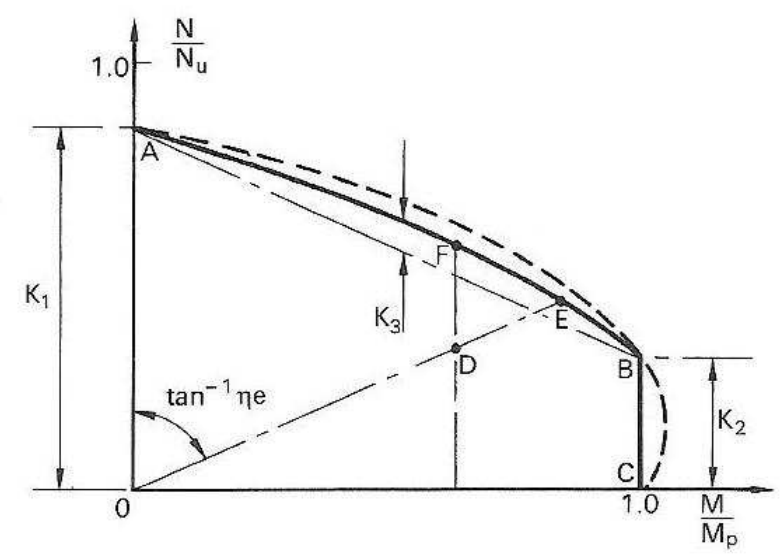

Figura 1.3-1 Diagrama de interacción de la barra. Método de Basu y Sommerville

En el caso de soportes metálicos el diagrama se puede idealizar mediante un tramo recto $(\mathrm{CB})$ y un arco parabólico $(\mathrm{AB})$. En el caso de soportes mixtos, el hormigón del recubrimiento modifica esta curva, interviniendo en la determinación de los mencionados parámetros.

${ }^{41}$ Esta Figura se ha extraído de la publicación "Composite structures of steel and concrete" de R. P. Johnson. 
La ordenada del punto A, denominada $K_{1}$ en la figura, corresponde a la relación entre la carga axial última $N_{a}$ de una barra esbelta y la carga axial última $N_{u}$ de un soporte corto de la misma sección $\left(K_{1}=N_{a} / N_{u}\right)$. Su abscisa es nula por tratarse de un caso de esfuerzo axil centrado.

La carga de agotamiento de un soporte corto de sección mixta se puede obtener mediante la ecuación:

$$
N_{u}=A_{s} \cdot f_{s}+A_{r} \cdot f_{r}+A_{c} \cdot f_{c}
$$

siendo $A_{s}, A_{r}$ y $A_{c}$, respectivamente, las áreas del perfil metálico, de los barras de acero de refuerzo longitudinal y del hormigón, y $f_{s}, f_{r}$ y $f_{c}$, las resistencias a compresión de los respectivos materiales.

Para un soporte de acero la mencionada relación $K_{1}$, para pandeo elástico (Euler), se puede expresar:

$$
K_{1}=\frac{N_{a}}{N_{u}}=\frac{\pi \cdot E_{s}}{f_{y} \cdot(L / r)^{2}}
$$

La curvatura inicial y la plastificación local de la barra, la curva que relaciona $K_{1}$ con la esbeltez queda por debajo que la teórica (fórmula anterior).

En el caso de secciones mixtas, se puede encontrar una expresión obtener un radio de giro equivalente que permita seguir utilizando la misma ecuación. Si se adopta un módulo de elasticidad del hormigón $E_{c}=300 \cdot f_{c u} / \gamma_{m}$, el valor del radio de giro para una sección mixta, teniendo en cuenta las imperfecciones iniciales y el comportamiento inelástico del material, se podría obtener mediante la expresión:

$$
r^{2}=\left(f_{y}^{*} \cdot l_{s}+0,24 \cdot f_{c u} \cdot l_{c}\right) / N_{u}
$$


donde $I_{s}$ y $I_{c}$ son, respectivamente, los momentos de inercia del acero y del hormigón de la sección mixta y $f_{y}{ }^{*}$ el límite elástico del hierro dulce.

A continuación se determina la abscisa del punto $\mathrm{C}$, correspondiente a la capacidad resistente última a flexión o momento plástico de la sección $M_{p}$ (su ordenada es nula por tratarse de un caso de flexión simple). Para ello es necesario obtener la posición de la fibra neutra para la que se produce el equilibrio de fuerzas entre los bloques traccionados y comprimidos. La Figura 1.3-2 $2^{42}$ muestra la disposición de bloques para el eje fuerte de la sección, según Basu y Sommerville, en la que se han realizado las siguientes hipótesis y simplificaciones: las áreas traccionadas y comprimidas de los armaduras de acero son iguales, el límite elástico a compresión y a tracción del acero de los refuerzos es la misma y no se deduce el área de las armaduras y de la parte del perfil comprimido del área del bloque comprimido de hormigón ${ }^{43}$.

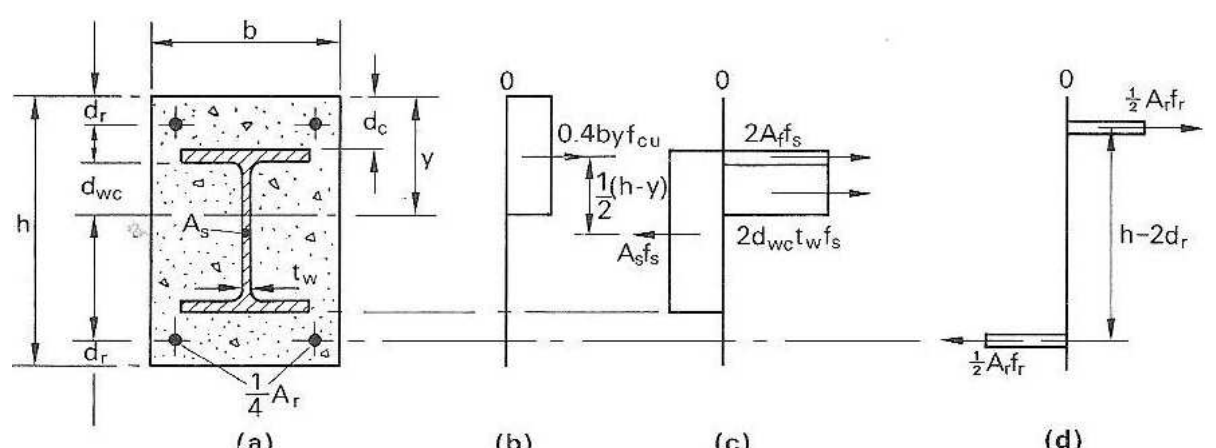

Figura 1.3-2 Esquema para la determinación del momento plástico de la sección

\footnotetext{
${ }^{42}$ Esta Figura se ha extraído de la publicación "Composite structures of steel and concrete" de R. P. Johnson.

${ }^{43}$ El error, del lado de la inseguridad cometido por esta última simplificación se ha tenido en cuenta en el coeficiente corrector de la resistencia característica del hormigón.
} 
Bajo los supuestos anteriores y, si el eje neutro corta el alma del perfil, lo que es bastante frecuente, el equilibrio de fuerzas en los bloques se produce cuando la resultante del bloque comprimido coincide con la resultante de tracción de una zona del alma del pefil de altura $(h-2 \cdot y)$ es decir, cuando se cumple la igualdad:

$$
0,4 \cdot b \cdot y \cdot f_{c u}=(h-2 \cdot y) \cdot t_{w} \cdot f_{s}
$$

De donde se puede despejar la altura de la fibra neutra y. El momento plástico de la sección se obtiene tomando momentos respecto de la resultante del bloque comprimido:

$$
M_{p}=\left[\frac{1}{2} A_{s}(h-y)-A_{f}\left(d_{c}-d_{w c}\right)-d_{w c} t_{w}\left(y-d_{w c}\right)\right] \cdot f_{s}+A_{r} f_{r}\left(\frac{1}{2} h-d_{r}\right)
$$

Mediante un proceso similiar se puede obtener el momento plástico para el eje débil de la sección.

El punto $B$ está definido por una abscisa igual a la del punto $C$, es decir $\left(M / M_{p}\right)$ y una ordenada equivalente al parámetro $K_{2}$, que representa el axil máximo que es capaz de soportar la barra actuando simultáneamente con el momento plástico $\left(M_{p}\right)$. La expresión empírica que permite su cálculo, tanto para el caso de secciones recubiertas como de secciones rellenas de hormigón es:

$$
\frac{K_{2}}{K_{20}}=\frac{90-25 \cdot(2 \cdot \beta-1) \cdot(1,8-\alpha)-L / r}{30 \cdot(2,5-\beta)} \quad(\geq 0 \quad y \leq 1)
$$

siendo:

$K_{20}$ : valor correspondiente para una esbeltez nula $(L / r=0)$ definido por: $K_{20}=0,9 \cdot \alpha^{2}+0,2 \quad(\leq 0,75)$

$\alpha$ : coeficiente de contribución del hormigón, en la capacidad resistente de la sección al esfuerzo axil centrado: $\alpha=A_{c} \cdot f_{u} / N_{u}$ 
$\beta$ : coeficiente que relaciona el momento flector en los dos extremos de la barra en un plano de simetría: $\beta=M_{i} / M_{f}$

Con estos tres puntos se puede representar el diagrama formado por las rectas $A B$ y $B C$ (ver Figura 1.3-2), suficiente para soportes recubiertos con flexión respecto del eje fuerte de la sección, ya que en estos casos el parámetro $K_{3}$ se puede despreciar $\left(K_{3} \approx 0\right)$.

Una vez determinados los puntos que definen el diagrama, la comprobación de una barra puede realizarse de forma gráfica, comprobando que el punto definido por las coordenadas $\left(M / M_{p}, N / N_{u}\right)$ queda en el interior de la superficie encerrada por el diagrama, (punto $D$ de la Figura 1.3-1) o bien de forma analítica mediante la verificación de las siguientes condiciones:

$$
\begin{aligned}
& M \leq M_{p} \\
& \frac{N}{N_{u}} \leq K_{1}-\left(K_{1}-K_{2}-4 K_{3}\right) \cdot\left(M / M_{p}\right)-4 K_{3}\left(M / M_{p}\right)^{2}
\end{aligned}
$$

\section{Flexocompresión esviada. Método de Virdi y Dowling}

Dado que el método de Basu y Sommerville únicamente es aplicable en soportes mixtos sometidos a flexocompresión recta, era necesario el desarrollo de algún método que permitiera la comprobación de soportes cuando aparecen simultáneamente momentos en los dos planos. Los ensayos realizados por Virdi y Dowling ${ }^{44}$ les permitieron desarrollar un método de cálculo de soportes mixtos sometidos a flexocompresión esviada y que tiene en cuenta tanto las tensiones residuales del perfil metálico como las imperfecciones iniciales de la barra. No obstante, el método desarrollado era de compleja aplicación por lo que optaron por comparar los resultados de los ensayos con la ecuación:

44 "The ultimate strength of composite columns in biaxial bending". Virdi, K.S., Dowling, P.J. (1973) 


$$
\frac{1}{N_{x y}}=\frac{1}{N_{x}}+\frac{1}{N_{y}}-\frac{1}{N_{a x}}
$$

Donde $N_{x y}$ es el axil último en flexocompresión esviada, $N_{x}$ y $N_{y}$, respectivamente, los axiles últimos en flexocompresión recta del soporte teniendo en cuenta su esbeltez en cada plano (que se pueden obtener a partir del método de Basu y Sommerville) y $N_{a x}$ el axil último cuando el pandeo está impedido en el plano "y-z".

Esta expresión tiene en cuenta las imperfecciones iniciales respecto del eje débil cuando la flexión se produce únicamente en el eje fuerte, pero que desprecia las imperfecciones respecto del eje fuerte si la flexión se aplica sólo en el eje débil.

Su aplicación conduce a resultados suficientemente aproximados en soportes cortos pero, por diferentes motivos, excesivamente conservadores para soportes esbeltos ${ }^{45}$.

\subsubsection{NORMA AMERICANA AISC LRFD}

Esta normativa del American Institute of Steel (AISC), incluye ya el concepto de los "estados límites", Load and Resistance Factor Design (LRFD) en la terminología americana y recoge algunas propuestas del Structural Specifications Liaison Committe (SSLC) ${ }^{46,47}$.

Su aplicación exige el cumplimiento de una serie de reglas de diseño del soporte compuesto:

\footnotetext{
45 "Colonnes mixtes acier-béton". Glück, R., Clenin, D. (1987)

46 "1986 AISC LRFD Design for composite buildings". Composite construction in steel and concrete proceedings of an engineering foundation conference. Pinkham, C.W. (1988)

47 "Diseño de estructuras de acero" A.J. Rokach. (1992)
} 
- El área del perfil metálico será al menos el 4\% del área total de la sección.

- Se incluirán armaduras de refuerzo longitudinal y transversal, estas últimas con una separación máxima $e \leq 2 / 3 d_{\min }$ (siendo $d_{\min }$ la menor de las dos dimensiones de la sección transversal).

- La resistencia a compresión del hormigón estará comprendida entre 20 y $55 \mathrm{MPa}$.

- El límite de fluencia del acero del perfil metálico no superará los $380 \mathrm{MPa}$.

- Si se dispusieran dos o más perfiles metálicos en una sección mixta, éstos se deberán conectar entre sí.

- La parte del axil que debe soportar el hormigón se transmitirá por apoyo directo en las uniones.

La resistencia última de un soporte mixto sometido a compresión simple se puede obtener de la expresión:

$$
F_{u}=\phi_{c} \cdot P_{n}
$$

En ella $\phi_{c}$ es el coeficiente de minoración de la resistencia del hormigón que adopta un valor $\phi_{c}=0,85$, y $P_{n}$ la resistencia de cálculo a compresión, determinada por la expresión $P_{n}=A_{s} \cdot F_{c r}$, siendo $A_{s}$ el área del perfil estructural y $F_{c r}$ el esfuerzo crítico en compresión dado por:

$$
\begin{array}{ll}
F_{c r}=\left(0,658 / \lambda_{c}^{2}\right) \cdot F_{m y} & \text { para } \lambda_{c} \leq 1,5 \\
F_{c r}=\left(0,877 / \lambda_{c}^{2}\right) \cdot F_{m y} & \text { para } \lambda_{c}>1,5
\end{array}
$$

La esbeltez $\lambda_{c}$, el esfuerzo de fluencia modificado $F_{m y}$ y el módulo de elasticidad modificado $E_{m}$, vienen definidos por: 


$$
\begin{aligned}
\lambda_{c} & =\frac{K \cdot L}{r_{m} \cdot \pi} \cdot \sqrt{\frac{F_{m y}}{E_{m}}} \\
F_{m y} & =F_{y}+c_{1} \cdot F_{y r} \cdot\left(A_{r} / A_{s}\right)+c_{2} \cdot f^{\prime}{ }_{c} \cdot\left(A_{c} / A_{s}\right) \\
E_{m} & =E+c_{3} \cdot E_{c} \cdot\left(A_{c} / A_{s}\right)
\end{aligned}
$$

siendo:

K: $\quad$ coeficiente de ponderación de la longitud efectiva del soporte

$L: \quad$ longitud del soporte

$r_{m}$ : radio de giro del perfil estructural y no menor de 0,3 el espesor de la sección mixta en el plano de pandeo

$A_{c}, A_{r}, A_{s}$ : áreas, respectivamente, del hormigón, armadura longitudinal y perfil estructural

$E, E_{c}$ : módulos de elasticidad, respectivamente, del acero estructural y del hormigón

$F_{y}, F_{y r}$ : límite de fluencia, respectivamente, del acero estructural y de las armaduras

$f_{c}^{\prime}$ : resistencia a compresión especificada del hormigón

$c_{1}, c_{2}, c_{3}$ : coeficientes que toman el valor, respectivamente, de $0,7,0,6$ y 0,2 en el caso de secciones de acero recubiertas

En el caso de flexocompresión, cuando la sección tiene uno o dos ejes de simetría, la comprobación de la barra se realiza verificando las desigualdades:

$$
\begin{array}{ll}
\frac{P_{u}}{\phi_{c} \cdot P_{n}}+\frac{8}{9} \cdot\left[\frac{M_{u x}}{\phi_{b} \cdot M_{n x}}+\frac{M_{u y}}{\phi_{b} \cdot M_{n y}}\right] \leq 1,0 & \text { para } \frac{P_{u}}{\phi_{c} \cdot P_{n}} \geq 0,2 \\
\frac{P_{u}}{2 \cdot \phi_{c} \cdot P_{n}}+\left[\frac{M_{u x}}{\phi_{b} \cdot M_{n x}}+\frac{M_{u y}}{\phi_{b} \cdot M_{n y}}\right] \leq 1,0 & \text { para } \frac{P_{u}}{\phi_{c} \cdot P_{n}}<0,2
\end{array}
$$


siendo:

$P_{u}: \quad$ esfuerzo axil con cargas ponderadas

$P_{n}: \quad$ resistencia de cálculo a compresión $\left(P_{n}=A_{s} \cdot F_{c r}\right)$

$M_{u}$ : $\quad$ momento flector con cargas ponderadas

$M_{n}$ : resistencia de cálculo a flexión

$\phi_{c}: \quad(0,85)$ coeficiente minorador de la resistencia a compresión

$\phi_{b}: \quad(0,90)$ coeficiente minorador de la resistencia a flexión

Los valores de los momentos $M_{u}$ que se indican en las expresiones anteriores deben incluir los efectos de segundo orden (producidos por los desplazamientos horizontales de la estructura). Si el análisis estructural se ha realizado en primer orden, la norma plantea un procedimiento simplificado que permite corregir el momento obtenido en el cálculo y determinar el momento de cálculo $M_{u}$ mediante la ecuación: $M_{u}=B_{1} \cdot M_{n t}+B_{2} \cdot M_{l t}$.

En ella, $M_{n t}$ corresponde al momento de primer orden producido por las cargas gravitarorias suponiendo que no existe desplazamiento lateral de la estructura y $M_{l t}$ el momento producido por el desplazamiento lateral, que se considerará nulo si la estructura es intraslacional. El valor de los coeficientes $B_{1}$ y $B_{2}$ se obtienen de las espresiones:

$$
\begin{aligned}
& B_{1}=\frac{C_{m}}{\left[1-\frac{P_{u}}{P_{e}}\right]} \geq 1,0 \\
& B_{2}=\frac{1}{\sum P_{u} \cdot\left[\frac{\Delta_{o h}}{\Sigma H \cdot L}\right]} \quad \text { ó } \quad B_{2}=\frac{1}{1-\frac{\Sigma P_{u}}{\Sigma P_{e}}}
\end{aligned}
$$

El coeficiente $C_{m}$ adopta los siguientes valores: 
$C_{m}=0,6-0,4 \cdot\left(M_{1} / M_{2}\right)$ : para el caso de soportes empotrados en sus extremos y sin cargas transversales, y siendo $M_{1}$ y $M_{2}$ el momento menor y mayor en los estremos y expresados con su signo.

$C_{m}=0,85: \quad \quad \quad$ cuando existen cargas transversales en el soporte y los extremos de la barra tienen rotación restringida

$C_{m}=1,00: \quad \quad \quad$ cuando existen cargas transversales en el soporte y los extremos de la barra no tienen rotación restringida

En las expresiones anteriores:

$P_{u}: \quad$ esfuerzo axil con cargas ponderadas

$P_{e}$ : carga crítica del soporte, dada por $P_{e}=\pi^{2} \cdot E \cdot I /\left(K \cdot L^{2}\right)$, con $K=1,0$

$\Sigma P_{u}$ : suma de los axiles mayorados de todos los soportes de una determinada planta.

$\Delta_{o h}: \quad$ desplazamiento lateral de la planta considerada

$\Sigma H$ : suma de todas las fuerzas horizontales que producen $\Delta_{o h}$

$L: \quad$ altura de la planta

$\Sigma P_{e}:$ suma de las cargas críticas de los soportes de la planta.

\subsubsection{NORMA AMERICANA ACI 318}

En esta normativa desarrollada por el American Concrete Institute $(\mathrm{ACl})^{48}$, el criterio de agotamiento de la sección consiste en alcanzar

48 "Building code requirements for structural concrete $(A C l$ 318M-95) and commentary (ACl 318M-95)" ACl (1995) 
en cualquier punto de ella, una deformación del 0,3\%, producida por compresión.

Cuando se trata de compresión simple, esta deformación se alcanza simultáneamente en todos los puntos. La carga de agotamiento de la sección $P_{0}$ se obtiene de la ecuación:

$$
P_{0}=A_{s} \cdot f_{y}+A_{s r} \cdot f_{y r}+0,85 \cdot f_{c}^{\prime} \cdot A_{c}
$$

siendo:

$A_{s}, A_{s r}, A_{c}: \quad$ áreas, respectivamente, del perfil estructural, de la armadura longitudinal y del hormigón.

$f_{y}, f_{y r}$ límite elástico, respectivamente, del acero estructural y de las armaduras

$f_{c}^{\prime}$ : $\quad$ resistencia a compresión del hormigón en probeta cilíndrica

Los casos de flexocompresión recta y esviada requieren, tras la imposición de la citada condición de agotamiento, la deducción de la tensión de cada punto de la sección a partir de las relaciones $\sigma$ - $\varepsilon$ de cada uno de los materiales y su integración para averiguar las solicitaciones $N_{n}-M_{n}$ que, actuando conjuntamente, han producido dicha situación de agotamiento.

Es frecuente la comprobación de soportes sometidos a flexocompresión recta a partir de la representación del diagrama de interacción. La comprobación de un soporte corto consiste en la verificación de la desigualdad:

$$
\left(P_{u}, M_{u}\right) \leq\left(\varphi \cdot P_{n}, \varphi \cdot M_{n}\right)
$$

siendo:

$N_{d}, M_{d}$ : axil y momento mayorados, incluidos los efectos de segundo orden. 
$\varphi$ : coeficiente de minoración de los valores $N_{n}-M_{n}$ de agotamiento. Adopta los siguientes valores:

$\varphi=0,70 \quad$ para perfiles metálicos recubiertos de hormigón

$\varphi=0,75 \quad$ para perfiles metálicos rellenos de hormigón

$\varphi=0,90 \quad$ para vigas en flexión pura

En el tramo inferior de la curva, para valores $0,10 \cdot P_{0} \leq N_{n} \leq 0$, el coeficiente $\varphi$ se puede incrementar linealmente, con una serie de condiciones, dando lugar al diagrama representado en la Figura $1.3-3^{49}$ para $\varphi=0,70$.

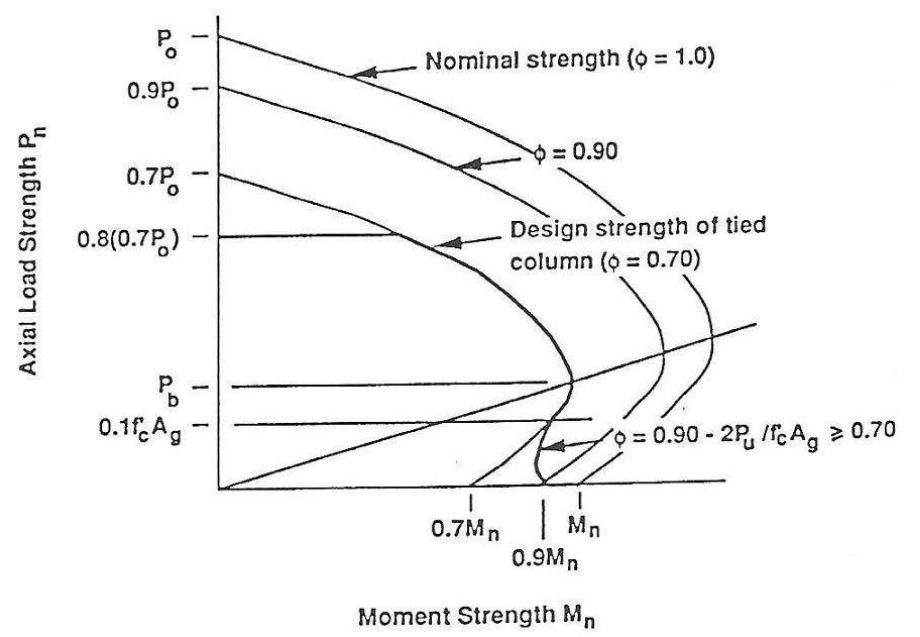

Figura 1.3-3 Diagramas de interacción

Si el análisis estructural no incluye los efectos de segundo orden, la norma $\mathrm{ACl}$ propone un método simplificado, similar al anteriormente descrito en la norma AISC, para corregir el valor del momento de primer orden. El momento $M_{c}$ que tiene en cuenta los efectos de segundo orden se obtiene mediante la suma de dos términos afectados de un coeficiente $\delta$ : el primer término $\left(M_{2 b}\right)$ corresponde al

49 "Notes on ACl 318-89 with Design Applications". Portland Cement Association. (1990) 
momento en el extremo del soporte producido por las cargas gravitatorias sobre el pórtico indesplazable y el segundo $\left(M_{2 s}\right)$ corresponde al momento en mismo extremo producido por los desplazamientos laterales ${ }^{50}$.

$$
M_{c}=\delta_{b} \cdot M_{2 b}+\delta_{s} \cdot M_{2 s}
$$

Los coeficientes $\delta$ representan factores de amplificación de los momentos de primer orden, dados por:

$$
\begin{aligned}
& \delta_{b}=\frac{C_{m}}{1-\frac{P_{u}}{\varphi \cdot P_{c b}}} \geq 1,0 \\
& \delta_{s}=\frac{1}{1-\frac{\Sigma P_{u}}{\varphi \cdot \Sigma P_{c s}}} \geq 1,0
\end{aligned}
$$

siendo:

$P_{c b}$ : carga crítica del soporte dada por:

$$
P_{c b}=\frac{\pi^{2} \cdot E \cdot I}{k_{b} \cdot l_{u}^{2}}
$$

$C_{m}$ : coeficiente que se evaluará a partir de las solicitaciones reales del cálculo mediante la expresión:

$$
C_{m}=0,6+0,4 \cdot \frac{M_{1 b}}{M_{2 b}} \geq 0,4
$$

si el momento en alguno de los extremos fuera nulo, se adoptará $M_{1 b} / M_{2 b}=1$

${ }^{50}$ Ambos momentos adoptarán un valor mínimo, para cada plano de flexión de: $M_{2 b} \geq P_{u} \cdot(0,6+0,03 \cdot h)$ y $M_{2 s} \geq P_{u} \cdot(0,6+0,03 \cdot h)$, siendo $h$ el canto de la sección. 
$\Sigma P_{u}$ : suma de los axiles mayorados de todos los soportes de la planta considerada

$\Sigma P_{c s}$ : suma de la carga crítica de todos los soportes de la planta considerada, dada por la expresión:

$$
P_{c s}=\frac{\pi^{2} \cdot E \cdot I}{\left(k_{s} \cdot I_{u}\right)^{2}}
$$

La norma indica que, en la determinación de la rigidez para la obtención de las cargas críticas $P_{c b}$ y $P_{c s}$, se tenga en cuenta el efecto de la fisuración del hormigón, que para soportes mixtos se puede estimar mediante la expresión:

$$
E \cdot I=\frac{E_{c} \cdot l_{g}}{5} \cdot \frac{1}{1+\beta_{d}}+E_{s} \cdot I_{t}
$$

siendo:

$I_{g}, l_{t:}$ momentos de inercia respecto del centro de gravedad de la sección, de hormigón y del perfil estructural, respectivamente.

$\beta_{d}$ : $\quad$ coeficiente de minoración de la rigidez del hormigón, que adopta los siguientes valores:

Para cargas gravitatorias:

$$
\beta_{d}=\frac{\text { Peso propio }}{\text { Carga total }}
$$

Para desplazamiento lateral:

$$
\beta_{d}=\frac{\text { Carga lateral prolongada }}{\text { Carga lateral total }}
$$

Las cargas utilizadas en las expresiones anteriores deben ser ponderadas por los coeficientes correspondientes. 
Respecto del procedimiento de cálculo propuesto por el AISC, expuesto en el apartado anterior, este método del $\mathrm{ACl}$ resulta de más laboriosa aplicación, aunque es aplicable a secciones de cualquier tipo. También resulta reseñable que sus resultados son excesivamente conservadores en el caso de soportes mixtos esbeltos $^{51}$.

\subsection{EUROCODIGO 4}

Esta norma, entre las más recientes y completas sobre la materia, dedica el apartado 4.8 a los "Pilares mixtos" "2,53,54,55.

Aunque la recopilación de sus prescripciones, y las referencias a otras normativas que de ellas se derivan, representa un pesado y arduo trabajo, dada su importancia al tratarse del marco de referencia del presente trabajo, se ha decidido su exposición con amplio detalle en el presente apartado, con especial detenimiento en lo que afecta a las comprobaciones de la barra a flexocompresión recta y esviada.

Se describirán los dos procedimientos de cálculo que contempla: uno, que denomina "general", del que sólo establece las hipótesis de

51 "Column rules of ACI, SSLC, and LRFD compared" Furlong, R.W. (1983)

52 UNE ENV 1994-1-1 Eurocódigo 4: Proyecto de estructuras mixtas de hormigón y acero. Parte 1-1: Reglas generales y reglas para edificación AENOR 1995

53 "Composite structures" European Convention for Constructional Steelwork Joint Committee on Composite Structures. (1981)

54 "Composite beams and columns to Eurocode 4" European Convention for Constructional Steelwork. Technical Committee 11. Composite Structures (1993)

55 "Comprobación de soportes compuestos según EC4". Quintero, F. (1991) 
cálculo y que resulta de compleja aplicación práctica, aunque es aplicable inclusive a pilares con secciones asimétricas o de canto variable a lo largo de su longitud, y otro, que denomina "simplificado", mucho más sencillo, aunque con mayores restricciones en su campo de utilización (secciones doblemente simétricas y constantes a lo largo del pilar).

\subsubsection{GENERALIDADES}

El apartado 1.4.2 "Términos especiales de la Parte 1-1 del Eurocódigo 4", realiza las siguientes definiciones:

Elemento mixto: Elemento estructural compuesto por hormigón y acero estructural o conformado en frío, interconectados por conectadores para limitar el desplazamiento longitudinal entre el hormigón y el acero, y el despegue de un componente del otro.

Pilar mixto: Elemento mixto sometido, principalmente, a compresión y flexión.

\subsubsection{CAMPO DE APLICACIÓN}

El campo de aplicación del cálculo de Pilares mixtos se especifica en el artículo 4.8.1 del EC4.

En su epígrafe (1) se clasifican las secciones mixtas típicas, que se describen con mayor detalle en el siguiente apartado del presente trabajo.

En su epígrafe (2) se establece que éste es aplicable a pilares aislados intraslacionales que pueden ser: 
- Elementos comprimidos que son parte integrante de pórticos intraslacionales pero que se consideran aislados a efectos de cálculo, o

- Elementos comprimidos aislados que atienden a la clasificación de "intraslacionales" que se da en los apartados 4.3.5.3.3 del Eurocódigo 2 ó 5.2.5.2 del Eurocódigo 3, según corresponda $^{56}$.

\subsubsection{CLASIFICACIÓN DE SECCIONES MIXTAS}

Las secciones típicas en este tipo de soportes, según la clasificación que establece EC4, en su apartado 4.8.1, se recogen en la Figura 1.4-1:

- secciones recubiertas de hormigón (a): un perfil metálico completamente embebido en la sección de hormigón, que incluye armadura longitudinal y transversal.

Presentan un eje de inercia fuerte y otro débil, coincidentes con los del elemento metálico. Pueden ser prefabricados $u$ hormigonados "in situ" y también se suelen emplear como refuerzo de una estructura metálica existente, que posteriormente se recubre de hormigón.

- secciones parcialmente recubiertas o enrasadas (b) y (c): el hormigón queda exterior al perfil pero no lo rodea completamente, de forma que algunas caras del soporte quedan de hormigón y

${ }^{56}$ En el apartado 8.2 del presente trabajo se expone el criterio de los Eurocódigos al respecto de la traslacionalidad de las estructuras y la consideración de los efectos de segundo orden 
otras metálicas. Al igual que el primer tipo, el hormigón también puede incluir armaduras.

Este tipo de elementos suelen ser prefabricados, requiriendo el hormigón la disposición de una armadura longitudinal conectada al acero estructural, para evitar el deslizamiento entre los materiales.

Para el diseño de los nudos, la existencia de caras exteriores de hormigón y acero puede resultan una ventaja o un inconveniente en función del material del elemento con el que se realice el nudo.

- secciones rellenas de hormigón (d), (e) y (f): tubo metálico relleno de hormigón, que puede incluir en su interior otro perfil (f) o armaduras.

En este caso el perfil metálico hace a la vez de encofrado y mejora la resistencia debido al confinamiento del hormigón.
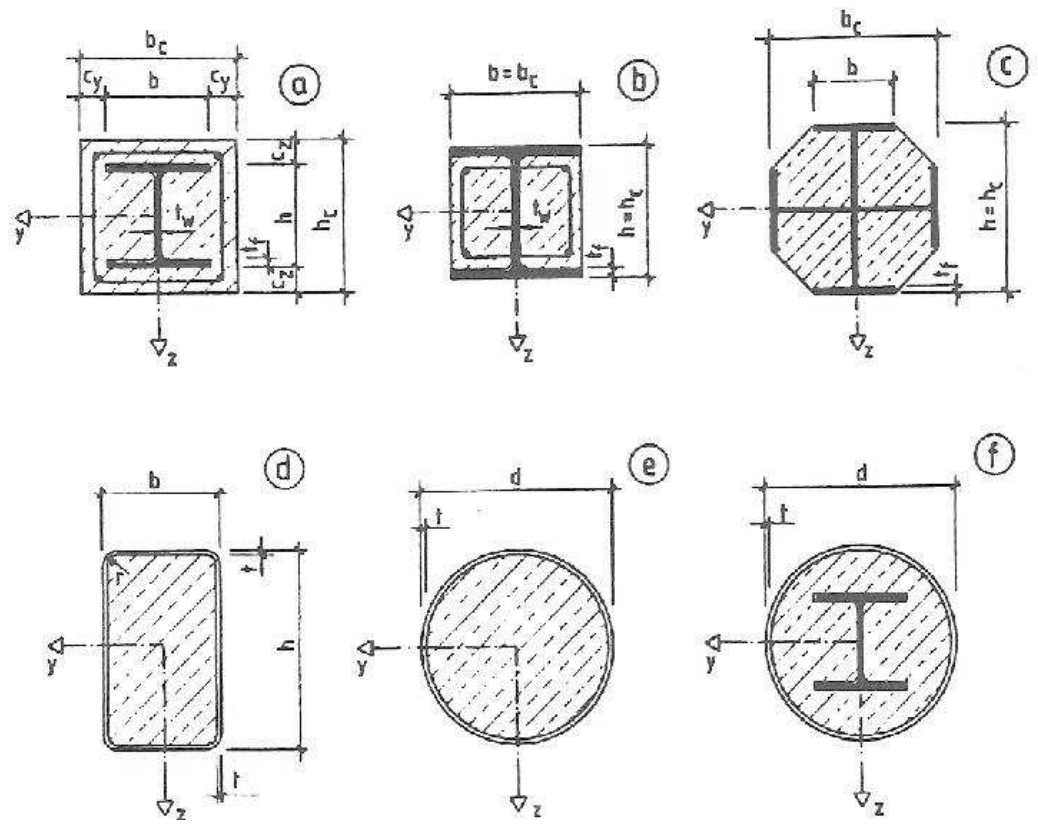

Figura 1.4-1. Secciones típicas de Soportes Mixtos. 
Como características de estos elementos, podemos señalar ${ }^{57}$ :

- Proporcionan resistencia suficiente para cargas elevadas con secciones relativamente pequeñas (es necesario que la solicitación dominante sea de compresión).

- Los soportes recubiertos presentan buen comportamiento frente a la corrosión y sobre todo al fuego, siendo necesario en algunos casos recurrir a una solución de este tipo, para alcanzar los tiempos de estabilidad al fuego requeridos por la normativa vigente.

- En cualquier caso presentan buena resistencia al choque.

Como principales inconvenientes cabe citar que:

- En soportes rellenos, al quedar el acero exterior, no se obtiene la protección contra el fuego que se consigue en los soportes recubiertos, y además no es posible observar visualmente el aspecto acabado del hormigón, que es un mínimo control necesario, independientemente de que también haya que aplicar otras técnicas más fiables.

- Los métodos de cálculo, por la complejidad propia de estos elementos y la falta de tablas y gráficos de ayuda, resultan laboriosos.

- En el caso de piezas prefabricadas, presenta todas las ventajas de este tipo de construcción, con el inconveniente del peso considerable de los elementos que dificulta su transporte y puesta en obra.

57 "Estructuras mixtas para edificación : según criterios del Eurocódigo 4" Monfort Lleonart, J.(2002) 
Dado que los inconvenientes afectan, especialmente a los soportes rellenos y que en la práctica profesional es mas frecuente el uso de los soportes recubiertos, en el presente trabajo, se decide desarrollar los planteamientos teóricos para ésta última tipología citada.

No obstante, el trabajo que aquí se desarrolla, sería aplicable a los soportes metálicos rellenos sin más que considerar el incremento de resistencia del hormigón por el efecto de confinamiento que produce el tubo metálico.

\subsubsection{ENLACES}

Las uniones de las barras de sección mixta con el resto de elementos de la estructura requieren diseños específicos para garantizar que las solicitaciones de las vigas se transmiten correctamente a los materiales que componen el soporte.

A continuación se plantean y comentan algunas soluciones de nudos $^{58}$ entre soportes mixtos de los distintos tipos descritos en el apartado anterior y vigas mixtas, cuando éstas deben comportarse como articuladas o rígidas, ya que las uniones semi-rígidas se excluyen según el apartado 4.10.5.2 del EC4.

\section{Soporte de hormigón recubierto: enlace articulado.}

La unión se realiza mediante elementos metálicos, angulares y pletinas que enlazan los perfiles metálicos de la viga y el soporte, que, generalmente, quedan embebidos en el hormigón.

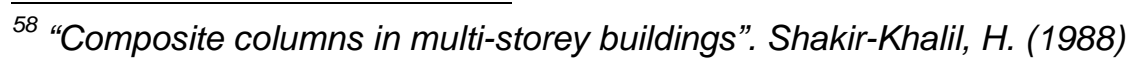



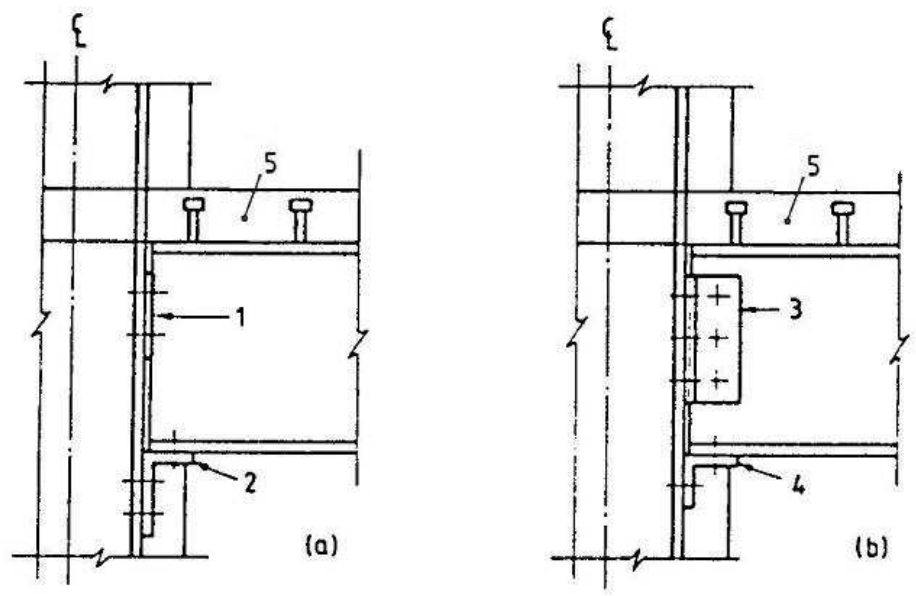

Figura 1.4-2 Soporte de hormigón recubierto: enlace articulado

El cortante de la viga puede transmitirse al soporte mediante el angular inferior (2) de la Figura 1.4-2 (a) en cuyo caso el elemento que sirve de apoyo provisional es el elemento del alma (1); o bien puede transmitirse mediante los angulares conectados al alma (3), tal como se muestra en la Figura 1.4-2 (b) en cuyo caso el angular inferior (4) es un apoyo provisional de montaje.
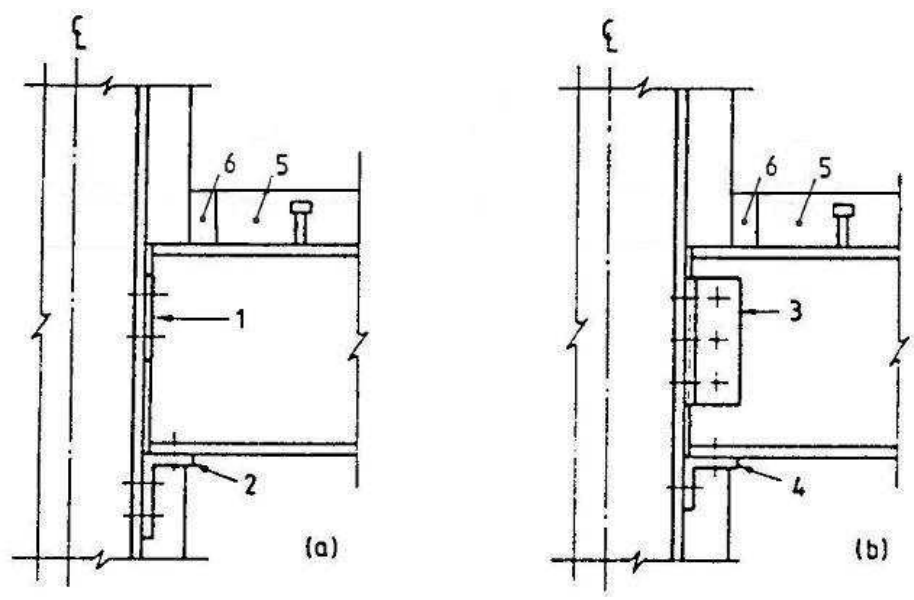

Figura 1.4-3 Junta entre el soporte y la cabeza de hormigón. 
Cuando se emplean vigas mixtas, la continuidad de la cabeza de hormigón (5) influye en la rigidez del nudo por quedar solidarizada con el hormigón del soporte, por lo que se puede disponer una junta (6) para evitarla (Figura 1.4-3).

\section{Soporte de hormigón recubierto: enlace rígido.}

En el caso de vigas mixtas, se requiere la continuidad de la cabeza de hormigón (5), cuyas armaduras son necesarias para transmitir los esfuerzos y evitar la fisuración.
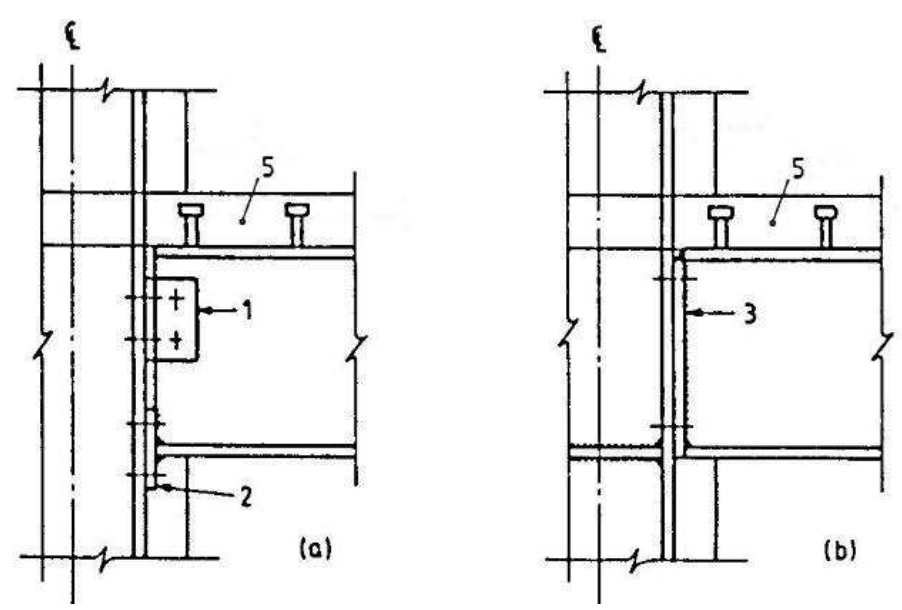

Figura 1.4-4 Soporte de hormigón recubierto: enlace rígido

En la disposición que se muestra en la Figura 1.4-4 (a), la compresión del ala inferior de la viga se transmite al soporte metálico por medio de una chapa (2), que puede hacer innecesaria la colocación de rigidizadores; la reacción vertical se transmite por los angulares (1) conectados al alma.

En la disposición (b) la chapa frontal (3) soldada a lo largo de toda la altura de la viga, transmite tanto el esfuerzo axil como la reacción 
vertical. La unión entre los elementos metálicos puede exigir el uso de rigidizadores del alma del soporte.

Tubos rellenos: enlace articulado.

Puesto que la unión se realiza entre elementos metálicos, se puede utilizar cualquier articulación usual entre estos materiales, si bien, los angulares deberán unirse al soporte mediante soldadura, ya que no pueden atornillarse a él.
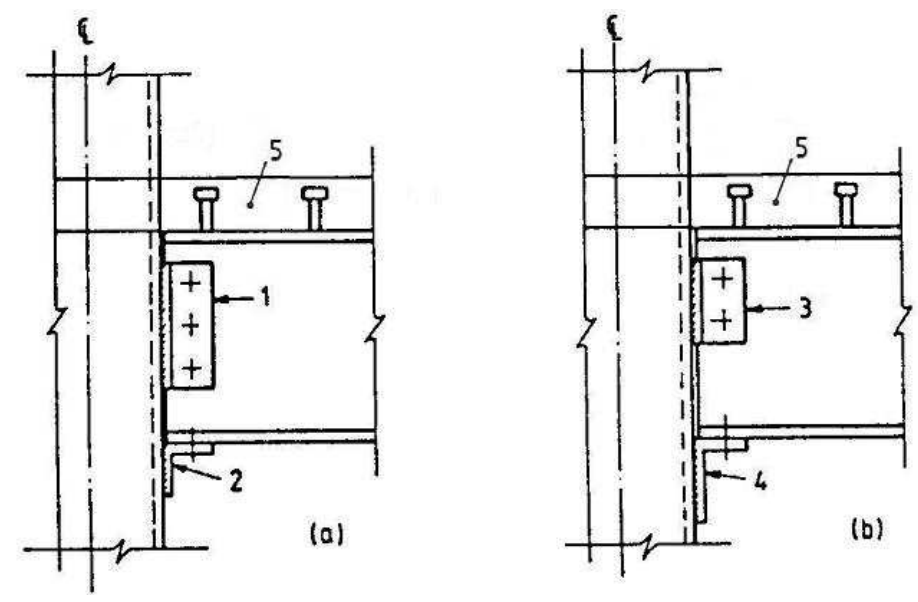

Figura 1.4-5 Tubos rellenos: enlace articulado

El cortante de la viga puede transmitirse al soporte mediante los angulares conectados al alma (1), tal como se muestra en la Figura 1.4-5 (a), en cuyo caso el angular inferior (2) es un apoyo provisional de montaje; o bien puede transmitirse mediante el angular inferior (4) de la disposición (b) en cuyo caso el elemento que sirve de apoyo provisional es el angular del alma (3).

Cuando se emplean vigas mixtas, la influencia de la continuidad de la cabeza de hormigón (5) en la rigidez del nudo es mínima (se sigue 
considerando articulado), aunque se debe disponer armadura para evitar la fisuración de la zona traccionada.

Tubos rellenos: enlace rígido.
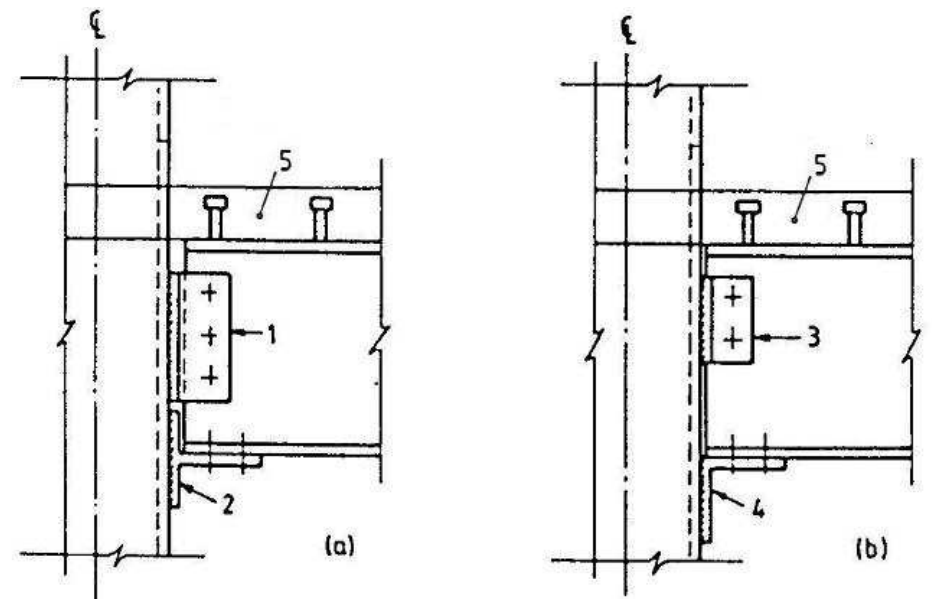

Figura 1.4-6 Tubos rellenos: enlace rígido

En la disposición (a) la fuerza de compresión del ala inferior es transmitida por la pieza en T (2), y la reacción vertical, por medio de los angulares (1) conectados al alma de la viga. Puede ser necesario comprobar la rigidez del soporte por el axil transmitido por el angular.

En la disposición (b) el par de fuerzas generadas por el momento se transmiten por la cabeza de hormigón y por el angular (4) conectado al ala inferior de la viga, mientras que el angular (3) conectado al alma de la viga hace solo de apoyo provisional de montaje. 


\subsubsection{MÉTODO GENERAL}

\subsubsection{GENERALIDADES}

Un pilar mixto de cualquier sección, cargado con fuerzas normales y momentos flectores, debe comprobarse frente a:

- Resistencia del elemento

- Resistencia frente al pandeo local

- Introducción de cargas

- Resistencia a cortante

\subsubsection{PROCESO DE CÁLCULO}

El cálculo de la estabilidad estructural debe tener en cuenta los efectos de segundo orden, incluyendo imperfecciones, y debe asegurar que, para la combinación de acciones más desfavorables en el estado límite último, no existe inestabilidad y no se supera la resistencia de las secciones individuales sometidas a flexión y a fuerza axil.

Los coeficientes de seguridad parciales serán los especificados en el apartado 3.1.4, con la excepción de $\gamma_{c}$ cuando se utilicen elementos prefabricados de hormigón.

Los efectos de segundo orden se considerarán en cualquier dirección en la pueda producirse la rotura, si afectan significativamente a la estabilidad estructural.

Se considerará la influencia de los efectos de segundo orden cuando el incremento sobre los momentos de primer orden, debidos a los movimientos en el pilar, excede del 10\%. Esta comprobación debe realizarse mediante un análisis elástico de segundo orden del pilar 
aislado, suponiendo que sus extremos están articulados y sometidos a las solicitaciones determinados en el análisis global, y con cargas transversales, si existieran.

Se supondrá que las secciones planas permanecen planas tras la deformación. Se supondrá una respuesta conjunta perfecta hasta la rotura entre los componentes de acero y hormigón del elemento.

Se recomienda utilizar las siguientes relaciones tensióndeformación ${ }^{59}$ en el análisis (no lineal):

- Para el hormigón, la dada en la Figura 3.1-2

- Para el acero de armar, la dada en la Figura 3.1-4

- Para el acero estructural, la dada en la Figura 3.1-5

Cuando se están calculando deformaciones de segundo orden, es recomendable utilizar el diagrama tensión deformación del hormigón dado en el apartado 4.2.1.3.3 del Eurocódigo $2^{60}$, en lo sucesivo EC2, con $f_{c d}=f_{c k} / \gamma_{c}$ y $E_{c d}=E_{c m} / \gamma_{c}$.

Será necesario considerar los efectos de retracción y fluencia si son capaces de reducir significativamente la estabilidad estructural.

Como simplificación, se pueden despreciar los efectos de la fluencia si el incremento de los momentos flectores de primer orden debido a las deformaciones por fluencia y a la fuerza longitudinal resultante de las cargas permanentes, no excede del $10 \%$.

Se pueden despreciar las deformaciones debidas a la fluencia de elementos esbeltos en compresión, en pórticos intraslacionales de

59 Las relaciones tensión-deformación cuyas figuras se indican a continuación se exponen en el apartado 3.1 .5 del presente trabajo.

${ }^{60}$ UNE ENV 1992-1-1 Eurocódigo 2: Proyecto de estructuras de hormigón. Parte 1-1: Reglas generales y reglas para edificación. AENOR 1993 
edificación con uniones rígidas a las losas o vigas en sus dos extremos (apartado A.3.4 (9) del EC2).

Puede considerarse la contribución de la resistencia a tracción del hormigón entre fisuras.

En elementos prefabricados de hormigón los coeficientes parciales de seguridad se tomarán de acuerdo con los apartados correspondientes del EC2.

\subsubsection{IMPERFECCIONES}

Las imperfecciones a lo largo del pilar se tendrán en cuenta en el cálculo de las solicitaciones.

Las imperfecciones geométricas iniciales equivalentes deben referirse a las curvas de pandeo especificadas en el apartado 5.5.1 del Eurocódigo $3^{61}$, en lo sucesivo EC3, en función del tipo de sección y de la relación de la flexión respecto el eje mayor de la sección de acero.

\subsubsection{PANDEO LOCAL DE ELEMENTOS DE ACERO}

Será necesario considerar en el cálculo de la resistencia del pilar, la influencia del pandeo local de elementos de acero.

${ }^{61}$ ENV 1993-1-1: Eurocodigo 3: Proyecto de estructuras de acero. Parte 11: Reglas generales y reglas para edificación. AENOR 1996 
Los efectos del pandeo local de elementos de acero en pilares mixtos pueden tratarse según el apartado siguiente para secciones embebidas, así como para otros tipos de pilares, siempre que:

- Para secciones de acero circulares huecas: $d / t \leq 90 \varepsilon^{2}$

- Para secciones de acero rectangulares huecas: $h / t \leq 52 \varepsilon$

- Para secciones en I parcialmente embebidas: $b / t_{f} \leq 44 \varepsilon$

Donde $d$ es el diámetro exterior de la sección de acero hueca circular, $h$ es la dimensión mayor de la sección, paralela al eje principal, $t$ es el espesor de la pared de una sección hueca rellena de hormigón, $t_{f}$ y $b$ son el espesor y el ancho total del ala de una sección de acero en I o similar, y $\varepsilon=\sqrt{235 / f_{y}}$ ( $f_{y}$ es el límite elástico del acero en $\mathrm{N} / \mathrm{mm}^{2}$ )

Si se superan los valores anteriormente especificados, los efectos del pandeo local deben tenerse en cuenta mediante un método apropiado, confirmado experimentalmente.

\subsubsection{RECUBRIMIENTO Y ARMADURA}

En secciones de acero embebidas debe disponerse un recubrimiento mínimo de hormigón armado, para asegurar la adecuada transmisión de las fuerzas de adherencia, la protección del acero contra la corrosión, que no se producirán desconchones en el hormigón y garantizar una resistencia adecuada al fuego, de acuerdo con el EC4.

Se recomienda que el recubrimiento de hormigón de un ala de acero de una sección embebida no sea menor de $40 \mathrm{~mm}$, ni menor de la sexta parte del ancho b del ala. 
El recubrimiento de la armadura debe estar de acuerdo con el apartado 4.1.3.3 del EC2 (se reproducen únicamente los aspectos que afectan en el presente trabajo):

Para transmitir las tensiones de adherencia de forma segura y garantizar a la vez una compactación adecuada, el recubrimiento de hormigón de la barra o el tendón considerados nunca deberá ser menor de $\phi \circ \phi_{n}$, o $(\phi+5 \mathrm{~mm}) \circ\left(\phi_{n}+5 \mathrm{~mm}\right)$ si $d_{g}>32 \mathrm{~mm}$, (siendo $\phi$ el diámetro de la barra, $\phi_{\mathrm{n}}$ el diámetro equivalente en el caso de grupos de barras y $d_{g}$ la dimensión nominal del tamaño máximo del árido).

El recubrimiento mínimo de hormigón de la armadura, incluyendo cercos y estribos no deber ser menor que los valores que se indican en la Tabla $1.4-1^{62}$.

\begin{tabular}{|c|c|c|c|c|c|c|c|c|c|c|}
\hline \multicolumn{2}{|c|}{} & \multicolumn{10}{|c|}{ Tipo de exposición } \\
\cline { 3 - 11 } & 1 & $2 a$ & $2 b$ & 3 & $4 a$ & $4 b$ & $5 a$ & $5 b$ & $5 c$ \\
\hline $\begin{array}{c}\text { Recubri- } \\
\text { miento } \\
\text { mínimo } \\
(\mathrm{mm})\end{array}$ & $\begin{array}{c}\text { Hormigón } \\
\text { armado }\end{array}$ & $|15|$ & $|20|$ & $|25|$ & $|40|$ & $|40|$ & $|40|$ & $|25|$ & $|30|$ & $|40|$ \\
\cline { 2 - 10 } & $\begin{array}{c}\text { Hormigón } \\
\text { pretensado }\end{array}$ & $|25|$ & $|30|$ & $|35|$ & $|50|$ & $|50|$ & $|50|$ & $|35|$ & $|40|$ & $|50|$ \\
\hline
\end{tabular}

Tabla 1.4-1 Recubrimientos mínimos para hormigones de peso normal

Los recubrimientos mínimos indicados en el apartado anterior también serán de aplicación para la armadura de piel, o bien se adoptarán medidas especiales de protección (por ejemplo, capas protectoras).

${ }^{62}$ Los tipos de exposición en ella mencionados se pueden consultar en la tabla 4.1 del artículo 4.1.3.3 del Eurocódigo 2. 
En el caso de elementos prefabricados, el margen de tolerancia se situará generalmente, en el intervalo $|0 \mathrm{~mm} \leq \Delta h \leq 5 \mathrm{~mm}|^{63}$, siempre que el control de ejecución pueda asegurar estos valores y que su obtención se verifique mediante control de calidad. En el caso de construcciones de hormigón armado in situ, el margen se situará en el intervalo | $5 \mathrm{~mm} \leq \Delta h \leq\left. 10 \mathrm{~mm}\right|^{64}$

El área de armadura longitudinal en pilares embebidos, que se supone colaborante en la resistencia de la sección, no debe ser menor del 0,3\% del área de la sección de hormigón.

La armadura transversal en pilares embebidos en hormigón debe calcularse según el apartado 5.4.1.2.2 del EC2 (se reproducen los aspectos más relevantes):

El diámetro de las armaduras transversales (cercos, horquillas o armaduras en espiral helicoidal) no será en ningún caso menor de $|6 \mathrm{~mm}|$ o bien |una cuarta parte| del máximo diámetro de las barras longitudinales, cualquiera que sea el mayor; el diámetro de los alambres de una malla para las armaduras transversales no será menor de $|5 \mathrm{~mm}|$

La armadura transversal se anclará adecuadamente.

La separación de la armadura transversal a lo largo del soporte no excederá nunca la menor de las tres distancias siguientes: 12 veces el diámetro mínimo de las barras longitudinales; la menor dimensión del soporte; y $300 \mathrm{~mm}$.

La separación se reducirá mediante un factor $|0,6|$ en los dos siguientes casos: secciones localizadas por encima o por debajo de

\footnotetext{
${ }^{63}$ Se utilizan módulos, siguiendo la notación utilizada por los Eurocódigos

${ }^{64}$ El capítulo 6 del EC2 indica las disposiciones adicionales relativas a la ejecución y la mano de obra (incluyendo tolerancias)
} 
una viga o losa en una altura igual a la mayor dimensión de la sección transversal del soporte; y cerca de un empalme por solapo si el máximo diámetro de las barras longitudinales es mayor de $|14 \mathrm{~mm}|$

Para la distancia entre armaduras es aplicable el apartado 5.2 del EC2 (se reproducen los aspectos más relevantes):

La separación de las barras será tal que el hormigón pueda ser colocado y compactado satisfactoriamente, quedando asegurado el desarrollo de la adecuada adherencia entre hormigón y armadura.

El tamaño máximo del árido, $d_{g}$, se elegirá de modo que permita una adecuada compactación del hormigón alrededor de las barras.

La distancia libre (horizontal y vertical) entre barras aisladas paralelas o capas horizontales de barras paralelas no deberá ser menor del |máximo diámetro de barra o $20 \mathrm{~mm}$. Además, si $d_{g}>32$ $\mathrm{mm}$, estas distancias no deberán ser menores de $d_{g}+5 \mathrm{~mm}$.

Las armaduras solapadas podrán tocar una con otra en la longitud de solapo.

La distancia libre entre las barras de la armadura longitudinal y la sección de acero estructural puede ser menor que la requerida, e incluso cero.

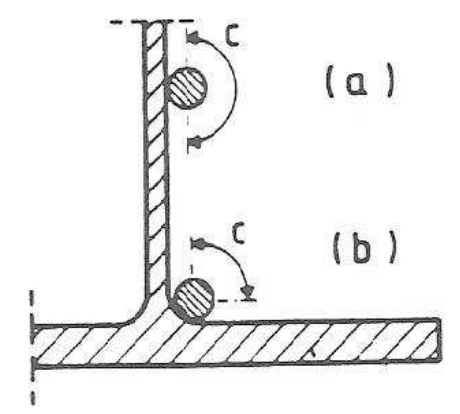

Figura 1.4-7 Perímetro eficaz c de un redondo 
En este caso, a efectos de adherencia, el perímetro eficaz $c$ de la armadura se tomará igual a la mitad o a una cuarta parte de su perímetro, tal como se muestra en la Figura 1.4-7 en (a) y (b) respectivamente.

La armadura de mallas electrosoldadas puede utilizarse como elemento de unión entre pilares embebidos, pero no debe contribuir o sustituir a la armadura longitudinal.

En secciones huecas rellenas generalmente no es necesaria la armadura longitudinal.

\subsubsection{RASANTE ENTRE LOS COMPONENTES DE ACERO Y HORMIGON}

Se tomarán medidas para que las fuerzas y momentos internos, provenientes de elementos unidos a los extremos de un pilar, sean distribuidos entre los componentes de acero y hormigón del pilar, considerando la resistencia a rasante en la superficie de contacto entre acero y hormigón, de acuerdo con el siguiente apartado "Resistencia al esfuerzo rasante"

Debe establecerse un recorrido claro y bien definido de transferencia de cargas que no implique un gran deslizamiento entre las superficies de contacto que invalidaría las hipótesis de cálculo.

No debe suponerse una longitud de transferencia del esfuerzo rasante mayor que el doble de la dimensión transversal pertinente del pilar.

En una sección en I, en la que sólo hay hormigón entre las alas, el hormigón debe estar sujeto con estribos y se debe establecer un recorrido de transmisión de la carga claramente definido entre el 
hormigón y el alma de acero (esto es, los estribos deben atravesar el alma, o estar soldados a la misma, o estar entrelazados a los conectadores).

Cuando los pilares mixtos están sometidos a un cortante transversal significativo, como por ejemplo en caso de cargas horizontales localizadas, se tomaran medidas para que el correspondiente esfuerzo rasante sea transmitido a través de la superficie de contacto entre el acero y el hormigón.

En ausencia de otro método más preciso, se debe utilizar el análisis elástico de la sección no fisurada, considerando la secuencia constructiva, para estimar el esfuerzo rasante producido por el cortante transversal entre el acero y el hormigón.

El esfuerzo rasante resultante, calculado en la superficie de contacto entre el acero y el hormigón, no debe ser excesivo en ningún punto, de acuerdo con el siguiente apartado.

\subsubsection{RESISTENCIA AL ESFUERZO RASANTE}

La resistencia a rasante debe venir proporcionada por las tensiones de adherencia y el rozamiento en la superficie de contacto, o por conexión mecánica, de forma que no haya un deslizamiento relativo significativo.

La resistencia para cálculo a rasante debida a adherencia y fricción debe tomarse como:

- Para secciones completamente embebidas en hormigón: $\quad 0,6 \mathrm{~N} / \mathrm{mm}^{2}$

- Para secciones huecas rellenas de hormigón: $\quad 0,4 \mathrm{~N} / \mathrm{mm}^{2}$

- Para alas en secciones parcialmente embebidas: $0,2 \mathrm{~N} / \mathrm{mm}^{2}$ 
- Para almas en secciones parcialmente embebidas: cero

Alternativamente, cabe mostrar mediante ensayos que se puede contar con una interacción plena acero-hormigón hasta la rotura del elemento.

\subsubsection{PERNOS DE CONEXION UNIDOS AL ALMA DE UN PILAR MIXTO}

Cuando los pernos de conexión están anclados al alma de una sección de acero en I embebida en hormigón o sección similar (ver Figura 1.4-8), la expansión lateral del hormigón contra el que se apoyan está impedida por las alas del acero adyacentes. Las fuerzas de rozamiento resultantes proporcionan una resistencia al rasante adicional a la dada por el apartado 6.3.2 del EC4 "Pernos conectadores en losas macizas"

Esta resistencia adicional puede suponerse igual a $\mu \cdot P_{R d} / 2$ en cada ala, por cada fila de pernos, como muestra la Figura 1.4-8, donde $P_{R d}$ es la resistencia de cálculo de un perno y $\mu$ el coeficiente de rozamiento correspondiente ${ }^{65}$.

En ausencia de mejor información obtenida de ensayos, estos valores sólo deben permitirse cuando la distancia libre entre las alas, como se muestra en la Figura 1.4-8, no exceda de:

- $300 \mathrm{~mm}$ utilizando un perno por fila;

- 400 mm utilizando dos pernos por fila;

- $600 \mathrm{~mm}$ utilizando tres pernos por fila;

${ }^{65}$ Definidos, respectivamente, en el apartado 6.3.2 y en el apartado 6.5.2 del EC4 


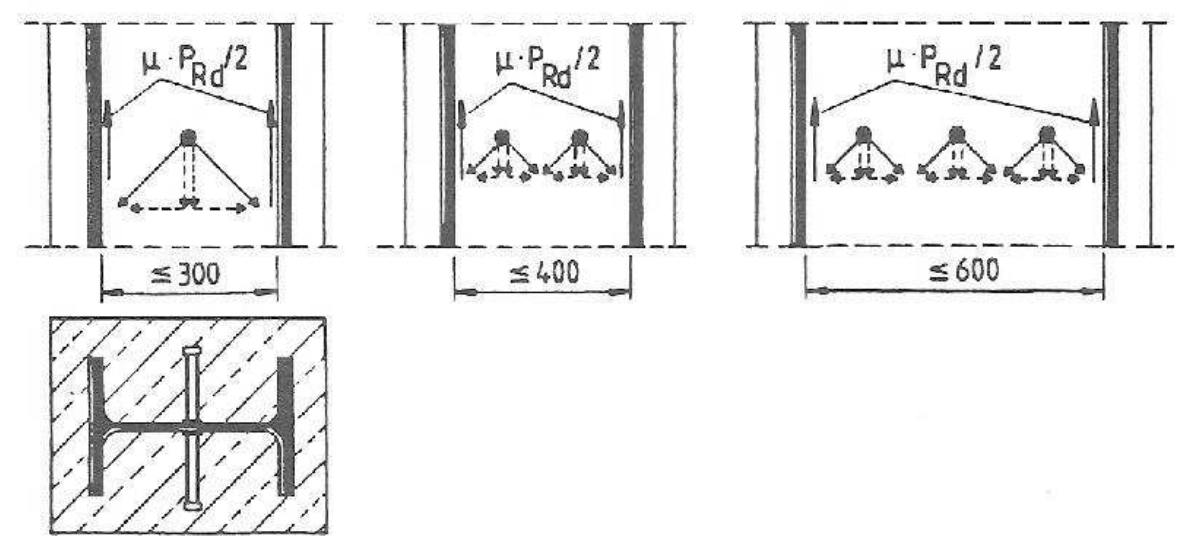

Figura 1.4-8 Pernos de conexión en un pilar mixto

\subsubsection{MÉTODO SIMPLIFICADO DE CÁLCULO}

\subsubsection{CAMPO DE APLICACIÓN}

En caso de aplicarse este método, debe utilizarse en su conjunto, de acuerdo con el apartado 4.8.1 (2) del EC4. Si se utilizan artículos concretos como parte de otro método, es preciso comprobar su validez.

Como este método tiene en cuenta las imperfecciones a lo largo del pilar, éstas no tienen que considerarse de nuevo; pero todas las demás prescripciones del "Método General" son aplicables cuando se utiliza el "Método Simplificado de Cálculo".

El campo de aplicación de este método simplificado está limitado a los casos en que: 
a) El pilar es de sección uniforme y doblemente simétrica a lo largo de toda su longitud ${ }^{66}$

b) El factor de contribución del acero $\delta$, tal como se definirá posteriormente, debe estar comprendido entre 0,2 y 0,9. Los elementos de acero pueden ser laminados o soldados.

c) La esbeltez adimensional $\bar{\lambda}$ tal como se definirá posteriormente, no debe ser mayor de 2,0.

d) Para secciones de acero embebidas, los límites de espesor del recubrimiento de hormigón que pueden utilizarse en los cálculos, son:

- En la dirección y: $40 \mathrm{~mm} \leq c_{y} \leq 0,4 \mathrm{~b}$

- En la dirección z: $40 \mathrm{~mm} \leq c_{z} \leq 0,3 \mathrm{~h}$

con la notación indicada en la Figura 1.4-1. Se puede utilizar un recubrimiento mayor, pero no se debe tener en cuenta en el cálculo.

e) El área de la sección transversal de la armadura longitudinal que puede considerarse en los cálculos no debe exceder del 4\% del área de hormigón.

f) Si la armadura longitudinal se desprecia en los cálculos de la resistencia del pilar y si el ambiente al que está expuesta la estructura corresponde a un ambiente seco para edificación

\footnotetext{
${ }^{66}$ Por tanto, los centros de gravedad de la sección de acero y de la sección de hormigón no fisurada, coinciden. Este punto se define como el baricentro mecánico de la sección, incluso cuando el momento flector es capaz de fisurar el hormigón. Algunas secciones monosimétricas se tratan en el anexo $D$ del EC4.
} 
(interiores de edificios para viviendas y oficinas), pueden admitirse como adecuadas las siguientes armaduras:

- Barras longitudinales de diámetro mínimo igual a $8 \mathrm{~mm}$ y con una separación máxima de 250 mm;

- Cercos de diámetro mínimo igual a $6 \mathrm{~mm}$ y con una separación máxima de 200 mm;

- Para armaduras de malla electrosoldada, los diámetros mínimos pueden reducirse a $4 \mathrm{~mm}$.

Las secciones típicas y su correspondiente notación se muestran en la Figura 1.4-1.

Se recomienda realizar las comprobaciones de cálculo de un pilar mixto siguiendo la siguiente secuencia:

a) Comprobar las limitaciones generales.

b) Comprobar el pandeo local.

c) Comprobar los recubrimientos y la armadura.

d) Calcular la carga crítica del pilar $N_{c r}$ y la esbeltez adimensional $\bar{\lambda}$ y determinar el coeficiente de seguridad parcial para el acero estructural $\gamma_{M a}$

e) Decidir si es necesario realizar un análisis de segundo orden para los momentos flectores.

f) Comprobar la resistencia del pilar.

g) Comprobar la introducción de cargas y el rasante. 


\subsubsection{COEFICIENTES DE SEGURIDAD PARCIALES}

Según el artículo 4.83.2 del EC4 y de acuerdo con el apartado 4.1.1 (5) del mismo Eurocódigo el coeficiente de seguridad para el acero estructural $\gamma_{M a}$ adopta uno de los dos valores siguientes ${ }^{67}$ :

a) $\gamma_{\mathrm{Ma}}=\gamma_{a}=1,00$ para pilares cuya esbeltez adimensional $\bar{\lambda} \leq 0,2$ ó con el cociente entre el axil de cálculo y su carga crítica $N_{S d} / N_{c r} \leq 0,1$

b) $\gamma_{M a}=\gamma_{R d}=1,00$ en los demás casos.

Las excepciones a los casos anteriores se exponen en los apartados correspondientes.

\subsubsection{RESISTENCIA PLÁSTICA A COMPRESIÓN DE LA SECCIÓN}

Este valor $N_{p l, R d}$ se obtiene sumando las resistencias plásticas de los componentes de la sección mixta:

$$
N_{p l, R d}=\left(A_{c} \cdot 0,85 \cdot \frac{f_{c k}}{\gamma_{c}}\right)+\left(A_{a} \cdot \frac{f_{y}}{\gamma_{a}}\right)+\left(A_{s} \cdot \frac{f_{s k}}{\gamma_{s}}\right)
$$

Ecuación 1.4-1

donde

$A_{a} A_{c} A_{s}$ áreas de las secciones transversales de acero estructural, hormigón y armadura, respectivamente.

\footnotetext{
${ }^{67}$ Aunque en los dos casos se adopta un valor $\gamma_{M a}=1,00$, esta clasificación permite identificar $\gamma_{M a}=\gamma_{R d}$, coeficiente de seguridad específico cuando los efectos de la inestabilidad pueden influir en la resistencia, o, en caso contrario, $\gamma_{\mathrm{Ma}}=\gamma_{\mathrm{a}}$ (coeficiente de seguridad parcial del acero estructural)
} 
$f_{y} f_{c k} f_{s k}$ resistencias características respectivas (para los aceros pueden tomarse su límite elástico).

$\gamma_{a} \gamma_{c} \gamma_{s}$ coeficientes de seguridad parciales en estados límite últimos.

En el caso de secciones huecas rellenas de hormigón, la resistencia plástica $N_{p l, R d}$ se puede calcular con $f_{c k}$ en vez de $0,85 f_{c k}$.

Para tubos de sección circular rellenos de hormigón, se puede tener en cuenta el incremento en la resistencia del hormigón debido al efecto de zunchado, siempre que:

- la esbeltez relativa $\bar{\lambda} \leq 0,5$ (ver Ecuación 1.4-5.)

- el mayor momento flector de cálculo obtenido con la teoría de primer orden $M_{\max , S d}$ no exceda de $\left(N_{s d} \cdot d\right) / 10$, donde $d$ es el diámetro exterior del pilar.

En este caso (tubos de sección circular), la resistencia plástica a compresión se puede calcular a partir de:

$$
N_{p l, R d}=\left(A_{c} \cdot \frac{f_{c k}}{\gamma_{c}}\right) \cdot\left[1+\eta_{1} \cdot(t / d) \cdot\left(f_{y} / f_{c k}\right)\right]+\left(A_{a} \cdot \eta_{2} \cdot \frac{f_{y}}{\gamma_{a}}\right)+\left(A_{s} \cdot \frac{f_{s k}}{\gamma_{s}}\right)
$$

Ecuación 1.4-2

donde $t$ es el espesor de la pared de acero del tubo y $\eta_{1}$ y $\eta_{2}$ se definen a continuación.

Los valores de los coeficientes $\eta$, cuando la excentricidad ${ }^{68} e=0$ (en este caso se designan $\eta_{10}$ y $\eta_{20}$ ), pueden obtenerse de la Tabla 1.4-2 o de forma analítica mediante las expresiones:

$\overline{{ }^{68} \text { La excentricidad e de la carga se define por el cociente } N_{\text {max.Sd }} / N_{S d}}$ 


$$
\begin{array}{ll}
\eta_{10}=4,9-18,5 \cdot \bar{\lambda}+17 \cdot \bar{\lambda}^{2} & (\text { pero siempre } \geq 0) \\
\eta_{20}=0,25 \cdot(3+2 \cdot \bar{\lambda}) & (\text { pero siempre } \leq 1)
\end{array}
$$

\begin{tabular}{|c|c|c|c|c|c|c|}
\hline$\lambda$ & 0 & 0,1 & 0,2 & 0,3 & 0,4 & $\geq 0,5$ \\
\hline$\eta_{10}$ & 4,90 & 3,22 & 1,88 & 0,88 & 0,22 & 0,00 \\
\hline$\eta_{20}$ & 0,75 & 0,80 & 0,85 & 0,90 & 0,95 & 1,00 \\
\hline
\end{tabular}

Tabla 1.4-2 Valores de $\eta_{10} y \eta_{20}$ cuando e $=0$

Para otros valores de $e$, los coeficientes $\eta_{1}$ y $\eta_{2}$ se determinan en función de los anteriores:

- $\quad$ para $0<e \leq d / 10$

$$
\begin{aligned}
& \eta_{1}=\eta_{10} \cdot(1-10 \cdot e / d) \\
& \eta_{2}=\eta_{20} \cdot\left(1-\eta_{20}\right) \cdot(10 \cdot e / d)
\end{aligned}
$$

- $\quad$ para $e>d / 10$

$$
\begin{aligned}
& \eta_{1}=0 \\
& \eta_{2}=1,0
\end{aligned}
$$

\subsubsection{FACTOR DE CONTRIBUCIÓN DEL ACERO}

El factor de contribución del perfil de acero se define como la relación del axil máximo que es capaz de resistir el perfil respecto del axil máximo que es capaz de resistir la sección completa:

$$
\delta=\frac{A_{a} \cdot f_{y} / \gamma_{a}}{N_{p l . R d}}
$$

donde $N_{p l . R d}$ se calcula con $\gamma_{M a}=\gamma_{a}$. 


\subsubsection{RIGIDEZ A FLEXIÓN ELÁSTICA EFICAZ (A CORTO PLAZO)}

La rigidez elástica eficaz a flexión $(E \cdot I)_{e}$, para cargas instantáneas, de una sección de un pilar mixto se calcula mediante la expresión:

$$
\left(E \cdot l_{e}=E_{a} \cdot l_{a}+0,8 \cdot E_{c d} \cdot l_{c}+E_{s} \cdot I_{s}\right.
$$

Ecuación 1.4-3

donde:

$l_{a} I_{c} I_{s} \quad$ momentos de inercia para el plano de flexión considerado del acero estructural, del hormigón (supuesto no fisurado) y la armadura, respectivamente.

$E_{a} E_{s} \quad$ módulos de elasticidad del acero estructural y de la armadura

$0,8 \cdot E_{c d} \cdot I_{c}$ rigidez eficaz de la parte de hormigón siendo $E_{c d}=E_{c m} / \gamma_{c}$.

$\gamma_{c}=1,00$ coeficiente de seguridad para la rigidez, dado por EC2 y $E_{c m}$ módulo secante del hormigón, dado por la tabla siguiente:

\begin{tabular}{|c|c|c|c|c|c|c|c|c|c|}
\hline $\boldsymbol{f}_{\boldsymbol{c k}}$ & $\mathbf{C 1 2 / 1 5}$ & $\mathbf{C 1 6 / 2 0}$ & $\mathbf{C 2 0 / 2 5}$ & $\mathbf{C 2 5 / 3 0}$ & $\mathbf{C 3 0 / 3 5}$ & $\mathbf{C 3 5 / 4 0}$ & $\mathbf{C 4 0 / 4 5}$ & $\mathbf{C 4 5 / 5 0}$ & $\mathbf{C 5 0 / 6 0}$ \\
\hline$E_{c m}$ & 26 & 27,5 & 29 & 30,5 & 32 & 33,5 & 35 & 36 & 37 \\
\hline
\end{tabular}

Tabla 1.4-3 Módulo secante del hormigón $\left(\mathrm{kN} / \mathrm{mm}^{2}\right)$

La influencia de la carga mantenida debe tenerse en cuenta de forma más precisa en la rigidez elástica eficaz a flexión, cuando la esbeltez relativa $\lambda$ en el plano de la flexión considerado excede el 
límite dado en la Tabla 1.4-4 y el cociente e/d es menor de 2, donde:

e excentricidad de la carga

d canto total de la sección en el plano de flexión considerado

$\delta$ factor de contribución del acero definido en el apartado anterior

$\bar{\lambda}$ es el definido en la Ecuación 1.4-5. Para comparar con los límites dados en la Tabla $1.4-4, \bar{\lambda}$ puede calcularse sin considerar la influencia de la carga mantenida en la rigidez a flexión.

\begin{tabular}{|l|c|c|}
\hline & $\begin{array}{c}\text { Pórticos intraslacionales } \\
\text { arriostrados }\end{array}$ & $\begin{array}{c}\text { Pórticos traslacionales y/o } \\
\text { pórticos no arriostrados }\end{array}$ \\
\hline $\begin{array}{l}\text { Secciones embebidas en } \\
\text { hormigón. }\end{array}$ & 0,8 & 0,5 \\
\hline $\begin{array}{l}\text { Tubos rellenos de } \\
\text { hormigón }\end{array}$ & $0,8 /(1-\delta)$ & $0,5 /(1-\delta)$ \\
\hline
\end{tabular}

Tabla 1.4-4 Valores límites de $\bar{\lambda}$

Bajo estas condiciones, el módulo de elasticidad eficaz del hormigón debe reducirse al valor:

$$
E_{c}=E_{c d} \cdot\left(1-0,5 \cdot N_{G . S d} / N_{S d}\right)
$$

siendo $N_{S d}$ la carga axil de cálculo del pilar y $N_{G . S d}$ la parte permanente de esta carga. 


\subsubsection{LONGITUD DE PANDEO DEL PILAR}

La longitud de pandeo I de un pilar mixto aislado intraslacional puede tomarse, de forma conservadora, igual a su propia longitud $L$.

Alternativamente, la longitud de pandeo puede determinarse utilizando el anexo E del EC3 y las siguientes reglas:

- Las rigideces a flexión de elementos adyacentes vinculados mediante elementos de unión rígidos, deben ser las utilizadas en el análisis del pórtico de acuerdo con el apartado 4.9.6.2 del EC4

- La tabla E.2 del EC3 puede aplicarse cuando las vigas son mixtas, o de acero, o de hormigón armado, y también cuando se utilizan losas de hormigón sin vigas.

\subsubsection{ESBELTEZ RELATIVA Y ADIMENSIONAL}

La carga crítica del pilar, $N_{c r}$, debe calcularse mediante la expresión:

$$
N_{c r}=\pi^{2} \cdot \frac{(E \cdot I)_{e}}{l^{2}}
$$

Ecuación 1.4-4

siendo $(E \cdot I)_{e}$ la determinada mediante la Ecuación 1.4-3 y I la longitud de pandeo del pilar, de acuerdo con el apartado anterior.

La esbeltez adimensional en el plano de la flexión considerado se calcula mediante a expresión:

$$
\bar{\lambda}=\sqrt{N_{p l . R} / N_{c r}}
$$


donde $N_{p l, R}$ es el valor de $N_{p l, R d}$, cuando los coeficientes $\gamma_{a}, \gamma_{c}$ y $\gamma_{s}$ se toman iguales a 1,0 .

\subsubsection{COMPRESIÓN SIMPLE}

Para garantizar la resistencia a compresión simple de estas piezas, se debe verificar en ambos ejes que

$$
N_{S d} \leq \chi \cdot N_{p l, R d}
$$

siendo $N_{S d}$ el axil considerado, afectado de los correspondientes coeficientes de mayoración de acciones y $\chi$ el coeficiente reductor por pandeo, función de la esbeltez adimensional $\bar{\lambda} \mathrm{y}$ de la curva de pandeo correspondiente ${ }^{69}$, debiendo considerarse:

- curva a para secciones huecas rellenas de hormigón.

- curva b para secciones en I, total o parcialmente recubiertas de hormigón, con flexión sobre el eje fuerte de la sección de acero.

- curva c para secciones en I, total o parcialmente recubiertas de hormigón, con flexión sobre el eje débil de la sección de acero.

\subsubsection{FLEXOCOMPRESIÓN}

El caso se refiere a la existencia de un momento flector en uno de los ejes principales de inercia de la sección, realizándose la comprobación respecto del mismo. Adicionalmente, se deben

${ }^{69}$ Curvas europeas de pandeo definidas en el artículo 5.5.1 del EC3. Parte 1-1: Reglas generales y reglas para edificación 
verificar las condiciones exigidas en el caso de la flexocompresión biaxial, considerando nulo el momento en la otra dirección.

Como norma general, los pilares se comprobarán teniendo en cuenta los efectos de segundo orden.

Los pilares aislados intraslacionales no requieren comprobación de los efectos de segundo orden, si:

- $\quad N_{S d} / N_{c r} \leq 0,1$ donde la carga crítica, $N_{c r}$ se determina según la Ecuación 1.4-4

- $\quad \bar{\lambda}_{\text {crit }} \leq 0,2$ para cualquier caso de carga

- no hay cargas transversales y la esbeltez adimensional $\bar{\lambda}_{\text {crit }} \leq 0,2 \cdot(2-r)$ siendo $r$ la relación entre los momentos en los extremos de la barra (diagrama lineal).

\begin{tabular}{|c|c|c|c|}
\hline Línea & Distribución de momentos & Factores $\boldsymbol{\beta}$ & Comentario \\
\hline 1 & $\begin{array}{l}\text { Momentos flectores de primer } \\
\text { orden de cargas laterales en } \\
\text { pilares aislados intraslacionales }\end{array}$ & $\beta=1,0$ & $\begin{array}{l}\text { Msd es el momento flector } \\
\text { máximo del pilar debido a } \\
\text { fuerzas laterales ignorando } \\
\text { efectos de segundo orden }\end{array}$ \\
\hline 2 & $\begin{array}{l}\text { Momentos en las extremos de un } \\
\text { pórtico intraslacional }\end{array}$ & $\begin{array}{c}\beta=0,66+0,44 r \\
\operatorname{con} \beta \geq 0,44\end{array}$ & $\begin{array}{l}\text { MSd y }_{S M_{S d} \text { son los momentos }} \\
\text { extremos de un análisis del } \\
\text { pórtico, de acuerdo con el } \\
\text { apartado } 4.9\end{array}$ \\
\hline
\end{tabular}

Tabla 1.4-5 Factores $\beta$ para la determinación de momentos.

Cuando no se cumplen estas condiciones, los efectos de segundo orden en un pilar aislado intraslacional, se pueden considerar de forma simplificada, incrementando los momentos flectores mayores 
de primer orden $M_{S d}$ mediante un factor corrector $k$ dado por la expresión:

$$
k=\frac{\beta}{1-N_{S d} / N_{c r}} \geq 1,0
$$

donde $N_{c r}$ es la carga crítica (Ecuación 1.4-4) para el eje correspondiente, con la longitud de pandeo / igual a la longitud del pilar, y $\beta$ es un factor de momento equivalente que adopta el valor

$$
-\beta=0,66+0,44 \cdot r \geq 0,44
$$

cuando no actúa carga transversal a lo largo de la longitud del soporte.

$$
-\beta=1,0
$$

Cuando actúa una acción combinada de momentos extremos y momentos de cargas laterales, salvo cálculos más precisos.

\subsubsection{RESISTENCIA DE SECCIONES SOMETIDAS A FLEXO- COMPRESIÓN MONOAXIAL}

La curva de interacción axil-flector para una sección sometida a flexocompresión simple, se representa en la Figura 1.4-9. Esta curva se considera definida por cinco puntos $A, B, C, D$ y E, simplificación que equivale a sustituirla por una poligonal (línea de puntos de la figura) que pueden calcularse suponiendo diagramas rectangulares de tensión. Estos puntos representan los valores de las solicitaciones (axil y momento) que agotan la sección en estado límite último; los puntos comprendidos entre esta curva y los ejes de coordenadas, corresponden a valores del axil y momento que no agotan la sección 
que estemos considerando. Esta curva no tiene en cuenta la esbeltez de la pieza, que se introduce posteriormente.

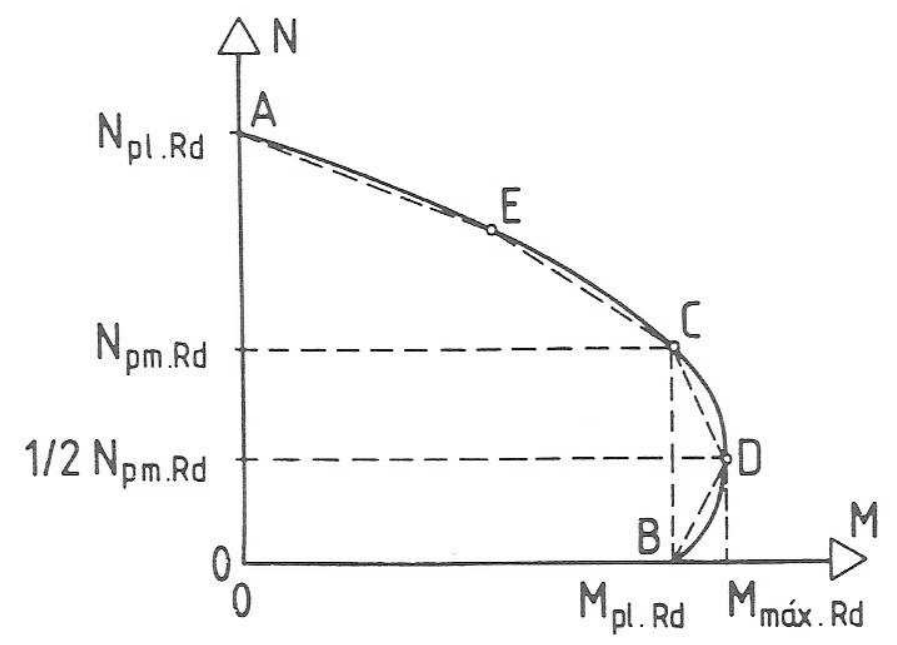

Figura 1.4-9. Diagrama de interacción para compresión y flexión monoaxial

En la Figura 1.4-10 se indican las distribuciones de tensiones que definen a los puntos $A, B, C$ y $D$ del diagrama de interacción, para una sección típica en $\mathrm{H}$ recubierta de hormigón con flexión respecto del eje fuerte de la sección de acero.

Cuando la resistencia del soporte a compresión ( $\left.\chi N_{p l . R d}\right)$ es mayor que la resistencia plástica de la sección de hormigón aislada $\left(N_{p m . R d}\right)$, debe obtenerse un punto adicional $E$ entre los puntos A y $C$ (la obtención de este punto no es necesaria para perfiles en $\mathrm{H}$ embebidos, con flexión según su eje fuerte). 

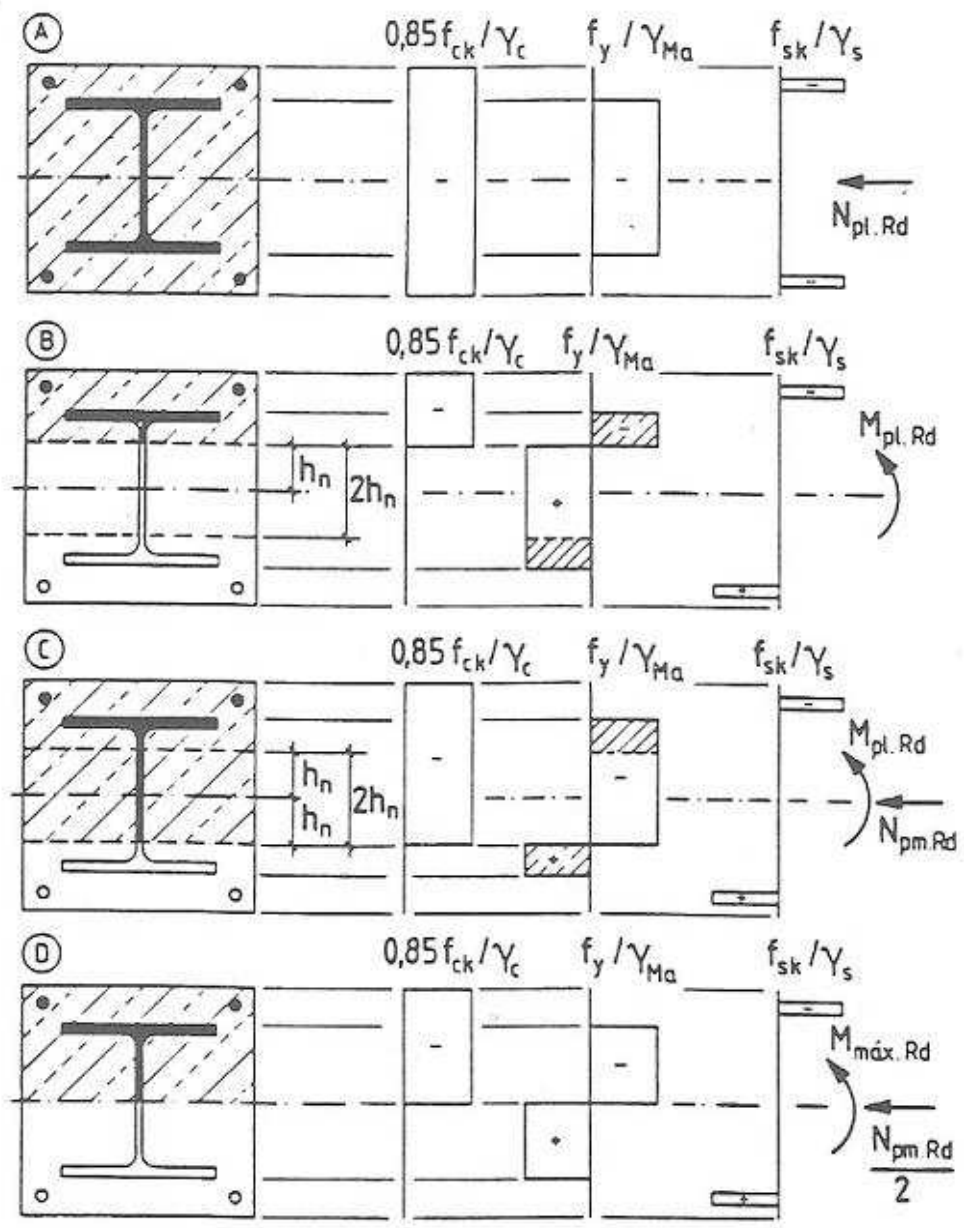

Figura 1.4-10. Distribución de tensiones en la sección

Los citados puntos del diagrama de interacción se determinan con los siguientes criterios:

- Punto A: corresponde al agotamiento de la sección en compresión simple $(M=0)$. La resistencia plástica a compresión $N_{\text {pl.Rd }}$ se calcula sumando la resistencia plástica de sus componentes. 


$$
N_{p l, R d}=\left(A_{c} \cdot 0,85 \cdot \frac{f_{c k}}{\gamma_{c}}\right)+\left(A_{a} \cdot \frac{f_{y k}}{\gamma_{a}}\right)+\left(A_{s} \cdot \frac{f_{s k}}{\gamma_{s}}\right)
$$

- Punto B: corresponde al agotamiento de la sección por flexión pura $(N=0)$. La distancia $h_{n}$ que define la posición de la fibra neutra, se calculará imponiendo la condición de equilibrio de fuerzas (sin considerar la resistencia a tracción del hormigón): la zona que aparece sin sombrear en las tracciones del acero estructural, de amplitud $2 \cdot h_{n}$, corresponde al esfuerzo axil que debe equilibrar las compresiones del hormigón.

Cuando la fibra neutra corta al alma de perfil metálico, la distancia $h_{n}$ se puede obtener mediante la expresión ${ }^{70}$ :

$$
h_{n}=\frac{N_{p m, R d}-A_{s n} \cdot\left(2 \cdot f_{s d}-f_{c d}\right)}{2 \cdot b_{c} \cdot f_{c d}+2 \cdot t_{w} \cdot\left(2 \cdot f_{y d}-f_{c d}\right)}
$$

siendo $N_{p m . R d}$ la resistencia a compresión de todo el área de hormigón:

$$
N_{p m, R d}=A_{c} \cdot f_{c d}
$$

Obtenido este valor, el momento resultante $M_{p l, R d}$ nos define la abscisa del punto se puede obtener mediante la expresión:

$$
M_{p l . R d}=M_{\max . R d}-M_{n \cdot R d}
$$

Ecuación 1.4-6

donde:

70 UNE ENV 1994-1-1 Eurocódigo 4: Proyecto de estructuras mixtas de hormigón y acero. Parte 1-1: Reglas generales y reglas para edificación. Anexo C. Método simplificado para el cálculo de la resistencia de secciones mixtas doblemente simétricas en flexión compuesta. AENOR 1995 


$$
M_{n . R d}=W_{p a n} \cdot f_{y d}+W_{p s n} \cdot f_{s d}+W_{p c n} \cdot f_{c d} / 2
$$

siendo $W_{p a n}, W_{p s n}$ y $W_{p c n}$ los módulos plásticos de la sección de acero estructural, la armadura y las partes de hormigón de la sección que están dentro de la región central de la sección de altura $2 \cdot h_{n}$, y que pueden obtenerse mediante las siguientes expresiones:

$$
\begin{aligned}
& W_{p a n}=t_{w} \cdot h_{n}^{2} \\
& W_{p n s}=\sum_{i=1}^{n}\left|A_{s n i} \cdot e_{z i}\right| \\
& W_{p c n}=b_{c} \cdot h_{n}^{2}-W_{p a n}-W_{p s n}
\end{aligned}
$$

- Punto C: su abscisa es la misma que la del punto B $\left(M_{p l . R d}\right)$, anteriormente determinada, y la ordenada viene definida por el valor del axil $N_{p m, R d}$ que agota la banda central de altura $2 \cdot h_{n}$ de la sección mixta.

A nivel tensional, este punto corresponde a una posición del eje neutro situada a una distancia $h_{n}$, pero al otro lado del eje. Comparando este diagrama de tensiones con el correspondiente al punto $\mathrm{B}$, vemos que las compresiones en la zona central del acero estructural son iguales a las del hormigón en la zona superior a la banda que estamos considerando (ver el equilibrio de fuerzas en el diagrama de B); por ello, estas compresiones en la sección central de acero equivalen, por simetría, a las que se producirían en la parte inferior del hormigón en el caso de que estuviese comprimida.

De aquí podemos concluir que $N_{p m, R d}$ es igual a la resistencia plástica de toda la sección de hormigón.

$$
N_{p m, R d}=A_{c} \cdot f_{c d}
$$


- Punto D: si hacemos coincidir la fibra neutra con el centro de gravedad de la sección completa (baricentro mecánico), el axil será la mitad del valor $N_{p m, R d}$ al que hemos hecho referencia en la determinación del punto anterior (los esfuerzos en el acero se anulan por simetría), y el momento resultante, que en este caso denominaremos $M_{\max , R d}$, vendrá dado por:

$$
M_{\text {max. } R d}=W_{p a} \cdot f_{y d}+W_{p s} \cdot f_{s d}+W_{p c} \cdot f_{c d} / 2
$$

donde $W_{p a}, W_{p c}$ y $W_{p s}$ son los módulos plásticos de las secciones de acero estructural, hormigón y armadura, respectivamente, que pueden determinarse mediante las siguientes expresiones:

$$
\begin{aligned}
& W_{p a}=\frac{\left(h-2 \cdot t_{f}\right) \cdot t_{w}^{2}}{4}+b \cdot t_{f} \cdot\left(h-t_{f}\right) \\
& W_{p s}=\sum_{i=1}^{n}\left|A_{s i} \cdot e_{i}\right| \\
& W_{p c}=\frac{b_{c} \cdot h_{c}^{2}}{4}-W_{p a}-W_{p s}
\end{aligned}
$$

- Punto E: cuando haya que determinar este punto, (no es necesario para perfiles en $\mathrm{H}$ con flexión según su eje fuerte), se supondrá la fibra neutra en una posición intermedia entre la correspondiente al punto $\mathbf{C}$ y el borde inferior de la sección.

Para secciones huecas rellenas de hormigón, las resistencias plásticas pueden ser calculadas sustituyendo $0,85 \cdot f_{c k}$ por $f_{c k}$.

Como simplificación en cualquier caso, la curva puede ser sustituida por un diagrama poligonal (línea de puntos de la Figura 1.4-9). En este caso, se debe determinar un punto adicional E, entre el A y el C, si la resistencia del pilar a compresión $\left(\chi \cdot N_{p l, R d}\right)$ es mayor que $N_{c, R d}$ siendo este último valor la resistencia plástica de la sección de 
hormigón por sí sola; esto no es necesario para perfiles en I con flexión sobre el eje fuerte de la sección de acero.

Determinada la curva de interacción, la comprobación resistente de una sección mixta consiste en verificar si el punto definido por las solicitaciones $\left(M_{s d}, N_{s d}\right)$ queda en su interior.

\subsubsection{INFLUENCIA DEL ESFUERZO CORTANTE}

Se puede considerar que el esfuerzo cortante transversal de cálculo $V_{S d}$ lo absorbe únicamente la sección de acero estructural, o que es compartido entre el acero y el hormigón.

Si se supone resistido solamente por el acero (caso más frecuente), no es necesario considerar su influencia sobre el momento plástico resistente, a menos que su valor exceda del $50 \%$ de la resistencia plástica a cortante $V_{p l, R d}$ dada por

$$
V_{p l . R d}=A_{v} \cdot\left(f_{y} / \sqrt{3}\right) \cdot \gamma_{a}
$$

Ecuación 1.4-7

donde $A_{v}$ es el área de cortante del elemento de acero estructural (prácticamente, el área del alma).

El criterio de interacción, cuando se supera el porcentaje anteriormente indicado, viene dado por la expresión:

$$
M_{S d} \leq M_{f . R d}+\left(M_{R d}-M_{f . R d}\right) \cdot\left[1-\left(2 V_{S d} / V_{p l . R d}-1\right)^{2}\right]
$$

donde:

$M_{S d}$ y $V_{S d} \quad$ momento y cortante de cálculo 
$V_{\text {pl.Rd }}$ resistencia plástica al cortante dada por la Ecuación 1.4-7

$M_{R d}$ resistencia de cálculo a flexión dada por la Ecuación 1.4-6

$M_{\text {f.Rd }}$ momento resistente plástico de una sección transversal que consta sólo de alas, con las mismas secciones eficaces que las usadas para el cálculo de $M_{R d}$.

\subsubsection{RESISTENCIA DE ELEMENTOS SOMETIDOS A COMPRESIÓN Y FLEXIÓN MONOAXIAL}

Conocida la curva de interacción de la sección mixta, la comprobación resistente de un soporte de esa misma sección, teniendo en cuenta sus imperfecciones y su esbeltez, consiste en verificar la siguiente ecuación:

$$
M_{S d} \leq 0,9 \cdot \mu \cdot M_{p l, R d}
$$

siendo:

$M_{S d}$ el máximo momento flector que actúa sobre el pilar considerado, calculado, cuando sea necesario, teniendo en cuenta los efectos de segundo orden,

0,9 coeficiente tiene en cuenta los errores introducidos por el método simplificado que se ha utilizado.

$M_{p l, R d}$ es el momento flector sobre la sección del pilar, calculado utilizando la distribución de tensiones que se indica en la Figura 1.4-10, punto B.

$\mu \quad$ capacidad resistente de la sección, una vez descontada la capacidad invertida en absorber los momentos debidos a las 
imperfecciones de la pieza. Este coeficiente se puede obtener de forma analítica mediante la expresión:

$\mu=\mu_{d}-\frac{\mu_{k} \cdot\left(\chi_{d}-\chi_{n}\right)}{\chi-\chi_{n}}$

donde:

$\mu_{d} \quad$ representa el momento flector que agota la sección actuando simultáneamente con el axil $N_{S d}$.

$\frac{\mu_{k} \cdot\left(\chi_{d}-\chi_{n}\right)}{\chi-\chi_{n}}$ capacidad resistente invertida en absorber los momentos debidos a las imperfecciones de la pieza.

Los parámetros que intervienen en la ecuación anterior, que se representan gráficamente en la Figura 1.4-11, se pueden determinar mediante el proceso siguiente:

- El diagrama de interacción axil-flector se representa de forma adimensional; para ello, las ordenadas del diagrama de la Figura 1.4-9, se dividen por $N_{p l, R d}$ y las abscisas por $M_{p l, R d}$. Resulta así la curva de la Figura 1.4-11, donde en ordenadas tenemos los axiles reducidos $\left(N_{R d} / N_{p l, R d}\right)$ y en abscisas los momentos reducidos $\left(M_{R d} / M_{p l, R d}\right)$.

- Obtenemos el valor del coeficiente reductor por pandeo $\chi$ de la pieza que queremos comprobar, con los criterios que se han indicado para compresión simple, apartado 1.4.3.8, (que tiene en cuenta las esbeltez de la pieza). A partir de este valor, y, entrando en la curva de interacción, obtenemos la abscisa correspondiente $\mu_{k}$. 


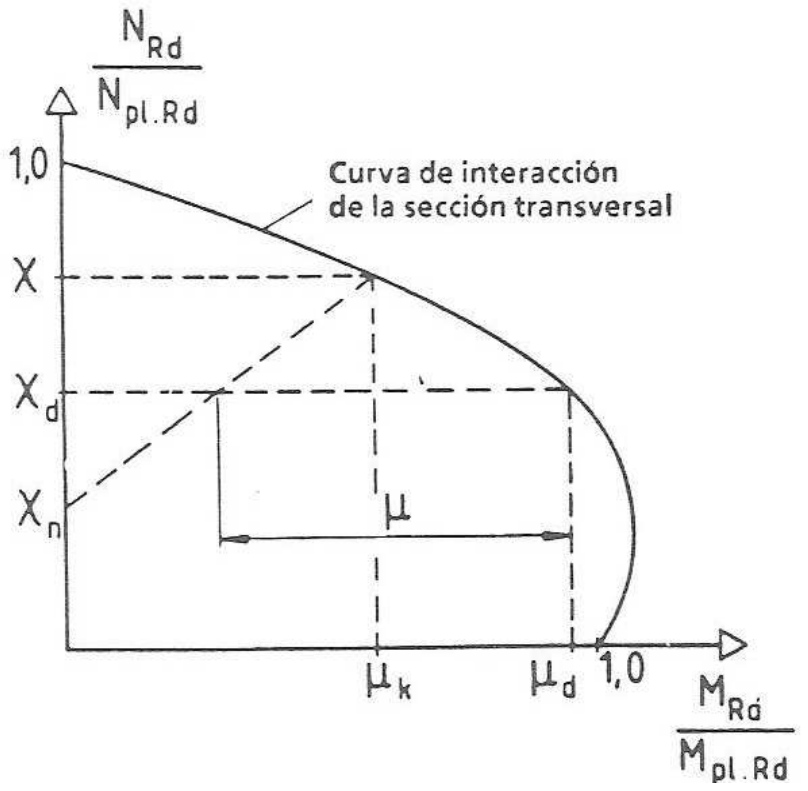

Figura 1.4-11. Diagrama de interacción adimensional

- Si la pieza está sometida a un axil $N_{S d}$, determinamos

$\chi_{d}=N_{S d} / N_{p l, R d}$

y la abscisa correspondiente $\mu_{d}$ entrando en la curva de interacción. Este valor representa el momento que agota la sección actuando simultáneamente con el axil $N_{S d}$.

- Determinamos el valor $\chi_{n}$ que, cuando la variación del momento flector a lo largo del pilar es lineal (no hay carga transversal), viene dada por $\chi_{n}=\chi \cdot(1-r) / 4$, con $\chi_{n} \leq \chi_{d}$, donde $r$ es la relación entre el menor y el mayor momento en los extremos de la barra; en caso contrario (soporte con cargas transversales), $\chi_{n}$ debe tomarse igual a cero. 


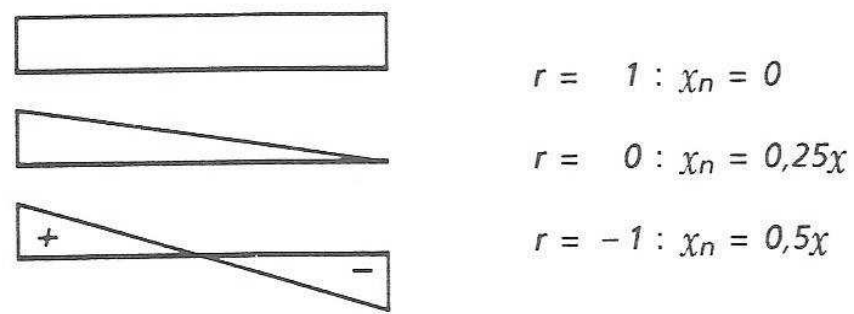

Figura 1.4-12. Valores típicos de $\chi_{n}$

- Se traza la recta que une los puntos de coordenadas $\left(0, \chi_{n}\right)$ y $\left(\mu_{k}, \chi\right)$, determinando el punto de intersección con la horizontal trazada por $\chi_{d}$.

Cabe tener en cuenta, además, las dos siguientes consideraciones

1. Cuando $N_{S d}<N_{p m, R d}$ (Figura 1.4-9), el incremento de resistencia a flexión debido a la fuerza normal (abombamiento del tramo inferior de la curva), puede ser sobrestimado si el axil actuante $N$ y el momento flector $M$ son independientes. En este caso se reduce el coeficiente de seguridad de la componente favorable $N_{S d}$ en un $20 \%$.

2. El valor de $\mu$ no debe ser superior a 1,0 a no ser que el momento flector $M_{S d}$ sea debido exclusivamente a la acción de la excentricidad de la fuerza $N_{S d}$ (por ejemplo, en un pilar aislado en el que no actúan cargas transversales entre sus extremos).

\subsubsection{COMPRESIÓN Y FLEXIÓN BIAXIAL}

En el caso de flexocompresión biaxial, la solicitación viene definida por tres valores: $N_{S d}, M_{y, S d}$ y $M_{z . S d}$. La interacción entre las 
solicitaciones da lugar a una superficie como la representada en la Figura 1.4-13.

Dada la compleja representación gráfica, es habitual trabajar con sus proyecciones sobre los planos de referencia, y comprobando el efecto del axil con cada uno de los momentos, por separado, y después los dos momentos se consideran conjuntamente.

Si se conoce el plano en que se agota la pieza, el efecto de las imperfecciones se considera únicamente en éste. En caso contrario, es necesario repetir el planteamiento para los dos planos.

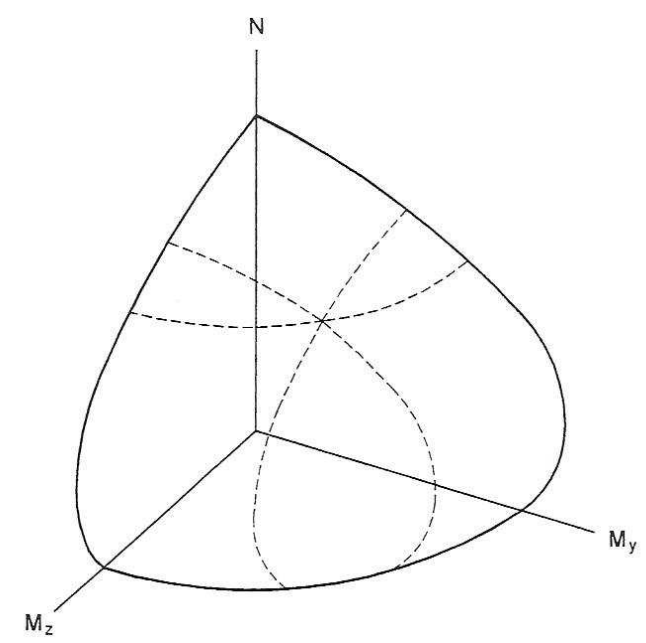

Figura 1.4-13. Representación tridimensional del diagrama de interacción

\section{Proceso de comprobación}

Se representan las dos curvas de interacción adimensionales, Figura 1.4-14, correspondientes al axil con cada uno de los momentos, siguiendo los criterios indicados en el apartado anterior. Estas curvas la son la intersección de la superficie de la Figura 1.4-13 con los planos coordenados verticales, representadas de forma adimensional. 


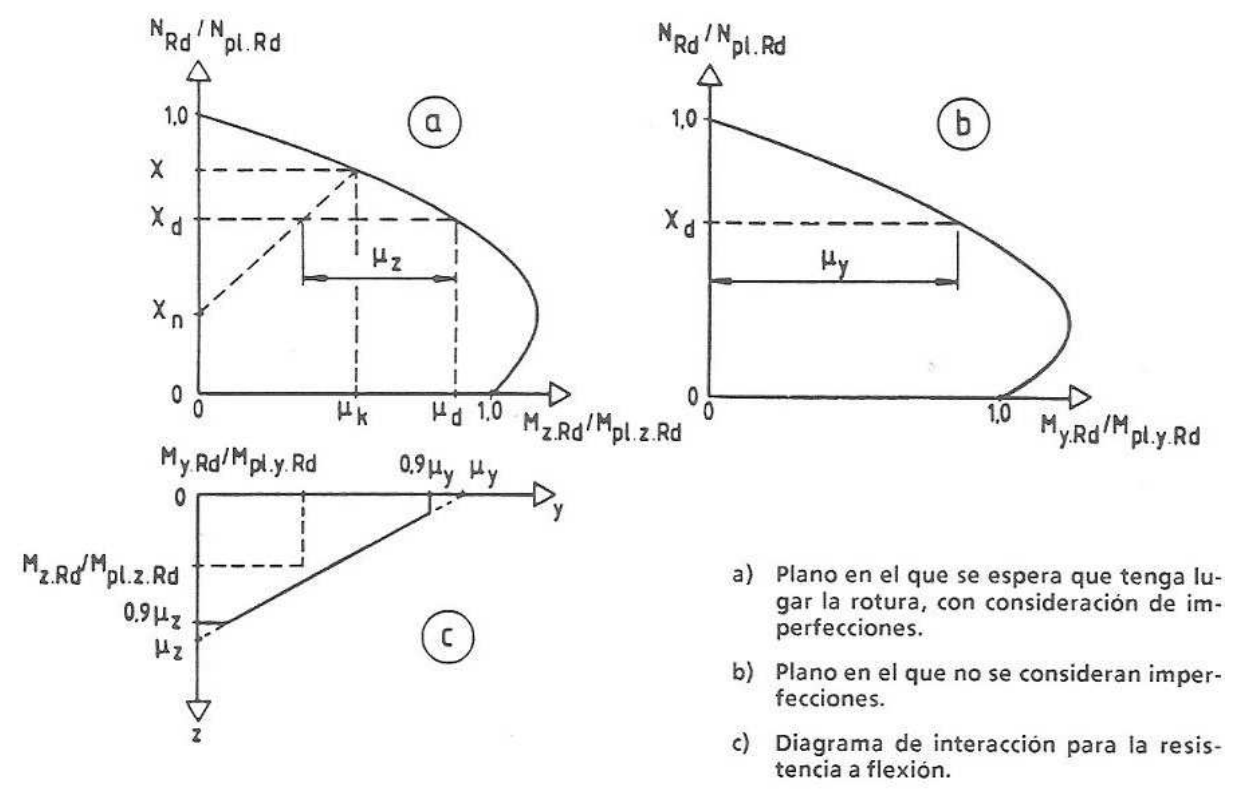

Figura 1.4-14. Curvas de interacción adimensionales

Los sistemas de referencia, tal como se aprecia en la figura, serán:

Figura a): Ordenadas: $N_{R d} / N_{p l, R d}$ y abscisas $M_{z . R d} / M_{p l, z, R d}$

Figura b): Ordenadas: $N_{R d} / N_{p l, R d}$ y abscisas $M_{y, R d} / M_{p l, y, R d}$

A continuación se obtiene el coeficiente reductor $\chi_{d}$ correspondiente al axil $N_{S d}$ mediante la Ecuación 1.4-8. Entrando en ambas gráficas se determinan las resistencias reducidas $\mu_{z}$ y $\mu_{y}$, incluyendo el efecto de las imperfecciones de la pieza sólo en el plano que se supone de agotamiento, en este caso considerado en la curva a). Para cada plano se deben cumplir las siguientes desigualdades:

$$
\begin{aligned}
& M_{y, S d} \leq 0,9 \cdot \mu_{y} \cdot M_{p l, y, R d} \\
& M_{z, S d} \leq 0,9 \cdot \mu_{z} \cdot M_{p l, z, R d}
\end{aligned}
$$


El análisis del efecto conjunto de los dos momentos flectores se construye una nueva curva de interacción, recta que pasa por los siguientes puntos coordenados: $\left(\mu_{y}, 0\right)$ y $\left(0, \mu_{z}\right)$, aunque truncada por los valores $0,9 \cdot \mu_{y}$ y $0,9 \cdot \mu_{z}$, tal como se aprecia en la Figura 1.4-14 c).

Si el punto que representa a los momentos reducidos $\left(M_{z . R d} / M_{p l, z, R d}\right.$, $\left.M_{y, R d} / M_{p l, y, R d}\right)$ queda comprendido entre las rectas y los ejes coordenados, la comprobación es correcta. Dicha comprobación también puede realizarse de forma analítica mediante la ecuación:

$$
\frac{M_{y, S d}}{\mu_{y} \cdot M_{p l, y, R d}}+\frac{M_{z, S d}}{\mu_{z} \cdot M_{p l, z, R d}} \leq 1,0
$$

Ecuación 1.4-9

La aceptación del dimensionamiento de la sección exige el cumplimiento simultáneo de las dos condiciones anteriores (Ecuación 1.4-8 y Ecuación 1.4-9). 
2. OBJETIVOS DEL TRABAJO 
El objetivo global del presente trabajo podría definirse genéricamente como el análisis de soportes mixtos sometidos a flexocompresión recta y esviada incluyendo el estudio de las diferentes variables que les afecta como la longitud de la barra, su esbeltez, imperfecciones iniciales, excentricidad de la carga, relaciones tensión-deformación de los materiales o los efectos reológicos en el hormigón.

Como resultado de este amplio objetivo planteado se pretende conseguir la representación de gráficos de interacción de soportes mixtos de varias secciones usuales en el campo de la edificación, de barras de distintas longitudes y diferentes excentricidades de la carga en los extremos.

No obstante, el desarrollo del trabajo ha ido planteando una serie de objetivos parciales sencillos, basados en el estudio y cálculo de la Sección, pero que a su vez sirvieran de base para la consecución de otros objetivos más ambiciosos, como el análisis de la Barra y, finalmente, el de la Estructura.

Y es así como finalmente ha quedado redactado el trabajo realizado, con los objetivos concretos que se describirán a continuación y cuyos resultados parciales y conclusión final se expondrán en los Capítulos posteriores.

\subsection{ESTUDIO DE LA SECCION MIXTA}

El "Método simplificado de cálculo" del Eurocódigo 4 contiene un proceso para la obtención del diagrama de interacción de la sección. $\mathrm{Si}$ bien la aplicación de este método simplificado exige el cumplimiento de una serie de condiciones, las que afectan a la sección de la barra, como son la doble simetría, un factor de 
contribución del acero comprendido entre 0,2 y 0,9, los espesores del recubrimiento de hormigón en los soportes embebidos o la limitación de la armadura longitudinal, son razonables y sencillas de cumplir.

El proceso propuesto consiste en el trazado de la curva a partir de 5 puntos cuyo cálculo se especifica en el apartado 1.4.3.10. Disponiendo de las curvas de interacción, la comprobación de una sección frente a un estado de flexocompresión recta consiste en comprobar que el punto $N_{d}-M_{d}$ queda en la superficie encerrada por la curva.

Dados los numerosos parámetros que se pueden modificar en el proceso de comprobación de una sección mixta como las dimensiones de la sección de hormigón, el tipo y tamaño del perfil estructural, el número y diámetro de las barras de refuerzo longitudinal o la resistencia carcterística de cada uno de los materiales, se ha considerado oportuno, como primer objetivo, el desarrollo de un programa informático que permita elaborar el diagrama de interacción de cualquier sección mixta embebida con similar proceso al indicado en el método simplificado del Eurocódigo, pero introduciendo dos matices:

- Puesto que el proceso es informático, se pueden obtener tantos puntos de paso del diagrama como se desee, simplemente con fijar una nueva posición del eje neutro.

- Las tensiones de cada punto no se consideran constantes e iguales a la tensión de cálculo de cada material, sino que a partir de la imposición de la condición de rotura, el valor de la tensión se obtiene entrando en la ley que relaciona la tensión con la deformación en cada material.

Además introduciendo una nueva variable, el giro del eje neutro respecto de los ejes de la sección, desplazándolo respecto del centro 
de gravedad e imponiendo la misma condición de agotamiento, se podrá obtener la superficie de interacción para el caso de la flexocompresión esviada.

De esta forma se han elaborado, como ejemplo de aplicación, los diagramas que se muestran en el apartado 4.6 de este trabajo.

Cabe indicar que si bien la representación del diagrama para el caso de la flexocompresión recta es plana y su uso práctico es sencillo, la representación en el caso de la flexocompresión esviada es tridimensional, y de compleja aplicación práctica. Para facilitar su uso, la superficie se ha seccionado por planos de axil constante y representado como un conjunto de curvas planas.

El programa desarrollado permite además la comprobación automática de la sección mixta que se desee sometida a flexocompresión plana o esviada, sin necesidad de pasar por los diagramas de interacción.

Las hipótesis comprendidas en este programa, el planteamiento conceptual para su desarrollo, la forma de entrada de datos y de cálculo de la sección, así como la verificación de su fiabilidad y la muestra de varios ejemplos como resultado de su uso, se exponen en el Capítulo 4 del trabajo que nos ocupa.

\subsection{EFECTOS REOLÓGICOS}

Uno de factores que intervienen en la capacidad resistente de los soportes mixtos son los efectos reológicos del hormigón.

El Eurocódigo 4, cuando se refiere al "Método General" para el cálculo de soportes mixtos, establece que deberán considerarse los efectos reológicos cuando éstos puedan reducir significativamente la 
estabilidad estructural, mientras que podrán despreciarse en dos casos:

1. cuando el incremento de los momentos flectores de primer orden debido a las deformaciones por fluencia y a la fuerza longitudinal resultante de las cargas permanentes, no excede del 10\%

2. cuando se trate de pórticos intraslacionales de edificación con uniones rígidas a las losas o vigas en sus extremos.

Puede consultarse la discusión sobre el criterio de los Eurocódigos en el apartado 5.3 de este trabajo.

Dado que el primer caso no puede verificarse a priori y puesto que el presente trabajo pretende extender su aplicación a soportes de estructuras traslacionales, se plantea también como objetivo, el estudio de sus efectos y de la forma de abordar su cálculo, así como el desarrollo de una opción en el programa informático que permita su consideración en el cálculo de los soportes. De esta forma se podrán elaborar gráficos de interacción para poder cuantificar sus efectos.

\subsection{ANÁLISIS DE LA BARRA}

El "Método simplificado de cálculo" del Eurocódigo 4 contiene un proceso para la comprobación de barras sometidas a flexocompresión recta o esviada, que tiene en cuenta la esbeltez de la pieza, las imperfecciones de la directriz y la relación entre los momentos en los extremos. Su aplicación exige el cumplimiento de las siguientes condiciones: el análisis se realizará sobre barras aisladas e intraslacionales ${ }^{71}$, la esbeltez reducida de la barra no debe

${ }^{71}$ Artículo 4.8.1 del Eurocódigo 4 
ser mayor de 2 y el efecto del esfuerzo cortante se considerará desacoplado respecto del resto de solicitaciones ${ }^{72}$.

En el caso de flexocompresión plana, el proceso de comprobación con este método simplificado, comienza por la representación de forma adimensional del diagrama de interacción de la sección. A continuación se localizan tres puntos; uno sobre el propio diagrama que tiene en cuenta la esbeltez de la pieza, y otros dos sobre el eje de ordenadas, uno que tiene en cuenta la variación de los momentos a lo largo de la barra y otro correspondiente al axil al que está sometido la barra (expresados de forma adimensional). A partir de ellos se obtiene la reducción de la capacidad resistente producida por las imperfecciones, consistiendo la comprobación de la barra en verificar que el momento solicitación no supera al $90 \%$ del momento reducido de la sección.

En el caso de la flexocompresión esviada, el Eurocódigo propone una extensión del método simplificado expuesto anteriormente, que exige la comprobación, de forma independiente para cada uno de los planos de flexión, más la comprobación simultánea con los dos momentos.

La explicación detallada de ambos procesos, se expone en los apartados 1.4.3.12 y 1.4.3.13, respectivamente.

Como objetivo en cuanto al análisis de la barra, se pretenden obtener los diagramas de interacción, hasta cierto punto similares a los de la sección, que permitan de una forma sencilla, la comprobación de soportes de sección mixta. Las variables adicionales de cálculo, que ahora se tendrán en cuenta, son la longitud de la barra, las imperfecciones iniciales, los efectos de segundo orden y la relación de momentos en los extremos de la barra. Por lo tanto, en este objetivo se conservan los dos

\footnotetext{
${ }^{72}$ Artículo 4.8.3.1 del Eurocódigo 4.
} 
condicionantes del tratamiento aislado de la barra y de su intraslacionalidad, requeridos en método simplificado del Eurocódigo 4 , anteriormente descrito.

En cuanto a la limitación de la esbeltez de la barra, el proceso de cálculo planteado en el presente trabajo no utiliza el método simplificado expuesto, sino que corresponde a lo que el Eurocódigo denomina "método general". En la determinación de la resistencia de un soporte se tendrán en cuenta los efectos que realmente se derivan de su esbeltez, por lo que no es necesario limitarla a un valor máximo ( $\bar{\lambda} \leq 2)$. La comprobación de un soporte podrá realizarse utilizando la tabla correspondiente o bien directamente en el programa informático si no se dispone de su gráfico.

Dada la cantidad de variables involucradas y la dificultad de la representación gráfica de los resultados, especialmente si se pretende un uso práctico de los mismos, para la obtención de los diagramas que se incluyen en el presente trabajo, se ha decidido adoptar una misma sección para todos los casos, fijar tres casos típicos de cargas que darán lugar a tres grupos de gráficas y variar la longitud del soporte desde los $3 \mathrm{~m}$ hasta los $10 \mathrm{~m}$ con intervalos de 1 metro.

La exposición de distintos métodos de resolución del problema no lineal de la barra comprimida, el método adoptado y los diagramas de interacción desarrollados como muestra del trabajo realizado se encuentran en los apartados $7.4,7.5$ y 7.6 , respectivamente.

Como herramienta de cálculo se ha utilizado un programa de análisis matricial no lineal en cuanto a la geometría y el material, en el cual se ha implementado el programa de cálculo de secciones mixtas desarrollado. 


\subsection{EL CONJUNTO ESTRUCTURAL}

La integración del programa de cálculo de secciones mixtas en el programa de análisis matricial de estructuras, AV_CID, que dispone de la posibilidad de realizar el cálculo no lineal, tanto geométrico como de material, abre la posibilidad de plantear dos nuevos objetivos:

1.- Disponer de un programa informático, que tras el análisis de una estructura, en régimen lineal o no lineal según se desee, permita la comprobación de los soportes mixtos y su re-dimensionamiento en caso necesario. Un programa de tales características sería de gran utilidad en las oficinas técnicas, especialmente de aquellas involucradas en el proyecto de estructuras de edificios "en altura".

2.- Disponer de un programa de tales características permite superar las dos limitaciones exigidas por el método simplificado del Eurcódigo 4: el análisis del soporte aislado y la intraslacionalidad de la estructura. Se trataría de un programa de indudable valor para el desarrollo de proyectos de investigación. Como muestra de sus posibilidades se plantean dos líneas, que tienen que ver con cada una de las mencionadas limitaciones que en esta situación se pueden obviar:

a) La condición de intraslacionalidad de la barra (desplazamiento pequeño y no significativo de sus extremos respecto de la posición inicial) implica, habitualmente, la necesidad de disponer de sistemas de arriostramiento horizontal, triangulaciones, pantallas o núcleos de hormigón, etc, para conseguir la intraslacionalidad del pórtico, ya que conseguirla con la propia rigidez del pórtico conduce, cuando el número de plantas es considerable, a un sobredimensionamiento de los elementos. 
La primera línea de investigación consiste en comprobar el efecto de la ausencia de sistemas específicos de arriostramiento, confiando únicamente en la propia rigidez del pórtico y por lo tanto asumiendo que se producirán movimientos horizontales, tanto mayores cuanto mayores sean la altura del edificio y las acciones horizontales y cuanto menor sea la rigidez del pórtico.

b) El hecho de considerar el soporte aislado implica que el efecto del resto del entramado del que forma parte, es sustituido por las solicitaciones, fuerzas y momentos, en los extremos de cada barra. Este planteamiento impide valorar los efectos de la plastificación progresiva de las barras que produce una variación de su rigidez y modifica, por tanto, el reparto de las solicitaciones.

La segunda línea de investigación consiste en comprobar la incidencia en la variación de las solicitaciones por efecto de la plastificación de las barras, observando como afecta a la seguridad global del edificio. 


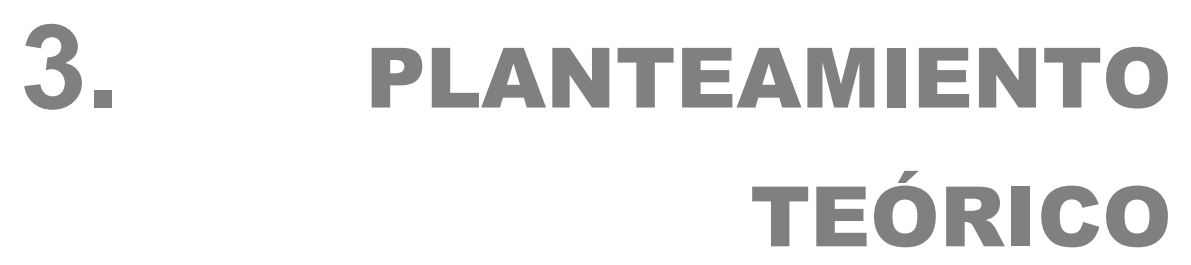




\subsection{HIPÓTESIS DE CÁLCULO}

\subsubsection{SOBRE EL ELEMENTO}

Se debe garantizar que, para la más desfavorable combinación de acciones en el estado límite último, no se supera la resistencia de los materiales en ninguna de las secciones y que además no se produce inestabilidad de la barra debido a los efectos de segundo orden.

En cuanto a las imperfecciones iniciales a tener en cuenta en la evaluación de los efectos de segundo orden, se tendrá en cuenta el artículo 4.8.2.3(2) del EC4:

Las imperfecciones geométricas iniciales equivalentes, deben referirse a las siguientes curvas de pandeo dadas en el apartado 5.5.1 del EC3:

curva b para secciones en I total o parcialmente embebidas en hormigón, con flexión alrededor del eje mayor de la sección de acero.

curva c para secciones en I total o parcialmente embebidas en hormigón, con flexión alrededor del eje menor de la sección de acero.

Estas imperfecciones, según el artículo 5.5.1.3(5) del EC3, vienen definidas por la flecha $e_{0, d}$ de la Figura 3.1-1, que, cuando en la sección consideramos un comportamiento plástico no lineal, y en la estructura aplicamos análisis elastoplástico, adopta el valor:

eje de referencia y-y (fuerte), curva b

$$
e_{0, d}=0,34 \cdot(\bar{\lambda}-0,2) \cdot(0,85+0,30 \bar{\lambda}) \cdot W_{p l} / A
$$

eje de referencia z-z (débil), curva c 


$$
e_{0, d}=(0,89+0,22 \bar{\lambda}) \cdot 1 /(270 \cdot \varepsilon)
$$

donde la designación de ejes corresponde a la la Ecuación 1.4-8, y $\varepsilon=\left(235 / f_{y}\right)^{0,5}$ con $f_{y}$ en $\mathrm{N} / \mathrm{mm}^{2}$.

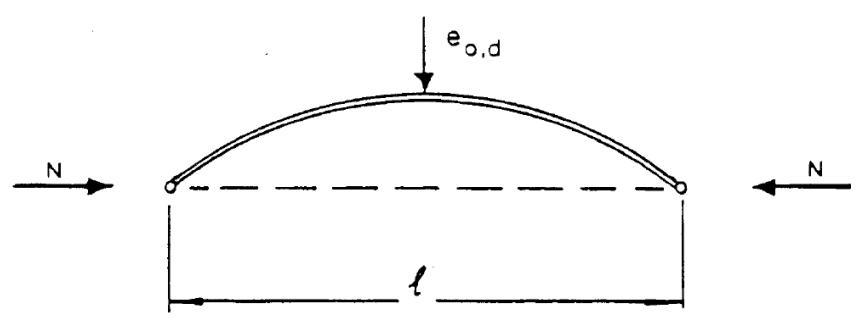

Figura 3.1-1. Imperfecciones geométricas iniciales equivalentes

\subsubsection{COMBINACION DE ACCIONES}

Según EC4, para cada hipótesis de carga, los valores de cálculo de los efectos $E_{d}$ de las acciones se determinarán a partir de las reglas de combinación en las que intervengan los valores de cálculo de aquellas acciones que se indican en la Tabla 3.1-1:

\begin{tabular}{|c|c|c|c|c|}
\hline \multirow{2}{*}{$\begin{array}{c}\text { Situación de } \\
\text { proyecto }\end{array}$} & \multirow{2}{*}{$\begin{array}{c}\text { Acciones } \\
\text { permanentes } \\
\mathrm{G}_{\mathrm{d}}\end{array}$} & \multicolumn{2}{|c|}{ Acciones variables $Q_{d}$} & \multirow{2}{*}{$\begin{array}{c}\text { Acciones } \\
\text { accidentales } \\
\mathrm{A}_{d}\end{array}$} \\
\hline & & $\begin{array}{c}\text { Acción } \\
\text { variable } \\
\text { principal }\end{array}$ & $\begin{array}{c}\text { Acciones } \\
\text { variables } \\
\text { concomitantes }\end{array}$ & \\
\hline $\begin{array}{c}\text { Persistente y } \\
\text { transitoria }\end{array}$ & $\gamma_{G} \cdot G_{k}$ & $\gamma_{\mathrm{Q}} \cdot \mathrm{Q}_{\mathrm{k}}$ & $\psi_{0} \cdot \gamma_{\mathrm{Q}} \cdot \mathrm{Q}_{\mathrm{k}}$ & - \\
\hline $\begin{array}{l}\text { Accidental (si } \\
\text { no se } \\
\text { especifica de } \\
\text { otra manera } \\
\text { en otro sitio) }\end{array}$ & $\gamma_{G A} \cdot G_{k}$ & $\psi_{1} \cdot Q_{k}$ & $\psi_{2} \cdot Q_{k}$ & $\begin{array}{c}\gamma_{A} \cdot A_{k} \\
\text { Si } A_{d} \text { no se } \\
\text { especifica } \\
\text { directamente }\end{array}$ \\
\hline
\end{tabular}

Tabla 3.1-1 Valores de cálculo de las acciones a utilizar en su combinación

Los valores de cálculo de la tabla anterior, se combinarán utilizando las siguientes reglas (que se dan en forma simbólica): 
- Situaciones de proyecto persistentes y transitorias para comprobaciones que no sean de fatiga (combinaciones fundamentales):

$$
\sum_{j} \gamma_{G, j} \cdot G_{k, j}+\gamma_{Q, 1} \cdot Q_{k, 1}+\sum_{i>1} \gamma_{Q, i} \cdot \psi_{o, i} \cdot Q_{k, i}
$$

Ecuación 3.1-1

- Situaciones de proyecto accidentales (si no se especifica de otra forma en otra parte):

$$
\sum_{j} \gamma_{G A, j} \cdot G_{k, j}+A_{d}+\psi_{1,1} \cdot Q_{k, 1}+\sum_{i>1} \psi_{2, i} \cdot Q_{k, i}
$$

Ecuación 3.1-2

donde:

$G_{k, j} \quad$ son los valores característicos de las acciones permanentes

$Q_{k, 1} \quad$ es el valor característico de una de las acciones variables

$Q_{k, i} \quad$ son los valores característicos del resto de las acciones variables

$A_{d}$ es el valor de cálculo (valor especificado) de la acción accidental

$\gamma_{G, j}$ es el coeficiente de seguridad parcial para la acción permanente $G_{k, j}$

$\gamma_{G A, j}$ es como $\gamma_{G, j}$ pero para acciones accidentales de proyecto

$\gamma_{Q, i} \quad$ es el coeficiente de seguridad parcial para la acción variable $Q_{k, i}$

$\psi_{0}, \psi_{1}, \psi_{2}$, son coeficientes de combinación ${ }^{73}$.

${ }^{73}$ UNE ENV 1990 Eurocódigos: Bases de cálculo de estructuras. AENOR 2003 
Las combinaciones de situaciones accidentales de proyecto, o bien involucran a una acción accidental explícita $A$, o bien se refieren a una situación posterior a un suceso accidental $(A=0)$. Si no se especifica lo contrario se puede utilizar $\gamma_{G A}=1,0$

En las ecuaciones de combinación de acciones se introducirán las acciones indirectas cuando sea pertinente.

\subsubsection{VALORES DE CÁLCULO DE LAS ACCIONES PERMANENTES}

En las diferentes combinaciones, anteriormente definidas, aquellas acciones permanentes que aumentan el efecto de las acciones variables (es decir, que producen efectos desfavorables) deben representarse por sus valores superiores de cálculo; y aquellas que disminuyen el efecto de las acciones variables (es decir, que producen efectos favorables) por sus valores inferiores de cálculo.

Cuando los resultados de una comprobación puedan ser muy sensibles a variaciones en la magnitud de la misma acción permanente de un lugar a otro de la estructura, esta acción deberá tratarse como formada por una parte desfavorable y otra favorable. Esto es aplicable, en particular, a la comprobación del equilibrio estático.

Cuando una única acción permanente está descompuesta en dos, una desfavorable y otra favorable, se permite utilizar una relación entre estas partes adoptando valores especiales de cálculo.

Excepto para los casos anteriormente mencionados, el conjunto de cada acción permanente debe representarse en toda la estructura por su valor de cálculo superior o inferior, adoptando el que ofrezca un efecto más desfavorable. 
Para vigas y pórticos continuos, puede aplicarse a todos los vanos el mismo valor de cálculo del peso propio de la estructura, excepto para los casos en que se considera el equilibrio estático de ménsulas.

\subsubsection{COEFICIENTES DE SEGURIDAD}

a) Coeficientes de seguridad parciales para acciones

Los coeficientes de seguridad parciales para acciones en estructuras de edificación, para las situaciones persistentes y transitorias serán los definidos en la siguiente tabla:

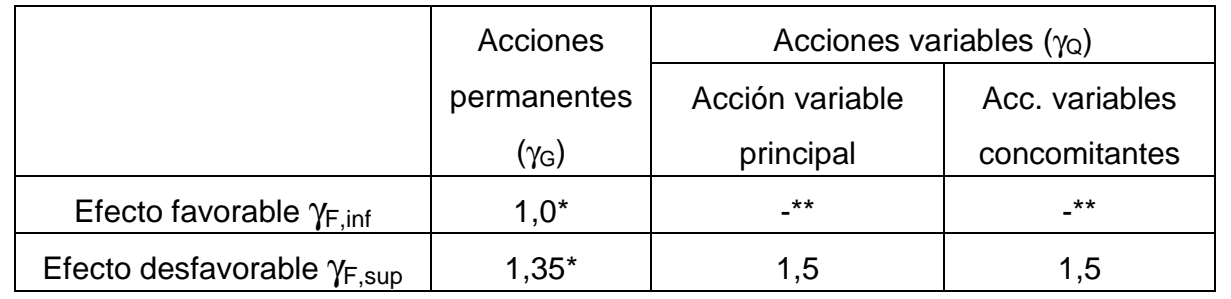

* Ver lo especificado en los párrafos siguientes

${ }^{*}$ Ver Eurocódigo 1: en casos normales para estructuras de edificación, $\gamma_{Q, i n f}=0$

Tabla 3.1-2 Coeficientes de seguridad parciales para acciones

Para situaciones accidentales de proyecto, a las que es aplicable la Ecuación 3.1-2, los coeficientes de seguridad parciales para acciones variables se tomarán iguales a 1,0. Para acciones permanentes, se tendrá en cuenta el apartado anterior.

Cuando una acción permanente debe ser descompuesta en dos, una desfavorable y otra favorable, la parte favorable puede multiplicarse, como alternativa, por $\gamma_{\text {Ginf }}=1,1$ y la parte desfavorable por $\gamma_{\text {Gsup }}=1,35$ con tal de que, al aplicar $\gamma_{\text {Ginf }}=1,0$ a la parte favorable y desfavorable, no se produzca un efecto más desfavorable. 
Para deformaciones impuestas, cuando se utilicen métodos de análisis no lineal, se aplicarán los coeficientes para acciones accidentales. Para un cálculo lineal, el coeficiente para efectos desfavorables se debe reducir en un $20 \%$.

Para efectos vectoriales (por ejemplo, axil y flector independientes) en pilares, si una de las componentes del efecto es favorable (caso del axil cuando $N_{S d}<N_{p m, R d}$ ) el coeficiente de seguridad de esta componente se reducirá en un $20 \%$.

En estructuras de edificación, como simplificación, la Ecuación 3.1-1 se puede sustituir por la más desfavorable de las dos expresiones siguientes:

Considerando sólo la acción variable más desfavorable:

$$
\sum_{j} \gamma_{G, j} \cdot G_{k, j}+\gamma_{Q, 1} \cdot Q_{k, 1}
$$

Considerando sólo la acción variable más desfavorable:

$$
\sum_{j} \gamma_{G, j} \cdot G_{k, j}+0,9 \cdot \sum_{i \geq 1} \gamma_{Q, i} \cdot Q_{k, i}
$$

b) Coeficientes de seguridad parciales para la resistencia y las propiedades de los materiales

Los coeficientes de seguridad parciales para la minoración de la resistencia característica y de otras propiedades de los materiales, serán los dados por la tabla siguiente: 


\begin{tabular}{|c|c|c|c|c|}
\hline Combinación & $\begin{array}{c}\text { Acero } \\
\text { estructural } \\
\gamma_{\mathrm{a}} \\
\left(=\gamma_{\mathrm{m} 0} \text { en EC3 }\right)\end{array}$ & $\begin{array}{c}\text { Hormigón } \\
\gamma_{c}\end{array}$ & $\begin{array}{c}\text { Acero de } \\
\text { armar } \\
\gamma_{\mathrm{s}}\end{array}$ & $\begin{array}{c}\text { Chapa } \\
\text { nervada } \\
\gamma_{\mathrm{ap}}\end{array}$ \\
\hline Fundamental & 1,10 & 1,5 & 1,15 & 1,10 \\
\hline Accidental $^{*}$ & 1,0 & 1,3 & 1,0 & 1,0 \\
\hline
\end{tabular}

Accidental excepto terremotos

Tabla 3.1-3 Coeficientes de seguridad parciales para la resistencia de los materiales

Los valores especificados en la Tabla 3.1-3 tienen en cuenta las diferencias entre la resistencia de las probetas de ensayo del material estructural y su resistencia in situ. Son aplicables a algunas propiedades mecánicas, pero sólo en los casos especificados en los apartados correspondientes; en otros casos deben ser sustituidos por $\gamma_{M}=1,0$. Para coeficientes físicos no mecánicos (por ejemplo, densidad, coeficiente de dilatación) $\gamma_{M}$ debe tomarse como 1,0.

\subsubsection{MATERIALES}

Como relaciones tensión-deformación de los materiales, se consideran las siguientes:

a) En el hormigón

Diagrama para el análisis estructural: ${ }^{74}$

Para el análisis estructural teniendo en cuenta los efectos no lineales, se adoptará como diagrama tensión-deformación del hormigón el de la Figura 3.1-2, definido por el módulo de elasticidad $E_{c, \text { nom }}$, por la resistencia a compresión del hormigón $f_{c}$ y

74 UNE ENV 1992-1-1 Eurocódigo 2: Proyecto de estructuras de hormigón. Parte 1-1: Reglas generales y reglas para edificación. Articulo 4.2.1.3.3 a). AENOR 1993 
por la deformación $\varepsilon_{c 1}$ correspondiente a la resistencia a compresión $f_{c}$.

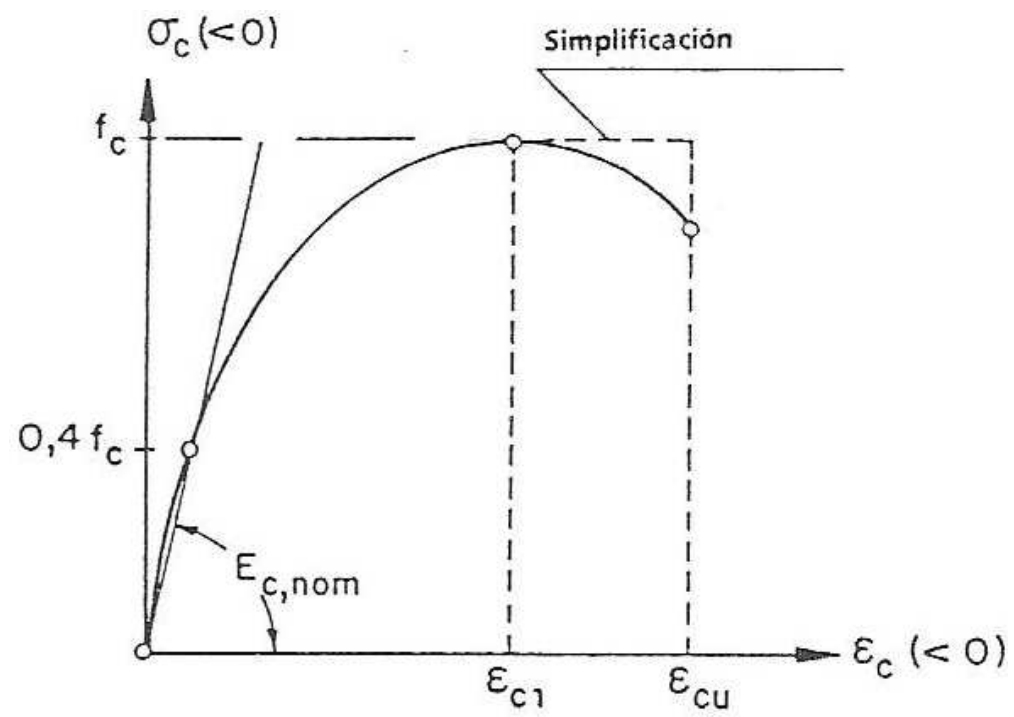

Figura 3.1-2 Diagrama tensión - deformación.

Como valor nominal del módulo de elasticidad $E_{c, n o m}$, se tomará el valor medio $E_{c m}$ definido por la tabla siguiente:

\begin{tabular}{|c|c|c|c|c|c|c|c|c|c|}
\hline $\begin{array}{c}\text { Clase de } \\
\text { hormigón }\end{array}$ & C12/15 & C16/20 & C20/25 & C25/30 & C30/37 & C35/45 & C40/50 & C45/55 & C50/60 \\
\hline$E_{c m}$ & 26 & 27,5 & 29 & 30,5 & 32 & 33,5 & 35 & 36 & 37 \\
\hline
\end{tabular}

Tabla 3.1-4 Valores del módulo de elasticidad secante $E_{c m}\left(\mathrm{kN} / \mathrm{mm}^{2}\right)$ 
y como valor de la resistencia a compresión $f_{c}$, el valor medio $f_{c m}$ dado por:

\begin{tabular}{|c|c|c|c|c|c|c|c|c|c|}
\hline $\begin{array}{c}\text { Clase de } \\
\text { hormigón }\end{array}$ & $12 / 15$ & $16 / 20$ & $20 / 25$ & $25 / 10$ & $30 / 37$ & $35 / 45$ & $40 / 45$ & $45 / 55$ & $50 / 60$ \\
\hline $\begin{array}{c}\mathrm{f}_{\mathrm{cm}} \\
\left(\mathrm{N} / \mathrm{mm}^{2}\right)\end{array}$ & 20 & 24 & 28 & 33 & 38 & 43 & 48 & 53 & 58 \\
\hline$\varepsilon_{\mathrm{cu}} 10^{-3}$ & $-3,6$ & $-3,5$ & $-3,4$ & $-3,3$ & $-3,2$ & $-3,1$ & $-3,0$ & $-2,9$ & $-2,8$ \\
\hline
\end{tabular}

Tabla 3.1-5 Valores nominales de $\varepsilon_{c u}$ (secciones rectangulares)

Como valores de cálculo de estas magnitudes, se adoptará

$$
\begin{gathered}
E_{c d}=\frac{E_{c m}}{\gamma_{c}} \\
f_{c d}=\frac{f_{c k}}{\gamma_{c}}
\end{gathered}
$$

Ecuación 3.1-1.

siendo $f_{c k}$ la resistencia característica del hormigón, y $\gamma_{c}$ el coeficiente de seguridad de este material, para el que adoptaremos el valor reducido $\gamma_{c}=1,35$ que establece EC2 (anexo 3, A.3.1(6)) cuando se analizan pórticos por el método general.

La relación tensión-deformación de la Figura 3.1-2, se puede expresar por la función

$$
\frac{\sigma_{c}}{f_{c}}=\frac{\kappa \cdot \eta-\eta^{2}}{1+(\kappa-2) \cdot \eta}
$$


donde:

$\eta=\varepsilon_{c} / \varepsilon_{c 1} \quad$ siendo $\varepsilon_{c}$ y $\varepsilon_{c 1}$ ambos menores que cero

$\varepsilon_{c 1}=-0,0022 \quad$ deformación correspondiente a la tensión de compresión máxima $f_{c}$

$\kappa=\left(1,1 . E_{c, \text { nom }}\right) \cdot \varepsilon_{c 1} / f_{c} \quad$ con $f_{c}$ introducido como $-f_{c}$

$E_{c, \text { nom }} \quad$ representa el valor medio $E_{c m}$ del módulo de deformación longitudinal, o el correspondiente valor de cálculo $E_{c d}$

La relación anterior es válida siempre que

$0>\varepsilon_{c}>\varepsilon_{c u}$ donde $\mathcal{E}_{c u}$ representa la deformación última de la fibra extrema sometida a compresión (ver

Tabla 3.1-5). Como simplificación puede suponerse un valor constante de $\sigma_{c}=f_{c}$ en el intervalo $\varepsilon_{c 1}>\varepsilon_{c}>\varepsilon_{c u}$ (ver la Figura 3.1-2).

\section{Diagrama para el cálculo de secciones: ${ }^{75}$}

No obstante, para el cálculo de secciones, la idealización más frecuentemente utilizada es el diagrama parábola-rectángulo que se representa en la figura:

${ }^{75}$ UNE ENV 1992-1-1 Eurocódigo 2: Proyecto de estructuras de hormigón. Parte 1-1: Reglas generales y reglas para edificación. Articulo 4.2.1.3.3 b). AENOR 1993 


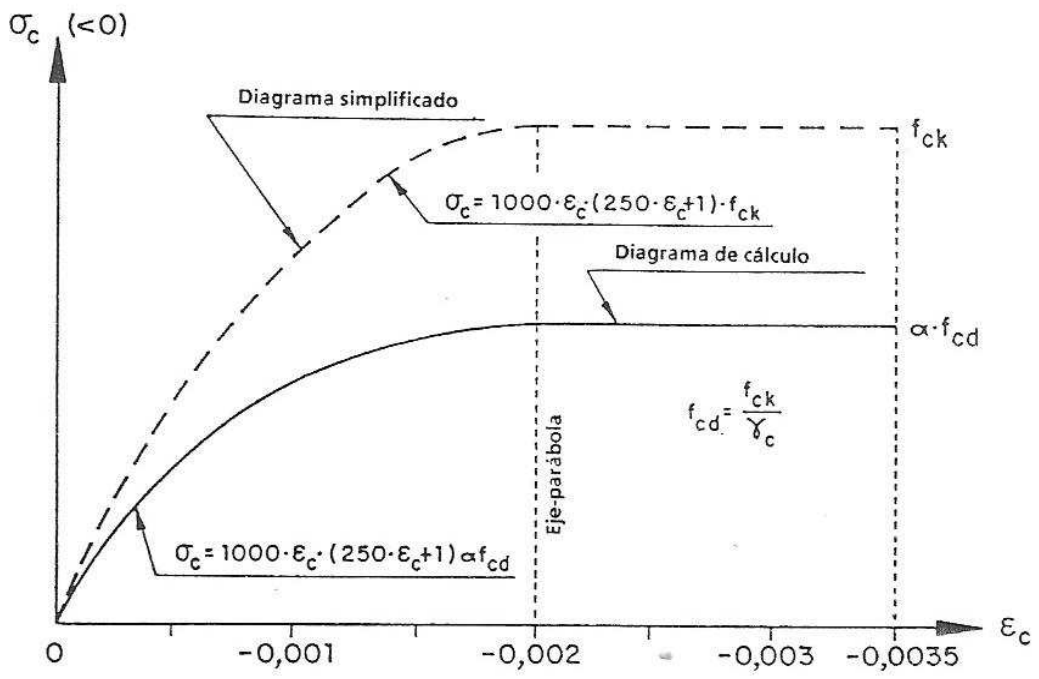

Figura 3.1-3 Diagrama tensión - deformación parábola-rectángulo

La deformación máxima $\varepsilon_{\mathrm{cu}}$ adoptada en este diagrama es del 3,5\% La relación tensión-deformación de la figura 4.1-2, se puede expresar por la función:

$$
\sigma_{c}=1000 \cdot \varepsilon_{c} \cdot\left(250 \cdot \varepsilon_{c}+1\right) \cdot f_{c k}
$$

Ecuación 3.1-2.

Como resistencia de cálculo del hormigón se adoptará:

$$
f_{c d}=\frac{f_{c k}}{\gamma_{c}}
$$

El diagrama de cálculo se deduce del diagrama idealizado mediante la reducción de la tensión por un factor $\alpha / \gamma_{c}$, donde:

$\gamma_{c}$ es el coeficiente parcial de seguridad del hormigón. 
$\alpha$ es un coeficiente que tiene en cuenta los efectos del cansancio del hormigón en la resistencia a compresión y los efectos desfavorables resultantes de la forma en que se aplica la carga. Generalmente se emplea el valor 0,85 como factor de reducción a para las compresiones permanentes.

b) En el acero de $\operatorname{armar}^{76}$

De acuerdo con EC4, adoptaremos un diagrama simplificado tensióndeformación constituido por dos ramas, una primera que parte del origen, con pendiente igual a $E_{s}$ (que se adopta $210 \mathrm{kN} / \mathrm{mm}^{2}$ ) hasta $f_{s k}$ (valor especificado del límite elástico característico, que depende del tipo de acero), o $f_{s k} / \gamma_{s}$ si se trata del diagrama de cálculo $\left(\gamma_{s}=1,15\right)$.

Una segunda rama horizontal, que, para cálculo por ordenador adoptaremos con una pendiente $E_{s} / 10000$, limitando la deformación unitaria al valor 0,01 .

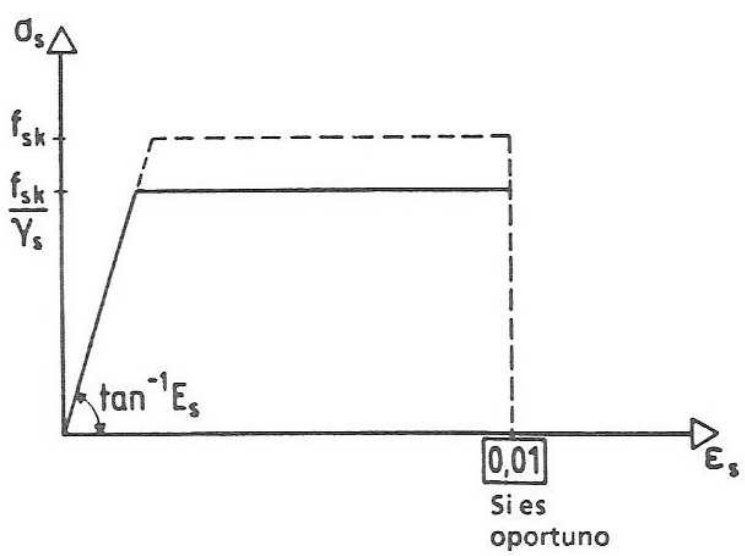

Figura 3.1-4 Diagrama tensión - deformación para el acero de armar

${ }^{76}$ UNE ENV 1994-1-1 Eurocódigo 4: Proyecto de estructuras mixtas de hormigón y acero. Parte 1-1: Reglas generales y reglas para edificación. AENOR 1995 
Para los valores característicos del límite elástico de este material, se adoptaran los valores propuestos por EHE.

c) Acero estructural ${ }^{77}$

Para los tipos usuales, en la tabla siguiente se dan los valores nominales del límite elástico $f_{y}$ y de la resistencia última a tracción $f_{u}$

\begin{tabular}{|c|c|c|c|c|}
\hline \multirow{2}{*}{ Tipo de acero } & \multicolumn{4}{|c|}{ Espesor $\mathrm{en} \mathrm{mm}^{*}$} \\
\cline { 2 - 5 } & \multicolumn{2}{|c|}{$\mathrm{t} \leq 40 \mathrm{~mm}$} & \multicolumn{2}{|c|}{$40 \mathrm{~mm}<\mathrm{t} \leq 100 \mathrm{~mm}$} \\
\cline { 2 - 5 } & $\mathrm{f}_{\mathrm{y}}\left(\mathrm{N} / \mathrm{mm}^{2}\right)$ & $\mathrm{f}_{\mathrm{u}}\left(\mathrm{N} / \mathrm{mm}^{2}\right)$ & $\mathrm{f}_{\mathrm{y}}\left(\mathrm{N} / \mathrm{mm}^{2}\right)$ & $\mathrm{f}_{\mathrm{u}}\left(\mathrm{N} / \mathrm{mm}^{2}\right)$ \\
\hline Fe 360 & 235 & 360 & 215 & 340 \\
Fe 430 & 275 & 430 & 255 & 410 \\
Fe 510 & 355 & 510 & 335 & 490 \\
\hline
\end{tabular}

* $t$ es el espesor nominal del elemento

Tabla 3.1-4. Valores nominales del límite elástico $f_{y}$ y de la resistencia última a tracción fu para acero estructural según la Norma EN 10025

Estos valores se pueden adoptar como característicos a efectos de cálculo.

Como diagrama tensión-deformación (EC4 adopta el criterio de EC3) se considerará el bilineal grafiado en la Figura 3.1-5, donde:

$$
\begin{aligned}
& E_{a}=210000 \mathrm{~N} / \mathrm{mm}^{2} \\
& \gamma_{a}=1,10
\end{aligned}
$$

77 UNE ENV 1994-1-1 Eurocódigo 4: Proyecto de estructuras mixtas de hormigón y acero. Parte 1-1: Reglas generales y reglas para edificación. AENOR 1995 


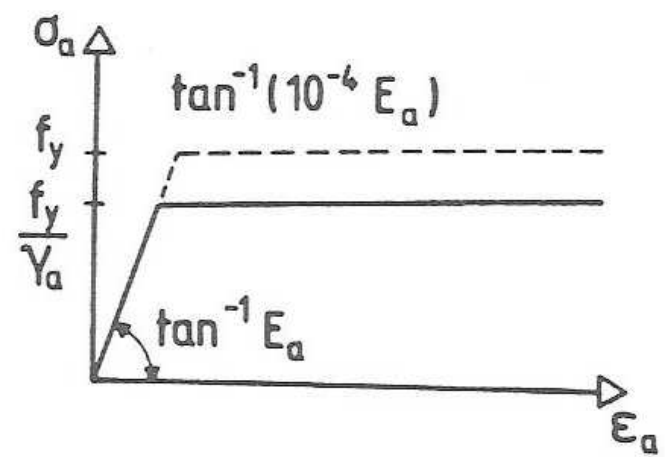

Figura 3.1-5. Diagrama tensión - deformación para el acero estructural

\subsubsection{RESISTENCIA AL PANDEO LATERAL}

Este efecto, en secciones recubiertas, puede evitarse cumpliendo las disposiciones especificadas en el artículo 4.8.2.5 de EC4, relativas a recubrimientos y armadura (ver los títulos 1.4.2.4 y 1.4.2.5 del presente trabajo)

\subsubsection{INTRODUCCIÓN DE CARGAS}

Se adoptarán disposiciones constructivas para que las fuerzas y momentos internos, transmitidas por elementos unidos a los extremos del pilar, se distribuyan entre sus componentes de acero y hormigón, considerando la resistencia a rasante en la superficie de contacto entre ambos materiales según se especifica en el punto siguiente.

Esta comprobación, en ausencia de otro método más preciso, se puede realizar mediante análisis elástico de la sección no fisurada, producido por el cortante transversal entre el acero y el hormigón. La 
longitud de transferencia de este rasante no debe ser mayor que el doble de la dimensión transversal pertinente del pilar.

\subsubsection{RESISTENCIA A CORTANTE}

Para el cálculo a rasante se tomará como resistencia debida a adherencia y fricción en secciones recubiertas, el valor $0,6 \mathrm{~N} / \mathrm{mm}^{2}$, según el artículo 4.8.2.7 del EC4 (ver el título 1.4.2.7 del presente trabajo)

Cuando este efecto sea insuficiente para asegurar que no hay deslizamiento relativo significativo, se puede emplear una conexión mecánica, generalmente formada por pernos soldados al alma del elemento metálico. En este caso, además de la resistencia del perno, se considerará un incremento debido a que el hormigón tiene su expansión lateral impedida por las alas del elemento metálico, cuyo valor es $\mu . P_{R d} / 2$ en cada ala y por cada fila de pernos, Figura 1.4-8, donde $P_{R d}$ es la resistencia de cálculo de un perno y $\mu$ el coeficiente de rozamiento (según artículo 4.8.2.8 del EC4). 


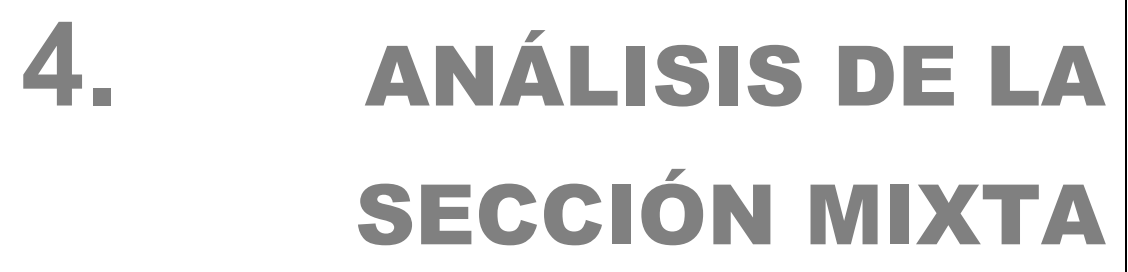




\subsection{INTRODUCCIÓN.}

A partir de las consideraciones expuestas en los capítulos anteriores se ha desarrollado un programa informático de cálculo de secciones mixtas con los siguientes objetivos:

1. Elaboración de gráficas de interacción de distintas secciones para los casos de flexocompresión $\left(N, M_{Z}\right) \circ\left(N, M_{Y}\right)$ y flexocompresión esviada $\left(N, M_{Z}, M_{Y}\right)$ que proporcionan una visión general del comportamiento de estas secciones y permiten comprobar su dimensionado sin necesidad de la aplicación del programa para cada caso.

En la actualidad se encuentran desarrolladas las rutinas que permiten el estudio de secciones en flexocompresión plana en cualquiera de los dos ejes principales y en flexocompresión esviada.

Las tablas que se obtienen de la aplicación del programa en distintos tipos de secciones se muestran en los apartados 4.6 .2 y 4.6.4.

2. Comprobación, o peritación, de la sección frente a unas solicitaciones dadas.

3. Conexión del programa de análisis de secciones mixtas con un programa general de cálculo de solicitaciones.

La fiabilidad del programa desarrollado ha sido verificada con la comparando sus resultados con los obtenidos por los programas informáticos y métodos simplificados de la forma que se indica a continuación: 
- En el caso de secciones de hormigón armado: con el prontuario informático desarrollado por el Instituto Español del Cemento y sus Aplicaciones (IECA ${ }^{78}$.

- En el caso de secciones mixtas con el "método simplificado para el cálculo de la resistencia de secciones mixtas doblemente simétricas en flexión compuesta" del EC4 (expuesto en el apartado 1.4.3 del presente trabajo) y con el programa de cálculo "Composite Column Design" (CCD) desarrollado para ARCELOR ${ }^{79}$.

Esta comparación de resultados se muestra en el apartado 4.6.1.

\subsection{HIPÓTESIS COMPRENDIDAS EN EL PROGRAMA DE CÁLCULO.}

Para la resolución del problema de la flexión esviada en soportes mixtos se ha recurrido a ciertas simplificaciones e idealizaciones ${ }^{80}$. Las que se indican a continuación son las más importantes:

1. Existe una completa interacción entre el acero y el hormigón, las tensiones en el acero y hormigón y sus superficies de contacto se suponen compatibles.

\footnotetext{
${ }^{78}$ Prontuario informático del hormigón estructural 3.0 desarrollado para el Instituto Español del Cemento y sus Aplicaciones (IECA) por Corres Peiretti, H.; Martínez Martínez, J.L.; Pérez Caldentey, A.; López Agüí, J.C

79 "Composite Column Design" versión 3.06 realizado para Arcelor Sections Commercial S.A. por Daedalus Engineering s.a.r.L.,

${ }^{80}$ Las simplificaciones e idealizaciones utilizadas en el presente trabajo, habitualmente utilizadas en el cálculo estructural, se basan en criterios de los Eurocódigos (ver artículo 4.8.2.2 del Eurocódigo 4 y artículo 4.3.1.2 del Eurocódigo 2).
} 
2. La deformación de la sección se supone lineal, variando proporcionalmente a la distancia a la fibra neutra: se supone que una seccion plana antes de la deformación, permanece plana incluso después de la aplicación de las cargas. Este supuesto no es estrictamente correcto, excepto cuando la flexión se produce respecto de un plano de simetria. En otro caso la seccion esta sujeta a efectos de torsión que producen alabeos, aunque pequeños, de la sección.

3. Las curvas tension-deformacion del hormigón, del acero para armar y del acero estructural, usadas en el análisis son las descritas en el apartado 3.1.5 del presente trabajo, basadas en relaciones observadas en probetas ensayadas experimentalmente.

4. Se desprecian la resistencia a tracción del hormigón y el efecto de tension por endurecimiento del acero, aunque unas ligeras correcciones en el programa informatico permitirían considerarlas.

5. Las teniones tangenciales son pequeñas, por lo que su efecto sobre las deformaciones y sobre la resistencia se ha despreciado.

\subsection{PLANTEAMIENTO CONCEPTUAL DEL PROGRAMA.}

La idea del programa informático parte del planteamiento desarrollado por Virdi y Dowling ${ }^{81}$

81 "The ultimate strength of composite columns in biaxial bending". K.S. Virdi, P.J. Dowling (1973) 
Esta consiste en subdividir la sección en una parrilla de pequeños elementos rectangulares. Se supone que cada una de estas superficies está compuesta de un único material: hormigón, acero para armar o acero estructural (podría incluirse cualquier otro material o incluso la ausencia del mismo).

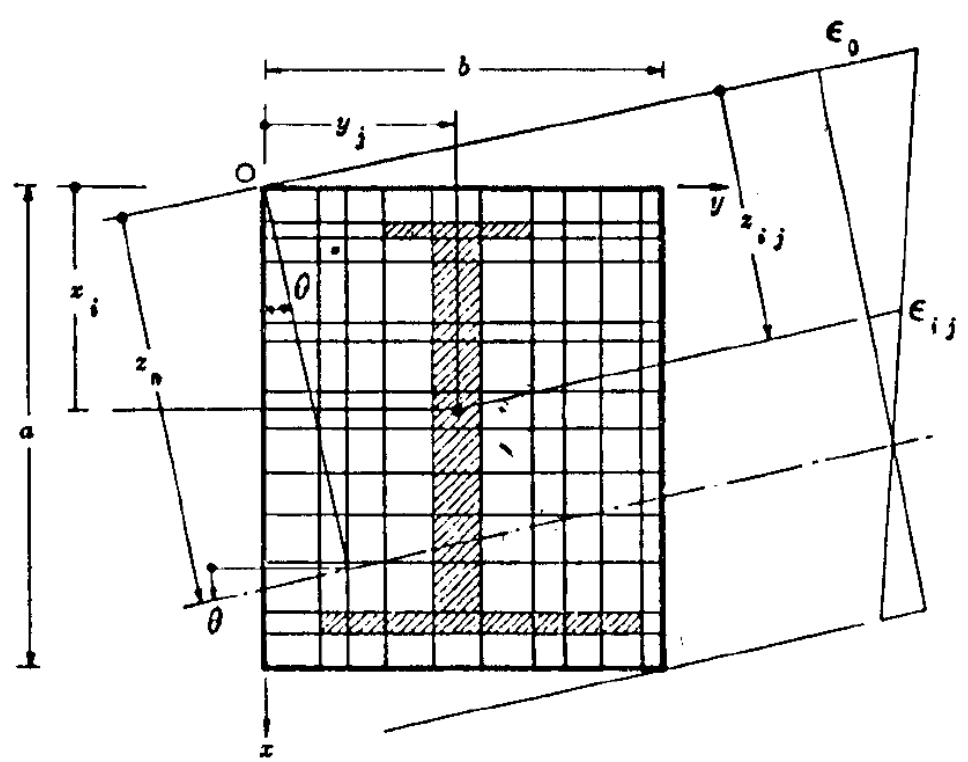

Figura 4.3-1 Discretización de una sección mixta

La Figura 4.3-1, muestra una sección tipica subdividida en una parrilla.

Aunque en el presente trabajo el método se ha aplicado a perfiles metálicos envueltos de hormigón y a secciones de hormigón armado (para la verificación de los resultados mediante la comparación con otros programas), también podría aplicarse a perfiles metalicos huecos rellenos de hormigón, incluyendo las correcciones oportunas para la consideración del efecto del confinamiento del hormigón. 
Las áreas circulares de las barras de acero se sustituyen por un área rectangular que cumple dos condiciones:

- El área sustituida es igual al área equivalente del rectángulo.

- El centroide del área sustituida coincide con el del rectángulo equivalente.

El área de la superficie de acuerdo entre el alma y las alas de los perfiles estructurales no se ha tenido en cuenta en el presente trabajo, aunque podría considerarse siguiendo las condiciones anteriormente indicadas.

En el caso de flexión esviada compuesta, la relación momento-axilcurvatura involucra seis variables, que respecto del sistema de referencia grafiado en la Figura 4.3-1 son: el axil $P$, el momento $M_{X}$, el momento $M_{Y}$, la distancia $z_{n}$ desde el eje neutro punto $O$ (que permite deducir su distancia al centro de gravedad de la sección), la curvatura $\phi$ del plano de deformación y el angulo $\theta$ que forma el eje neutro con el eje $\mathrm{Y}$.

Asignando valores a tres variables cualquiera, las otras tres se pueden encontrar, al menos en principio.

Conocidas las solicitaciones, $P, M_{X}$ y $M_{Y}$, el proceso para la comprobación de una sección, consiste en determinar, mediante un proceso iterativo, el resto de incógnitas $z_{n}, \phi$ y $\theta$, que conducen a una posición de equilibrio. Cabe reseñar que si las solicitaciones de partida correspondiensen a una situación de agotamiento, no se podría alzanzar la posición de equilibrio.

No obstante, el camino por el que se ha optado en el presente trabajo consiste en asignar valores a $\theta, \phi$ y $z_{n}$. De esta forma no se requiere el proceso iterativo, y el axil y los momentos se obtienen directamente. 
$\mathrm{Si}$, en la asignación del valor de $\phi$ (curvatura del plano de deformación) se impone la condición de agotamiento de la sección (deformación máxima en el punto más alejado del eje neutro para cada uno de los materiales ${ }^{82}$ ), las solicitaciones que se obtienen corresponden a situaciones de agotamiento para cada una de las posiciones del eje neutro.

De esta forma, mediante el progresivo desplazamiento y giro del eje neutro, $z_{n}$ y $\theta$ respectivamente, se obtendrán, por tanto, los puntos que conforman la superficie del diagrama de interacción.

Dado que el programa se pretende aplicar sobre secciones con doble eje de simetría, el ángulo $\theta$ que forma el eje neutro con el eje y se hace variar entre $0 \mathrm{y} \pi / 2$, lo cual permite reducir el número de posiciones a analizar.

El proceso, a partir de la determinación de la curvatura del plano de rotura, consiste en determinar la deformación de cada uno de los centros de gravedad de las celdillas y, por aplicación de la relación tensión-deformación del material que se le ha asignado, la obtención de la tensión y fuerza en cada celdilla. El sumatorio de las componentes fuerza de cada celdilla y la consideración de su excentricidad sobre los dos ejes principales de la sección da lugar a la resultante de fuerzas $P$ y momentos $M_{X}$ y $M_{Y}$ sobre la sección.

El desarrollo concreto de este planteamiento conceptual se expone con mayor detalle en los apartados siguientes.

${ }^{82}$ En el caso de secciones mixtas, con los recubrimientos habituales tanto del perfil metálico como de las armaduras, usualmente la condición de agotamiento se produce en el hormigón. 


\subsection{ENTRADA DE DATOS.}

\subsubsection{DATOS DE LA SECCIÓN}

La definición de los datos de la sección mixta comprende dos aspectos: la definición geométrica y la especificación del tipo de material.

\section{Geometría}

La sección mixta se discretiza mediante celdillas rectangulares, similar a la realizada por Virdi y Dowling en su planteamiento, Figura 4.3-1.

Como ejemplo, se representa en la Figura 4.4-1 una propuesta de discretización de una sección de $45 \times 45 \mathrm{~cm}$ compuesta por un perfil de acero laminado tipo HEB-260 embebido en hormigón con 8 barras de acero corrugado de $20 \mathrm{~mm}$ de diámetro.

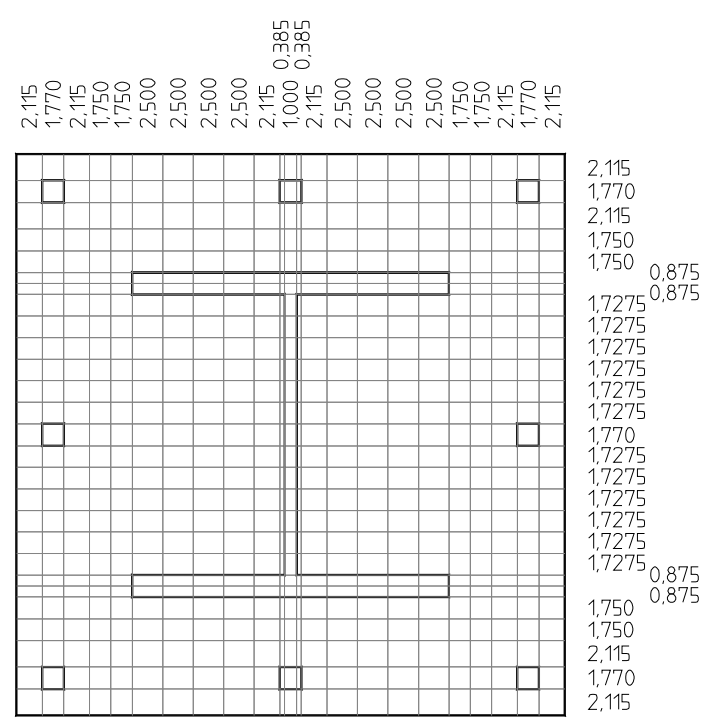

Figura 4.4-1 Discretización de una sección mixta 
A partir de esta discretización se generará el correspondiente listado de entrada de datos para el programa de cálculo (Figura 4.4-2), consistente, a grandes rasgos, en las dimensiones $x$ e $y$ de cada celdilla y el material que se le asigna.

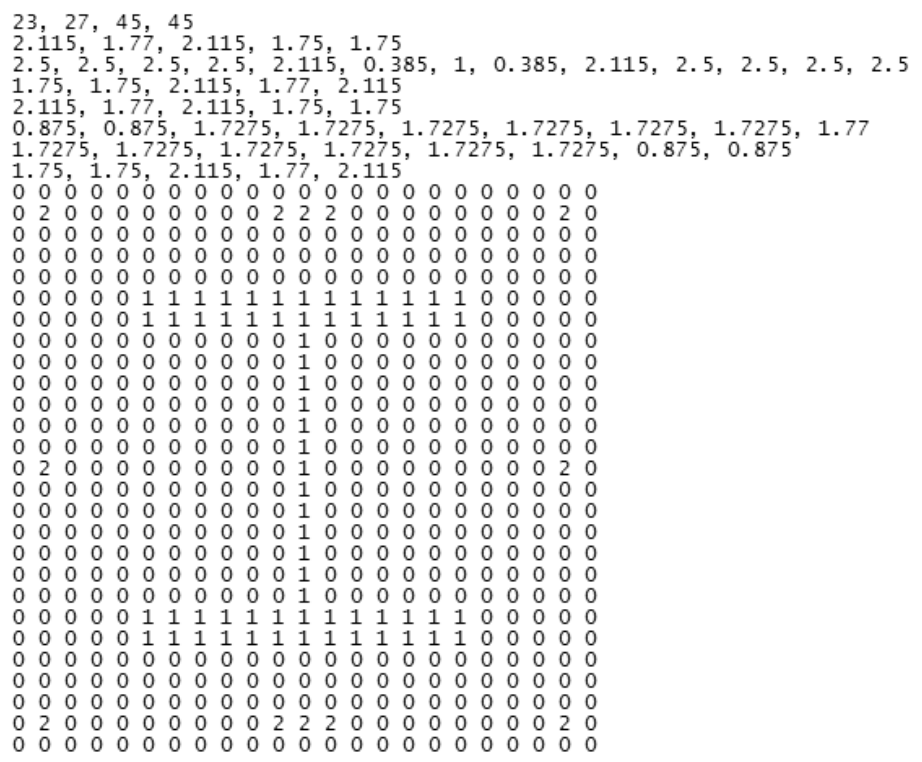

Figura 4.4-2 Listado de datos de una sección mixta

Los datos de la geometría de la sección del citado listado, Figura 4.4-2, se organizan con el siguiente orden y significado de su:

Línea 1: $\quad$ npasosx, npasosy, dimx, dimy npasosx: $\quad$ número de divisiones en la dirección $X$ npasosy: $\quad$ número de divisiones en la dirección $Y$ dimx: dimensión de la sección en la dirección $\mathrm{X}$ dimy: dimensión de la sección en la dirección $Y$

Línea 2 a línea $(n p a s o s x+1)$ : $\quad$ pasos $\quad$ pasosx: dimensión de cada división en la dirección $\mathrm{X}$ 
Línea $(n p a s o s x+1)$ a línea (npasosy+1): $\quad$ pasos $x y$

pasosy: dimensión de cada división en la dirección $Y$

Conocido el número de divisiones y las dimensiones de cada una de ellas, el programa determina la posición del centro de gravedad de cada celdilla, que referenciará a un nuevo sistema con origen en el centro de gravedad de la sección, con el eje $Z$ horizontal positivo hacia la derecha (eje fuerte del perfil metálico) y como eje $Y$ el vertical, positivo hacia abajo (eje débil del perfil).

Como se puede observar en la discretización expuesta en la Figura 4.4-2 para una sección de $45 \times 45 \mathrm{~cm}$, el tamaño medio de las celdillas es de unos $2 \mathrm{~cm}$. Se ha comprobado el efecto en los resultados del tamaño de estas celdillas, comparando esta misma sección con unas celdillas de un tamaño mitad (aproximadamente $1 \mathrm{~cm}$ ), verificando que para las citadas dimensiones la repercusión no es apreciable.

\section{Material}

A continuación se asigna el tipo de material a cada una de las celdillas mediante los siguientes códigos:

$$
\begin{array}{ll}
0 & \text { hormigón } \\
1 & \text { acero estructural } \\
2 & \text { acero de armar }
\end{array}
$$

En el listado de datos, Figura 4.4-2, dicha información se introduce mediante una matriz, cuyo número de filas es npasosy (número de divisiones de la sección en vertical) y el número de columnas es npasosx (número de divisiones de la sección en horizontal). Alternativamente se podría facilitar la misma información mediante una lista de códigos organizada por filas, con un número total de ellas igual a npasosx por npasosy. 
Por mayor comodidad en la generación del fichero de entrada de datos (geometría y materiales) de la sección, se ha elaborado un programa informático, que, a partir de las dimensiones de la sección, de las características del perfil (dimensiones y espesores de alma y alas), diámetro de las armaduras y su recubrimiento, realiza la discretización y genera el citado fichero.

A pesar de la ayuda de este programa, por motivos de tiempo de programación, la tipología de secciones queda limitada con las siguientes restricciones:

- Secciones rectangulares

- Perfiles metálicos tipo H (doble T)

- 8 armaduras longitudinales (3 por cara) dispuestas de forma simétrica.

\subsubsection{CARACTERÍSTICAS DE LOS MATERIALES}

Las características mecánicas de los materiales se definen mediante distintos campos, organizados en la ventana principal del programa de cálculo, Figura 4.4-3, de forma independiente de los datos de la sección y que corresponden a las siguientes variables:

\section{Hormigón:}

$f_{c k}\left(\right.$ Sigma_hor): $\quad$ resistencia característica $\left(\mathrm{N} / \mathrm{mm}^{2}\right)$

$\mathcal{E}_{c}$ (Epsilon_ult_hor): deformación última a compresión

$E_{c m}$ : módulo de elasticidad secante $\left(\mathrm{N} / \mathrm{mm}^{2}\right)$

$\gamma_{c}($ Coeficiente_hor): $\quad$ coeficiente de seguridad parcial

$\alpha($ Alfa): $\quad$ coef. cansancio y aplicación carga 


\section{Acero estructural:}

$F_{y k}\left(\right.$ Sigma_st): límite elástico $\left(\mathrm{N} / \mathrm{mm}^{2}\right)$

$\varepsilon_{y}\left(E p s i l o n \_y \_s t\right): \quad$ deformación límite régimen elástico

$\gamma_{y}($ Coeficiente_st): $\quad$ coeficiente de seguridad parcial

\section{Acero de armar:}
$f_{s k}($ Sigma_ar):
límite elástico $\left(\mathrm{N} / \mathrm{mm}^{2}\right)$
$\varepsilon_{s}$ (Epsilon_y_ar):
deformación límite régimen elástico
$\gamma_{s}$ (Coeficiente_ar):
coeficiente de seguridad parcial

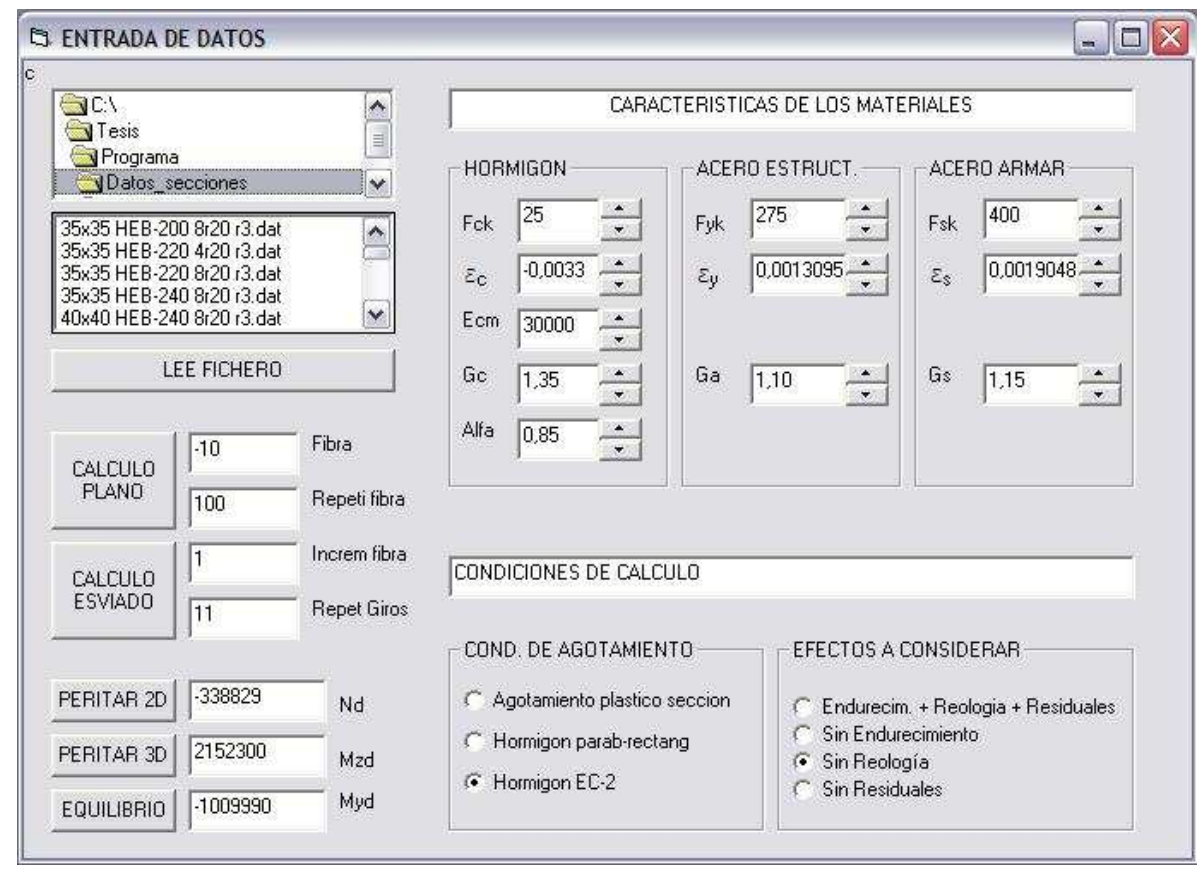

Figura 4.4-3 Ventana principal del programa de cálculo

Como se aprecia en la Figura 4.4-3, el programa dispone de valores por defecto para todos los datos mencionados, que pueden ser modificados por el usuario. 


\subsubsection{OPCIONES DE CÁLCULO}

El programa informático desarrollado proporciona la posibilidad de tener en cuenta las siguientes opciones y efectos:

\section{Sobre las condiciones de agotamiento de los materiales}

\section{Diagrama plástico de la sección}

Esta opción permite la consideración de la sección completamente plastificada, y por tanto con todos los puntos sometidos a la máxima tensión del material correspondiente: $f_{c d}, f_{y d} \circ f_{s d}$. Este es el estado que utiliza el el método simplificado para secciones mixtas propuesto por el Eurocódigo $4 \mathrm{y}$ nos ha permitido contrastar la validez del programa desde sus primeras etapas de desarrollo.

\section{Diagrama parábola - rectángulo en el hormigón}

En este caso, el diagrama tensión-deformación del hormigón adoptado para el cálculo de secciones, corresponde al denominado "parábola-rectángulo", que se muestra en la Figura 3.1-3, y los parámetros de cálculo (módulo de elasticidad, resistencia a compresión o deformación de rotura) y la expresión matemática que relaciona la tensión con la deformación, las especificadas en el correspondiente apartado.

\section{Diagrama para "análisis estructural" en el hormigón}

En este caso, el diagrama tensión-deformación del hormigón adoptado corresponde indicado por el EC4 para el "análisis estructural", Figura 3.1-2, y los parámetros de cálculo (módulo de elasticidad, resistencia a compresión o deformación de rotura) y la expresión matemática que relaciona la tensión con la deformación, las especificadas en el correspondiente apartado. 


\section{Sobre distintos efectos:}

En el momento actual, el programa desarrollado es capaz de evaluar, si el usuario lo desea, los efectos reológicos del hormigón ${ }^{83}$ y se espera que versiones posteriores puedan tener en cuenta en el análisis el efecto de las tensiones residuales del acero estructural y del endurecimiento de los aceros.

\subsection{ANÁLISIS DE LA SECCIÓN}

Esta opción, que realiza el análisis de la sección sin tener en cuenta la esbeltez de la barra, pretende obtener las distintas combinaciones axil $(M)$ y momentos $\left(M_{Y}, M_{Z}\right)$ que producen el agotamiento de la sección (bajo la consideración o no de los efectos reológicos en el hormigón y la elección del diagrama tensión-deformación deseado para el hormigón).

Para ello, el programa adopta como variable independiente la posición de la fibra neutra $\left(z_{n}\right)$, que se desplaza y gira, y como condición el agotamiento del material (que, en el caso de secciones mixtas, corresponde al hormigón).

Cuando la fibra neutra se desplaza paralelamente a los ejes principales $Z, Y$, casos de flexocompresión plana, las coordenadas de los centros de gravedad de cada celdilla se conocen a partir de los datos de partida de cada sección.

Cuando la fibra neutra se encuentra girada respecto de los ejes principales, el programa cambia las coordenadas del centro de

${ }^{83}$ Dada la importancia y complejidad de los efectos reológicos del hormigón, se ha considerado oportuno dedicarles completamente el capítulo 5 de este trabajo de investigación 
gravedad de todas las celdillas mediante la siguiente expresión de transformación de coordenadas:

$$
\left[\begin{array}{l}
z^{\prime} \\
y^{\prime}
\end{array}\right]=\left[\begin{array}{cc}
\cos \alpha & \sin \alpha \\
-\sin \alpha & \cos \alpha
\end{array}\right] \cdot\left[\begin{array}{l}
z \\
y
\end{array}\right]
$$

Siendo $\left(z, y^{\prime}\right)$ las coordenadas respecto del nuevo sistema de referencia, $\alpha$ el ángulo de giro de la fibra neutra respecto del eje $Z$ y $(z, y)$ las coordenadas del centro de gravedad de cada celdilla respecto del sistema $Z, Y$.

Dada la posición de la fibra neutra, se determina la curvatura de la sección por aplicación de la condición de agotamiento: la deformación en el centro de gravedad de la celdilla más alejada del eje neutro coincide con la deformación correspondiente a la tensión máxima del material $(0,0033$ en el diagrama tensión-deformación para el hormigón definido en la Figura 3.1-2). De esta forma, la curvatura se obtiene mediante el cociente entre la citada deformación y la distancia del punto mas alejado a la fibra neutra $\left(\phi=\varepsilon_{c 1} / z_{n}\right)$.

Conocida la curvatura de la sección, se determina la deformación a la que se encuentra sometido el centro de gravedad de cada una de las celdillas en que hemos discretizado la sección, mediante el producto de la curvatura por su distancia a la fibra neutra $\left(\varepsilon_{i}=\phi \cdot f_{i}\right)$

A partir de la deformación de cada celdilla, se obtiene su tensión, por aplicación de las leyes de comportamiento de cada uno de los materiales que componen la sección (definidas en el apartado 3.1.5). Esta tensión se considera constante para toda el área de la celdilla.

Las solicitaciones que han producido la configuración $\left(z_{n}-\theta-\phi\right)$ de partida, y que corresponden a una de las solicitaciones de agotamiento de la sección $\left(N_{u}, M_{u}\right)$, se obtienen por aplicación de las siguientes expresiones: 


$$
\begin{aligned}
& N_{u}=\sum_{i} \sigma_{i} \cdot A_{i} \\
& M_{Y u}=\sum_{i} \sigma_{i} \cdot A_{i} \cdot z_{i} \\
& M_{Z u}=\sum_{i} \sigma_{i} \cdot A_{i} \cdot y_{i}
\end{aligned}
$$

siendo:

$N_{u} \quad$ axil de agotamiento de la sección

$M_{Y u} \quad$ momento $Y$ de agotamiento de la sección

$M_{Z u} \quad$ momento $Z$ de agotamiento de la sección

$\sigma_{i} \quad$ tensión de cálculo de cada una de las celdillas

$A_{i} \quad$ área de cada una de las celdillas

$y_{i} \quad$ distancia del Centro de Gravedad de cada celdilla al eje Z de la sección.

$z_{i} \quad$ distancia del Centro de Gravedad de cada celdilla al eje Y de la sección.

Los resultados $\left(N_{u}, M_{Y u}, M_{Z u}\right)$ para las distintas posiciones del eje neutro se pueden obtener en forma de listado o de fichero tipo DXF (reconocible por programas CAD).

A partir de éstos se han elaborado los distintos diagramas de interacción que se exponen en el apartado 4.6 RESULTADOS: TABLAS DE DIMENSIONAMIENTO DE SECCIONES MIXTAS.

El organigrama del proceso de obtención de los puntos que definen las curvas de interacción (estado de solicitación plana) y la superficie de interacción (estados de flecocompresión esviada) de una sección mixta se representa en la siguiente figura: 


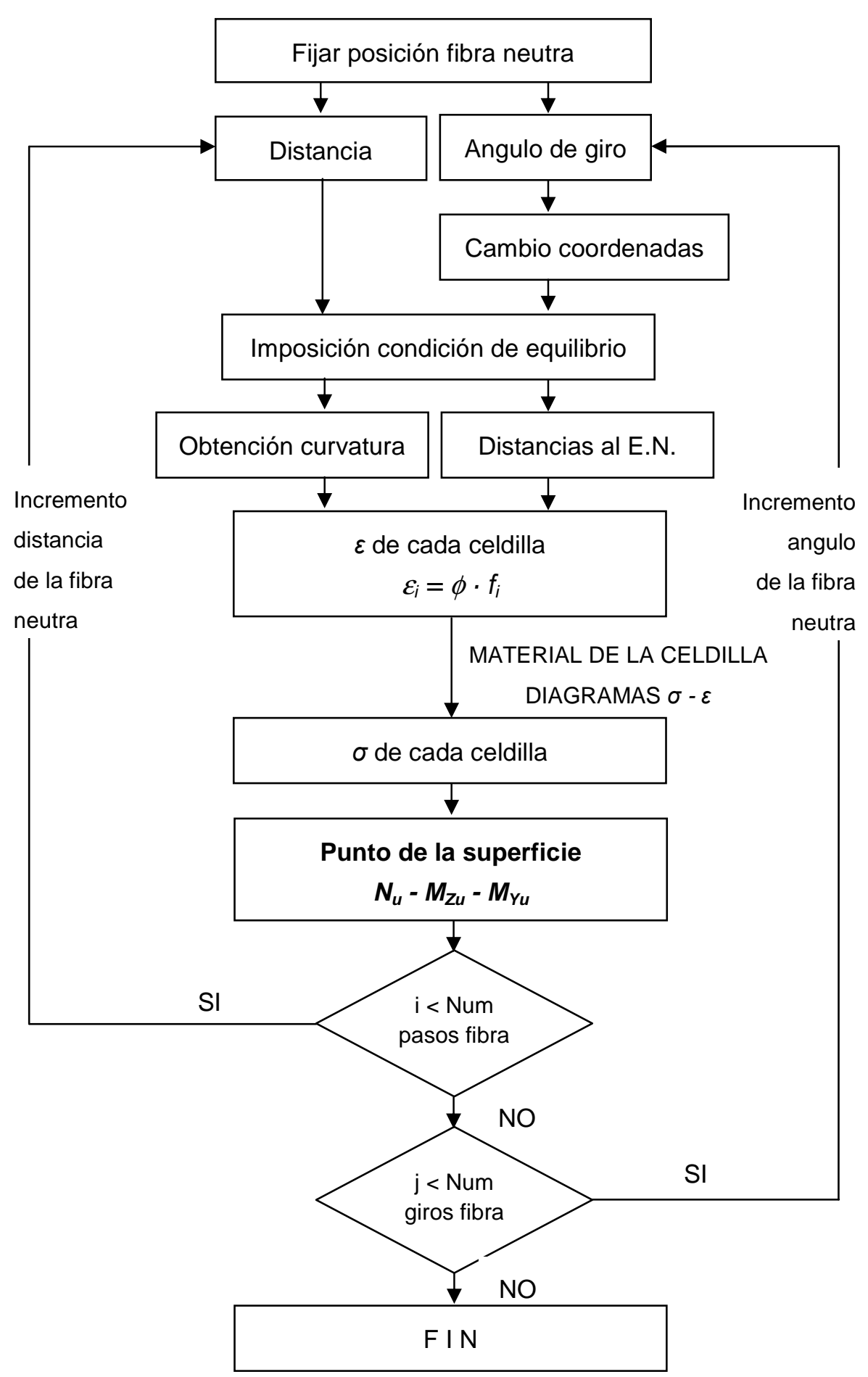




\subsubsection{COMPROBACIÓN DE LA SECCIÓN}

\section{Comprobación en flexocompresión recta}

Dado cualquier par de valores $\left(N_{d}, M_{z d}\right)$ el programa es capaz de comprobar si dichas solicitaciones producen o no el agotamiento de la sección.

Para ello, comienza comprobando si el axil de cálculo $\left(N_{d}\right)$ es mayor o menor que el axil máximo que es capaz de soportar la sección. En caso de ser mayor, termina el proceso de comprobación, puesto que se produce el agotamiento.

En caso contrario, el programa busca la combinación $\left(N_{u}, M_{Z u}\right)$ por aplicación de la condición $N_{u}=N_{d}$. A continuación se compara el momento flector de cálculo con el momento flector de agotamiento obtenido en el paso anterior: si es menor, la sección cumple, en caso contrario, se produce el agotamiento.

\section{Comprobación en flexocompresión esviada}

El programa también es capaz de comprobar la validez de la sección mixta frente a esfuerzos de flexocompresión esviada $\left(N_{d}, M_{Z d}, M_{Y_{d}}\right)$.

Al igual que en flexocompresión recta, el proceso se inicia con la comparación del axil de cálculo $\left(N_{d}\right)$ con el axil máximo que es capaz de soportar la sección. En caso de ser mayor, la sección es insuficiente; en caso contrario, a partir de los puntos de la superficie de interacción $\left(N_{u}, M_{Z u}, M_{Y u}\right)$, previamente determinados, se realiza el siguiente proceso:

1. Se determina el vector unitario $\vec{u}_{d}$ que define la dirección del vector solicitación $\vec{S}_{d}$ : 


$$
\vec{u}_{d}=\left(u_{x}, u_{y}, u_{z}\right)=\frac{\left(N_{d}, M_{Z d}, M_{Y d}\right)}{\sqrt{N_{d}^{2}+M_{Z d}^{2}+M_{Y d}^{2}}}
$$

2. Obtención, de forma iterativa, del vector unitario último $\vec{u}_{\underline{u}}$, para cada una de las solicitaciones de agotamiento $\vec{S}_{u}$ (correspondiente a cada uno de los puntos que definen la superficie de interacción) mediante la expresión:

$\vec{u}_{u}=\left(u_{x}, u_{y}, u_{z}\right)=\frac{\left(N_{u}, M_{Z u}, M_{Y u}\right)}{\sqrt{N_{u}^{2}+M_{Z u}^{2}+M_{Y u}^{2}}}$

comparándolo con el vector $\vec{u}_{d}$

3. Se acepta como solución el vector unitario de agotamiento $\vec{u}_{u}$, cuyas coordenadas coinciden, con un cierto margen de error, con las coordenadas del vector unitario solicitación $\vec{u}_{d}$.

4. A continuación se obtiene el módulo del vector solicitación y del vector de agotamiento lineal con áquel y se comparan. Si el módulo del vector solicitación es mayor que el del vector de agotamiento, la sección no cumple y en caso contrario se trataría de una sección insuficiente.

El proceso descrito se representa gráficamente, de forma plana por mayor sencillez de dibujo, en la Figura 4.5-1

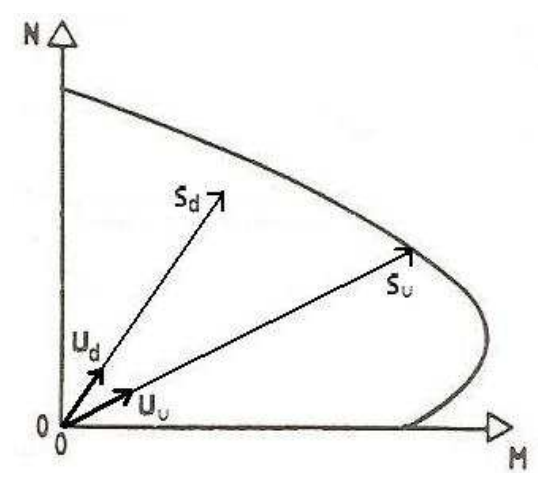

Figura 4.5-1 Proceso de comprobación de la sección 


\subsection{RESULTADOS: TABLAS DE DIMENSIONAMIENTO DE SECCIONES MIXTAS.}

\subsubsection{ANÁLISIS COMPARATIVO DIAGRAMAS INTERACCIÓN}

Con el objeto de comprobar la fiabilidad del programa informático desarrollado, y de forma progresiva con el proceso de programación, se han elaborado una serie gráficos (diagramas de interacción) de distintos tipos de secciones para poder compararlos con los correspondientes diagramas elaborados, bien por otros programas informáticos o bien mediante métodos simplificados de cálculo.

\section{Hormigón armado: Comparación con el "IECA"}

Los resultados obtenidos con el programa desarrollado, aplicado sobre una sección de hormigón armado, se han comparado con los que se obtienen del "Prontuario Informático del Hormigón Estructural"84 realizado por el Instituto Español del Cemento y sus Aplicaciones (en lo sucesivo IECA).

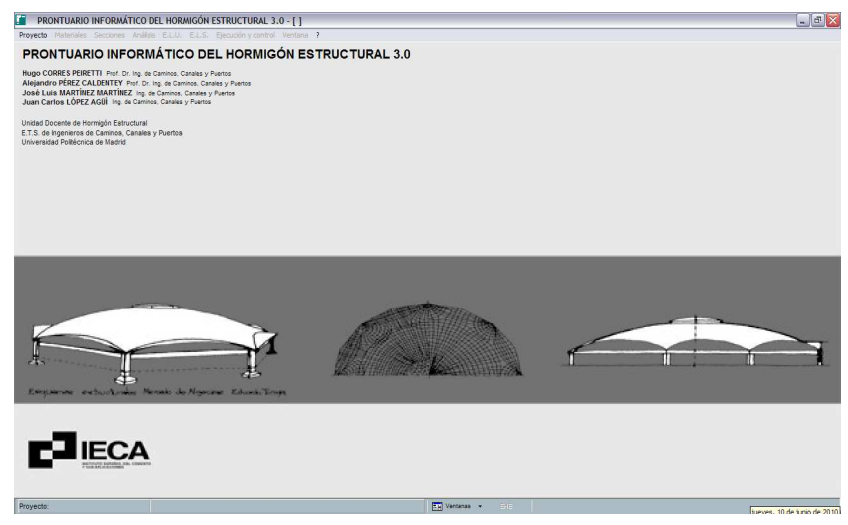

Figura 4.6-1 Ventana de acceso al programa del IECA

84 "TN3. Flexión Compuesta Recta" "Prontuario Informático del Hormigón Estructural" versión 3.0. realizado para el Instituto Español del Cemento y sus Aplicaciones por los Ingenieros de Caminos Canales y Puertos, $\mathrm{H}$. Corres Peiretti, A. Pérez Caldentey, J.L. Martínez Martínez y J.C. López. 
Como hipótesis y criterios de cáculo, este programa utiliza los contenidos en la norma EHE.

En la Figura 4.6-2 se comparan el diagrama obtenido mediante el programa del IECA y los diagramas obtenidos por el programa infórmatico desarrollado, utilizando dos diagramas tensióndeformación en el hormigón. Como ejemplo, se ha utilizado una sección de hormigón armado de $35 \times 35 \mathrm{~cm}$ y cuatro barras de diámetro $20 \mathrm{~mm}$ con un recubrimiento de $3 \mathrm{~cm}$ y las características de los materiales siguientes:

Resistencias de los materiales:

Hormigón. HA-25: $\mathrm{f}_{\mathrm{ck}}=25 \mathrm{~N} / \mathrm{mm}^{2}$

Acero de armar. B-400: $\mathrm{f}_{\mathrm{sk}}=400 \mathrm{~N} / \mathrm{mm}^{2}$

Coeficientes de minoración de resistencias:
Hormigón.
$\gamma_{\mathrm{c}}=1,50$
Acero de armar. $\quad \gamma_{s}=1,15$

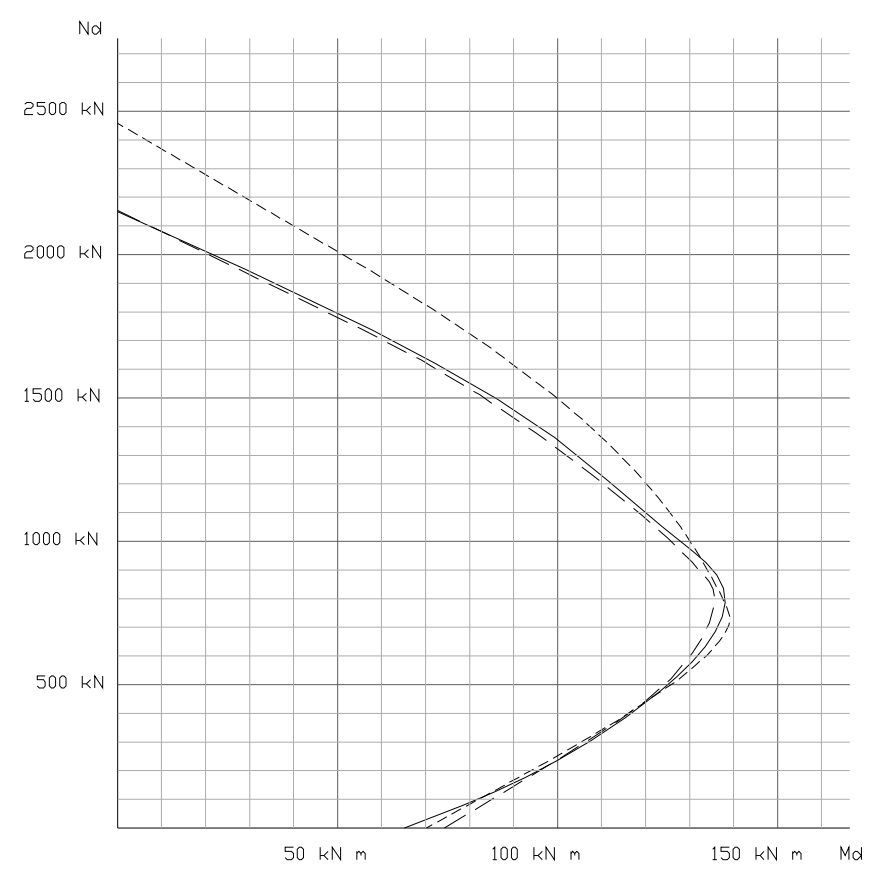

Figura 4.6-2 Comparativo IECA - Programa 
Leyenda de los diagramas de interacción:

1. Trazos largos: Diagrama obtenido con el Programa elaborado, utilizando para el hormigón la relación tensióndeformación parábola rectángulo (Figura 3.1-3) y un coeficiente alfa igual a 0,85

2. Línea continua: Diagrama obtenido con el programa del IECA

3. Trazos cortos: Diagrama obtenido con el Programa elaborado, utilizando para el hormigón la relación tensióndeformación según EC2 para cálculo estructural, (Figura 3.1-2) y un coeficiente alfa igual a 1,00

Se puede comprobar que el diagrama de interacción prácticamente se solapa con el obtenido con el IECA cuando el Programa utiliza como opciones de cálculo para el hormigón la relación tensióndeformación parábola rectángulo, (Figura 3.1-3) y un coeficiente alfa igual a 0,85. Cuando en el Programa desarrollado se utiliza la relación tensión-deformación según EC2 para análisis estructural (Figura 3.1-2) el diagrama de interacción coincide cuando los efectos de flexión son predominantes pero diverge conforme el axil predomina sobre la flexión (en estos casos se incrementa la capacidad resistente de la sección).

Secciones Mixtas: Comparación con el método simplificado del Eurocódigo 4 y con el programa de ARCELOR.

Los resultados obtenidos con el programa desarrollado, aplicado sobre una sección mixta, y utilizando distintas relaciones tensióndeformación de los materiales, se han comparado con los que se obtienen: 
- mediante la aplicación "Composite Column Design" versión 3.06 realizado para Arcelor Sections Commercial S.A. por Daedalus Engineering s.a.r.L., basado en los criterios del Eurocódigo 4.

- a partir de la aplicación del método simplificado para el cálculo de soportes mixtos expuesto en el EC4.

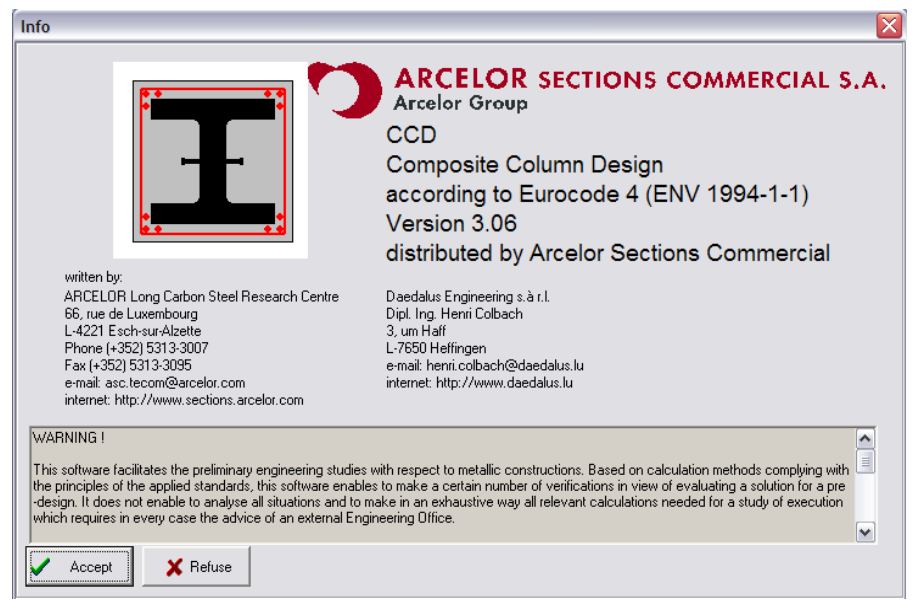

Figura 4.6-3 Ventana de acceso al programa de ARCELOR

Como ejemplo se ha utilizado una sección mixta formada por un perfil HEB-220 embebida en una sección de hormigón armado de 35X35 $\mathrm{cm}$ y cuatro barras (una por esquina) de diámetro $20 \mathrm{~mm}$, con un recubrimiento de $3 \mathrm{~cm}$

Resistencias de los materiales:
Hormigón.
HA-25: $f_{c k}=25 \mathrm{~N} / \mathrm{mm}^{2}$
Acero de armar.
B-400: $\mathrm{f}_{\mathrm{sk}}=400 \mathrm{~N} / \mathrm{mm}^{2}$
Acero estructural.
S-275: $f_{\mathrm{yk}}=275 \mathrm{~N} / \mathrm{mm}^{2}$

Coeficientes de minoración de resistencias:

Hormigón.

$$
\begin{aligned}
& \gamma_{c}=1,35 \\
& \alpha=1,00
\end{aligned}
$$

Acero de armar. $\quad \gamma_{\mathrm{s}}=1,15$

Acero estructural. $\quad \gamma_{y}=1,10$ 


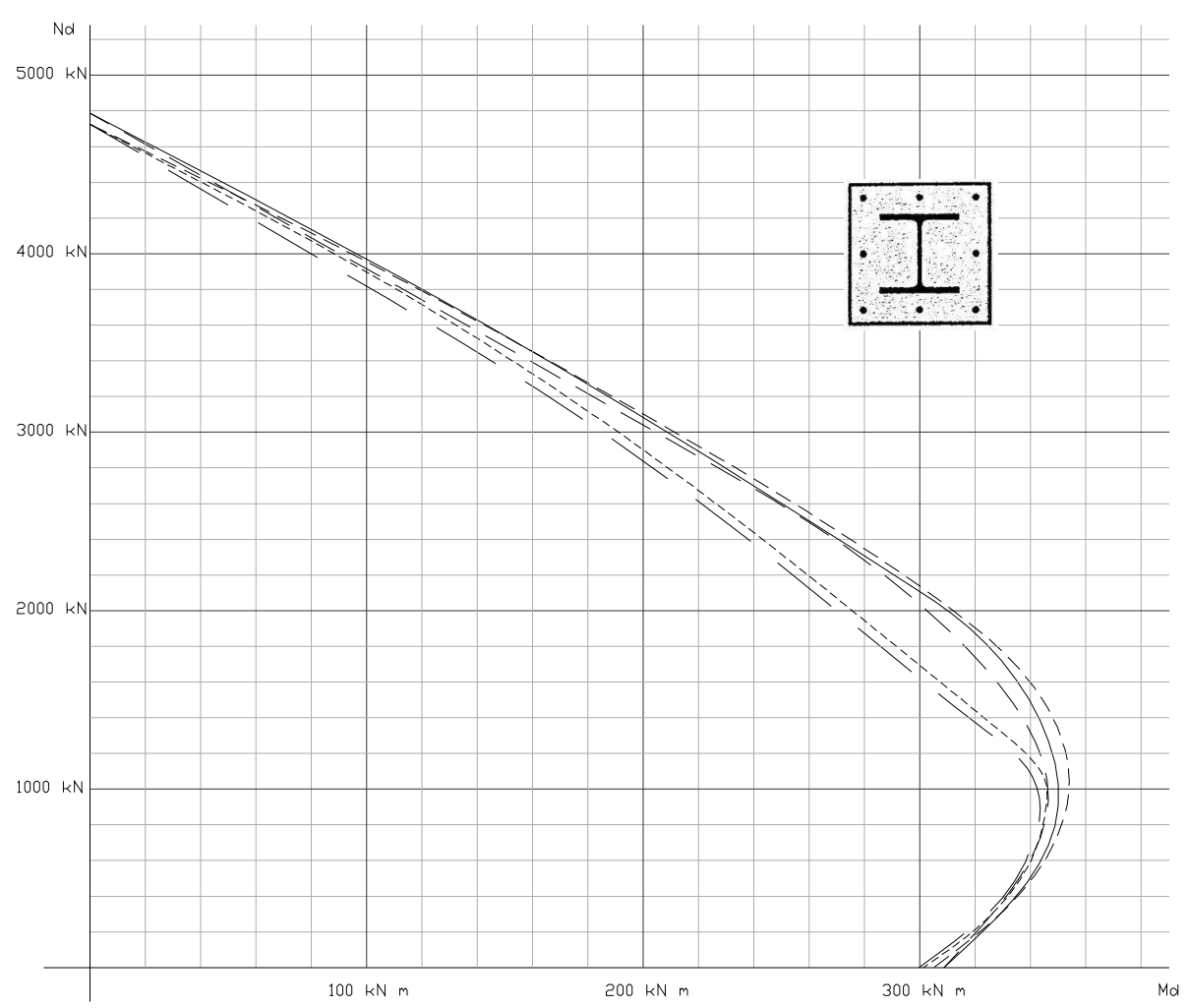

Figura 4.6-4 Comparativo Método Simplificado - ARCELOR - Programa

Leyenda de los diagramas de interacción:

1. Trazos cortos: Diagrama obtenido con el Programa desarrollado con todos los puntos solicitados a su máxima capacidad resistente

2. Línea continua: Diagrama obtenido por aplicación del Método Simplificado contenido en el EC4 (ver apartado 1.4.3.10)

3. Trazos medios: Diagrama obtenido con el programa de ARCELOR, anteriormente expuesto

4. Trazos pequeños: Diagrama obtenido con el Programa desarrollado utilizando para el hormigón la relación tensióndeformación parábola rectángulo (Figura 3.1-3) 
5. Trazos grandes: Diagrama obtenido con el Programa utilizando para el hormigón la relación tensión-deformación según EC2 para cálculo estructural, (Figura 3.1-2)

Como conclusiones del análisis de gráfico anterior, cabe citar:

a) Las dos curvas de interacción obtenidas con el programa desarrollado, utilizando distintas relaciones tensióndeformación para el hormigón (curvas 4 y 5 ) son muy similares.

b) Cuando se utiliza el programa con la sección totalmente plastificada (curva 1) los resultados prácticamente se solapan con los obtenidos por aplicación del método simplificado (curva 2) y con los obtenidos con el programa CCD de Arcelor (curva 3).

c) Todas las curvas se solapan cuando claramente predomina una solicitación sobre la otra: el axil sobre el flector (parte alta de los diagramas) o el flector sobre el axil (parte baja de los diagramas)

d) Las mayores diferencias se observan entre las curvas 4 y 5 respecto del resto cuando se combinan tanto axiles como flectores de valores intermedios, siendo desfavorables los primeros diagramas citados. Los motivos de las diferencias se pueden deber a:

- Las relaciones tensión-deformación adoptadas para los materiales, en especial, la consideración de la sección totalmente plastificada.

- El área de la superficie de acuerdo entre el alma y las alas del perfil estructural, que se ha despreciado, al no 
haber sido considerada en la discretización de la sección para el cálculo mediante el programa desarrollado.

- La discretización de la sección mediante celdillas para el cálculo mediante el programa desarrollado y la consideración de toda el área de la celdilla y por tanto de la tensión, en su centro de gravedad.

Este hecho tiene especial incidencia cuando el eje neutro corta a la sección y por tanto a algunas de las celdillas, en cuyo caso el total de la celdilla se considera traccionada o comprimida según la posición de su centro de gravedad. No obstante, el error será muy pequeño en la apreciación del momento, pues su brazo de palanca es mínimo.

\subsubsection{TABLAS DE DIMENSIONAMIENTO EN FLEXOCOMPRESIÓN RECTA}

Verificada la fiabilidad del programa de cálculo desarrollado, se han elaborado tablas que permiten el predimensionado de secciones mixtas sometidas a flexocompresión recta, para el caso de flexión respecto del Eje Fuerte y respecto del Eje Débil de la sección.

Las tablas que se muestran se han elaborado utilizando el diagrama tensión-deformación del hormigón según EC2 para cálculo estructural y las siguientes características para los materiales:

Resistencias de los materiales:

Hormigón.

HA-25: $f_{\mathrm{ck}}=25 \mathrm{~N} / \mathrm{mm}^{2}$

Acero de armar. $\quad$ B-400: $\mathrm{f}_{\mathrm{sk}}=400 \mathrm{~N} / \mathrm{mm}^{2}$

Acero estructural. S-275: $\mathrm{f}_{\mathrm{yk}}=275 \mathrm{~N} / \mathrm{mm}^{2}$ 
Coeficientes de minoración de resistencias:

Hormigón.

$\gamma_{\mathrm{c}}=1,35$

$\alpha=1,00$

Acero de armar. $\quad \gamma_{\mathrm{s}}=1,15$

Acero estructural. $\quad \gamma_{y}=1,10$

Leyenda de los diagramas de interacción:

1. Sección de $35 \times 35 \mathrm{~cm}$. HEB-200. 8ф20. Recubrimiento=3cm

2. Sección de 35×35 cm. HEB-220. 8ф20. Recubrimiento=3cm

3. Sección de 35×35 cm. HEB-240. 8ф20. Recubrimiento=3cm

4. Sección de 40x40 cm. HEB-240. 8ф20. Recubrimiento=3cm

5. Sección de 40x40 cm. HEB-260. 8ф20. Recubrimiento=3cm

6. Sección de 45×45 cm. HEB-260. 8ф20. Recubrimiento=3cm

7. Sección de $45 \times 45 \mathrm{~cm}$. HEB-280. 8ф20. Recubrimiento=3cm

8. Sección de 45×45 cm. HEB-300. 8ф20. Recubrimiento=3cm

9. Sección de 45×45 cm. HEB-320. 8ф20. Recubrimiento=3cm

10. Sección de $50 \times 50 \mathrm{~cm}$. HEB-300. 8ф20. Recubrimiento=3cm

11. Sección de 50x50 cm. HEB-320. 8ф20. Recubrimiento=3cm

12. Sección de 50x50 cm. HEB-340. 8ф20. Recubrimiento=3cm

13. Sección de 50x50 cm. HEB-360. 8ф20. Recubrimiento=3cm 


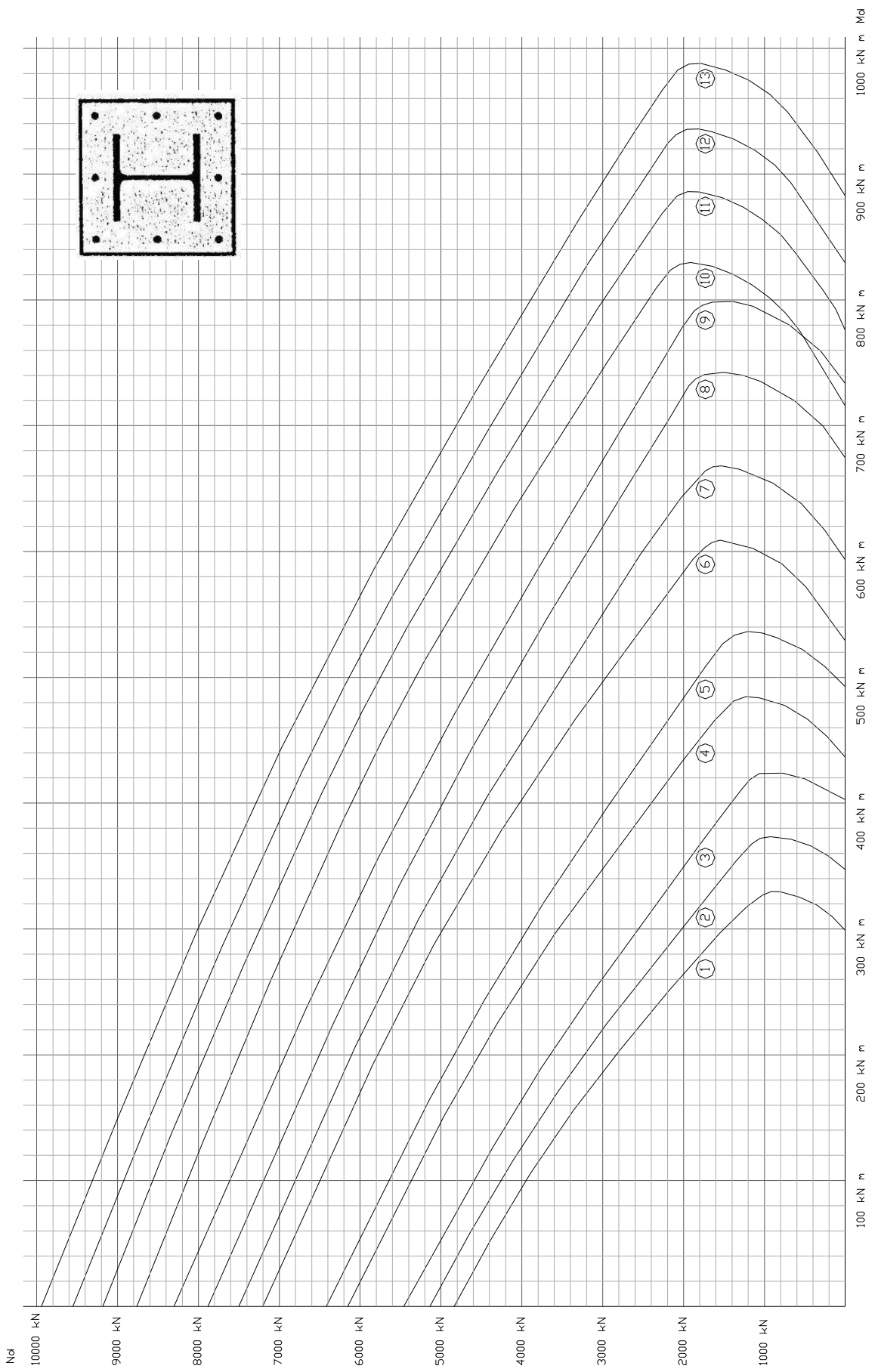

Figura 4.6-5 Tabla de dimensionamiento. Flexocompresión plana. Eje Fuerte 


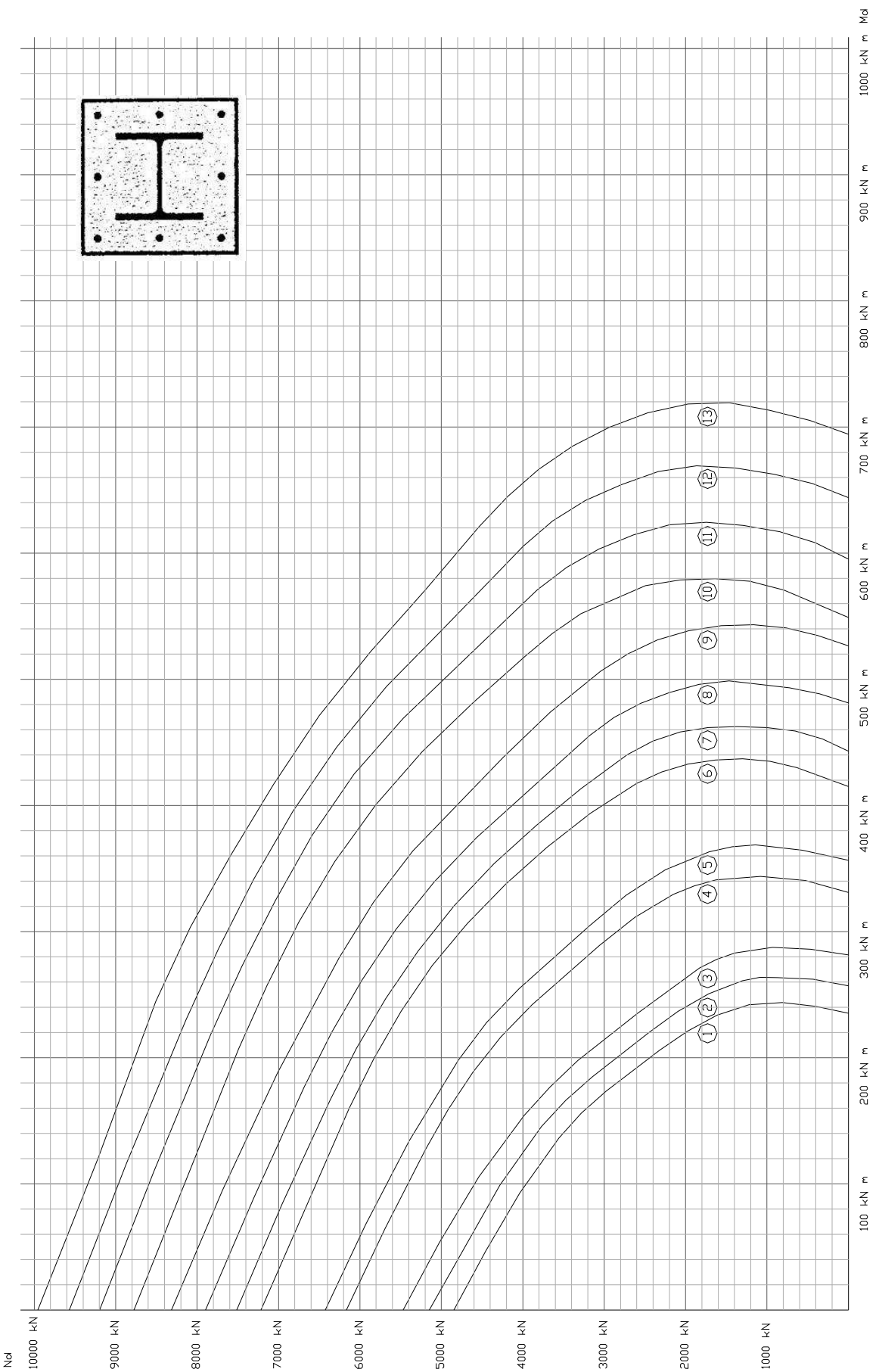

Figura 4.6-6 Tabla de dimensionamiento. Flexocompresión plana. Eje Débil 


\subsubsection{FLEXOCOMPRESIÓN ESVIADA}

En el caso de la flexocompresión esviada $\left(N+M_{Z}+M_{Y}\right)$, la curva de interacción se convierte en una superficie.

En la Figura 4.6-7 se representa la superficie correspondiente a una sección mixta, obtenida mediante el programa de cálculo desarrollado, a partir de las dos curvas de interacción respecto de los dos ejes principales de la sección $\left(N+M_{Z}\right.$ y $\left.N+M_{Y}\right)$ y otras 9 posiciones intermedias de la fibra neutra que ha ido girando desde una posición paralela al eje $Z$ hasta una posición paralela al eje $Y$ (meridianos). A continuación se representan una serie de curvas para axil constante (paralelos).

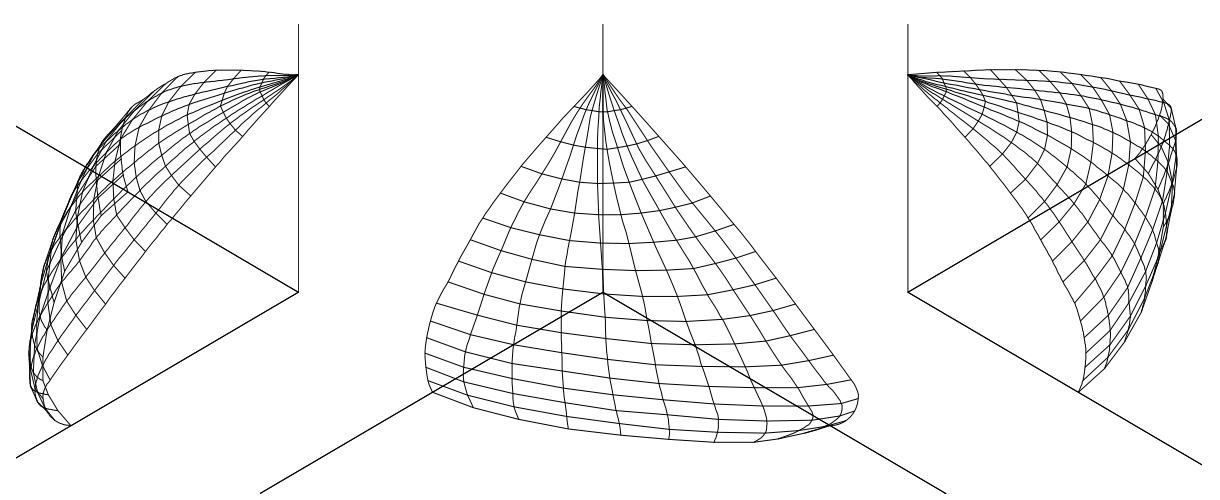

Figura 4.6-7. Representación tridimensional de la superficie de agotamiento

A partir de las mencionadas curvas, se ha modelizado en AutoCad la superficie por ellas definida. 


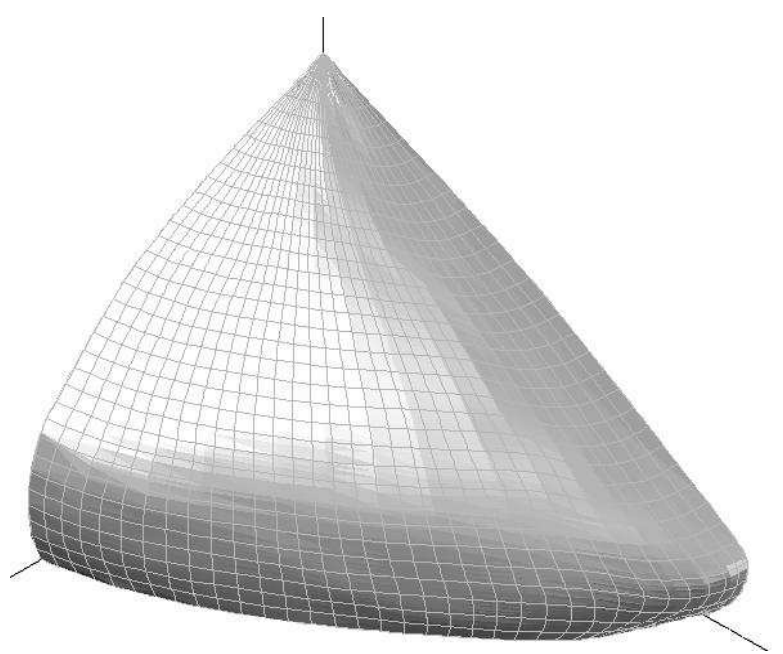

Figura 4.6-8. Superficie de agotamiento

\section{Verificación de resultados}

Para comprobar el correcto funcionamiento del programa de cálculo desarrollado en el caso de la flexocompresión esviada aplicada a secciones de hormigón armado, se han comparado sus resultados con los diagramas obtenidos mediante la aplicación "TN5. Flexión Esviada Compuesta" del anteriomente mencionado programa "Prontuario Informático del Hormigón Estructural" del IECA.

Los diagramas que se representan en la Figura 4.6-9, han sido obtenidos para una sección de hormigón armado de $35 \times 35 \mathrm{~cm}$ y cuatro barras de diámetro $20 \mathrm{~mm}$ con un recubrimiento de $3 \mathrm{~cm}$. y las características de los materiales siguientes:

Resistencias de los materiales:

Hormigón. HA-25: $f_{c k}=25 \mathrm{~N} / \mathrm{mm}^{2}$

Acero de armar. $\quad$ B-400: $\mathrm{f}_{\mathrm{sk}}=400 \mathrm{~N} / \mathrm{mm}^{2}$

Coeficientes de minoración de resistencias:

Hormigón. $\quad \gamma_{c}=1,50$

Acero de armar. $\quad \gamma_{\mathrm{s}}=1,15$ 
Para una mayor facilidad de comparación, las superficies tridimensionales de agotamiento han sido cortadas por planos correspondientes a cinco axiles distintos, de forma que se obtienen una serie de curvas que se representan en el plano.

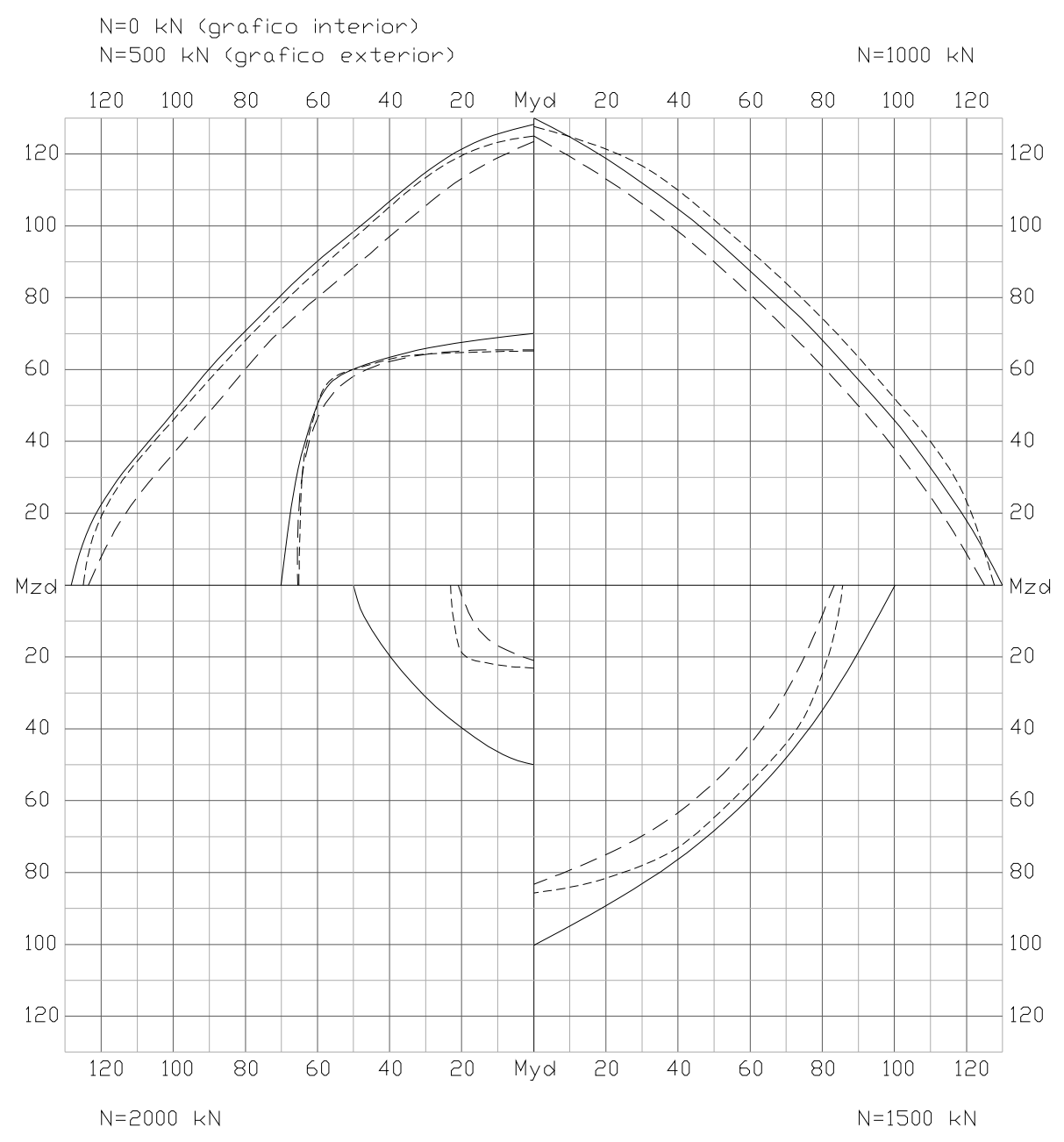

Figura 4.6-9 Análisis comparativo. Flexocompresión esviada.

Leyenda de los diagramas de interacción:

1. Línea discontinua de trazo largo: Diagrama obtenido con el Programa utilizando para el hormigón la relación tensión- 
deformación parábola rectángulo (Figura 3.1-3) y un coeficiente alfa igual a 0,85

2. Línea discontinua de trazo corto: Diagrama obtenido con el programa del IECA

3. Línea continua: Diagrama obtenido con el Programa utilizando para el hormigón la relación tensión-deformación según EC2 para cálculo estructural, (Figura 3.1-2) y un coeficiente alfa igual a 1,00

Del análisis de gráfico comparativo cabe puntualizar dos aspectos:

1. Las curvas prácticamente se solapan para los distintos valores del esfuerzo axil, salvo en el caso del mayor axil que se grafía (1.500 kN en la Figura 4.6-9) donde el programa informático desarrollado, cuando se utiliza la relación tensióndeformación del hormigón según EC2 para cálculo estructural, da como resultado momentos de agotamiento superiores a los otros dos casos representados.

Ello puede deberse la relación tensión-deformación empleada, junto a los coeficientes de minoración de la resistencia del hormigón $\left(\gamma_{c}=1,50\right.$ y $\left.\alpha=1,00\right)$

2. Las curvas generadas a partir de los datos obtenidos en el programa IECA son más abombadas (se obtienen mayores valores de solicitaciones en flexión a $45^{\circ}$ ) que las curvas generadas por el programa desarrollado.

Este hecho pasa prácticamente desapercibido para las curvas obtenidas para los menores axiles ( 0 kN, 500 kN y 1000 kN) y se acentúa con los mayores axiles 


\subsubsection{TABLAS DE DIMENSIONAMIENTO EN FLEXOCOMPRESIÓN ESVIADA}

Verificada la fiabilidad del programa de cálculo desarrollado, se han elaborado tablas que permiten el predimensionado de secciones mixtas sometidas a flexocompresión esviada.

A modo de ejemplo, se han obtenido las superficies de interacción en flexocompresión esviada de tres secciones mixtas formadas por un perfil HEB embebido en una sección cuadrada de hormigón armado y ocho barras (una por esquina y una en mitad de cada cara) de diámetro $20 \mathrm{~mm}$ con un recubrimiento de $3 \mathrm{~cm}$. Las dimensiones y perfiles de cada sección son las siguientes:

1. Sección de $35 \times 35 \mathrm{~cm}$. HEB-240. 8ф20. Recubrimiento $=3 \mathrm{~cm}$

2. Sección de $40 \times 40 \mathrm{~cm}$. HEB-260. 8ф20. Recubrimiento $=3 \mathrm{~cm}$

3. Sección de $45 \times 45 \mathrm{~cm}$. HEB-280. 8ф20. Recubrimiento=3cm

Las tablas que se muestran se han elaborado utilizando en el hormigón la relación tensión-deformación según EC2 para cálculo estructural y las siguientes características para los materiales:

Resistencias de los materiales:

Hormigón.

$\mathrm{HA}-25: \mathrm{f}_{\mathrm{ck}}=25 \mathrm{~N} / \mathrm{mm}^{2}$

Acero de armar. $\quad$ B-400: $\mathrm{f}_{\mathrm{sk}}=400 \mathrm{~N} / \mathrm{mm}^{2}$

Acero estructural. $\quad$ S-275: $\mathrm{f}_{\mathrm{yk}}=275 \mathrm{~N} / \mathrm{mm}^{2}$

Coeficientes de minoración de resistencias:

Hormigón.

$\gamma_{\mathrm{c}}=1,35$

$\alpha=1,00$

Acero de armar. $\quad \gamma_{\mathrm{s}}=1,15$

Acero estructural. $\quad \gamma_{y}=1,10$

Para su representación gráfica, esta superficie ha sido cortada por planos de axil constante, a intervalos de $500 \mathrm{kN}$. 
Curvas de dimensionamiento en flexocompresión esviada

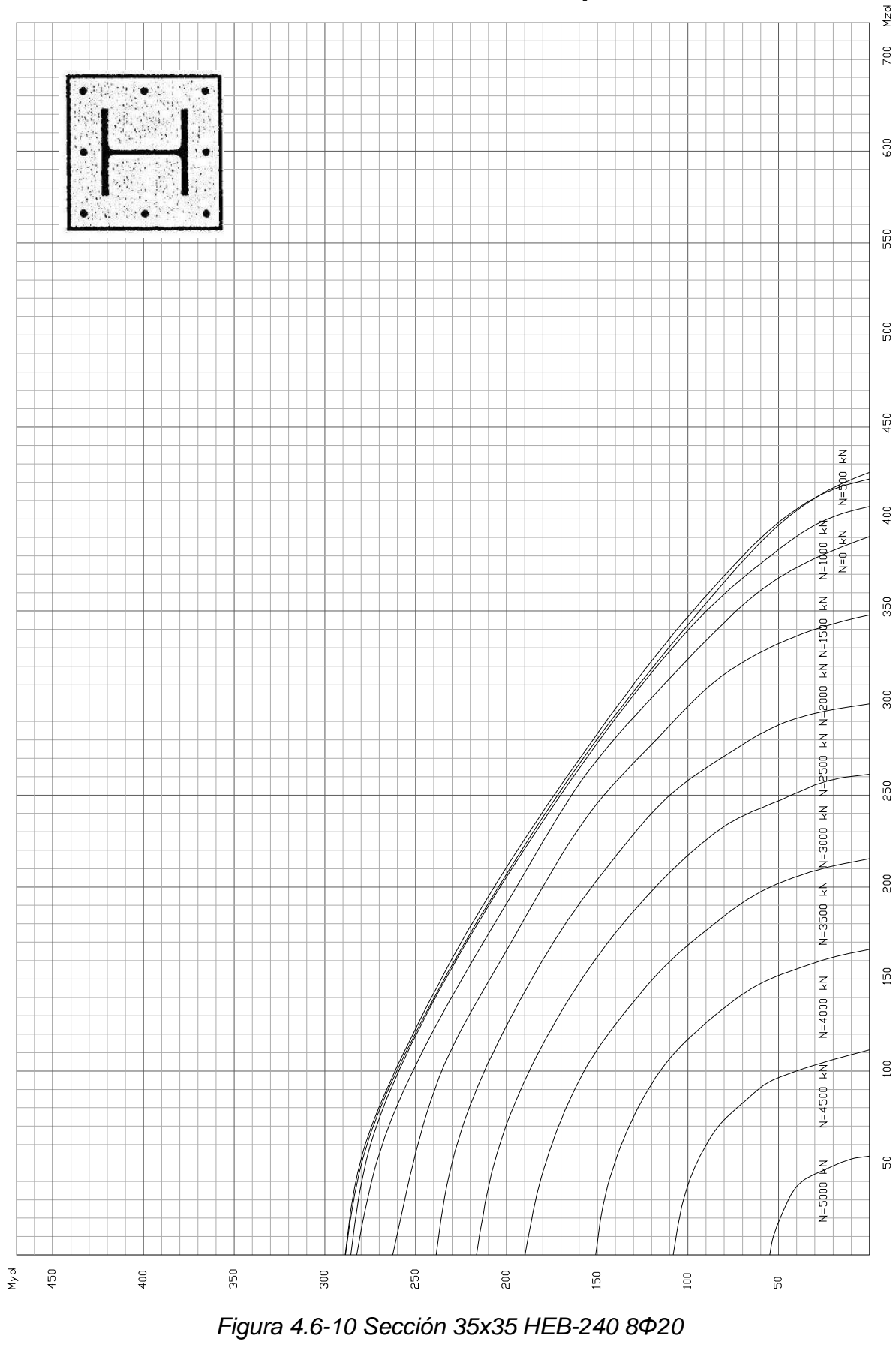




\section{Superficie mecánica}

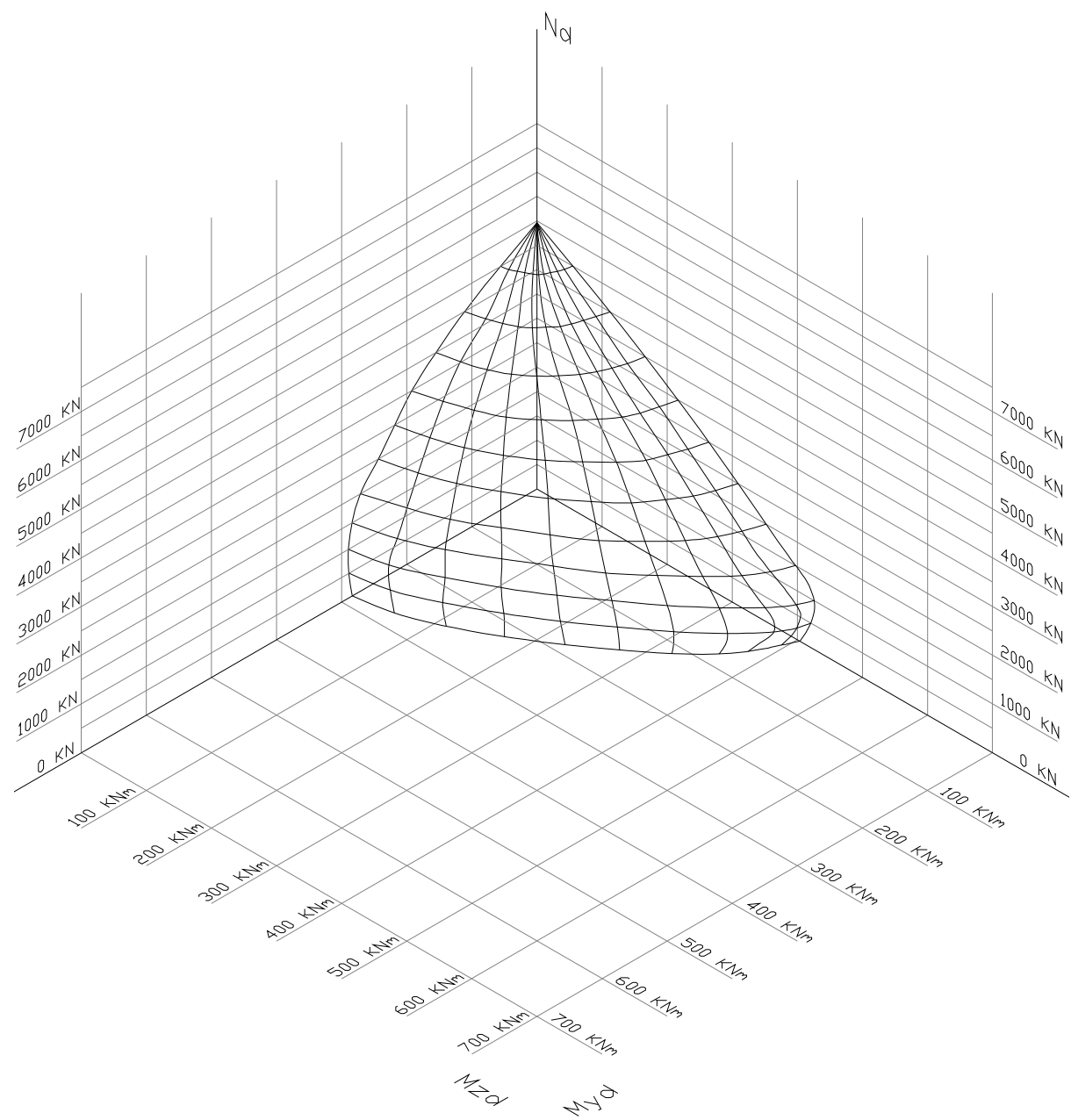

Figura 4.6-11 Sección 35×35 HEB-240 8Ф20 
Curvas de dimensionamiento en flexocompresión esviada

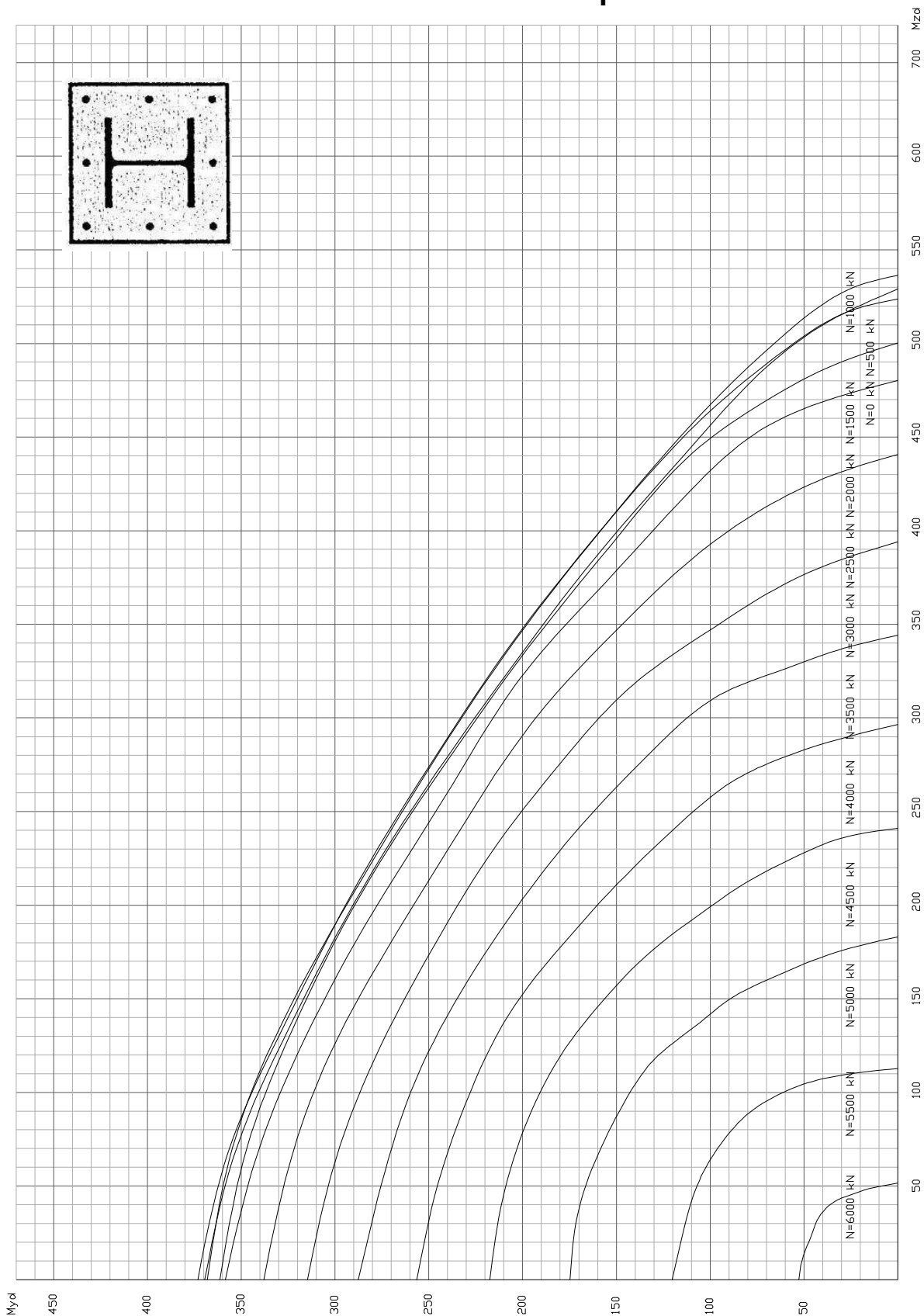

Figura 4.6-12 Sección 40x40 HEB-260 8\$20 


\section{Superficie mecánica}

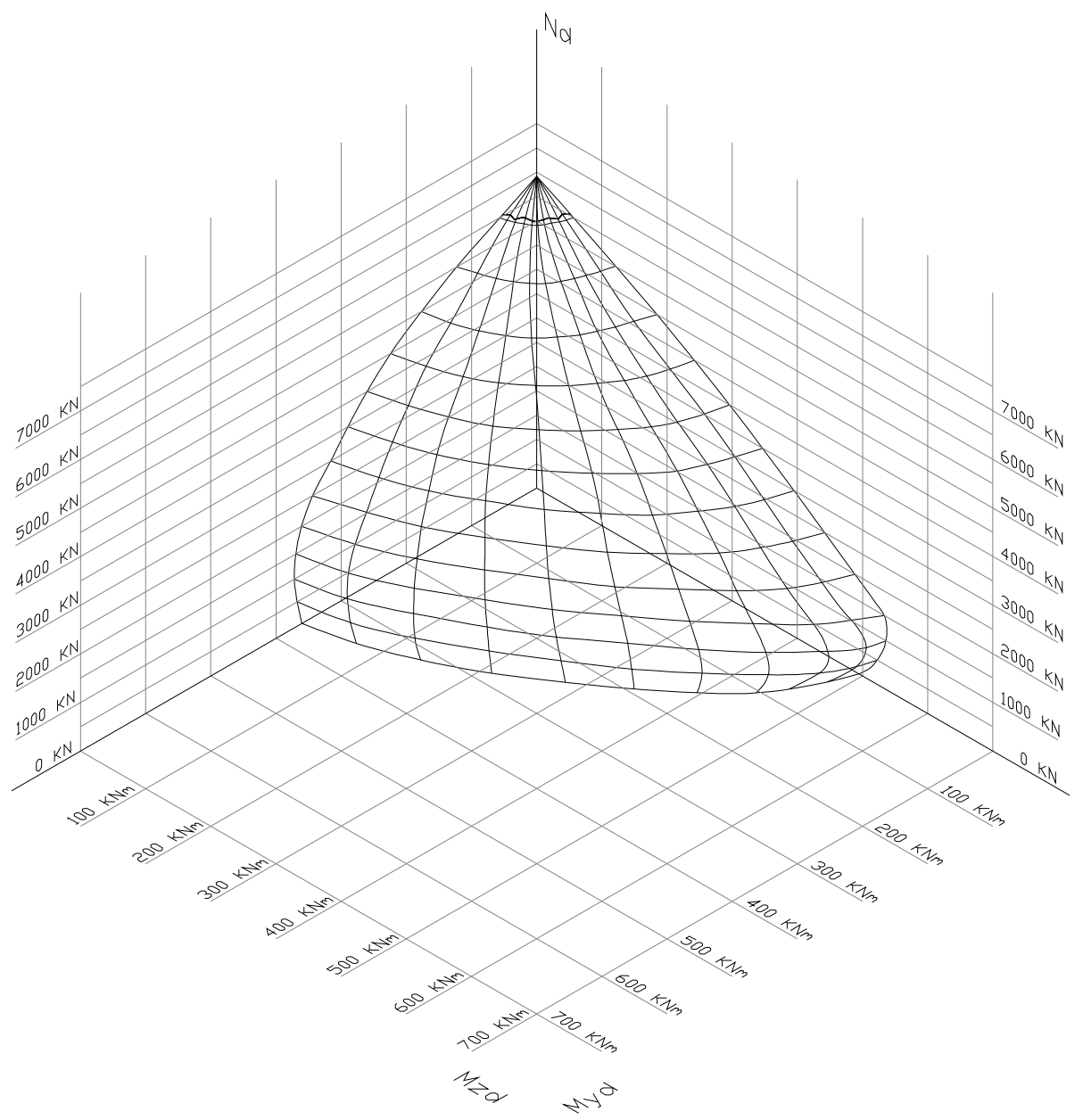

Figura 4.6-13 Sección 40x40 HEB-260 8Ф20 


\section{Curvas de dimensionamiento en flexocompresión esviada}

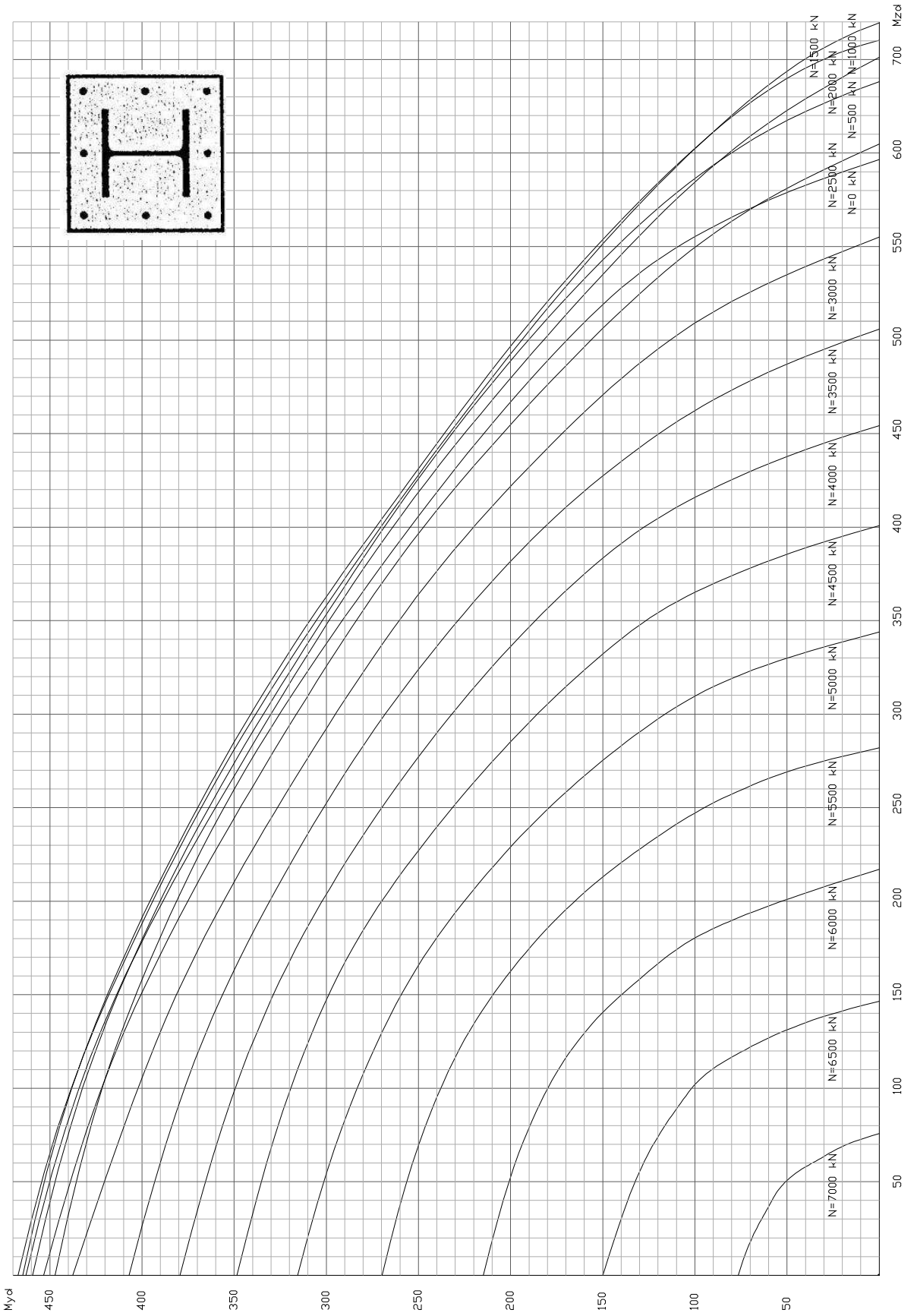

Figura 4.6-14 Sección 45×45 HEB-280 8Ф20 


\section{Superficie mecánica}

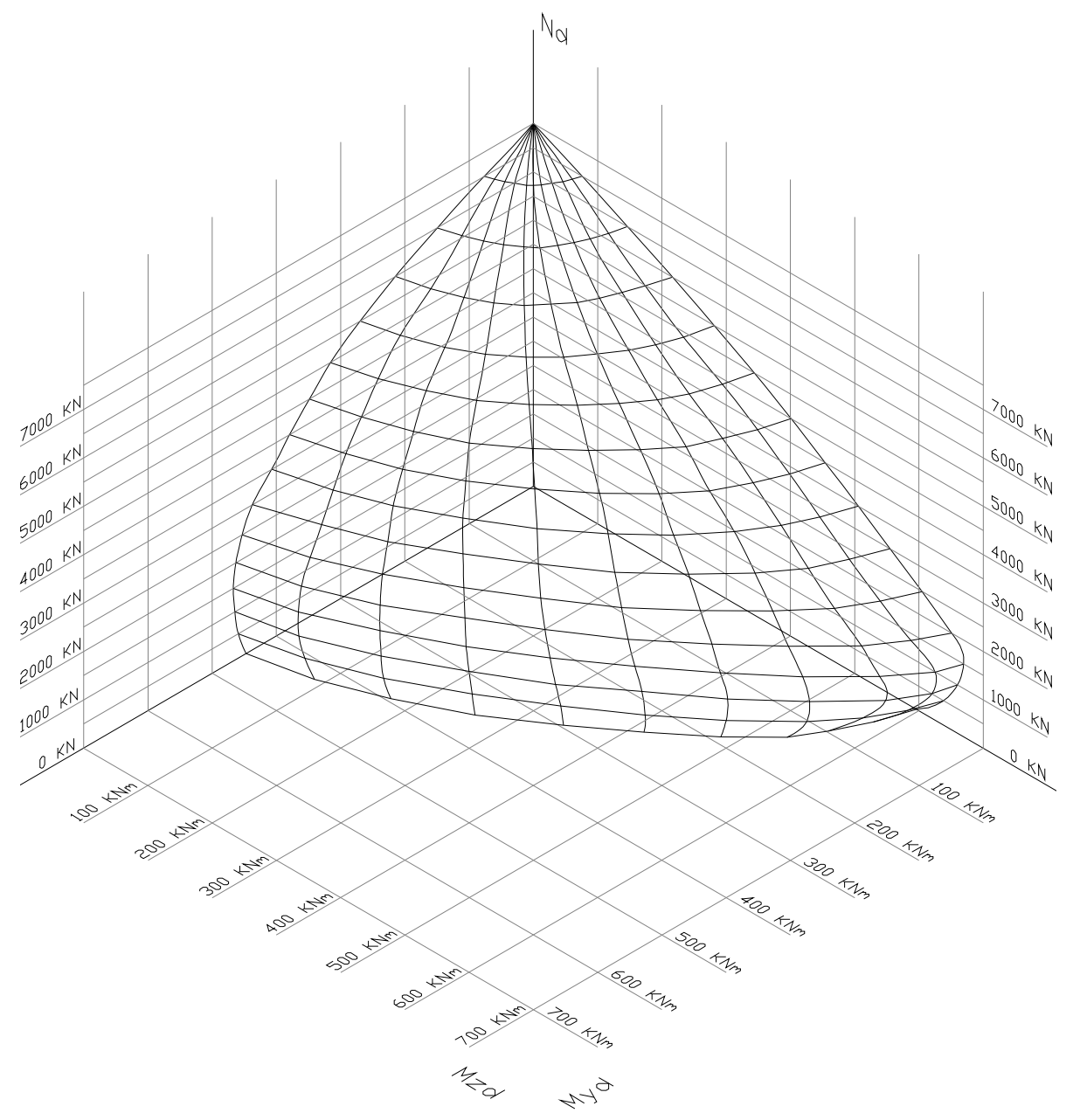

Figura 4.6-15 Sección 45x45 HEB-280 8Ф20. 


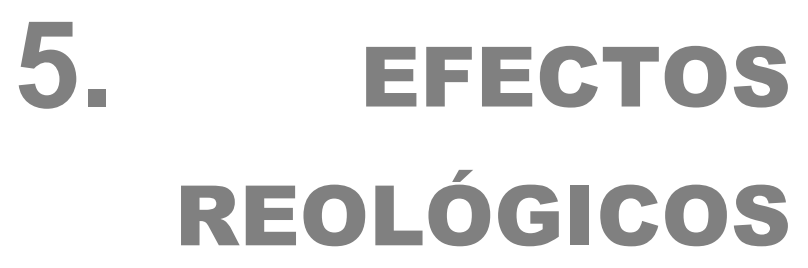




\subsection{INTRODUCCIÓN}

\subsubsection{RETRACCIÓN}

Se denomina retracción a la deformación que experimenta el hormigón a lo largo del tiempo, sin haber sido sometido a carga. Este efecto se debe, fundamentalmente, a la pérdida del agua aportada al gel del cemento que finalmente no se combina químicamente con éste.

Los factores que más influyen en la retracción son ${ }^{85}$ :

La humedad relativa. En gran medida, la retracción se debe a la pérdida de agua que experimenta el hormigón hasta alzanzar el equilibrio higrométrico con el ambiente exterior.

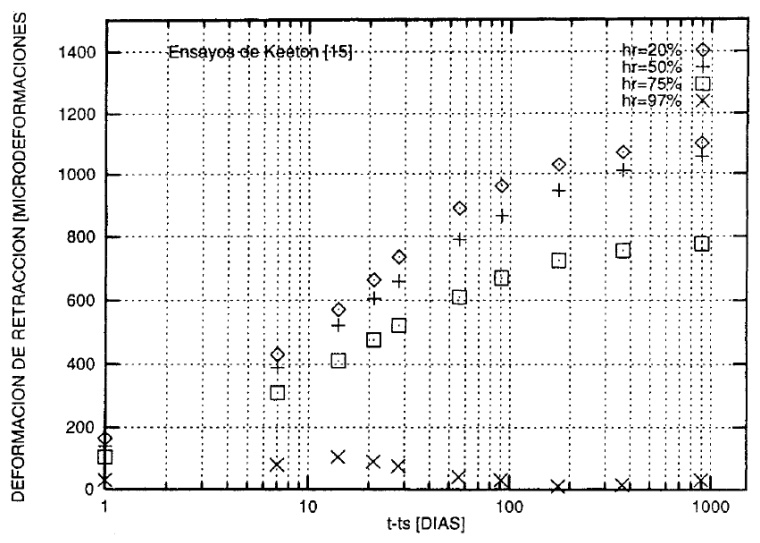

Figura 5.1-1 Influencia de la humedad relativa

85 "Caracterización de las propiedades diferidas del hormigón y su incidencia estructural" publicada por el G.E.H.O. (1998).

Todos los diagramas de resultados de ensayos contenidos en la presente Introducción a los Efectos Reológicos han sido extraídos de esta publicación. 
La influencia de este parámetro se observa en la Figura 5.1-1 obtenido a partir de los ensayos de Keeton. Además, se puede ver que la relación entre la retracción y la humedad no es lineal e incluso que, con atmósfera saturada, se produce un incremento de volumen, denominado entumecimiento.

La temperatura. De los resultados de los ensayos de Budelmann, Figura 5.1-2 realizados a una humedad relativa constante de $65 \%$ y distintas temperaturas, se observa que este factor no afecta significativamente a la retracción para temperaturas moderadas (entre $20^{\circ}$ y $50^{\circ}$ ), pero si es importante para temperaturas elevadas.

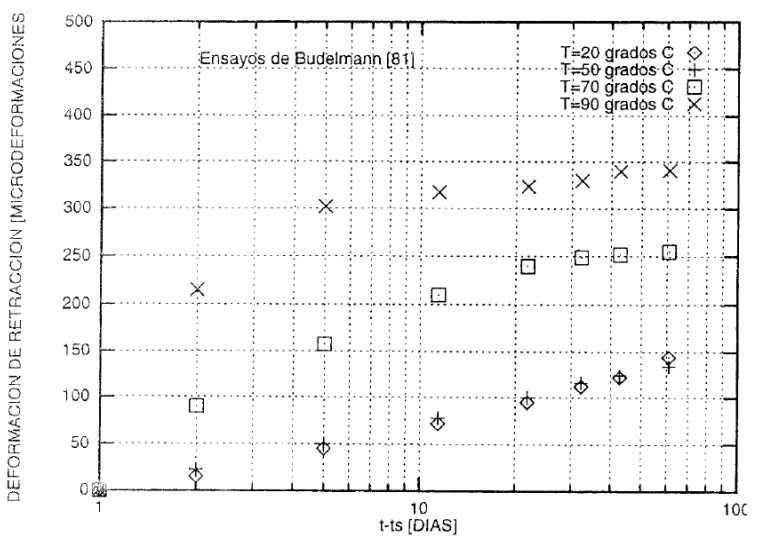

Figura 5.1-2 Influencia de la temperatura

La relación agua/cemento. Cuanto mayor sea la relación agua/cemento, mayor será la cantidad de agua existente en el gel del cemento que no podrá combinarse químicamente, mayor la cantidad de agua susceptible de evaporarse y, por lo tanto, mayor su influencia en la retracción. Esto queda constatado en los ensayos de Brooks, Figura 5.1-3, donde también se observa que la relación entre ambos no es lineal. 


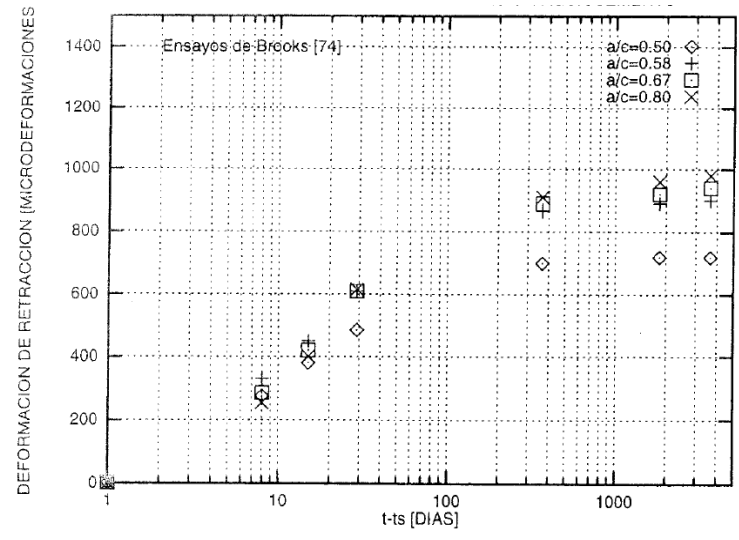

Figura 5.1-3 Influencia de la relación agua/cemento

Espesor de la pieza. La pérdida del agua existente en el gel de cemento se produce de una forma más rápida cuanto menor es el espesor de la pieza. Por ello, para edades cortas, la diferencia de retracción en piezas de distintos espesores puede ser muy importante, aunque la retracción tiende a valores similares a largo plazo. Este efecto se puede observar en la Figura 5.1-4 que representa los ensayos realizados por L'Hermitte y Mammillan, comparando los resultados a 100 y 1000 días respectivamente.

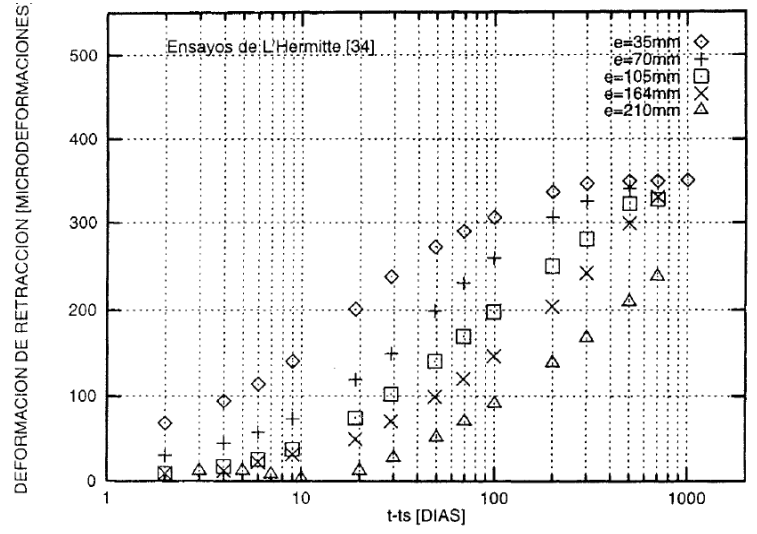

Figura 5.1-4 Influencia del espesor ficticio

La relación árido/cemento. Puesto que la retracción se produce en la pasta de cemento y no en los áridos, el incremento de la relación 
árido/cemento implicará una reducción de la deformación de retracción, como se observa en la Figura 5.1-5 obtenida de los ensayos de Wischer.

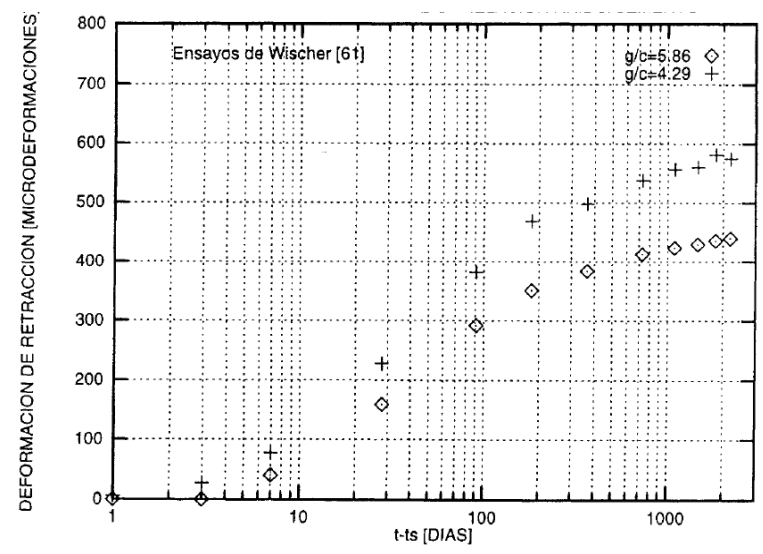

Figura 5.1-5 Influencia de la relación árido/cemento

El tiempo de curado. Aunque este factor no afecta significativamente a la deformación de retracción, si es importante puesto que la retracción se inicia al finalizar éste. Un largo tiempo de curado permite que el hormigón alcance suficiente resistencia a tracción que evite la fisuración.

\section{Caracterización de la retracción.}

Según el Model Code $1990^{86}$ y el Eurocódigo $2^{87}$, la retracción del hormigón en masa puede obtenerse a partir de la expresión:

$$
\varepsilon_{c s}\left(t, t_{s}\right)=\varepsilon_{c s 0} \cdot \beta_{s}\left(t-t_{s}\right)=\left(\varepsilon_{s} \cdot \beta_{H R}\right) \cdot\left(\frac{t-t_{s}}{0,035 \cdot e_{m}^{2}+\left(t-t_{s}\right)}\right)^{0,5}
$$

donde:

${ }^{86}$ Model Code 1990. Comité Eurointernacional del Hormigón (CEB). 1993

${ }^{87}$ UNE ENV 1992-1-1 Eurocódigo 2: Proyecto de estructuras de hormigón. Parte 1-1: Reglas generales y reglas para edificación. AENOR 1993 
$t$ es la edad del hormigón en el momento de cálculo, en días

$t_{s}$ es la edad del hormigón al inicio de la retracción, en días

$\varepsilon_{c s 0} \quad$ es el coeficiente básico de la retracción

$\beta_{s}\left(t-t_{s}\right) \quad$ representa la evolución de la retracción en el tiempo

$e_{m} \quad$ espesor medio de la pieza, en milímetros

con los valores siguientes:

$$
\begin{array}{ll}
\varepsilon_{s}=\left[160+\beta_{s c}\left(90-f_{c m}\right)\right] \cdot 10^{-6} & \\
\beta_{H R}=-1,55\left[1-(H R / 100)^{3}\right] & \text { si } 40 \% \leq H R<99 \% \cdot \beta_{s 1} \\
\beta_{H R}=0,25 & \text { si } H R \geq 99 \% \cdot \beta_{s 1} \\
\beta_{s 1}=\left(35 / f_{c m}\right)^{0,1} &
\end{array}
$$

siendo:

$\beta_{s c} \quad$ coeficiente en función de la velocidad intrínseca de hidratación del cemento que adopta los valores 4, 5 u 8 para los casos de velocidad lenta, normal o rápida, respectivamente.

$H R \quad$ Humedad relativa de la atmósfera, en tanto por ciento.

\subsubsection{FLUENCIA}

Se denomina deformación de fluencia reológica del hormigón, $\varepsilon_{c}(t)$ en la Figura $5.1-6,{ }^{88}$ a la deformación que experimenta el hormigón sometido a una carga exterior por el hecho que ésta se mantega a lo largo del tiempo.

${ }^{88}$ Esta figura se ha extraído de la publicación "Manual del hormigón estructural" de B. Perepérez y E. Barberá. 

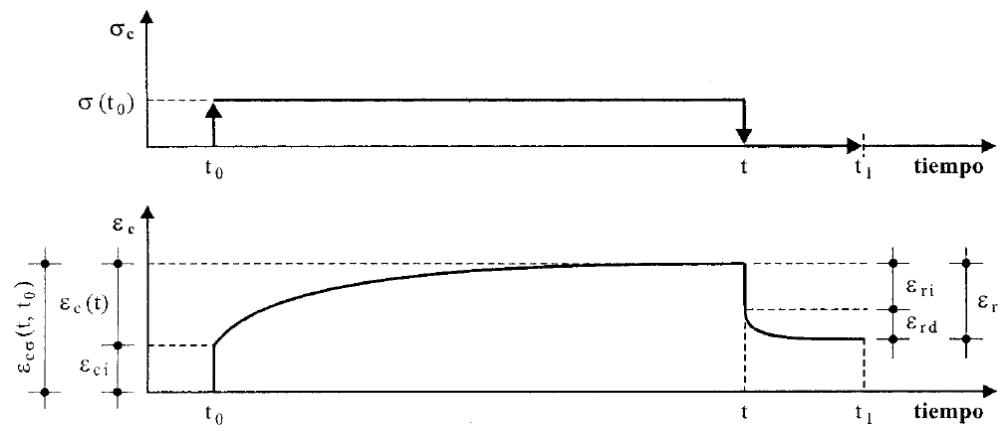

Figura 5.1-6 Gráfico de la fluencia.

Parte de la deformación total $\varepsilon_{c \sigma}\left(t, t_{0}\right)$ producida por la carga aplicada a lo largo del tiempo, se recupera si se retira esta carga $\varepsilon_{r}$. Así, una porción $\varepsilon_{r}$, cuyo valor coincide aproximadamente con la deformación elástica inicial $\varepsilon_{c i}$, se recupera de forma instantánea. Otra porción se recupera progresivamente a lo largo del tiempo $\varepsilon_{r d}$, quedando una deformación permanente.

Si las tensiones a las que se somete el hormigón no superan el $40 \%$ de su resistencia característica, la deformación de fluencia puede considerarse proporcional a la deformación instantánea. El factor que las relaciona se denomina coeficiente de fluencia. Para mayores valores de la tensión, la deformación de fluencia aumenta con mayor rapidez que aquélla.

La deformación de fluencia puede ser varias veces mayor que la instantánea, con efectos generalmente desfavorables: incrementa las flechas en elementos sometidos a flexión y las pérdidas de la fuerza de pretensado.

Varios de los factores que influyen en la fluencia coinciden con los mencionados para la retracción, ya que se trata de fenómenos estrechamente relacionados. No obstante, cabe $\operatorname{citar}^{89}$ :

89 "Caracterización de las propiedades diferidas del hormigón y su incidencia estructural” publicada por el G.E.H.O. (1998) 
La edad de la puesta en carga. Es uno de los principales factores junto con la relación agua/cemento. La Figura 5.1-7 representa los resultados de los ensayos de L'Hermitte y Mammillan con probetas cargadas a los 7, 21, 365 y 720 dias. Se aprecia que las deformaciones de fluencia son menores conforme se retrasa la puesta en carga: a una edad menor de 21 días, la carga produce una gran deformación de fluencia mientras que retrasar la carga de 1 a 2 años no produce un decremento de la deformación tan importante. El efecto se debe al proceso de hidratación de las partículas de cemento y al de polimerización de los silicatos a lo largo del tiempo, que incrementan los vínculos entre las partículas, disminuyendo la tensión unitaria.

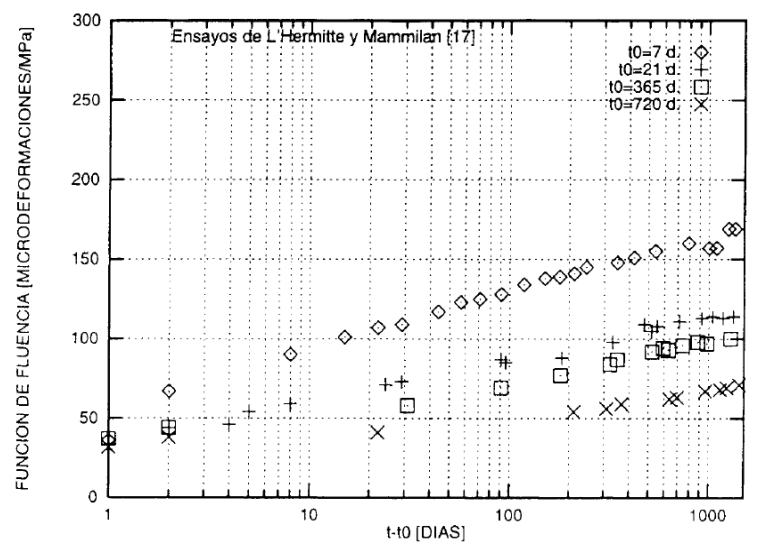

Figura 5.1-7. Influencia de la edad de puesta en carga

La resistencia del hormigón o la relación agua/cemento. Es frecuente que estos dos factores se analicen de forma conjunta, ya que la resistencia característica del hormigón depende fundamentalmente de la relación agua/cemento. El gráfico de la Figura 5.1-8 corresponde a los resultados de los ensayos de Brooks realizados con probetas de hormigón de distintas resistencias características sometidas a la misma relación entre resistencia y carga $(0,30)$. Se observa que las deformaciones de fluencia son mayores conforme aumenta la relación agua/cemento. Ello se debe a que con relaciones 
altas, la distancia entre las partículas de cemento en la sustancia coloidal será grande y se formarán vínculos débiles entre ellas, generando una mayor tensión entre los elementos resistentes.

Los dos factores se suelen tener en cuenta en los modelos reológicos a partir de la resistencia característica del hormigón, ya que ésta es un dato de partida en el cáculo.

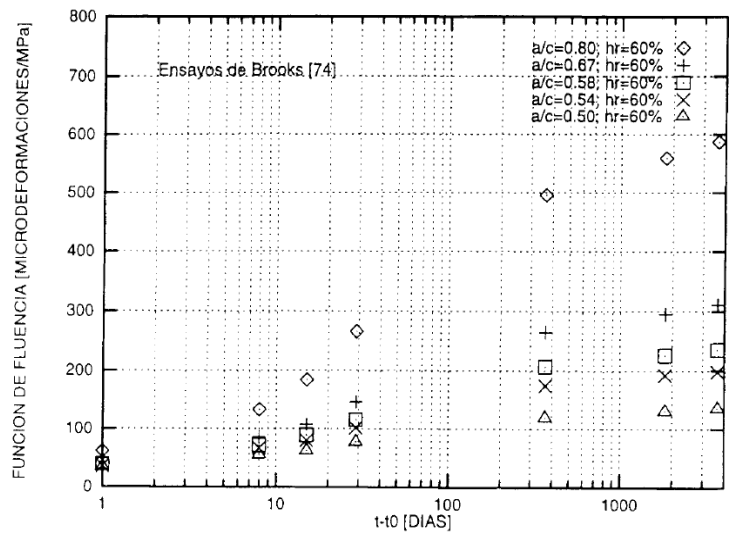

Figura 5.1-8. Influencia de la relación agua/cemento

La temperatura. El análisis independiente de los efectos de la temperatura sólo puede realizarse con probetas en cámara, ya que la variación de la temperatura afecta a la humedad relativa.

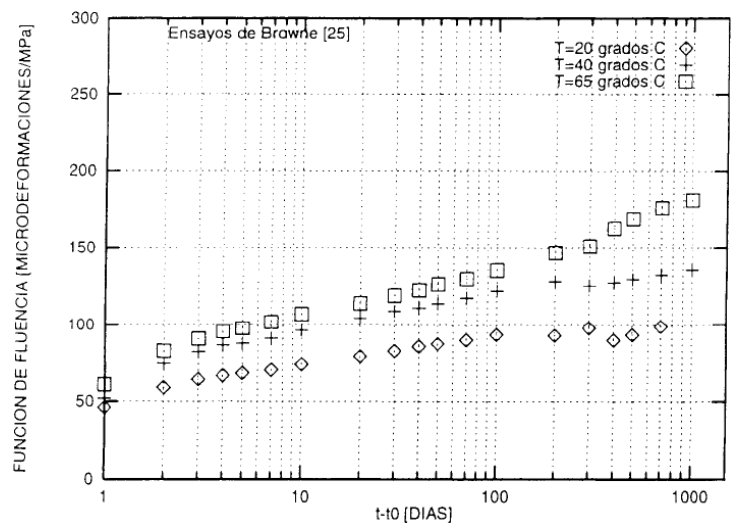

Figura 5.1-9. Influencia de la temperatura 
De los ensayos de Browne, Figura 5.1-9 se observa que las deformaciones de fluencia son mayores con temperaturas elevadas.

La humedad relativa. La gráfica de la Figura 5.1-10 representa los resultados de los ensayos de L'Hermitte realizados con probetas de hormigón a distintas humedades relativas. Se observa que a mayor grado de humedad, menores son las deformaciones de fluencia. Su influencia tiene una explicación similar a la expuesta anteriormente en el caso de la retracción.

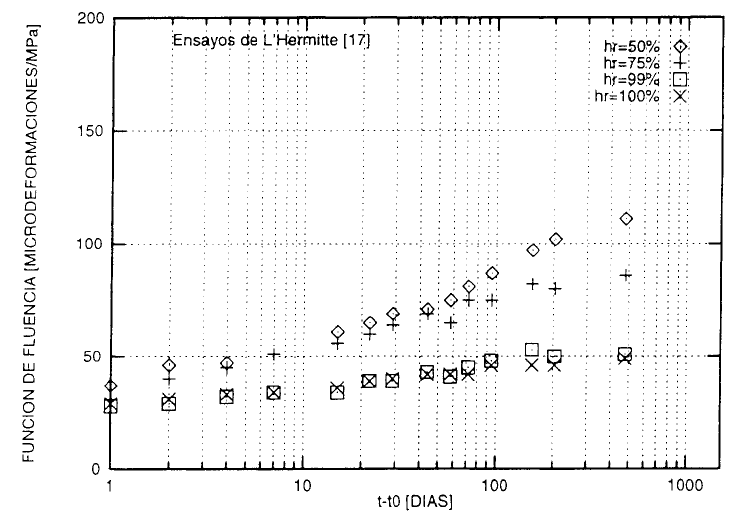

Figura 5.1-10. Influencia de la humedad relativa

El espesor de la pieza. Se entiende por espesor ficticio a la relación entre el área de la sección de hormigón y su perímetro.

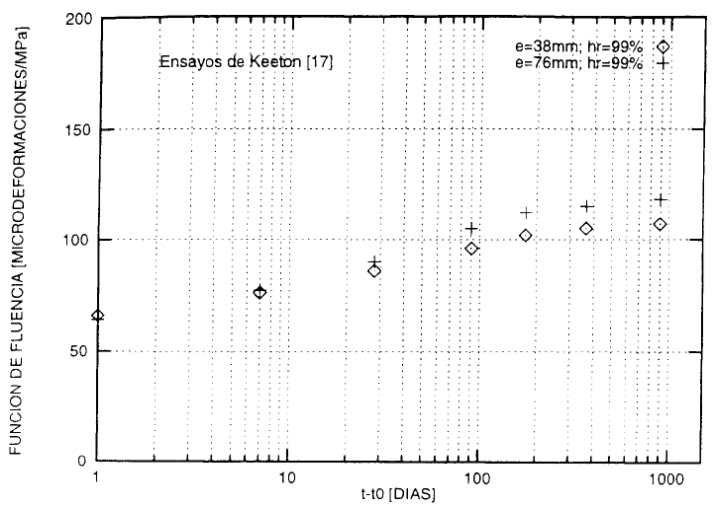

Figura 5.1-11. Influencia del espesor ficticio 
De los resultados de los ensayos de Keeton, Figura 5.1-11, realizados con espesores de 38 y $76 \mathrm{~mm}$, se observa que la influencia de este factor es menos relevante que los expuestos anteriormente.

El tipo de cemento. Los resultados de los ensayos de Wesche demuestran que la deformación de fluencia es menor en hormigones fabricados con cementos con una mayor velocidad de endurecimiento.

La tensión. La deformación de fluencia es sensiblemente proporcional a la tensión hasta que se alcanza una relación tensión/resistencia característica en el entorno del 40\%. A partir de este valor se pierde la proporcionalidad, aumentando de una forma más rápida la deformación conforme se incrementa la tensión.

La relación árido/cemento y el tipo de árido. Los ensayos de Rüsch demuestran que la deformación de fluencia disminuye conforme se incrementa el módulo de deformación del árido. A pesar de la gran importancia de este factor, no es considerado en los modelos reológicos dada la incertidumbre sobre el tipo de árido en el momento del cálculo.

\section{Caracterización de la fluencia.}

La caracterización de la fluencia según el Model Code $1990^{90}$ para hormigones de densidad ordinaria, sometidos a tensiones de compresión constantes que no superen el $40 \%$ de su resistencia característica, una humedad relativa comprendida entre el $40 \%$ y el $100 \%$ y una temperatura entre $5^{\circ}$ y $30^{\circ}$ es la siguiente:

${ }^{90}$ Model Code 1990. Comité Eurointernacional del Hormigón (CEB). 1993 


$$
\varepsilon_{c \sigma}\left(t, t_{0}\right)=\varepsilon_{c i}+\varepsilon_{c}(t)=\frac{\sigma\left(t_{0}\right)}{E_{0}\left(t_{0}\right)}+\frac{\sigma\left(t_{0}\right)}{E_{0}(28)} \varphi\left(t, t_{0}\right)
$$

siendo:

$$
\begin{array}{ll}
\mathcal{E}_{c s}\left(t, t_{0}\right) & \text { deformación total } \\
\varepsilon_{c i} & \text { deformación instantánea } \\
\varepsilon_{c}(t) & \text { deformación de fluencia } \\
\sigma\left(t_{0}\right) & \text { tensión aplicada sobre el elemento } \\
E_{0}\left(t_{0}\right) & \begin{array}{l}
\text { módulo de deformación longitudinal inicial del } \\
\text { hormigón a la edad } t_{0} .
\end{array} \\
E_{0}(28) & \begin{array}{l}
\text { módulo de deformación longitudinal inicial del } \\
\text { hormigón a } 28 \text { días. }
\end{array} \\
\varphi\left(t, t_{0}\right) \quad \begin{array}{l}
\text { coeficiente de fluencia que se puede determinar } \\
\text { mediante la expresión: } \varphi\left(t, t_{0}\right)=\varphi_{0} \cdot \beta_{c}\left(t, t_{0}\right)
\end{array}
\end{array}
$$

Siendo, en esta última expresión $\varphi_{0}$ el coeficiente básico de fluencia que se puede obtener de la siguente ecuación:

$$
\varphi_{0}=\varphi_{H R} \cdot \beta\left(f_{c m}\right) \cdot \beta\left(t_{0}\right)=\left[1+\frac{100-H R}{9,9 \cdot e_{m}^{1 / 3}}\right] \cdot\left[\frac{16,8}{\left(f_{c k}+8\right)^{0,5}}\right] \cdot\left[\frac{1}{0,1+t_{0}^{0,2}}\right]
$$

y $\beta_{c}\left(t, t_{0}\right)$ función hiperbólica del tiempo que modula la evolución de la fluencia definida por:

$$
\beta_{c}\left(t, t_{0}\right)=\left[\frac{t-t_{0}}{\beta_{H}+\left(t-t_{0}\right)}\right]^{0,3}
$$

Con:

$$
\begin{aligned}
& \beta_{H}=1,5 \cdot e_{m} \cdot\left[1+(0,012 \cdot H R)^{18}\right\rfloor+250 \leq 1500 \\
& e_{m} \quad \text { espesor medio de la pieza, en milímetros } \\
& H R \quad \text { Humedad relativa de la atmósfera, en tanto por ciento. }
\end{aligned}
$$




\subsection{CRITERIO DE LA EHE-08}

La Instrucción de Hormigón Estructural EHE-08 ${ }^{91}$, en el artículo 25 "Análisis en el tiempo", contenido en el Capítulo 5, "Análisis Estructural", aborda los efectos estructurales de la fluencia, retracción y envejecimiento del hormigón, y de la relajación del acero de pretensado. Según se indica, estos efectos pueden ser deformaciones y desplazamientos diferidos, así como variaciones en el valor o en la distribución de esfuerzos, reacciones o tensiones.

En el apartado 25.2 se propone un método general, bajo la aplicación de las siguientes hipótesis:

- Se considera un comportamiento lineal de los distintos aceros frente a las cargas instantáneas.

Para aceros de pretensado, con tensiones superiores a 0,5 $f_{p \max }$ se tendrá en cuenta la relajación y el hecho de que ésta se produce a deformación variable.

- Se considera que existe adherencia perfecta entre el hormigón y las armaduras adherentes y entre los distintos hormigones que pudieran existir en la sección.

- En el caso de elementos lineales, se considera válida la hipótesis de deformación plana de las secciones.

- Se deben verificar las condiciones de equilibrio a nivel de cualquier sección.

- Se debe verificar el equilibrio a nivel de estructura teniendo en cuenta las condiciones de apoyo.

${ }^{91}$ EHE-08 Instrucción de hormigón estructural. Real decreto/2008, de 18 de julio, por el que se aprueba la instrucción de hormigón estructural (EHE-08) 
- La ecuación que determina las deformaciones del hormigón a lo largo del tiempo es:

- $\varepsilon_{c}(t)=\frac{\sigma\left(t_{0}\right)}{E_{c}(t)}+\varphi\left(t, t_{0}\right) \frac{\sigma_{0}}{E_{c}(28)}+\sum_{i=1}^{n}\left(\frac{1}{E_{c}\left(t_{i}\right)}+\frac{\varphi\left(t, t_{i}\right)}{E_{c}(28)}\right) \Delta \sigma\left(t_{i}\right)+\varepsilon_{r}\left(t, t_{s}\right)$

Ecuación 5.2-1

Significado de los términos de la ecuación constitutiva:

- El primer sumando representa la deformación instantánea debida a una tensión aplicada en $t_{0}$.

- El segundo sumando representa la fluencia del hormigón, debida a dicha tensión.

- El tercer sumando representa la suma de la deformación instantánea y de fluencia debida a la variación de tensiones que se produce en un instante $t_{i}$.

- El cuarto sumando representa la deformación de retracción

El significado de los símbolos utilidados en la Ecuación 5.2-1 se pueden encontrar en el Anejo 1 de la EHE-08:

- $\varepsilon_{c}(t)$ deformación del hormigón en el tiempo $t$

- $\quad \varepsilon_{r}\left(t, t_{s}\right)$ deformación de retracción del hormigón desde el inicio

- $\sigma(t) \quad$ tensión normal en el tiempo $t$.

- $\sigma_{0} \quad$ tensión normal inicial.

- $E_{c}(t)$ módulo de deformación del hormigón a la edad $t$.

- $\quad E_{c}(28)$ módulo de deformación del hormigón a 28 días. 
- $\varphi\left(t, t_{i}\right)$ coeficiente de evolución de la fluencia en en periodo de tiempo considerado

\subsection{CRITERIO DE LOS EUROCÓDIGOS}

Al respecto de la consideración de los efectos de fluencia y retracción, el artículo 4.8.2.2 incluido en el apartado "Método General" para el cálculo de soportes mixtos, del Eurocódigo 4, en virtud de los párrafos que se enumeran, establece:

(8) Se considerarán los efectos de retracción y fluencia si son capaces de reducir significativamente la estabilidad estructural.

(9) Como simplificación, pueden despreciarse los efectos de la fluencia si el incremento de los momentos flectores de primer orden debido a las deformaciones por fluencia y a la fuerza longitudinal resultante de las cargas permanentes, no excede del $10 \%$.

(10) De acuerdo con el apartado A.3.4(9) del EC2, pueden despreciarse normalmente las deformaciones debidas a la fluencia de elementos esbeltos en compresión, en pórticos intraslacionales de edificación con uniones rígidas a las losas o vigas en sus dos extremos.

El artículo aludido en el párrafo anterior se puede encontrar en el Anexo 3 del Eurocódigo 2, y en él se indica:

En edificios intraslacionales, las deformaciones debidas a la fluencia de piezas esbeltas comprimidas unidas monolíticamente a losas o vigas en sus dos extremos pueden despreciarse en general porque sus efectos suelen verse compensados por otras 
influencias que se desprecian en el cálculo. En soportes interiores, los empotramientos de sus extremos reducen en gran manera las deformaciones debidas a la fluencia, por lo que pueden despreciarse. En soportes exteriores con excentricidades diferentes en cada uno de sus extremos, la fluencia aumenta las deformaciones, pero esto no disminuye la capacidad de carga porque estas deformaciones no se suman a las del soporte en el estado de agotamiento correspondiente.

Si tenemos en cuenta que el uso de soportes mixtos en estructuras de edificación se suelen proyectar formando parte de pórticos con sistemas de arriostramiento que garantizan su intraslacionalidad y enlazados con las vigas mediante uniones rígidas, y por lo tanto cumpliendo las condiciones establecidas en el artículo 4.8.2.2(10) de EC4, no sería necesaria la consideración de los efectos de fluencia y retracción para el cálculo de soportes mixtos en estructuras de edificación.

No obstante, la ambigüedad en la redacción de estos artículos puede ocasionar ciertas dudas en su aplicación:

- Si no se evalúan los efectos de retracción y fluencia, ¿cómo puede saberse si son capaces de reducir significativamente la estabilidad estructural (8), o si el incremento de los momentos flectores de primer orden debido a sus deformaciones excede o no del $10 \%$ (9) ?

- No queda claro que siempre puedan despreciarse estos efectos en estructuras intraslacionales de nudos rígidos, ya que (10) indica "pueden despreciarse normalmente las deformaciones..."

Por ello, se considera necesario recurrir a otros artículos de los propios Eurocódigos que abordan específicamente los efectos de la 
deformación diferida del hormigón, en los que, de una forma más clara, se deduce la consideración de estos efectos.

Por ejemplo, el artículo A.1.2 (1) del anexo 1 del EC2 indica:

Cuando se considere que la influencia de las deformaciones diferidas vaya a ser de tal importancia que se requiera para su evaluación el uso de procedimientos avanzados de cálculo (por ejemplo en presencia de coacciones elásticas; cuando ocurran deformaciones impuestas; cuando se considere la presencia de armaduras simétricas 0 asimétricas; para construcciones mixtas de hormigón y acero; etc.) deberá hacerse uso de la literatura especializada correspondiente, de acuerdo con $\mathrm{P}(1), \mathrm{P}(2)$ y (5) de 2.5.5.

En la ejecución de tales cálculos, no deberá olvidarse la precisión implícita en A.1.1.2(3) y A.1.1.3(1). Para realizar previsiones con exactitud es necesario un conocimiento de la historia del medio ambiente y de la composición y propiedades de los materiales.

y en los mencionados párrafos del artículo 2.5.5.1 del mismo Eurocódigo se especifica:

$\mathrm{P}(1) \quad$ La precisión de los procedimientos para el cálculo de los efectos de la fluencia y de la retracción del hormigón deberá ser consecuente con la fiabilidad de los datos disponibles para la descripción de dichos fenómenos y con la importancia de sus efectos en el estado límite considerado.

$\mathrm{P}(2) \quad$ En general, los efectos de la fluencia y la retracción solo se considerarán en los estados límite de servicio. Una excepción importante son los efectos de segundo orden. 
Por todo ello, se concluye en la oportunidad de la consideración de los efectos reológicos del hormigón:

- Porque de la consideración del artículo 2.5.5.1 del EC2, se puede deducir que se debe tener en cuenta los citados efectos en el caso de estructuras mixtas de hormigón y acero, y cuando se plantee el análisis por un procedimiento de segundo orden.

- Porque el trabajo que nos ocupa, además de la obtención de resultados que faciliten la aplicación práctica al dimensionamiento, pretende realizar un estudio lo más exhaustivo posible de soportes mixtos con la evaluación de los diversos parámetros que en él intervienen.

- Porque, del análisis comparativo, podrá cuantificarse su efecto y de esta forma verificar el alcance de la aplicación del artículo 4.8.2.2 del EC4.

\subsection{EVALUACIÓN DE LOS EFECTOS REOLÓGICOS}

Para la evaluación de los efectos reológicos aplicaremos el artículo 2.5.5.1 de EC2:

Artículo 2.5.5.1

(5) Se pueden hacer las siguientes hipótesis para mejor estimar el comportamiento de una sección de hormigón si las tensiones se mantienen dentro de los límites correspondientes a las condiciones normales de servicio:

- la retracción y la fluencia son independientes;

- existe una relación lineal entre la fluencia y la tensión que produce la fluencia; 
- los efectos de la humedad y de la distribución no uniforme de temperaturas son despreciables;

- el principio de superposición a las acciones ocurridas en diferentes edades del hormigón es aceptable;

- estas hipótesis son también aceptables con el hormigón a tracción.

(7) La función de fluencia viene dada por la relación:

$$
J\left(t, t_{0}\right)=1 / E_{c}\left(t_{0}\right)+\phi\left(t, t_{0}\right) / E_{c 28}
$$

Ecuación 5.4-1

donde:

$t_{0}$ es el tiempo al iniciar la carga del hormigón

$t$ es el tiempo considerado

$J\left(t, t_{0}\right) \quad$ es la función de fluencia en el tiempo $t$.

$E_{c}\left(t_{0}\right) \quad$ es el módulo de elasticidad tangente en el tiempo $t_{0}$.

$E_{c 28} \quad$ es el módulo de elasticidad tangente a 28 días.

$\phi\left(t, t_{0}\right) \quad$ es el coeficiente de fluencia relativo a la deformación elástica a los 28 días, calculada con $E_{c 28}$

(9) Según las hipótesis enunciadas en (5), la deformación total del hormigón sometido a una carga inicial en el tiempo $t_{0}$ con una tensión $\sigma\left(t_{0}\right)$ y sometido a las subsiguientes variaciones de tensión $\Delta \sigma\left(t_{i}\right)$ en el tiempo $t_{i}$, puede expresarse de la siguiente manera:

$$
\varepsilon_{\text {tot }}\left(t, t_{0}\right)=\varepsilon_{n}(t)+\sigma\left(t_{0}\right) \cdot J\left(t, t_{0}\right)+\sum J\left(t, t_{i}\right) \Delta \sigma\left(t_{i}\right)
$$


En esta expresión $\varepsilon_{n}(t)$ es una deformación impuesta independiente de las tensiones (por ejemplo retracción, efectos de la temperatura).

(10) A efectos del análisis estructural, la Ecuación 5.4-2 puede escribirse de la siguiente forma:

$$
\varepsilon_{\text {tot }}\left(t, t_{0}\right)=\varepsilon_{n}(t)+\sigma\left(t_{0}\right) \cdot J\left(t, t_{0}\right)+\left\{\sigma(t)-\sigma\left(t_{0}\right)\right\} \cdot\left\{\frac{1}{E_{0}\left(t_{0}\right)}+\chi \cdot \frac{\phi\left(t, t_{0}\right)}{E_{c 28}}\right\}
$$

Ecuación 5.4-3

Donde el coeficiente de edad $\chi$ depende de la evolución de la deformación con el tiempo.

(11) En los casos normales, $\chi$ puede tomarse como 0,8. Esta simplificación es adecuada tanto en los casos de relajación pura de los efectos de una deformación impuesta constante, como en los casos en los que solo se consideren los efectos a largo plazo.

Los valores de los distintos parámetros que intervienen en la Ecuación 5.4-3 los obtendremos de la consideración del artículo 3.1.2.5.5 del EC2.

Artículo 3.1.2.5.5

(1) La fluencia y retracción del hormigón dependen fundamentalmente de la humedad ambiental, de las dimensiones del elemento y de la composición del hormigón. La fluencia también está influida por la madurez del hormigón al aplicarle la carga por primera vez y por la duración y magnitud de la carga. Cualquier estimación del coeficiente de fluencia $\phi\left(t, t_{0}\right)$ y de la 
deformación de retracción $\varepsilon_{c s}$ debe tener en cuenta estos parámetros.

(2) Cuando no se requiera gran precisión, los valores que se dan en la Tabla 5.4-1 y Tabla 5.4-2 pueden considerarse como el coeficiente final de fluencia $\phi\left(\infty, t_{0}\right)$ y la deformación final de retracción $\varepsilon_{\mathrm{cs} \infty}$ respectivamente, para un hormigón de peso normal sometido a compresión que no exceda de $|0,45| \cdot f_{c k}$ a partir del tiempo $t_{0}$ de la primera carga. En la Tabla 5.4-1, el coeficiente de fluencia $\phi\left(\infty, t_{0}\right)$ se refiere a $E_{c m}$ de acuerdo con la Tabla 3.1-4 y la ecuación $E_{c m}=9,5 \cdot\left(f_{c k}+8\right)^{1 / 3}$. Si se requiere mayor precisión debe consultarse el anexo 1.

\begin{tabular}{|c|c|c|c|c|c|c|}
\hline & \multicolumn{6}{|c|}{ Espesor ficticio $2 A_{c} / u(e n ~ m m)$} \\
\hline & 50 & 150 & 600 & 50 & 150 & 600 \\
\hline $\begin{array}{l}\text { Edad al } \\
\text { cargar } \\
t_{0} \text { (dias) }\end{array}$ & \multicolumn{3}{|c|}{$\begin{array}{l}\text { Condiciones atmosféricas } \\
\text { secas (interiores) } \\
\text { (RH =50\%) }\end{array}$} & \multicolumn{3}{|c|}{$\begin{array}{l}\text { Condiciones atmostéricas } \\
\text { húmedas (exteriores) } \\
\text { (RH = } 80 \%)\end{array}$} \\
\hline 1 & 5,5 & 4,6 & 3,7 & 3,6 & 3,2 & 2,9 \\
\hline 7 & 3,9 & 3,1 & 2,6 & 2,6 & 2,3 & 2,0 \\
\hline 28 & 3,0 & 2,5 & 2,0 & 1,9 & 1,7 & 1,5 \\
\hline 90 & 2,4 & 2,0 & 1,6 & 1,5 & 1,4 & 1,2 \\
\hline 365 & 1,8 & 1,5 & 1,2 & 1,1 & 1,0 & 1,0 \\
\hline
\end{tabular}

Tabla 5.4-1 Coeficiente final de fluencia $\phi\left(\infty, t_{0}\right)$ para hormigones de peso normal

\begin{tabular}{|c|c|cc|}
\hline $\begin{array}{c}\text { Situación del } \\
\text { elemento }\end{array}$ & $\begin{array}{c}\text { Humedad relativa } \\
(\%)\end{array}$ & \multicolumn{2}{|c|}{$\begin{array}{c}\text { Espesor ficticio } 2 \mathbf{A}_{\mathrm{c}} \mathbf{u}(\mathrm{mm}) \\
\mathbf{6 0 0}\end{array}$} \\
\hline Interior & 50 & $-0,60$ & $-0,50$ \\
\hline Exterior & 80 & $-0,33$ & $-0,28$ \\
\hline
\end{tabular}

Tabla 5.4-2 Deformación final de retracción $\varepsilon_{c s_{\infty}}($ en \%) para hormigones de peso normal 
El mencionado Anexo 1 del EC2 permite evaluar, con mayor precisión, el coeficiente de fluencia (Tabla 5.4-1) y las deformaciones debidas a la retracción (Tabla 5.4-2), teniendo en cuenta, mediante una serie de coeficientes, el efecto de distintos parámetros que les afectan, tales como el tipo de cemento, las temperaturas elevadas o reducidas en el curado del hormigón o la humedad relativa.

Evidentemente, y tal como se indica en el articulado del Anexo 1, "Para realizar previsiones con exactitud es necesario un conocimiento de la historia del medio ambiente y de la composición y propiedades de los materiales"

Puesto que el trabajo que nos ocupa tiene un carácter genérico y no se dispone de los datos concretos necesarios para poder evaluar con precisión los coeficientes de fluencia y las deformaciones debidas a la retracción, se ha optado por aplicar el citado artículo 3.1.2.5.5 y utilizar los valores aproximados de las tablas en él contenidas.

\subsection{VALORES ADOPTADOS}

Para la aplicación de la Ecuación 5.4-3 es necesario adoptar una serie de valores previos, que, dado el carácter genérico el trabajo han sido:

$$
\begin{array}{llr}
t_{0} & 28 \text { días } & \text { (edad al cargar) } \\
2 \cdot A_{d} U & 150 \mathrm{~mm} . & \text { (espesor ficticio) } \\
R H & 50 \% & \text { (condiciones atmosféricas secas, interiores) }
\end{array}
$$

A partir de los cuales, y entrando en la Tabla 5.4-1 y Tabla 5.4-2 se obtienen, respectivamente, los siguientes valores: 
- coeficiente final de fluencia

$$
\begin{aligned}
& \phi\left(\infty, t_{0}\right)=2,5 \\
& \varepsilon_{c s \infty}=-0,60 \cdot 10^{-3}
\end{aligned}
$$

- deformación final de retracción

Si además consideramos que $\sigma(t)=\sigma\left(t_{0}\right)$, lo que implica que:

$$
E_{c}\left(t_{0}\right)=E_{28}=E_{c m}
$$

Ecuación 5.5-1

la Ecuación 5.4-3 queda simplificada de la siguiente forma:

$$
\varepsilon_{\text {tot }}\left(t, t_{0}\right)=\varepsilon_{n}(t)+\sigma\left(t_{0}\right) \cdot J\left(t, t_{0}\right)
$$

Si sustituimos en ésta la función de la fluencia en el tiempo según la Ecuación 5.4-1, obtenemos:

$$
\varepsilon_{\text {tot }}\left(t, t_{0}\right)=\varepsilon_{n}(t)+\sigma\left(t_{0}\right) \cdot J\left(t, t_{0}\right)=\varepsilon_{n}(t)+\sigma\left(t_{0}\right) / E_{c}\left(t_{0}\right)+\sigma\left(t_{0}\right) \cdot \phi\left(t, t_{0}\right) / E_{c 28}
$$

Y haciendo uso de la Ecuación 5.5-1, podemos reordenar de la siguiente forma:

$$
\varepsilon_{\text {tot }}\left(t, t_{0}\right)=\varepsilon_{n}(t)+\frac{\sigma\left(t_{0}\right)}{E_{c m}} \cdot\left(1+\phi\left(t, t_{0}\right)\right)
$$

Sustituyendo en la ecuacón anterior, los valores del coeficiente final de fluencia y de la deformación final de retracción, anterioremente indicados y refiriendo $t_{0}=28$ días y $t=\infty$, resulta:

$$
\varepsilon_{t o t}=-0,6 \cdot 10^{-3}+\frac{\sigma_{c 0}}{E_{c m}} \cdot(1+2,5)=-0,6 \cdot 10^{-3}+3,5 \cdot \frac{\sigma_{c 0}}{E_{c m}}
$$

siendo $\sigma_{0}$ la tensión inicial ${ }^{92}$ en cada punto de la sección de hormigón producida por las cargas exteriores consideradas de corta duración.

92 Las compresiones se considerarán de signo negativo 
Suponiendo que únicamente el $70 \%$ de las cargas totales se pueden considerar de carácter permanente (cargas que influyen en la fluencia) y considerando una relación lineal entre la fluencia y la tensión que la ocasiona (según EC2), podemos corregir la expresión anterior de la siguiente forma:

$$
\varepsilon_{\text {tot }}=-0,6 \cdot 10^{-3}+0,70 \cdot 3,5 \cdot \frac{\sigma_{c 0}}{E_{c m}}=-0,6 \cdot 10^{-3}+2,45 \cdot \frac{\sigma_{c 0}}{E_{c m}}
$$

Ecuación 5.5-2

\section{Efecto sobre el diagrama reológico del hormigón:}

Como se puede observar en la ecuación anterior, la deformación total es el resultado de la suma de dos términos:

- El primer sumando, correspondiente al efecto de la retracción, es un valor constante, lo que implica que, respecto del diagrama noval, la deformación inicial, producida por la tensión inicial, se incrementa de forma constante. Por tanto, el diagrama reológico sufre una traslación debida a la retracción.

- El segundo sumando, correspondiente al efecto de la fluencia, no es un valor constante sino proporcional a la tensión inicial, de forma que, respecto del diagrama noval, la deformación inicial producida por la tensión inicial, se incrementa de una forma tanto mayor cuanto mayor es la tensión inicial. Por tanto, el diagrama tensión-deformación sufre un desplazamiento debido a la fluencia ${ }^{93}$.

El resultado final de ambos efectos se representa gráficamente en la Figura 5.5-1

93 "Pandeo de estructuras de hormigón armado". Jordi Maristany. (1996) 


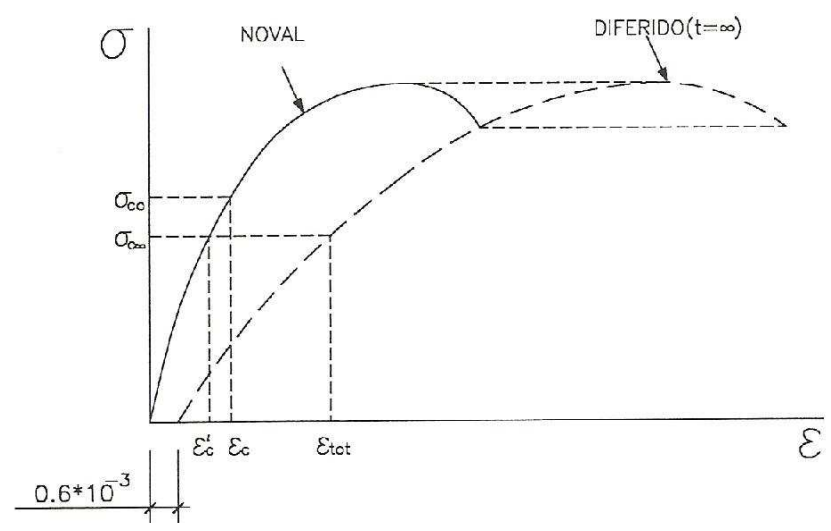

Figura 5.5-1 Diagrama tensión-deformación noval y diferido del hormigón

La aplicación informática desarrollada con motivo del presente trabajo permite considerar los efectos reológicos del hormigón en la capacidad resistente de la sección, mediante el empleo de la relación tensión-deformación mostrada en trazos discontínuos en la figura anterior.

A efectos prácticos, el proceso operativo para la determinación de la tensión y deformación final en el hormigón sería el siguiente:

1. Para una determinada tensión $\sigma_{c 0}$ se obtiene la deformación $\varepsilon_{c}$ en el diagrama reológico noval del hormigón, por aplicación de la función que relaciona tensión-deformación (Ecuación $3.1-2)^{94}$.

2. Se calcula una "deformación ficticia" mediante la expresión:

$$
\varepsilon_{c}^{\prime}=\frac{\varepsilon_{c}+0,6 \cdot 10^{-3}}{2,45}
$$

Tanto la deformación inicial como la ficticia se consideran de signo negativo.

94 El proceso también puede ser inverso, es decir, a partir de una deformación $\varepsilon_{c}$ conocida, se puede determinar, mediante la relación citada, el valor de la tensión $\sigma_{c 0}$ 
3. Entrando en el diagrama reológico noval con esta deformación ficticia $\mathcal{E}_{c}^{\prime}$ se determina la tensión final en el hormigón $\sigma_{c^{\infty}}{ }^{95}$

4. La deformación final, sobre la gráfica que incluye los efectos reológicos se puede determinar sustituyendo en la Ecuación 5.5-2 el valor de la tensión final en el hormigón, $\sigma_{c^{\infty}}$, quedando de la siguiente forma:

$$
\varepsilon_{t o t}=-0,6 \cdot 10^{-3}+2,45 \cdot \frac{\sigma_{c \infty}}{E_{c m}}
$$

\subsection{RESULTADOS: ESTUDIO COMPARATIVO DEL EFECTO REOLÓGICO}

Para la comprobación del efecto reológico sobre el diagrama de interacción se ha utilizado una sección mixta formada por un perfil HEB-220 embebida en una sección de hormigón armado de 35×35 $\mathrm{cm}$ y cuatro barras (una por esquina) de diámetro $20 \mathrm{~mm}$ con un recubrimiento de $3 \mathrm{~cm}$

Resistencias de los materiales:
Hormigón.
HA-25: $f_{\mathrm{ck}}=25 \mathrm{~N} / \mathrm{mm}^{2}$
Acero de armar.
B-400: $\mathrm{f}_{\mathrm{sk}}=400 \mathrm{~N} / \mathrm{mm}^{2}$
Acero estructural.
S-275: $\mathrm{f}_{\mathrm{yk}}=275 \mathrm{~N} / \mathrm{mm}^{2}$

Coeficientes de minoración de resistencias:

$\begin{array}{ll}\text { Hormigón. } & \gamma_{c}=1,35 \\ \text { Acero de armar. } & \gamma_{s}=1,15 \\ \text { Acero estructural. } & \gamma_{y}=1,10\end{array}$

95 Se puede observar que la tension final en el hormigón $\sigma_{c \infty}$ disminuye respecto de la tensión inicial $\sigma_{c o}$ ya que al incrementarse la deformación debido a los efectos diferidos, parte de la carga que inicialmente soporta el hormigón se transfiere al acero. 


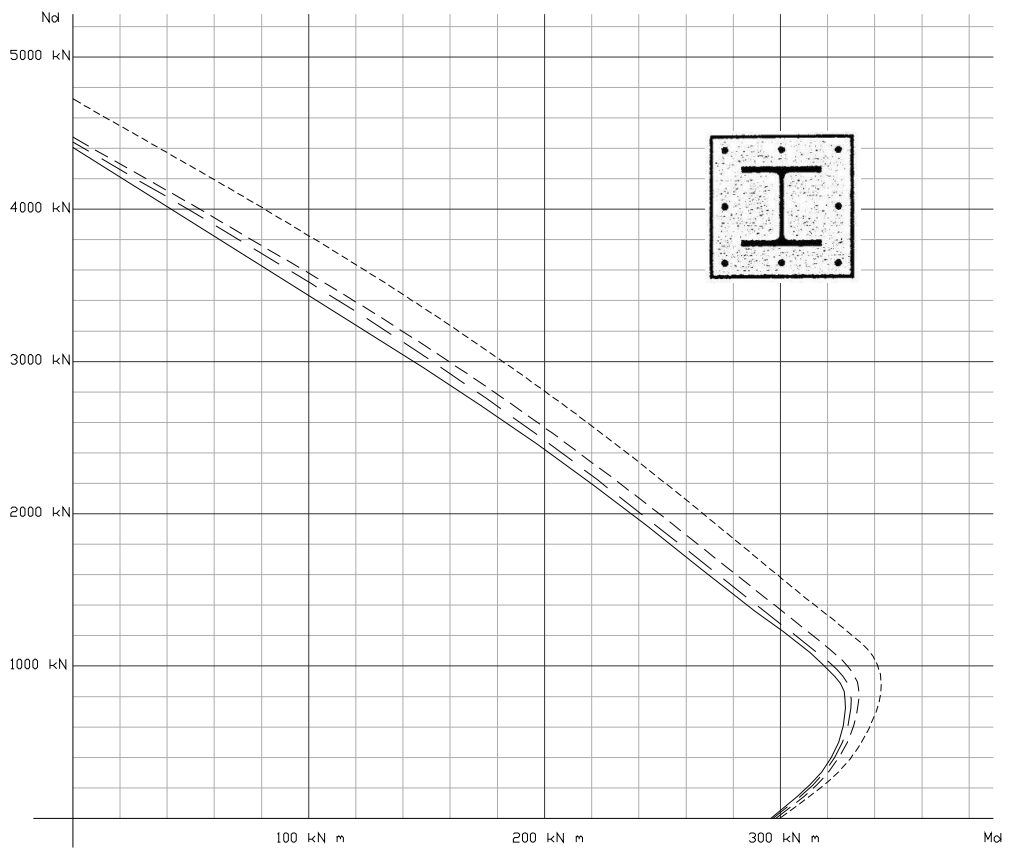

Figura 5.6-1 Eje Fuerte. Comparativo: Efecto Reológico

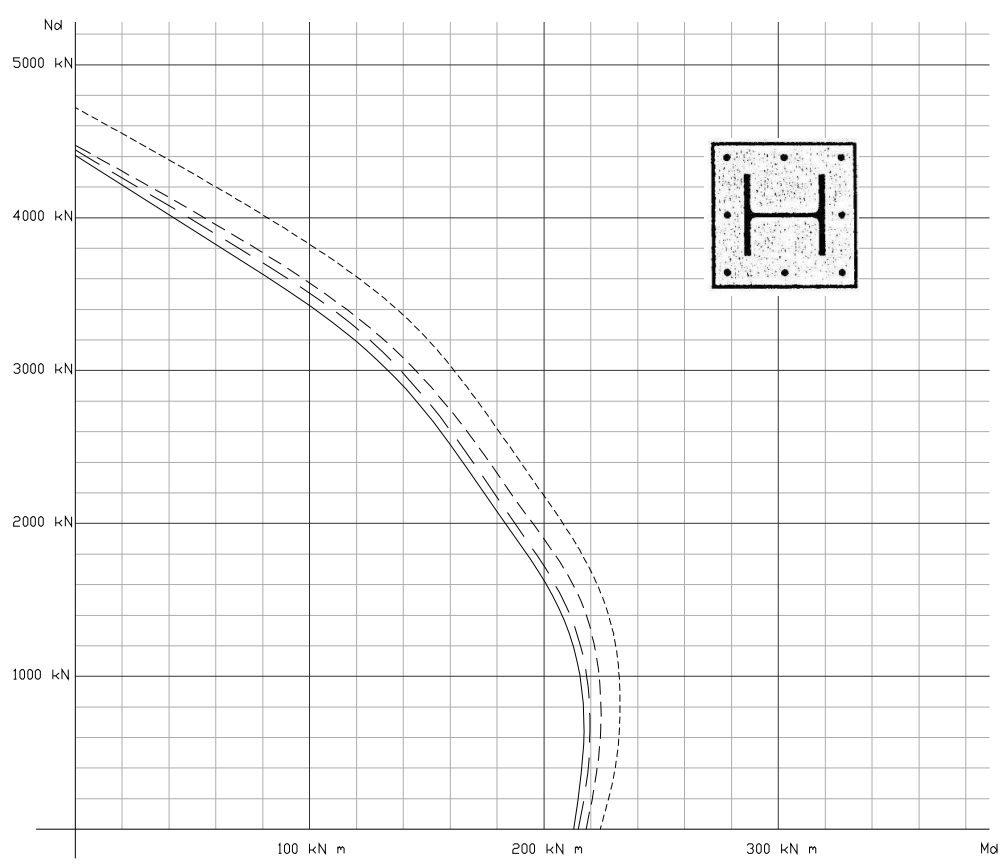

Figura 5.6-2 Eje Débil. Comparativa: Efecto Reológico. 
Leyenda de los diagramas de interacción:

1. Trazos cortos: Diagrama obtenido SIN Efectos Reológicos, utilizando para el hormigón el diagrama tensión-deformación según EC2 para cálculo estructural y un coeficiente alfa igual a 1,0

2. Trazos medios: Diagrama obtenido SIN Efectos Reológicos, utilizando para el hormigón el diagrama tensión-deformación parábola-rectángulo y un coeficiente alfa igual a 0,85

3. Trazos largos: Diagrama obtenido SIN Efectos Reológicos, utilizando para el hormigón el diagrama tensión-deformación según EC2 para cálculo estructural y un coeficiente alfa igual a 0,85 .

4. Trazo continuo: Diagrama obtenido CON Efectos Reológicos, utilizando para el hormigón el diagrama tensión-deformación según EC2 para cálculo estructural y un coeficiente alfa igual a 1,0

Como conclusiones de la comparación de los gráficos de la figura anterior, se puede observar que la curva que tiene en cuenta los efectos reológicos conduce a una menor capacidad resistente de la sección, especialmente cuando predomina el axil sobre el flector. Esta curva prácticamente coincide, con la obtenida sin la consideración de los efectos reológicos, con el mismo diagrama tensión-deformación según EC2 y aplicando un coeficiente alfa igual a 0,85 .

En el caso analizado, la reducción de la capacidad resistente de la sección varía entre el $7 \%$ en el caso de la compesión centrada y el $1,3 \%$ - $5 \%$, para el eje fuerte y el débil respectivamente, en la flexión simple. 


\subsection{INTRODUCCIÓN.}

En el anterior capítulo 4 se ha realizado el estudio de la sección mixta y se han mostrado las curvas y superficies de interacción de distintas secciones mixtas, para el caso de la flexocompresión plana y esviada, respectivamente, generadas mediante un programa informático desarrollado para la ocasión.

En el Capítulo 5 se han estudiado los efectos reológicos en el hormigón cuya consideración se ha implementado como una opción de cálculo en el mencionado programa.

En los Capítulos siguientes se va a realizar el salto conceptual de la Sección al estudio de la Barra y a continuación al Conjunto Estructural. De los distintos métodos para abordar su análisis, que se exponen, en el apartado 7.4 para el caso de la Barra y en los apartados 8.3 y 8.4 para la Estructura, se ha optado por ensamblar el módulo de cálculo desarrollado en esta tesis, junto con un programa de análisis estructural, denominado $\mathrm{AV} \_\mathrm{CID}^{96}$, capaz de realizar su cálculo en régimen no lineal.

Por ello, se incluye el presente Capítulo con el objeto de mostrar el enlace entre el programa de cálculo de la Sección Mixta y el entorno del Cálculo Estructural. En él se expondrán, brevemente, las características del mencionado entorno y las intervenciones que en él se han realizado para poder ensamblar el programa desarrollado en el presente trabajo.

${ }^{96}$ Programa informático "AV_CID: Calcul i Diseny d'estructures". Autor: D. Adolfo Alonso Durá.

Aunque el programa puede emplearse profesionalmente, la versión utilizada esta destinada a la investigación. 


\subsection{EL PROGRAMA AV_CID.}

Con carácter general, se puede definir el programa AV_CID como un programa informático de diseño y cálculo de estructuras, compuesto por los siguientes módulos ${ }^{97}$ :

Módulo de generación del modelo de diseño de la estructura.

Se trata del módulo de entrada de datos, que permite la completa definición del modelo estructural (geometría, materiales, vínculos externos y cargas) en un entorno CAD (Computer Asist Design), en concreto en AutoCAD. La elección de este entorno se debe a su amplia utilización como programa de dibujo en el ámbito de la arquitectura y a que su diseño permite incorporarle aplicaciones que amplían sus posibilidades gráficas.

Módulo de generación de la base de datos del modelo de cálculo.

Una vez definido el modelo de diseño, el presente módulo se encarga de procesarlo y de generar un fichero que contenga todos sus datos estructurales para su posterior análisis.

\section{Módulo de cálculo.}

Se trata del módulo que realiza el análisis de la estructura y que presenta dos opciones: cálculo en régimen elástico y lineal y cálculo no lineal.

El análisis lineal de las estructuras de barras se realiza por el método matricial de las rigideces y en el caso de las estructuras superficiales

${ }^{97}$ Una más amplia descripción de estos módulos se puede consultar en el ANEXO del presente trabajo. 
y volumétricas, el procedimiento de cálculo de deformaciones, solicitaciones y tensiones se basa en el método de los elementos finitos.

El procedimiento de resolución del problema no lineal consiste por un lado en un tratamiento incremental de la aplicación de la carga, planteándose en cada uno de los pasos una variante de la ecuación de equilibrio: $\left[K_{t}\right]\{d \Delta\}=\{d P\}$ que se resuelve en el campo lineal; y por otro, en un proceso iterativo, por desarrollo del método NewtonRaphson, para la búsqueda de la solución en cada incremento de carga.

Con el objeto de reducir el ancho de banda de la matriz de rigidez, un procedimiento basado en el algoritmo de Cuthill y McKee, incluido en el presente módulo, realiza una renumeración de los nudos de la estructura. Los datos de la matriz de rigidez se almacenan por columnas y únicamente hasta la altura de valores no nulos, según el denominado método del perfil o sky-line.

La obtención del vector de movimientos de los nudos $\{\delta\}$, por resolución de la ecuación $[K] \cdot\{\delta\}=\{F\}$, donde $K$ representa la matriz de rigidez global de la estructura y $\{\mathrm{F}\}$ las acciones aplicadas en los nudos, se realiza por aplicación del método de Crout modificado ${ }^{98}$.

\section{Módulo de visualización gráfica.}

Distintos procedimientos permiten la visualización tanto del modelo de diseño como de los resultados obtenidos tras su cálculo. Entre estos resultados cabe citar la deformada de la estructura, diagramas de solicitaciones en estructuras de barras, líneas de isovalores de momentos en estructuras superficiales y gráficos de tensiones en

\footnotetext{
${ }^{98}$ La descripción del método y el proceso de aplicación se pueden consultar en el apartado 0 del ANEXO del presente trabajo.
} 
estructuras superficiales y volumétricas y sobre el terreno en losas de cimentación ${ }^{99}$.

\section{Módulo de dimensionamiento de elementos y de comprobación de elementos de acero.}

El programa incluye una serie de aplicaciones que, a partir de las solicitaciones y deformaciones obtenidas por el módulo de análisis, y en base a una serie de opciones elegidas por el usuario, tales como la resistencia de los materiales, realizan:

- el ajuste de las dimensiones de las zapatas y riostras.

- el dimensionamiento de las armaduras necesarias de distintos elementos (soportes, vigas, zapatas, riostras,...) de hormigón armado.

- la comprobación de la validez de las secciones de acero propuestas en el modelo de diseño.

\subsection{INTERVENCIONES REALIZADAS.}

La integración del módulo de cálculo de soportes mixtos en el programa de análisis estructural ha exigido la intervención en distintas partes de este programa y el desarrollo de diversas rutinas de ensambaje que se aportan como méritos de la presente tesis doctoral.

Las intervenciones más importantes consisten en la inclusión del tipo de secciones mixtas en el modelo de diseño (entorno AutoCAD) y en el ensamblaje del módulo de cálculo para la comprobación de las

99 Algunos ejemplos de la visualización de resultados se muestra en el apartado 0 del ANEXO del presente trabajo. 
secciones mixtas (en el entorno del cálculo y visualización). No obstante, se han desarrollado otros procedimientos que permiten la verificación de datos y resultados, que, junto con los anteriores, se describirán a continuación.

\section{Intervenciones en el módulo de diseño.}

El menú de definición del tipo de sección contemplaba, entre otros, las secciones rectangulares, en $\mathrm{T}$, circulares y genéricas para el caso del hormigón armado y secciones doble $T$ y perfiles tubulares para el caso de estructuras metálicas.

La integración del módulo de cálculo de secciones mixtas ha permitido ampliar este catálogo de secciones con la inclusión de dos nuevos iconos (ver recuadro de la Figura 6.3-1): uno correspondiente a la sección mixta para soportes, formada por un perfil doble $\mathrm{T}$ embebido en hormigón armado y otra a la sección mixta para vigas ${ }^{100}$, formada por un perfil doble $\mathrm{T}$ con una cabeza rectangular de hormigón armado.

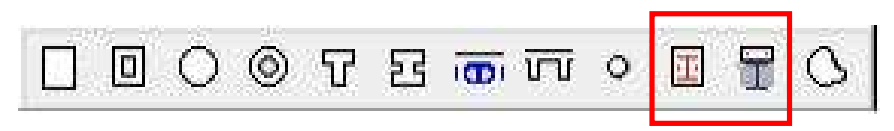

Figura 6.3-1 Catálogo de tipos de secciones

Seleccionado este icono, el programa solicita dimensiones $\mathrm{BxH}$ de la sección de hormigón así como tipo y serie del perfil metálico. A partir de estos datos, el módulo desarrollado para la generación de datos de la sección, realiza su descomposición en celdillas ${ }^{101}$ para su posterior análisis.

${ }^{100}$ En previsión de que en un futuro se desarrolle un módulo de cálculo de vigas mixtas integrado en el entorno AV_CID.

${ }^{101}$ Procedimiento descrito en el apartado 4.4 del presente trabajo. 
Los datos de partida de la sección se almacenan en una variable del tipo grupo, denominada "d_secc_pmixto", mientras que los valores obtenidos tras la discretización, dimensiones $x$ e y de cada celdilla, distancias $x$ e y de su centro de gravedad y material, se guardan, respectivamente en las variables "pasosx()", "pasosy()", "distx_cdg()", "disty_cdg()", "m_aterial(,)", todas ellas del tipo lista, salvo la del material que pertenece al tipo matriz. Para una mejor organización, éstas se agrupan en el conjunto de variables que definen los datos de la sección, denominada "d_geosecpmixt".

Al mismo tiempo, una rutina realiza el cálculo de las inercias de la sección de cada parte que compone la sección, hormigón, acero estructural y acero de armar, y obtiene su inercia total equivalente de la sección en hormigón ${ }^{102}$, con el objeto de que el módulo de análisis disponga de los datos necesarios para poder evaluar la rigidez de las barras para el ensamblaje de la matriz de rigidez.

Todos estos valores, tanto por partes como globales, que representan propiedades de la sección, se almacenan en una variable del tipo grupo, denominada "d_prop_pmixto".

Otro aspecto de la generación del modelo de diseño en que se ha intervenido en la información que se facilita al usuario.

Una vez definidas las características de la sección mixta, el usuario asigna sus valores a determinadas barras del modelo geométrico, cuya aparencia en el modelo continúa siendo el de una línea. El icono "información", mostrado en la Figura 6.3-2, permite recordar al

102 El análisis de estructuras que incluyen soportes de sección mixta se realiza como estructuras de hormigón armado. 
usuario las características asignadas a la barra: dimensiones $\mathrm{BxH}$ de la sección de hormigón así como tipo y serie del perfil metálico.

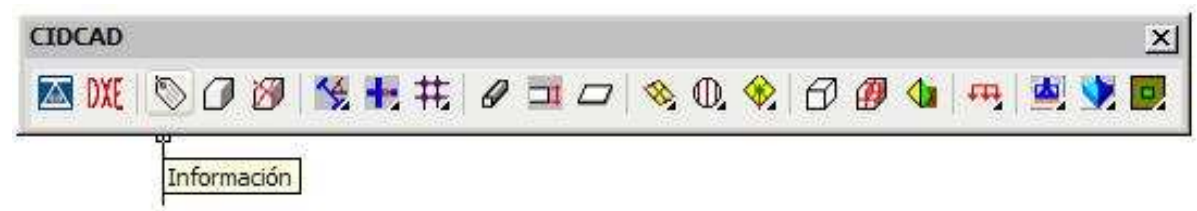

Figura 6.3-2 Ventana para la solicitud de información

\section{Intervención en el modelo de cálculo.}

La otra intervención más importante corresponde a la integración del programa de cálculo de soportes de sección mixta en el módulo de dimensionamento de elementos.

El ensamblaje de este módulo ha afectado a distintos aspectos:

1. Visualización de datos de las barras.

El icono del menú del programa que se resalta con un recuadro en la Figura 6.3-3, facilita la información de las propiedades de cada una de las barras.

A esta opción se le ha añadido un procedimiento que también proporciona estas propiedades, cuando al soporte seleccionado se le ha asignado una sección mixta.

Las propiedades de la barra que se ofrecen al usuario son: esquema geométrico de la sección, número de los nudos inicial y final, longitud del soporte, nombre de la capa en que se ha dibujado la barra en el modelo de diseño, área, inercias y datos del material. 


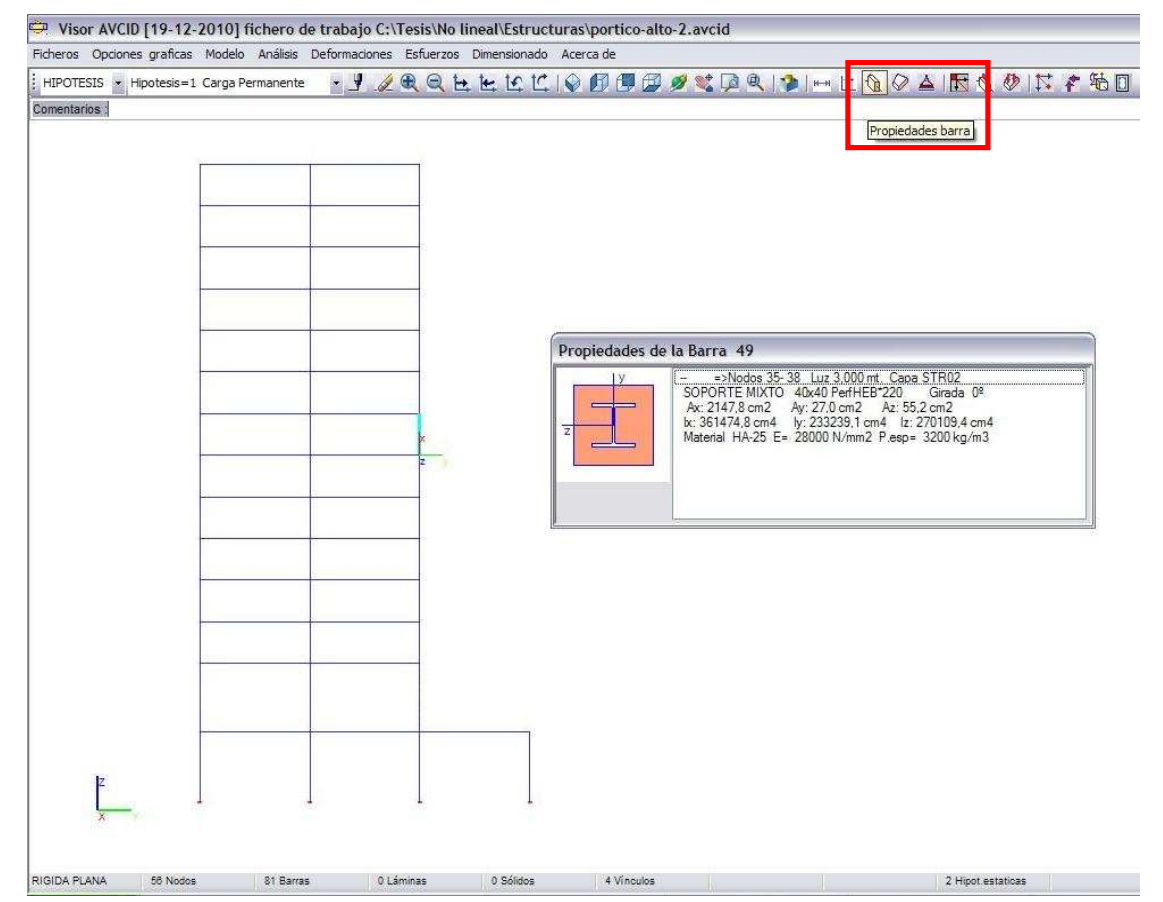

Figura 6.3-3 Ventana de información de las propiedades de la barra

2. Lectura de los parámetros de cálculo.

Se trata del valor de la resistencia característica del hormigón, del acero del perfil estructural y del acero de armar. Los coeficientes de minoración de las resistencias de dichos materiales y la consideración de los efectos reológicos del hormigón se definen en la programación y no están, de momento, abiertos al usuario.

Con el objeto de no duplicar ventanas de características de los materiales, los valores que afectan al hormigón y al acero de armar se obtienen del cuadro correspondiente a las estructuras de hormigón armado, que se muestra en la Figura 6.3-4, y los que afectan al acero estructural de la ventana correspondiente a las estructuras metálicas, Figura 6.3-5. 


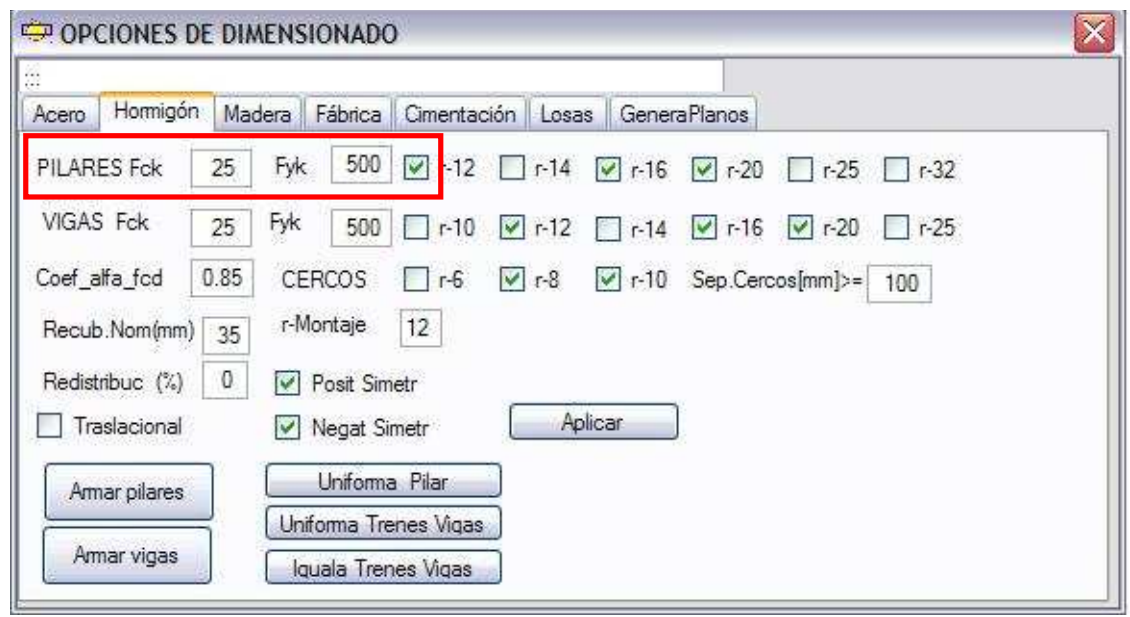

Figura 6.3-4 Cuadro de especificaciones del hormigón armado

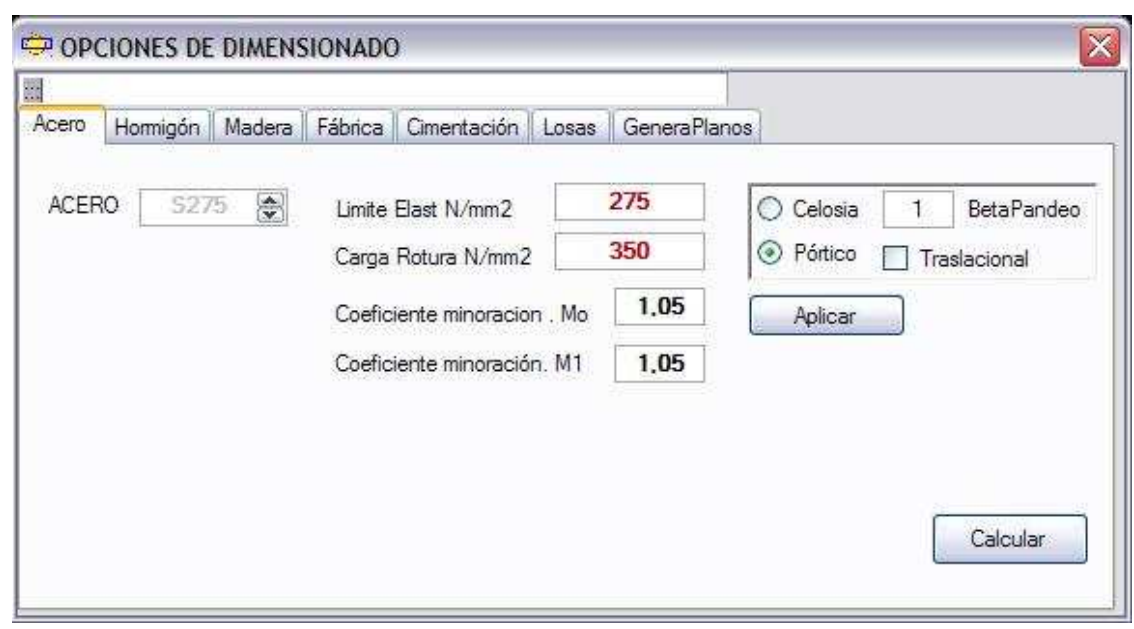

Figura 6.3-5 Cuadro de especificaciones del acero estructural

Los valores de todos estos parámetros generales de cálculo se almacenan en la variable del tipo grupo, denominada "d_hor_s_mixta" 
3. Comprobación de la sección.

Dada la diferencia de los efectos que se producen, y la trascendencia conceptual en el desarrollo del presente trabajo, cabe distinguir dos casos:

a) Análisis de la estructura en régimen lineal

Tras el cálculo de movimientos y solicitaciones de la estructura en régimen lineal, la opción "Dimensionar" del menú "Dimensionado", realiza el armado de las barras de hormigón armado y la comprobación de los perfiles de acero estructural.

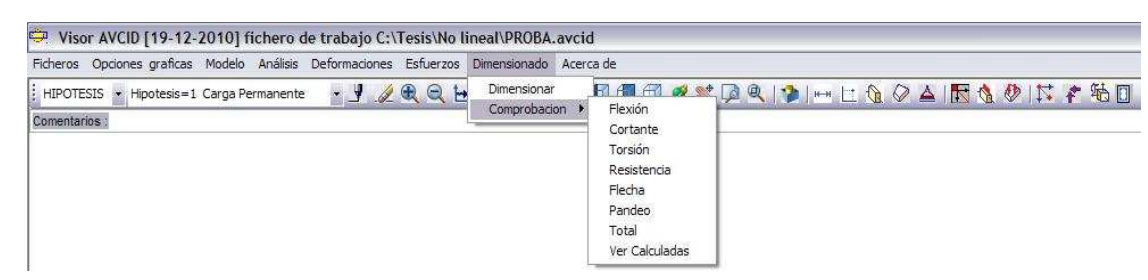

Figura 6.3-6. Menú: Dimensionado de barras.

En esta opción se ha añadido un procedimiento que lee la base de datos y resultados generada por el módulo de análisis estructural, discriminando todas aquellas barras de sección mixta. Para cada una de ellas, realiza una llamada al programa de cálculo de soportes mixtos frente a flexocompresión esviada, enviándole como datos las características de la sección y las solicitaciones $\left(\mathrm{N}-\mathrm{M}_{\mathrm{z}}-\mathrm{M}_{\mathrm{y}}\right)$, para comprobar la idoneidad de la sección.

Como resultado, el módulo de cálculo de secciones mixtas devuelve al programa de análisis estructural la validez de cada sección comprobada, o en su defecto, la insuficiencia de la misma. 
b) Análisis de la estructura en régimen no lineal geométrico y del material $^{103}$

Si se opta por un análisis de la estructura en régimen no lineal, la comprobación de los soportes de sección mixta es automática, realizándose en cada iteración de cada incremento de carga si la solución se busca utilizando el método de Newton-Raphson o al final de cada incremento de carga si se utiliza el método de Newton-Raphson modificado.

Cuando el resultado de la comprobación es favorable, una rutina incluida en el modulo de soportes mixtos busca la posición de la fibra neutra $(f)$ y la curvatura del plano deformado $(\theta)$ que conducen al equilibrio entre la solicitación exterior y los esfuerzos internos de la sección, devolviendo estos resultados al programa de análisis estructural.

De esta forma, tras cada comprobación, se dispone de los datos necesarios para poder evaluar el módulo de rigidez de cada barra $(E I=M / \theta)$ con objeto de corregir los valores de la matriz de rigidez en la resolución de la siguiente iteración o incremento de carga.

Un resultado negativo en la comprobación de cualquier soporte mixto conduce a la interrupción automática e inmediata del proceso de cálculo en régimen no lineal.

\section{Intervención en el módulo de visualización de resultados.}

Por último, se ha intervenido en el módulo de visualización de los resultados obtenidos tras el análisis de la estructura y

103 La consideración de la no linealidad del material únicamente se encuentra desarrollada en la actualidad para estructuras planas y soportes mixtos. 
dimensionamiento de sus elementos, de forma que ahora también se grafían los resultados obtenidos en el cálculo de los soportes mixtos.

El procedimiento recupera el resultado de la comprobación de los soportes de sección mixta y envía un código para que el módulo de visualización de resultados de AV_CID sea capaz de grafiar en color rojo los soportes que no cumplen los estados límites.

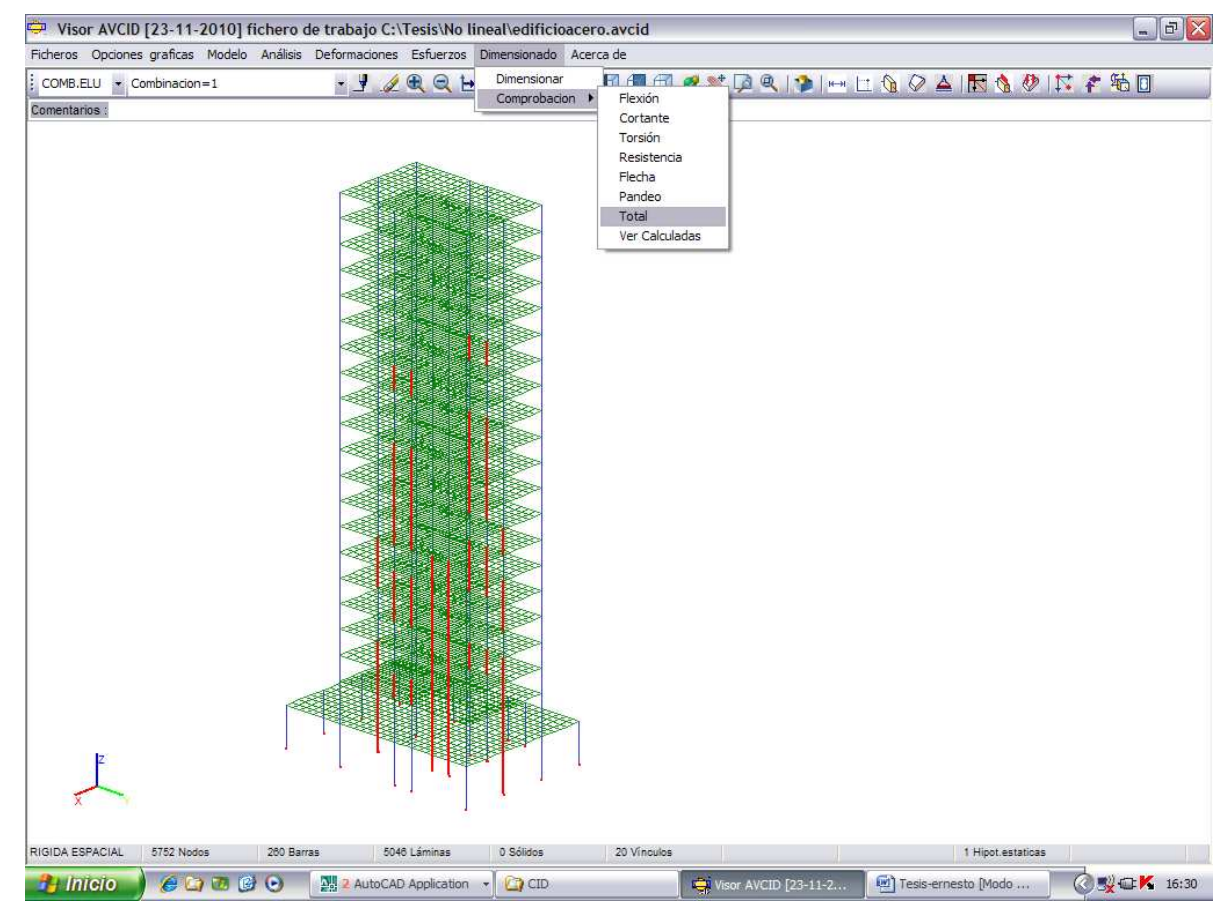

Figura 6.3-7 Visualización de resultados de una estructura con soportes mixtos 


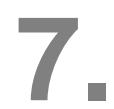

ANÁLISIS DE LA BARRA 


\subsection{INTRODUCCIÓN. RESEÑA HISTORICA}

Los efectos de segundo orden que se van a estudiar en el presente capítulo se producen en barras sometidas a compresión, ya que el esfuerzo de tracción tiende a corregir las imperfecciones iniciales de las barras mientras que la compresión las amplifica.

Partimos de una barra perfectamente recta, solicitada por un esfuerzo axil centrado, a la que le provocamos una excentricidad lateral. Si el axil es de tracción, además de que la barra trata de recuperar su forma inicial, el propio esfuerzo tiende a corregir la excentricidad impuesta. Si el axil es de compresión pueden ocurrir tres casos:
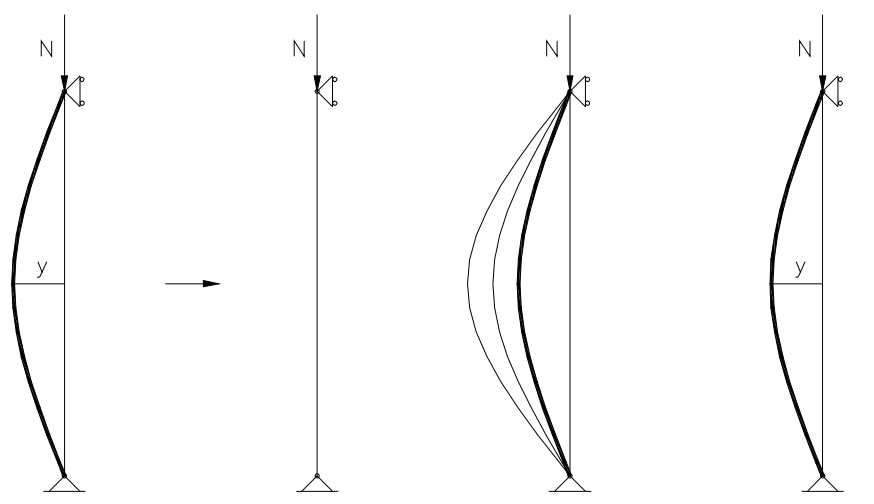

Figura 7.1-1 Estados de equilibrio: estable, inestable e indiferente

1.- Si el momento solicitación $N \cdot y$ es menor que el momento interno surgido como consecuencia de que la barra trata de enderezarse, volverá a su posición recta inicial. Se trata de un equilibrio estable.

2.- Si el momento solicitación $N \cdot y$ es mayor que el momento interno, la barra incrementará su deformación hasta que se produzca el colapso. Se trata de un equilibrio inestable.

3.- Si el momento solicitación $N \cdot y$ es igual que el momento interno, la deformación impuesta ni se corrige ni se incrementa. Se trata de un 
equilibrio indiferente. Al valor de la carga que conduce a esta situación se le denomina carga crítica.

Dada la importancia del asunto que se va a tratar, merece la pena recordar que los primeros ensayos de compresión sobre columnas esbeltas de madera fueron realizados por Musschenbroek en 1729, observando experimentalmente que la carga de colapso por pandeo es inversamente proporcional al cuadrado de la longitud de la barra $^{104}$.

No obstante, es Euler en 1744 quien plantea de forma teórica el análisis del efecto del pandeo sobre una barra ideal: de geometría recta con sección constante y material perfectamente elástico, con sus extremos articulados y sometida a un esfuerzo axil centrado a la que se aplica una perturbación horizontal ${ }^{105,106}$ (Figura 7.1-2).

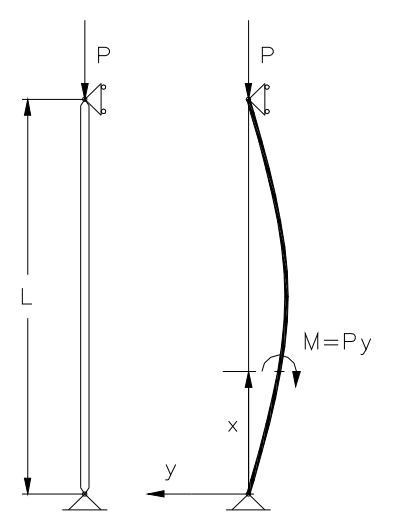

Figura 7.1-2 Barra recta sometida a compresión

104 "Coulomb's memoir on statics. An essay in the history of civil engineering". Jacques Heyman (1972)

105 "Mecánica de sólids". Popov, E.P. (2000)

106 "Resistencia de materiales". Gere, J. (2002) 
Imponiendo el equilibrio entre el momento externo y el momento interno en una sección situada a una distancia $x$ cualquiera respecto del origen:

$$
M_{\text {ext }}=P \cdot y=-E \cdot r \cdot y^{\prime \prime}=M_{\text {int }}
$$

Despejando se llega a la siguiente ecuación diferencial:

$$
P \cdot y+E \cdot l \cdot y^{\prime \prime}=0 \rightarrow y^{\prime \prime}+\frac{P}{E \cdot l} \cdot y=0
$$

Cuya solución es la siguiente:

$$
y=A \cdot \operatorname{sen}\left(\sqrt{\frac{P}{E \cdot I}}\right) \cdot x+B \cdot \cos \left(\sqrt{\frac{P}{E \cdot I}}\right) \cdot x
$$

Imponiendo las condiciones de contorno particulares podremos resolver los valores de $\mathrm{A}$ y $\mathrm{B}$.

$$
\begin{aligned}
& y_{(x=0)}=0 \rightarrow B=0 \\
& y_{(x=I)}=0 \rightarrow A \cdot \operatorname{sen}\left(\sqrt{\frac{P}{E \cdot I}}\right) \cdot L=0 \rightarrow\left\{\begin{array}{l}
A=0 \text { ó } \\
\left(\sqrt{\frac{P}{E \cdot I}}\right) \cdot L=n \cdot \pi
\end{array}\right.
\end{aligned}
$$

Dado que la primera posible solución del valor de $\mathrm{A}(A=0)$ conduce a una ecuación de la línea elástica nula, la solución que nos interesa es la segunda, de donde se puede despejar $P$ :

$$
\left(\sqrt{\frac{P}{E \cdot I}}\right) \cdot L=n \cdot \pi \rightarrow \sqrt{\frac{P}{E \cdot I}}=\frac{n \cdot \pi}{L} \rightarrow P=\frac{n^{2} \cdot \pi^{2}}{L^{2}} \cdot E \cdot I
$$

Siendo $n$ un número entero que representa los arcos de sinusoide compatible con las condiciones de contorno. En la figura siguiente se muestran los tres primeros modos de pandeo que puede adoptar una barra biarticulada: 

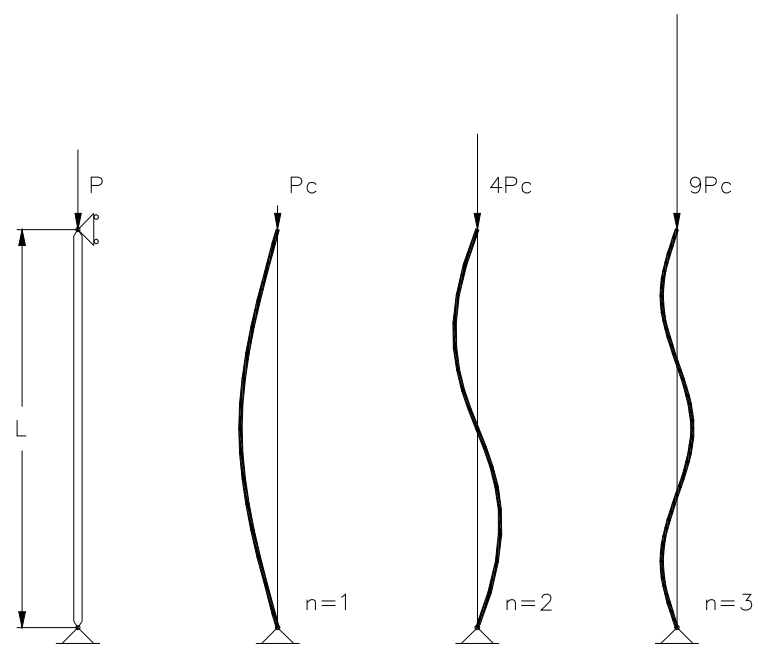

Figura 7.1-3 Tres primeros modos de pandeo de una barra biarticulada ${ }^{107}$

La menor de las cargas con la que se puede producir el pandeo, la que exige un mínimo consumo de energía en la barra, corresponde a un valor $n=1$. Es a ésta a la que se denomina "carga crítica de Euler":

$$
P_{\text {crit }}=\frac{\pi^{2} \cdot E \cdot I}{L^{2}}
$$

El valor de la carga crítica deducida para una barra biarticulada se puede aplicar a otras condiciones de contorno sustituyendo la longitud real de la barra por la denominada longitud de pandeo (longitud entre dos puntos de inflexión de la curva sinusoide compatible con las condiciones de contorno). Utilizando el concepto de la longitud de pandeo, la carga crítica se puede generalizar, para distintas condiciones de contorno de la forma:

$$
P_{\text {crit }}=\frac{\pi^{2} \cdot E \cdot I}{L_{P}^{2}}
$$

107 "Mecánica de sólidos" Popov, E.P. (2000) 
A continuación se indican las longitudes de pandeo de distintas barras con las condiciones de contorno teóricas más comunes (articulada-empotrada, biempotrada y en voladizo) comparadas con la barra biarticulada.
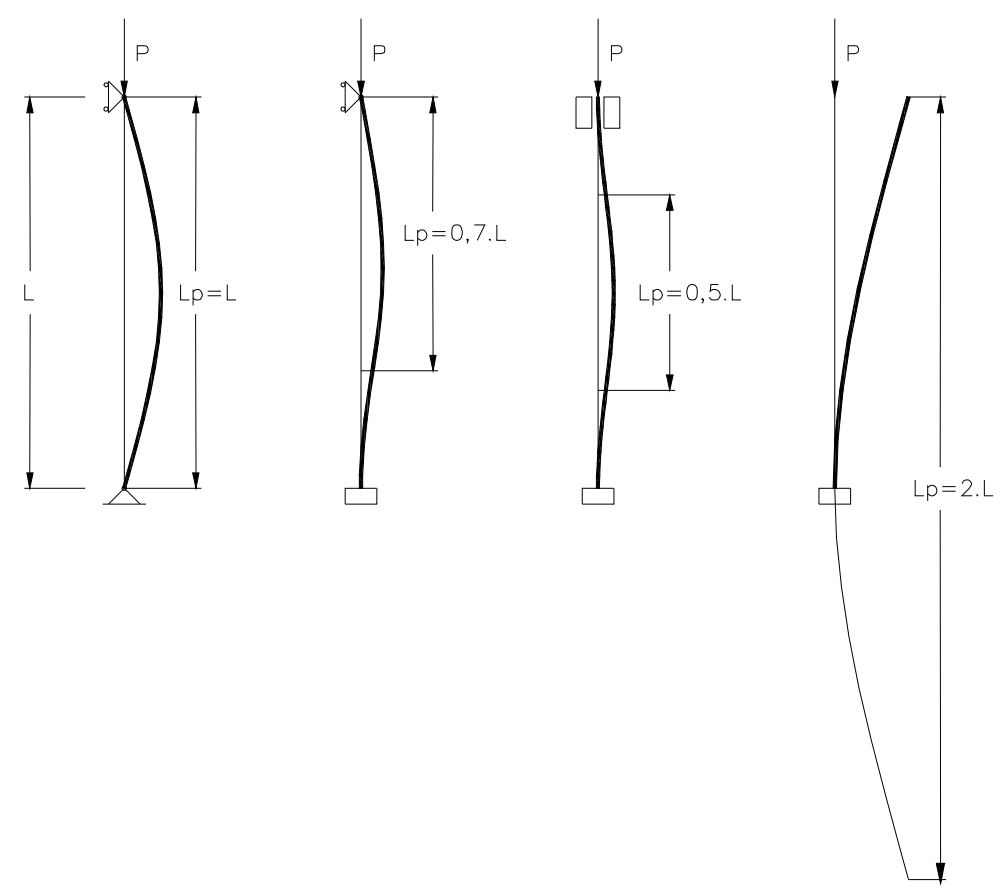

Figura 7.1-4 Longitud de pandeo para distintas condiciones de contorno.

Cuando la barra forma parte de un entramado de nudos rígidos, la longitud de pandeo dependerá de la traslacionalidad de la estructura y de la rigidez relativa entre vigas y soportes.

Volviendo la carga crítica, si dividimos ésta por el área de la sección podremos expresar el problema en términos de tensión:

$$
\sigma_{\text {crit }}=\frac{P_{\text {crit }}}{A}=\frac{\pi^{2} \cdot E \cdot I / A}{L^{2}}=\frac{\pi^{2} \cdot E}{(L / i)^{2}}=\frac{\pi^{2} \cdot E}{\lambda^{2}}
$$


Siendo $\lambda$ la esbeltez mecánica de la barra, definida como la relación entre la longitud de la barra y el radio de giro de la sección, en el plano de pandeo considerado.

Si representamos el valor de la carga crítica en función de la esbeltez, podemos observar que cuando la esbeltez tiende a cero, la tensión crítica tiende a infinito mientras que cuando la esbeltez tiende a infinito, la carga crítica tiende a cero.

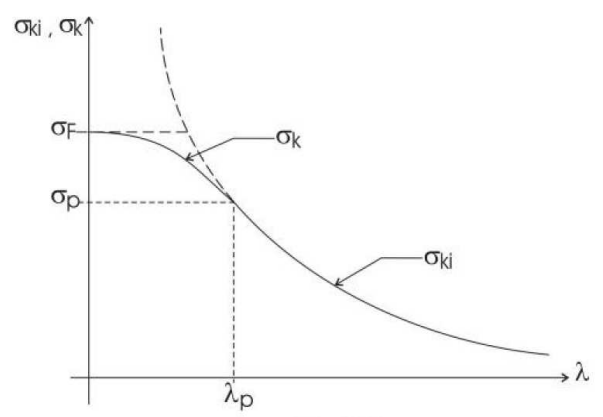

Figura 7.1-5. Relación tensión crítica - esbeltez

En 1845 Lamarle demostró que el colapso de las barras esbeltas sometidas a compresión se producía por inestabilidad, pero que en las barras cortas se producía por aplastamiento del material, ya que la tensión crítica superaba a la resistencia a compresión.

Bauschinger y Tetmajer comprobaron experimentalmente este hecho, verificando que las piezas cortas se agotaban por aplastamiento y que la fórmula de Euler tiene un límite superior definido por la resistencia a compresión del material. Por tanto la esbeltez sólo es condicionante para piezas esbeltas cuyo límite fijó Tetmajer en 110.

Ademas, puesto que la fórmula de Euler ha sido deducida bajo la hipótesis del cumplimiento de la ley de Hooke, sólo será válida mientras la tensión crítica sea menor que el límite de proporcionalidad $\sigma_{P}$. En la zona comprendida entre la esbeltez nula y el límite de 
proporcionalidad, la fórmula de Euler debe sustituirse por otra que tenga en cuenta el comportamiento elasto-plástico del material.

Fue Engesser el primero en estudiar el comportamiento de las barras biarticuladas de sección constante sometidas a un esfuerzo axil centrado, cuando las tensiones superaban el límite de proporcionalidad. Para la determinación de la carga crítica propuso en 1889, la denominada "Teoría del módulo tangente", consistente en sustituir el módulo de elasticidad utilizado en el tramo elástico, por el módulo de elasticidad tangente a la curva $\sigma-\varepsilon$ en el punto correspondiente a la tensión considerada ${ }^{108}$.

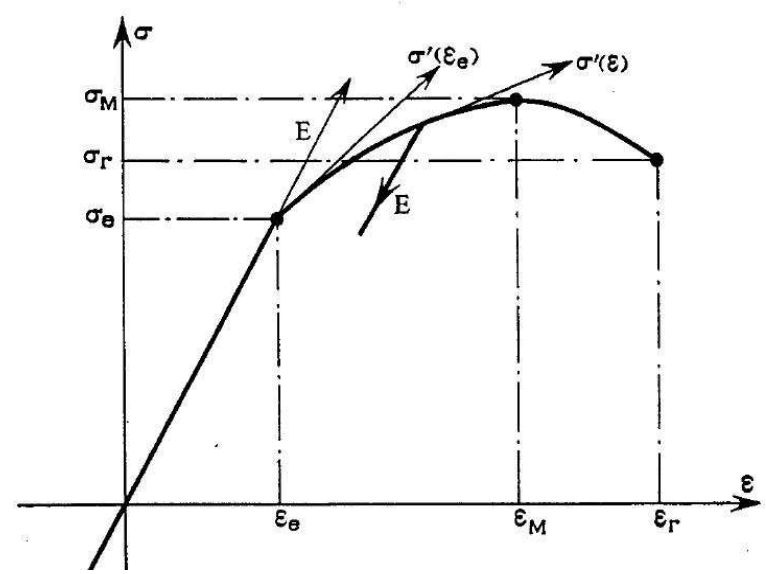

Figura 7.1-6. Diagrama tensión-deformación no lineal

Se obtiene así la llamada fórmula generalizada para la carga de pandeo de Euler o fórmula del módulo tangente, que hace aplicable las fórmulas del pandeo elástico en el rango plástico:

$$
N_{\text {crit }}=\frac{\pi^{2} \cdot E_{t} \cdot l}{L^{2}}
$$

108 "Contribution a l'etude du flambement dans le domaine plastique" Virlogeux. Annales de l'Institute Technique du Batiment et des Travaux Publics. № 312 
En 1.891 fue publicada una memoria, incluida como apéndice a los proceedings del Congrés International des Procédés de Constructions, celebrado en París del 9 al 14 de septiembre de 1.889, en la cual $A$. Considère señalaba que, cuando una columna ideal cargada más allá de su límite de proporcionalidad comenzaba a flectar, la tensión en el lado cóncavo se incrementa de acuerdo con el diagrama tensión-deformación, y la tensión en el lado convexo disminuye de acuerdo con la Ley de Hooke.

Por ello consideró que no era correcto el uso del módulo tangente para la determinación de la carga crítica en el rango plástico, sino que era necesaria la aplicación un módulo reducido, $\bar{E}$ cuyo valor estaba comprendido entre el módulo de elasticidad y el módulo tangente. La fórmula para la determinación de la carga crítica quedaba de la siguiente forma ${ }^{109}$ :

$$
N_{\text {crit }}=\frac{\pi^{2} \cdot \bar{E} \cdot I}{L^{2}}
$$

Engesser reconoció el error en su teoría original, y replicó sobre la posibilidad de determinar teóricamente el valor del módulo reducido. En 1910 von Kármán presentó de nuevo la teoría, añadiendo la evaluación del módulo tangente $\bar{E}$ para el caso de sección rectangular y para una sección en $\mathrm{H}$ idealizada (suponiendo infinitamente delgadas las alas y despreciando el alma), junto a los resultados de una serie de ensayos realizados para verificar la teoría.

Otro aspecto a considerar son los efectos de la aplicación excéntrica de la carga, cuyo estudio fue iniciado por von Kármán en 1.908, y desarrollado por Chwalla en 1.928, ambos en el campo elástico. A

109 "Contribution a l'etude du flambement dans le domaine plastique" Virlogeux. Annales de l'Institute Technique du Batiment et des Travaux Publics. № 312 
partir de 1.934, Jezek comenzó a utilizar diagramas simplificados bilineales de tensión-deformación para piezas metálicas, incluyendo los efectos de la plastificación.

Resulta interesante la reflexión final realizada por Osgood, incluida en el artículo de Shanley ${ }^{110}$ y que se reproduce a continuación:

"Aunque la teoría del módulo reducido (o del doble módulo) ha sido aceptada como exacta para el comportamiento de la columna, la teoría del módulo tangente resulta mucho más fácil de aplicar, puesto que en ello no influye la forma de la sección; como proporciona, además, valores más bajos de la carga crítica, es preferida por los ingenieros en base a criterios de seguridad. Finalmente, los ensayos indican que la carga real de pandeo es más próxima a la que indica la teoría del módulo tangente, que la del módulo reducido."

El problema principal en la aplicación de la teoría del módulo tangente surge en la determinación de los límites de las zonas de comportamiento elástico y plástico.

Para estudiar el problema, Shanley propone un soporte formado por dos tramos infinitamente rígidos unidos, en su zona central, por dos barras cortas de longitud despreciable frente a los tramos rígidos, donde se concentra toda la deformación producida por la flexión y realizadas con un material de módulo tangente constante.

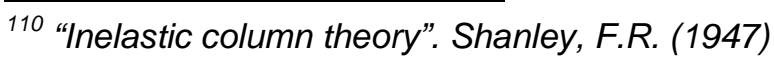




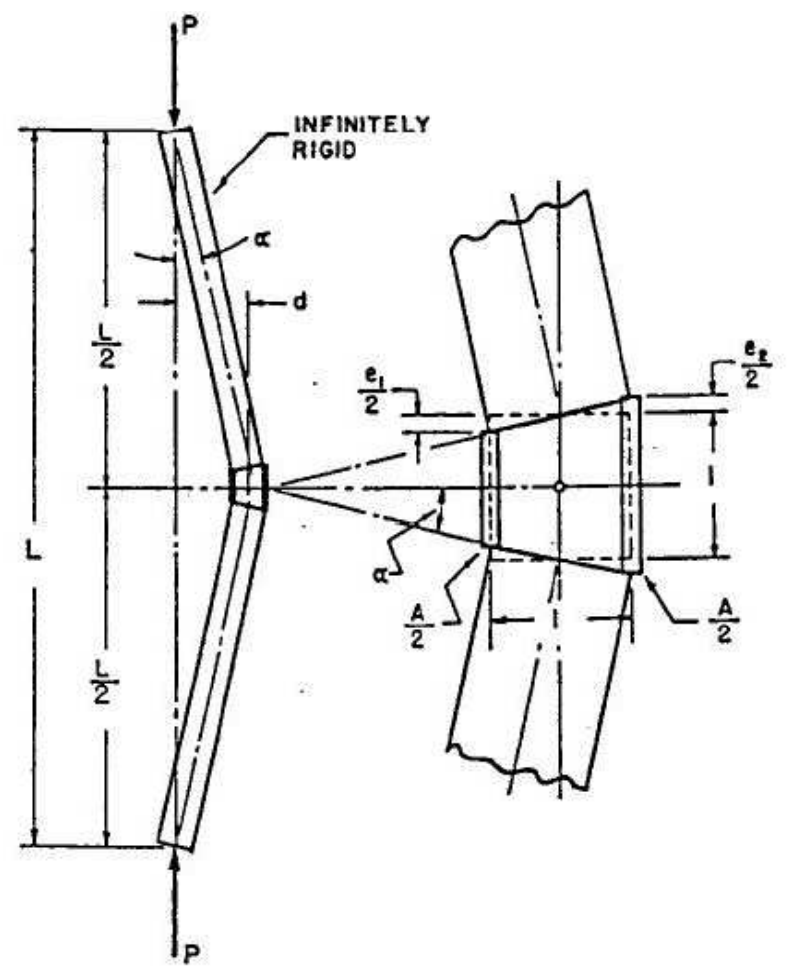

Figura 7.1-7 Modelo de Shanley

Las conclusiones de los resultados obtenidos con el modelo de Shanley se pueden extraer de la "discusión" de von Kármán, publicada al final del mismo texto de Shanley"111:

"Dos aspectos de la cuestión son dignos de mención:

a) Mi análisis original, y también de Engesser, es una generalización del razonamiento utilizado en la teoría del pandeo elástico. ¿Por qué no comprende todas las posibles posiciones de equilibrio en el rango enelástico? Obviamente, por el carácter no reversible del diagrama tensión-deformación en el rango inelástico; hay infinitos valores de deformación permanente que pueden corresponder a la misma

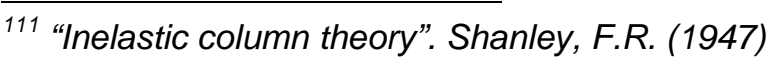


tensión, dependiendo de la historia de carga y descarga del proceso. Por ello, la definición del límite de estabilidad debe ser revisada para procesos no reversibles. Esta necesidad es reconocida intuitivamente por Mr. Shanley, lo que es, en mi opinión, el gran mérito de su escrito.

b) Aunque la fórmula de Euler con módulo tangente, no proporciona, en general, el valor máximo de la carga sin que se produzcan grandes deformaciones, es conservadora para la evaluación práctica de la carga en columnas. Como indiqué en mi escrito de 1909, también la curva carga-deformación que parte del límite superior de la carga expermenta pronto giros negativos, por lo que es difícil determinar el valor máximo del esfuerzo axil. Ciertamente estará entre los dos valores que corresponden al módulo tangente y el reducido, que pueden ser considerados como límite superior e inferior de la carga crítica".

El modelo de Shanley fue adoptado por Virlogeux ${ }^{12,113}$, y el planteamiento generalizado considerando inicialmente, en el tramo deformable, una ley de comportamiento de módulo tangente constante y después con un módulo tangente variable, analizando de esta forma el comportamiento de distintos tipos de barras, en función de su esbeltez y excentricidad.

Otras aportaciones sobre el estudio del pandeo lateral y los efectos de torsión que por efecto del esfuerzo axil se producen en secciones

112 "Contribution a l'etude du flambement dans le domaine plastique" Virlogeux. Annales de l'Institute Technique du Batiment et des Travaux Publics. № 312

113 "Analyse des problèmes de stabilite d'une colonne dans le domaine plastique”. Virlogeux.(1975) 
abiertas de pared delgada fueron realizadas por Wagner y Kappus, siendo recogidas y completadas en el tratado de Vlasov.

El análisis de la pieza real, denominado como "problema de la vigacolumna", exige la consideración conjunta de todos estos aspectos que intervienen en el pandeo tales como flexión esviada, condiciones de enlace entre las barras de los entramados, imperfecciones geométricas de barras y pórticos, efectos reológicos en el hormigón y tensiones residuales en el acero, ...

Vista la complejidad del problema, de la consideración de todos los aspectos no cabe esperar soluciones sencillas y prácticas, mientras que la obtención de expresiones de fácil aplicación práctica obliga a la no consideración de ciertos aspectos, lo cual reduce su campo de aplicación.

En referencia al presente estudio, el de los soportes de sección mixta, y más en concreto de las secciones en $\mathrm{H}$ recubiertas de hormigón, cabe indicar que tratándose de secciones doblemente simétricas podemos ignorar los efectos de pandeo por torsión, y que el recubrimiento por el hormigón proporciona al perfil metálico una rigidización que evita los problemas de abollamiento. Siguen existiendo los aspectos relacionados con la rigidez del nudo en función del tipo de enlace del soporte con el pórtico, la excentricidad en la aplicación de la carga y el proceso de carga, para los que se tratará de aplicar hipótesis razonables que permitan obtener soluciones de aplicación práctica para las estructuras de edificación, uno de los objetivos del trabajo que nos ocupa. 


\subsection{INESTABILIDAD Y AGOTAMIENTO}

El presente apartado se va a detener en el estudio de las dos posibles formas de colapso de la barra, aspectos a los que se considera necesario prestar especial atención, puesto que condiciona el método de resolución a utilizar.

El colapso de una barra sometida a un esfuerzo de compresión excéntrica puede producirse de dos formas ${ }^{114}$ :

- por agotamiento de su sección más solicitada. En este caso la resistencia de la barra coincide con la de su sección y es función de la geometría de la sección, de las características resistentes de los materiales y de las solicitaciones (fuerzas y momentos) obtenidos en el análisis de la estructura. A los soportes con este tipo de colapso se les suele clasificar como "piezas cortas"

- Por inestabilidad. Las deformaciones laterales producidas por la excentricidad de la carga produce un incremento de los momentos que crecen más deprisa que el momento resistido por la sección, transformando el proceso en dinámico. El colapso de la pieza se reduce respecto de la capacidad resistente de su sección, habiéndose producido sin que ninguna sección alcance las condiciones de rotura. A los soportes con este tipo de colapso se les suele clasificar como "piezas esbeltas"

Puede observarse que la clasificación de los soportes en cortos y esbeltos $^{115}$ se ha realizado en función del tipo de colapso y no

114 "Cálculo de soportes rectangulares de hormigón armado en teoría de segundo orden". Juan J. Arenas de Pablo. (1980)

115 "Design of slender concrete columns" Mac Gregor, J.G; Breen, J.E. y Pfrang, E.O. (1970) 
únicamente según su esbeltez, ya que en el fenómeno de la inestabilidad intervienen otros parámetros como por ejemplo las coacciones existentes en los extremos de la barra.

La Figura 7.2-1 $1^{116}$ ilustra los posibles caminos de colapso de un soporte comprimido excéntricamente, respecto del diagrama de interacción de la sección (curva que limita superiormente la capacidad resistente de la barra).
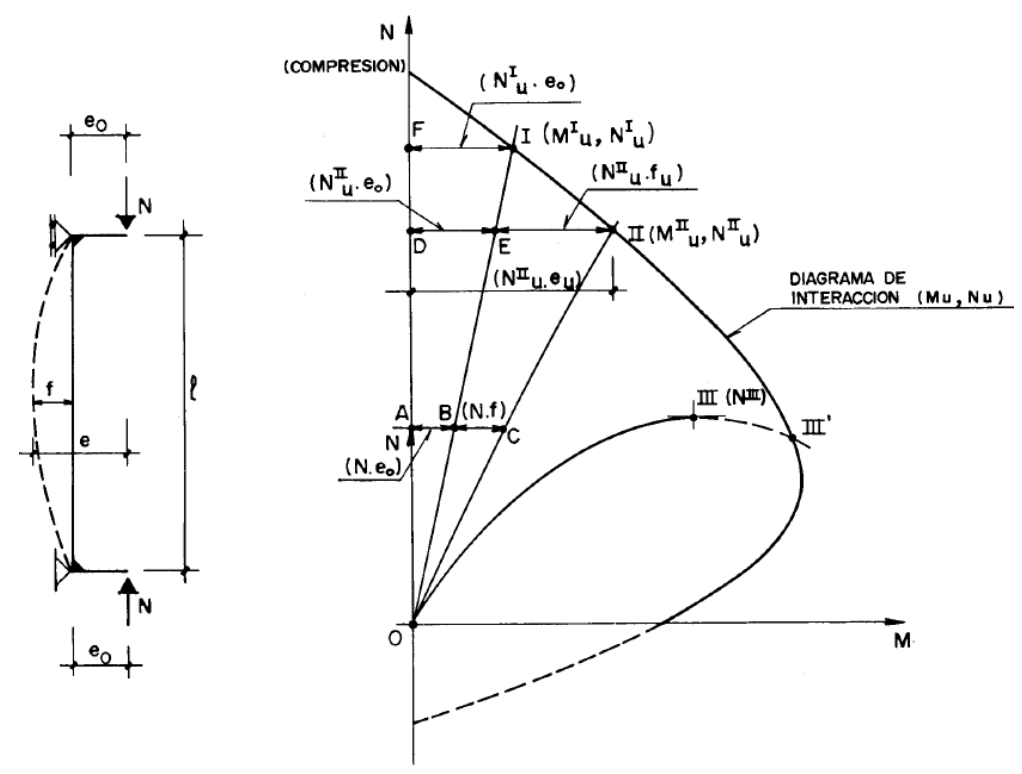

Figura 7.2-1 Caminos de agotamiento de un soporte sometido a compresión excéntrica

Si consideramos la barra como un sólido rígido (la aplicación de la carga no produce deformaciones respecto de la posición inicial) el camino hasta el colapso quedaría representado en el diagrama N-M, por una recta inclinada (OBEI), en la que el factor de proporcionalidad

\footnotetext{
${ }^{116}$ Esta figura y la siguiente han sido extraidas de la publicación "Cálculo de soportes rectangulares de hormigón armado en teoría de segundo orden" de Juan J. Arenas de Pablo.
} 
entre el axil y el momento es la excentricidad inicial $e_{0}$. El colapso de la barra se produce cuando se agota su sección, es decir, cuando la recta alcanza el diagrama de interacción (punto I).

En realidad, la excentricidad de la carga produce deformaciones laterales, flechas, que dependen, entre otros factores, del valor de la carga, de la excentricidad inicial y de la esbeltez de la barra. Si aplicamos una carga creciente hasta el colapso sobre un "soporte corto" según la clasificación que hemos establecido anteriormente, el camino del agotamiento quedaría representado por la curva OCII. El momento máximo, para cada escalón de carga, sería igual a la suma del momento de primer orden $\left(N \cdot e_{0}\right)$ más el de segundo orden $(N \cdot f)$. El colapso de la barra se produce cuando la curva alcanza el diagrama de interacción (punto II). Se observa que se ha producido una reducción de la capacidad resistente de la barra respecto de la capacidad de su sección, representada en la recta descrita anteriormente, ya que $N_{u}^{\prime \prime}<N_{u}^{\prime}$.

Si se repite este proceso de carga sobre un "soporte esbelto", la curva que representa el camino del agotamiento (OIII) podría alcanzar una tangente horizontal, siendo la barra incapaz de soportar un axil mayor e iniciándose el proceso de inestabilidad. A partir de este punto, el equilibrio sólo es posible si se reduce el esfuerzo axil, situación que no se produce en la edificación. La capacidad resistente máxima del soporte se produce antes de que la curva haya alcanzado el diagrama de interacción.

Cabe indicar que existen otros fenómenos, como la fluencia, que al mantener la carga a lo largo del tiempo, producen deformaciones diferidas que amplifican las deformaciones existentes y por lo tanto con efectos desfavorables como se puede explicar a partir de la Figura 7.2-2. 

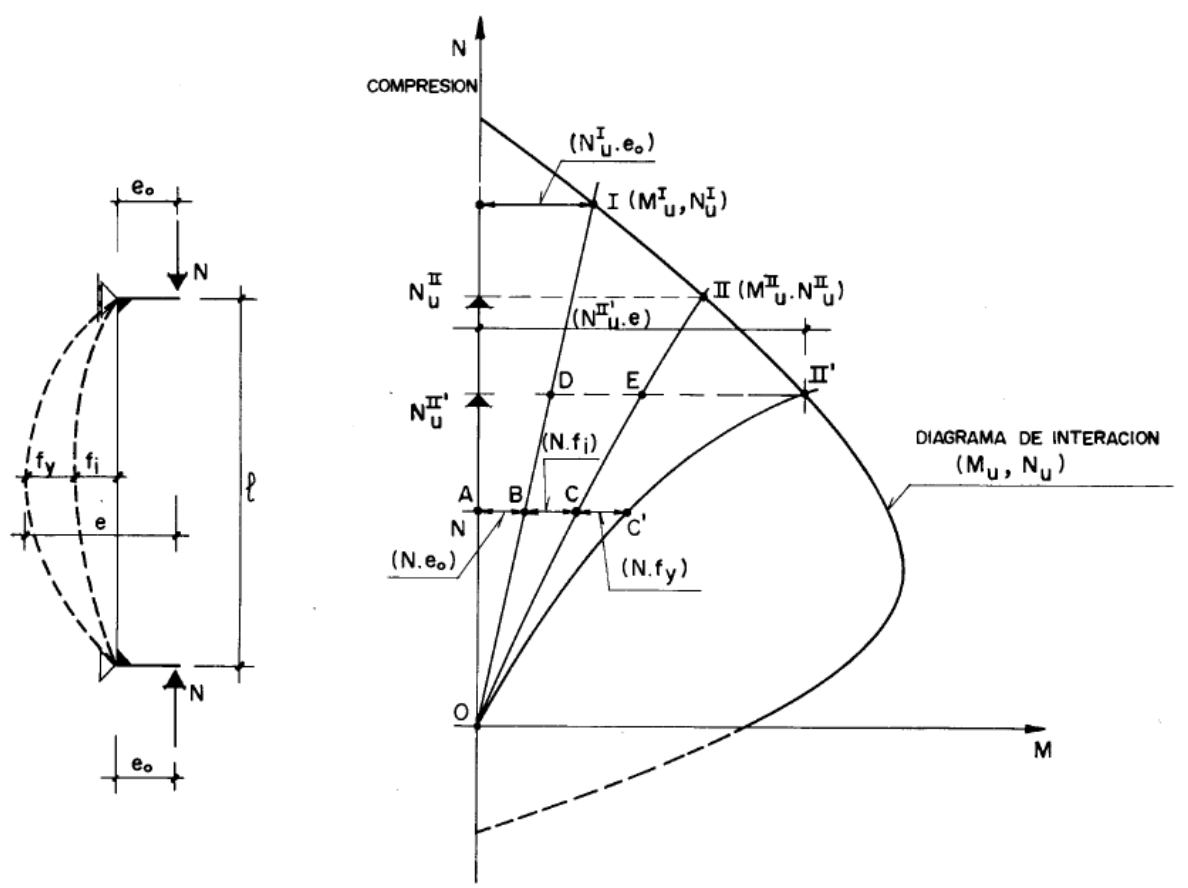

Figura 7.2-2 Caminos de agotamiento de un soporte sometido a compresión excéntrica a largo plazo

La curva OCEII representa un posible camino de agotamiento del soporte habiendo tenido en cuenta las deformaciones de segundo orden. El agotamiento se produce en el punto II para un axil de valor $N_{u}{ }_{u}^{\prime \prime}$. El punto $\mathrm{E}$ corresponde a un instante de equilibrio y estabilidad en dicho camino, ya que se encuentra en el interior del diagrama de interacción. El momento que lo solicita en la sección más desfavorable es $N_{u}^{\prime \prime \prime} \cdot\left(e_{0}+f_{i}\right)$, es decir, la suma del momento producido por la excentricidad del axil más el momento producido debido a la deformación instantánea de primer orden de la barra.

Si se mantiene la carga a lo largo del tiempo, se producen deformaciones diferidas ( $f_{y}$ en la figura) debido al efecto de la fluencia. Si se tienen en cuenta estas deformaciones diferidas, el momento que solicita al punto $\mathrm{E}$ es $N_{u}{ }^{\prime \prime \prime} \cdot\left(e_{0}+f_{i}+f_{y}\right)$ lo que produce que 
la curva de agotamiento sea mas tendida, pudiendo alcanzarse el diagrama de interacción (punto II' en la figura), y por tanto el colapso de la sección, para un axil menor al obtenido anteriormente $\left(N_{u}{ }^{\prime \prime}<N_{u}{ }^{\prime \prime}\right)$ lo que pone de manifiesto el efecto desfavorable del fenómeno de la fluencia.

Los parámetros que más influyen en el fenómeno de la inestabilidad son la esbeltez $(h / t)$ de la barra, la relación de la excentricidad de la carga $(e / t)$ y la relación entre las excentricidades de las cargas en los extremos $\left(e_{1} / e_{2}\right)^{117}$, siendo $h$ la altura del soporte, $t$ el canto de la sección y e la excentricidad del axil respecto de la directriz de la barra.

En la Figura 7.2-3 se representan las curvas correspondientes a los tres casos de relación de excentricidades en la aplicación de la carga en los extremos.

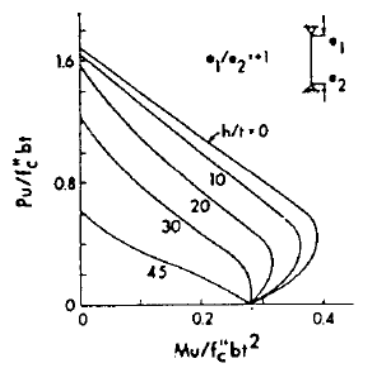

(a)

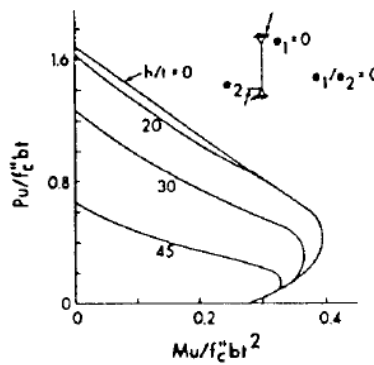

(b)

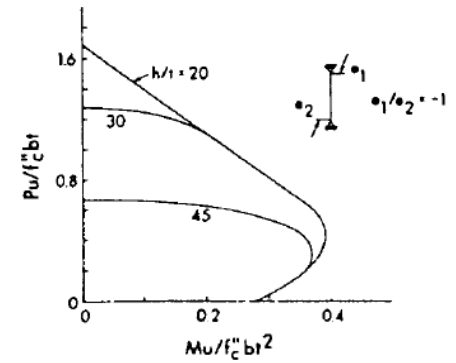

(c)

Figura 7.2-3 Efecto de la esbeltez del soporte en el agotamiento para distintas excentricidades en la aplicación del axil

117 "Design of slender concrete columns" Mac Gregor, J.G; Breen, J.E. y Pfrang, E.O. (1970).

La Figura 7.2-3, que se muestra a continuación, se ha extraido de este artículo. 
En los diagramas a) de la Figura 7.2-3, donde las dos excentricidades son del mismo signo $\left(e_{1} / e_{2}=1\right)$, la consideración de la deformación de la directriz produce siempre un incremento de los momentos, con efectos desfavorables. Es por ello que las curvas de agotamiento por inestabilidad para las distintas esbelteces consideradas son siempre interiores al diagrama de interacción de la sección $(h / t=0)$

En los diagramas c) donde se consideran dos excentricidades de signos contrarios $\left(e_{1} / e_{2}=-1\right)$ cabe distinguir dos casos:

- si se trata de un "soporte corto", hasta esbelteces $h / t=20$, aún considerando la deformada de la directriz, los momentos máximos se siguen produciendo en los extremos de la barra y coinciden con los iniciales. El colapso de la barra únicamente puede producirse por agotamiento resistente de la sección, por lo que las curvas se solapan con el diagrama de interacción de la sección.

- En el caso de soportes con mayor esbeltez, en determinados valores el momento máximo seguirá produciéndose en los extremos (agotamiento de la sección) y en otros en un punto intermedio (inestabilidad), por lo que la curva coincidirá parcialmente con el diagrama de interacción de la sección.

En los diagramas $b$ ), donde se considera que una excentricidad es nula $\left(e_{1} / e_{2}=0\right)$, los efectos son similares a los diagramas $\left.c\right)$ aunque con resultados menos visibles.

\subsection{HIPÓTESIS SOBRE LA DEFORMADA DE LA DIRECTRIZ}

El análisis riguroso de un soporte que forma parte de un entramado de nudos rígidos, y por tanto sometido a un exfuerzo axil de 
compresión más dos momentos en los extremos, generalmente de distinto valor, transmitidos por las vigas, es muy complejo.

Es por ello que se suele recurrir a hipótesis simplificadoras que faciliten su cálculo.

Una simplificación consiste en sustituir los empotramientos elastoplásticos con los que el soporte se enlaza al entramado de nudos rígidos, por dos articulaciones. Los momentos que, en el caso real transmiten las vigas, se sustituirán por la aplicación de un esfuerzo axil excéntrico sobre dichas articulaciones. Cabe señalar que las excentricidades del axil sobre los dos extremos no deben ser necesariamente iguales ni del mismo signo. Por convención, cuando las excentricidades tienen distinto valor, la excentricidad mayor se aplica sobre el extremo superior de la barra.

La segunda simplificación consiste en predeterminar la forma que adopta la deformada del soporte, asimilándola a un arco de tipo senoidal.

La Figura 7.3-1, obtenida a partir de la publicación de Juan J. Arenas, se muestra el caso de una barra comprimida por un axil con excentricidades distintas pero del mismo signo en ambos extremos.
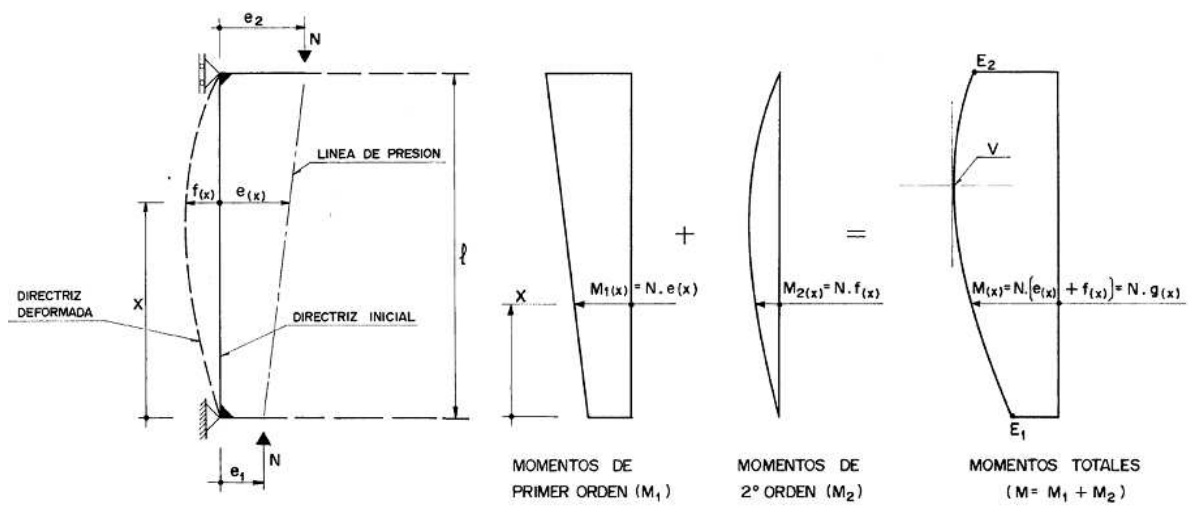

Figura 7.3-1 Momentos de primer y segundo orden en un soporte sometido a compresión excéntrica. 
Los momentos de primer orden son lineales y proporcionales a la excentricidad $e_{(x)}$ de la carga respecto de la geometría sin deformar de la barra. Los momentos de segundo orden están representados por una curva y son proporcionales a la deformación $f_{(x)}$ de la barra producida por los momentos de primer orden. Mediante la suma de ambos obtenemos los momentos totales, en los que la existencia de un valor máximo relativo (punto $\mathrm{V}$ ) dependerá de las excentricidades y de la intensidad de la carga.

A continuación se describe la evolución de la deformada por el incremento progresivo de la carga para los distintos casos de excentricidad $^{118}$ :

a) Excentricidades del mismo signo

La deformada siempre es un arco que no contiene ningún punto de inflexión. Si las excentricidades son de distinto valor y la carga pequeña, imagen a), la deformada no presenta ningún máximo relativo en la longitud de la barra. Si se incrementa progresivamente la carga, se alcanza un valor crítico, imagen b), en el cual el máximo relativo se sitúa en el extremo superior, donde la excentricidad de la carga es mayor. En este intervalo el colapso de la barra únicamente podría producirse por agotamiento de su sección más defavorable (extremo superior).

118 "Le flambement des poteaux en béton armé chargés avec des excentricités différentes a leurs extremités”. J.R. Robinson, B. Fouré y A.Y. Bourghli. (1975) 

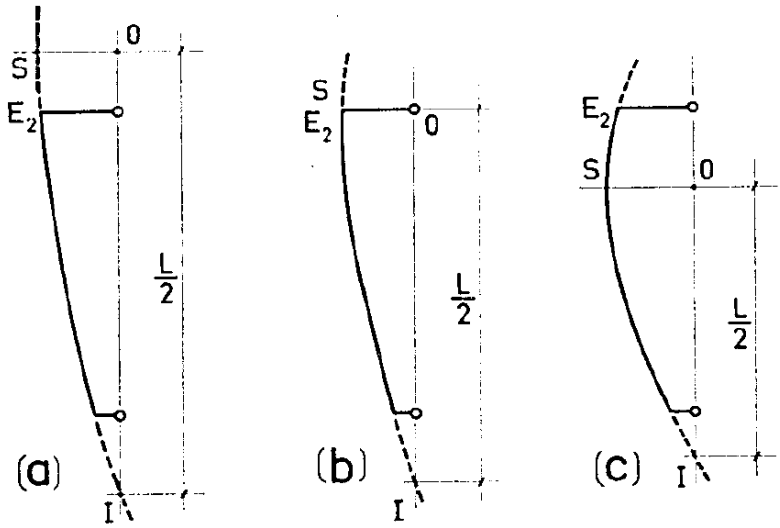

Figura 7.3-2 Evolución de la deformada de un soporte sometido a un axil con dintintas excentricidades pero del mismo signo

Si se sigue incrementando la carga, el máximo relativo se producirá en el intervalo de la longitud de la barra (punto O) de la imagen c). En este caso, dicha sección será la de mayor solicitación y por tanto en la que se producirá la rotura que podrá ser debida al agotamiento resistente de la sección, o bien a la inestabilidad de la pieza ya que las deformaciones de primer orden amplifican las solicitaciones.

En el caso particular de excentricidades de la carga iguales en ambos extremos, el momento máximo siempre se producirá en el punto medo de la barra debido a la simetría. Ello implica que la excentricidad adicional interviene desde el primer momento en el agotamiento de la barra.

b) Excentricidades signos contrarios

La deformada es un arco que siempre contiene un punto de inflexión en el intervalo de la longitud de la barra. 

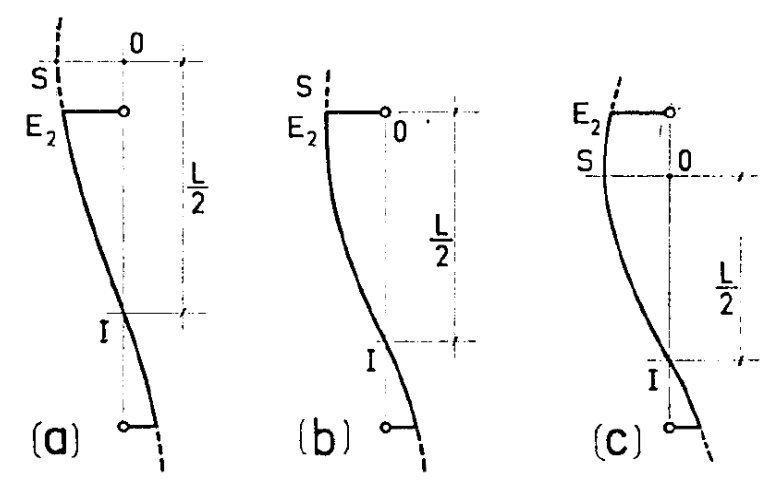

Figura 7.3-3 Evolución de la deformada de un soporte sometido a un axil con dintintas excentricidades y de signos contrarios

El proceso de incremento de la carga produce tres estados similares, en cuando a la posición de las deformaciones máximas relativas y posible forma de colapso de la barra que las expuestas en el caso de excentricidades del mismo signo. Al igual que antes, sólo a partir del estado c), las excentricidades adicionales intervienen en la resistencia de la barra.

Es interesante el caso particular de excentricidades iguales y de signo contrario.

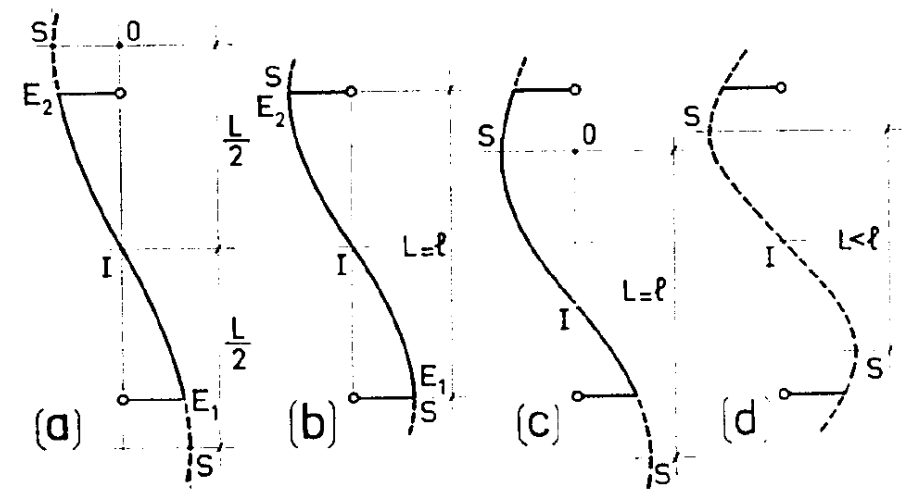

Figura 7.3-4 Evolución de la deformada de un soporte sometido a un axil con iguales excentricidades pero de signos contrarios 
Debido a la simetría, la deformada tiene un punto de inflexión situado en el centro de la barra y, o bien no existen máximos relativos, imagen a) en su longitud (para cargas reducidas) o bien existen dos, imagen b) situados en los extremos de la barra (situación crítica conforme se incrementa la carga). Si se sigue incrementando la carga, el punto de inflexión se desplaza, desapareciendo la simetría, para que la deformada tenga un único máximo relativo, imagen c). La deformada simétrica con dos máximos relativos, imagen d), nunca puede alcanzarse, puesto que implica una energía de deformación mayor que la de c).

Asumida ya la hipótesis de deformada senoidal, vamos a continuación a deducir la ecuación que la caracteriza en un caso general. Tal como se representa en la Figura 7.3-5, adoptaremos como eje $\mathrm{X}$ del sistema de referencia, la línea de acción de la carga que pasa por las articulaciones, con excentricidades $e_{1}$ y $e_{2} ;$ y como eje $\mathrm{Y}$, el ortogonal al $\mathrm{X}$ que pasa por el punto de deformación máxima.

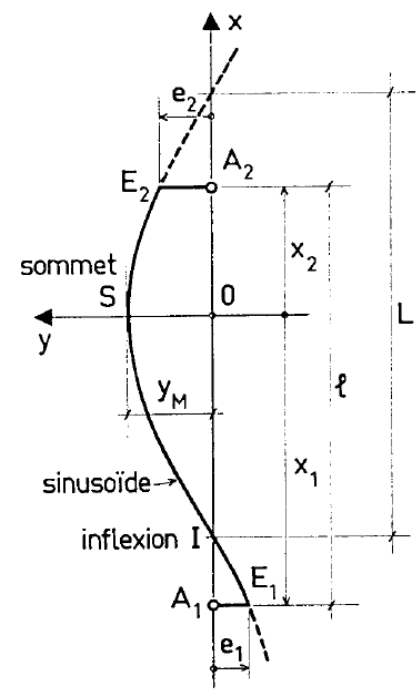

Figura 7.3-5 Representación de la deformada senoidal 
De esta forma, la ecuación general que define a la línea deformada será:

$$
y=y_{M} \cdot \cos \frac{\pi \cdot x}{L}
$$

Ecuación 7.3-1

siendo:

$L \quad$ semilongitud de onda

$y_{M}$ deformación máxima, situada en el origen y con signo positivo dado el sistema de referencia adoptado.

La relación entre las excentricidades de la carga de los extremos y la deformación máxima será:

$$
\begin{aligned}
& e_{1}=y_{M} \cdot \cos \frac{\pi \cdot x_{1}}{L} \\
& e_{2}=y_{M} \cdot \cos \frac{\pi \cdot x_{2}}{L} \\
& x_{2}-x_{1}=I
\end{aligned}
$$

Ecuación 7.3-2

Despejando $x_{1}$ y $x_{2}$ de las ecuaciones anteriores:

$$
\begin{aligned}
& x_{1}=\frac{L}{\pi} \cdot \arccos \frac{e_{1}}{y_{M}} \\
& x_{2}=\frac{L}{\pi} \cdot \arccos \frac{e_{2}}{y_{M}}
\end{aligned}
$$

Ecuación 7.3-3

Sustituyendo estos valores en la tercera igualdad de la Ecuación 7.3-2 y despejando: 


$$
\frac{L}{\pi} \cdot\left(\arccos \frac{e_{2}}{y_{M}}-\arccos \frac{e_{1}}{y_{M}}\right)=I \rightarrow L=I \cdot \frac{\pi}{\arccos \frac{e_{2}}{y_{M}}-\arccos \frac{e_{1}}{y_{M}}}
$$

Y sustituyendo esta expresión en las dos igualdades de la Ecuación 7.3-3 podemos expresar el valor de $x_{1}$ y $x_{2}$ en función de $y_{M}$

$$
\begin{aligned}
& x_{1}=1 \cdot \frac{\arccos \frac{e_{1}}{y_{M}}}{\arccos \frac{e_{2}}{y_{M}}-\arccos \frac{e_{1}}{y_{M}}} \\
& x_{2}=1 \cdot \frac{\arccos \frac{e_{2}}{y_{M}}}{\arccos \frac{e_{2}}{y_{M}}-\arccos \frac{e_{1}}{y_{M}}}
\end{aligned}
$$

\subsection{DISTINTOS MÉTODOS DE ANÁLISIS}

Una vez asumida la hipótesis de la deformada inicial, y conocidas las solicitaciones de flexo-compresión simple o esviada en sus extremos, el problema consiste en cómo poder determinar la deformada final de la barra a partir de la que se puedan obtener las solicitaciones a lo largo de su longitud.

A continuación se exponen varios métodos para resolver el problema $^{119}$.

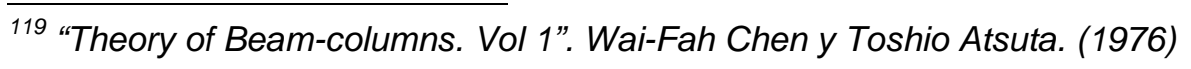




\subsubsection{MÉTODOS DE INTEGRACIÓN NUMÉRICA}

Uno de las formas de determinar las solicitaciones de agotamiento de una viga-columna consiste en obtener el punto donde se produce el cambio de curvatura de la gráfica carga-deformación, utilizando alguno de los métodos de integración numérica.

Uno de los más utilizados, el método de Newmark, permite, mediante un proceso iterativo, obtener los desplazamientos de una serie de puntos determinados, a partir de los cuales se puede representar la deformada de la barra. Este método, como paso intermedio para determinar las deformaciones, necesita calcular la curvatura de los puntos de referencia. Mientras la barra tiene un comportamiento elástico, la curvatura se puede obtener, de una forma sencilla, mediante la relación $\phi=M / E l$. Sin embargo, cuando se entra en la fase de comportamiento no lineal, las curvaturas ya no siguen la expresión indicada y su determinación requiere el uso de los diagramas $M-\phi-P$ (Momento-curvatura-axil) de la sección de la barra, cuyo conocimiento se requiere previamente a la aplicación del método.

El proceso, que se ilustra en la Figura $7.4-1^{120}$, parte de una deformación inicial conocida, definida por la función $w_{l}(x)$, a partir de la cual se pueden obtener los momentos en una serie de nodos mediante la expresión:

$$
M(x)=\bar{M}(x)+P \cdot w_{l}(x)
$$

siendo $\bar{M}(x)$ la ley de momentos inicial en la barra de directriz recta y $P$ el esfuerzo axil.

\footnotetext{
${ }^{120}$ Esta figura y las siguientes han sido extraídas de la publicación "Theory
} of Beam-columns. Vol 1" de Wai-Fah Chen y Toshio Atsuta. 
Curvature
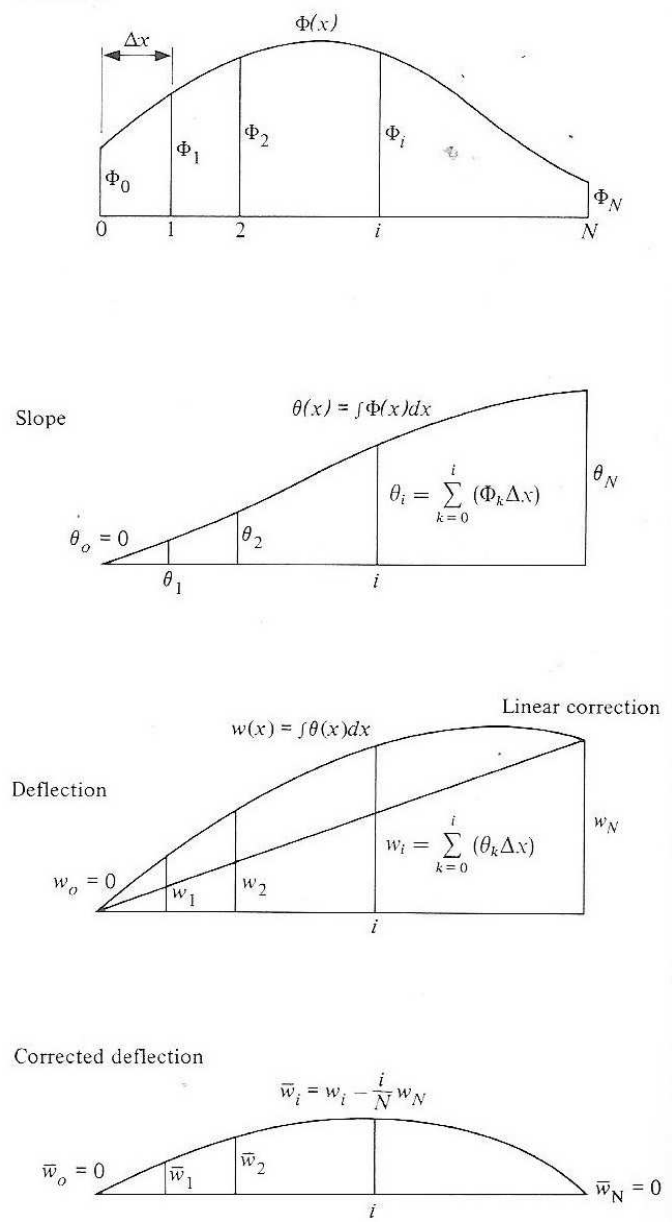

Figura 7.4-1 Proceso de determinación de la línea deformada

La curvatura de cada nodo se obtiene, conocidas las solicitaciones, utilizando los diagramas $M-\phi-P$, y mediante la integración numérica de ésta, se obtiene el giro. Los desplazamientos se obtienen integrando numéricamente los giros. 


$$
\begin{aligned}
& \theta_{i}=\sum_{k=0}^{i} \Phi_{k} \cdot \Delta x \\
& w_{i}=\sum_{k=0}^{i} \theta_{k} \cdot \Delta x
\end{aligned}
$$

Tanto los giros como los desplazamientos se consideran nulos en el origen, aunque estos valores no coincidan con las verdaderas condiciones de contorno. La nueva deformada, $w_{I I}(x)$, con los desplazamientos reales en los extremos, se obtiene aplicando una corrección lineal de los desplazamientos obtenidos:

$$
\bar{w}_{i}=w_{i}-\frac{i}{N} w_{N}
$$

A continuación se repite el método con la nueva deformada, en un proceso iterativo que finaliza con la convergencia entre la deformada inicial y final de la iteración.

La resistencia máxima de la barra se obtiene incrementando progresivamente las cargas e iniciando de nuevo el proceso descrito. Los valores para los que no se consigue la convergencia en la deformación corresponden a solicitaciones de agotamiento, y por tanto a un punto del diagrama de interacción.

Repitiendo todo el proceso para distintas relaciones $N-M$ se pueden obtener suficientes puntos para la representación del mencionado diagrama de interacción.

\subsubsection{MÉTODOS BASADOS EN LAS CURVAS DE DEFORMACIÓN}

Las curvas de deformación, en inglés Column Deflexión Curves $(C D C)$, se podrían definir como el conjunto de curvas que puede 
adoptar la deformada de la "columna equivalente", entendida ésta como la barra imaginaria sometida a esfuerzo axil centrado.

A partir de las dimensiones y esfuerzos en los extremos de una barra, se podrán determinar los parámetros, axil, longitud e inclinación, que definen su columna equivalente.

En la figura, se muestra la columna equivalente de una barra, esquema (a), de longitud I sometida a una carga axial $P$ y unos momentos $M_{A}$ y $M_{B}$ en sus extremos.

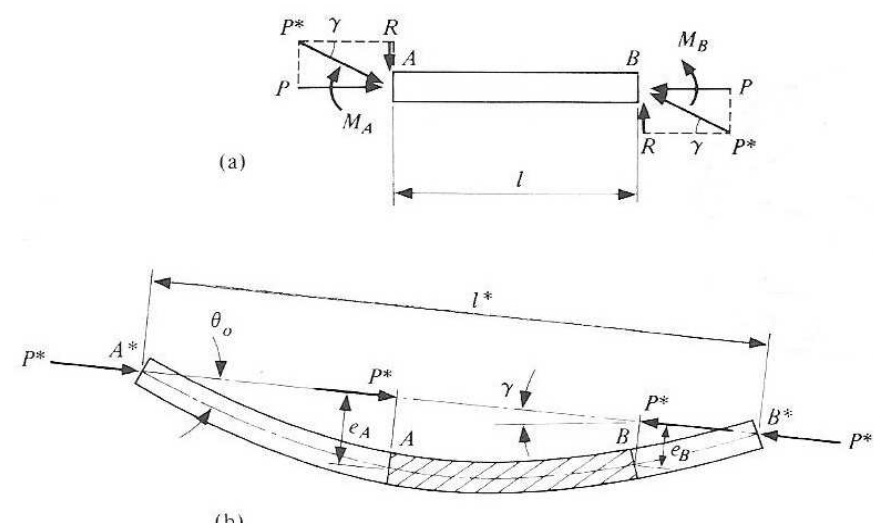

Figura 7.4-2 Barra flexocomprimida y su columna equivalente

Las reacciones verticales $R$ que producen el equilibrio, así como la resultante de la fuerza en los extremos y la inclinación de la barra, se pueden obtener, respectivamente, mediante las expresiones:

$$
\begin{aligned}
& R=\frac{M_{A}-M_{B}}{l} \\
& P^{*}=\sqrt{P^{2}+R^{2}} \\
& \gamma=\operatorname{arctg} \frac{R}{P}
\end{aligned}
$$


Si se grafía una barra, esquema (b), de longitud $l^{*}$ y una curvatura tal que las excentricidades entre la línea de acción del axil y los puntos $A$ y $B$ produzcan las mismas condiciones que en la situación inicial, cuyos valores se pueden obtener mediante las expresiones:

$$
e_{A}^{*}=\frac{M_{A}}{P^{*}} \quad y \quad e_{B}^{*}=\frac{M_{B}}{P^{*}}
$$

Dicha barra imaginaria será la denominada "columna equivalente" de la barra flexocomprimida original.

Determinada, por tanto, una CDC, se podrán extraer de ella tantas porciones como se desee, correspondiente, cada una de ellas a una combinación distinta de momentos en los extremos.

A modo de ejemplo se ha representado en la Figura 7.4-3, una CDC formada por dos semiondas de una barra sometida, en sus extremos, a un esfuerzo axil $P^{*}$, y con un giro en el origen y final de la barra $\theta_{0}$. De ella se han extraido tres casos de barras comprimidas: una con el extremo izquierdo articulado y el derecho sometido a un momento, otra sometida en sus extremos a dos momentos del mismo sentido de giro y otra sometida en sus extremos a dos momentos iguales y de sentido de giro contrario.

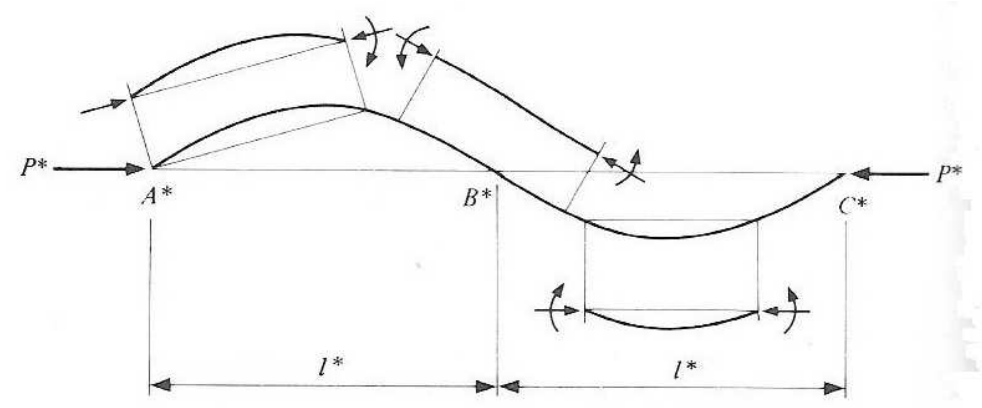

Figura 7.4-3 Ejemplo de CDC 
La representación de una CDC exige el conocimiento del desplazamiento $w$ y del giro $\theta$ de cada uno de sus puntos, a partir de los que se puede determinar el momento $M=P^{*} \cdot w$. La resolución de estas deformaciones cuando nos encontramos en el rango inelástico exige el conocimiento de las relaciones momento-axil-curvatura y recurrir a procedimientos de integración númerica como el descrito en el apartado anterior.

Conocida la familia de curvas de deformación de una columna del mismo material, la misma sección y el mismo axil que la barra objeto de análisis, es posible representar la curva momento-rotación o momento-desplazamiento. La Figura 7.4-4 muestra un ejemplo de cómo utilizar las CDC's en el caso de una barra sometida a flexocompresión.

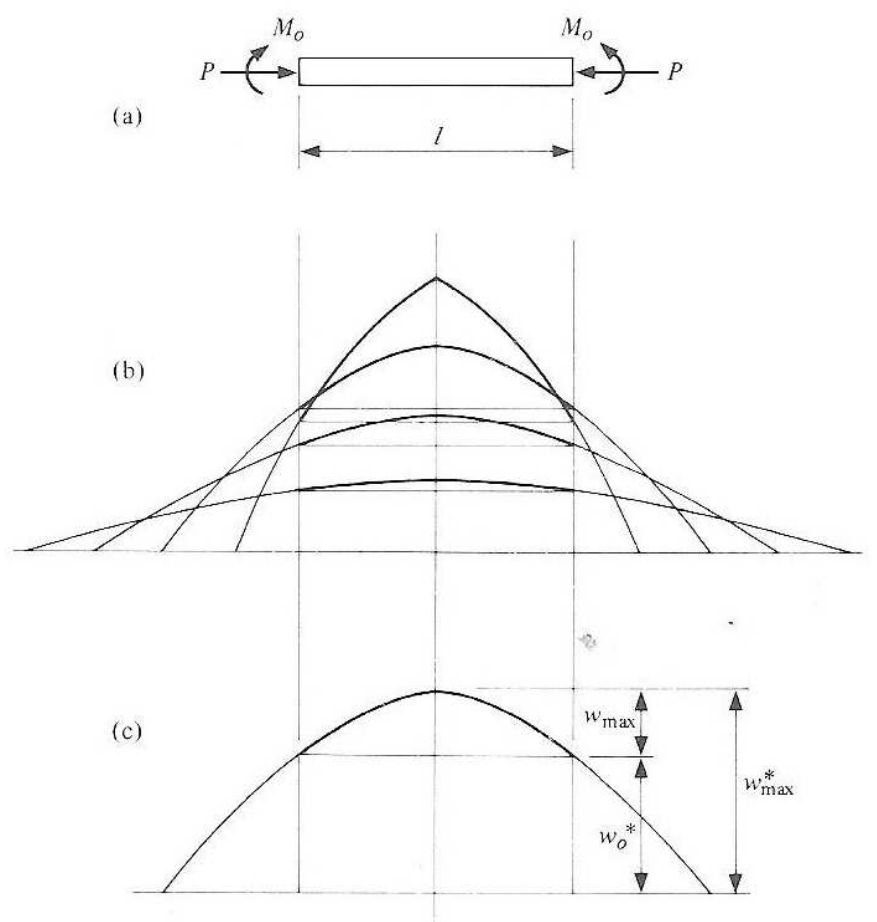

Figura 7.4-4 Ejemplo de aplicación de las CDC's 
Cada una de las curvas que forman la familia de CDC's, se obtiene fijando el valor del momento aplicado en el extremo $\left(M_{0}\right)$ al que le corresponde el giro $\theta_{0}$. Para obtener las distintas curvas, Figura 7.4-4 (b), el momento se incrementa progresivamente mientras que el esfuerzo axil $P$ se mantiene constante en toda la familia de CDC's.

La zona central de longitud I de la familia de CDC's corresponde a las distintas deformaciones que adopta la barra flexocomprimida en estudio, sometida a distintos momentos $M_{0}$ en los extremos. Elegida una determinada curva, como se observa en la Figura 7.4-4 (c), se puede obtener la deformación máxima $w_{\max }^{*}$ en el centro del vano y la deformación en los extremos de la longitud $l, w_{0}$. Las expresiones que nos permiten calcular la deformación máxima y los momentos de la barra flexocomprimida en estudio son las siguientes:

$$
\begin{aligned}
& M_{0}=P \cdot w_{0}^{*} \\
& M_{\max }=P \cdot w_{\max }^{*} \\
& w_{\max }=w^{*}-w_{0}^{*}
\end{aligned}
$$

Este planteamiento teórico, expuesto por Von Kármán en 1910 y desarrollado por Chwalla, Horne y Ellis entre otros, junto a los resultados de numerosos ensayos de barras de distintas esbelteces y secciones, ha sido la base para la determinación en la universidad de Lehigh de las curvas de máxima resistencia de distintos tipos de soportes de acero, conocidas como Curvas Europeas de Pandeo, que en la actualidad sirven como base para la comprobación de estos elementos. 


\subsubsection{MÉTODOS BASADOS EN LAS FUNCIONES DE CURVATURA}

En este método, las funciones de curvatura, en inglés Column Curvature Curves (CCC), sustituyen a las curvas de deformación del análisis expuesto en el apartado anterior.

Como en el método anterior, se requiere el conocimiento de las relaciones momento-axil-curvatura y como en aquél, es el concepto de la "columna equivalente" quien permite el desarrollo del método de las funciones de curvatura. El problema, dado un soporte sometido a flexocompresión, consistirá en encontrar su correspondiente columna equivalente y en localizar que porción de ésta se toma como solución.

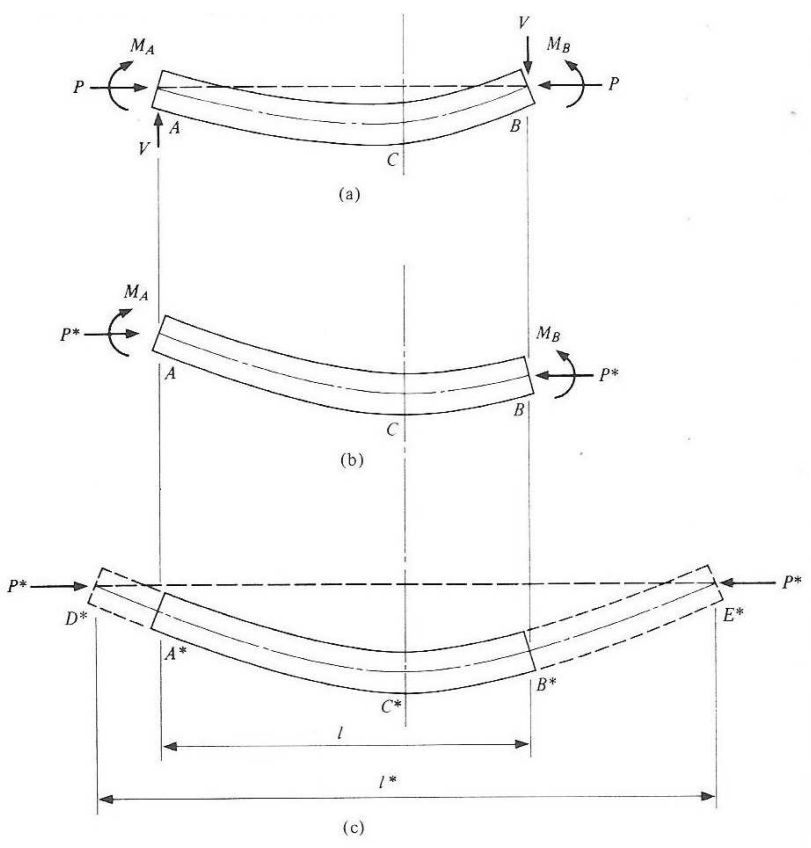

Figura 7.4-5 Columna equivalente

La diferencia principal entre el método de las CDC's y el de las CCC's consiste en que el primero únicamente resuelve, utilizando un procedimiento de integración numérica, la curva de deformación en la longitud de la barra flexocomprimida, mientras que las CCC's, cuando 
las leyes de comportamiento del material son sencillas, se resuelven analíticamente en el total de la columna equivalente y se selecciona una porción de ella como solución.

Puesto que el concepto de "columna equivalente", así como el proceso para su determinación ya ha sido descrito en el apartado 7.4.2, partiremos aquí, de una barra sometida a flexocompresión y su correspondiente columna equivalente sometida a compresión centrada, de la que queda por determinar su longitud $l^{*}$.

Del análisis de esta columna equivalente en estado elasto-plástico podemos encontrarnos en tres situaciones distintas, grafiadas en la Figura 7.4-6, de las que se expresan a continuación las funciones de curvatura para su mitad izquierda:
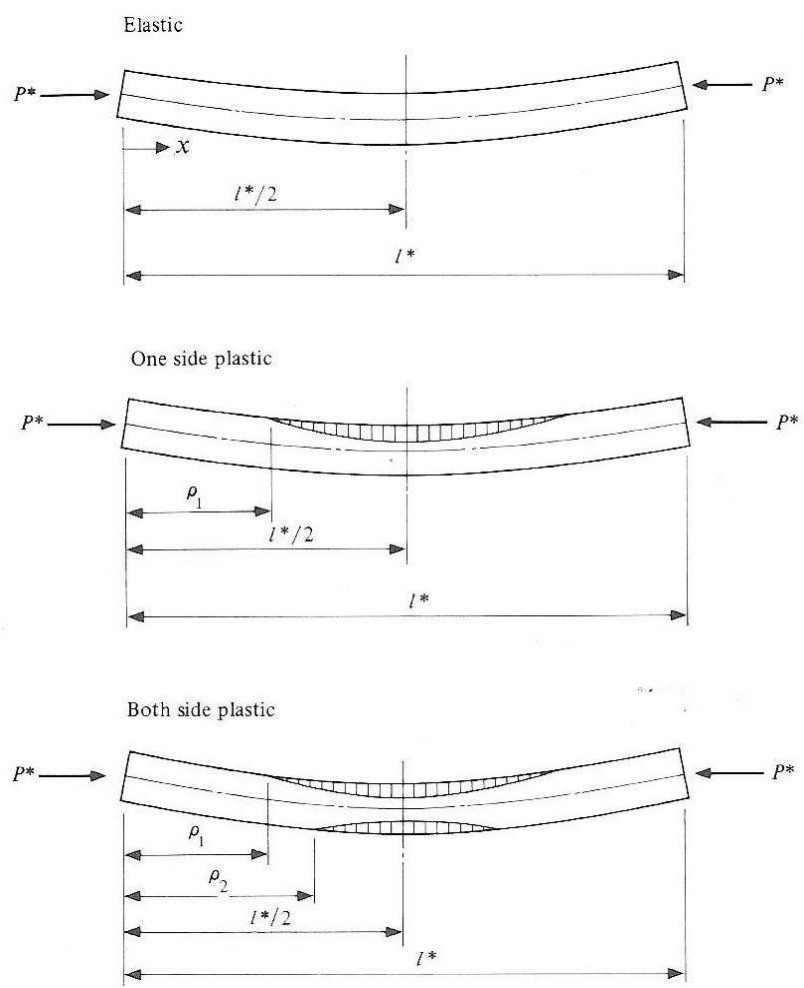

Figura 7.4-6 Estados de plastificación en una barra flexocomprimida 
Columna en estado elástico: $\left(0 \leq \varphi_{m} \leq \varphi_{1}\right)$

$$
k \cdot x=\sqrt{a} \cdot \operatorname{sen}^{-1}\left(\frac{\varphi}{\varphi_{m}}\right)
$$

La longitud de la columna equivalente se puede obtener mediante la expresión:

$$
k \cdot I^{*}=\pi \cdot \sqrt{a}
$$

Columna plastificada en una cara: $\left(\varphi_{1} \leq \varphi_{m} \leq \varphi_{2}\right)$

Zona elástica $\left(0 \leq x \leq \rho_{1}\right)$

$$
k x=\sqrt{a} \cdot \operatorname{sen}^{-1}\left(\frac{\varphi}{\varphi_{1}} \operatorname{sen} \frac{k \cdot \rho_{1}}{\sqrt{a}}\right)
$$

Zona plástica primaria $\left(\rho_{1} \leq x \leq I^{*} / 2\right)$

$$
k \cdot x=\frac{k \cdot l^{*}}{2}-\sqrt{\frac{c}{2}} \cdot \varphi_{m}^{-3 / 4}\left(\sqrt{\frac{\varphi_{m}}{\varphi}} \sqrt{1-\sqrt{\frac{\varphi}{\varphi_{m}}}}+\tan ^{-1} \sqrt{1-\sqrt{\frac{\varphi}{\varphi_{m}}}}\right)
$$

Columna plastificada en las dos caras: $\left(\varphi_{2} \leq \varphi_{m}\right)$

Zona elástica $\left(0 \leq x \leq \rho_{1}\right)$

$$
k x=\sqrt{a} \cdot \operatorname{sen}^{-1}\left(\frac{\varphi}{\varphi_{1}} \operatorname{sen} \frac{k \cdot \rho_{1}}{\sqrt{a}}\right)
$$

Zona plástica primaria $\left(\rho_{1} \leq x \leq \rho_{2}\right)$

$$
k \cdot x=C_{4}-\sqrt{\frac{c}{2}} \cdot C_{3}^{-3 / 4}\left(\sqrt{\frac{C_{3}}{\varphi}} \sqrt{1-\sqrt{\frac{\varphi}{C_{3}}}}+\tan ^{-1} \sqrt{1-\sqrt{\frac{\varphi}{C_{3}}}}\right)
$$


Zona plástica secundaria ${ }^{121}\left(\rho_{2} \leq x \leq I^{*} / 2\right)$

$$
k \cdot x=\frac{k \cdot l^{*}}{2}-\frac{2}{3} \sqrt{f} \cdot \varphi_{m}^{-3 / 2}\left(2+\frac{\varphi_{m}}{\varphi}\right) \cdot \sqrt{\frac{\varphi_{m}}{\varphi}-1}
$$

El proceso de resolución de un problema de flexocompresión mediante las funciones de curvatura, esquematizado en la Figura 7.4-7, se inicia determinando $\rho^{*}, \varphi_{A}, \varphi_{B}, y=\cdot l$ a partir de las condiciones particulares del problema $\left(M_{A}, M_{B}, P, I\right.$ y $\left.E \cdot l\right)$ y utilizando las relaciones momento-axil-curvatura.

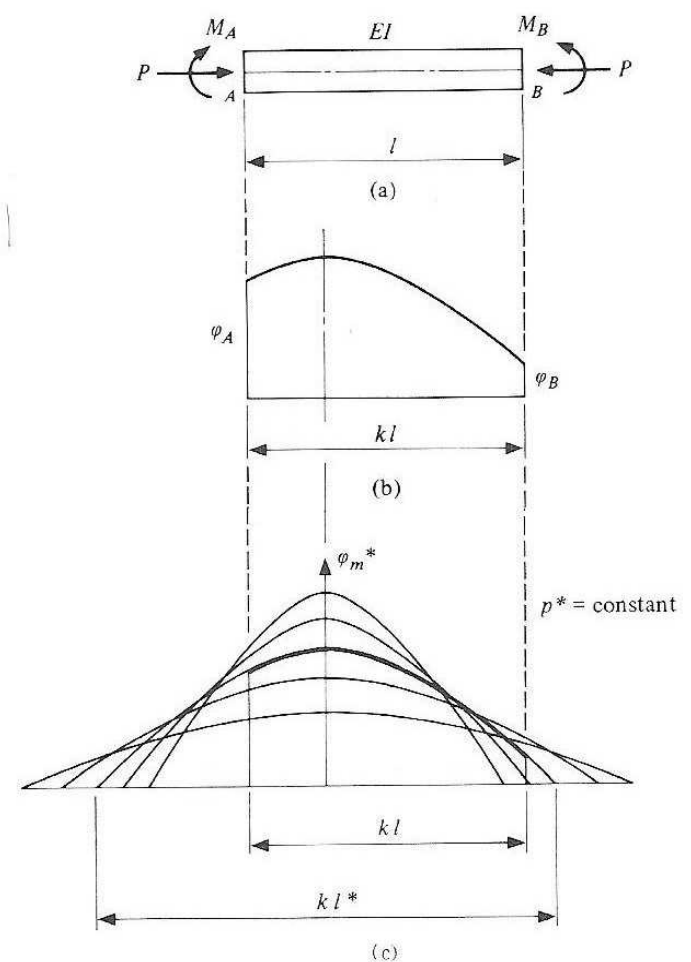

Figura 7.4-7 Proceso de resolución mediante las CCC.

${ }^{121}$ Las expresiones que permiten determinar el valor de $k \rho_{1}, k \rho_{2}, k l^{*}, C_{3}$ y $C_{4}$ utilizados en las ecuaciones anteriores, se pueden consultar en la publicación "Theory of Beam-columns. Vol 1" de Wai-Fah Chen y Toshio Atsuta. 
Para cada valor de $\rho^{*}$ se puede obtener una CCC, (Figura 7.4-7 (c)). La porción de curva donde coinciden las curvaturas en los extremos y la longitud con las de la barra objeto de análisis, proporciona la columna equivalente. De ella se puede determinar su longitud $\left(I^{*}\right)$, la curvatura máxima $\left(\varphi_{m}^{*}\right)$ así como la función de curvatura $\varphi(x)$.

\subsubsection{MÉTODOS BASADOS EN TÉCNICAS ITERATIVAS}

La deformada final de la barra sometida a flexo-compresion plana o esviada, se obtiene mediante el siguiente proceso iterativo, basado en el método de Newmark ${ }^{122}$, que se expone de forma gernérica:

1. Discretización del soporte en tramos suficientemente cortos, habitualmente de la misma longitud o que responden a posibles cambios de sección.

2. Desplazamiento de los nodos de la discretización inicial para estimar las imperfecciones iniciales, habitualmente estableciendo una hipótesis de deformación inicial de tipo senoidal, que puede asimilarse a una línea poligonal

3. Aplicación de la carga de forma incremental.

4. Ajuste de la deformada en cada escalón de carga mediante un proceso iterativo como el que a continuación se describe:

- Imposición de las condiciones de equilibrio y compatibilidad, en los extremos de cada tramo de la barra definidos por los nodos y a lo largo de toda la longitud del soporte.

122 "Cálculo matricial de estructuras en $1^{\text {er }}$ y $2^{\circ}$ orden. Teoría y problemas". Ramón Argüelles Álvarez. (2005) 
En las mencionadas condiciones se tendrán en cuenta las leyes de comportamiento que relacionan tensiones y deformaciones en cada uno de los materiales.

- Planteamiento del criterio de convergencia, tanto a nivel de equilibrio de tensional de la sección como de deformaciones en los distintos nodos de la barra.

- Resolución del sistema de ecuaciones no lineales, por aplicación de un proceso iterativo (habitualmente por el método de Newton-Raphson). Se acepta como solución del problema aquélla que cumple las condiciones de convergencia planteadas.

5. Aplicación del siguiente escalón de carga sobre la nueva geometría deformada: imperfecciones iniciales más deformaciones producidas por el primer escalón de carga. Reajuste de la deformada según se expone en el paso 4.

6. El proceso finaliza con la obtención de la deformada para el último escalón de carga.

\subsection{MÉTODO DE ANÁLISIS ADOPTADO}

La complejidad para plantear de forma analítica las funciones que definen el comportamiento de la barra a lo largo del proceso, agravado por el hecho de tener tres materiales que componen una sección no homogénea, explica que sea necesario recurrir a una discretización de la barra (división en porciones) para poder evaluar numéricamente los efectos de inestabilidad.

El objetivo que se pretende alcanzar con el proceso que a continuación se describirá, consiste en obtener las distintas 
combinaciones de valores axil-momento que producen el colapso de la barra, con los que se representará la curva de agotamiento ${ }^{123}$.

Los datos de partida para la obtención de cada una de las mencionadas curvas, serán la longitud del soporte, la sección de la barra y las características resistentes de los materiales.

El proceso consta de los siguientes pasos:

1. Considerada la barra, inicialmente de directriz recta, se discretiza en una serie de tramos (distinto número en función de la longitud de la barra analizada) y se obtienen las coordenadas iniciales de los nodos.

2. A partir de la longitud y de la sección de la barra se determinan las imperfecciones iniciales, definidas por la flecha $e_{0, d}$ según EC4 (ver apartado 3.1.1 del presente trabajo) y asimilando la forma de la deformada a un arco de tipo senoidal, se obtienen las coordenadas de los nodos sobre la directriz deformada inicial.

3. Se aplica un esfuerzo axil, mayor que el de colapso de la sección, a una determinada excentricidad respecto de la directriz recta de la barra.

La geometría y vínculos exteriores de la barra junto con las cargas aplicadas constituyen el modelo de cálculo cuyo análisis se realizará mediante el programa AV_CID. Las características de este programa, introducción de datos, método de cálculo, forma de integración del módulo de cálculo

123 En la actualidad esta opción está desarrollada para estados de flexocompresión plana, con la intención de poder generalizarla para el caso de flexocompresión esviada con la intervención de una nueva variable, el giro de la fibra neutra. 
de secciones mixtas desarrollado y representación de resultados, se describen en el capítulo 9.

4. Se realiza el análisis matricial no lineal de la estructura, con un tratamiento incremental de la carga, mediante el mencionado programa. La matriz de rigidez en el primer escalón de carga se determina sobre la geometría de la deformada inicial (por imperfecciones) y con la inercia bruta de la sección. Para cada escalón de carga se busca el equilibrio mediante la aplicación del método de Newton-Raphson o Newton-Raphson modificado $^{124}$.

5. La búsqueda del equilibrio de un determinado escalón de carga, puede finalizar de dos formas:

- Si no se alcanza el equilibrio, el par de valores $N-M$ corresponde a un punto de la curva de agotamiento de la barra, en este caso por inestabilidad de la barra. En este caso, el proceso de cálculo ha finalizado.

- Si se alcanza el equilibrio, se obtienen los desplazamientos de los distintos nodos de la barra discretizada a partir de los cuales se calculan las solicitaciones de los extremos de las barras. Las nuevas coordenadas de los nodos se guardan, de forma que el siguiente escalón de carga se aplicará sobre esta nueva geometría.

6. Con las solicitaciones obtenidas se comprueba el agotamiento de la sección mediante el programa informático desarrollado. Podemos encontrarnos en varias situaciones:

\footnotetext{
${ }^{124}$ La descripción del planteamiento matricial para la resolución del problema no lineal, así como de distintas técnicas iterativas y de la forma concreta adoptada, se expone en el Apartado 8.4 del presente trabajo.
} 
- Si la sección no es resistente, el par de valores $N-M$ corresponde a un punto de la curva de agotamiento de la barra, en este caso por colapso de su sección. En este caso, el proceso de cálculo ha finalizado.

- Si la sección es capaz de soportar las solicitaciones, se determina, en cada extremo de barra, la posición de la fibra neutra y de la curvatura del plano deformado, correspondientes al equilibrio. Con ello se determina la inercia fisurada de la sección en el caso de que la fibra neutra la corte, y el nuevo término de rigidez del tramo a partir de la curvatura y del momento flector: $(E \cdot I=M / \theta)$.

7. Se establece un nuevo escalón de carga (incremento del axil) y se vuelve al paso 4, teniendo en cuenta que las componentes de la matriz de rigidez de la estructura se determinan a partir la geometría deformada del escalón de carga anterior y con el módulo de rigidez $(E \cdot l)$ obtenido para cada sección. Se trata de un proceso iterativo hasta que se consigue el colapso de la barra, bien por inestabilidad, bien por agotamiento de su sección.

8. Finalizado el proceso para una determinada excentricidad, y por tanto, obtenido un punto de la curva de agotamiento, se incrementa la excentricidad de la aplicación de la carga, volviendo al punto 3 y repitiendo todo el proceso.

9. En el gráfico se representa, para cada longitud de la barra, los pares de valores $N-M$ siendo $M=N \cdot e$ ( $e=$ excentricidad inicial respecto de la geometría recta sin deformación). Se trata de una curva que se traza a partir de un número significativo de puntos.

El proceso descrito se representa en el diagrama siguiente: 


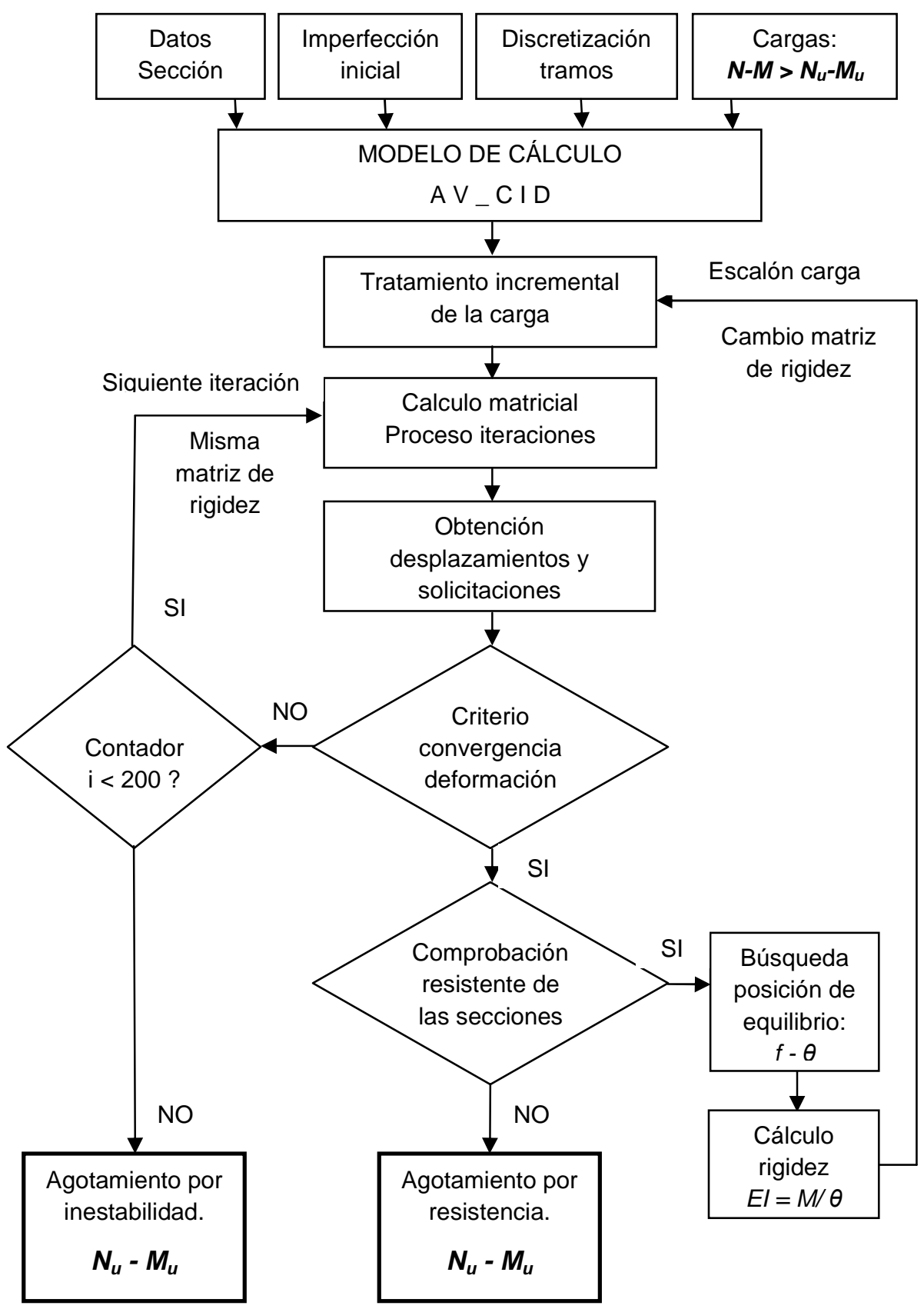

Figura 7.5-1 Organigrama de cálculo del soporte 


\subsection{RESULTADOS:}

\subsubsection{CURVAS DE INTERACCION DE LA BARRA}

Aunque el módulo informático desarrollado, junto con el programa AV_CID en su modo de cálculo no lineal, permite la comprobación de soportes de cualquier longitud y de sección mixta formada por un perfil $\mathrm{H}$ recubierto de hormigón armado, sometido a flexocompresión recta, como ejemplo se ha querido mostrar su funcionamiento con la representación de las curvas de iteracción de una serie de casos particulares.

El parámetro principal que se modificará progresivamente, en cada conjunto de curvas, será la longitud del soporte. La primera de ellas corresponde al análisis de la sección $(L=0 \mathrm{~m})$, la siguiente a una longitud del soporte de $3 \mathrm{~m}$ y en cada una del resto se incrementa 1 $\mathrm{m}$ su longitud, hasta los $10 \mathrm{~m}$, lo cual incluye las alturas que pueden plantearse en estructuras de edificación.

El parámetro que distingue cada conjunto de curvas es el tipo de carga, caracterizado por el valor relativo y el signo entre las excentricidades del esfuerzo axil en los dos extremos de la barra. Dado que la casuística es ilimitada, se han escogido tres situaciones que puedan ser representativas desde el punto de vista teórico y cuyos resultados puedan ser aplicables a las situaciones que se producen en los casos reales.
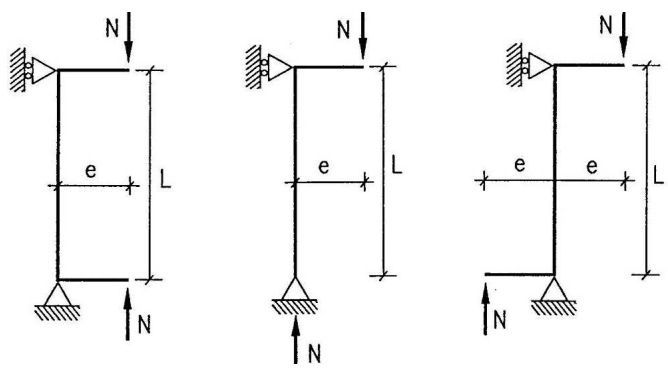

Figura 7.6-1 Casos de cargas considerados 
La Figura 7.6-1 muestra gráficamente los tres casos de cargas considerados y que se caracterizan por:

Caso a) Excentricidades de la carga iguales y del mismo signo en ambos extremos de la barra, lo que produce momentos iguales y del mismo signo en los extremos.

Caso b) Excentricidad de la carga nula en uno de los extremos, es decir, momento nulo en un extremo

Caso c) Excentricidades de la carga iguales y de signos contrarios en ambos extremos de la barra, lo que produce momentos iguales y del signo contrario en los extremos.

\section{Datos empleados en la elaboración de las gráficas:}

Sección:

Hormigón. $\quad 45 \times 45 \mathrm{~cm}^{2}$

Perfil de acero. HEB-260

Armaduras. $\quad 8 \phi 20$

Recubrimiento: $\quad 3 \mathrm{~cm}$

Resistencias de los materiales:

Hormigón. HA-25: $\mathrm{f}_{\mathrm{ck}}=25 \mathrm{~N} / \mathrm{mm}^{2}$

Acero de armar. B-400: $\mathrm{f}_{\mathrm{sk}}=400 \mathrm{~N} / \mathrm{mm}^{2}$

Acero estructural. $\quad$ S-275: $\mathrm{f}_{\mathrm{yk}}=275 \mathrm{~N} / \mathrm{mm}^{2}$

Coeficientes de minoración de resistencias:

$\begin{array}{ll}\text { Hormigón. } & \gamma_{c}=1,35 \\ & \alpha=1,00 \\ \text { Acero de armar. } & \gamma_{s}=1,15 \\ \text { Acero estructural. } & \gamma_{y}=1,10\end{array}$

En todos los casos se han tenido en cuenta los efectos reológicos en el hormigón, de la forma expuesta en el apartado 5.5 del presente trabajo. 
Las imperfecciones iniciales se han obtenido a partir del valor de la flecha $e_{0, d}$ calculada según EC4 (ver apartado 3.1.1 del presente trabajo) a partir de la longitud de la barra y de las características de la sección y de los materiales. La forma de la deformada inicial se ha asimilado a un arco de forma senoidal. Los valores de las flechas, así calculadas y su relación respecto de la longitud de la barra se indican en la tabla siguiente:

\section{Valores de las imperfecciones iniciales}

\begin{tabular}{|c|c|c|}
\hline Long. $(\mathrm{m})$ & \multicolumn{2}{|c|}{$\mathrm{e}_{0, \mathrm{~d}}$ eje fuerte $(\mathrm{cm})$} \\
\hline 3,00 & 0,345 & $\mathrm{~L} / 845$ \\
\hline 4,00 & 0,735 & $\mathrm{~L} / 544$ \\
\hline 5,00 & 1,138 & $\mathrm{~L} / 439$ \\
\hline 6,00 & 1,564 & $\mathrm{~L} / 383$ \\
\hline 7,00 & 2,010 & $\mathrm{~L} / 348$ \\
\hline 8,00 & 2,484 & $\mathrm{~L} / 322$ \\
\hline 9,00 & 2,978 & $\mathrm{~L} / 302$ \\
\hline 10,00 & 3,495 & $\mathrm{~L} / 286$ \\
\hline
\end{tabular}

Los soportes se han discretizado en tramos, con distinto número y dimensión según la longitud de la barra: los soportes de 3 y $4 \mathrm{~m}$ se han dividido en 8 tramos, los de 5 y $6 \mathrm{~m}$ en 10 tramos, los de 7 y $8 \mathrm{~m}$ en 12 tramos y los de 9 y $10 \mathrm{~m}$ en 14 tramos. A partir del número de tramos y la flecha $e_{0, d}$ se han determinado las coordenadas de cada uno de los nodos de la deformada inicial.

Las curvas de interacción que a continuación se muestran se han obtenidos por puntos, a partir de la geometría de la barra y la aplicación del axil con una determinada excentricidad. El tiempo de cálculo de cada uno de ellos varía entre 2 y 3 minutos, dependiendo del número de tramos en que se ha discretizado la barra y el número de escalones de carga empleado.

A partir de las curvas de interacción se han representando las correspondientes superficies mecánicas. 
Curvas de interacción de la barra. Excentricidades iguales.

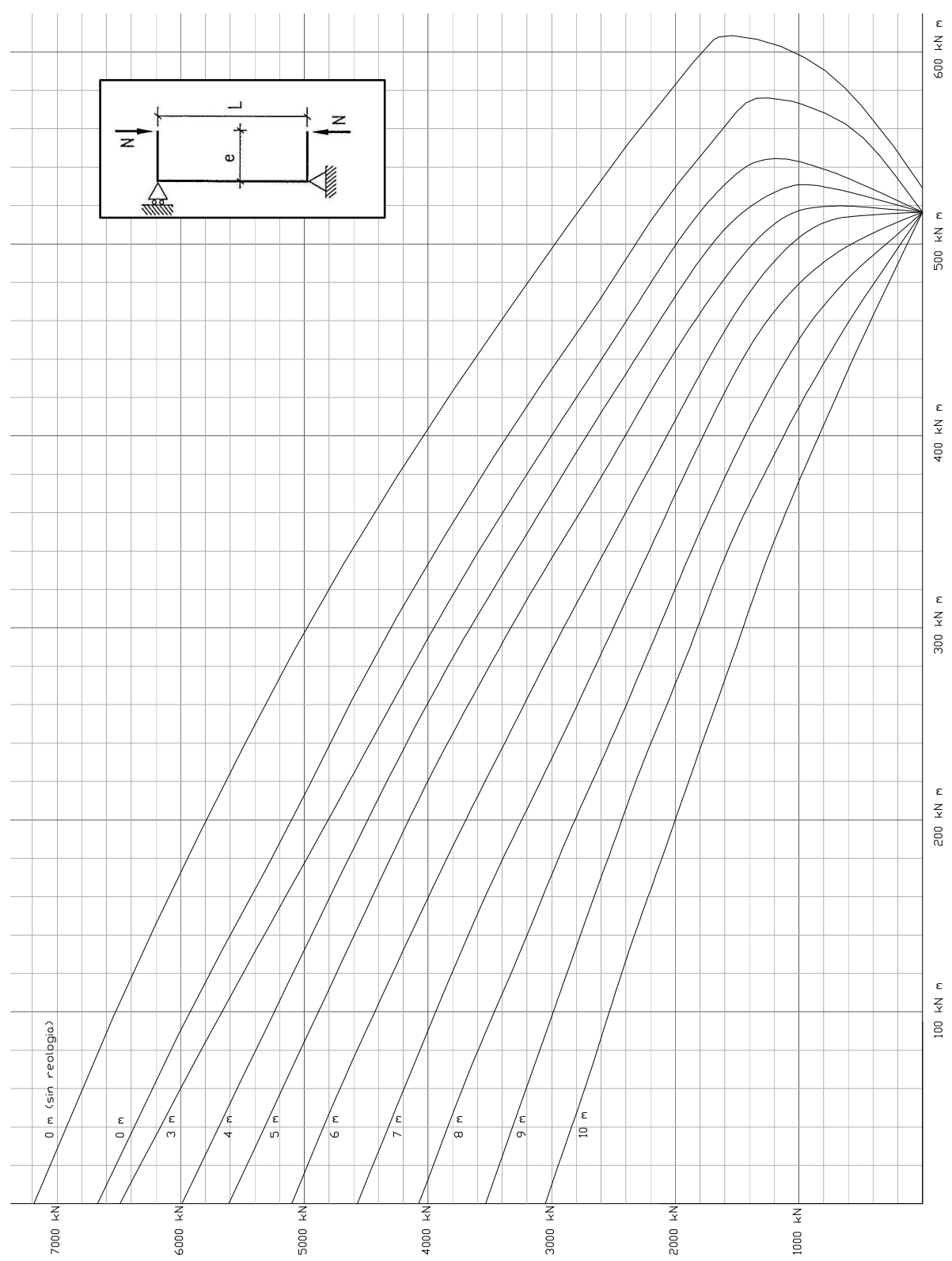




\section{Superficie mecánica. Excentricidades iguales.}

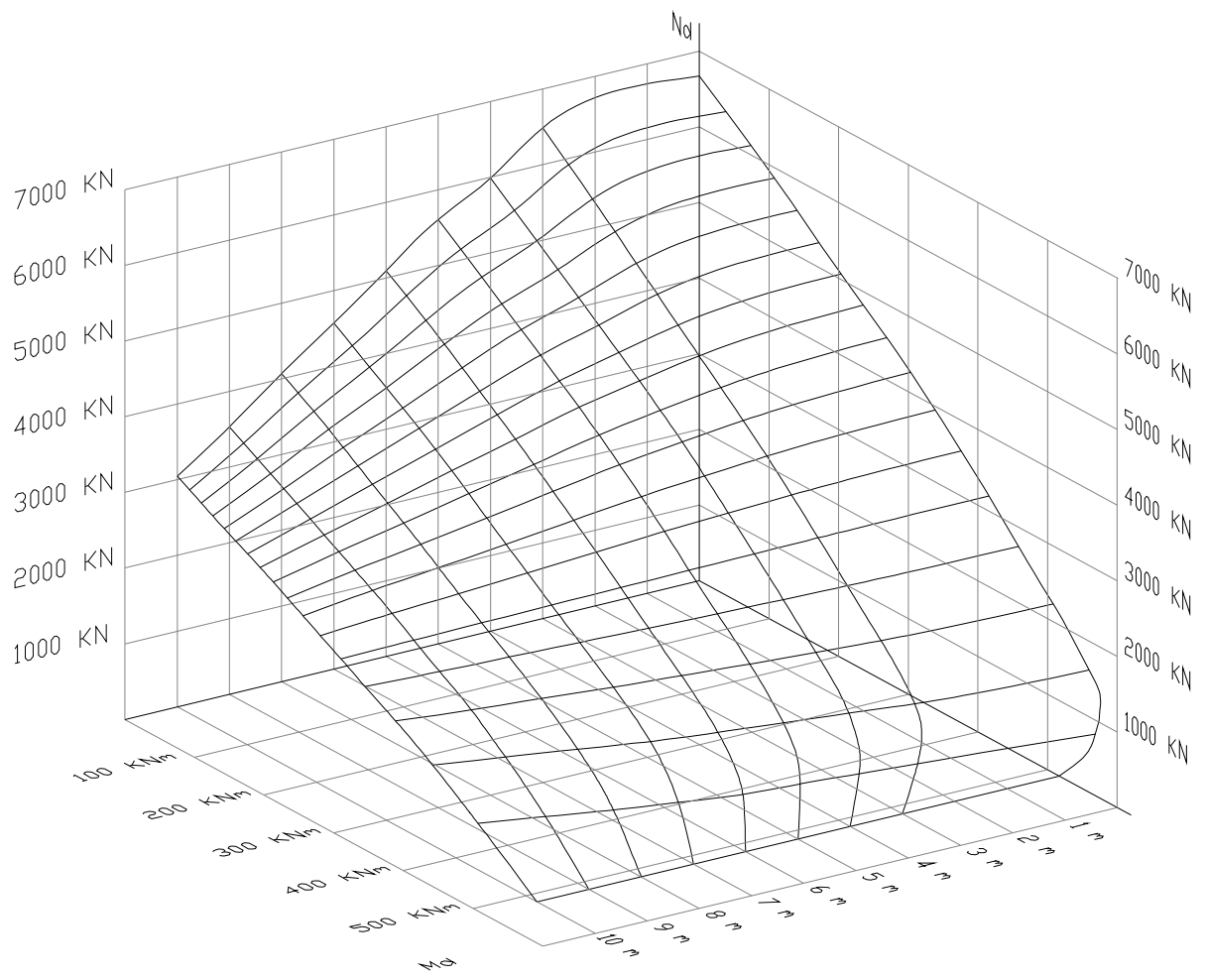

Tanto en las curvas de interacción como en la superficie mecánica, se observa que de las solicitaciones de agotamiento, $\left(N_{u}, M_{u}\right)$, son menores para el soporte, que los respectivos valores obtenidos en la sección, salvo para el caso de la flexión pura, $N_{u}=0$. 
Curvas de interacción de la barra. Una excentricidad nula.

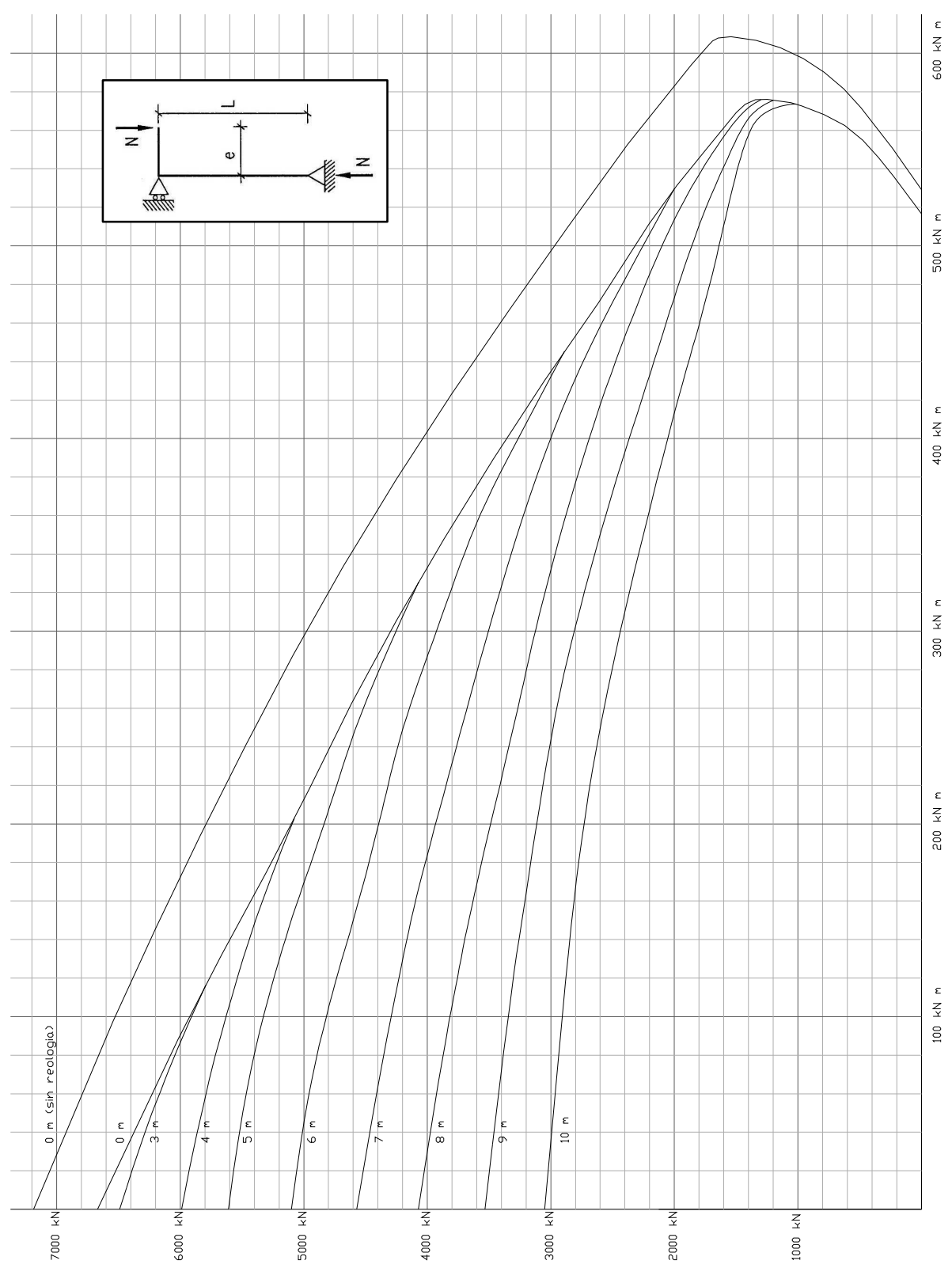


Superficie mecánica. Una excentricidad nula.

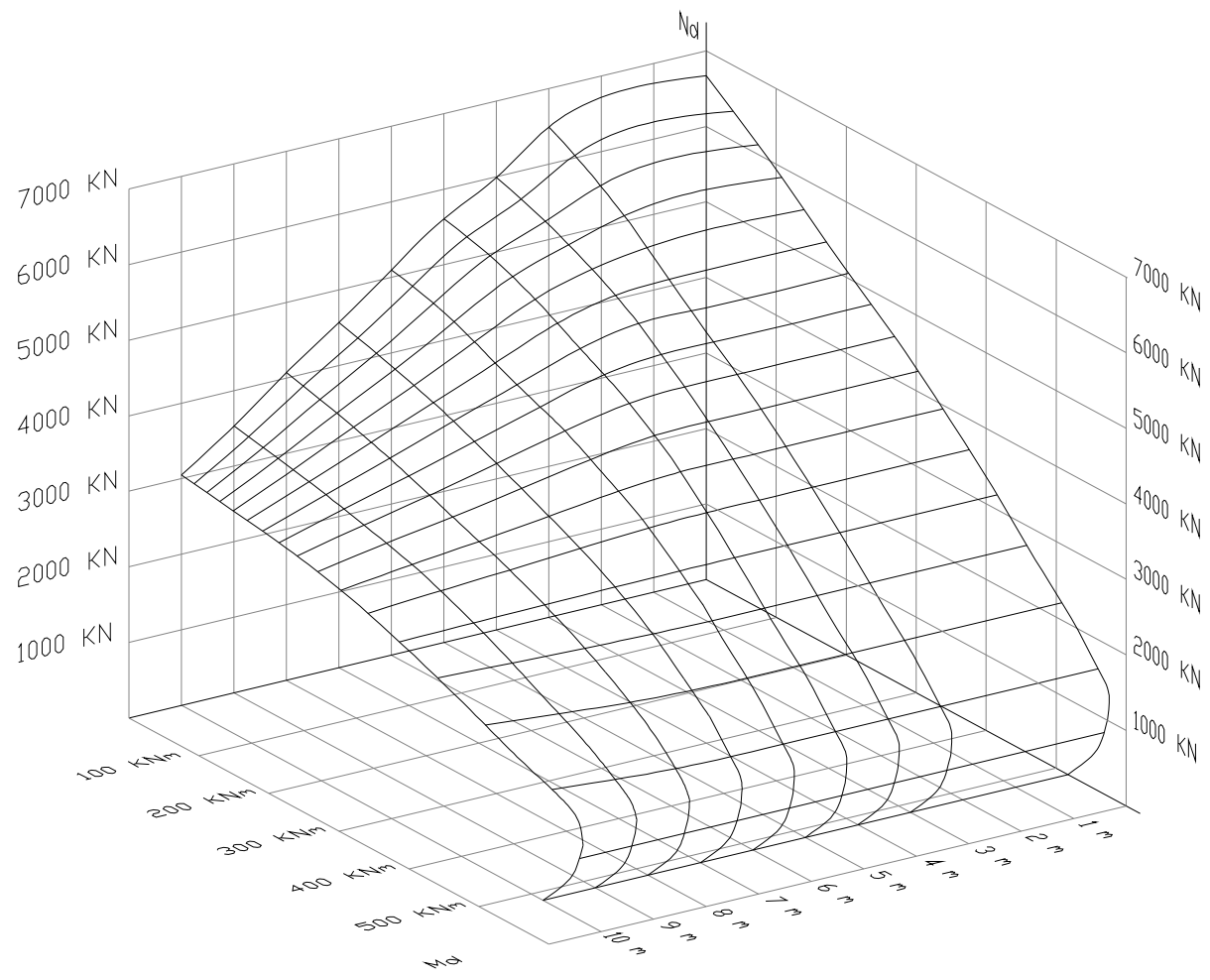

Gran parte de las curvas de interacción de los soportes, hasta longitudes de $5 \mathrm{~m}$., coinciden con la curva de la sección y todas ellas se superponen cuando predomina el claramente el momento frente al axil. 
Curvas de interacción de la barra. Excentricidades contrarias.

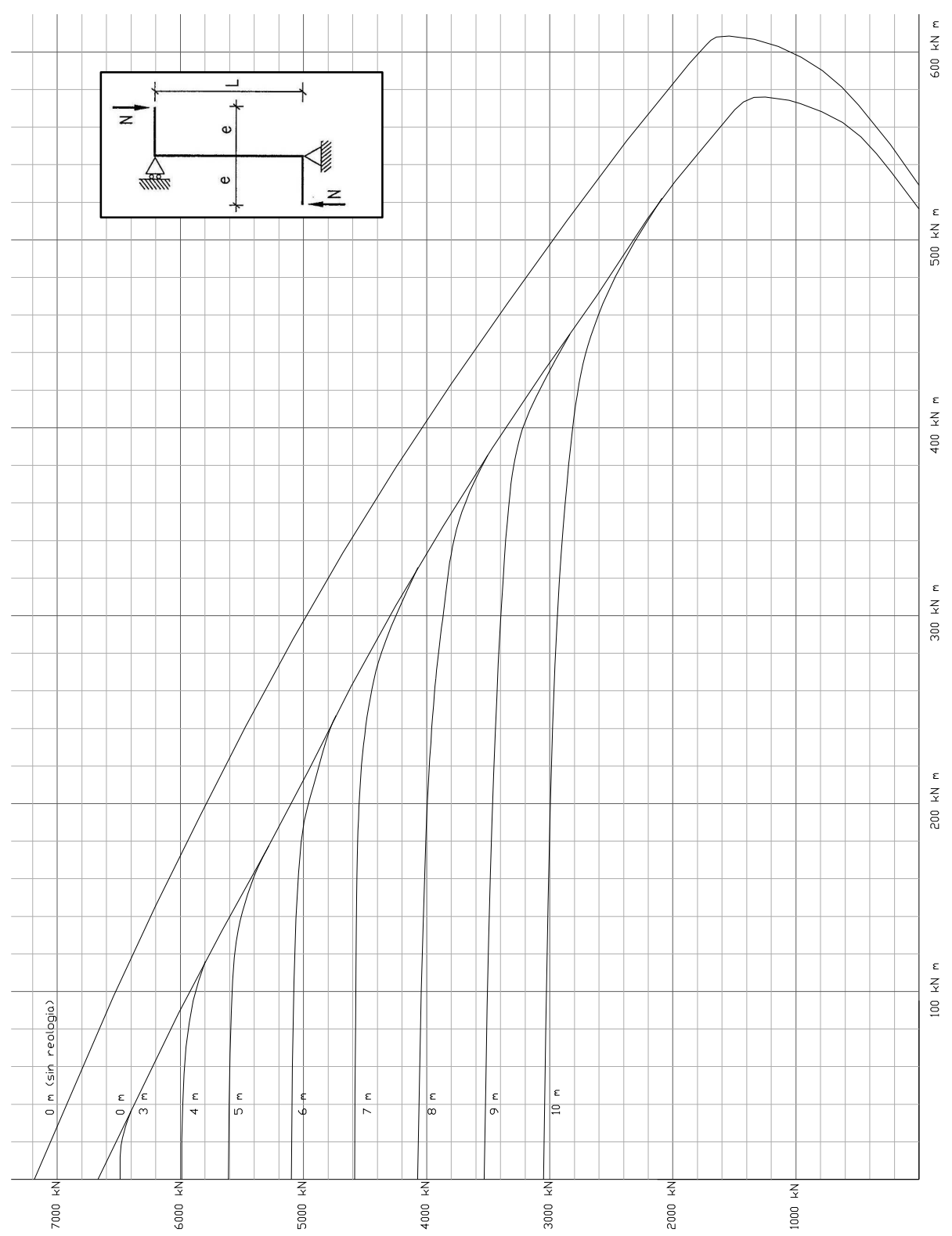




\section{Superficie mecánica. Una excentricidad nula.}

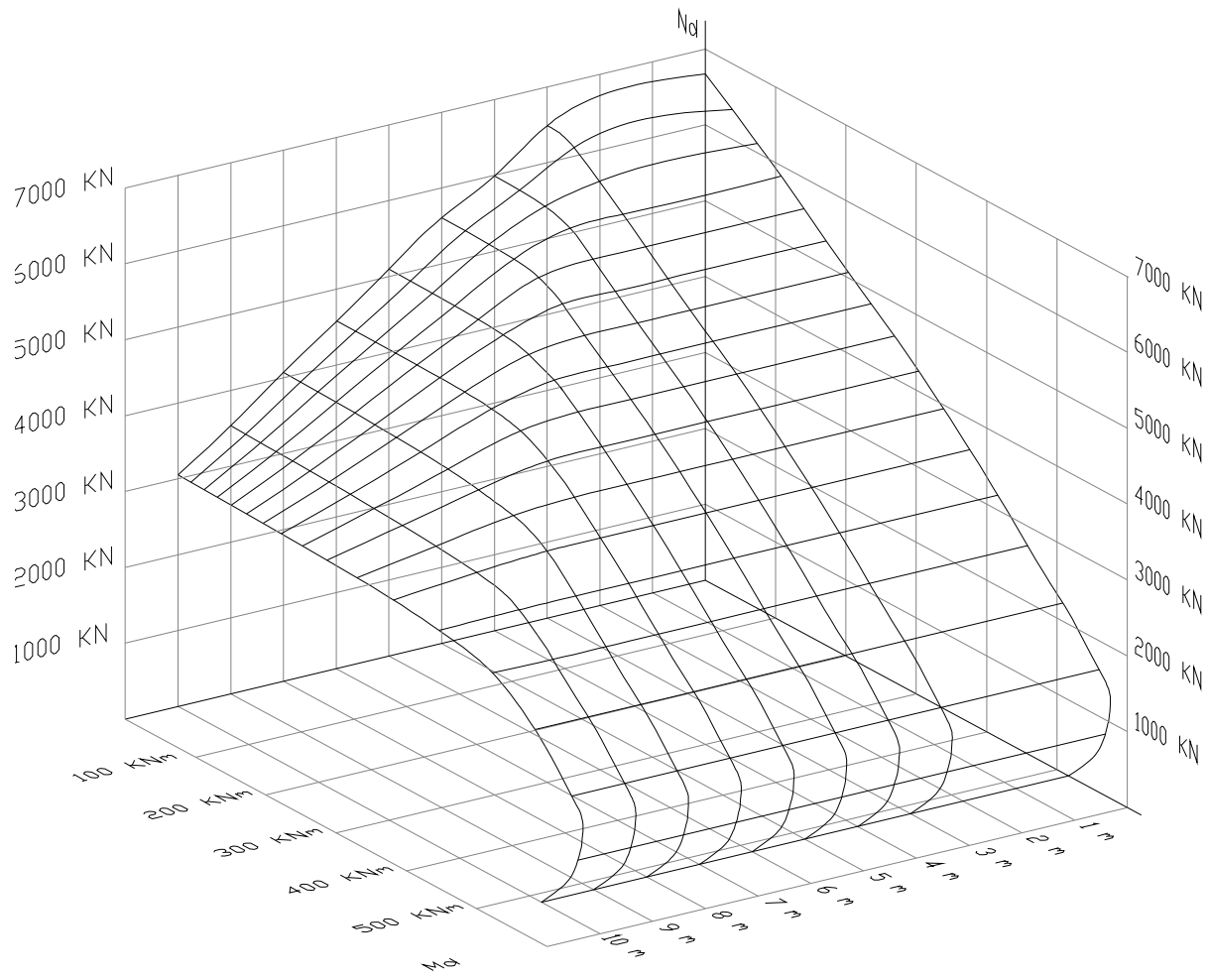

La superficie mecánica se pliega cuando predomina el axil sobre el momento, con más intensidad cuanto mayor es la esbeltez del soporte. En esta zona, se puede ver que las curvas de interacción tienen un tramo sensiblemente horizontal, mientras que coinciden con la curva de la sección cuando predomina el momento frente al axil. La coincidencia de las curvas en estos tramos implica que no se produce una reducción de la capacidad resistente de la barra respecto de la sección. 


\section{Análisis de resultados}

Tras los cálculos realizados resulta interesante cuantificar la pérdida de capacidad resistente. Para ello se han extraído los datos de los distintos casos de carga, correspondientes a los soportes de 3, 6 y 10 m. de longitud y varias excentricidades del axil. Los puntos considerados se muestran en el esquema siguiente:
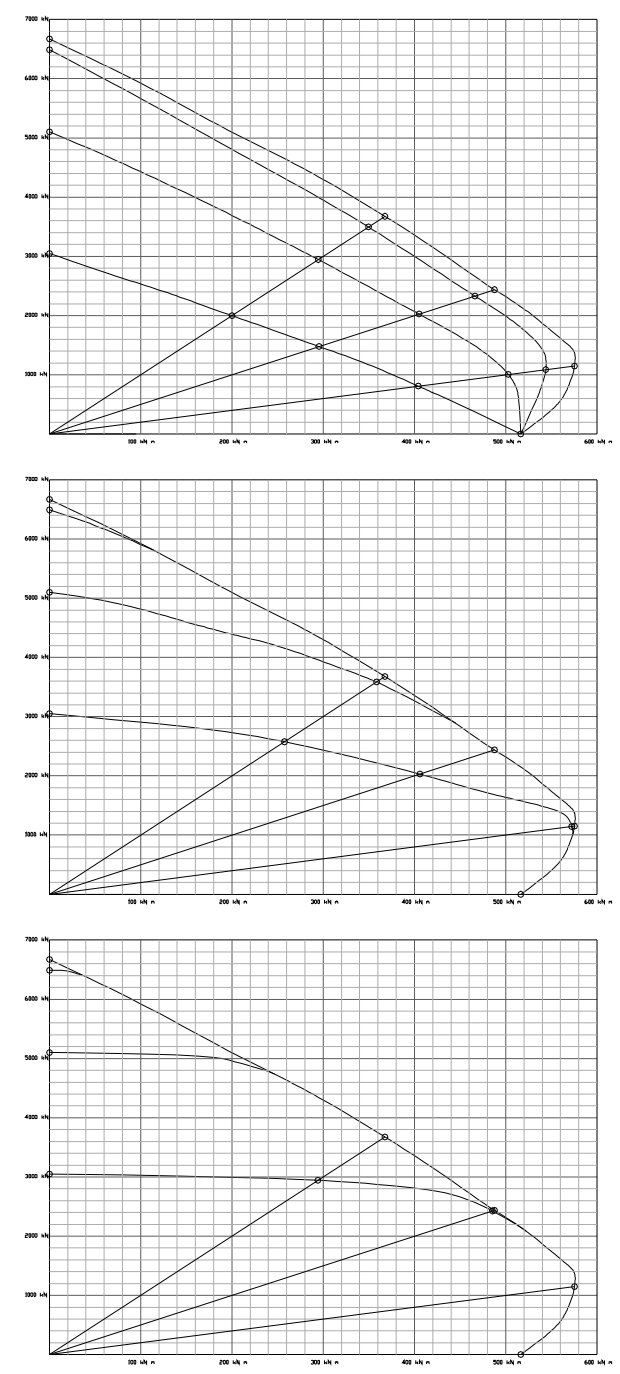

Figura 7.6-2 Esquema de los valores analizados 
Con estos valores se ha elaborado la tabla que se muestra a continuación, en la que se evalúa, la reducción de la capacidad de carga de, mediante la expresión:

$$
\text { pérdidade capacidad }(\%)=\left(\frac{N_{\text {sec ción }}-N_{\text {barra }}}{N_{\text {sec ción }}}\right) \cdot 100
$$

\begin{tabular}{|c|c|c|c|c|c|c|}
\hline $\mathrm{L}$. & excen & $\mathrm{e}=0$ & $\mathrm{e}=0,10$ & $\mathrm{e}=0,20$ & $\mathrm{e}=0,50$ & $\mathrm{e}=\infty$ \\
\hline \multirow{3}{*}{$3 \mathrm{~m}$} & Iguales & $2,7 \%$ & $4,9 \%$ & $4,4 \%$ & $5,4 \%$ & $0 \%$ \\
\cline { 2 - 7 } & Una nula & $2,7 \%$ & $0 \%$ & $0 \%$ & $0 \%$ & $0 \%$ \\
\cline { 2 - 7 } & Contrarias & $2,7 \%$ & $0 \%$ & $0 \%$ & $0 \%$ & $0 \%$ \\
\hline \multirow{3}{*}{$6 \mathrm{~m}$} & Iguales & $23,5 \%$ & $19,8 \%$ & $16,9 \%$ & $12,6 \%$ & $0 \%$ \\
\cline { 2 - 7 } & Una nula & $23,5 \%$ & $2,5 \%$ & $0 \%$ & $0 \%$ & $0 \%$ \\
\cline { 2 - 7 } & Contrarias & $23,5 \%$ & $0 \%$ & $0 \%$ & $0 \%$ & $0 \%$ \\
\hline \multirow{3}{*}{$10 \mathrm{~m}$} & Iguales & $54,3 \%$ & $45,6 \%$ & $39,4 \%$ & $30,3 \%$ & $0 \%$ \\
\cline { 2 - 7 } & Una nula & $54,3 \%$ & $29,9 \%$ & $16,7 \%$ & $12,0 \%$ & $0 \%$ \\
\cline { 2 - 7 } & Contrarias & $54,3 \%$ & $19,9 \%$ & $0,1 \%$ & $0 \%$ & $0 \%$ \\
\hline
\end{tabular}

Tabla 7.6-1 Evaúación de la pérdida de capacidad resistente

A partir de los gráficos anteriores y de la tabla, se puede concluir:

- Cuando las excentricidades del esfuerzo axil son del mismo signo, la consideración de los efectos de segundo orden siempre reducen la capacidad resistente del soporte, salvo para el caso concreto de la flexión simple.

- Cuando una excentricidad es nula o las dos excentricidades son contrarias, las curvas de interacción de la barra coinciden parcialmente con la de la sección cuando predomina el momento sobre el axil. El tramo de superposición de las curvas es mayor cuando las excentricidades son de signo contrario, y, en ambos casos, cuanto menor es la longitud del soporte. 
- A la vista de las curvas de interacción de los distintos soportes, el caso de cargas más desfavorable consiste en dos excentricidades iguales y del mismo signo (salvo para el punto particular del esfuezo axil centrado que coincide en cualquier caso)

- La pérdida de capacidad resistente debido a los efectos de segundo orden disminuye conforme se reduce la longitud del soporte, siendo prácticamente despreciable para longitudes menores de 3,00 metros.

\subsubsection{EFECTO DE LA NO LINEALIDAD DEL MATERIAL}

El programa de análisis estructural en el que se ha integrado el módulo de dimensionamiento de soportes mixtos contempla la posibilidad de realizar el cálculo en segundo orden de dos formas:

- Teniendo únicamente en cuenta la no linealidad geométrica, en cuyo caso, en cada intervalo de carga se corrige la geometría de la estructura pero se mantiene la rigidez de la barra $E \cdot I$ a lo largo del proceso de cálculo.

- Teniendo en cuenta simultáneamente la no linealidad geométrica y la del material ${ }^{125}$. Además de modificar la geometría de la estructura, el modulo de rigidez de la barra se corrige en cada intervalo de carga al tener en cuenta el equilibrio de cada sección, según se describe en el apartado 7.5 y se esquematiza en el organigrama de la Figura 7.5-1.

125 Únicamente para el caso de soportes mixtos mediante el módulo de cálculo de soportes mixtos desarrollado. 
Con el objeto de comprobar el efecto de la reducción de la rigidez de la barra sobre la capacidad resistente del soporte, se han elaborado las curvas de agotamiento de dos soportes, uno de $5 \mathrm{~m}$ y otro de 10 $\mathrm{m}$ de longitud, para las dos situaciones de cálculo no lineal descritas: geométrico y geométrico-material.

En cada una de las tablas se aplica un tipo de excentricidad de la carga: dos excentricidades iguales, una única excentricidad y dos excentricidades contrarias. $Y$ en cualquier caso se han considerado los efectos reológicos en el hormigón.

\section{Datos empleados en la elaboración de los gráficos comparativos}

Soportes:

$\begin{array}{lr}\text { Longitud 1: } & 5,00 \mathrm{~m} \\ \text { Longitud 2: } & 10,00 \mathrm{~m}\end{array}$

Sección:

Hormigón. $\quad 45 \times 45 \mathrm{~cm}^{2}$

Perfil de acero. HEB-260

Armaduras. $\quad 8 \phi 20$

Recubrimiento: $\quad 3 \mathrm{~cm}$

Resistencias de los materiales:

Hormigón. HA-25: $f_{c k}=25 \mathrm{~N} / \mathrm{mm}^{2}$

Acero de armar. B- $400: \mathrm{f}_{\mathrm{sk}}=400 \mathrm{~N} / \mathrm{mm}^{2}$

Acero estructural. S-275: $\mathrm{f}_{\mathrm{yk}}=275 \mathrm{~N} / \mathrm{mm}^{2}$

Coeficientes de minoración de resistencias:

Hormigón. $\quad \gamma_{c}=1,35$

$\alpha=1,00$

Acero de armar. $\quad \gamma_{\mathrm{s}}=1,15$

Acero estructural. $\quad \gamma_{y}=1,10$ 
Diagramas comparativos de no linealidad geométrica y material
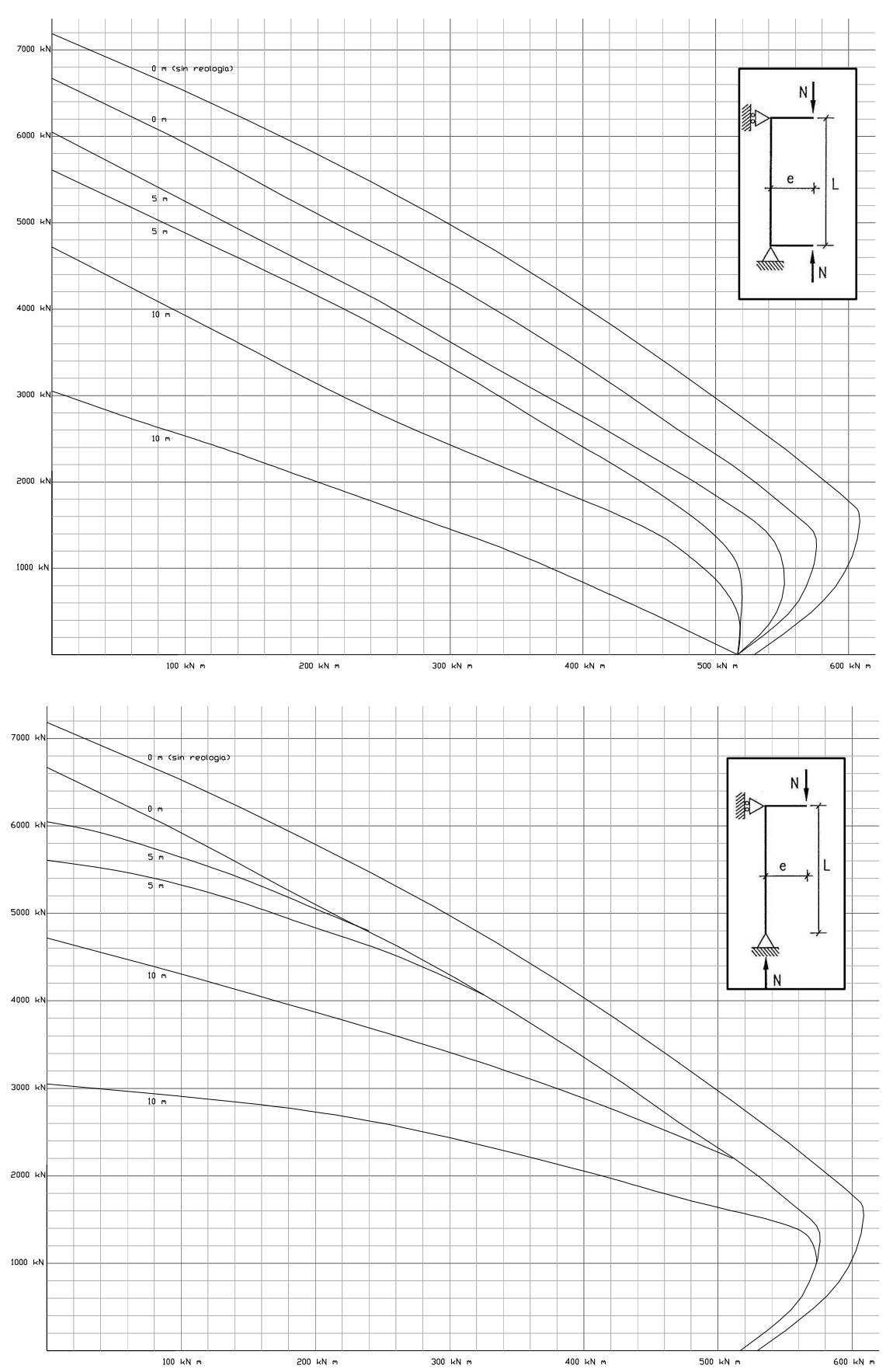


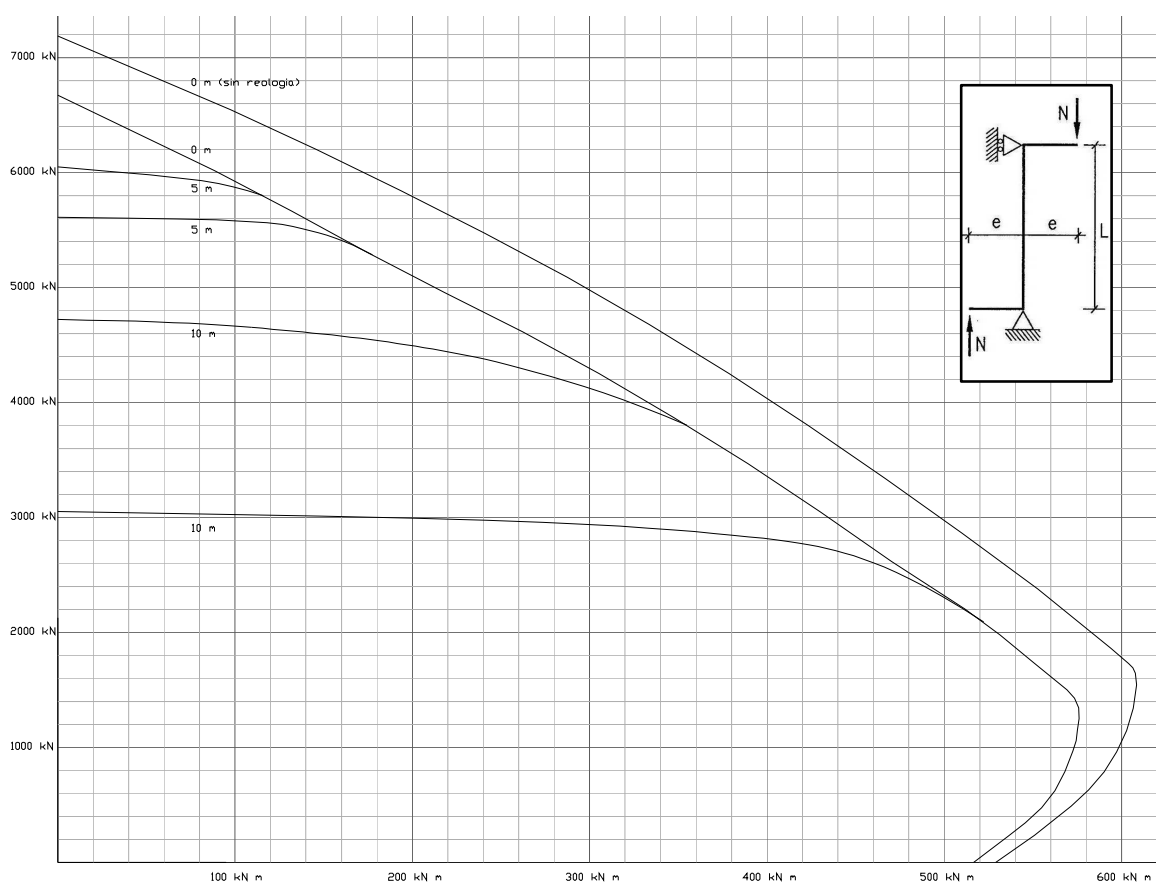

Figura 7.6-3 Comparación distintos tipos de no linealidad

\section{Análisis de resultados}

De la observación de los gráficos obtenidos, se puede concluir:

- La pérdida de capacidad resistente debida a la consideración de la no linealidad del material es mayor en el soporte de mayor longitud. En el caso del esfuerzo axil centrado, la reducción es del $7,5 \%$ para el soporte de $5,00 \mathrm{~m}$ y se incrementa hasta el $35 \%$ para el de $10,00 \mathrm{~m}$.

- La pérdida de capacidad resistente es mayor cuando predomina el esfuerzo axil y menor conforme predomina la flexión. Este aspecto se pone especialmente de manifiesto en el caso del soporte de $10,00 \mathrm{~m}$

- En el caso del soporte de 5,00 m, las dos curvas obtenidas son sensiblemente paralelas en las tres situaciones de carga. 


\subsubsection{INFLUENCIA DE LOS EFECTOS REOLOGICOS}

En el apartado 5.6 de presente trabajo se mostraba el análisis del diagrama de interacción de una sección mixta comparándolo con el obtenido al tener en cuenta los efectos reológicos en el hormigón.

Con el objeto de cuantificar la influencia de estos mismos efectos sobre la barra y compararlos, de forma relativa, con el caso de la sección, se han elaborado las curvas de interacción, para un caso de carga correspondiente al de dos excentricidades iguales respecto del eje fuerte de una sección mixta con las siguientes características dimensionales y de los materiales:

Datos empleados en la elaboración de los gráficos comparativos:

Soporte:

Longitud: $\quad 5,00 \mathrm{~m}$

Sección:

Hormigón. $\quad 45 \times 45 \mathrm{~cm}^{2}$

Perfil de acero. HEB-260

Armaduras. $\quad 8 \phi 20$

Recubrimiento: $\quad 3 \mathrm{~cm}$

Resistencias de los materiales:

Hormigón. HA-25: $f_{c k}=25 \mathrm{~N} / \mathrm{mm}^{2}$

Acero de armar. B-400: $f_{\mathrm{sk}}=400 \mathrm{~N} / \mathrm{mm}^{2}$

Acero estructural. S-275: $\mathrm{f}_{\mathrm{yk}}=275 \mathrm{~N} / \mathrm{mm}^{2}$

Coeficientes de minoración de resistencias:

Hormigón. $\quad \gamma_{c}=1,35$

$\alpha=1,00$

Acero de armar. $\quad \gamma_{\mathrm{s}}=1,15$

Acero estructural. $\quad \gamma_{y}=1,10$ 


\section{Diagramas comparativos de los efectos reológicos}

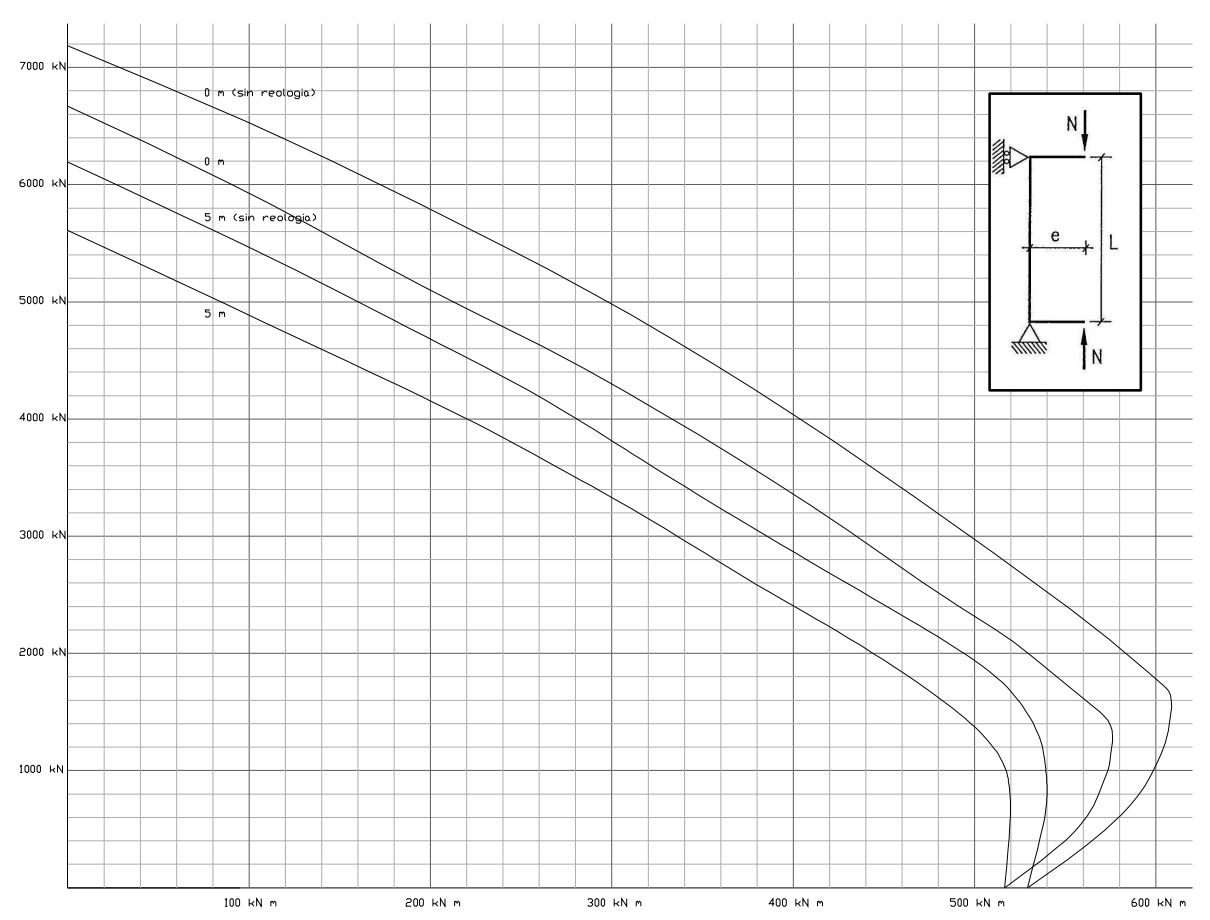

Figura 7.6-4 Comparacion efectos reológicos sobre la barra

\section{Análisis de resultados}

Tal como se aprecia en la figura, la toma en consideración de los efectos reológicos del hormigón en la obtención de la curva de interacción del soporte, reproduce de forma aproximada, la pérdida de la capacidad resistente ya observada en la sección.

Esta reducción de la capacidad resistente de la barra varía entre el 10 $\%$ en el caso de la compesión centrada y el $3 \%$ en la flexión simple, mientras que para la sección la reducción varía entre el 7\% y el 3 \%, respectivamente. 
8. EL CONJUNTO ESTRUCTURAL 


\subsection{INTRODUCCIÓN}

En el capítulo anterior se han analizado los efectos de segundo orden en soportes aislados e intraslacionales. Como se ha visto, es un problema complejo con numerosas variables implicadas que ha dado lugar a distintos métodos que abordan su cálculo de una forma más o menos simplificada en función de la aproximación que se pretende conseguir.

Si el estudio no se limita al análisis de la barra aislada sino que se considera que ésta forma parte de un entramado, como sucede en la realidad, el problema se complica dado que a las variables analizadas se incorporan otros efectos que se deben tener en cuenta como los desplazamientos transversales de los extremos del soporte o la variación de la rigidez de las barras por efecto de la plastificación de los materiales y de la fisuración del hormigón.

A pesar de la amplitud y complejidad del campo que ahora se aborda, y de que se excede del objetivo general inicialmente planteado, no se ha querido dejar pasar la ocasión de realizar una aproximación al problema del análisis global de los efectos de segundo orden en la estructura, mostrar las diferencias que se obtienen de la consideración del soporte mixto como un elemento del conjunto estructural y plantear una serie de cuestiones que pudieran servir de líneas de estudio posteriores.

En los siguientes apartados se expondrá el criterio de los Eurocódigos para la clasificación de las estructuras como traslacionales o intraslacionales y la necesidad de la consideración de los efectos de segundo orden, se expondrá el denominado "método P- $\Delta$ " adoptado por numerosos programas de cálculo para la evaluación de los efectos de segundo orden, el método matricial 
general, desarrollado por el programa utilizado en el presente trabajo y algunos de los resultados con él obtenidos.

\subsection{CRITERIO DE LOS EUROCÓDIGOS}

El epígrafe (2) del Campo de Aplicación del método de cálculo de soportes mixtos establece que éste es aplicable a pilares aislados intraslacionales que pueden ser:

- Elementos comprimidos que son parte integrante de pórticos intraslacionales pero que se consideran aislados a efectos de cálculo, o

- Elementos comprimidos aislados que atienden a la clasificación de "intraslacionales" que se da en los apartados 4.3.5.3.3 del EC2 ó 5.2.5.2 del EC3, según corresponda.

Veamos por tanto el criterio del EC2 de estructuras de hormigón y el EC3 de estructuras de acero para la consideración de una estructura traslacional o intraslacional y cuando se deben tener en cuenta los efectos de segundo orden.

El artículo 4.3.5.3.3 del EC2 ${ }^{126}$ define que se entiende por estructuras intraslacionales y el criterio para su clasificación:

(1) Las estructuras o elementos estructurales, con 0 sin elementos de arriostramiento, para las que la influencia de los desplazamientos de los nudos debidos a los momentos y esfuerzos de cálculo se puede despreciar, se clasifican como

${ }^{126}$ Eurocódigo 2: Proyecto de estructuras de hormigón. Parte 1-1: Reglas generales y reglas para edificación. AENOR 1993 
intraslacionales. En cualquier otro caso se clasifican como traslacionales.

(2) Las estructuras de edificación arriostradas, en las que el arriostramiento viene dado por pantallas o núcleos de importancia, pueden considerarse como intraslacionales. En otros casos, las estructuras pueden clasificarse mediante la aplicación de las reglas del anexo 3, apartado A.3.2.

(3) Los pórticos pueden clasificarse como intraslacionales si los desplazamientos de primer orden de los nudos no aumentan los esfuerzos calculados sin considerar estos desplazamientos en más de un 10\%. En general, es suficiente considerar únicamente los momentos flectores relevantes (véase apartado 2.5.1.4).

Las mencionadas reglas del anexo 3 del EC2 son las siguientes:

(1) Suponiendo que los elementos de arriostramiento están distribuidos de una forma razonablemente simétrica, los pórticos arriostrados pueden clasificarse en intraslacionales si la rigidez lateral de los elementos arriostradores satisface los criterios siguientes:

$$
\begin{array}{lll}
\text { para } & n \leq 3: & h_{t o t} \cdot \sqrt{\left(F_{v} / E_{c m} I_{c}\right)} \leq 0,2+0,1 \cdot n \\
\text { para } & n \geq 4: & h_{t o t} \cdot \sqrt{\left(F_{v} / E_{c m} I_{c}\right)} \leq 0,6
\end{array}
$$

donde:

n es el número total de plantas

$h_{\text {tot }}$ es la altura total de la estructura medida en metros desde la superficie superior de los cimientos o desde un estrato indeformable 
$E_{c m} I_{c}$ es la suma de las rigideces a flexión nominales de todos los elementos de arriostramiento verticales tal y como se define en el apartado 4.3.5.3.2 (1) que actúan según la dirección considerada. En los elementos de arriostramiento, la tensión de tracción en el hormigón bajo la acción de una combinación relevante de cargas en condiciones de servicio no ha de ser mayor que $f_{c t k, 0,05}$ definida en 3.1.2.3. Si la rigidez de los elementos de arriostramiento varía con la altura, se tomará una rigidez equivalente.

$F_{v} \quad$ es la suma de todas las cargas verticales (esto es, las que actúan tanto en los elementos de arriostramiento como en el subconjunto arriostrado) en condiciones de servicio (es decir $\gamma_{t}=1$ )

Debe señalarse que existen casos en los que las ecuaciones anteriores serán excesivamente conservadoras.

(2) Si las ecuaciones anteriores no se verifican, la estructura se clasificará como traslacional y se calculará de acuerdo con esto.

(3) Los pórticos sin elementos de arriostramiento pueden considerarse como estructuras intraslacionales siempre que cada elemento vertical que resista más del $70 \%$ del esfuerzo axil medio $N_{S d, m}=\gamma_{\uparrow} \cdot F_{v} / n$ ( $n$ es el número de elementos verticales que hay en una planta) tenga una esbletez $\lambda$ menor o igual que el mayor de los valores siguientes: $25015 / \sqrt{v_{u}}$ (siendo $v_{u}=N_{S d} / A_{c} \cdot f_{c d}$ )

Por otro lado, el anteriormente mencionado apartado 2.5.1.4 del EC2 aclara cuando se deben tener en cuenta los efectos de segundo orden: 
(1) Los efectos de segundo orden se tendrán en cuenta cuando puedan afectar significativamente a la estabilidad del conjunto de la estructura o cuando puedan provocar que se alcance un estado límite último en alguna sección crítica

(2) En edificios normales, los efectos de segundo orden se pueden despreciar cuando no incrementen en más de un $10 \%$ los momentos calculados prescindiendo de los desplazamientos

De forma similar, el artículo 5.2.5.2 del EC3 ${ }^{127}$ proporciona un criterio para la clasificación como traslacional o intraslacional de los pórticos. En él se indica:

(1) Se clasificará un pórtico como intraslacional cuando su respuesta a los esfuerzos horizontales contenidos en su plano es tal que sean despreciables las solicitaciones adicionales debidas a los deplazamientos horizontales de sus nudos.

(2) En caso contrario se considerará el pórtico como traslacional $y$, en su dimensionamiento se considerarán los efectos de los desplazamientos horizontales de sus nudos.

(3) Se considerará un pórtico como intraslacional para un determinado estado de cargas, si la relación $V_{s d} / V_{c r}$ para ese estado de cargas, satisface la condición:

$V_{S d} / V_{c r} \leq 0,1$

${ }^{127}$ ENV 1993-1-1. Eurocodigo 3: Proyecto de estructuras de acero. Parte 11: Reglas generales y reglas para edificación. AENOR 1996 
donde

$V_{S d}$ es el valor de cálculo de la carga vertical total

$V_{c r}$ es su valor elástico crítico de la carga vertical total considerando un estado traslacional.

(4) Los pórticos planos de edificación con vigas unidas a los pilares en cada planta, se considerarán como intraslacionales para un cierto estado de cargas, si se satisface lo siguiente: Cuando se utilice la teoría de primer orden, los deplazamientos horizontales de cada planta producidos por las cargas horizontales y verticales de cálculo, y a las imperfecciones debidas a un desplazamiento inicial, aplicadas como esfuerzos horizontales equivalentes, deberán cumplir el criterio:

$\left(\frac{\delta}{h}\right) \cdot\left(\frac{V}{H}\right) \leq 0,1$

donde

$\delta \quad$ es el desplazamiento horizontal relativo entre una planta y la superior

$h \quad$ es la altura entre plantas

$H$ es la reacción horizontal total en la planta

$v \quad$ es la reacción vertical total en la planta

(5) En el caso de pórticos traslacionales, también deberán cumplirse los requisitos relativos a la estabilidad global, recogidos en el apartado 5.2.6 


\section{$\underline{\text { Reflexión sobre el criterio de los Eurocódigos }}$}

Teniendo en cuenta que el uso de soportes mixtos en estructuras de edificación se suelen proyectar formando parte de pórticos con sistemas de arriostramiento, siempre que éstos cumplan las condiciones de rigidez exigidas por el EC2, apartados 4.3.5.3.3(2) y A.3.2(1), dichos pórticos podrían considerarse intraslacionales. Cuando no existen estos elementos de arriostramiento, también pueden considerarse intraslacionales si se verifican los apartados 5.2.5.2 (3) y (4) del EC3. En estos casos no sería necesario el análisis en segundo orden de la estructura al ser despreciables los desplazamientos horizontales de sus nudos.

No obstante, junto a estos epígrafes existen otros de mayor ambigüedad en su redacción que pueden ocasionar dudas respecto a su aplicación. Por ejemplo el apartado 4.3.5.3.3(1) del EC2 clasifica como estructuras intraslacionales aquellas "para las que la influencia de los desplazamientos de los nudos debidos a los momentos y esfuerzos de cálculo se puede despreciar" y el apartado 5.2.5.2 (3) del EC3 clasifica un pórtico como intraslacional "cuando su respuesta a los esfuerzos horizontales contenidos en su plano es tal que sean despreciables las solicitaciones adicionales debidas a los deplazamientos horizontales de sus nudos".

Resulta complejo saber si las "solicitaciones adicionales" producidas por los desplazamientos horizontales de los nudos son despreciables, si no son evaluadas.

De forma similar, en este caso al referirse a las consideraciones de los efectos de segundo orden, resulta complicado saber si se debe aplicar el apartado 2.5.1.4 del EC2 cuando indica (1) "se tendrán en cuenta cuando puedan afectar significativamente a la estabilidad del conjunto de la estructura" y (2) "se pueden despreciar cuando no incrementen en más de un $10 \%$ los momentos calculados 
prescindiendo de los desplazamientos", si previamente no se ha realizado un análisis de la estructura en segundo orden

De ello, se concluye en la oportunidad de la consideración de los efectos de segundo orden sobre el conjunto estructural:

- Porque el trabajo que nos ocupa, además de la obtención de resultados que faciliten la aplicación práctica al dimensionamiento, pretende realizar un estudio lo más exhaustivo posible de soportes mixtos con la evaluación de los diversos parámetros que les afecta.

- Porque, del análisis comparativo, de al menos algún caso, podrá cuantificarse su efecto y de esta forma verificar el alcance de la aplicación de los mencionados artículos.

\subsection{METODO P- $\Delta$}

La necesidad de tener en cuenta los efectos de segundo orden producidos por los desplazamientos horizontales de los nudos en las estructuras traslacionales, unido a la complejidad de abordar el problema por métodos "exactos", ha conducido al desarrollo de métodos iterativos de mayor sencillez de aplicación que a la vez proporcionan resultados suficientemente fiables. Uno de estos métodos es el conocido como "P- $\Delta$ " que ha sido adoptado como método de cálculo de muchos programas de estructuras de barras que realizan su análisis en teoría de segundo orden ${ }^{128}$.

Cuando sobre una estructura porticada, como la de la Figura 8.3-1, actúan simultáneamente cargas verticales y horizontales, las primeras se acoplan con los desplazamientos horizontales producidos por las segundas, de forma que estos desplazamientos

128 "Pandeo de estructuras de hormigón armado". Jordi Maristany. (1996) 
se incrementan hasta que se alzanza una posición de equilibrio. Esta interacción entre cargas verticales con los desplazamientos horizontales de la estructura, efectos de segundo orden, es la conocida como efecto "P- $\Delta$ ".

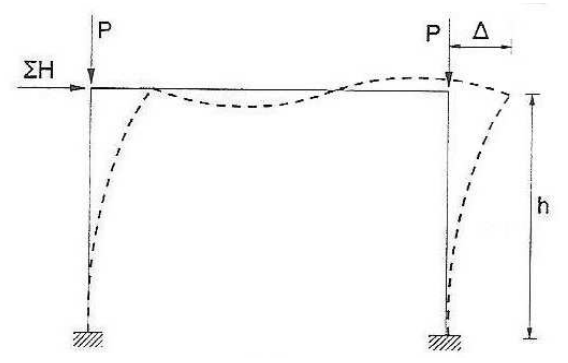

Figura 8.3-1 Efecto "P- $\triangle$ "

Resulta evidente que estos efectos de segundo orden globales de la estructura sólo se producen en estructuras traslacionales (Figura 8.3-2 B) y a ellos hay que añadir los efectos de segundo orden de la propia barra comprimida que ya han sido analizados en el Capítulo 7 del presente trabajo. En el caso de estructuras intraslacionales (Figura 8.3-2 A), al no producirse desplazamientos horizontales de los nudos, únicamente es necesaria la evaluación de los efectos de segundo orden en la "viga-columna".
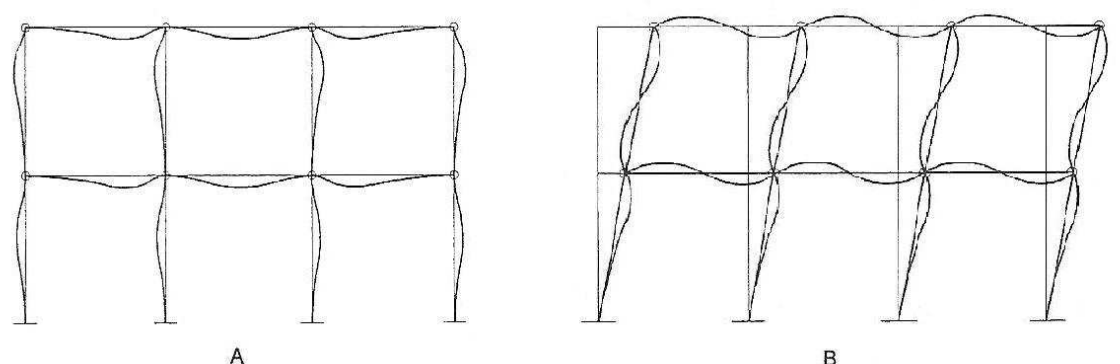

Figura 8.3-2 Estructuras intraslacionales y traslacionales 
A continuación se exponen dos procedimientos simplificados basados en el método P- $\Delta$ que permiten aproximarse suficientemente a los efectos de segundo orden sobre la estructura:

Método de la carga lateral equivalente

El planteamiento conceptual consiste en sustituir el desplazamiento horizontal de los nudos de la estructura, por unas fuerzas horizontales ficticias.

El proceso de resolución requiere un primer análisis de la estructura en la que se evalúan los esfuerzos y deformaciones de primer orden. Si se aisla una barra de una estructura plana, se observa que se encontrará solicitada por un axil, cortante y momento flector en cada uno de sus extremos.

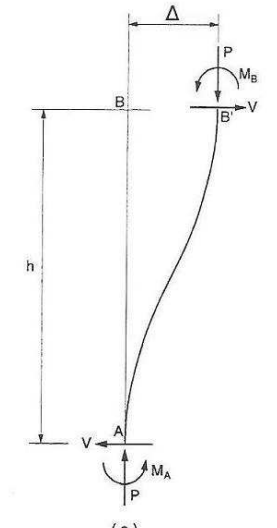

(a)

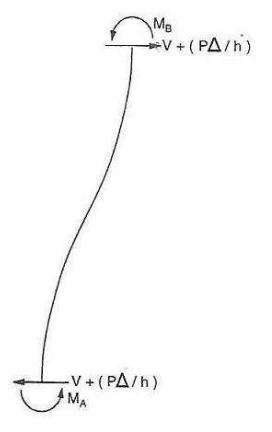

(b)

Figura 8.3-3 Desplazamientos y esfuerzos en la barra

Suponiendo que se produce un desplazamiento horizontal relativo entre los dos extremos de la barra y que no existen cargas axiales ni transversales a la misma, el equilibrio se produciría de la forma: 


$$
\begin{array}{ll}
\sum F_{V}=0 & P_{A}=P_{B} \\
\sum F_{H}=0 & V_{A}=V_{B} \\
\sum M_{Z}=0 & M_{A}+M_{B}=V_{A} \cdot h+P \cdot \Delta
\end{array}
$$

De la ecuación de equilibrio de momentos se deduce el valor de la fuerza horizontal ficticia equivalente:

$$
V^{\prime}=\frac{P \cdot \Delta}{h}
$$

Que corresponde al cortante adicional que equilibra el momento producido por la excentricidad de aplicación de la carga gravitatoria.

Si este pilar corresponde a un pórtico de una única planta, el cortante ficticio obtenido sería la fuerza horizontal adicional que habría que añadir a las acciones exteriores para, en un segundo análisis de la estructura, obtener unas solicitaciones y deformaciones que simularan los efectos de segundo orden.

Cuando el pilar analizado forma parte de un pórtico de varias plantas, Figura 8.3-4, la expresión equivalente que nos permite obtener el cortante ficticio de un pilar de la planta $i$, sería:

$$
V^{\prime}=\frac{\Sigma P_{i}}{h_{i}}\left(\Delta_{i+1}-\Delta_{i}\right)
$$

Donde $\Sigma P_{i}$ es la suma de axiles que actúan sobre todos los soportes de la planta $i, h_{i}$ es la altura de la planta, y $\left(\Delta_{i+1}-\Delta_{i}\right)$ el desplazamiento horizontal relativo de los forjados superior e inferior de la planta $i$. 


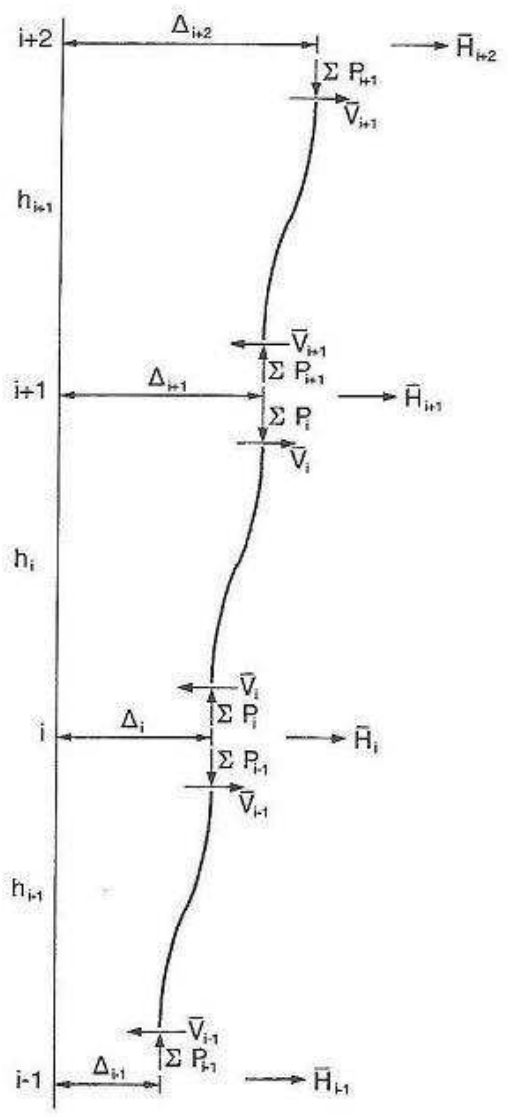

Figura 8.3-4 Fuerzas horizontales equivalentes en un pórtico de varias plantas

La fuerza horizontal ficticia a aplicar en el forjado $i$ de cada planta se obtiene de la diferencia entre los cortantes de los soportes concurrentes en cada forjado:

$$
H_{i}=V_{i-1}^{\prime}-V_{i}^{\prime}
$$

Estas fuerzas horizontales obtenidas se añaden a las cargas iniciales y se realiza un segundo análisis de la estructura en primer orden de donde se obtienen unos nuevos desplazamientos horizontales con los que se corrige el valor de las fuerzas horizontales ficticias para realizar un nuevo análisis. 
Se adoptan como valores finales de las deformaciones y solicitaciones de la estructura, los obtenidos en un ciclo donde el incremento de las deformaciones respecto del ciclo anterior sea despreciable.

\section{Método de la carga gravitatoria iterativa}

El planteamiento de este método consiste en aplicar las cargas gravitatorias sobre la estructura previamente deformada por las fuerzas horizontales.

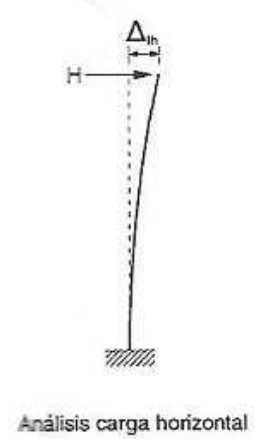

Anălisis carga horizontal

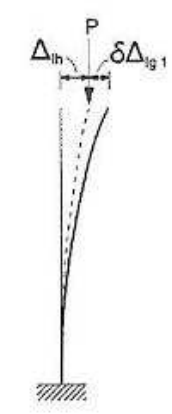

Análisis carga gravedad

ciclo 1

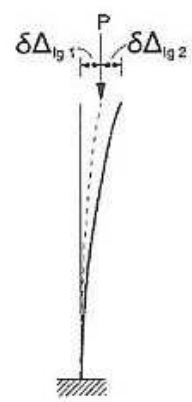

Análisis carga gravedad

$$
\text { cicio } 2
$$

Figura 8.3-5 Proceso de aplicación de I método de la carga gravitatoria iterativa

El proceso de resolución requiere un primer análisis de la estructura en la que se evalúan los esfuerzos y deformaciones de primer orden producidos únicamente por las fuerzas horizontales. A continuación se realiza un segundo análisis considerando la geometría deformada de la estructura sobre la que se aplican las cargas verticales que producirán unas nuevas deformaciones, iniciándose un proceso iterativo en la aplicación de las cargas verticales sobre la geometría deformada del ciclo anterior. El proceso concluye con la obtención de la posición de equilibrio cuando el incremento de las deformaciones de un ciclo respecto a las del anterior sean despreciables. 
Alcanzada la posición de equilibrio, el desplazamiento horizontal final se puede obtener como la suma del desplazamiento producido por las fuerzas horizontales más los producidos por la aplicación de las cargas gravitatorias en los $n$ ciclos:

$$
\Delta=\Delta_{1 h}+\sum_{i=1}^{n}\left(\delta \cdot \Delta_{1 g}\right)_{i}
$$

\subsection{MÉTODOS DE ANÁLISIS MATRICIAL DE LOS EFECTOS DE SEGUNDO ORDEN.}

\subsubsection{INTRODUCCIÓN.}

En el problema elástico, la relación de equilibrio se define de forma lineal entre fuerzas y desplazamientos, en la que los valores de la matriz de rigidez de la estructura son valores constantes. Su resolución consiste en obtener, a partir de la relación $\left[K_{e}\right]\{\Delta\}=\{P\}$ el vector de movimientos de los nudos $\{\Delta\}$ siendo $\left[K_{e}\right]$ la matriz de rigidez elástica y lineal y $\{\mathrm{P}\}$ el vector de cargas ${ }^{129} \quad 130131$.

La diferencia fundamental cuando se trata de abordar los efectos de segundo orden consiste en que la matriz de rigidez de la estructura no es constante sino que presenta valores que dependen de los movimientos y de las cargas aplicadas. La resolución de la ecuación de equilibrio presenta una mayor complejidad, para lo cual se han desarrollado diversos métodos.

\footnotetext{
129 "Matrix structural analysis". W. McGuire, R.H. Gallagher y R.D. Ziemian (2000)

130 "Análisis matricial de estructuras de barras" Lluis Moya Ferrer

131 "Estudio no lineal del comportamiento resistente de soportes de hormigón armado solicitados a flexo-compresión esviada" J.R. Atienza, R. Irles. (1985)
} 
Destaca, en la mayor parte de ellos, el tratamiento incremental de la aplicación de las cargas y la búsqueda de la solución mediante un proceso iterativo cuyo resultado se espera sea convergente en la solución del problema. De esta forma, en cada uno de los incrementos e iteraciones se puede plantear una variante de la ecuación de equilibrio: $\left[K_{t}\right]\{d \Delta\}=\{d P\}$ cuya resolución se plantea en el campo lineal. En ella $\{d \Delta\}$ y $\{d P\}$ representan, respectivamente, los vectores de los diferenciales de desplazamiento y de cargas y $\left[K_{t}\right]$ la matriz de rigidez tangente, formada por una componente elástica y lineal y una o más componentes no lineales. La resupuesta total de la estructura se obtiene mediante la suma de los incrementos.

En función de los efectos que se tengan en cuenta en la deducción de la matriz de rigidez tangente se pueden distinguir distintos tipos de análisis $^{132}$ :

Análisis elástico en segundo orden: Las deformaciones y desplazamientos de la estructura se tienen en cuenta en la construcción de la matriz de rigidez tangente, quedando la ecuación de equilibrio: $\left\lfloor K_{e}+K_{g}\right\rfloor\{d \Delta\}=\{d P\}$ siendo $\left\lfloor K_{g}\right\rfloor$ las componentes de la matriz de rigidez que tienen en cuenta la no linealidad geométrica.

Análisis inelástico en primer orden: La matriz de rigidez tangente se plantea con la geometría inicial, es decir, con la estructura descargada, pero se incluyen los efectos de la no linealidad mecánica de los materiales a lo largo del proceso de carga. La ecuación de equilibrio queda: $\left[K_{e}+K_{m}\right]\{d \Delta\}=\{d P\}$ siendo $\left[K_{m}\right]$ las componentes de la matriz de rigidez que tienen en cuenta la no linealidad del material.

132 "Matrix structural analysis". W. McGuire, R.H. Gallagher y R.D. Ziemian (2000) 
Análisis inelástico en segundo orden: La matriz de rigidez tangente tiene en cuenta tanto los efectos de las variaciones geométricas como la no linealidad mecánica de los materiales, expresándose la ecuación de equilibrio de la forma: $\left\lfloor K_{e}+K_{g}+K_{m}\right\rfloor\{d \Delta\}=\{d P\}$

En cuanto al tratamiento incremental de la aplicación de las cargas, cabe decir que es el proceso que nos permite trazar la historia de los movimientos de una estructura desde su posición inicial no deformada hasta su configuración final, planteándose en cada escalón de carga un problema particular de no linealidad. La carga total se aplicará mediante una serie de incrementos que matemáticamente se puede expresar de la siguiente forma:

$$
\{P\}=\sum_{i=1}^{n}\left\{d P_{i}\right\}
$$

donde $\left\{d P_{i}\right\}$ es el incremento de carga y $n$ el número total de incrementos. Los movimientos totales de la estructura se pueden obtener como suma de los movimientos diferenciales $\left\{d \Delta_{i}\right\}$ producidos por cada incremento de carga. La desviación entre la solución de estas funciones lineales respecto de la solución real de equilibrio depende de la relación de no linealidad entre cargas $\left\{d P_{i}\right\}$ y desplazamientos $\left\{d \Delta_{i}\right\}$.

La forma y el número de análisis realizados para obtener los desplazamientos $\left\{d \Delta_{i}\right\}$ es lo que distingue los distintos métodos de resolución del problema no lineal. La Figura 8.4-1 ${ }^{133}$ representa el proceso incremental de aplicación de las cargas y la variación entre los desplazamientos obtenidos y los reales.

\footnotetext{
${ }^{133}$ Esta figura y las siguientes han sido extraídas de la publicación "Matrix structural analysis" de William McGuire
} 


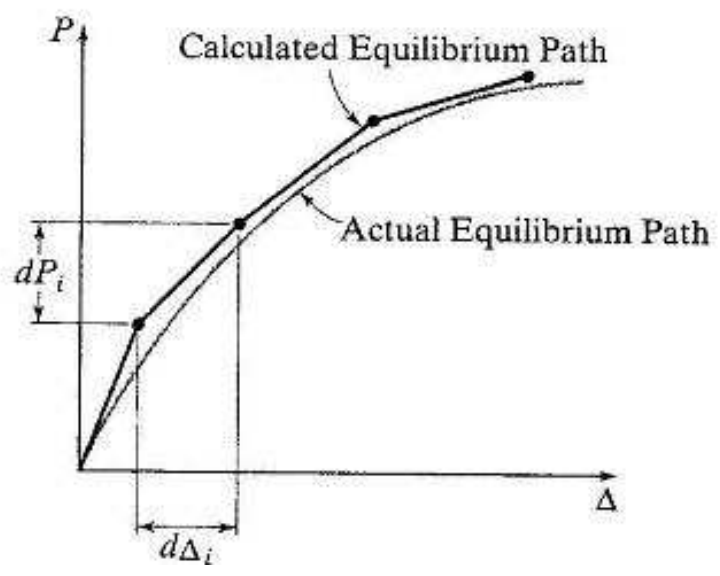

Figura 8.4-1 Esquema del proceso incremental de cargas.

Con carácter general los métodos más usuales se pueden clasificar en dos grandes grupos: métodos de paso único, entre los que se encuentran el método de Euler y los métodos Runge-Kutta, y los métodos iterativos, donde se sitúa, entre otros, el método NewtonRaphson.

En la descripción de estos métodos que a continuación se expone, se entenderá que se realiza un análisis incremental de la forma en que se ha descrito anteriormente.

\subsubsection{MÉTODOS INCREMENTALES DE PASO ÚNICO}

Los desplazamientos $\left\{d \Delta_{i}\right\}$ para un determinado incremento de carga se obtienen, en un paso único, mediante la resolución del sistema de ecuciones lineales $\left|\bar{K}_{i}\right|\left\{d \Delta_{i}\right\}=\left\{d P_{i}\right\}$, siendo $\left[\bar{K}_{i}\right]$ la matriz de rigidez representativa del intervalo de cargas, obtenida a partir de los resultados obtenidos en el incremento de carga anterior.

Dado que la relación entre cargas y desplazamientos no es lineal, la exactitud de la solución mejora cuantos más escalones de carga se 
utilicen. Dependiendo de la forma de obtención de la matriz de rigidez y de los puntos de referencia utilizados se derivan distintos tipos de estrategias, como las que se enuncian a continuación:

\section{Método de Euler}

Representa la estrategia más elemental del método de paso único. Como matriz representativa del incremento de carga se adopta la matriz de rigidez tangente obtenida con la geometría deformada del inicio del incremento (deducida a partir de los resultados calculados en el incremento de carga anterior). Los desplazamientos en el intervalo se obtienen de la resolución de un sistema de ecuaciones lineal. La Figura 8.4-2 representa el proceso de aplicación del Método de Euler para un incremento de carga.

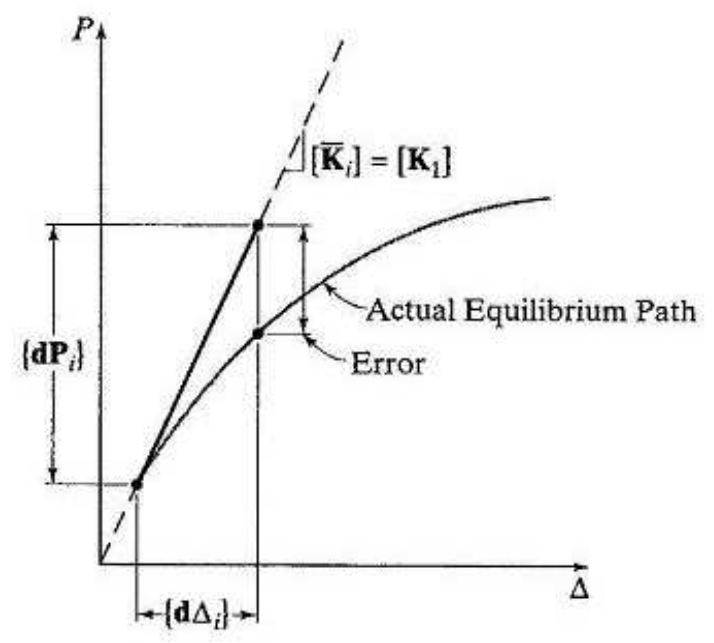

Figura 8.4-2 Esquema del Método de Euler.

\section{Método Runge-Kutta de segundo orden}

La diferencia consiste en la matriz de referencia del intervalo que, en este caso se obtiene como un porcentaje de la rigidez tangente al inicio del intervalo $\left[K_{1}\right]$ y la rigidez calculada a partir de la geometría y 
las fuerzas correspondientes en un punto intermedio del incremento $\left[K_{2}\right]$, mediante la aplicación de la expresión: $\left[K_{i}\right]=\alpha_{1}\left[K_{1}\right]+\alpha_{2}\left[K_{2}\right]$. La geometría y las fuerzas de este punto intermedio, inicialmente desconocidas, necesitan ser calculadas mediante un segundo análisis global de la estructura, realizado utilizando la matriz de rigidez tangente en el inicio del incremento y una parte de la carga del intervalo mediante las dos siguientes expresiones:

$$
\begin{aligned}
& \left\lfloor\bar{K}_{i}\right\rfloor\left\{d \Delta_{\mu}\right\}=\mu\left\{d P_{i}\right\} \\
& \left\{\Delta_{2}\right\}=\left\{\Delta_{i-1}\right\}+\left\{d \Delta_{\mu}\right\}
\end{aligned}
$$

Determinada la matriz de referencia del intervalo, las deformaciones al final del incremento de cargas, se calculan desde el punto de inicio aplicando el total de las cargas del mismo: $\left[\bar{K}_{i} \mid\left\{d \Delta_{i}\right\}=\left\{d P_{i}\right\}\right.$.

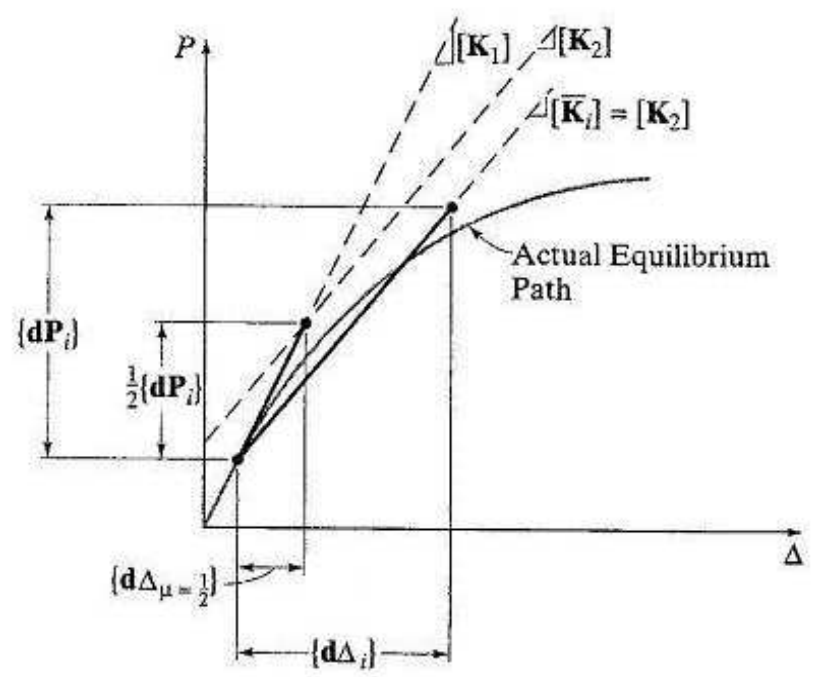

Figura 8.4-3 Esquema del Método del medio punto de Runge-Kutta.

Una de las particularizaciones más conocidas consiste en utilizar como punto intermedio de referencia, el punto medio del intervalo y unos coeficientes $\alpha_{1}=0$ y $\alpha_{2}=1$, lo que conduce a utilizar como matriz de rigidez representativa del intervalo la rigidez tangente del 
punto medio. Esta aplicación se conoce como método del punto medio de Runge-Kutta y el esquema de su proceso se esquematiza en la Figura 8.4-3.

\subsubsection{MÉTODOS INCREMENTALES ITERATIVOS}

La diferencia de los métodos iterativos respecto de los de paso único consiste en que, en este caso, en cada incremento de carga se entra en un proceso iterativo al que se le exige el cumplimiento de las condiciones de equilibrio, con una cierta tolerancia.

Los desplazamientos de un determinado incremento de carga se obtienen mediante la resolución del siguiente sistema de ecuaciones lineales $\left[K_{i}^{j-1}\right]\left\{d \Delta_{i}^{j}\right\}=\left\{d P_{i}^{j}\right\}+\left\{R_{i}^{j-1}\right\}$ donde $\left[K_{i}^{j-1}\right]$ es la matriz de rigidez utilizada en la iteración que se determina teniendo en cuenta la geometría deformada y las solicitaciones de la iteración previa, $\left\{d \Delta_{i}^{j}\right\}$ el vector de desplazamientos a obtener de la resolución de la iteración, $\left\{d P_{i}^{j}\right\}$ el vector de cargas aplicadas en la iteración y $\left\{R_{i}^{j-1}\right\}$ el vector de cargas de desequilibrio entre las fuerzas externas e internas.

El proceso iterativo finaliza con el cumplimiento del criterio de convergencia (condiciones de equilibrio) y la obtención de los desplazamientos producidos por la aplicación del incremento de la carga. Los desplazamientos totales de la estructura al final de un escalón de carga, se obtienen a partir de la ecuación:

$$
\left\{\Delta_{i}\right\}=\left\{\Delta_{i-1}\right\}+\sum_{j=1}^{m_{i}}\left\{d \Delta_{i}^{j}\right\}
$$

Entre los métodos iterativos más conocidos se pueden citar el método de iteración directa y el método de Newton-Raphson. 


\section{Método de iteración directa}

El proceso en cada escalón de carga se inicia adoptando la rigidez del punto final del escalón anterior con la que, aplicando el total de la carga del incremento, se determina una primera aproximación del movimiento de los nudos mediante la resolución de la ecuación $\left[K_{i}^{o}\right]\left\{d \Delta_{i}^{i}\right\}=\left\{d P_{i}\right\}$. A partir de estos movimientos se calcula una nueva matriz de rigidez secante, con la que, aplicando de nuevo el total de la carga del incremento y resolviendo una ecuación similar a la anterior $\left[K_{i}^{1}\right]\left\{d \Delta_{i}^{2}\right\}=\left\{d P_{i}\right\}$ se puede obtener una mejor aproximación de los movimientos de los nudos. Cuando el proceso es convergente, las iteraciones se dan por finalizadas cuando la diferencia entre el vector de movimientos de dos iteraciones consecutivas se considera suficientemente pequeña, con un margen de error previamente determinado.

La Figura 8.4-4 ilustra el proceso iterativo descrito.

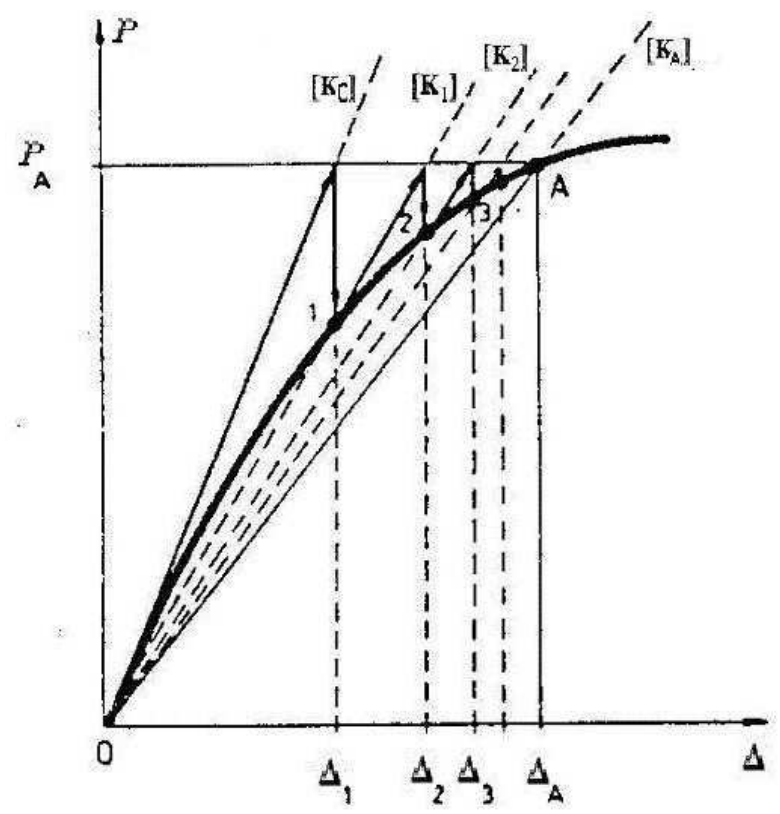

Figura 8.4-4 Esquema de iteración directa. 
Este método, de gran aplicación práctica en problemas de baja no linealidad, pierde su eficacia en problemas de acusada no linealidad debido a que su escasa convergencia exige gran cantidad de iteraciones para obtener una aceptable aproximación.

\section{Método de Newton-Raphson}

Para un determinado escalón de carga, el proceso, cuyo esquema se representa en la Figura 8.4-5, comienza determinando la matriz de rigidez tangente. La primera iteración del siguiente escalón utiliza esta matriz y, mediante la aplicación del total de la carga del siguiente escalón, permite obtener un primer valor del incremento del movimiento de los nudos mediante la resolución de la ecuación $\left[K_{i}^{1}\right]\left\{d \Delta_{i}^{1}\right\}=\left\{d P_{i}\right\}$ y determinar la parte del vector de cargas residual que no se encuentra en equilibrio con el movimiento obtenido. A continuación se recalcula la matriz de rigidez tangente asociada a los movimientos $\left\{d \Delta_{i}^{\dagger}\right\}$, y cargando la estructura con el vector de cargas residual, podemos obtener un incremento de movimiento adicional mediante la resolución de la ecuación $\left[K_{i}^{2}\right]\left\{d \Delta_{i}^{2}\right\}=\left\{d R_{i}^{1}\right\}$, de la que se obtendrá un nuevo vertor de cargas residual. Las iteraciones se repiten hasta que, en caso de que el proceso sea convergente, el vector de cargas residual sea menor que un valor predeterminado. El vector de movimientos de la estructura al final del escalón de cargas se determina mediante el sumatorio de movimientos obtenidos en cada una de las iteraciones:

$$
\left\{d \Delta_{i}\right\}=\sum_{j=1}^{m_{i}}\left\{d \Delta_{i}^{j}\right\}
$$




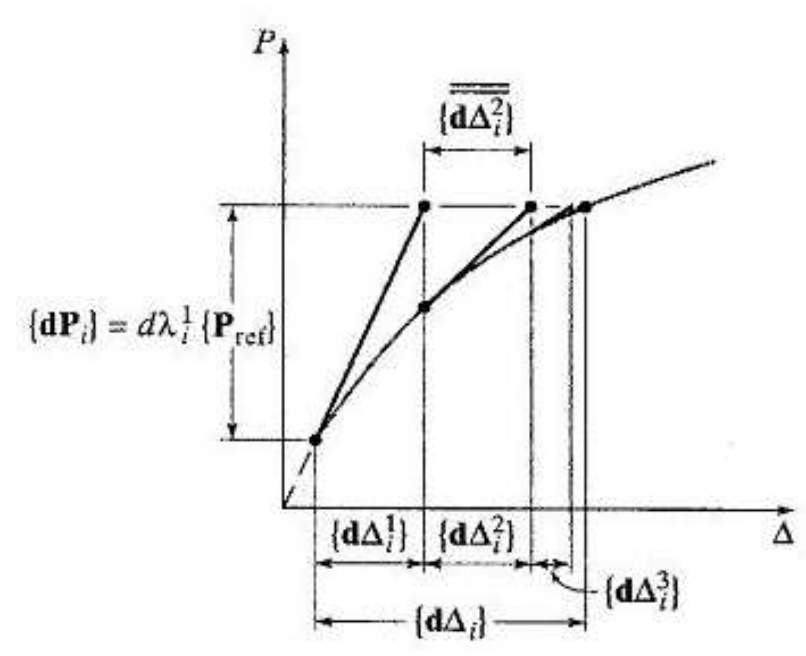

Figura 8.4-5 Esquema del Método de Newton-Raphson.

Como ventaja de este método se puede citar que presenta una convergencia mucho más rápida que el método de iteración directa anteriormente expuesto. Además la convergencia se puede garantizar siempre que el punto de inicio de la iteración se sitúe suficientemente cerca del punto final del incremento, correspondiente a la solución buscada. Con el propósito de acelerar la obtención del estado final de deformaciones, y dado que en general las estructuras presentan una mayor linealidad en las primeras etapas de carga, es frecuente plantear escalones de carga con mayores incrementos de la misma en las etapas iniciales e ir progresivamente reduciendo los incrementos de carga conforme se acera al agotamiento de la estructura, donde las no linealidades son frecuentemente mayores.

El inconveniente principal del método expuesto radica en el desmesurado problema operacional que se requiere, ya que para la resolución de cada iteración es necesaria la determinación de la matriz de rigidez tangente y el cálculo de su inversa. Para facilitar el proceso de cálculo, es posible modificar este método de forma que en las distintas iteraciones de un incremento de carga se sustituye la 
matriz de rigidez tangente de cada punto por la matriz de rigidez tangente en el origen del intervalo.

Aunque este método de la matriz de rigidez inicial o de NewtonRaphson modificado requiere de un mayor número de iteraciones para alcanzar el equilibrio, el tiempo total de cálculo queda reducido al operar con una única matriz que una vez invertida, operación más costosa del cálculo matricial, se aplica en el total de las iteraciones del escalón de carga.

\subsection{MÉTODO ADOPTADO EN ESTE TRABAJO}

El método adoptado para la obtención de las solicitaciones y deformaciones finales de la estuctura reproduce el proceso iterativo expuesto para el caso de la barra, tal como a continuación se indica:

1. Definición del modelo de la estructura.

Cuando únicamente se desee realizar un análisis no lineal geométrico, los elementos (pilares y vigas) constarán de una sóla barra.

Si además se desea realizar un análisis no lineal del material, con $E \cdot I$ variable $^{134}$ o bien se quieren tener en cuenta las imperfecciones iniciales, cada elemento deberá ser discretizado en una serie de tramos a los que se les aplicarán dichas imperfecciones.

2. Aplicación de la carga de forma incremental.

${ }^{134}$ La posibilidad de contemplar la no linealidad mecánica únicamente se encuentra desarrollada en la actualidad para el caso de los soportes mixtos, elemento estructural objeto de la presente Tesis Doctoral, y en estados planos de solicitación. 
La matriz de rigidez para el primer escalón de carga se obtiene considerando la geometría inicial (recta o con imperfecciones iniciales), los módulos de elasticidad $E$ de los distintos materiales y la inercia / global de la sección.

3. Ajuste de la deformada en cada escalón de carga mediante la imposición de las condiciones de equilibrio y compatibilidad y la aplicación de un proceso iterativo (Newton-Raphson).

Se acepta como solución del problema aquella que cumple las condiciones de convergencia planteadas: incremento de deformaciones despreciable entre dos iteraciones consecutivas

4. Aplicación del siguiente escalón de carga.

La matriz de rigidez para los sucesivos escalones de carga se obtiene considerando la geometría deformada obtenida en el escalón anterior.

Cuando además se trata de soportes mixtos, la matriz de rigidez tiene en cuenta la no linealidad mecánica, mediante la variación del término $(E \cdot)$ tras la imposición de las condiciones de equilibrio en las distintas secciones entre las solicitaciones exteriores y la respuesta interna.

5. El proceso puede finalizar de varias formas:

- Colapso por inestabilidad: no se consigue el equilibrio de la estructura. El programa muestra el paso de carga para el que se produce el colapso, del cual se obtiene el porcentaje de carga aplicado en ese instante, y conserva el estado de deformaciones y solicitaciones del paso anterior, cuyos valores se pueden consultar.

- Colapso por agotamiento resistente de la sección más desfavorable de alguno de los soportes mixtos. Al igual que el el caso anterior se guardan los datos del paso anterior al del colapso. 
- Equilibrio en el último escalón de carga y obtención de deformaciones y solicitaciones finales de la estructura.

Cabe indicar que si bien en el cálculo lineal o en el no lineal geométrico ${ }^{135}$, la comprobación de los elementos estructurales es posterior al propio análisis de las solicitaciones, cuando se realiza un cálculo no lineal geométrico y del material, la comprobación se realiza de una forma simultánea al proceso de cálculo al buscar el equilibrio de la sección en cada escalón de carga para poder determinar la rigidez de la barra. En este caso, el agotamiento de alguna barra produce la interrupción del proceso, obteniéndose el estado de deformaciones y solicitaciones del intervalo de carga anterior y la barra objeto del colapso pero no se consigue información del estado resistente del resto de barras frente al total de la carga.

El proceso de cálculo no lineal geométrico y del material de la estructura, cuando se utiliza el método de Newton-Raphson modificado, sigue el organigrama que se representa en la Figura 8.5-1.

${ }^{135}$ El programa de cálculo AV_CID no realiza la comprobación de la sección en cada intervalo de carga, salvo que el análisis incluya la no linealidad del material. 


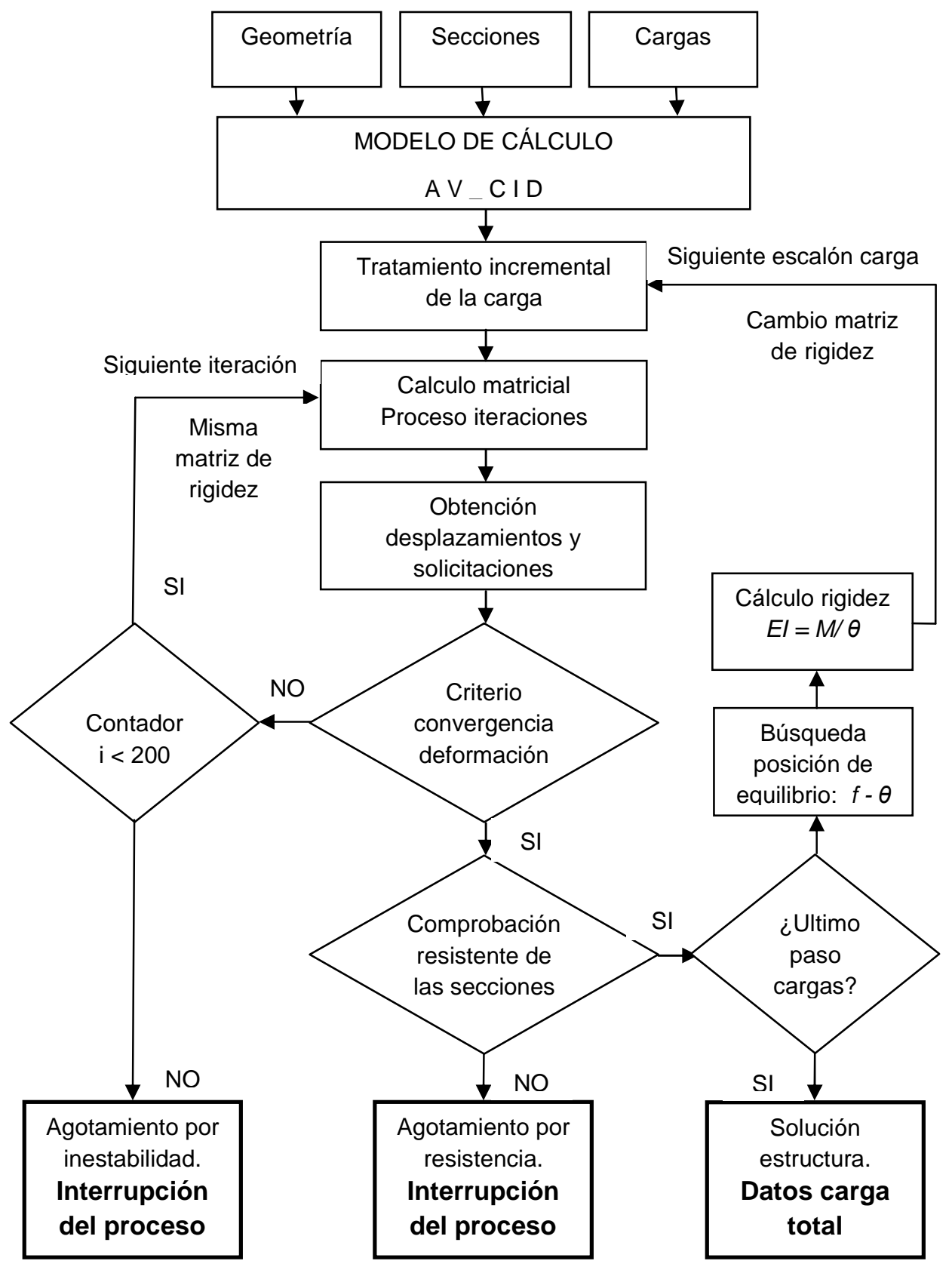

Figura 8.5-1 Organigrama de cálculo de la estructura 


\subsection{RESULTADOS}

\subsubsection{ANÁLISIS COMPARATIVO DE LOS EFECTOS DE SEGUNDO ORDEN}

Con el objeto de mostrar el efecto y la trascendencia de la consideración de los efectos de segundo orden sobre las estructuras traslacionales se han desarrollado y comparado dos casos opuestos: un pórtico de una altura y otro de un edificio "alto".

En ambos casos se han aplicado cargas gravitatorias y fuerzas horizontales y se han comparado los resultados obtenidos de someter sus modelos a distintos tipos de cálculo.

\section{Pórtico de una planta}

Se ha modelizado un pórtico que podría formar parte de la estructura de una nave industrial de un vano de $18,00 \mathrm{~m}$ de luz entre pilares y de una única planta de $5,00 \mathrm{~m}$ de altura, como se muestra en la Figura 8.6-1.

Ambos soportes han sido discretizados mediante 10 tramos de 0,50 $m$ de longitud a los que se ha asignado una sección mixta formada por un perfil de acero HEB-160, dispuesto con su eje fuerte perpendicular al plano de la estructura, embebido en hormigón $(25 \times 25 \mathrm{~cm})$. A la viga se le ha asignado una sección de hormigón de $25 \times 180 \mathrm{~cm}$.

Las dimensiones de la sección de las distintas barras se han escogido tras la modelización y cálculo de distintos casos y su posterior discusión que brevemente se reproduce. Si a la viga se le asigna un canto en el entorno del 1/20 de la luz del pórtico, los momentos que transmite a los soportes obligan a unas dimensiones 
del mismo que proporcionan tal rigidez al pórtico que hacen despreciables los desplazamientos horizontales. En la opción elegida, el comportamiento del pórtico se asemeja al de una viga apoyada sobre los soportes. Su dimensión puede ser relativamente pequeña, ya que se encuentran sometidos, principalmente a esfuerzo axil, acompañado de un pequeño momento flector. Su escasa rigidez conduce a desplazamientos horizontales no despreciables, tal como se verá en el análisis posterior.

Las características dimensionales de la sección mixta de los soportes, así como las resistencias características de los materiales y sus correspondientes coeficientes de minoración que se han utilizado son:

Sección soportes:

Hormigón.

$25 \times 25 \mathrm{~cm}$

Acero de armar. $\quad 8 \phi 20(\mathrm{r}=3 \mathrm{~cm})$

Acero estructural. HEB-160

Resistencias de los materiales:

Hormigón.

Acero de armar.

Acero estructural.
HA-30: $f_{c k}=30 \mathrm{~N} / \mathrm{mm}^{2}$

B-500: $\mathrm{f}_{\mathrm{sk}}=500 \mathrm{~N} / \mathrm{mm}^{2}$

S-355: $\mathrm{f}_{\mathrm{yk}}=355 \mathrm{~N} / \mathrm{mm}^{2}$

Coeficientes de minoración de resistencias:

Hormigón.

$\gamma_{c}=1,35$

$\alpha=1,00$

Acero de armar. $\quad \gamma_{\mathrm{s}}=1,15$

Acero estructural. $\quad \gamma_{y}=1,10$

No se han tenido en cuenta los efectos reológicos del hormigón.

Las cargas gravitatorias aplicadas en la hipótesis 1, simulan unos forjados realizados con paneles alveolares de hormigón pretensado de $8 \mathrm{~m}$ de luz entre vigas más el revestimiento de cubierta. Las cargas horizontales, hipótesis 2 , aplicadas como una fuerza puntual 
sobre el extremo superior del soporte, corresponden al empuje del viento sobre la fachada.

A efecto de las comprobaciones en Estados Límites Últimos, se ha utilizado una combinación de cargas en la que actúan simultáneamente las cargas gravitatorias (de carácter permanente) afectadas de un coeficiente de mayoración de acciones de valor 1,35 más las cargas horizontales (de carácter variable) afectadas de un coeficiente de mayoración de 1,50. Se ha aplicado la simplificación de combinaciones de acciones para casos de edificación ${ }^{136}$.

Con el objeto de comparar resultados, se han analizado dos modelos geométricos: en el primero no se han considerado imperfecciones en ninguna de las barras mientras que en el segundo modelo se han considerado en los dos soportes las imperfecciones iniciales, según las prescripciones del EC4 expuestas en el apartado 3.1.1. Las coordenadas $^{137}$ de los nudos de los tramos en que se han discretizado ambos soportes, expresadas en centímetros, se indican en la tabla siguiente:

\begin{tabular}{|c|c|c|c|c|c|c|c|c|c|c|c|}
\hline Nudo & 0 & 1 & 2 & 3 & 4 & 5 & 6 & 7 & 8 & 9 & 10 \\
\hline$y$ & 0,00 & 0,76 & 1,44 & 1,98 & 2,33 & 2,45 & 2,33 & 1,98 & 1,44 & 0,76 & 0,00 \\
\hline$z$ & 0,00 & 50 & 100 & 150 & 200 & 250 & 300 & 350 & 400 & 450 & 500 \\
\hline
\end{tabular}

Tabla 8.6-1 Coordenadas de los nodos de los soportes

Se ha realizado un cálculo en régimen lineal en el modelo sin imperfecciones y otro cálculo en régimen no lineal geométrico y del

${ }^{136}$ Combinaciones de acciones de acuerdo con lo prescrito por el EC4, expuesto en los apartados 3.1.2 y 3.1.4 del presente trabajo

137 " $z$ " representa la coordenada vertical de cada uno de los nodos e " $y$ " el desplazamiento horizontal respecto de un eje vertical, debido a las imperfecciones iniciales. 
material en los dos modelos (sin y con imperfecciones en los soportes). El número de incrementos de carga utilizados en el cálculo no lineal ha sido de 10.

La Figura 8.6-1 muestra, superpuestas, las deformaciones obtenidas mediante los dos tipos de cálculo realizados sobre el modelo sin imperfecciones. Los cuadros laterales indican el valor de los deplazamientos y giros del nudo del encuentro entre la viga y el soporte derecho.

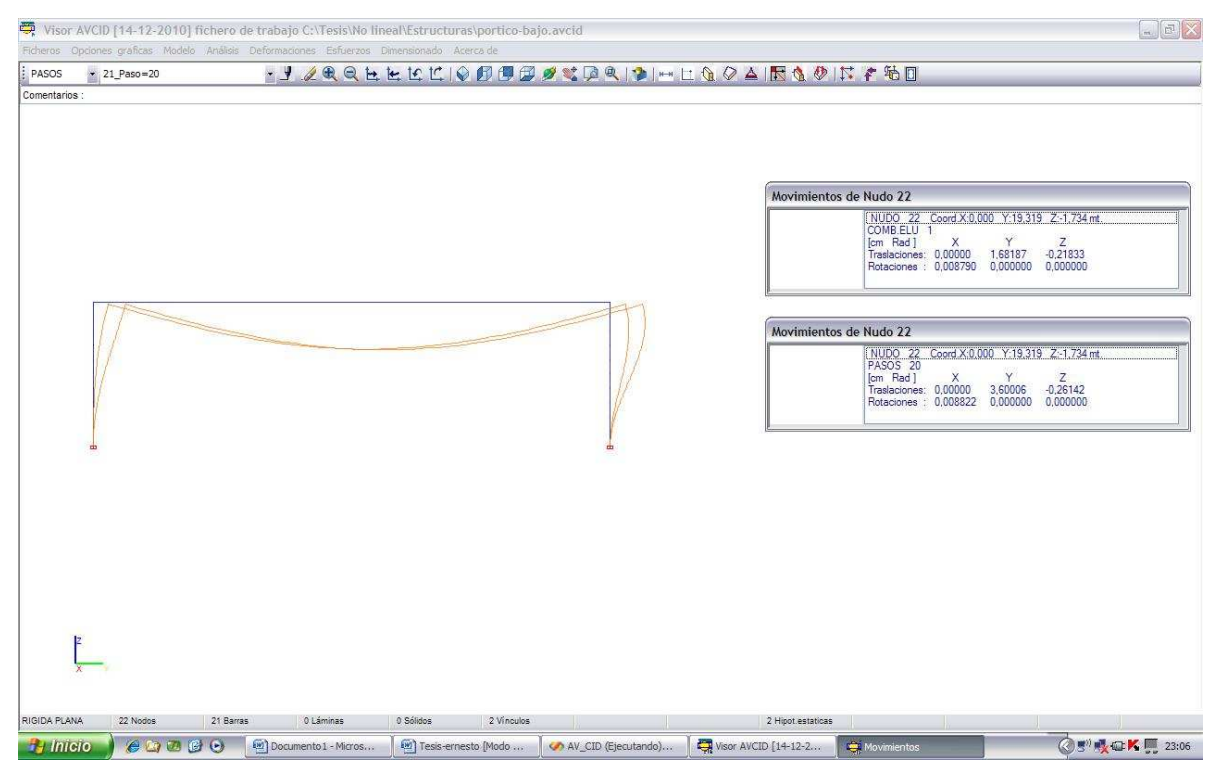

Figura 8.6-1 Comparación en el pórtico sin imperfecciones.

Los tres diagramas de la Figura 8.6-5 muestran el valor del momento flector, para la anteriormente mencionada combinación de acciones, en los nodos donde se ha discretizado la barra: los diagramas $A$ y $B$ corresponden al cálculo lineal y al no lineal, respectivamente, ambos del modelo sin imperfecciones y el diagrama $C$ al cálculo no lineal del modelo con imperfecciones iniciales en el soporte. 


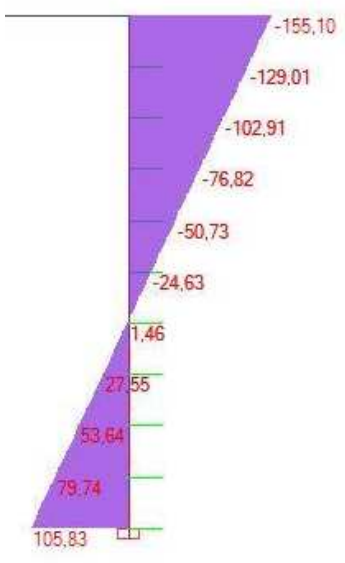

A

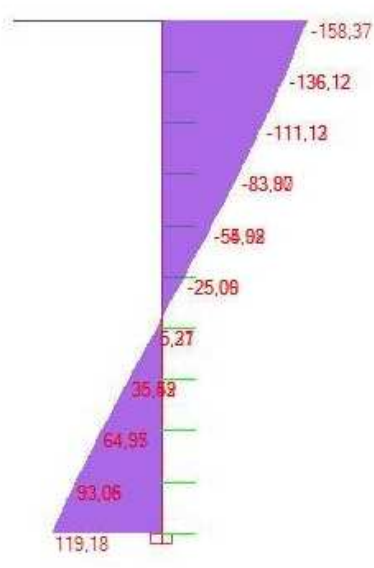

B

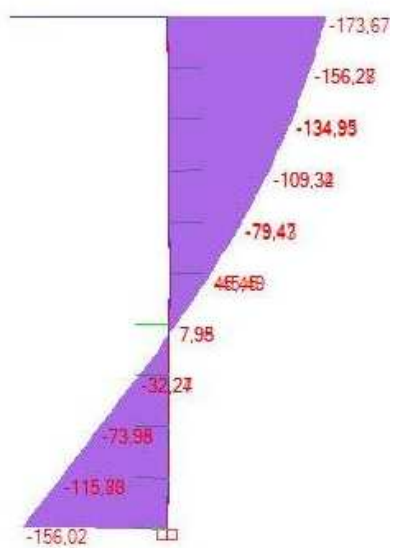

C

Figura 8.6-2 Diagramas de momentos flectores

El esfuerzo axil para la misma combinación de acciones es sensiblemente igual en los tres casos, con un valor de $1.150 \mathrm{kN}$.

El valor absoluto de los momentos máximos y su incremento en los dos cálculos en segundo orden respecto del cálculo lineal se muestra en la siguiente tabla:

\begin{tabular}{|l|l|c|c|c|c|}
\hline Modelo & Cálculo & $\begin{array}{c}\text { Extremo } \\
\text { Inferior }\end{array}$ & Incremento \% & $\begin{array}{c}\text { Extremo } \\
\text { Superior }\end{array}$ & Incremento \% \\
\hline \multirow{2}{*}{$\begin{array}{l}\text { Sin } \\
\text { imperfecciones }\end{array}$} & Lineal & 105,83 & & 155,10 & \\
\cline { 2 - 6 } & No lineal & 119,18 & 12,61 & 158,37 & 2,11 \\
\hline $\begin{array}{l}\text { Con } \\
\text { imperfecciones }\end{array}$ & No lineal & 156,02 & 47,42 & 173,67 & 11,97 \\
\hline
\end{tabular}

Tabla 8.6-2 Comparativa de los momentos flectores máximos

El incremento de los momentos que se indica en la tabla anterior está calculado respecto de los momentos obtenidos en el cálculo lineal.

En la Figura 8.6-3 se ha representado el diagrama de interacción de la mencionada sección, obtenida mediante el programa de cálculo de secciones mixtas desarrollado y utilizando los siguientes valores: 


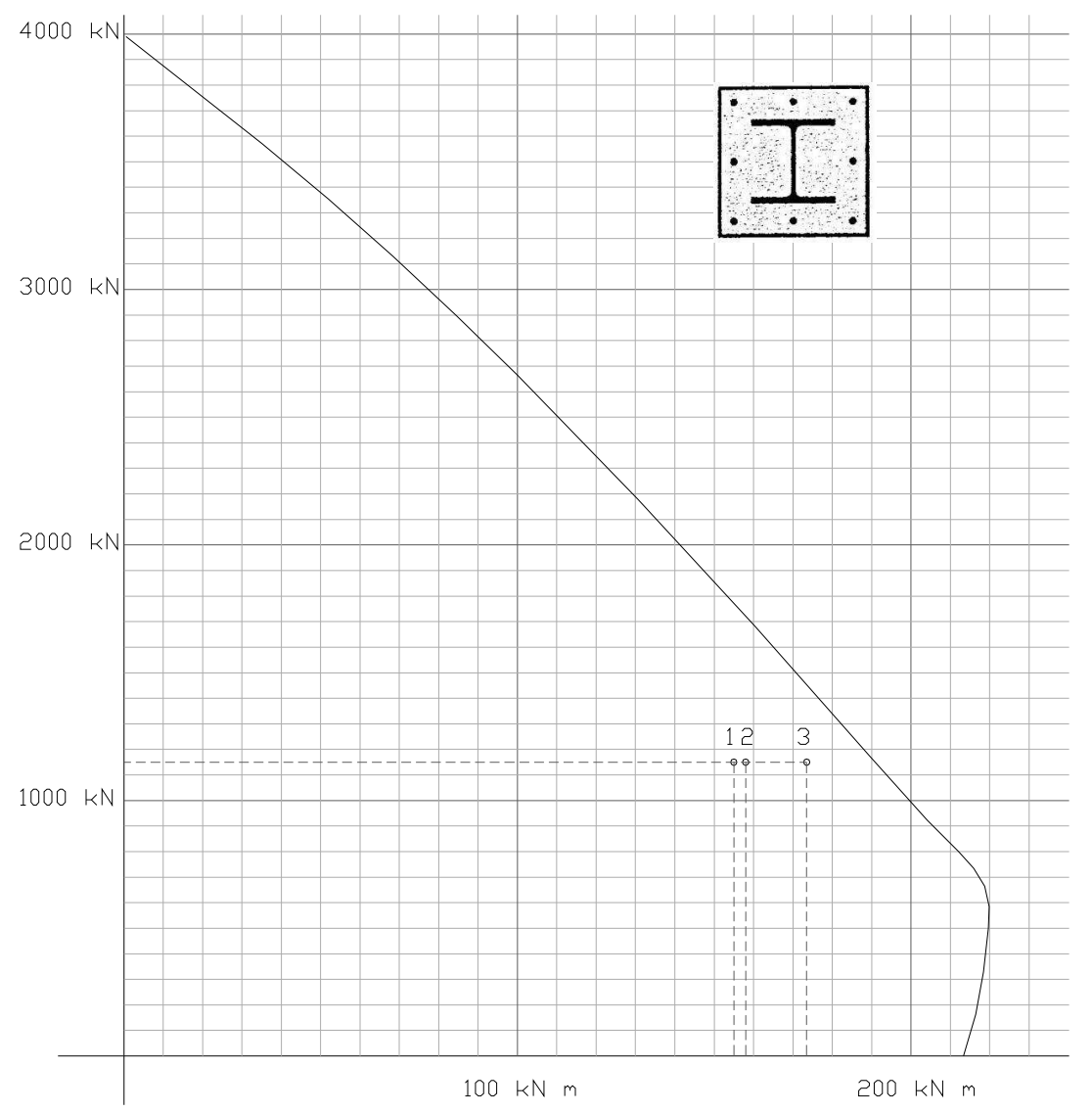

Figura 8.6-3 Diagrama de interacción

Sobre dicho diagrama se han indicado los pares de solicitaciones $\left(N_{d}, M_{d}\right)$ obtenidos en cada uno de los cálculos realizados:

Pto 1: Modelo sin imperfecciones y cálculo lineal

Pto 2: Modelo sin imperfecciones y cálculo no lineal

Pto 3: Modelo con imperfecciones en los soportes y cálculo no lineal.

Se puede observar que los tres puntos quedan en el interior de la superficie encerrada por la curva, por lo que en todos los casos se podría dar por aceptable el dimensionamiento del soporte. 
No obstante la capacidad resistente adicional, entendida como la relación entre las solicitaciones de agotamiento $\left(N_{u}, M_{u}\right)$ y las solicitaciones de cálculo $\left(N_{d}, M_{d}\right)$, lineales entre sí, varía en los tres casos, ya que las distancias de los tres puntos grafiados respecto de la curva de interacción son distintas. El valor de la capacidad resistente adicional en los tres casos es la siguiente:

Caso 1: $\quad Y=1,162$

Caso 2: $\quad Y=1,145$

Caso 3: $\quad Y=1,072$

El hecho de que los tres valores sean superiores a la unidad indica que el coeficiente de seguridad global es mayor que los aplicados como mayoración de las acciones.

\section{Análisis de resultados}

De la obseración de los resultados expuestos, para el pórtico analizado, se pueden extraer las siguientes conclusiones:

- El incremento de los momentos flectores obtenidos en el análisis no lineal respecto del lineal superan el $10 \%$, al menos en algún nodo intermedio en el modelo sin imperfecciones.

- El incremento de los momentos flectores es sustancialmente mayor si se parte de un modelo donde se consideran imperfecciones iniciales en los soportes.

- La capacidad resistente adicional se reduce de una forma notable en el modelo con imperfecciones iniciales respecto del modelo sin imperfecciones. 
- Por aplicación de los artículos 4.3.5.3.3 del EC2 y 5.2.5.2 del EC3 (ver apartado 8.2 del presente trabajo), sería necesario el análisis en segundo orden del pórtico ensayado.

\section{Pórtico de 12 plantas de altura}

Se ha modelizado un pórtico que podría formar parte de la estructura de un edificio de oficinas de planta baja más 11 plantas de altura (de $5,00 \mathrm{~m}$. de altura las plantas baja y primera y de 3,00 $\mathrm{m}$ el resto), con dos vanos de $8,00 \mathrm{~m}$ de luz entre pilares.

Todas las vigas se han predimensionado con secciones de hormigón armado de $30 \times 50 \mathrm{~cm}$. Los soportes han sido predimensionados con secciones mixtas formadas por perfiles HEB embebidos en hormigón, a partir de las solicitaciones obtenidas mediante un primer cálculo en régimen lineal, dando lugar a distintas dimensiones y perfiles. Todos los soportes, salvo el soporte central de la planta baja (número 2) se han discretizado en 4 tramos de igual longitud, con el objeto de obtener una mejor precisión en la determinación del módulo de rigidez a lo largo de la puesta en carga en el cálculo en régimen no lineal.

El citado soporte 2, se ha predimensionado con una sección mixta formada por un perfil HEB-260, dispuesto con su eje fuerte perpendicular al plano de la estructura, embebido en una sección de hormigón de $45 \times 45 \mathrm{~cm}$, se ha discretizado en 10 tramos de igual longitud $(0,50 \mathrm{~m})$ para poder precisar el valor de las imperfecciones iniciales, (único soporte en el que se consideran) evaluadas según las prescripciones del EC4 expuestas en el apartado 3.1.1. Las 
coordenadas $^{138}$ de los nudos de los tramos en que se han discretizado este soportes, expresadas en centímetros, se indican en la tabla siguiente:

\begin{tabular}{|c|c|c|c|c|c|c|c|c|c|c|c|}
\hline Nudo & 0 & 1 & 2 & 3 & 4 & 5 & 6 & 7 & 8 & 9 & 10 \\
\hline$y$ & 0,00 & 0,35 & 0,67 & 0,92 & 1,08 & 1,14 & 1,08 & 0,92 & 0,67 & 0,35 & 0,00 \\
\hline$z$ & 0,00 & 50 & 100 & 150 & 200 & 250 & 300 & 350 & 400 & 450 & 500 \\
\hline
\end{tabular}

Tabla 8.6-3 Coordenadas de los nodos del soporte

No se ha dispuesto ningún elemento de arriostramiento lateral, quedando las deformaciones horizontales de la estructura únicamente condicionadas por la propia rigidez del pórtico.

Las cargas gravitatorias aplicadas simulan unos forjados unidireccionales realizados con nervios de hormigón in situ de $6 \mathrm{~m}$ de luz entre vigas más revestimientos y cargas de uso. Las cargas horizontales, aplicadas como una fuerza puntual sobre los nudos del pórtico, corresponden al empuje del viento sobre la fachada. En el modelo de cálculo, las cargas gravitatorias han sido introducidas en la hipótesis 1 y las horizontales en la hipótesis 2 .

A efecto de las comprobaciones en Estados Límites Últimos, se ha utilizado una combinación de cargas en la que actúan simultáneamente las cargas gravitatorias (de carácter permanente) afectadas de un coeficiente de mayoración de acciones de valor 1,35 más las cargas horizontales (de carácter variable) afectadas de un coeficiente de mayoración de 1,50. Se ha aplicado la simplificación de combinaciones de acciones para casos de edificación ${ }^{139}$.

\footnotetext{
138 " $z$ " representa la coordenada vertical de cada uno de los nodos e " $y$ " el desplazamiento horizontal respecto de un eje vertical, debido a las imperfecciones iniciales.

${ }^{139}$ Combinaciones de acciones de acuerdo con lo prescrito por el EC4, expuesto en los apartados 3.1.2 y 3.1.4 del presente trabajo
} 
Los valores de las resistencias características de los materiales y sus correspondientes coeficientes de minoración que se han utilizado son:

Resistencias de los materiales:
Hormigón.
HA-30: $f_{c k}=30 \mathrm{~N} / \mathrm{mm}^{2}$
Acero de armar.
B-500: $f_{\mathrm{sk}}=500 \mathrm{~N} / \mathrm{mm}^{2}$
Acero estructural.
S-355: $\mathrm{f}_{\mathrm{yk}}=355 \mathrm{~N} / \mathrm{mm}^{2}$

Coeficientes de minoración de resistencias:

Hormigón.

$\gamma_{c}=1,35$

$\alpha=1,00$

Acero de armar. $\quad \gamma_{\mathrm{s}}=1,15$

Acero estructural. $\quad \gamma_{y}=1,10$

En el cálculo no se han tenido en cuenta los efectos reológicos del hormigón.

El modelo descrito se ha sometido a tres tipos de cálculo: lineal, no lineal geométrico y no lineal geométrico y del material. En los dos últimos casos mencionados la carga se ha aplicado en 25 pasos.

Como avance de los resultados, cabe indicar que en el cálculo no lineal geométrico se ha llegado a aplicar la carga total en los 25 pasos, ya que en todo el proceso se utiliza el módulo de rigidez inicial $E \cdot I$ sin comprobar el agotamiento de las barras. Sin embargo, el cálculo no lineal geométrico y del material no ha llegado a finalizar el proceso de carga completo, al producirse el colapso se la sección inferior del soporte central de la planta baja en el último paso, por lo que los movimientos y solicitaciones de la carga máxima aplicada corresponden al paso 24 de 25, es decir al 0,964 de la carga total.

En la Figura 8.6-4 se muestran, superpuestas, las deformadas de la estructura obtenidas en los tres tipos de cálculo mencionados. 


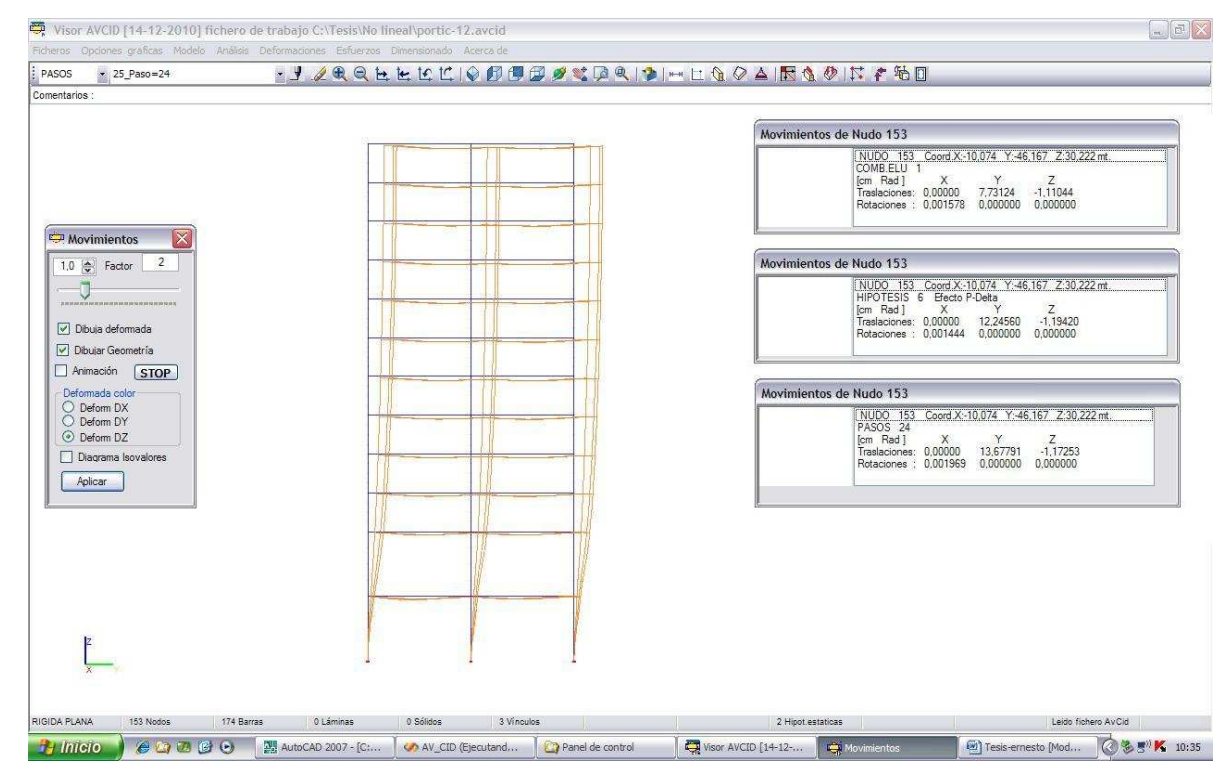

Figura 8.6-4 Deformadas de la estructura superpuestas.

Los desplazamientos horizontales del nudo 153 (nudo del encuentro del soporte derecho con la viga de la última planta) mostrados en los cuadros de la Figura 8.6-4, se reproducen en la tabla siguiente:

\begin{tabular}{|l|c|}
\hline Cálculo realizado & Desplazamiento horizontal \\
\hline Lineal & $7,73 \mathrm{~cm}$ \\
\hline No lineal geométrico & $12,24 \mathrm{~cm}$ \\
\hline $\begin{array}{l}\text { No lineal geométrico y de material } \\
\text { (paso 24 de 25) }\end{array}$ & $13,68 \mathrm{~cm}$ \\
\hline
\end{tabular}

Los momentos flectores de los dos extremos de todos los soportes correspondientes a la combinación de cargas descrita, obtenidos como resultado del cálculo lineal y del no lineal geométrico se reproducen, en valor absoluto, en la Tabla 8.6-4. En ella, además se ha incluido la variación de los momentos flectores obtenidos en el cálculo no lineal geométrico respecto del lineal, con valores positivos 
cuando se produce un incremento y negativos cuando se produce una disminución. No se incluyen los valores del cálculo no lineal geométrico y del material puesto que al haberse producido el colapso de la barra número 2, el soporte intermedio de la planta baja, antes de la aplicación total de la carga, los valores que se disponen, correspondientes al $96 \%$ de la carga (paso 24 de 25) no son totalmente comparables.

\begin{tabular}{|l|c|c|c|c|c|c|}
\cline { 2 - 7 } \multicolumn{1}{c|}{} & \multicolumn{2}{c|}{34} & \multicolumn{2}{c|}{35} & \multicolumn{2}{c|}{36} \\
\cline { 2 - 7 } \multicolumn{1}{c|}{ PLANTA 12} & Inf. & Sup. & Inf. & Sup. & Inf. & Sup. \\
\hline Lineal & 208,74 & 304,47 & 8,80 & 22,03 & 207,94 & 328,44 \\
\hline No lineal & 207,42 & 303,72 & 9,12 & 23,25 & 206,11 & 328,93 \\
\hline$\%$ & $-0,63$ & $-0,25$ & 3,64 & 5,54 & $-0,88$ & 0,15 \\
\hline
\end{tabular}

\begin{tabular}{|l|c|c|c|c|c|c|}
\cline { 2 - 7 } \multicolumn{1}{c|}{} & \multicolumn{2}{c|}{31} & \multicolumn{2}{c|}{32} & \multicolumn{2}{c|}{33} \\
\cline { 2 - 7 } \multicolumn{1}{c|}{ PLANTA 11} & Inf. & Sup. & Inf. & Sup. & Inf. & Sup. \\
\hline Lineal & 174,74 & 141,71 & 19,08 & 33,63 & 192,76 & 186,46 \\
\hline No lineal & 173,69 & 141,07 & 19,65 & 35,69 & 183,88 & 188,25 \\
\hline$\%$ & $-0,60$ & $-0,45$ & 2,99 & 6,13 & $-4,61$ & 0,96 \\
\hline
\end{tabular}

\begin{tabular}{|l|c|c|c|c|c|c|}
\cline { 2 - 7 } \multicolumn{1}{c|}{} & \multicolumn{2}{c|}{28} & \multicolumn{2}{c|}{29} & \multicolumn{2}{c|}{30} \\
\cline { 2 - 7 } \multicolumn{1}{c|}{ PLANTA 10 } & Inf. & Sup. & Inf. & Sup. & Inf. & Sup. \\
\hline Lineal & 169,28 & 153,08 & 31,89 & 47,66 & 192,76 & 212,09 \\
\hline No lineal & 167,96 & 151,39 & 33,19 & 51,09 & 191,58 & 214,50 \\
\hline$\%$ & $-0,78$ & $-1,10$ & 4,08 & 7,20 & $-0,61$ & 1,14 \\
\hline
\end{tabular}

\begin{tabular}{|l|c|c|c|c|c|c|}
\cline { 2 - 7 } \multicolumn{1}{c|}{} & \multicolumn{2}{c|}{25} & \multicolumn{2}{c|}{26} & \multicolumn{2}{c|}{27} \\
\cline { 2 - 7 } \multicolumn{1}{c|}{} & Inf. & Sup. & Inf. & Sup. & Inf. & Sup. \\
\hline Lineal & 161,28 & 143,70 & 44,96 & 60,85 & 198,39 & 216,83 \\
\hline No lineal & 159,54 & 140,94 & 47,39 & 66,04 & 197,71 & 220,27 \\
\hline$\%$ & $-1,08$ & $-1,92$ & 5,40 & 8,53 & $-0,34$ & 1,59 \\
\hline
\end{tabular}




\begin{tabular}{|l|c|c|c|c|c|c|}
\cline { 2 - 7 } \multicolumn{1}{c|}{ PLANTA 8 } & \multicolumn{2}{c|}{22} & \multicolumn{2}{c|}{23} & \multicolumn{2}{c|}{24} \\
\cline { 2 - 7 } \multicolumn{1}{c|}{} & Inf. & Sup. & Inf. & Sup. & Inf. & Sup. \\
\hline Lineal & 80,81 & 135,35 & 58,17 & 74,10 & 203,72 & 222,29 \\
\hline No lineal & 150,50 & 131,26 & 62,15 & 81,49 & 203,75 & 226,99 \\
\hline$\%$ & 86,24 & $-3,02$ & 6,84 & 9,97 & 0,01 & 2,11 \\
\hline
\end{tabular}

\begin{tabular}{|l|c|c|c|c|c|c|}
\cline { 2 - 7 } \multicolumn{1}{c|}{ PLANTA 7 } & \multicolumn{2}{c|}{19} & \multicolumn{2}{c|}{20} & \multicolumn{2}{c|}{21} \\
\cline { 2 - 7 } \multicolumn{1}{c|}{} & Inf. & Sup. & Inf. & Sup. & Inf. & Sup. \\
\hline Lineal & 144,16 & 126,71 & 71,39 & 87,36 & 208,72 & 227,48 \\
\hline No lineal & 141,03 & 120,99 & 77,37 & 97,48 & 209,66 & 233,72 \\
\hline$\%$ & $-2,17$ & $-4,51$ & 8,38 & 11,58 & 0,45 & 2,74 \\
\hline
\end{tabular}

\begin{tabular}{|l|c|c|c|c|c|c|}
\cline { 2 - 7 } \multicolumn{1}{c|}{} & \multicolumn{2}{c|}{16} & \multicolumn{2}{c|}{17} & \multicolumn{2}{c|}{18} \\
\cline { 2 - 7 } \multicolumn{1}{c|}{ PLANTA 6 } & Inf. & Sup. & Inf. & Sup. & Inf. & Sup. \\
\hline Lineal & 135,31 & 117,59 & 84,48 & 100,82 & 213,23 & 232,46 \\
\hline No lineal & 131,31 & 109,82 & 92,88 & 114,34 & 215,21 & 240,65 \\
\hline$\%$ & $-2,96$ & $-6,61$ & 9,94 & $\mathbf{1 3 , 4 1}$ & 0,93 & 3,52 \\
\hline
\end{tabular}

\begin{tabular}{|l|c|c|c|c|c|c|}
\cline { 2 - 7 } \multicolumn{1}{c|}{ PLANTA 5 } & \multicolumn{2}{c|}{13} & \multicolumn{2}{c|}{14} & \multicolumn{2}{c|}{15} \\
\cline { 2 - 7 } \multicolumn{1}{c|}{} & Inf. & Sup. & Inf. & Sup. & Inf. & Sup. \\
\hline Lineal & 126,90 & 107,60 & 97,39 & 115,11 & 216,50 & 237,60 \\
\hline No lineal & 122,26 & 97,04 & 108,76 & 133,26 & 219,32 & 248,47 \\
\hline$\%$ & $-3,66$ & $-9,81$ & $\mathbf{1 1 , 6 7}$ & $\mathbf{1 5 , 7 7}$ & 1,30 & 4,57 \\
\hline
\end{tabular}

\begin{tabular}{|l|c|c|c|c|c|c|}
\cline { 2 - 7 } \multicolumn{1}{c|}{} & \multicolumn{2}{c|}{10} & \multicolumn{2}{c|}{11} & \multicolumn{2}{c|}{12} \\
\cline { 2 - 7 } \multicolumn{1}{c|}{ PLANTA 4 } & Inf. & Sup. & Inf. & Sup. & Inf. & Sup. \\
\hline Lineal & 119,74 & 95,15 & 107,65 & 132,11 & 216,56 & 244,68 \\
\hline No lineal & 114,91 & 79,99 & 121,88 & 157,32 & 219,79 & 260,04 \\
\hline$\%$ & $-4,03$ & $-15,93$ & $\mathbf{1 3 , 2 2}$ & $\mathbf{1 9 , 0 8}$ & 1,49 & 6,28 \\
\hline
\end{tabular}




\begin{tabular}{|l|c|c|c|c|c|c|}
\cline { 2 - 7 } \multicolumn{1}{c|}{ PLANTA 3 } & \multicolumn{2}{c|}{7} & \multicolumn{2}{c|}{8} & \multicolumn{2}{c|}{9} \\
\cline { 2 - 7 } \multicolumn{1}{c|}{} & Inf. & Sup. & Inf. & Sup. & Inf. & Sup. \\
\hline Lineal & 141,69 & 76,06 & 118,90 & 162,25 & 216,95 & 259,78 \\
\hline No lineal & 140,14 & 52,32 & 140,32 & 201,84 & 216,88 & 283,63 \\
\hline$\%$ & $-1,09$ & $-31,21$ & $\mathbf{1 8 , 0 2}$ & $\mathbf{2 4 , 4 0}$ & $-0,03$ & 9,18 \\
\hline
\end{tabular}

\begin{tabular}{|l|c|c|c|c|c|c|}
\cline { 2 - 7 } \multicolumn{1}{c|}{ PLANTA 2 } & \multicolumn{2}{c|}{4} & \multicolumn{2}{c|}{5} & \multicolumn{2}{c|}{6} \\
\cline { 2 - 7 } \multicolumn{1}{c|}{} & Inf. & Sup. & Inf. & Sup. & Inf. & Sup. \\
\hline Lineal & 42,86 & 0,50 & 232,74 & 226,10 & 288,17 & 286,57 \\
\hline No lineal & 3,63 & 44,14 & 294,73 & 290,96 & 326,52 & 331,44 \\
\hline$\%$ & $-91,53$ & $\mathbf{8 7 2 8 , 0 0}$ & $\mathbf{2 6 , 6 3}$ & $\mathbf{2 8 , 6 9}$ & $\mathbf{1 3 , 3 1}$ & $\mathbf{1 5 , 6 6}$ \\
\hline
\end{tabular}

\begin{tabular}{|l|c|c|c|c|c|c|}
\cline { 2 - 7 } \multicolumn{1}{c|}{ PLANTA 1 } & \multicolumn{2}{c|}{1} & \multicolumn{2}{c|}{2} & \multicolumn{2}{c|}{3} \\
\cline { 2 - 7 } \multicolumn{1}{c|}{} & Inf. & Sup. & Inf. & Sup. & Inf. & Sup. \\
\hline Lineal & 177,54 & 50,64 & 330,98 & 120,80 & 303,16 & 198,84 \\
\hline No lineal & 224,41 & 35,88 & 384,25 & 155,41 & 351,45 & 212,60 \\
\hline$\%$ & $\mathbf{2 6 , 4 0}$ & $-29,15$ & $\mathbf{1 6 , 0 9}$ & $\mathbf{2 8 , 6 5}$ & $\mathbf{1 5 , 9 3}$ & 6,92 \\
\hline
\end{tabular}

Tabla 8.6-4 Tabla comparativa de momentos flectores

En el caso del "soporte 2", se ha elaborado un estudio más detallado, posibilitado por la discretización realizada y el haber considerado, en el modelo de la estructura, las imperfecciones iniciales de la barra. Los tres diagramas de la Figura 8.6-5 muestran el valor del momento flector en los nodos donde se ha discretizado la barra: el diagrama $A$ corresponde al cálculo lineal, el diagrama $B$ al cálculo no lineal geométrico y el diagrama $C$ al paso 24 de $25^{140}$ del cálculo no lineal geométrico y del material.

${ }^{140}$ En el paso 24 se ha aplicado el 0,964 de la carga total. El cálculo en el siguiente paso de carga (100\%) no llega a completarse al producirse el colapso de la barra número 2. 


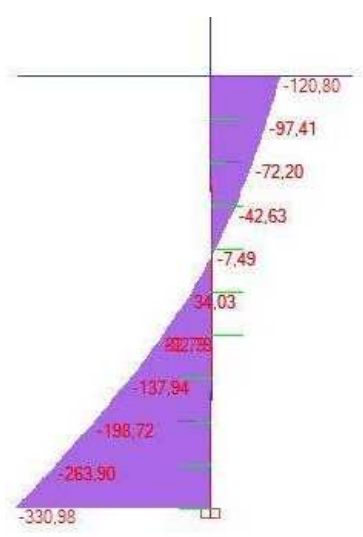

A

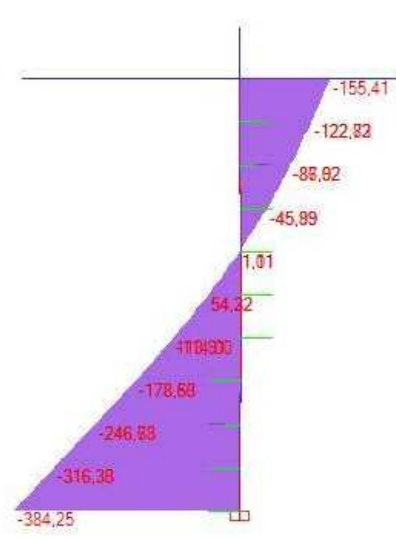

B

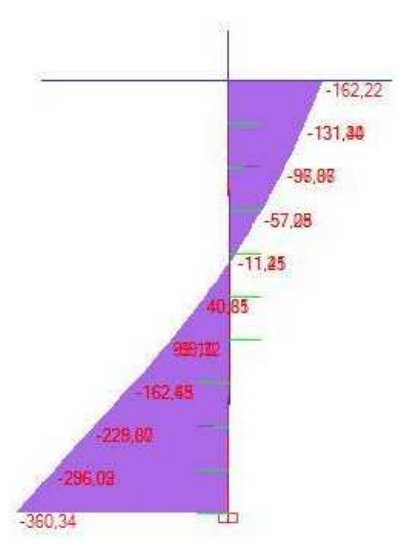

C (paso 24 de 25)

Figura 8.6-5 Diagramas de momentos flectores en el soporte 2

El valor absoluto de los esfuerzos axiles y de momentos máximos y su incremento en los dos cálculos en segundo orden respecto del cálculo lineal se muestra en la siguiente tabla:

\begin{tabular}{|c|c|c|c|c|c|c|}
\hline Modelo & Cálculo & Solicitación & $\begin{array}{l}\text { Extremo } \\
\text { Inferior }\end{array}$ & $\begin{array}{c}\text { Incremento } \\
\%\end{array}$ & $\begin{array}{l}\text { Extremo } \\
\text { Superior }\end{array}$ & $\begin{array}{c}\text { Incremento } \\
\%\end{array}$ \\
\hline \multirow{6}{*}{$\begin{array}{l}\text { Con } \\
\text { imper- } \\
\text { fecciones } \\
\text { en soporte } \\
2\end{array}$} & \multirow[b]{2}{*}{ Lineal } & $\mathrm{Md}(\mathrm{kN} \cdot \mathrm{M})$ & 330,98 & & 120,80 & \\
\hline & & $\mathrm{Nd}(\mathrm{kN})$ & 6.270 & & & \\
\hline & \multirow[t]{2}{*}{ N.L.G. } & $\mathrm{Md}(\mathrm{kN} \cdot \mathrm{M})$ & 384,25 & 16,09 & 155,41 & 28,65 \\
\hline & & $\mathrm{Nd}(\mathrm{kN})$ & 6.270 & & & \\
\hline & \multirow{2}{*}{$\begin{array}{c}\text { N.L. } \\
\text { G.+M. } \\
\text { (paso } \\
24 / 25 \text { ) }\end{array}$} & $\mathrm{Md}(\mathrm{kN} \cdot \mathrm{M})$ & 360,34 & 8,87 & 162,22 & 34,29 \\
\hline & & $\mathrm{Nd}(\mathrm{kN})$ & 6.050 & & & \\
\hline
\end{tabular}

Tabla 8.6-5 Comparativa de momentos flectores

En la Figura 8.6-6 se ha representado el diagrama de interacción de la mencionada sección, obtenida mediante el programa de cálculo de secciones mixtas desarrollado: 


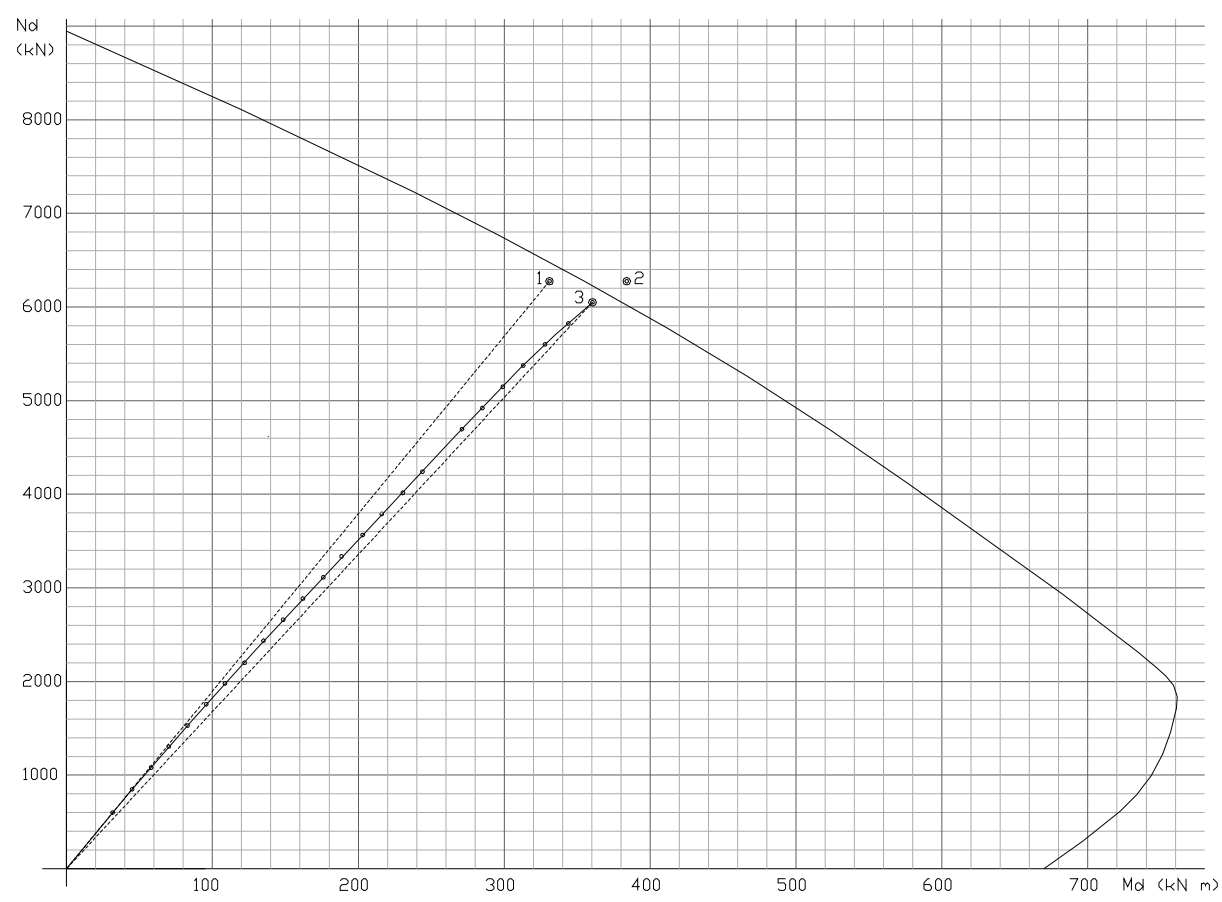

Figura 8.6-6 Referencia a la curva de interacción

Sobre dicho diagrama se han indicado los pares de solicitaciones $\left(N_{d}, M_{d}\right)$ obtenidos en extremo inferior del soporte número 2, en cada uno de los cálculos realizados:

Pto 1: cálculo lineal

Pto 2: cálculo no lineal geométrico (módulo de rigidez $E \cdot I$ constante)

Pto 3: cálculo no lineal geométrico y del material.

También se ha representado la trayectoria de la puesta en carga en el cálculo lineal, definida en este caso por una línea recta, y en el 
cálculo no lineal geométrico y del material ${ }^{141}$, gracias a la aplicación de la carga de forma incremental que permite analizar toda la historia de solicitaciones. Se puede observar que en este caso la trayectoria está definida por una curva que finalmente alcanzaría al diagrama de interacción de la sección entre el paso 24 y 25, entre el 96,4\% y el $100 \%$ de la carga total.

\section{Análisis de resultados}

De la obseración de los resultados expuestos, para el pórtico analizado, se pueden extraer las siguientes conclusiones:

- El incremento de los momentos flectores obtenidos en el análisis no lineal geométrico (ver tabla de valores) respecto del lineal superan el 10\%, en más de una sección.

De ello se concluye que, por aplicación de los artículos 4.3.5.3.3 del EC2 y 5.2.5.2 del EC3 (ver apartado 8.2 del presente trabajo), sería necesario el análisis en segundo orden del pórtico ensayado.

- El incremento de los momentos flectores obtenidos en el análisis no lineal geométrico (ver tabla de valores) respecto del lineal es mayor en las plantas inferiores del pórtico.

- Tanto el cálculo no lineal geométrico como el no lineal geométrico y del material conducen al agotamiento de la sección inferior del soporte central de la planta baja.

141 Trayectoria representada hasta el paso 24 de los 25 en que se ha aplicado la carga total. No se disponen de datos del paso 25 al haberse producido el colapso de la sección. 
En el primero de los casos queda verificado al observar que el punto 2 queda situado en el exterior de la curva de interacción de la sección, ya que este análisis se realiza sin la comprobación simúltánea de la sección, que debe realizarse con posterioridad al cálculo tal como se expone en el apartado 8.6.2 que se expone a continuación.

En el segundo caso, el programa se interrumpe en el paso 25 al producirse el agotamiento resistente de la mencionada sección. El punto 3 corresponde a las solicitaciones del paso 24, anterior al colapso.

- Se observa que la línea que une los puntos correspondientes a las solicitaciones de los distintos pasos de la historia de carga tienen una forma curva, lo que muestra la no linealidad del proceso.

\subsubsection{DIMENSIONAMIENTO DE SOPORTES MIXTOS}

La integración del módulo de cálculo desarrollado, en el programa de análisis estructural AV_CID, permite el diseño y la comprobación de soportes de sección mixta hormigón-acero sometidos a flexocompresión esviada, de una forma muy ágil, lo que simplifica su uso en las oficinas técnicas.

De esta forma, tras la modelización de la estructura, plana o espacial, y su análisis, como es frecuente, en régimen lineal, el módulo desarrollado es capaz de comprobar la validez de la sección predimensionada. 


\section{Estructura bidireccional de baja más 20 plantas}

Como ejemplo del cálculo, se ha modelizado una estructura de planta baja más 20 plantas de altura, con una de las tipologías estructurales más usuales de la construcción compuesta: forjados reticulares de hormigón y soportes mixtos, de distintas dimensiones.

Los datos de los soportes de sección mixta, dimensión de la sección de hormigón y el tipo y dimensión del perfil metálico se introducen en el modelo de la estructura realizado en AutoCAD, mediante el icono de secciones mixtas del menú de "tipos de secciones" (ver Figura 6.3-1).

Con el modelo así definido, se ha realizado un análisis de solicitaciones en régimen elástico y lineal, como se observa en la figura:

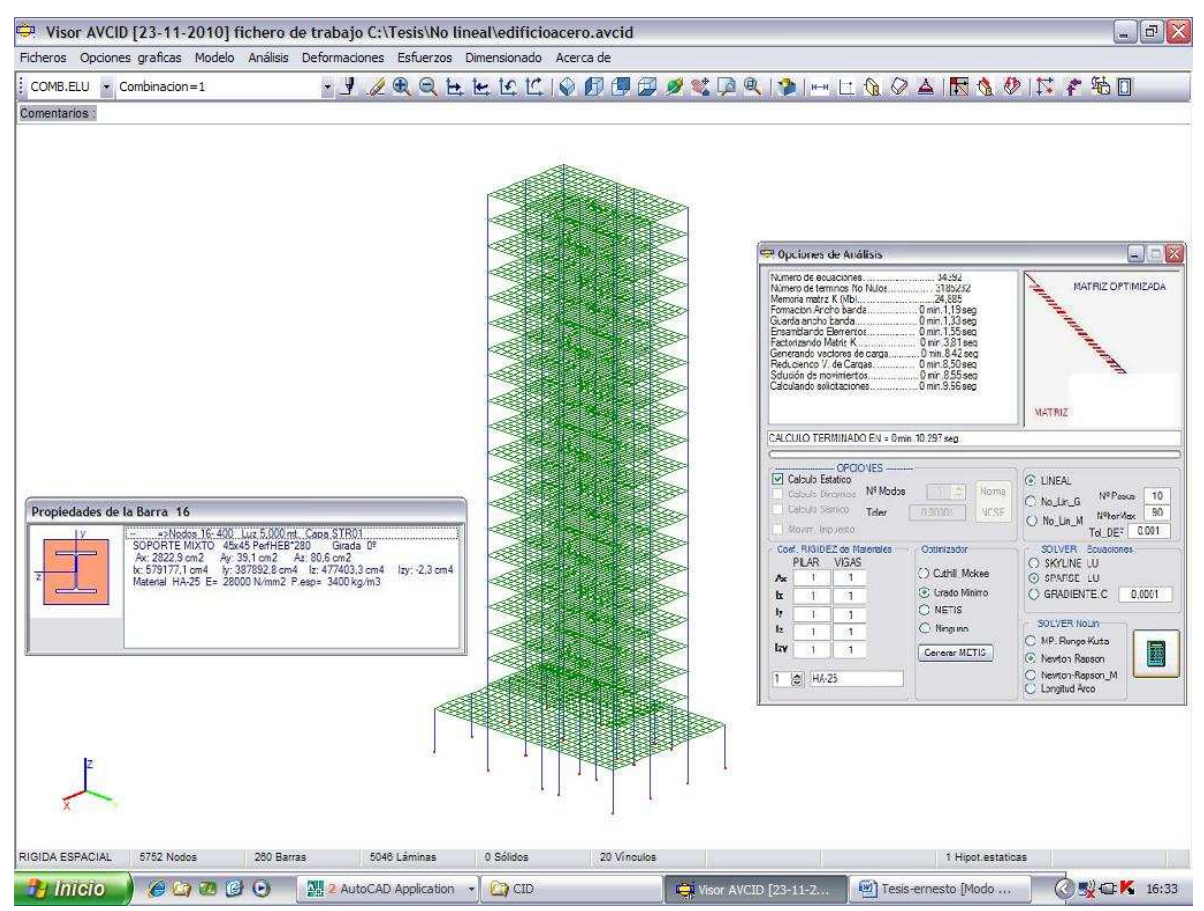

Figura 8.6-7 Análisis de la estructura 


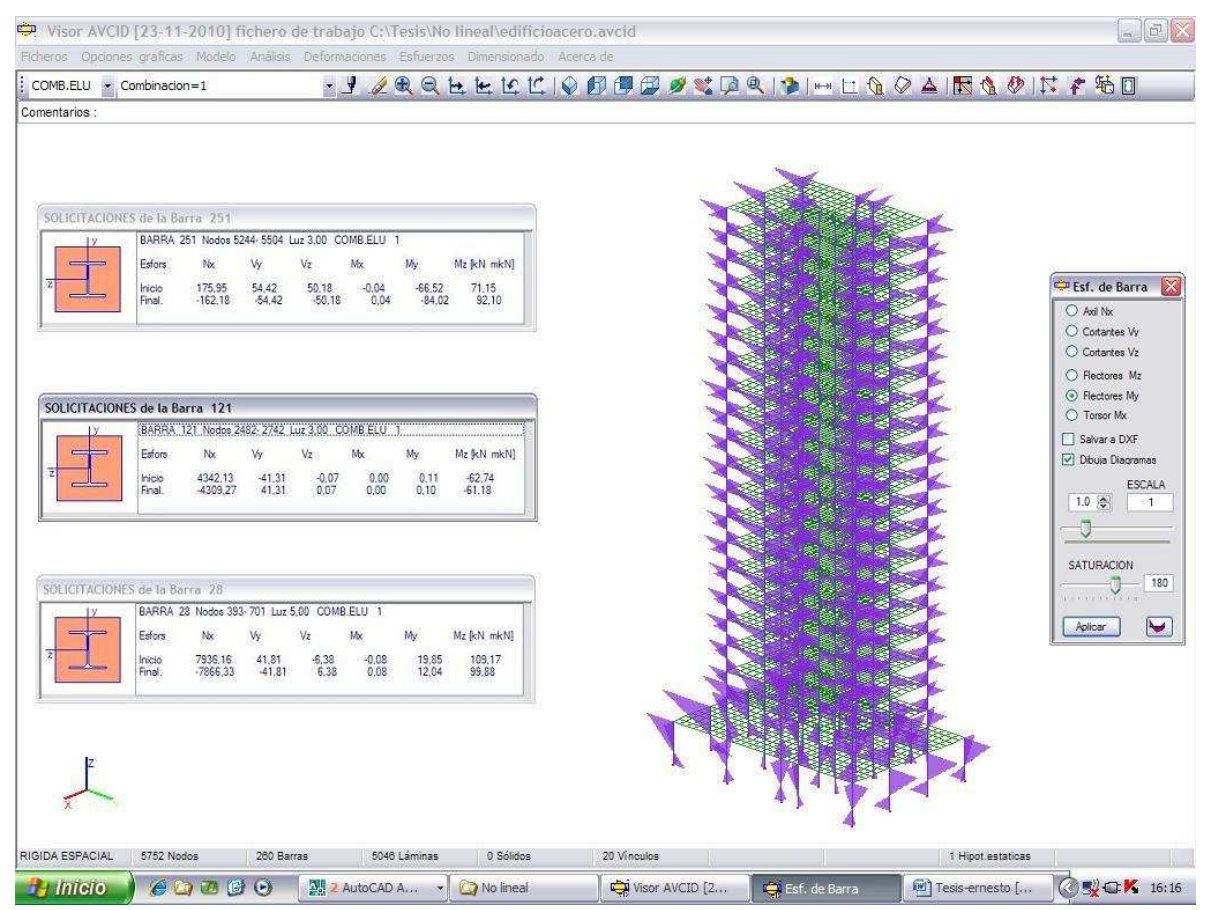

Figura 8.6-8 Diagramas de momentos flectores en soportes

La resistencia característica del hormigón y de las armaduras, así como sus coeficientes de minoración se definen en el cuadro de características de cálculo en hormigón armado de la ventana "Opciones de dimensionado" (ver Figura 10.6-1), mientras que la resistencia característica del acero del perfil metálico y su coeficiente de minoración se definen en el cuadro de características de cálculo en acero de la misma ventana (ver Figura 10.6-3)

La opción "Calcular" de la mencionada ventana que realiza la comprobación de las barras de acero y dimensiona las armaduras de los elementos de hormigón armado, ambas frente a los estados límites últimos y de servicio, analiza ahora también los soportes de sección mixta. 
A continuación, la opción de "comprobación" del menú "Dimensionado", permite verificar el estado de agotamiento de cada elemento y la idoneidad de la solución de forma visual, al grafiar en color rojo los elementos que no cumplen los estados límites.

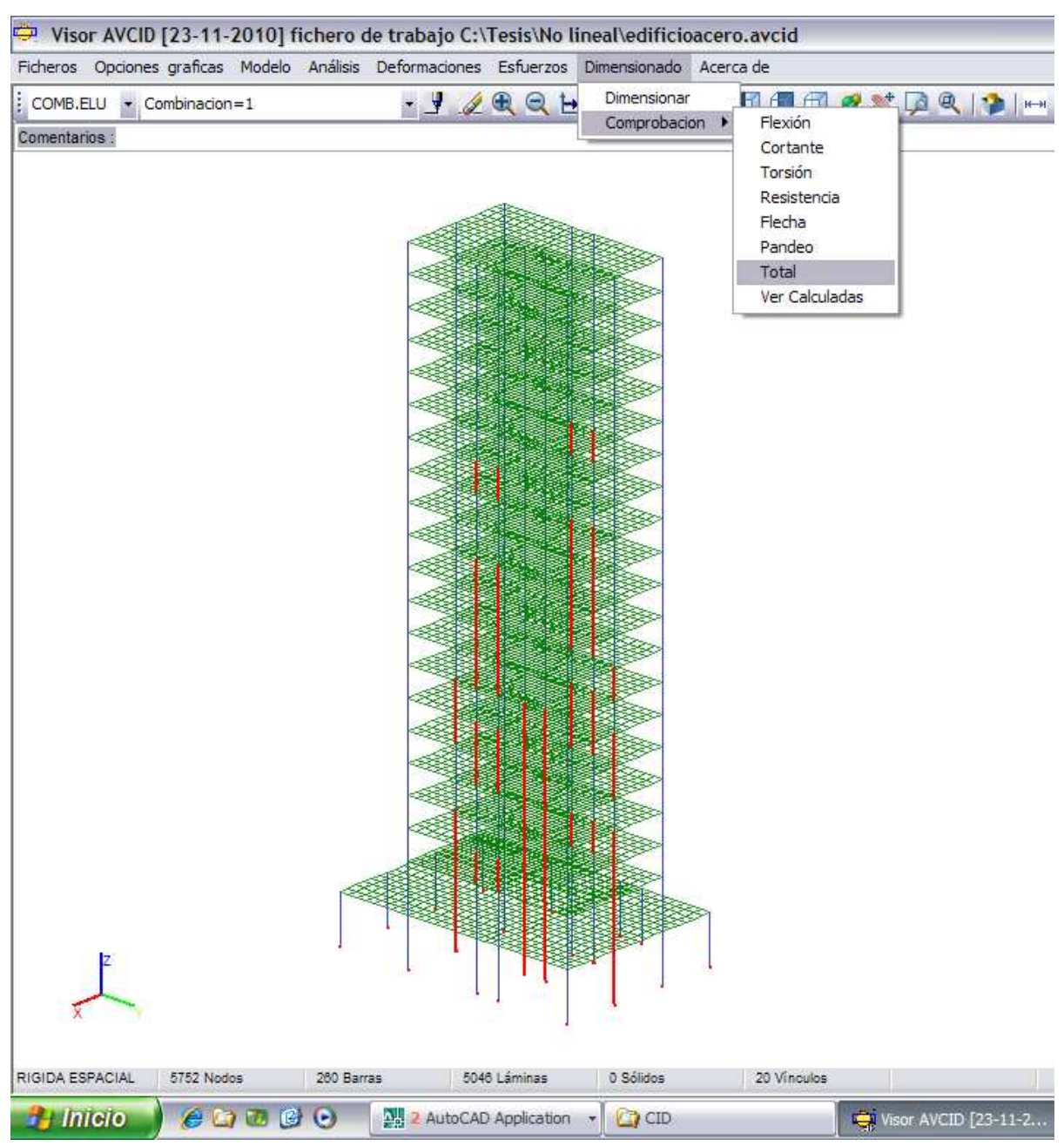

Figura 8.6-9 Ejemplo de comprobación de soportes mixtos

La aplicación de la visualización por colores, también al caso de los soportes mixtos, facilita al técnico la labor de supervisión de resultados en el proceso de diseño y cálculo de la estructura. 


\subsection{CONCLUSIONES}

El presente trabajo, consistente en el estudio de los soportes mixtos, se inicia con el comportamiento de la sección, continúa con la barra como elemento aislado, y finaliza con el análisis del conjunto de la estructura.

La aproximación teórica, se ha realizado a la vista de la normativa que le afecta, Eurocódigo 4, y de las hipótesis recogidas en la literatura técnica especializada. Con estos criterios se han establecido unos modelos de cálculo y se han desarrollado los oportunos métodos de análisis.

Los resultados de cada uno de los aspectos se exponen a continuación, siguiendo el mismo orden en que se ha estructurado todo el proceso de trabajo:

- Para la sección mixta hemos realizado un método lógico del estudio de su equilibrio y deformación, que se ha materializado en una aplicación informática que contempla:

- Las ecuaciones constitutivas de los materiales, modelizadas de forma no lineal de acuerdo con los criterios del Eurocódigo 4.

- La influencia de los efectos reológicos, modelizando la fluencia del hormigón según los criterios del Eurocódigo 2.

- Como resultado de esta aplicación se han elaborado las curvas y superficies de interacción de una serie de secciones mixtas. 
- Para la viga-columna se ha modelizado la barra de forma discreta con un conjuto de divisiones longitudinales, recogiendo todos los criterios utilizados en la sección y que incluye:

- Las imperfecciones iniciales de la barra, modelizadas de acuerdo con el articulado del Eurocódigo 4.

- El estudio de los casos habituales de excentricidades del esfuezo axil en cabeza y pie de la viga-columna.

- Comparación de la influencia de los efectos reológicos del hormigón, no habiéndose alcanzado una reducción mayor del $10 \%$ de la capacidad resistente, en los casos estudiados.

- El conjunto de la estructura se ha modelizado teniendo en cuenta el comportamiento de la sección mixta dentro de un avanzado programa de análisis no lineal de estructuras que permite:

- Analizar las estructuras como traslacionales, realizando un cálculo no lineal geométrico y del material.

- Considerar la influencia de las imperfecciones iniciales de las barras.

- Mostrar los efectos de la traslacionalidad en dos tipologías estructurales: edificio no arriostrado de gran luz y una planta y edificio en altura.

- Comprobar la seguridad de cada elemento, indicando los que no cumplen.

Por todo ello, hay que reseñar que se ha alcanzado el objetivo global propuesto inicialmente, consistente en el estudio y análisis de 
soportes mixtos sometidos a flexocompresión recta y esviada, incluyendo la cuantificación de las diferentes variables que les afecta.

A continuación todas las conclusiones y resultados obtenidos se exponen de una forma más detallada.

\section{Estudio de la sección:}

1.1. En la sección se ha tenido en cuenta la no linealidad en las ecuaciones constitutivas de los materiales que la componen. En las relaciones entre tensión y deformación se han incluido los efectos reológicos del hormigón, que incluso han merecido un capítulo aparte.

Como primera conclusión, se ha desarrollado un procedimiento lógico, para su análisis. Este algoritmo integra las tensiones a partir del planteamiento Virdi y Dowling (apartado 4.3)

1.2. A partir de este algoritmo, se ha elaborado un programa de cálculo por ordenador que permite el estudio de las piezas mixtas de hormigón y acero. La sección puede estar sometida tanto a flexocompresión recta como esviada.

Sus resultados se han contrastado con los obtenidos mediante:

- el programa de hormigón estructural desarrollado por el IECA,

- el programa "CCD" desarrollado por Arcelor, para diseño y cálculo de soportes mixtos,

- el método simplificado propuesto por el Eurocódigo 4.

Las diferencias entre los diferentes programas pueden considerarse despreciables cuando parten de las mismas hipótesis. 
La aplicación del programa de cálculo de secciones mixtas desarrollado para la presente tesis, proporciona:

- La representación de la curva o superficie de interacción de secciones mixtas, según se trate de casos de flexocompresión recta (respecto de cada uno de los ejes principales de la sección) o esviada. En los apartados 4.6.2 y 4.6.4, se muestran varios de estos gráficos uno de los cuales se reproduce a continuación.

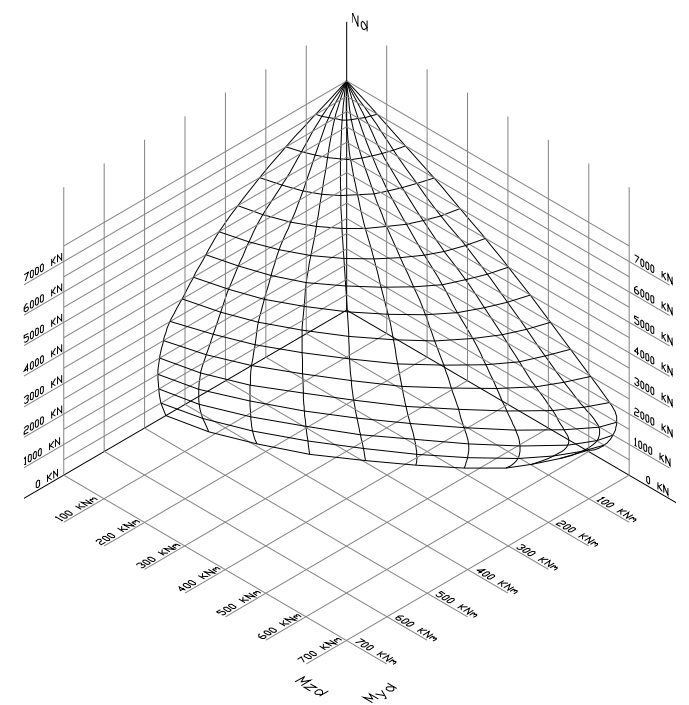

Figura 9.1-1 Superficie mécanica de una sección mixta

- La comprobación de cualquier sección mixta solicitada a flexocompresión plana o esviada, bajo los parámetros de cálculo definidos por el usuario.

- La posición de equilibrio (fibra neutra y curvatura del plano deformado) para el caso de flexocompresión plana, mediante un proceso iterativo. Este aspecto adquiere importancia especial en el análisis en régimen no lineal de la barra y de la estructura. 


\section{Efectos reológicos:}

2.1. Se han estudiado los distintos parámetros que afectan al comportamiento reológico del hormigón y los criterios para su evaluación según las normativas EHE-08, EC2 y EC4. A partir del criterio del Eurocódigo 2, se ha elaborado un método que se ha incluido en el algoritmo de análisis (apartado 5.5)

2.2. La cuantificación de los efectos reológicos se ha contemplado como una opción en el programa informático desarrollado con motivo del presente trabajo, de forma que el usuario los pueda tener en cuenta en el cálculo del soporte.

2.3. Se han elaborado los gráficos comparativos, mostrados en el apartado 5.6, para evaluar la influencia de los efectos reológicos en la capacidad resistente en un soporte mixto.

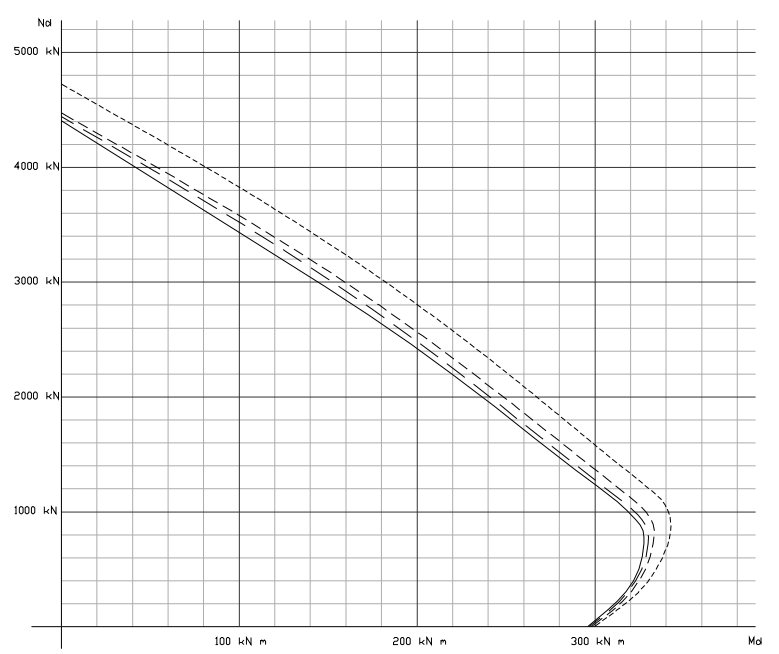

Tabla 9.1-1Influencia de los efectos reológicos del hormigón

Como conclusión de este estudio, se puede decir que la consideración de los efectos reológicos del hormigón: 
- Afectan más en compesión centrada que en flexión simple, con reducciones de la capacidad resistente del $7 \%$ y $1,3 \%$ respectivamente, cuando la flexión se produce respecto del eje fuerte. La capacidad resistente disminuye del orden de un $5 \%$ en el caso de flexión con respecto al eje débil.

- La utilización del diagrama tensión-deformación diferido en el hormigón, según la figura Figura 5.5-1, conduce a resultados similares a la aplicación del diagrama noval afectando a la resistencia con un coeficiente de cansancio del hormigón, alfa, igual a 0,85.

\section{La "viga-columna":}

3.1. El análisis de la sección mixta se ha extendido a la "vigacolumna", entendida como pieza aislada e intraslacional, sometida a flexocompresión recta o esviada. Se han estudiado, entre otros, el efecto de la longitud de la barra, las imperfecciones iniciales, la forma que adopta la directriz deformada y distintos métodos para cuantificar los efectos de segundo orden. Como conclusión del estudio, en el apartado 7.5, se desarrolla un procedimiento para abordar su cálculo, basado en el método de Newmark.

3.2. El análisis de la barra se realiza mediante la integración del módulo de cálculo de secciones mixtas en un avanzado programa de análisis no lineal de estructuras.

El comportamiento de la sección contempla la no linealidad del material, incluso con el estado de fisuración. Esto permite corregir el módulo de rigidez, E·I, en cada tramo de la barra y en cada iteración. 
Como aplicación, se han elaborado las curvas de interacción en flexocompresión recta, de una barra de sección mixta en la que se han considerado distintas longitudes y diferentes momentos aplicados en los extremos (apartado 7.6.1).

A modo de ejemplo, se reproduce a continuación, una de las superficies mecánicas obtenidas.

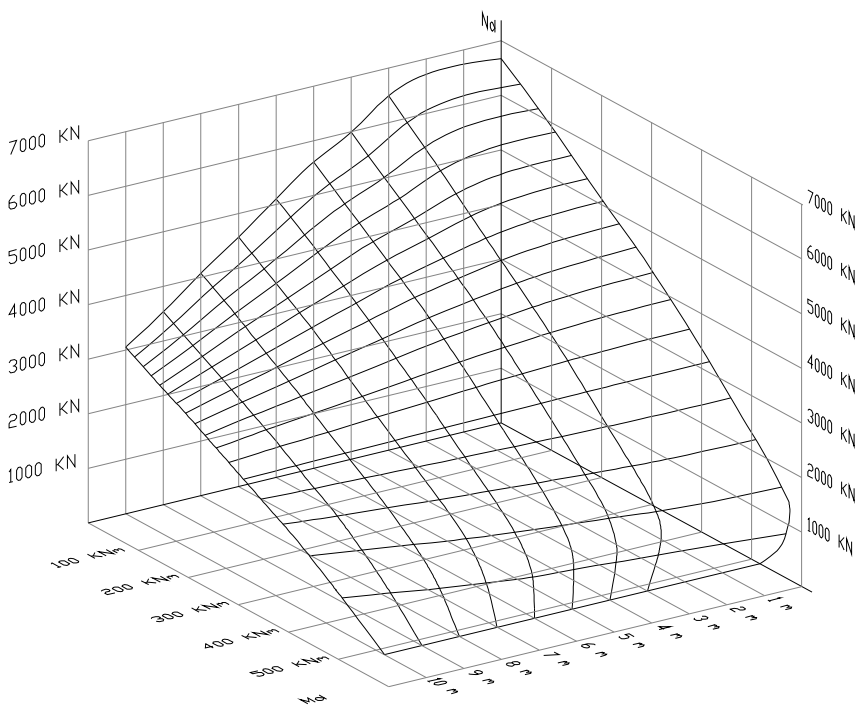

Figura 9.1-2 Superficie mecánica de un soporte mixto

3.3. Utilizando el programa, podemos calcular la capacidad resistente de cualquier otro tipo de soporte. Únicamente requiere la adecuada modelización de la geometría, características de los materiales y del estado de carga.

3.4. Además, se han elaborado dos tipos de gráficos comparativos, basados en las curvas de interacción de la "viga-columna" que se exponen en los apartados 7.6.2 y 7.6.3. 
El primer conjunto de gráficos, ver Figura 7.6-3, muestra el efecto de la no linealidad del material. Los casos estudiados son un soporte de $5 \mathrm{~m}$ y otro de $10 \mathrm{~m}$ de longitud. De su análisis se puede concluir que:

- Afecta más a los soportes de mayor longitud. En el caso del esfuerzo axil centrado, la reducción es del $7,5 \%$ en el soporte de $5,00 \mathrm{~m}$ y del $35 \%$ en el de $10,00 \mathrm{~m}$

- La pérdida de capacidad resistente es mayor cuando predomina el esfuerzo axil que cuando lo hace la flexión.

El segundo gráfico, Figura 7.6-4, contempla la influencia de los fenómenos de retracción y fluencia del hormigón en un soporte de $5 \mathrm{~m}$. de longitud. De su estudio se concluye que:

- La pérdida de la capacidad resistente ya observada en la sección se reproduce, aproximadamente, en la curva de interacción del soporte.

- Esta reducción de la capacidad resistente de la barra se cuantifica entre el $10 \%$ en compesión centrada y el $3 \%$ en flexión simple.

\section{El conjunto estructural:}

4.1. Para modelizar el comportamiento de los soportes de la forma más exacta posible hemos abordado el cálculo de un conjunto estructural, donde algunos o todos los elementos son de sección mixta.

En los soportes se han considerado las imperfecciones iniciales de acuerdo con el Eurocódigo 4. 
El equilibrio se plantea en la estructura deformada. La rigidez en cada tramo de la barra es evaluada teniendo en cuenta la fisuración de la sección y los diagramas de comportamiento de los tres materiales que la componen, para cada una de las iteraciones realizadas en la búsqueda del equilibrio.

4.2. Como aplicación práctica, hemos analizado el efecto de la traslacionalidad de los dos sistemas estructurales más representativos (apartado 8.6.1).

Uno de tipología de una planta de gran luz, en una nave no arriostrada de $18 \mathrm{~m}$. de vano, comparando el análisis de un modelo que considera las imperfecciones iniciales en los soportes y planteando el equilibrio en la estructura deformada, respecto del análisis tradicional con tramos rectos y linealidad geométrica.

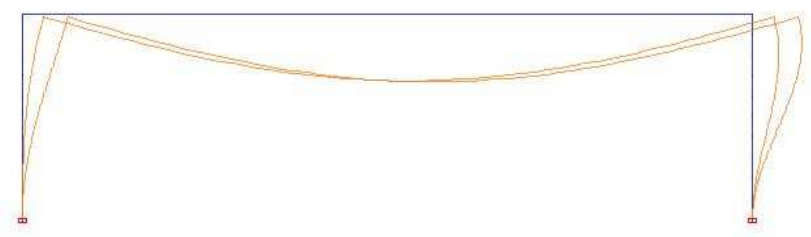

Figura 9.1-3 Comparación de deformaciones. Análisis lineal y no lineal

Como conclusiones más importantes cabe citar:

- El incremento de los momentos flectores obtenidos en el modelo sin imperfecciones mediante un análisis no lineal supera el $10 \%$ al del cálculo tradicional, al menos en algún nodo del soporte. 
- El incremento de los momentos flectores es significativamente mayor si se parte de un modelo donde se consideran imperfecciones iniciales en los soportes.

- Recordemos que el 10\% representa el límite a partir del cual todas las normas, $\mathrm{ACl}$, AISC y EC, impiden el cálculo lineal de la estructura. Luego, en estos casos, sería necesario algún tipo de arriostramiento, como es práctica imprescindible en cualquier edificación

El segundo caso es de una tipología en altura, donde suele ser más habitual el uso de secciones mixtas en los soportes.

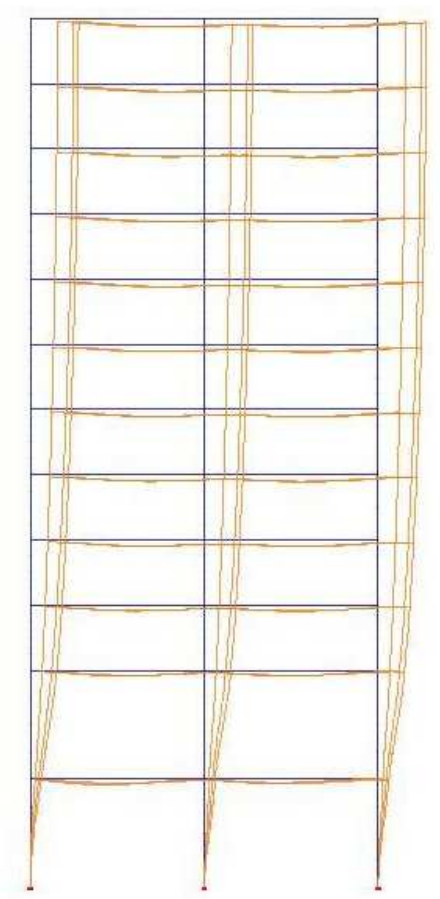

Figura 9.1-4 Comparación de deformaciones. 
Sin considerar ningún tipo de arriostramiento y contemplando siempre las imperfecciones iniciales, hemos realizado tres análisis:

No linealidad geométrica y mecánica

No linealidad geométrica y módulo de rigidez constante

Cálculo lineal tradicional

Las conclusiones obtenidas son las siguientes:

- El incremento de los momentos flectores obtenidos en el análisis no lineal geométrico respecto del cálculo tradicional, supera el $10 \%$, incluso en algún soporte de la sexta planta, amplificándose por encima del $25 \%$ en varios soportes de la planta baja.

- A diferencia del análisis en régimen lineal, el cálculo no lineal de la estructura conduce al agotamiento del soporte central de la planta baja, incluso sin considerar la no linealidad mecánica.

- A la vista de estos resultados, el pórtico ensayado requiere un análisis en segundo orden, por aplicación de los artículos 2.5.1.4 y 4.3.5.3.3 del EC2 y 5.2.5.2 del EC3.

4.3. Finalmente, en el apartado 8.6.2, se muestra una aplicación práctica que permite el diseño y cálculo de una estructura tridimensional de varias plantas y la comprobación de sus soportes mixtos. 


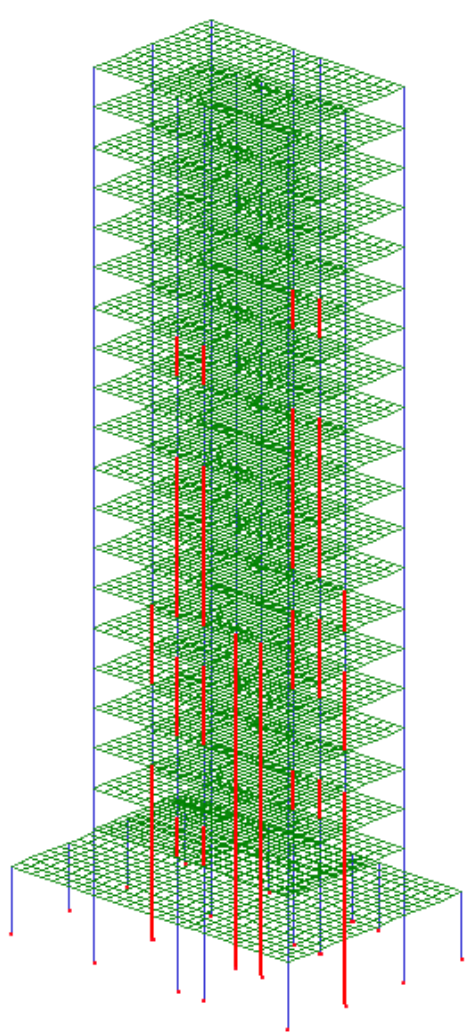

Figura 9.1-5 Visualización de resultados del cálculo de una estructura

Como conclusión general, cabe indicar que el cálculo no lineal de pórticos dimensionados con sección estricta, conduce fácilmente a incrementos de momentos flectores superiores al 10\%, incluso en los casos mostrados, buscados intencionadamente, que no son de gran dimensión o altura.

Por lo que, o bien se realiza un cálculo en segundo orden, o se diseñan los adecuados sistemas constructivos que garanticen el arriostramiento de la estructura. 


\subsection{DESARROLLO POSTERIOR}

Si bien las conclusiones expuestas en el apartado anterior muestran que se ha alcanzado tanto el objetivo global como los parciales inicialmente planteados, durante el proceso de desarrollo de cualquier trabajo de investigación surgen nuevas ideas para ampliar y completar el problema.

Desde este punto de vista, el trabajo realizado, además de conseguir sus propios objetivos, pasa a formar parte de un proyecto de investigación más amplio en el que se plantean los siguientes aspectos:

1. La elaboración de un catálogo de curvas de interacción de soportes de distintas secciones, longitudes, materiales, etc., a modo de prontuario, que pudiera ser de fácil aplicación práctica en el diseño y cálculo de soportes mixtos.

No obstante, dado el considerable número de gráficos, si se quiere abarcar los casos más usuales en edificación, sería interesante el estudio de la forma de organizar las tablas, por ejemplo, según la esbeltez de la barra.

2. La extensión, al caso de la flexocompresión esviada, de la rutina desarrollada que localiza la posición de la fibra neutra y la curvatura de equilibrio entre las solicitaciones exteriores y los esfuerzos internos.

Esto únicamente requiere añadir una nueva variable, la oblicuidad de la fibra neutra respecto de los ejes principales de la sección, en un proceso iterativo actualmente disponible para el caso plano. 
3. La integración del programa de cálculo de secciones mixtas en el mencionado programa de análisis no lineal representa una potente herramienta con la que se pueden abordar otros estudios sobre el conjunto estructural, que podrían ser motivo de otras tesis o de proyectos de investigación más amplios

Entre ellos, se puede citar el estudio sobre la repercusión en el coeficiente de seguridad global, de la formación de rótulas plásticas en función de si éstas se inician en las vigas o en el propio soporte, o el efecto del instante en que se aplican los coeficientes de seguridad: durante el proceso de análisis 0 , al dimensionar, finalizado el análisis.

4. El reajuste de la rutina de comprobación de secciones mixtas que permita redimensionar las secciones automáticamente. Estos cambios se realizarían siempre bajo el criterio del proyectista que decidirá las variables sobre las que intervenir: dimensiones de la sección y del perfil metálico, número y diámetro de las armaduras de refuerzo longitudinal o resistencia de los materiales.

Con respecto del trabajo realizado que aquí se ha expuesto, esto únicamente implica corregir la discretización de la sección modificada. 


\section{0. \\ ANEXO 1 \\ CARACTERISTICAS \\ AV_CID}




\subsection{INTRODUCCIÓN}

\section{INTRODUCCIÓN}

A grandes rasgos, se podría describir el programa AV_CID ${ }^{142}$ como un programa informático de diseño y cálculo de estructuras compuesto por los siguientes módulos:

1. Módulo de generación del modelo de diseño de la estructura en un entorno CAD (Computer Asist Design).

2. Módulo que procesa los datos incluidos en el modelo de diseño y genera la base de datos del modelo de cálculo.

3. Módulo de cálculo de estructuras (solicitaciones y deformaciones) por aplicación del método de los elementos finitos.

4. Módulo de visualización gráfica de resultados.

5. Módulo de dimensionamiento de elementos de hormigón armado y de comprobación de elementos de acero.

El desarrollo de este programa de diseño y cálculo de estructuras, así como la explicación completa y exhaustiva de sus bases de cálculo y procedimientos empleados dio lugar la Tesis Doctoral ${ }^{143}$ del profesor D. Adolfo Alonso Durá, por lo que a continuación se realizará una exposición únicamente de los aspectos que se consideran más relevantes.

142 Programa informático "AV_CID: Calcul i Diseny d'estructures". Autor: D. Adolfo Alonso Durá

${ }^{143}$ Tesis Doctoral: "Un modelo de integración del análisis estructural en entornos CAD para estructuras de edificación." Adolfo Alonso Durá (2003) 


\subsection{MÓDULO DE DISEÑO EN ENTORNO CAD}

Aunque el planteamiento es aplicable a cualquier entorno CAD, la aplicación para la generación del modelo estructural (entrada de datos) fue desarrollado en el entorno concreto de AutoCAD. La elección de este entorno se debe a su amplia utilización como programa de dibujo en el ámbito de la arquitectura y a que su diseño permite incorporarle aplicaciones que amplían sus posibilidades gráficas.

Para facilitar la generación del modelo de diseño de la estructura, se han desarrollado una serie de menús e iconos integrados en el propio programa de dibujo AutoCAD.

\section{Sistemas de referencia}

El programa utiliza dos sistemas de referencia: un sistema de referencia de ejes globales para el conjunto de la estructura y otro de ejes locales para cada barra.

El sistema de referencia de ejes globales coincide con el definido en el espacio modelo de AutoCAD, de forma que los elementos horizontales tipo viga se sitúan en el plano $X Y$ y los elementos verticales tipo soporte se sitúan según el eje $Z$.

A partir de las coordenadas de los extremos de cada barra, el módulo de cálculo genera un segundo sistema de referencia de ejes locales de barra.

\section{Tipos estructurales}

Aunque el módulo de análisis realiza un cálculo tridimensional de la estructura, con el objeto de simplificar la estructura y reducir el 
tiempo de cálculo, se han definido 5 tipos estructurales, dos de ellos planos (tanto los elementos estructurales como las cargas se encuentran en un único plano, el plano $\mathrm{YZ}$ de AutoCAD) y tres espaciales (tanto los elementos estructurales como las cargas pueden estar en cualquier plano del espacio):

Celosías planas: Disponen de dos grados de libertad por nudo (dos traslaciones). El programa asume que todos los nudos son articulaciones.

Rígidas planas: Disponen de tres grados de libertad por nudo (dos traslaciones y un giro). El programa asume que todos los nudos son rígidos, aunque el usuario puede modificar alguna de sus características).

Emparrillados: Estructuras de nudos rígidos cuya geometría se encuentra exclusivamente en un plano horizontal y cuyas cargas son verticales. Disponen de tres grados de libertad por nudo (una traslación y dos giros)

Celosías espaciales: Estructuras tridimensionales de nudos articulados. Disponen de tres grados de libertad por nudo (tres giros).

Rígidas espaciales: Estructuras de nudos rígidos definidas tridimensionalmente, tanto en su geometría como en sus cargas. Disponen de seis grados de libertas por nudo (tres traslaciones y tres giros).

\section{Elementos estructurales}

Elemento lineal o barra: Definido como elemento lineal recto orientado en cualquier dirección del espacio y con un nodo en cada extremo. Con carácter general, en una barra pueden producirse esfuerzos axiles, cortantes, momentos flectores y momento torsor. El 
enlace de la barra al resto de la estructura se puede realizar mediante un nudo rígido, articulado o con un cierto grado de rigidez.

La entidad gráfica de AutoCAD que define la barra es la "Línea", que representa el eje de la barra. Las características estructurales de la barra (material, tipo y dimensiones de la sección y giro de la sección) se codifica en un valor alfanumérico que se asocia al tipo de línea.

Se pueden utilizar hasta 28 materiales distintos cuyas características mecánicas se definen a través de "bloques" con atributos.

Los tipos de sección posibles son los siguientes: secciones rectangulares y circulares macizas o huecas, secciones en $T$, perfiles normalizados HEB, IPE, IPN, HEA, HEM, 2UPN en cajón, perfiles tubulares circulares, cuadradas y rectangulares o secciones definidas por el usuario tales como las armadas. Las dimensiones de los perfiles normalizados y las secciones definidas por el usuario se obtienen a partir de ficheros ASCII que se incorporan a la base de datos del programa.

En cuanto al giro de la sección, se refiere al ángulo que forman los ejes principales de la sección respecto de los ejes locales de la barra.

Una vez asignadas las características de las barras, el módulo de diseño permite visualizar su volumen.

Elemento superficial: Elemento superficial plano triangular (3 nodos en sus vértices) o cuadrilátero (4 nodos), orientados en cualquier plano del espacio. Se distinguen elementos superficiales tipo membrana, cuando únicamente se producen deformaciones y solicitaciones en el propio plano del elemento, tipo placa, con flexiones en la dirección perpendicular al plano del elemento y tipo lámina, generado por la unión de los elementos tipo membrana y placa. 
La entidad gráfica de AutoCAD que define los elementos superficiales es la "3DCara", a las que se les asigna propiedades (material y espesor). Al igual que las barras, una vez asignado el espesor, puede visualizarse su volumen.

Elemento volumétrico: Elementos sólidos formados por tetraedros (4 nodos en los vértices) o hexaedros (8 nodos), ubicados en cualquier posición del espacio.

La entidad gráfica de AutoCAD que define el elemento volumétrico es la "Malla Poligonal", a la que únicamente se le asigna el material, ya que el resto de características quedan ya definidas por la entidad gráfica.

\section{Tipos de materiales}

La especificación de las propiedades de los materiales que componen la estructura se realiza mediante un "bloque" con atributos llamado "Material". Las propiedades que se definen en este bloque son: denominación, número, módulo de deformación, coeficiente de Poisson, densidad y coeficiente de dilatación térmica.

Se debe insertar un bloque distinto para cada material que compone la estructura.

\section{Vínculos exteriores}

Los vínculos exteriores de la estructura se especifican mediante distintas entidades gráficas "Bloque" (uno distinto en función de los movimientos que se pretendan coartar). Su punto de inserción indica el nudo cuyos movimientos se coartan.

El programa dispone de dos tipos de vínculos: 
Los genéricos, elementos teóricos que definen los movimientos que se coartan. Pueden ser planos o espaciales y entre ellos podemos citar los empotramientos, apoyos articulados fijos o deslizantes, muelles elásticos. El vínculo espacial genérico permite al usuario definir las condiciones de cada grado de libertad: libre, fijo (impedido) o aplicarle un movimiento elástico. Otro vínculo permite modelizar el comportamiento del suelo elástico a través del parámetro "módulo de balasto". El programa obtiene la constante de muelle del vínculo mediante el producto del coeficiente de balasto y la superficie asociada al vínculo.

Los constructivos, o cimentaciones (zapatas aisladas o combinadas y riostras), que el programa es capaz de dimensionar. En todas las zapatas se asume que el vínculo es del tipo empotramiento, es decir, una coacción total de los seis grados de libertad. Los atributos de estos bloques permiten definir criterios de dimensionamiento como el canto mínimo, la relación entre los lados o una dimensión fija de uno de los lados.

\section{Cargas}

Los distintos tipos de acciones se introducen en el modelo mediante diferentes entidades gráficas propias del programa AutoCAD. Todas ellas deben ser dibujadas en capas cuyo nombre comenzará por HIP (iniciales de "hipótesis" para indicar que se trata de una capa que contiene cargas) seguido de un número de dos dígitos (de 01 a 99) que indicará la hipótesis a la que se asocia la carga para su posterior combinación. ${ }^{144}$

El módulo de diseño permite definir cuatro tipos de carga: puntual, lineal, superficial y variaciones de temperatura. Todas ellas se

\footnotetext{
144 Para evitar errores, el módulo de diseño impide introducir cargas en capas cuyo nombre no comience por HIP.
} 
pueden situar en cualquier posición del espacio y actuar en cualquier dirección.

Gráficamente, las cargas puntuales y repartidas corresponden a "Polilíneas 3D", de tres vértices en el primer caso y de 6 en el segundo. Las cargas superficiales se definen mediante un "Bloque", que determina el valor y dirección de la carga, y una o varias "3Dcara" que determinan la superficie de actuación de la carga. La carga por variación de temperatura se define mediante un "Bloque" con dos atributos, uno para determinar la hipótesis en la que actúa y otro que especifica el valor de la variación térmica.

Si se requiere el cálculo dinámico de la estructura, es necesaria la definición de la carga sísmica mediante un "Bloque" con atributos que especifican la hipótesis de carga a la que se asigna y las hipótesis y coeficientes que intervienen para la obtención de la masa vibrante total.

Si además se desea realizar un cálculo no lineal, es necesario definir, mediante los atributos de otro "Bloque" la hipótesis de carga a la que se asigna este cálculo y las hipótesis de carga que se combinan y los respectivos coeficientes para la obtención del estado deformado que sirve de punto de partida.

\subsection{MODULO DE GENERACIÓN DE LA BASE DE DATOS DEL MODELO DE CÁLCULO}

\section{El fichero DXE}

Una vez definido el modelo de diseño (epígrafe anterior), el sistema requiere la generación de un fichero que contenga a todos los datos estructurales para su posterior análisis. 
Con este objetivo se ha desarrollado una aplicación que genera un fichero, con formato ASCII, en cierta manera semejante al fichero $D X F$, cuya extensión se ha denominado $D X E^{145}$, y que contiene, de forma estructurada y sistemática, todos los parámetros que definen el modelo estructural. El contenido y forma de ordenación de los datos permitirá una sencilla y rápida lectura desde el módulo de análisis de la estructura.

Este módulo se ha programado para generar el citado fichero desde el programa de dibujo AutoCAD, aunque podría desarrollarse en cualquier programa CAD en función de su propia estructura interna.

El contenido del fichero DXE se organiza en cuatro secciones:

Proyecto: Contiene los datos generales de la estructura.

Geometría: Contiene una información preliminar sobre el número de barras, de elementos superficiales y volumétricos, de materiales, etc. A continuación se facilita la información (posición, dimensión y material) de cada elemento estructural.

Contorno: Inicialmente se especifica el número de apoyos, zapatas, riostras, etc. A continuación se indica la posición y las coacciones que impone cada uno de los vínculos externos.

Acciones: Primero se indican el número de hipótesis y combinaciones de carga, el número de cargas puntuales, lineales y superficiales, el número de superficies de carga y de movimientos

\footnotetext{
${ }^{145}$ La diferencia con el formato DXF, (formato estándar de intercambio de ficheros de dibujo entre distintas plataformas $C A D$ ), consiste en que el DXF contiene todos los datos del dibujo, y no únicamente la información estrictamente estructural. Además la información de las entidades gráficas aparecen de forma aleatoria, lo que exige laboriosos algoritmos de lectura y selección de la información.
} 
forzados. A continuación, y para cada carga, se especifica el valor, posición e hipótesis a la que pertenece.

\section{La base de datos del programa de cálculo.}

El paso siguiente consiste en la lectura del fichero de datos DXE por el programa de cálculo, con el objeto de generar la base de datos de cálculo, ya que las entidades, a efectos de cálculo, poseen unas relaciones distintas a las que tienen a efectos de dibujo. Todos los datos se guardan en variables y matrices de una, dos o tres dimensiones.

El proceso de generación de la base de datos del modelo de cálculo consiste en tres etapas:

1.- Generación de los nudos y las conectividades de los elementos. A partir de la definición gráfica de las entidades gráficas, los dos extremos de las barras y los vértices de láminas y sólidos, se generan los nudos de la estructura ${ }^{146}$ y se establecen las conectividades entre los elementos y los nudos. Puesto que, inicialmente, la numeración de los nudos se ha realizado de una forma aleatoria, se ha desarrollado un algoritmo que los reordena para darles un orden lógico.

2.- Proceso de búsqueda de nudos para la asignación o indexado de diversos parámetros (coacción de movimientos en los vínculos exteriores, articulaciones internas y cargas, momentos o movimientos forzados aplicados en los nudos). Ello se debe a que estas propiedades o acciones se introducen en el modelo de diseño mediante elementos independientes y es necesario referenciarlas a un determinado nudo a través de su punto de inserción.

146 Cuando dos o más vértices coinciden, se genera un único nudo estructural 
3.- Proceso de búsqueda de elementos, barras y placas, para asignarles las acciones. El método de búsqueda consiste en localizar la barra que contiene las coordenadas que definen a cada una de las cargas.

\subsection{MÓDULO DE ANÁLISIS}

\subsubsection{ANÁLISIS LINEAL EN MODELOS DE BARRAS}

El módulo de análisis estructural de los modelos de barras, realiza el cálculo estático y lineal por el método matricial de las rigideces ${ }^{147}$, que se expone a continuación de forma general.

En un sistema con comportamiento lineal se considera que existe una proporcionalidad entre las acciones y las deformaciones que producen. Esta relación se puede expresar mediante la ecuación:

$$
s=k \cdot \delta
$$

Siendo $k$ la rigidez del elemento que relaciona en un determinado nodo la solicitación $s$ con su movimiento $\delta$.

El número de nodos varía entre 2 para los elementos lineales hasta 8 para los elementos volumétricos.

La solicitación $s_{i}$ en el nudo i, se expresa como un vector de fuerzas $(f)$ y momentos $(m)$, y el movimiento $\delta_{i}$ como un vector con tres traslaciones $(\delta)$ y tres giros $(\varphi)$, en ambos casos expresados respecto de los ejes locales de la barra.

147 Tesis Doctoral "Un modelo de integración del análisis estructural en entornos de CAD para estructuras de edificación" presentada por $D$. Adolfo Alonso Durá en diciembre de 2003 


$$
S_{i}=\left|\begin{array}{l}
f_{x} \\
f_{y} \\
f_{z} \\
m_{x} \\
m_{y} \\
m_{z}
\end{array}\right| ; \quad \delta_{i}=\left|\begin{array}{l}
\delta_{x} \\
\delta_{y} \\
\delta_{z} \\
\varphi_{x} \\
\varphi_{y} \\
\varphi_{z}
\end{array}\right|
$$

La solicitación en el nudo $\boldsymbol{i}$ producida por movimientos del nudo $\boldsymbol{j}$, suponiendo el resto de nudos indeformables se puede obtener mediante la expresión

$$
s_{i}=k_{i j} \cdot \delta_{j} \quad ; \quad \text { Para } i=1, \ldots n ; \quad y \quad j=1, \ldots n
$$

Si por el contrario, todos los nodos de un elemento experimentan movimientos, la solicitación en uno de ellos producida por dichos movimientos se puede expresar en forma de sumatorio:

$$
s_{i}=\sum_{j=1}^{n} k_{i j} \cdot \delta_{j}
$$

Las solicitaciones de todos los nodos de un elemento se pueden obtener mediante la siguiente expresión matricial:

$$
\left|\begin{array}{c}
s_{1} \\
\ldots \\
s_{i} \\
\ldots \\
s_{n}
\end{array}\right|=\left|\begin{array}{ccccc}
k_{11} & \ldots & k_{1 i} & \ldots & k_{1 n} \\
\ldots & \ldots & \ldots & \ldots & \ldots \\
k_{i 1} & \ldots & k_{j i} & \ldots & k_{i n} \\
\ldots & \ldots & \ldots & \ldots & \ldots \\
k_{n 1} & \ldots & k_{n i} & \ldots & k_{n n}
\end{array}\right| \cdot\left|\begin{array}{c}
\delta_{1} \\
\ldots \\
\delta_{i} \\
\ldots \\
\delta_{n}
\end{array}\right|
$$

Para poder imponer las condiciones de equilibrio es necesario transformar la matriz de rigidez de cada elemento de sus ejes locales, en que se expresaba en las ecuaciones anteriores, al sistema de referencia de ejes globales: 


$$
K_{i j}=R \cdot k_{i j} \cdot R^{T}
$$

Siendo $R$ la matriz de rotación entre ambos sistemas de referencia.

Al imponer la condición de equilibrio de todos los nudos de la estructura queda formada la matriz de rigidez total mediante un ensamblaje de las matrices de rigidez de cada elemento.

Los movimientos de todos los nudos $(\Delta)$ de una estructura se pueden obtener mediante la resolución de la siguiente expresión:

$$
S=K \cdot \Delta \rightarrow \Delta=K^{-1} \cdot S
$$

Donde $K$ representa la matriz de rigidez global de la estructura y $S$ las acciones aplicadas en los nudos.

Conocidos estos movimientos las solicitaciones de cada elemento se determinan a partir de su propia matriz de rigidez.

\subsubsection{MODELO DE ELEMENTOS FINITOS SUPERFICIALES $Y$ VOLUMÉTRICOS}

El procedimiento de cálculo de deformaciones, solicitaciones y tensiones en el caso de las estructuras superficiales y volumétricas se basa en el método de los elementos finitos.

Para poder efectuar el análisis, el modelo de diseño de la estructura continua debe "discretizarse" en un conjunto de elementos, de dimensión "finita", bidimensionales 2D (en las estructuras superficiales) 0 de elementos 3D (en el caso de estructuras volumétricas). 
La diferencia principal respecto del modelo de barras, consiste en la obtención de la matriz de rigidez local de los elementos. El proceso sigue las etapas que se describen a continuación:

1. Definición del campo de desplazamientos. Se trata de la obtención de unas expresiones denominadas funciones de interpolación que, conocida la geometría y/o los desplazamientos de los nudos de un elemento, permiten deducir los valores correspondientes en cualquier punto del elemento.

2. Obtención del campo de deformaciones. Se trata de las expresiones que relacionan las deformaciones unitarias del material con los movimientos de cualquier punto del elemento.

3. Determinación del campo de tensiones. Consiste en una expresión, particularizada para cada elemento, que relaciona las tensiones con las deformaciones a través de la matriz constitutiva del material.

4. Obtención de las fuerzas nodales equivalentes. Por tales se definen las fuerzas que, actuando sobre los nudos, son estáticamente equivalentes a las fuerzas que actúan sobre el elemento.

5. Aplicación del principio de los trabajos virtuales. Sobre el elemento en equilibrio entre cargas externas y tensiones internas, se aplica un desplazamiento virtual infinitesimal en los nodos, compatibles con las condiciones de contorno. Por imposición del equilibrio interno y externo, extendidos por integración a todo el volumen del elemento, y sustituyendo la relación entre tensiones y deformaciones, es posible deducir 
la matriz de rigidez del elemento, que se particulariza para cada uno de ellos.

Una vez determinada la matriz de rigidez de los elementos en ejes locales, se transforma a ejes globales para poderlas ensamblar en la matriz de rigidez global $K$ de la estructura. De la resolución de la expresión $\Delta=K^{-1} \cdot F$ se obtienen los movimientos de los nudos, a partir de los cuales se deducirán las tensiones en determinados puntos del elemento para transformarlas en las tensiones sobre los nudos donde se promedian los valores obtenidos en los distintos elementos que concurren en el nudo.

\subsubsection{MÓDULO DE ANÁLISIS EN SEGUNDO ORDEN}

La diferencia fundamental respecto del análisis lineal consiste en que la matriz de rigidez de la estructura no presenta valores constantes sino que sus componentes dependen de los movimientos y de las cargas aplicadas.

El procedimiento de resolución del problema no lineal desarrollado en el programa AV_CID consiste por un lado en un tratamiento incremental de la aplicación de la carga y por otro en un proceso iterativo para la búsqueda de la solución en cada paso.

Así, en cada uno de los incrementos e iteraciones se plantea una variante de la ecuación de equilibrio: $\left[K_{t}\right]\{d \Delta\}=\{d P\}$ que se resuelve en el campo lineal. La resupuesta total de la estructura se obtiene mediante la suma de los incrementos.

En la actualidad se encuentran desarrolladas dos opciones de análisis no lineal (ver recuadro en la Figura 10.4-1):

Análisis no lineal geométrico: 
Desarrollado con carácter general para cualquier tipo de estructura.

La matriz de rigidez para la resolución de cada uno de los intervalos de carga tiene en cuenta la geometría deformada producida en el intervalo anterior, pero mantiene a lo largo de todo el proceso el módulo de rigidez inicial $E \cdot I$.

Análisis no lineal geométrico y del material:

Disponible únicamente para el caso de soportes mixtos gracias a la integración en el entorno AV_CID, del módulo de cálculo desarrollado con motivo del presente trabajo.

La matriz de rigidez para la resolución de cada uno de los intervalos de carga tiene en cuenta tanto la geometría deformada producida en el intervalo anterior como las variaciones en el módulo de rigidez E.I producidas por la plastificación de los materiales y la fisuración del hormigón (ver el organigrama de la Figura 8.5-1)

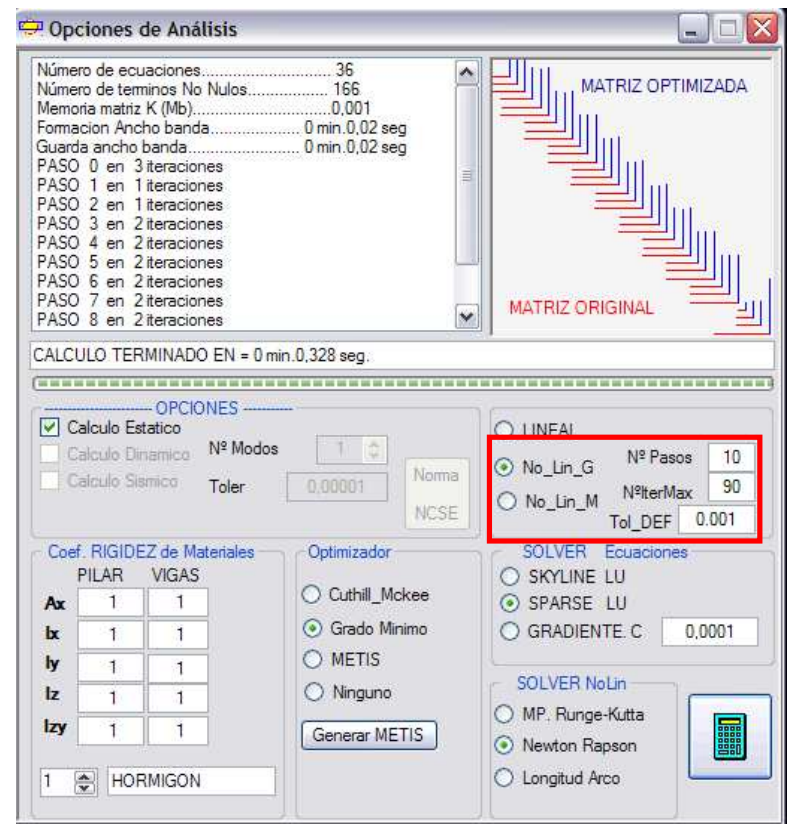

Figura 10.4-1 Ventana para definición de Opciones de Análisis 
El análisis en régimen no lineal exige la definición del número de intervalos ( $n^{\circ}$ pasos) en que se aplica la carga y del número de iteraciones ( $n^{\circ}$ iter max) que se realizarán en la búsqueda de la solución de cada uno de ellos.

Finalizado el cálculo, el programa permitirá recuperar las deformaciones y solicitaciones de la estructura en cada intervalo de cargas, lo que permite trazar toda la historia de movimientos desde su posición inicial no deformada hasta su configuración final.

En cuanto al proceso iterativo ${ }^{148}$ para la resolución de cada intervalo de carga, el programa AV_CID dispone de tres alternativas: el método del Punto Medio de Runge-Kutta, el método NewtonRaphson modificado y el de la Longitud de Arco.

La aplicación de una carga total superior a la de colapso, la adopción de un adecuado número de pasos para su aplicación y la comprobación del agotamiento de la sección en cada uno de los pasos, permitirá determinar el elemento crítico, el valor de la carga última y si el colapso se produce por inestabilidad o por agotamiento del material.

\subsubsection{ALMACENAMIENTO DE LA MATRIZ DE RIGIDEZ}

Uno de los factores que más influye en la reseva de memoria para el almacenamiento y estructuración de la matriz de rigidez $y$, posteriormente en los tiempos de cálculo, es la numeración de los nudos de la estructura.

\footnotetext{
${ }^{148}$ La descripción de distintos métodos de resolución iterativos se expone en el apartado 8.4 del presente trabajo.
} 
El programa AV_CID realiza una numeración inicial de los nudos, en función del valor de sus coordenadas. Esta numeración suele conducir a un gran ancho de banda en la matriz de rigidez global, por lo que se han implementado distintos procedimientos de renumeración, basados en el algoritmo de Cuthill y McKee, con el objetivo de minimizarlo.

Como la numeración óptima depende de la elección del primer nudo y es imposible saber que nudo inicial conducirá a la mejor ordenación de todos los nudos de la estructura, y con ello al menor ancho de banda de la matriz, el programa AV_CID realiza tres ordenaciones asignando como primer nudo al nudo inicial de la ordenación geométrica, al nudo final y a un nudo intermedio. De las tres, se adopta aquella que produce el nemor ancho de banda.

Con el objeto de reducir la cantidad de memoria necesaria para el almacenamiento de datos, puesto que la matriz de rigidez de la estructura es simétrica, sólo es necesario guardar una semibanda. Ésta se almacena por columnas y únicamente hasta la altura de valores no nulos, según el denominado método del perfil o sky-line.

Como alternativa, la matriz de rigidez también puede almacenarse utilizando la teoría de las matrices dispersas (sparse).

\subsubsection{RESOLUCION DEL SISTEMA DE ECUACIONES}

El programa AV_CID utiliza para la resolución del sistema de ecuaciones el método de Crout modificado, variante del de eliminación de Gauss, consistente el la transformación de la ecuación inicial $[K]\{\delta\}=\{F\}$ en una triangular tal como se indica: 


$$
\begin{aligned}
& k_{11} \cdot \delta_{1}+k_{12} \cdot \delta_{2}+k_{13} \cdot \delta_{3}+\ldots . .+k_{1, n-1} \cdot \delta_{n-1}+k_{1 n} \cdot \delta_{n}=f_{1} \\
& k_{22} \cdot \delta_{2}+k_{23} \cdot \delta_{3}+\ldots . .+k_{2, n-1} \cdot \delta_{n-1}+k_{2 n} \cdot \delta_{n}=f_{2} \\
& k_{n-1, n-1} \cdot \delta_{n-1}+k_{n-1, n} \cdot \delta_{n}=f_{n-1} \\
& k_{n n} \cdot \delta_{n}=f_{n}
\end{aligned}
$$

Ecuación 10.4-1

De forma que la última incógnita se pueda despejar directamente:

$$
\delta_{n}=\frac{f_{n}}{k_{n n}}
$$

Ecuación 10.4-2

Conocido este valor, y sustituyendo progresivamente en la ecuación anterior, en un proceso denominado sustitución hacia atrás, se resulven el resto de incognitas:

$$
\delta_{n-1}=\frac{f_{n-1}-\left(k_{n-1, n} \cdot \delta_{n}\right)}{k_{n-1, n-1}}
$$

Ecuación 10.4-3

Para poder llega al sistema de ecuaciones mostrado, es necesario descomponer la matriz de rigidez en dos matrices triangulares, inferior y superior:

$$
[K]:\{\delta\}=\{F\} \rightarrow[L] \cdot[U] \cdot\{\delta\}=[L]\{\{y\}=\{F\}
$$

Siendo: 


$$
\begin{aligned}
& {[U]\{\delta\}=\{y\}} \\
& {[L]=\left[\begin{array}{cccc}
1 & 0 & 0 & \ldots \\
L_{21} & 1 & 0 & \ldots \\
L_{31} & L_{32} & 1 & \ldots \\
\ldots & \ldots & \ldots & \ldots
\end{array}\right] \quad[U]=\left[\begin{array}{cccc}
u_{11} & u_{12} & u_{13} & \ldots \\
0 & u_{22} & u_{23} & \ldots \\
0 & 0 & u_{33} & \ldots \\
\ldots & \ldots & \ldots & \ldots
\end{array}\right]}
\end{aligned}
$$

Donde los términos de estas matrices triangulares se obtienen:

$$
L_{j i}=\frac{K_{j i}-\sum_{m=1}^{i-1} L_{j m} \cdot u_{m i}}{u_{i i}} \quad u_{i j}=K_{i j}-\sum_{m=1}^{i-1} L_{i m} \cdot u_{m j}
$$

Los términos de la matriz $[L]$ se pueden colocar en función de los términos de la matriz $[u]$, por lo que se puede operar únicamente con los términos de ésta matriz previamente reducidos:

$$
u_{i j}=K_{i j}-\sum_{m=1}^{i-1} \frac{u_{m i}}{u_{m m}} \cdot u_{m j}
$$

Los términos de la diagonal de la matriz $[u]$ se obtienen mediante la expresión:

$$
u_{i j}=K_{j j}-\sum_{m=1}^{j-1} \frac{u_{m j}}{u_{m m}} \cdot u_{m j}
$$

Las componentes del vector $\{y\}$ se obtienen de:

$$
\begin{aligned}
& y_{1}=F_{1} \\
& y_{i}=F_{i}-\sum_{j=1}^{i-1} L_{i j} \cdot y_{j}=F_{i}-\sum_{j=1}^{i-1} \frac{u_{j i}}{u_{i j}} \cdot y_{j}
\end{aligned}
$$

Finalmente, conocida la matriz [u] y el vector $\{y\}$, las incógnitas $\{\delta\}$ se obtienen a partir de la ecuación $[U]\{\delta\}=\{y\}$, de dónde, dada su expresión triangular, que conduce a expresiones equivalentes a la 
Ecuación 10.4-1, se puede calcular la última incógnita y por susutitución hacia atrás, el resto mediante expresiones similares a la Ecuación 10.4-2 y Ecuación 10.4-3

\subsection{MÓDULO DE VISUALIZACIÓN DE RESULTADOS}

La visualización de la volumetría del modelo CAD puede obtenerse a partir de una opción que muestra el volumen de las barras a partir de los datos de su sección y de las órdenes renderización de AutoCad.

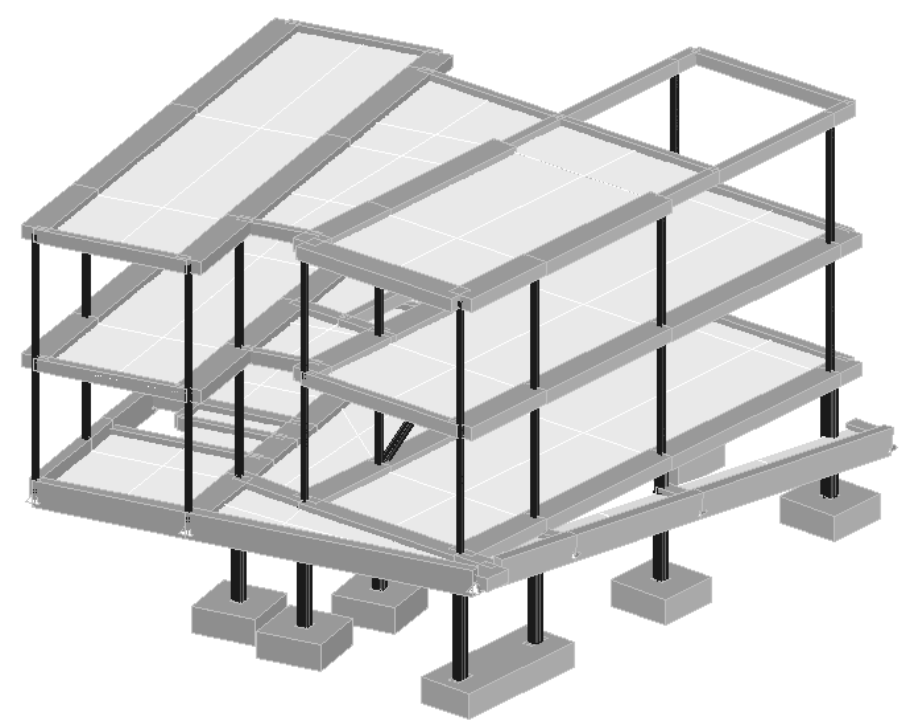

Figura 10.5-1 Modelo de la estructura. Volumetría.

El módulo de cálculo incluye distintas opciones de visualización que permiten el control y verificación de los resultados del análisis.

A modo de ejemplo, a continuación se muestran varias de las multiples opciones gráficas del programa, como la visualización de la geometría de la estructura, con los cuadros de características de la sección y cargas aplicadas sobre una de una de las barras y las 
propiedades de uno de los apoyos, el diagrama de momentos flectores y la visualización de la deformada de una estructura de barras, y por otro lado, el mapa de colores de los momentos flectores de una estructura superficial modelizada mediante elementos finitos.

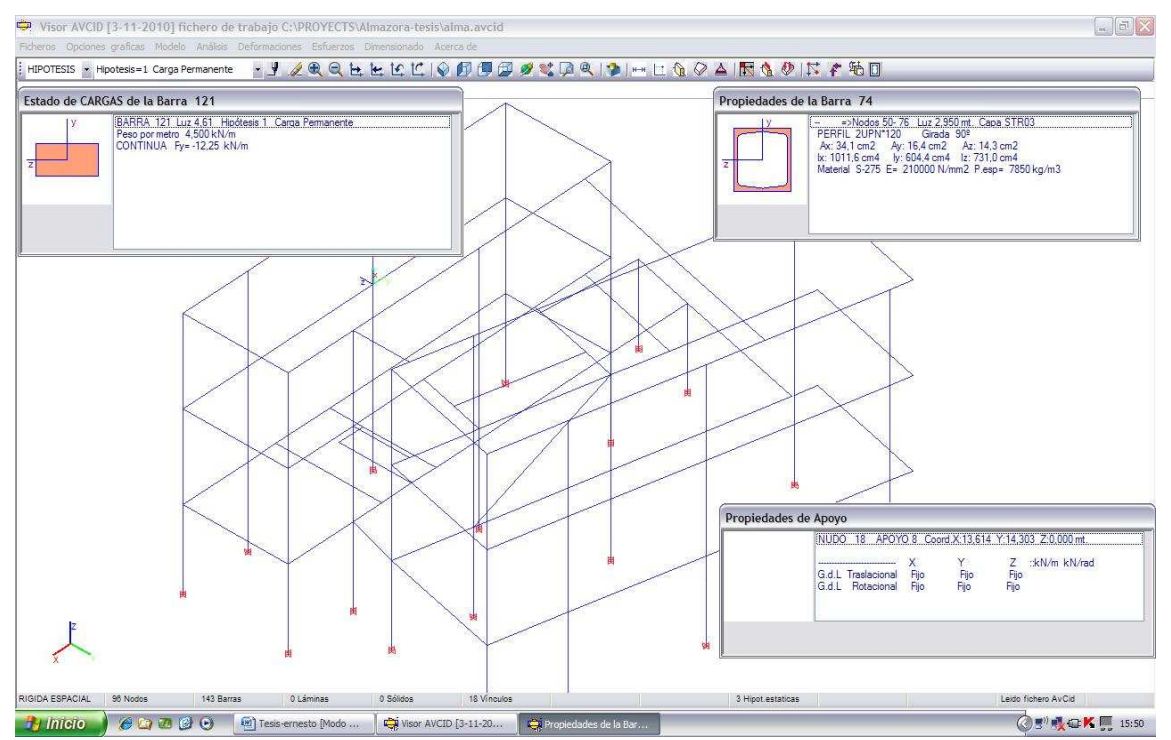

Figura 10.5-2 Visualización de la geometría.

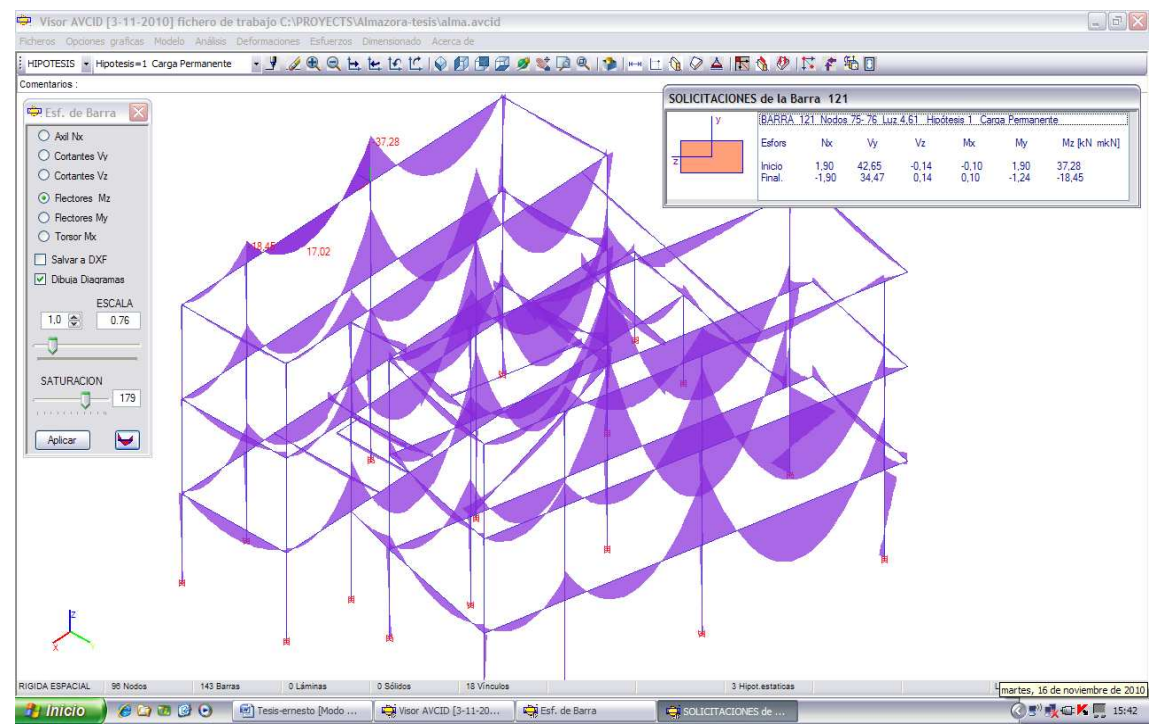

Figura 10.5-3 Visualización de solicitaciones. Diagrama de momentos flectores 


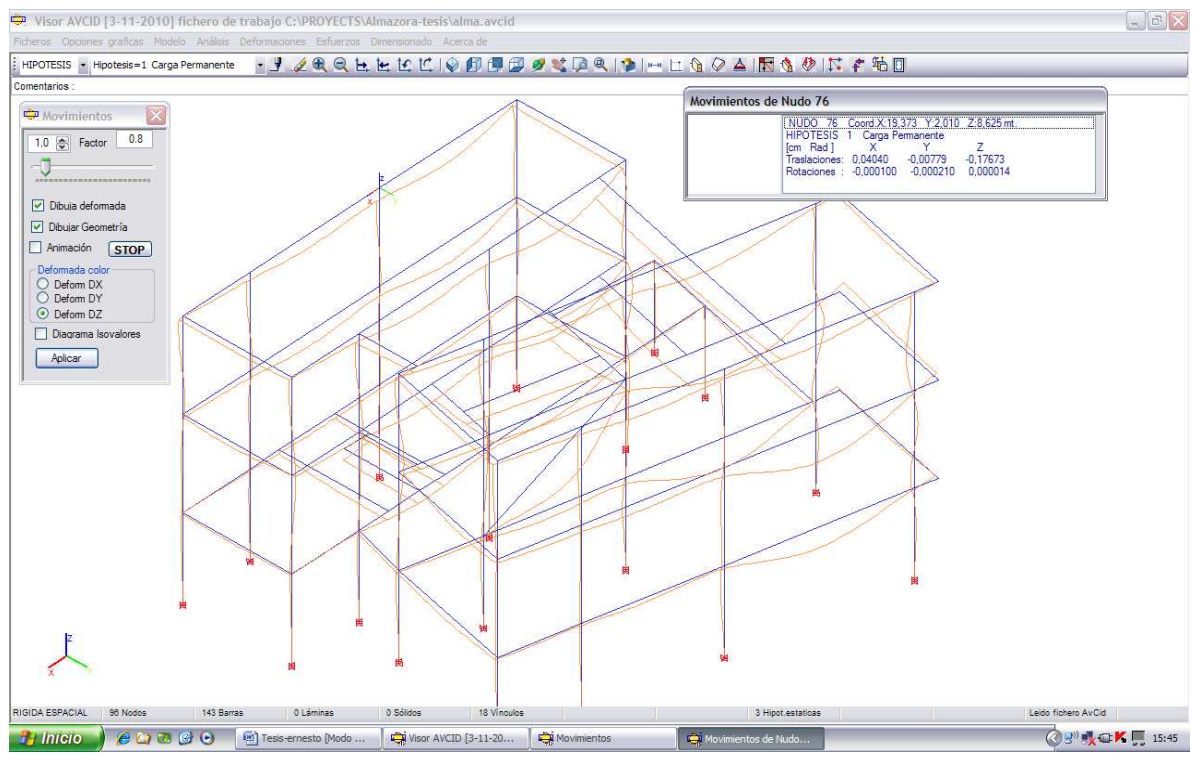

Figura 10.5-4 Visualización de deformaciones

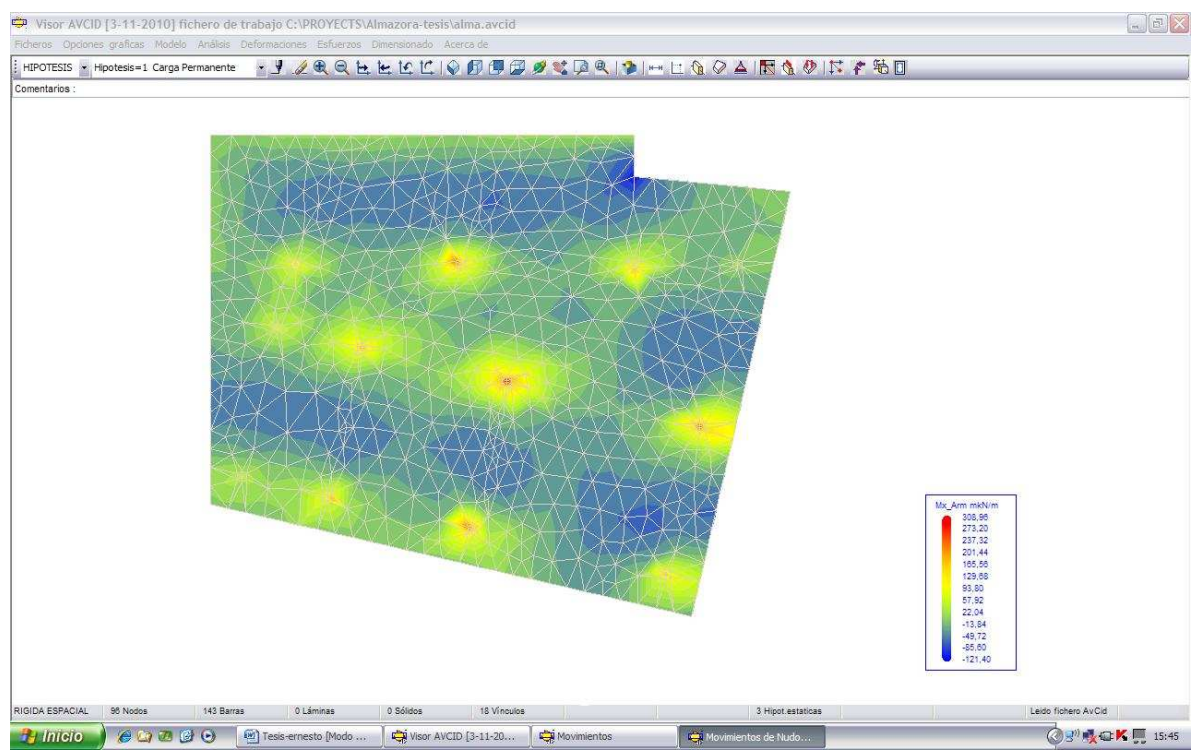

Figura 10.5-5. Solicitaciones en una losa de cimentación 
Otras posibilidades de este modulo de visualización se muestran en la Figura 10.6-2, en dicho caso aplicada a la visualización de los resultados obtenidos tras el dimensionamiento en hormigón armado.

\subsection{MÓDULO DE DIMENSIONAMIENTO}

Se han desarrollado una serie de aplicaciones que, a partir de las solicitaciones y deformaciones obtenidas en el módulo de cálculo, realizan el dimensionamiento de distintos elementos estructurales.

\section{Elementos de hormigón armado}

Esta aplicación realiza el dimensionamiento, según los criterios de la EHE (opcional según Eurocódigo 2) de elementos de hormigón armado: vigas de sección rectangular o en $\mathrm{T}$ y soportes de sección rectangular o circular.

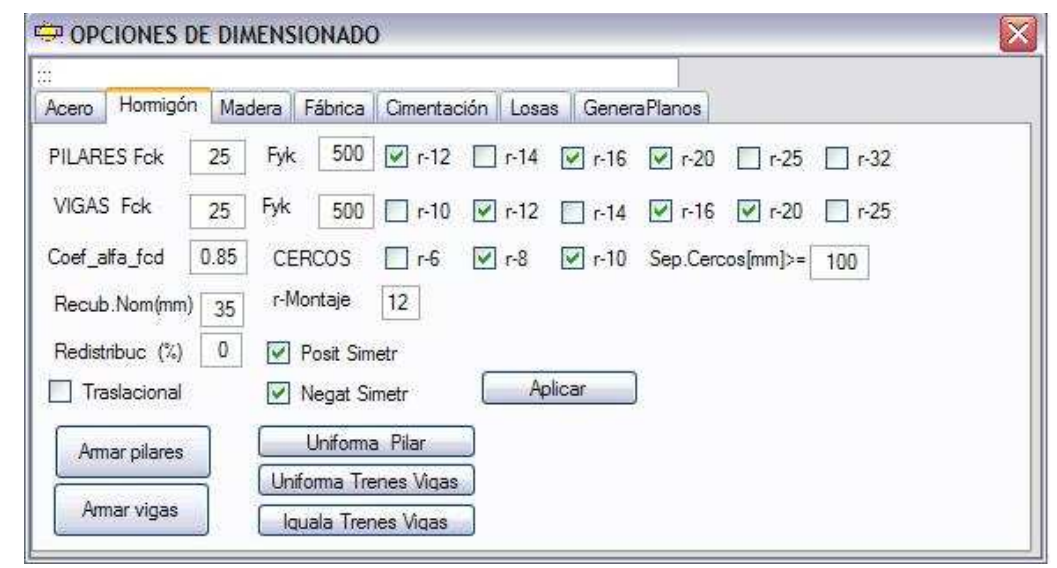

Figura 10.6-1. Cuadro de especificaciones técnicas para el dimensionamiento de elementos de hormigón armado.

La ventana de dimensionamiento en hormigón armado, (ver Figura 10.6-1) permite al usuario definir las resistencias del hormigón y de acero, los coeficientes de seguridad, parámetros de armado (como la 
redistribución de momentos, separación mínima de los cercos, simetría en las armaduras o grupos de armaduras), especificaciones de los diámetros con los que dimensionar vigas y soportes y los parámetros para el cálculo de la flecha.

Una vez realizado el dimensionamiento general de las barras de la estructura, la opción de peritación permite el análisis pormenorizado, barra a barra, y la modificación, a criterio del usuario, del resultado del cálculo. En la ventana flotante se accede a la modificación del armado, mientras que en la parte derecha de la pantalla se muestra la repercusión, respecto de las solicitaciones y coeficientes de seguridad, de los cambios realizados.

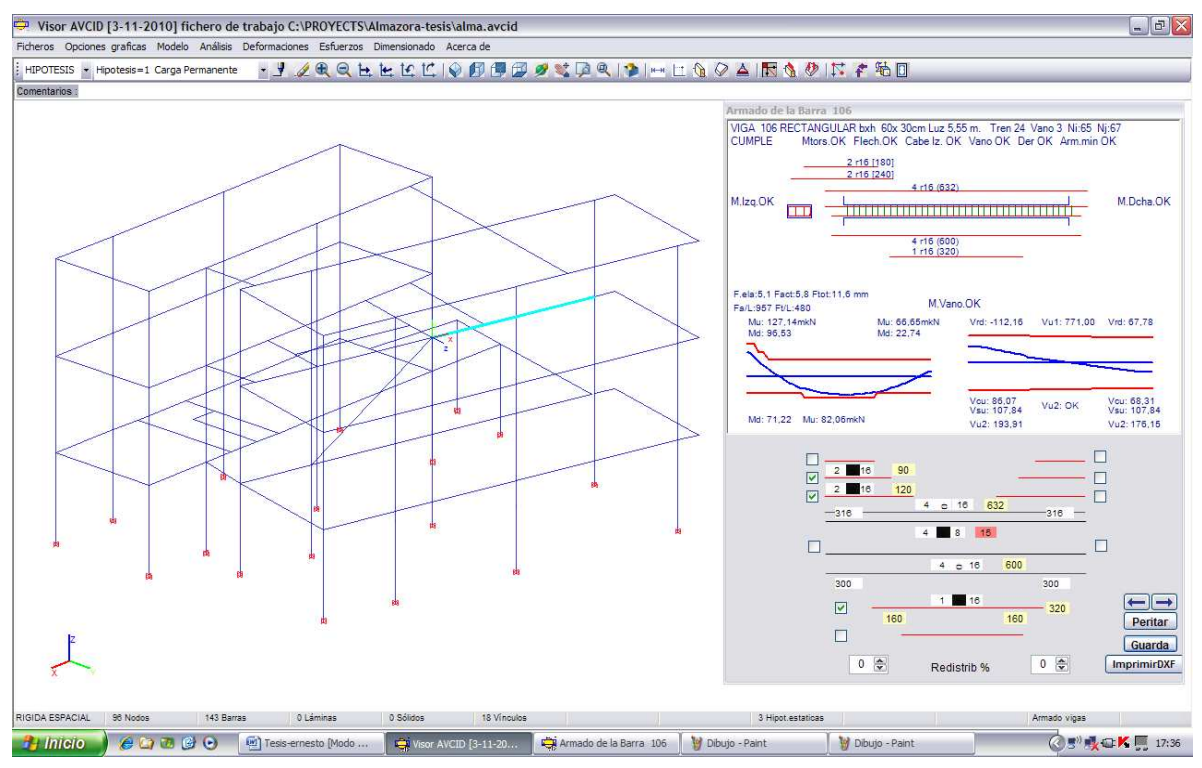

Figura 10.6-2. Peritación de una barra de hormigón armado

Una vez realizado y aceptado el dimensionamiento, la aplicación genera los planos de despiece de armaduras y el cuadro de pilares. 


\section{Elementos de acero}

Esta aplicación permite realizar la comprobación tensional, según criterios del Código Técnico (CTE SE-A), de las barras de acero formados por perfiles normalizados.

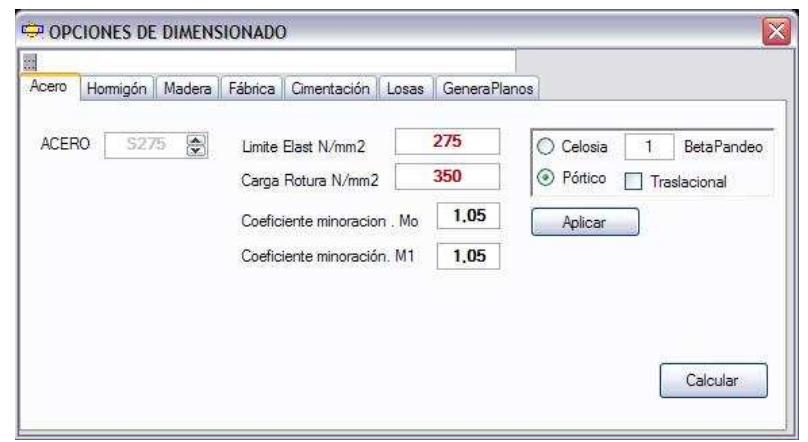

Figura 10.6-3 Cuadro de especificaciones técnicas para la comprobación de elementos de acero.

La ventana de dimensionamiento en acero, (ver Figura 10.6-3) permite al usuario definir la resistencia del acero, los coeficientes de seguridad y los parámetros para el cálculo de la flecha.

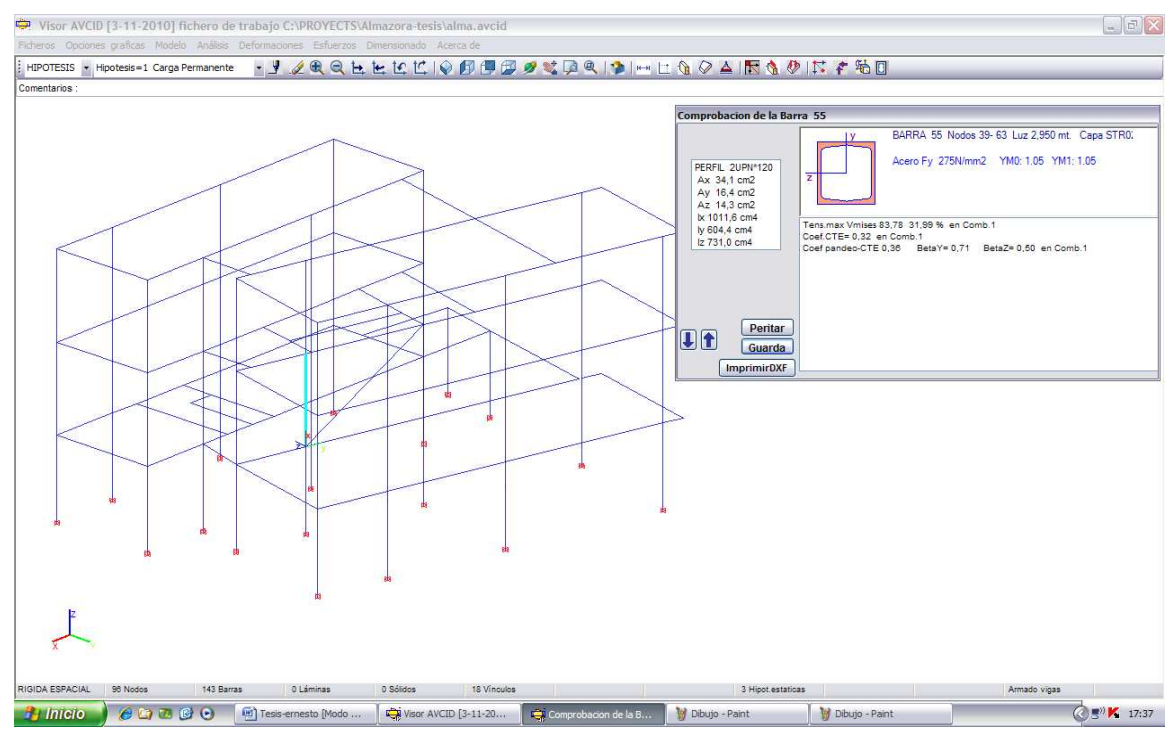

Figura 10.6-4 Peritación de una barra de acero laminado 
Para el caso de cimentaciones superficiales, otra aplicación permite realizar las comprobaciones geotécnicas, redimensionar las zapatas y riostras en caso necesario y calcular el armado de las mismas.

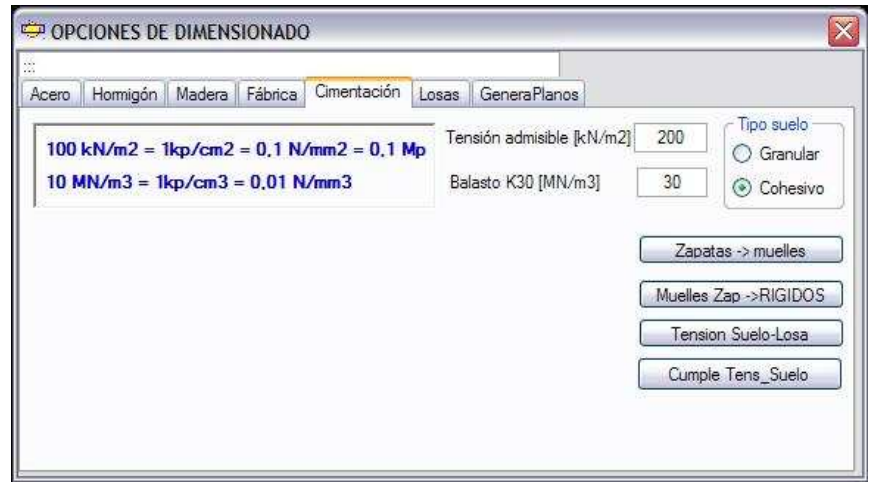

Figura 10.6-5 Cuadro de especificaciones técnicas para la comprobación y el dimensionamiento de cimentaciones.

La ventana de comprobación y dimensionamiento de cimentaciones, (ver Figura 10.6-5) permite al usuario definir la resistencia del hormigón y del acero, los coeficientes de seguridad, parámetros de armado (como los diámetros de las barras con las que dimensionar, recubrimientos y separaciones máxima de las armaduras o los parámetros para la comprobación de la fisuración) y las características del suelo (como la tensión admisible, el ángulo de rozamiento o el coeficiente de balasto).

Una vez realizado el dimensionamiento, la aplicación genera el plano de cimentación y los cuadros de armados de zapatas y riostras. 


\section{1. ANEXO 2 \\ LISTADO PROGRAMA SOPORTES MIXTOS}




\section{Variables generales}

Public cami\$, Nom\$

Public disty_cdg!(), distx_cdg!(), Disty_fibra!(), distx_fibra!(), angulo_giro!, incremento_angulo_giro!, repeticiones $\%$, giros $\%$

Public disty_g_cdg!(), distx_g_cdg!()

Public npasos $\mathrm{x} \%$, npasosy $\%$, dimx $\%$, dimy $\%$

Public pasosx!(), pasosy!(), material\%()

Public sigma_hor!, epsilon_ult_hor!, Ecm!, coeficiente_hor!, alfa!

Public sigma_st!, epsilon_y_st!, coeficiente_st!

Public sigma_ar!, epsilon_y_ar!, coeficiente_ar!

Public vert_mx!(), vert_my!(), result_x!(), result_y!(), result_3d!(), paralelos!()

Public cur!, eps!

Public axil!, mto_x!, mto_y!

Public area_x!, area_horm!, area_perfil!, area_redondos!

Public inercia_x_horm!, inercia_x_perfil!, inercia_x_redondos!

Type vert_tramos

$x$ As Single

y As Single

z As Single

n As Integer

End Type

Public coord_tramos() As vert_tramos

\section{Rutina de entrada de datos}

Private Sub Command1_Click()

Open cami\$ + "I" + Nom\$ For Input As \#1

Call leefichero

Close 
Ecm! $=$ Text6. Text

fibra! $=200$

Call calcula_inercia (Ecm!, fibra!, area_x!, inercia_x!, inercia_y!, area_horm!, area_perfil!, area_redondos!, inercia_x_horm!, inercia_x_perfil!, inercia_x_redondos!)

End Sub

\section{Rutina para leer fichero con datos de discretización de la sección}

Public Sub leefichero()

' Entrada de no divisiones y dimensiones de la sección

Input \#1, npasosx \%, npasosy\%, dimx\%, dimy\%

' Entrada de las dimensiones $x$ de cada división

ReDim pasosx!(1 To npasosx $\%+1$ )

For ni\% $=1$ To npasosx $\%$

Input \#1, pasosx!(ni\%)

Next ni\%

' Entrada de las dimensiones y de cada división

ReDim pasosy!(1 To npasosy\% + 1)

For ni\% = 1 To npasosy\%

Input \#1, pasosy!(ni\%)

Next ni\%

' Comprobacion de las dimensiones de la seccion

suma_pasosx $!=0$

suma_pasosy! =0

For ni\% $=1$ To npasosx $\%$

suma_pasosx! = suma_pasosx! + pasosx!(ni\%)

Next ni\%

For ni\% $=1$ To npasosy $\%$

suma_pasosy! = suma_pasosy! + pasosy!(ni\%) 
Next ni\%

' Distancia $x$ del cuadradito al CDG de la sección

ReDim distx_cdg!(1 To npasos $\%$ + 1)

distx_cdg! $(1)=($ pasosx $!(1) / 2)-(\operatorname{dim} x \% / 2)$

For ni\% $=1$ To $($ npasosx $\%-1)$

distx_cdg! $(\mathrm{ni} \%+1)=$ distx_cdg! (ni\%) + (pasosx!(ni\%) / 2) + (pasosx!(ni\% $+1) / 2)$

Next ni\%

' Distancia y del cuadradito al CDG de la sección

ReDim disty_cdg!(1 To npasosy\% + 1)

disty_cdg! $(1)=($ pasosy! $(1) / 2)-($ dimy\% / 2)

For ni\% $=1$ To $($ npasosy $\%-1)$

disty_cdg! $(\mathrm{ni} \%+1)=$ disty_cdg! $(\mathrm{ni} \%)+($ pasosy! $(\mathrm{ni} \%) / 2)+$ (pasosy! $(\mathrm{ni} \%$ $+1) / 2)$

Next ni\%

' Distancia x del cuadradito a la fibra

ReDim distx_fibra!(1 To npasosx\% + 1)

' Distancia y del cuadradito a la fibra

ReDim Disty_fibra!(1 To npasosy\% + 1)

' Entrada del material de cada división

ReDim material\%(1 To npasosx $\%+1,1$ To npasosy $\%+1$ )

For $n j \%=1$ To npasosy $\%$

For ni\% = 1 To npasosx $\%$

Input \#1, material\%(ni\%, nj\%)

Next ni\%

Next nj\%

End Sub 


\section{Rutina para el cálculo de áreas e inercias}

Public Sub calcula_inercia(Ecm!, fibra!, area_x!, inercia_x!, inercia_y!, area_horm!, area_perfil!, area_redondos!, inercia_x_horm!, inercia_x_perfil!, inercia_x_redondos!)

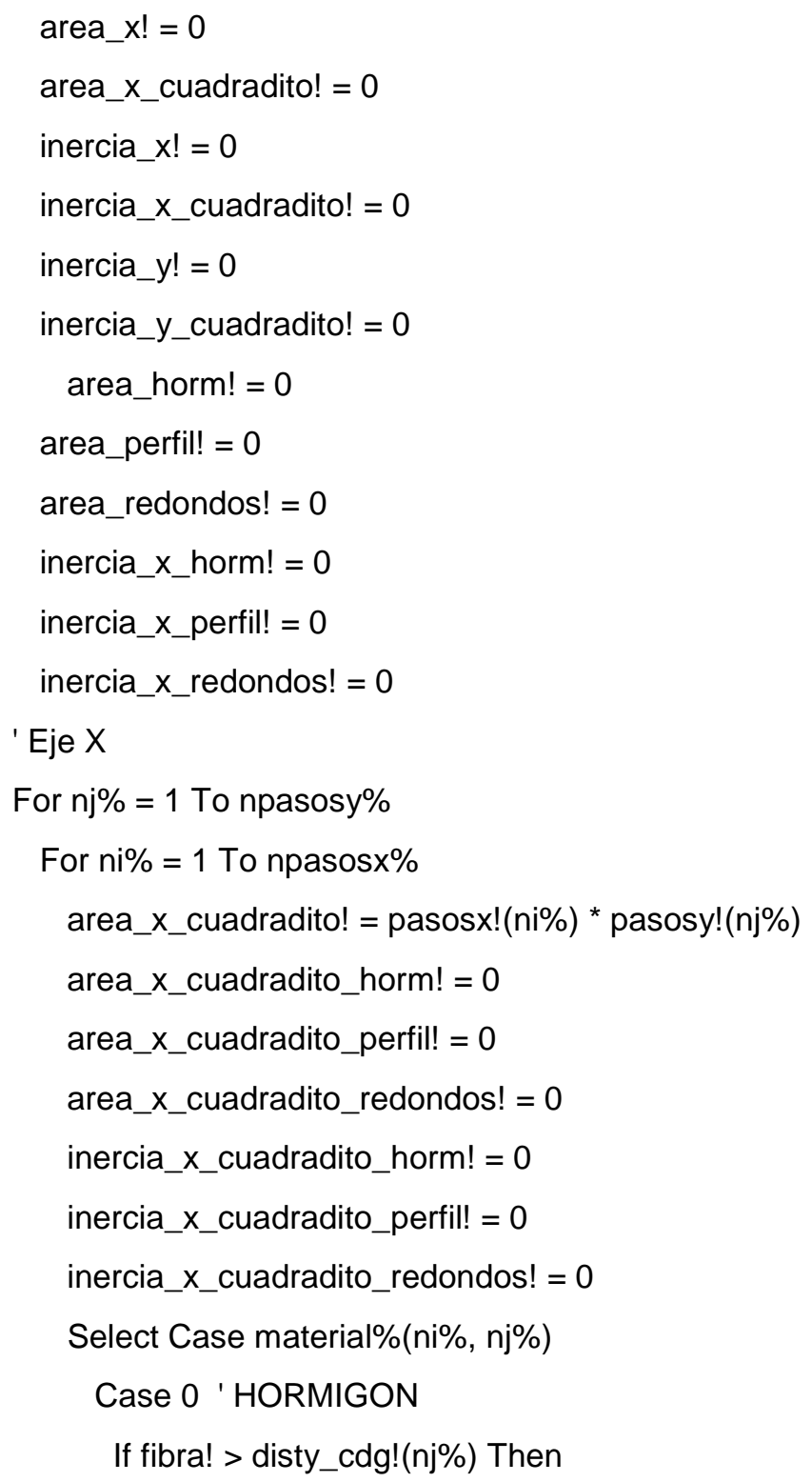




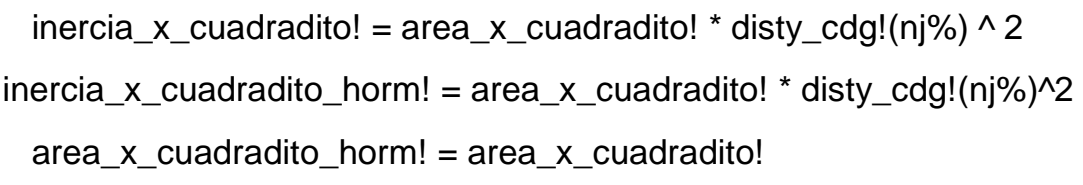

Else: End If

\section{Case 1 ' ACERO ESTRUCTURAL}

inercia_x_cuadradito! $=($ area_x_cuadradito! * disty_cdg! $(n j \%) \wedge 2)$ * $2100000 / \mathrm{Ecm}$ !

inercia_x_cuadradito_perfil! =(area_x_cuadradito! * disty_cdg! $\left.(\mathrm{nj} \%)^{\wedge} 2\right)$

area_x_cuadradito_perfil! = area_x_cuadradito!

Case 2 ' ACERO PARA ARMAR

inercia_x_cuadradito! $=\left(\right.$ area_x_cuadradito! * disty_cdg! $\left.(\mathrm{nj} \%)^{\wedge} 2\right)$ * $2100000 / \mathrm{Ecm}$ !

inercia_x_cuadradito_redondos! = (area_x_cuadradito! disty_cdg! $\left.(\mathrm{nj} \%)^{\wedge} 2\right)$

area_x_cuadradito_redondos! = area_x_cuadradito!

End Select

area_x! = area_x! +area_x_cuadradito!

inercia_x! = inercia_x! + inercia_x_cuadradito!

area_horm! =area_horm! + area_x_cuadradito_horm!

area_perfil! = area_perfil! + area_x_cuadradito_perfil!

area_redondos! = area_redondos! + area_x_cuadradito_redondos!

inercia_x_horm! = inercia_x_horm! + inercia_x_cuadradito_horm!

inercia_x_perfil! = inercia_x_perfil! + inercia_x_cuadradito_perfil!

inercia_x_redondos! = inercia_x_redondos! + inercia_x_cuadradito_redondos!

Next ni\%

Next nj\%

' Eje $Y$

For $n j \%=1$ To npasosy\%

For ni\% = 1 To npasosx $\%$

area_y_cuadradito! = pasosx!(ni\%) * pasosy!(nj\%) 
Select Case material\%(ni\%, nj\%)

Case 0 ' HORMIGON

inercia_y_cuadradito! = area_y_cuadradito! * distx_cdg!(ni\%)^ 2

Case 1 ' ACERO ESTRUCTURAL

inercia_y_cuadradito! = (area_y_cuadradito! * distx_cdg!(ni\%) ^ 2) * 2100000 / Ecm!

Case 2 ' ACERO PARA ARMAR

inercia_y_cuadradito! $=\left(\right.$ area_y_cuadradito! * distx_cdg! $\left(\right.$ ni\% $\left.\%{ }^{\wedge} 2\right)$ * 2100000 / Ecm!

End Select

area_y! = area_y! + area_y_cuadradito!

inercia_y! = inercia_y! + inercia_y_cuadradito!

Next ni\%

Next nj\%

End Sub

Rutina que inicia el cálculo de la sección en flexocompresión recta

'Calculo de la respuesta de la seccion: AXIL Y MOMENTO

'Para una FIBRA y una CURVATURA determinada

Open "c:Itesis|programalresultadosl" + Nom\$ For Output As \#1

'Entrada de las caracteristicas de los materiales

'Hormigón

sigma_hor! $=$ Text 4 . Text ${ }^{*} 10$

epsilon_ult_hor! = Text5.Text

Ecm! $=$ Text6. Text $* 10$

alfa! $=$ Text18. Text

'Acero estructural

sigma_st! $=$ Text8. Text ${ }^{*} 10$

epsilon_y_st! $=$ Text9.Text 
'Acero para armar

sigma_ar! = Text11. Text ${ }^{*} 10$

epsilon_y_ar! = Text12.Text

' Coeficientes de seguridad de los materiales

coeficiente_hor! $=$ Text7. Text

coeficiente_st! $=$ Text10.Text

coeficiente_ar! = Text13.Text

'MOMENTOS X: Bucle para desplazar la fibra neutra

Write \#1, "FIBRA - AXIL - MOMENTO X"

fibra! $=$ Text2.Text

repeticiones $\%=$ Text3.Text

incremento_fibra! $=$ Text14.Text

ReDim vert_mx!(0 To repeticiones\%, 1 To 3 )

ReDim vert_my!(0 To repeticiones\%, 1 To 3 )

ReDim result_x!(0 To repeticiones\%, 1 To 3 )

vert_mx! $(0,1)=$ repeticiones $\%$

vert_my! $(0,1)=$ repeticiones $\%$

For $i \%=1$ To repeticiones $\%$

If Abs(fibra!) $<20$ Then

fibra! = fibra! + (incremento_fibra!) / 2

Else

If Abs(fibra!) $<60$ Then

fibra! $=$ fibra! $+($ incremento_fibra! $){ }^{*} 2$

Else

fibra! = fibra! + (incremento_fibra!) * 2

End If

End If

' Cálculo de la distancia y del codg de cada cuadradito a la fibra neutra

ReDim Disty_fibra!(1 To npasosy\%) 
For ni\% $=1$ To npasosy $\%$

Disty_fibra!(ni\%) = disty_cdg!(ni\%) - fibra!

Next ni\%

'Determinación de la curvatura de agotamiento para cada posición de la fibra neutra

Call curvatura(fibra!, dimx\%, cur!)

'Determinación de la curva de interacción en el eje $X$

Call calcula_seccion_x

Write \#1, fibra!

Write \#1, axil!

vert_mx! $(i \%, 2)=$-axil!

result_x! $(i \%, 1)=$ axil!

Write \#1, mto_x!

vert_mx! $(i \%, 1)=m t o \_x !$

result_x! $(i \%, 2)=$ mto_ $x !$

result_x! $(i \%, 3)=$ fibra!

vert_mx! $(i \%, 3)=0$

Next i\% 'Fin del citado bucle

'MOMENTOS Y: Bucle para desplazar la fibra neutra

Write \#1, "FIBRA - AXIL - MOMENTO Y"

fibra! $=$ Text2. Text

For $i \%=1$ To repeticiones $\%$

If Abs(fibra!) $<20$ Then

fibra! = fibra! + (incremento_fibra!) / 2

Else

fibra! = fibra! + (incremento_fibra!) *2

End If

' Cálculo de la distancia y del codg de cada cuadradito a la fibra neutra

ReDim distx_fibra!(1 To npasosx\%) 


$$
\begin{aligned}
& \text { For ni\% = } 1 \text { To npasosx\% } \\
& \text { distx_fibra!(ni\%) = distx_cdg!(ni\%) - fibra! }
\end{aligned}
$$

Next ni\%

'Determinación de la curvatura de agotamiento para cada posición de la fibra neutra

Call curvatura(fibra!, dimy\%, cur!)

'Determinación de la curva de interacción en el eje $Y$

Call calcula_seccion_y

Write \#1, fibra!

Write \#1, axil!

vert_my! $(i \%, 2)=-$ axil!

Write \#1, mto_y!

vert_my! $(i \%, 1)=$ mto_y!

vert_my! (i\%, 3) =0

Next i\% 'Fin del citado bucle

Close

Open "c:Itesis|programa|resultados\curva_interaccion.dxf" For Output As \#9 Call prindxf("c:Itesis/programa\resultadoslcurva_interaccion.dxf")

Call polilin("0", vert_mx!(), "0", "0", "7", continuous\$, 0)

Call polilin("0", vert_my!(), "0", "0", "2", continuous\$, 0)

Call fidxf

Close

End Sub

Rutina que determina el valor de la curvatura en función del criterio de agotamiento en flexocompresión recta

Public Sub curvatura(fibra!, dimx\%, cur!)

' $\operatorname{dim} x \%$ es la base o el canto según el plano a calcular 
If Entrada.Option3. Value $=$ True Then ' Diagrama EC2 calculo estructural cur! $=0.0033 /($ fibra! $+(\operatorname{dim} x \% / 2))$

Else

If Entrada. Option2. Value = True Then 'Diagrama parábola rectángulo

If fibra! < dimx\% / 2 Then 'Sección parcialmente traccionada. Agotamiento hormigón al 3,5 por mil

$$
\text { cur! }=0.0035 \text { / (fibra! + dimx\% / 2) }
$$

Else 'Sección totalmente comprimida. Pivote en 2 por mil

$$
\text { cur! }=0.002 \text { / (fibra! - }(\operatorname{dimx} \% / 2)+(2 * \operatorname{dim} x \% / 3.5))
$$

End If

Else ' Sección plastificada

End If

End If

End Sub

\section{Rutina que calcula Nu - Mxu para cada posición de la fibra neutra}

Public Sub calcula_seccion_x()

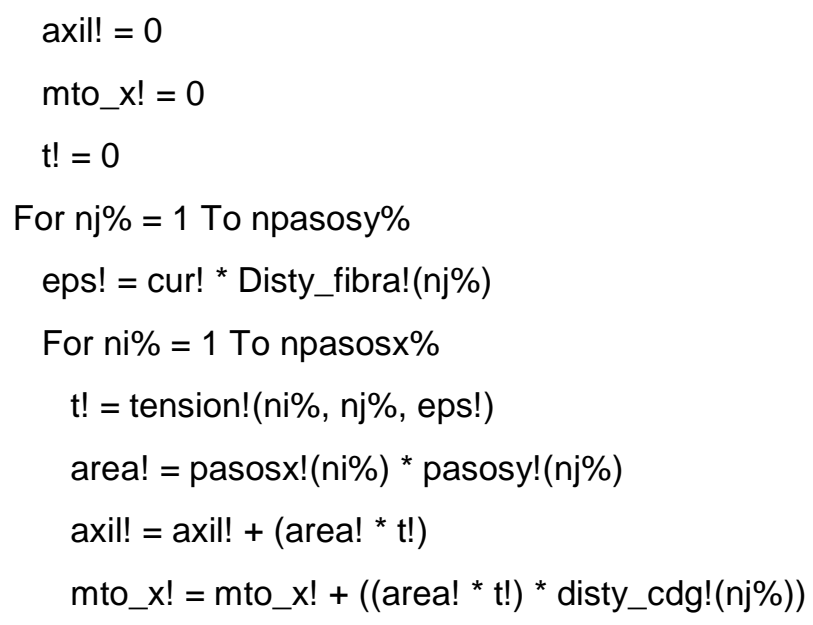

Next nj\% 
End Sub

\section{Rutina que calcula $\mathrm{Nu}$ - Myu para cada posición de la fibra neutra}

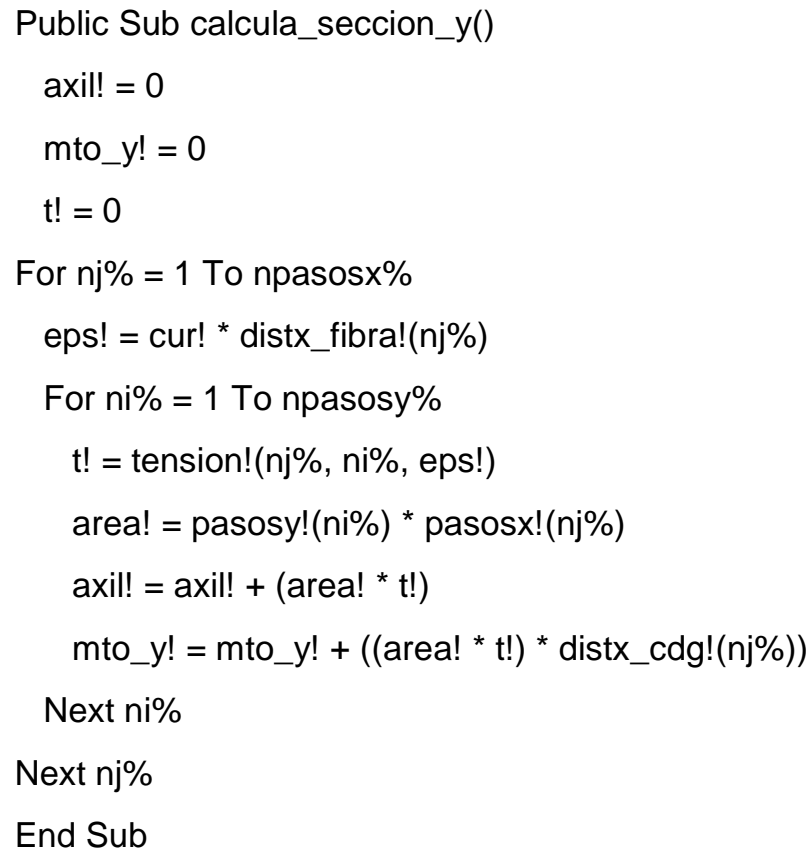

Rutina que calcula la tensión de cada celda en según el material y deformación (misma rutina en flexocompresión plana y esviada)

Select Case material\%(ni\%, nj\%)

Case 0 ' HORMIGON

If $($ eps! $<0)$ Then

If Entrada.Option3.Value $=$ True Then 'Diagrama EC2 cálculo estructural

If Entrada.Option6. Value $=$ True Then 'sin efectos reológicos

eta! = eps! / epsilon_ult_hor!

kapa! $=\left(1.1^{*}\right.$ Ecm! * epsilon_ult_hor! $) /$ sigma_hor! 
2) * eta!)))

$\mathrm{t} !=$ alfa! * sigma_hor! * $(((k a p a ! *$ eta!) - (eta! * eta!)) / $(1+((k a p a !-$

Else ' con efectos reologicos

eta! = eps! / epsilon_ult_hor!

kapa! $=\left(1.1{ }^{*}\right.$ Ecm! * epsilon_ult_hor!) / sigma_hor!

2) * eta!)))

$\mathrm{t} !=$ alfa! * sigma_hor! * $(((k a p a ! *$ eta!) - (eta! * eta!)) / (1 + ((kapa! -

'recalculo de la tension para una deformacion ficticia

eps_fict! $=($ eps! -0.0006$) / 2.45$

eta2! = eps_fict! / epsilon_ult_hor!

kapa! $=\left(1.1^{*} \mathrm{Ecm} !{ }^{*}\right.$ epsilon_ult_hor! $) /$ sigma_hor!

$\mathrm{t} !=$ alfa! * sigma_hor! * $(((k a p a ! *$ eta2!) $-($ eta2! * eta2!) $) /(1+$ $(($ kapa! - 2) * eta2!)))

End If 'de los efectos reologicos

Else

If Entrada. Option2. Value = True Then 'Diagrama parábola rectángulo

If eps! > -0.002 Then

$\mathrm{t} !=1000 *$ eps! * $(250$ * eps! +1$)$ * alfa! * (sigma_hor! * $(-1))$

Else

$\mathrm{t} !=$ alfa! * sigma_hor!

End If 'del diagrama parábola-rectángulo

Else 'Seccion plastificada

$\mathrm{t} !=$ alfa! * sigma_hor!

End If 'de la sección plastificada

End If 'del diagrama a utilizar

Else

$\mathrm{t} !=0$ 'celdilla traccionada

End If

$\mathrm{t} !=\mathrm{t} ! /$ coeficiente_hor!

Case 1 ' ACERO ESTRUCTURAL 
If Entrada.Option1.Value = False Then 'Diagrama elasto-plastico eps_abs! = Abs(eps!)

If eps_abs! < (epsilon_y_st! / coeficiente_st!) Then

$\mathrm{t} !=$ (eps_abs! / epsilon_y_st!) * sigma_st!

$\mathrm{t} !=$ eps_abs! * (sigma_st! / epsilon_y_st!)

Else

+ sigma_st!

$t !=\left(\left(e p s \_a b s !-e p s i l o n \_y \_s t !\right) *((\right.$ sigma_st! / epsilon_y_st! $\left.) / 10000)\right)$

End If

Else 'Seccion plastificada

$\mathrm{t} !$ = sigma_st!

End If ' del diagrama a utilizar

If eps! $<0$ Then

$t !=-t ! /$ coeficiente_st!

Else

$\mathrm{t} !=\mathrm{t} ! /$ coeficiente_st

End If

Case 2 ' ACERO PARA ARMAR

If Entrada.Option1. Value = False Then 'Diagrama elasto-plastico

eps_abs! = Abs(eps!)

If eps_abs! < (epsilon_y_ar! / coeficiente_ar!) Then

$\mathrm{t} !=$ (eps_abs! / epsilon_y_ar!) * sigma_ar!

$\mathrm{t} !=$ eps_abs! * (sigma_ar! / epsilon_y_ar!)

Else

$\mathrm{t} !=(($ eps_abs! - epsilon_y_ar!) * ((sigma_ar! / epsilon_y_ar!) / 10000)) + sigma_ar!

End If

Else 'Seccion plastificada

$\mathrm{t} !$ = sigma_ar! 
End If ' del diagrama a utilizar

$$
\begin{aligned}
& \text { If eps! }<0 \text { Then } \\
& \text { t! = -t! / coeficiente_ar! }
\end{aligned}
$$

Else

$\mathrm{t} !=\mathrm{t} ! /$ coeficiente_ar!

End If

Case 3 ' AIRE

$t !=0$

End Select

tension! $=\mathrm{t}$ !

End Function

\section{Rutina que inicia el cálculo de la sección en flexocompresión esviada}

'Bucle de giro de la fibra neutra

Open "c:|tesis|programa\resultados\resultado-3D.res" For Output As \#1

Open "c:Itesis/programalresultados/superficie_interaccion.dxf" For Output As \#9

Call prindxf("c:Itesis\programa\resultados\superficie_interaccion.dxf")

' Entrada de las características de los materiales

'Hormigón

sigma_hor! $=$ Text4. Text * 10

epsilon_ult_hor! = Text5.Text

Ecm! = Text6. Text * 10

alfa! $=$ Text18. Text

'Acero estructural

sigma_st! = Text8. Text * 10

epsilon_y_st! = Text9.Text 
'Acero para armar

sigma_ar! = Text11. Text * 10

epsilon_y_ar! = Text12.Text

' Coeficientes de seguridad de los materiales

coeficiente_hor! = Text7.Text

coeficiente_st! = Text10.Text

coeficiente_ar! = Text13.Text

giros\% = Text20.Text '101

angulo_giro! $=-(1.570796327 /($ giros\% - 1)) 'Divide pi/2 entre el numero de giros - 1

incremento_angulo_giro! $=(1.570796327 /($ giros\% - 1) $)$

fibra! = Text2.Text

repeticiones $\%=$ Text3. Text

incremento_fibra! = Text14.Text

ReDim result_3d!(1 To giros\%, 0 To repeticiones\%, 1 To 3)

For $n k \%=1$ To giros $\%$

angulo_giro! =angulo_giro! + incremento_angulo_giro!

Call cambia_coordenadas(angulo_giro!)

Call calculo_esviada(nk\%)

Next nk\%

Call calcula_paralelas(repeticiones\%, giros\%)

Call fidxf 'para terminar el fichero DXF de polilíneas

Close 'cierra el fichero DXF

Close 'cierra el fichero TXT

End Sub

Rutina que cambia las coordenadas de los CdG de las celdillas

Public Sub cambia_coordenadas(angulo_giro!)

ReDim distx_g_cdg!(1 To npasosx $\%+1,1$ To npasosy $\%+1)$ 


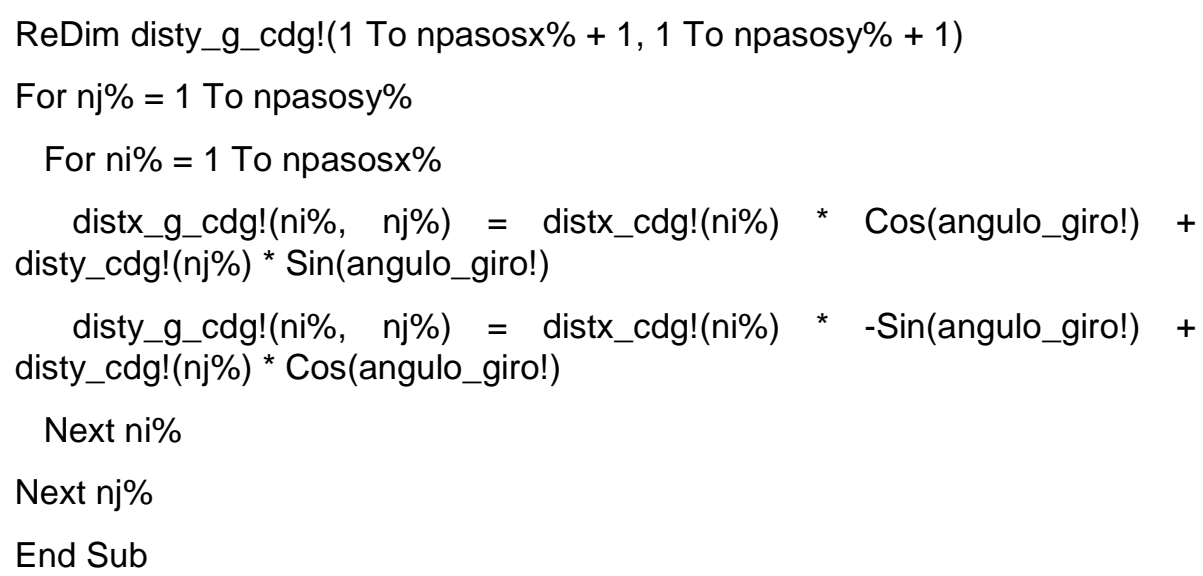

\section{Rutina que inicia el cálculo para un determinado giro de la fibra neutra}

'Calculo de una sección mixta en flexocompresión esviada para un ángulo de giro determinado

'Bucle de desplazamiento de la fibra neutra

Write \#1, "' 'Escribe en fichero de TXT de resultados

Write \#1, "ANGULO FIBRA, DISTANCIA FIBRA"

Write \#1, "AXIL - MOMENTO X - MOMENTO Y"

fibra! = Entrada.Text2.Text

repeticiones\% = Entrada.Text3.Text

incremento_fibra! = Entrada.Text14.Text

ReDim vert_sol!(0 To repeticiones\%, 1 To 3) ' N, Mz, My

ReDim result_sol!(0 To repeticiones\%, 1 To 3 )

vert_sol! $(0,1)=$ repeticiones $\%$

For $\mathrm{i} \%=1$ To repeticiones $\%$ ' INICIO DEL BUCLE para desplazar la fibra neutra

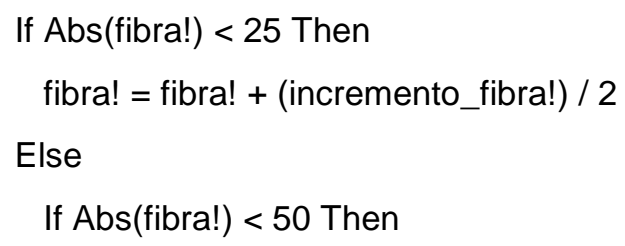




$$
\text { fibra! = fibra! + (incremento_fibra!) * } 3
$$

Else

fibra! = fibra! + (incremento_fibra! $) * 20$

End If

End If

' Cálculo de la distancia y del codg de cada cuadradito a la fibra neutra

ReDim Disty_fibra!(1 To npasosx\%, 1 To npasosy\%)

For nj\% = 1 To npasosy $\%$

For ni\% $=1$ To npasos $\%$

Disty_fibra!(ni\%, nj\%) = disty_g_cdg!(ni\%, nj\%) - fibra!

Next ni\%

Next nj\%

Call curvatura_3d(Disty_fibra!(), npasosx\%, cur!)

Call calculo_seccion_esviada(nk\%)

'Escribe fichero de TXT los resultados de un determinado angulo de giro

Write \#1, angulo_giro!, fibra!

Write \#1, axil!, mto_x!, mto_y!

'Vertices de las curvas para generar el DXF

vert_sol! $(i \%, 2)=$-axil!

result_sol! $(i \%, 1)=$ axil!

vert_sol! $(i \%, 1)=$ mto_x!

result_sol! $(i \%, 2)=$ mto_x!

vert_sol! $(i \%, 3)=$ mto_y!

result_sol! $(i \%, 3)=$ mto_y!

'Almacenamiento de todos los puntos de todas las curvas en una matriz

result_3d!(nk\%, i\%, 1) = axil!

result_3d! $(\mathrm{nk} \%, \mathrm{i} \%, 2)=$ mto_x!

result_3d! $(\mathrm{nk} \%, i \%, 3)=$ mto_y!

Next i\% 'Fin del citado bucle 
Call polilin("0", vert_sol!(), "0", "0", "7", continuous $\$, 0)$

End Sub

Rutina que determina el valor de la curvatura en función del criterio de agotamiento en flexocompresión esviada

Public Sub curvatura_3d(Disty_fibra!(), npasosx\%, cur!)

If Entrada.Option3.Value = True Then 'Diagrama EC2 para cálculo estructural

cur! = 0.0033 / -Disty_fibra!(npasosx\%, 1)

Else

If Entrada.Option2.Value = True Then 'Diagrama parábola rectángulo

If fibra! < Disty_fibra!(npasosx\%, 1) Then 'Sección parcialmente traccionada. Agotamiento hormigon al 3,5 por mil

cur! $=0.0035$ / -Disty_fibra!(npasosx\%, 1)

Else 'Sección totalmente comprimida. Pivote en 2 por mil cur! = 0.002 / -Disty_fibra!(npasosx\%, 1)

End If

Else 'Sección plastificada

End If

End If

End Sub

Rutina que calcula Nu - Mzu - Myu para cada posición de la fibra neutra

Public Sub calculo_seccion_esviada(nk\%)

$$
\begin{aligned}
& \text { axil! }=0 \\
& \text { mto_x } !=0 \\
& \text { mto_y! }=0 \\
& t !=0
\end{aligned}
$$




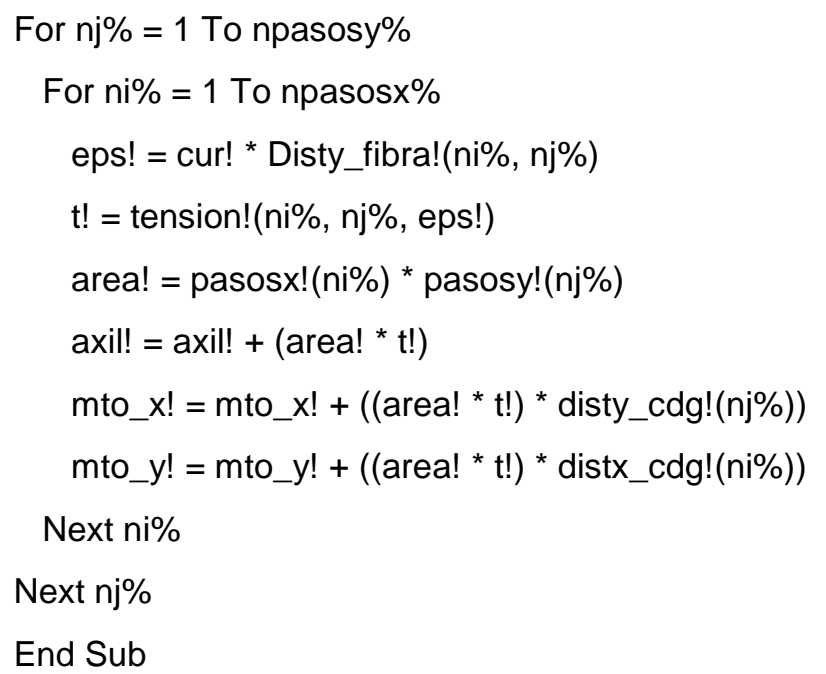

Rutinas para generar dibujo DXF con las curvas o superficie de interacción, con los resultados del cálculo de la sección

Public Sub prindxf(nomm\$)

Print \#9, " 0 "

Print \#9, "SECTION"

Print \#9, " 2"

Print \#9, "HEADER"

Print \#9, " 0 "

Print \#9, "ENDSEC"

Print \#9, " 0"

Print \#9, "SECTION"

Print \#9, " 2"

Print \#9, "TABLES"

Print \#9, " 0 "

Print \#9, "TABLE"

Print \#9, " 2"

Print \#9, "LTYPE" 
Call tablaltype

Print \#9, 0

Print \#9, "Table"

Print \#9, " 2"

Print \#9, "LAYER"

Print \#9, " 0"

Print \#9, "ENDTAB"

Print \#9, " 0"

Print \#9, "ENDSEC"

Print \#9, " 0"

Print \#9, "SECTION"

Print \#9, " 2"

Print \#9, "ENTITIES"

Exit Sub

End Sub

Public Sub tablaltype()

$\mathrm{nt} \&=12 \quad$ '15

ReDim tlin\$(1 To ntl\&), m_tipl\$(1)

$n t \mid \&=n t l \&+n t \&$

ReDim tlin\$(1 To ntl\&)

$\operatorname{tlin} \$(1)=$ "_FI5": $\operatorname{tlin} \$(2)=$ "_FI6": $\operatorname{tlin} \$(3)=$ "_FI8": $\operatorname{tin} \$(4)=$ "_FI10"

$\operatorname{tlin} \$(5)=$ "_Fl12": $\operatorname{tin} \$(6)=$ "_Fl14": $\operatorname{tin} \$(7)=$ "_Fl16": $\operatorname{tin} \$(8)=$ "_FI20"

$\operatorname{tlin} \$(9)=$ "_FI25": $\operatorname{tin} \$(10)=$ "_FI32": $\operatorname{tlin} \$(11)=$ "VOL_SEC": $\operatorname{tin} \$(12)=$ "CERC_CIR"

If $n t \&>0$ Then

$\mathrm{jj} \&=0$

For ii\& $=13$ To $n t l \&$

$\mathrm{jj} \&=\mathrm{jj} \&+1$

$\operatorname{tlin} \$(i i \&)=m_{-}$tip $\$(j \mathrm{j} \&)$ 
Next ii\&

End If

For ii\& $=1$ To ntl\& ' 12

Print \#9, 0

Print \#9, "LTYPE"

Print \#9, 5

Print \#9, 26

Print \#9, 100

Print \#9, "AcDbSymbolTableRecord"

Print \#9, 100

Print \#9, "AcDbLinetypeTableRecord"

Print \#9, 2

Print \#9, tlin\$(ii\&) '"_FI20"

Print \#9, 70

Print \#9, 0

Print \#9, 3

Print \#9, "CID Secciones__"

Print \#9, 72

Print \#9, 65

Print \#9, 73

Print \#9, 2

Print \#9, 40

Print \#9, 12.01

Print \#9, 49

Print \#9, "12\#"

Print \#9, 49

Print \#9, -0.01

Next ii\&

Print \#9, " 0"

Print \#9, "ENDTAB" 
Erase tlin\$, m_tipl\$

End Sub

Public Sub polilin(cerr\&, p!(), gros, capa\$, clor\&, tlin\$, alt!)

Print \#9, " 0"

Print \#9, "POLYLINE"

Print \#9, 8

Print \#9, capa\$

If clor $\&>0$ Then

ccolor $\$=" \quad "+\operatorname{Str} \$($ clor \& $)$

Print \#9, 62

Print \#9, ccolor\$

End If

Print \#9, 66

Print \#9, " 1"

Print \#9, 10

Print \#9, "0.0"

Print \#9, 20

Print \#9, "0.0"

Print \#9, 30

Print \#9, "0.0"

If $\operatorname{tin} \$<>$ "' Then

Print \#9, 6

Print \#9, thin\$

End If

If alt! $>0$ Then

Print \#9, 39

Print \#9, LTrim\$(Str\$(alt!))

End If

If gros $<>0$ Then 
$\operatorname{gro} \$=\operatorname{LTrim} \$(\operatorname{Str} \$($ gros $))$

Print \#9, 40

Print \#9, gro\$

Print \#9, 41

Print \#9, gro\$

End If

If cerr \& $=1$ Then

Print \#9, 70

Print \#9, " 1"

Else

Print \#9, 70

Print \#9, " 8"

End If

For $\mathrm{i} \&=1$ To $\mathrm{p} !(0,1)$

Print \#9, " 0"

Print \#9, "VERTEX"

Print \#9, 8

Print \#9, capa\$

puntox $\$=\operatorname{LTrim} \$(\operatorname{Str} \$(\mathrm{p} !(i \&, 1)))$

puntoy $\$=\operatorname{LTrim} \$(\operatorname{Str} \$(p !(i \&, 2)))$

puntoz $\$=\operatorname{LTrim} \$(\operatorname{Str} \$(p !(i \&, 3)))$

Print \#9, 10

Print \#9, puntox\$

Print \#9, 20

Print \#9, puntoy\$

Print \#9, 30

Print \#9, puntoz\$

If cerr \& $=0$ Then

Print \#9, 70

Print \#9, " 32" 
End If

Next i\&

Print \#9, " 0"

Print \#9, "SEQEND"

Print \#9, " 8"

Print \#9, "0"

End Sub

Public Sub lindxf(p!(), capa\$, clor, t_line\$)

Print \#9, "0"

Print \#9, "LINE"

Print \#9, 8

Print \#9, capa\$

If $t$ _line $\$<>$ "'" Then

Print \#9, 6

Print \#9, t_line\$

End If

If clor $>0$ Then

ccolor $\$=" \quad "+\operatorname{Str} \$($ clor $)$

Print \#9, 62

Print \#9, ccolor\$

End If

Print \#9, 10

Print \#9, Str $\$(p !(1,1))$

Print \#9, 20

Print \#9, Str $\$(p !(1,2))$

Print \#9, 30

Print \#9, Str $\$(p !(1,3))$

Print \#9, 11

Print \#9, Str $\$(p !(2,1))$ 
Print \#9, 21

Print \#9, Str\$(p! $(2,2))$

Print \#9, 31

Print \#9, Str\$(p! $(2,3))$

End Sub

Public Sub fidxf()

Print \#9, " 0"

Print \#9, "ENDSEC"

Print \#9, " 0"

Print \#9, "EOF"

Close \#9

End Sub

Rutinas para comprobar una sección mixta en flexocompresión recta

Private Sub Command4_Click()

$\mathrm{Nd} !=$ Text19.Text

$M d !=$ Text15.Text

repeticiones $\%=$ Text3.Text

Call Perita_2d(Nd!, Md!, repeticiones\%, resultado\$, fibra_sol!)

End Sub

Public Sub Perita_2d(Nd!, Md!, repeticiones\%, resultado\$, fibra_sol!)

If $\mathrm{Nd!}>$ result_x!(repeticiones\%, 1) Then

For $\mathrm{i} \%=1$ To repeticiones $\%$

If $(\mathrm{Nd!}$ - result_x! $(i \%, 1))>0$ Then

If (Md! - result_x! $(i \%, 2))<0$ Then

resultado\$ = "OK" 'result_x! (i\%, 2) 
fibra_sol! =result_x!(i\%, 3)

Else:

resultado\$ = "NO CUMPLE"

End If

$i \%=$ repeticiones $\%$

Else: End If

Next i\%

Else:

resultado\$ = "SE SUPERA EL AXIL MAXIMO"

End If

End Sub

\section{Rutinas para comprobar una sección mixta en flexocompresión esviada}

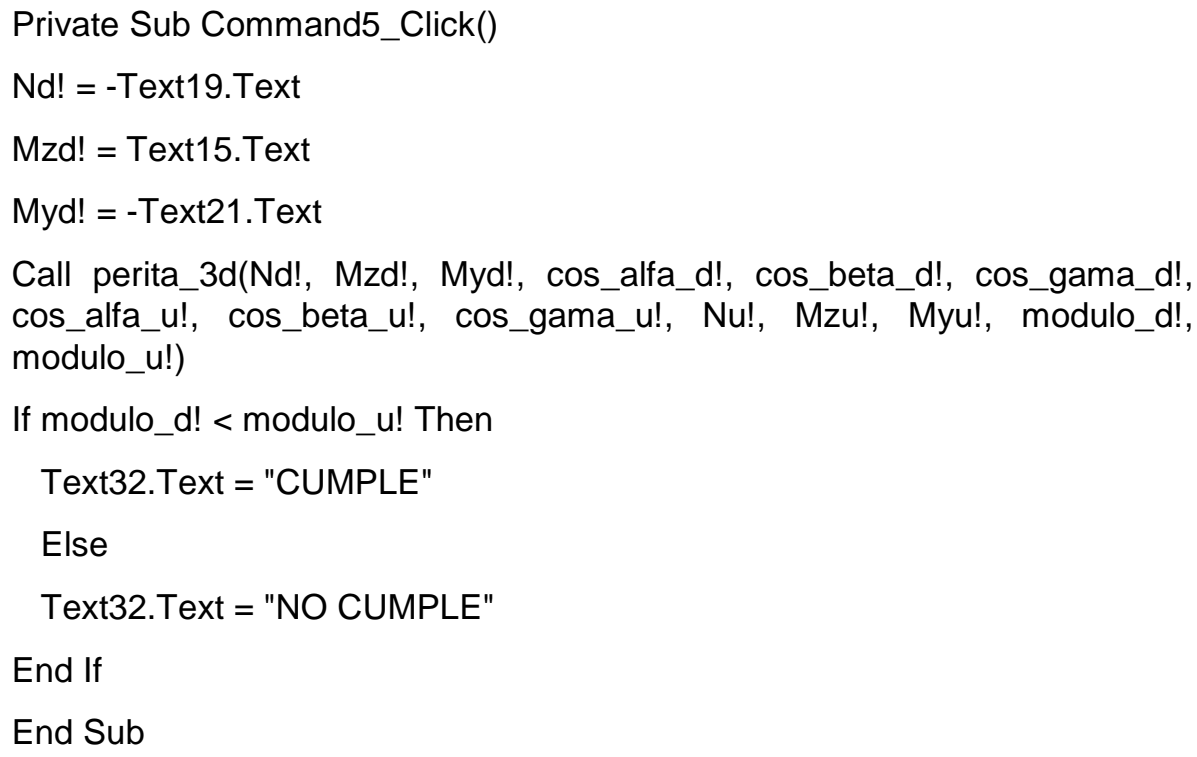


Public Sub perita_3d(Nd!, Mzd!, Myd!, cos_alfa_d!, cos_beta_d!, cos_gama_d!, cos_alfa_u!, cos_beta_u!, cos_gama_u!, Nu!, Mzu!, Myu!, modulo_d!, modulo_u!)

'(Nu, Mzu, Myu $)=$ vector agotamiento

' $(\mathrm{Nd}, \mathrm{Mzd}, \mathrm{Myd})=$ vector solicitacion

modulo_d! = Sqr(Nd!^^2+Mzd!^ $\left.2+\operatorname{Myd} !^{\wedge} 2\right)$

cos_alfa_d! $=(\mathrm{Nd} ! /$ modulo_d $)$

cos_beta_d! = (Mzd! / modulo_d! $)$

cos_gama_d! = (Myd! / modulo_d!)

dif_cos_alfa! $=60$

dif_cos_beta! $=60$

dif_cos_gama! $=60$

diferencia! $=60$

For $n k \%=1$ To giros $\%$ 'Comienza el bucle de busqueda

For $i \%=1$ To repeticiones $\%$

modulo_up! $=\operatorname{Sqr}\left(\text { result_3d! }(n k \%, i \%, 1)^{\wedge} 2+\text { result_3d!(nk\%, i } \%, 2\right)^{\wedge} 2$ + result_3d! $\left.(\mathrm{nk} \%, \mathrm{i} \%, 3)^{\wedge} 2\right)$

cos_alfa_up! = (Abs(result_3d! (nk\%, i\%, 1)) / modulo_up! $)$

cos_beta_up! $=(\operatorname{Abs}($ result_3d! $(\mathrm{nk} \%, \mathrm{i} \%, 2)) /$ modulo_up! $)$

cos_gama_up! $=($ Abs $($ result_3d! $(n k \%, i \%, 3)) /$ modulo_up! $)$

dif_cos_alfa_p! = Abs(cos_alfa_up! - cos_alfa_d!)

dif_cos_beta_p! = Abs (cos_beta_up! - cos_beta_d!)

dif_cos_gama_p! = Abs (cos_gama_up! - cos_gama_d!)

diferencia_p! = dif_cos_alfa_p! + dif_cos_beta_p! + dif_cos_gama_p!

If dif_cos_alfa_p! < dif_cos_alfa! And dif_cos_beta_p! < dif_cos_beta! And dif_cos_gama_p! < dif_cos_gama! Then

$\mathrm{Nu} !=$ result_3d!(nk\%, i\%, 1)

Mzu! = result_3d!(nk\%, i\%, 2)

Myu! = result_3d!(nk\%, i\%, 3)

diferencia! = diferencia_p!

dif_cos_alfa! =dif_cos_alfa_p! 


$$
\begin{aligned}
& \text { dif_cos_beta! = dif_cos_beta_p! } \\
& \text { dif_cos_gama! = dif_cos_gama_p! } \\
& \text { cos_alfa_u! = cos_alfa_up! } \\
& \text { cos_beta_u! = cos_beta_up! } \\
& \text { cos_gama_u! = cos_gama_up! } \\
& \text { modulo_u! = modulo_up! }
\end{aligned}
$$

Else:

If dif_cos_alfa_p! < dif_cos_alfa! And dif_cos_beta_p! < dif_cos_beta! And diferencia_p! < diferencia! Then

$$
\begin{aligned}
& \text { Nu! = result_3d!(nk\%, i\%, 1) } \\
& \text { Mzu! = result_3d!(nk\%, i\%, 2) } \\
& \text { Myu! = result_3d!(nk\%, i\%, 3) } \\
& \text { diferencia! = diferencia_p! } \\
& \text { dif_cos_alfa! = dif_cos_alfa_p! } \\
& \text { dif_cos_beta! = dif_cos_beta_p! } \\
& \text { dif_cos_gama! = dif_cos_gama_p! } \\
& \text { cos_alfa_u! = cos_alfa_up! } \\
& \text { cos_beta_u! = cos_beta_up! } \\
& \text { cos_gama_u! = cos_gama_up! } \\
& \text { modulo_u! = modulo_up! }
\end{aligned}
$$

\section{Else: End If}

If dif_cos_alfa_p! < dif_cos_alfa! And dif_cos_gama_p! < dif_cos_gama! And diferencia_p! < diferencia! Then

$$
\begin{aligned}
& \text { Nu! = result_3d!(nk\%, i\%, 1) } \\
& \text { Mzu! = result_3d!(nk\%, i\%, 2) } \\
& \text { Myu! = result_3d!(nk\%, i\%, 3) } \\
& \text { diferencia! = diferencia_p! } \\
& \text { dif_cos_alfa! = dif_cos_alfa_p! } \\
& \text { dif_cos_beta! = dif_cos_beta_p! } \\
& \text { dif_cos_gama! = dif_cos_gama_p! }
\end{aligned}
$$




$$
\begin{aligned}
& \text { cos_alfa_u! = cos_alfa_up! } \\
& \text { cos_beta_u! = cos_beta_up! } \\
& \text { cos_gama_u! = cos_gama_up! } \\
& \text { modulo_u! = modulo_up! }
\end{aligned}
$$

Else: End If

If dif_cos_gama_p! < dif_cos_gama! And dif_cos_beta_p! < dif_cos_beta! And diferencia_p! < diferencia! Then

$$
\begin{aligned}
& \text { Nu! = result_3d!(nk\%, i\%, 1) } \\
& \text { Mzu! = result_3d!(nk\%, i\%, 2) } \\
& \text { Myu! = result_3d!(nk\%, i\%, 3) } \\
& \text { diferencia! = diferencia_p! } \\
& \text { dif_cos_alfa! = dif_cos_alfa_p! } \\
& \text { dif_cos_beta! = dif_cos_beta_p! } \\
& \text { dif_cos_gama! = dif_cos_gama_p! } \\
& \text { cos_alfa_u! = cos_alfa_up! } \\
& \text { cos_beta_u! = cos_beta_up! } \\
& \text { cos_gama_u! = cos_gama_up! } \\
& \text { modulo_u! = modulo_up! }
\end{aligned}
$$

Else: End If

\section{End If}

Next i\%

Next nk\% 'Ciera el bucle de busqueda

End Sub

Rutina de obtención de la fibra neutra y la curvatura que proporcionan el equilibrio del momento interno de la sección con las solicitaciones $\mathrm{Nd}-\mathrm{Md}$

$\mathrm{Nd} !=$ Text19.Text
$\mathrm{Md} !=$ Text15.Text

Call Perita_2d(Nd!, Md!, repeticiones\%, resultado\$, fibra_sol!) 
If resultado $\$=$ "OK" Then ' La seccion cumple y se busca la posicion de equilibrio

fibra! = fibra_sol

cur! $=0.0022 /($ fibra $!+(\operatorname{dim} x \% / 2))$

ReDim Disty_fibra!(1 To npasosy\%)

For ni\% = 1 To npasosy $\%$

Disty_fibra! (ni\%) = disty_cdg!(ni\%) - fibra!

Next ni\%

Call calcula_seccion_x

Text32.Text $=$ fibra!

$\mathrm{i} \%=1$

Do ' INICIO DEL PRIMER BUCLE

If (mto_x! / Md!) > 1.2 Then ' Corrección de curvatura porque el momento se desvía más que el axil

$j \%=1$

Do

cur! $=0.98 *$ cur!

ReDim Disty_fibra!(1 To npasosy\%)

For ni\% $=1$ To npasosy $\%$

Disty_fibra! (ni\%) = disty_cdg!(ni\%) - fibra!

Next ni\%

Call calcula_seccion_x

$j \%=j \%+1$

Loop Until (Nd! / axil!) > 1.2 Or mto_x! $<$ Md! Or j\% $=300$

Else: End If

If (mto_x! / Md!) $<0.8$ Then ' Corrección de curvatura porque el momento se ha reducido demasiado

$\mathrm{j} \%=1$

Do

cur! $=1.02 *$ cur!

ReDim Disty_fibra!(1 To npasosy\%) 
For ni $\%=1$ To npasosy $\%$

Disty_fibra!(ni\%) = disty_cdg!(ni\%) - fibra!

Next ni\%

Call calcula_seccion_x

$\mathrm{j} \%=\mathrm{j} \%+1$

def_max! = cur! * $($ fibra! + $(\operatorname{dim} \times \% / 2))$

Loop Until (mto_x! / Md!) > 1 Or def_max! > 0.0033 Or j\% = 300 '(Nd! / axil!) $>1.2$ Or def_max! $>0.0033$ Or $\mathrm{j} \%=100$

Else: End If

If ( $\mathrm{Nd}$ ! / axil!) > 1.2 Then ' Corrección de fibra porque el axil se desvía más que el flector

$\mathrm{j} \%=1$

Do

$$
\begin{aligned}
& \text { fibra! }=\text { fibra! + } 0.5 \\
& \text { def_max! }=\text { cur! }{ }^{*}(\text { fibra! }-0.5+(\operatorname{dim} \times \% / 2)) \\
& \text { cur! = def_max! / (fibra! + }(\operatorname{dim} \times \% / 2))
\end{aligned}
$$

ReDim Disty_fibra!(1 To npasosy\%)

For $\mathrm{ni} \%=1$ To npasosy $\%$

Disty_fibra! (ni\%) = disty_cdg!(ni\%) - fibra!

Next ni\%

Call calcula_seccion_x

$\mathrm{j} \%=\mathrm{j} \%+1$

Loop Until (Nd! / axil!) $<1.05$ Or j\% $=300$

Else: End If

If (Nd! / axil!) < 0.8 Then ' Corrección de fibra porque el axil ha aumentado demasiado

$$
j \%=1
$$

Do

$$
\begin{aligned}
& \text { fibra! = fibra! - } 0.5 \\
& \text { def_max! }=\text { cur! }{ }^{*}(\text { fibra! }+0.5+(\operatorname{dim} x \% / 2)) \\
& \text { cur! = def_max! / (fibra! + (dimx\% / 2) })
\end{aligned}
$$


ReDim Disty_fibra!(1 To npasosy\%)

For ni\% $=1$ To npasosy\%

Disty_fibra!(ni\%) = disty_cdg!(ni\%) - fibra!

Next ni\%

Call calcula_seccion_x

$\mathrm{j} \%=\mathrm{j} \%+1$

Loop Until (Nd! / axil!) > 1 Or j\% $=300$

Else: End If

Loop Until $\%=1000$ Or $((\mathrm{Nd}$ ! / axil! < 1.2) And (Nd! / axil! > 0.8) And (mto_x! / Md! < 1.2) And (mto_x! / Md! > 0.8)) ' (( $0.95<(\mathrm{Nd} ! /$ axil!) $)$ And $((\mathrm{Nd} ! /$ axil!) < 1.05)) And (1.05 > mto_x! / Md!))

'FIN DEL PRIMER BUCLE

$i \%=1$

Do ' INICIO DEL SEGUNDO BUCLE

If (mto_x! / Md!) > 1.05 Then ' Corrección de curvatura porque el momento se desvía más que el axil

$j \%=1$

Do

cur! $=0.995 *$ cur!

ReDim Disty_fibra!(1 To npasosy\%)

For ni\% $=1$ To npasosy $\%$

Disty_fibra!(ni\%) = disty_cdg!(ni\%) - fibra!

Next ni\%

Call calcula_seccion_x

$\mathrm{j} \%=\mathrm{j} \%+1$

Loop Until (Nd! / axil!) > 1.05 Or mto_x! < Md! Or j\% $=300$

Else: End If

If (mto_x! / Md!) < 0.95 Then ' Corrección de curvatura porque el momento se ha reducido demasiado

$\mathrm{j} \%=1$ 
Do

cur! $=1.002$ * cur!

ReDim Disty_fibra!(1 To npasosy\%)

For ni\% $=1$ To npasosy $\%$

Disty_fibra!(ni\%) = disty_cdg!(ni\%) - fibra!

Next ni\%

Call calcula_seccion_x

$\mathrm{j} \%=\mathrm{j} \%+1$

def_max! = cur! * (fibra! + (dimx\% / 2) $)$

Loop Until (mto_x! / Md!) $>1$ Or def_max! $>0.0033$ Or j\% = 300 '(Nd! / axil!) $>1.2$ Or def_max! $>0.0033$ Or j $\%=100$

Else: End If

If (Nd! / axil!) > 1.05 Then ' Correccion de fibra porque el axil se desvia mas que el flector

$\mathrm{j} \%=1$

Do

fibra! $=$ fibra $!+0.2$

def_max! $=$ cur! * (fibra! $-0.2+(\operatorname{dim} \times \% / 2))$

cur! = def_max! / (fibra! + (dimx\% / 2))

ReDim Disty_fibra!(1 To npasosy\%)

For ni\% = 1 To npasosy $\%$

Disty_fibra!(ni\%) = disty_cdg!(ni\%) - fibra!

Next ni\%

Call calcula_seccion_x

$\mathrm{j} \%=\mathrm{j} \%+1$

Loop Until (Nd! / axil!) $<1.05$ Or j\% $=300$

Else: End If

If (Nd! / axil!) < 0.9 Then ' Corrección de fibra porque el axil se desvía más que el flector

$\mathrm{j} \%=1$

Do 


\section{fibra! $=$ fibra $!-0.2$}

def_max! $=$ cur! * $($ fibra! $+0.2+(\operatorname{dimx} \% / 2))$

cur! = def_max! $/($ fibra! $+(\operatorname{dim} \times \% / 2))$

ReDim Disty_fibra!(1 To npasosy\%)

For ni\% = 1 To npasosy\%

Disty_fibra!(ni\%) = disty_cdg!(ni\%) - fibra!

Next ni\%

Call calcula_seccion_x

$$
\mathrm{j} \%=\mathrm{j} \%+1
$$

Loop Until (Nd! / axil!) > 1 Or $j \%=300$

Else: End If

$i \%=i \%+1$

Loop Until $\%=1000$ Or $((\mathrm{Nd} ! /$ axil! $<1.05)$ And $(\mathrm{Nd!} /$ axil! $>0.95)$ And (mto_x! / Md! < 1.05) And (mto_x! / Md! > 0.95)) ' $(((0.95<(\mathrm{Nd} ! /$ axil! $))$ And $((\mathrm{Nd} ! /$ axil! $)<1.05))$ And $(1.05>$ mto_x! / Md!) $)$

'FIN DEL SEGUNDO BUCLE

\section{Else}

Text32. Text $=$ resultado $\$$

End If

End Sub 


\section{REFERENCIAS BIBLIOGRÁFICAS}


Addis, B.

Building: $\mathbf{3 0 0 0}$ years of design engineering and construction Phaidon Press Limited. 2007

Alonso Durá, A.

Tesis doctoral: Un modelo de integración del análisis estructural en entornos CAD para estructuras de edificación

Universidad Politécnica de Valencia, Dic-2003

Arenas de Pablo, Juan J.

Cálculo de soportes de hormigón armado en teoría de segundo orden

Editores técnicos asociados, S.A. Barcelona 1980

Argüelles Ávarez, R.; Argüelles Bustillo, R.; Arriaga Martitegui, F.; Argüelles Bustillo, J.M.; Esteban Herrero, M.

Cálculo matricial de estructuras en $1^{\text {er }}$ y $2^{\circ}$ orden. Teoría y problemas

Bellisco. Madrid 2005

Aroca Hernández-Ros, R.

Flexión compuesta y pandeo en barras rectas

Cuadernos del Instituto Juan de Herrera de la Escuela de Arquitectura de Madrid. 2001

Atienza Reales, J.R.; Irles Mas, R.

Estudio no lineal del comportamiento resistente de soportes de hormigón armado solicitados a Flexo-compresión esviada

Hormigón y acero. № 154.1985

Bernal, J. R.

Hormigón armado: Columnas

Nobuko. Buenos Aires, 2005 
Bonet, J.L.; Barros, M.H.F.M.; Romero, M.L.

Comparative study of analytical and numerical algorithms for designing reinforced concrete sections under biaxial bending

Computers \& Structures. Vol. 84, Num. 31-32. Dic-2006

Bridge, R.Q., Ansourian, P., Rotter, J.M., Patrick, M., Pham, L.

Australian standard for composite construction

Composite construction in steel and concrete proceedings of an engineering foundation conference.

American Society of Civil Engineers. 1988

Bridge, R.Q.

The long-term behavior of composite columns

Composite construction in steel and concrete proceedings of an engineering foundation conference.

American Society of Civil Engineers. 1988

Burr, WH.

Composite columns of concrete and steel

Proc. Institution of Civil Engineers. 1912

Castell Herrera, V.; Regalado Tesoro, F.; Farré Oro, B.

Biblioteca de detalles constructivos metálicos, de hormigón y mixtos en estructuras de edificación : 600 detalles constructivos de estructuras metálicas, mixtas y de hormigón armado, adaptados a la instrucción EHE

Alicante: CYPE Ingenieros, D.L. 2004

Charalampakis, A.E.; Koumousis, V.K.

Ultimate strength analysis of composite sections under biaxial bending and axial load

Advances in Engineering Software. Vol. 39. Num. 11. Nov-2008 
Chen, W.; Atsuta, T.

Interaction equations for biaxially loaded sections

Journal of the Structural Division ASCE. May-1972

Chen, W.; Atsuta, T.

Theory of beam-columns. Vol 1 In-plane behavior and design

McGraw-Hill, Inc. USA, 1976

Chen, W.; Atsuta, T.

Theory of beam-columns. Vol 2 Space behavior and design

McGraw-Hill, Inc. USA, 1977

Chen, C-C.; Li, J-M.; Weng, C.C.

Experimental behavior and strength of concrete-encased composite bem-columns with T-shaped steel section under cyclic loading

Journal of the Constructional Steel Research. Vol. 61, Num. 7. Jul2005

Chen, C-C.; Lin, N-J.

Analytical model for predicting axial capacity and behavior of concrete encased steel composite stub columns

Journal of Constructional Steel Research. Vol. 62, Num. 5. May-2006

Chen, W.F.; Richard Liew, J.Y.

The civil engineering handbook

CRC Press LLC. $2^{\underline{a}}$ edición. Florida. USA. 2003.

Cosenza, E.; Di Sarno, L.; Fabbrocino, G.; Pecce, M.

Composite steel and concrete structures: technology and design 
Corres Peiretti, H.; Martínez Martínez, J.L.; Pérez Caldentey, A.; López Agüí, J.C.

Prontuario informático del hormigón estructural 3.0 Instituto Español del Cemento y sus Aplicaciones (IECA). Cátedra de hormigón armado y pretensado. Escuela Técnica de Ingenieros de Caminos, Canales y Puertos.

Universidad Politécnica de Madrid. 2001

Degée, H.; Doneux, C.L.; Plumier, A.

Ductility of fully encased composite columns used to óbviate soft storey mechanisms in reinforced concrete frames

University of Liége. Bélgica

Elliot, C.D.

Technics and architecture

The MIT Press. Massachusetts. 1992

Ellobody, E.; Young, B.; Lam, D.

Eccentrically loaded concrete encased steel composite columns Thin-walled Structures. Pendiente de publicación. On line Sep-2010

European Convention for Constructional Steelwork Joint Committee on Composite Structures

Composite structures

ECCS Publication. № 28. 1981

European Convention for Constructional Steelwork

Technical Committee 11. Composite Structures

Composite beams and columns to Eurocode 4

ECCS publication. № 72.1993

Faber, O.; Mech, M.I.

Savings to be effected by the more rational design of cased stanchions as a result of recent full size tests

The Structural Engineer. Mar-1956 
Flavia Regina Bianchi

Análise do comportamento dos pilares mistos considerando a utilizaçäo de conectores de cisalhamento

Dissertaçäo apresentada ao programa de pós-graduaçäo em engenharia civil da universidade federal do Espírito Santo como parte dos requisitos para obtençäo do grau de mestre en engenharia civil.

Vitória, Brasil. 2002

Fouré, B.

Le flambement des poteaux compte tenu du fluage du béton

Annales de l'institut technique du batiment et des travaux publics, num 359. Mar-1978

Furlong, R.W.

Column rules of $\mathrm{ACl}$, SSLC, and LRFD compared

Journal of Structural Engineering ASCE, vol 109, num 9. 1983

Ghannam, S.

Behaviour of composite steel columns filled with normal concrete and others filled with light weight concrete. Comparative study

Faculty of Engineering. Al-Isra Private University. Jordan. Nov-2009

G.E.H.O. (Grupo Español del Hormigón)

Caracterización de las propiedades diferidas del Hormigón y su incidencia estructural. Boletín no 22

Coopegraf-visagrafic, S.L. 1998

Gere, J.

Resistencia de materiales

International Thomson editores Spain. 5ª edición. Madrid 2002

Glück, R.; Clenin, D.

Colonnes mixtes acier-béton

Construction métallique, num 4. 1987 
Goode, C.D.; Kuranovas, A.; Kvedaras, A.K.

Buckling of slender composite concrete-filled steel columns Journal of Civil Engineering and Management. 2010

Harstead, G.A.; Birnstiel, Ch.; Leu, K. Inelastic H-columns under biaxial bending Journal of the Structural Division ASCE. Oct-1968

Heyman, J.

Coulomb's memoir on statics. An essay in the history of civil engineering

Cambridge University Press. Cambridge, 1972

Heyman, J.

Structural analysis. A historical approach

Cambridge University Press. Cambridge, 1998

Horne, M.R.

The elastic-plastic theory of compression members

Journal of the Mechanics and Physics of Solids, vol 4. 1956

Jacobs, William P; Goverdhan, Arvind V.

Review and comparison of encased composite steel-concrete column detailing requirements

Proceedings: Composite Construction in Steel and Concrete VI. Pendiente de publicación.

Johnson, R.P.

Composite structures of steel and concrete.

Volume 1: Beams, columns, frames and applications in building

Blackwell Publishing. $3^{\text {a }}$ ed. 2004 
Jones, R; Rizk, A.A.

An investigation on the behaviour of encased steel columns under load

The Structural Engineer. Vol. 41. Ene-1963

Kim, D.K.

Tesis doctoral: A database for composite columns

Georgia Institute of Technology. Ago-2005

Kurrer, K.E.

The history of the theory of structures

Ernst \& Sohn. A Wiley Company. Alemania 2008

Lu, X.; Lu, W.

Seismic behaviour of concrete and steel composite columns under cyclic loading

12WCEE. 2000

Marco García, J.

Curso básico de cálculo y diseño de estructuras metálicas en ordenador. Adaptado al Eurocódigo 3 y al LRFD (AISC)

McGraw Hill. Madrid 2000

Maristany Carreras, J.

Pandeo de estructuras de hormigón armado

Edicions UPC (Universitat Politècnica de Catalunya). Barcelona 1996

Martinez Calzón, J.; Ortiz Herrera, J.

Construcción mixta. Hormigón-acero

Editorial Rueda. 1978

Mac Gregor, J.G.; Breen, J.E.; Pfrang, E.O.

Design of Slender Concrete Columns

ACI Journal, January-1970 
Mirza, SA.; Skrabek BW.

Reliability of short composite beam-column strength interaction Journal of Structural Engineering, ASCE. 1991

Mirza, SA.; Skrabek BW.

Statistical analysis of slender composite beam-column strength Journal of Structural Engineering, ASCE. 1992

Mc Guire, W.; Gallagher R.; Ziemian, R.

Matrix structural analysis

John Wiley \& Sons, Inc. USA. 2000

McGuire, W.

Steel structures

Prentice Hall International. 1968

Monfort Lleonart, J.

Estructuras metálicas para edificación: adaptado al CTE

Editorial UPV, Valencia. 2006

Monfort Lleonart, J.

Estructuras mixtas para edificación : según criterios del Eurocódigo 4

Editorial UPV, D.L. Valencia. 2002

Monfort Lleonart, J.

Trabajo de investigación: soportes mixtos

May-1996 (no publicado)

Moya Ferrer, L.

Análisis matricial de estructuras de barras

Edicions UPC (Universitat Politècnica de Catalunya). Barcelona,1995

Narayanan, R.

Steel-concrete composite structures. Stability and strength

Elservier Applied Science Publishers LTD. 1988 
Nethercot, D.A.

Composite construction

Spon Press. Londres, 2003

Neville, A.M.; Dilger, W.H.; Brooks, J.J.

Creep of plain \& structural concrete

Construction Press, 1983

Ortiz Berrocal, L.

Resistencia de materiales

McGraw Hill. Madrid 1994

Ortiz Herrera, J.; Martinez Calzón, J.

Análisis avanzado de estructuras mixtas. Resumen de un curso.

Revista de obras públicas, Ago-1979

Paz, Mario.

Dinámica estructural. Teoría y cálculo

Editorial Reverté, S.A. 2002

Perepérez Ventura, B.; Barberá Ortega, E.

Manual del hormigón estructural

Artes Gráficas Beracrom, S.L. 2005

Pfrang, Edward O.; Siess, Chester P.

Behavior of restrained reinforced concrete columns

Proceedings. ASCE. V. 90. Oct-1964

Pinkham, C.W.

1986 AISC LRFD Design for composite buildings

Composite construction in steel and concrete proceedings of an engineering foundation conference.

American Society of Civil Engineers. 1988 
Popov, E.P.

Mecánica de sólidos

Pearson Educación. México, 2000

Quintero, F.

Comprobación de soportes compuestos según EC-4

Jornada sobre Eurocódigo 4. Madrid, 1991

Robinson, J.R.; Modjabi, S.S.

La prévision des charges de flambement des poteaux en béton armé par la méthode de $M$. P. Faessel

Annales de l'institut thechnique du batiment et des travaux publics. № 249, Sep-1968

Robinson, J.R.; Fouré, B.; Bourghli, A.Y.

Le flambement des poteaux en béton armé chargés aves des excentricités différentes a leurs extrémités

Annales de l'institut thechnique du batiment et des travaux publics. № 333, Nov-1975

Rokach, A.J.

Diseño de estructuras de acero

McGraw Hill. 1992

Rosati, L.; Marmo, F.; Serpieri, R.

Enhanced solution strategies for the ultímate strength analsis of composite steel-concrete sections subject to axial forcé and biaxial bending

Computer Methods in Applied Mechanics and Engineering. Vol. 197 Num. 9-12. Feb-2008

Santathadaporn, S.; Chen, W.F.

Analysis of biaxially loaded steel H-columns

Journal of the Structural Division ASCE. Mar-1973 
Shakir-Khalil, $\mathrm{H}$.

Composite columns in multi-storey buildings

Composite construction in steel and concrete proceedings of an engineering foundation conference.

American Society of Civil Engineers. 1988

Shakir-Khalil, H.; Zeghiche, J.

Experimental behaviour of concrete-filled rolled rectangular hollow-section columns.

The Structural Engineer. 1989

Shakir-Khalil, H.; Mouli, M.

Further tests on concrete-filled rectangular hollow section columns.

The Structural Engineer. 1990

Shanmugam, N.E.; Choo, Y.S.

PSSC'95. 4th Pacific structural steel conference

Stuctural steel. Volume 3. Steel-concrete composite structures

Elservier Science Ltd. 1995

Shanmugam, N.E.; Lakshmi, B.

State of the art report on steel-concrete composite columns

Journal of the Constructional Steel Rresearch. Vol. 57, Num. 10. Oct2001

Shanley, F.R.

Inelastic column theory

Journal of the Aeronautical Sciences. Vol 14, num 5. May-1947

Sousa, J.B.M.; Muniz, C.F.D.G.

Analytical integration of cross section properties for numerical analysis of reinforced concrete, steel and composite frames

Engineering Structures. Vol. 29. Num. 4. Abr-2007 
Spacone, E.; El-Tawil, S.

Nonlinear analysis of steel-concrete composite structures: state of art

Journal of Structural Engineering. Vol. 130, Num 2. Feb-2004

Taylor, R.

Composite reinforced concrete

Thomas Telford Limited. Londres, 1979

Tebedge, N.; Chen, W.

Design criteria for H-columns under biaxial loading

Journal of the Structural Division ASCE. Mar-1974

Vazquez, M.

Resistencia de materiales

Editorial Noela. 3aㅡ edición. Madrid 1994

Viest, I.M.; Colaco, J.P.; Furlong, R.W.; Griffis, L.G.; Leon, R.T.; Wyllie, L.A.

Composite construction design for buildings

McGraw Hill. 1997

Virdi, K.S.; Dowling, P.J.

The ultimate strength of composite columns in biaxial bending

Proceedings Institution of Civil Engineers, vol. 55, Mar-1973

Virdi, K.S.; Dowling, P.J

The ultimate strength of biaxially restrained columns

Proceedings Institution of Civil Engineers, vol 61, Mar-1976

Virlogeux, M.

Analyse des problems de stabilite d'une colonne dans le domaine plastique

Journal de Mécanique, vol 14, num 2. 1975 
Virlogeux, M.

Justification règlementaire des pieces soumises aux effets du second ordre selon les principes des calculs aux états-limites ultimes: problems liés a la sécurité

Annales de l'institut technique du batiment et des travaux publics, num 359. Mar-1978

Wakabayasi, M.

Japanese standards for the design of composite buildings

Composite construction in steel and concrete proceedings of an engineering foundation conference.

American Society of Civil Engineers. 1988

Wang, Y.C.

Steel and composite structures. Behaviour and design for fire safety.

Spon Press. Londres, 2002

Wang, Y.C.

Test on slender composite columns

Journal of Constructional Steel Research. Vol. 49, Num 1. Ene-1999

Webb, J; Peyton, J.J.

Composite concrete filled steel tube columns

Estructural Engineering Conference. 1990

Weng, C.C.; Yen, S.I.

Comparisons of concrete-encased composite column strength provisions of $\mathrm{ACl}$ code and AISC specification

Engineering Structures. Vol. 24, Num 1. Ene-2002

Weng, C.C.; Yen, S.I.; Wang, H.S.

A relative rigidity approach for design of concrete-encased composite columns

Journal of the Chinese Institute of Engineers. Vol. 30, Num 4. 2007 
Normas, Códigos y Recomendaciones

Eurocódigo: Bases de cálculo de estructuras.

UNE ENV 1990. AENOR 2003

Eurocódigo 1: Acciones en estructuras.

Parte 1-1: Acciones generales. Pesos específicos, pesos propios y sobrecargas de uso en edificios

UNE ENV 1991-1-1. AENOR 2003

Eurocódigo 2: Proyecto de estructuras de hormigón.

Parte 1-1: Reglas generales y reglas para edificación

UNE ENV 1992-1-1. AENOR 1993

Eurocodigo 3: Proyecto de estructuras de acero

Parte 1-1: Reglas generales y reglas para edificación

ENV 1993-1-1. AENOR 1996

Eurocódigo 4: Proyecto de estructuras mixtas de hormigón y acero.

Parte 1-1: Reglas generales y reglas para edificación

UNE ENV 1994-1-1. AENOR 1995

EHE-08 Instrucción de hormigón estructural.

Real decreto/2008, de 18 de julio, por el que se aprueba la instrucción de hormigón estructural (EHE-08)

Building code requirements for structural concrete ( $\mathrm{ACI} 318 \mathrm{M}$ 95) and commentary (ACl 318M-95)

American Concrete Institute. USA 1995

CEB-FIP Model Code 1990.

Comité Eurointernacional del hormigón.

Bull. d'Information № 213/214.

Ed. Thomas Teldford. Londres 1993 
Notes on ACI 318-89 with Design Applications

Portland Cement Association. 1990

\section{Manual of steel construction}

American Institute of Steel Construction, Inc

Chicago. Illinois. 8ª edición 1984 